THE PREPARATION AND CHARACTERIZATION OF STRUCTURALLY STABLE

5-COORDINATE POL YSTANNANES

\author{
by \\ Aman Ullah Khan \\ Master of Science in Molecular Science, \\ Ryerson University, Toronto, Canada, 2010
}

A dissertation presented to Ryerson University

in partial fulfillment of the requirements for the degree of Doctor of Philosophy

in the Program of Molecular Science

Toronto, Ontario, Canada, 2014

C(Aman Ullah Khan) 2014 


\section{AUTHOR'S DECLARATION}

I hereby declare that I am the sole author of this dissertation. This is a true copy of the dissertation, including any required final revisions, as accepted by my examiners.

I authorize Ryerson University to lend this dissertation to other institutions or individuals for the purpose of scholarly research

I further authorize Ryerson University to reproduce this dissertation by photocopying or by other means, in total or in part, at the request of other institutions or individuals for the purpose of scholarly research.

I understand that my dissertation may be made electronically available to the public. 


\title{
THE PREPARATION AND CHARACTERIZATION OF STRUCTURALLY STABLE 5-COORDINATE POLYSTANNANES
}

Aman Ullah Khan, Molecular Science, Ryerson University, 2014

\begin{abstract}
Tetraorganotin compounds [2-( $\left.\left.\mathrm{MeOCH}_{2}\right) \mathrm{C}_{6} \mathrm{H}_{4}\right] \mathrm{SnR}_{3}(\mathrm{R}=\mathrm{Me}, n-\mathrm{Bu}, \mathrm{Ph})$ containing a $C, O$ chelating ligand were prepared in good yield from the reaction of the $\mathrm{R}_{3} \mathrm{SnCl}$ and [2$\left.\left(\mathrm{MeOCH}_{2}\right) \mathrm{C}_{6} \mathrm{H}_{4}\right] \mathrm{Li}$. Tethered organotin compounds $\mathrm{Ph}_{3} \mathrm{Sn}\left(\mathrm{CH}_{2}\right)_{3} \mathrm{OC}_{6} \mathrm{H}_{4} \mathrm{R}\left(\mathrm{R}=\mathrm{Ph}, \mathrm{H}, \mathrm{CF}_{3}, \mathrm{OCH}_{3}\right)$ were prepared in good yield from the hydrostannylation reactions of the corresponding vinyl ethers with $\mathrm{Ph}_{3} \mathrm{SnH}$. Conversion of two organotin compounds to triorganotin chlorides and diorganotin chlorides, $\left(\mathrm{Ph}_{3-\mathrm{n}} \mathrm{Cl}_{\mathrm{n}} \mathrm{Sn}\left(\mathrm{CH}_{2}\right)_{3} \mathrm{OC}_{6} \mathrm{H}_{4} \mathrm{R} ; \mathrm{R}=\mathrm{H}, \mathrm{Ph}: \mathrm{n}=1,2\right)$, was successfully carried out and characterisation afforded by NMR spectroscopy. X-ray crystallographic studies revealed a tetrahedral geometry for the tetraorganotin $\mathrm{Ph}_{3} \mathrm{Sn}\left(\mathrm{CH}_{2}\right)_{3} \mathrm{OC}_{6} \mathrm{H}_{4} \mathrm{CF}_{3}$, while five-coordinate trigonal bipyramidal structures with relatively short Sn-O (2.7-2.8 $\mathrm{A})$ interactions were observed for both mono- $\left(\mathrm{Ph}_{2} \mathrm{ClSn}\left(\mathrm{CH}_{2}\right)_{3} \mathrm{OC}_{6} \mathrm{H}_{4} \mathrm{R} ; \mathrm{R}=\mathrm{H}, \mathrm{Ph}\right)$ and dichloride $\left(\mathrm{PhCl}_{2} \mathrm{Sn}\left(\mathrm{CH}_{2}\right)_{3} \mathrm{OC}_{6} \mathrm{H}_{4} \mathrm{R} ; \mathrm{R}=\mathrm{H}, \mathrm{Ph}\right)$ species. Penta-coordinate diorganotin dichlorides containing a $C, N$ - chelating ligand[2$\left.\left(\mathrm{Me}_{2} \mathrm{NCH}_{2}\right) \mathrm{C}_{6} \mathrm{H}_{4}\right] \mathrm{RSnCl}_{2}(\mathrm{R}=\mathrm{Me}, n-\mathrm{Bu}, \mathrm{Ph})$ or $C, O$ - chelating ligand [2- $\left.\left(\mathrm{MeOCH}_{2}\right) \mathrm{C}_{6} \mathrm{H}_{4}\right] \mathrm{RSnCl}_{2}$ $(\mathrm{R}=\mathrm{Me}, n-\mathrm{Bu}, \mathrm{Ph})$ were prepared by treating $\mathrm{RSnCl}_{3}$ with the lithiated salts [2$\left.\left(\mathrm{Me}_{2} \mathrm{NCH}_{2}\right) \mathrm{C}_{6} \mathrm{H}_{4}\right] \mathrm{Li}$ and $\left[2-\left(\mathrm{MeOCH}_{2}\right) \mathrm{C}_{6} \mathrm{H}_{4}\right] \mathrm{Li}$ respectively. Organotin chlorides were successfully reduced with $\mathrm{LiAlH}_{4}$ or $\mathrm{NaBH}_{4}$ to produce novel hydrides. Catalytic dehydrocoupling of diorganotin dihydrides to yield polystannanes was explored using a variety of dehydrocoupling catalysts such as Wilkinson's catalyst, $\mathrm{Cp}_{2} \mathrm{ZrMe}_{2}$ or TMEDA. In almost every instance this resulted in the formation of yellow coloured gummy polymeric materials of moderate molecular weights $\left(\mathrm{Mw}=1 \times 10^{4}-1 \times 10^{5} \mathrm{Da}\right)$ and PDI's (1.3-2.0). The stability of polystannanes containing
\end{abstract}


tethered $\mathrm{O}$ or $\mathrm{C}, \mathrm{N}$ - or $\mathrm{C}, \mathrm{O}$-chelating ligands was investigated in both solid and in solution using NMR and UV-Vis spectroscopies. These studies revealed an enhanced stability to ambient light in the solid state and in solution in the dark when compared to known poly(dialkyl)stannanes. 


\section{ACKNOWLEDGEMENTS}

I would like to express my gratitude to my supervisor Dr. Daniel Foucher for his support throughout this study and confidence in me. I found his doors open whenever I needed his help. This dissertation would not have been possible without his guidance and insightful scientific discussions. His enthusiasm and passion towards research always provided the encouragement to achieve the goals.

I would like to thank members of my PhD supervisory committee Dr. Stephen Wylie and Dr. Robert Gossage for answering my questions and providing knowledgeable advice. I would like to give special thanks to Dr. Russell Viirre for his kindness and endless support during my studies at Ryerson. I am deeply in debt to Dr. Andrew McWilliams and Dr. Bryan Koivisto for providing suggestions to overcome the problems during research.

I am deeply grateful to Dr. Alan Lough, University of Toronto, for collecting the crystallographic data and increasing the strength of this study. I want to thanks Dr. Mathew Forbes, University of Toronto, for the mass spectrometry of the novel molecules. I would like to acknowledge the support and help offered by Shawn McFadden, Lab technologist. During my period of stay at Ryerson University, I would be particularly grateful to graduate colleagues Grace, Kamlesh, Khrystyna, Lukasz, Michelle, Jon, Justin and Shane for their help in solving the problems and undergraduate student Aagam, Christopher, Jeffery, Sarah, Tamara for their assistance.

I owe my deepest gratitude to my parents for their enormous support and encouragement throughout my life. My deepest appreciation would goes to my wife Gulshan Ara for her great tolerance and support during this period of study. I would like to offer my thanks and love to my sons Rizwan, Shahryar and Sheroze who missed me most weekends but never forget I am their father. 


\section{Dedication}

I dedicated this to my parents, my wife Gulshan Ara and sons Rizwan, Shahryar and Shehroze for their endless love, support and encouragement. 


\section{Table of Contents}

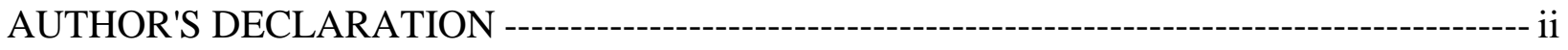

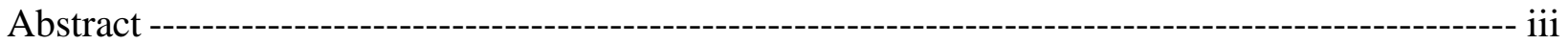

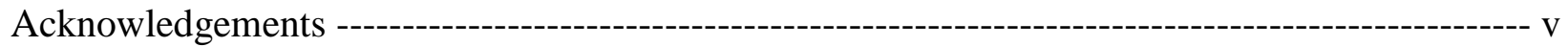

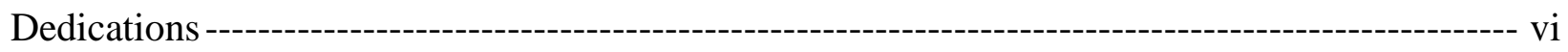

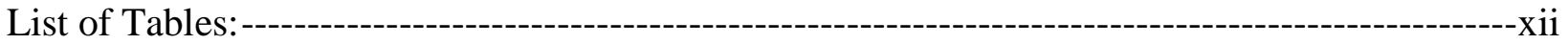

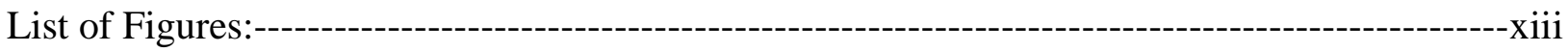

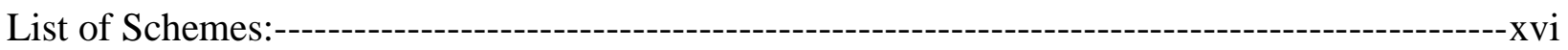

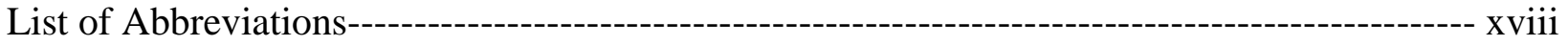

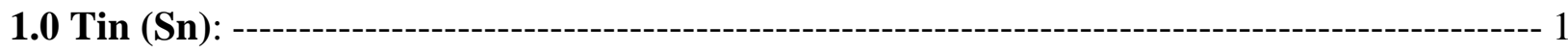

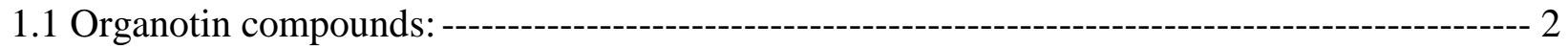

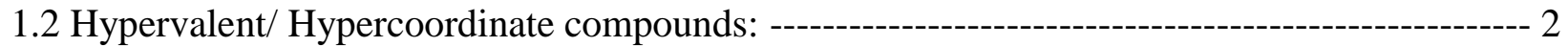

1.3 Hypervalent/ Hypercoordinate compounds of Group 14:--------------------------------------- 6

1.3.1 Hypercoordinate organotin compounds containing $C, N$-chelating ligands: ------------------- 6

1.3.1.1 Tetraorganotin compounds containing $C, N$-chelating ligands: ----------------------------- 7

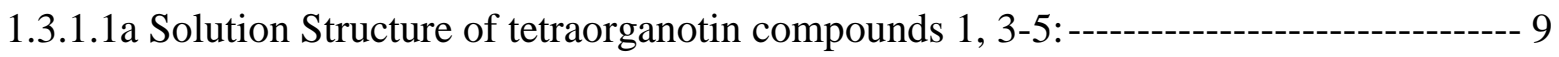

1.3.1.2 Synthesis of organotin halides containing $C, N$-chelating ligands: --------------------- 10

1.3.1.3 Triorganotin halides containing $C, N$-chelating ligands: -----------------------------------11

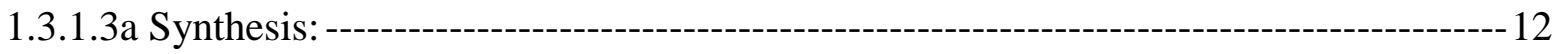

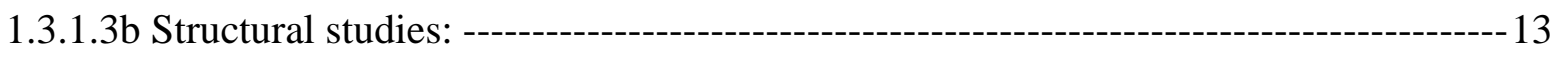

1.3.1.4 Diorganotin dihalides containing $C, N$-chelating ligands:----------------------------------- 18

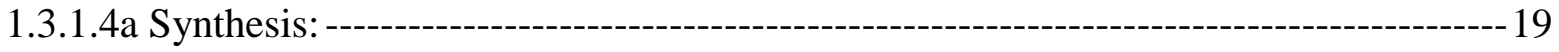

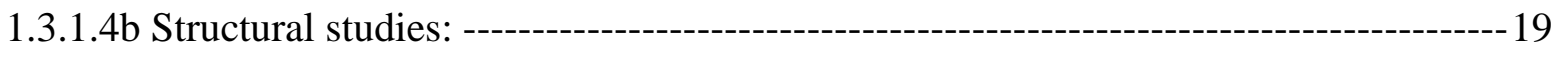

1.3.1.5 Monoorganotin trihalides containing $C, N$-chelating ligands: ------------------------------20

1.3.2 Organotin hydrides containing $C, N$-chelating ligands: -----------------------------------------21

1.3.2.1 Triorganotin hydrides containing $C, N$-chelating ligands: ---------------------------------22

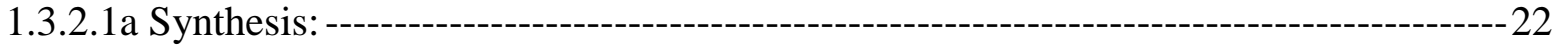

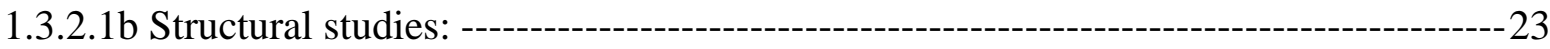

1.3.2.2 Diorganotin dihydrides containing $C, N$-chelating ligands:---------------------------------25

1.3.3 Si, $\mathrm{Ge}$ and $\mathrm{Pb}$ compounds containing a $C, N$-chelating ligand:----------------------------------26

1.4 Organotin compounds containing $C, O$-chelating ligands: ----------------------------------------31 


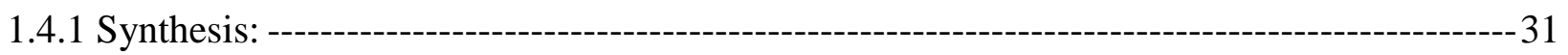

1.4.2 Structural studies:----_---_--- 32

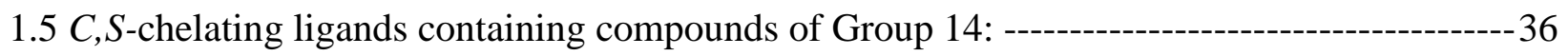

1.6 C,P-chelating ligand containing compounds of Group 14: ---

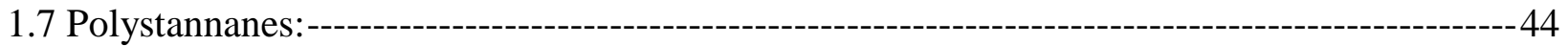

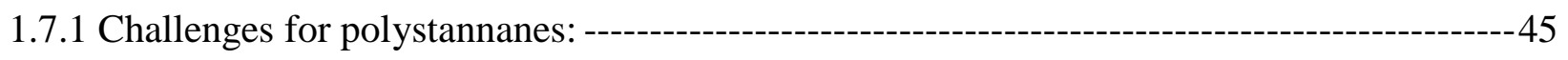

1.7.2 Synthesis of polystannanes: ---

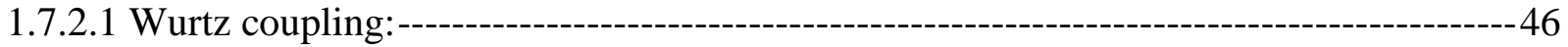

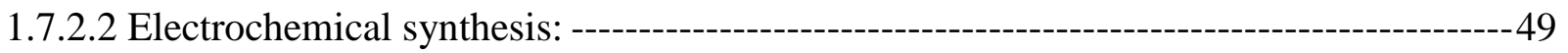

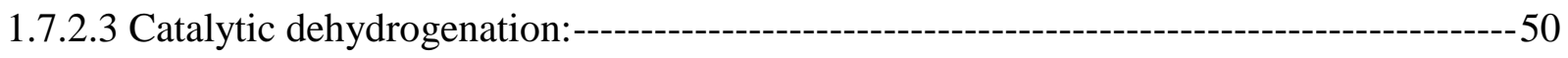

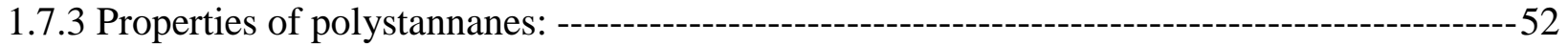

1.7.3.1 Photosensitivity: ------_-

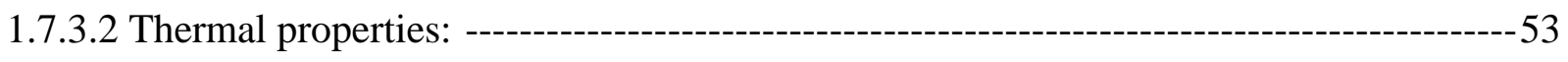

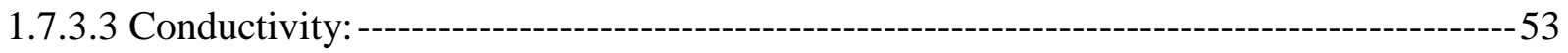

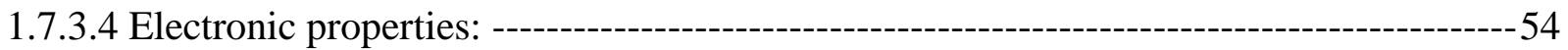

1.7.3.5 ${ }^{119}$ Sn NMR:-------

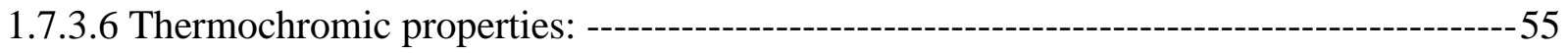

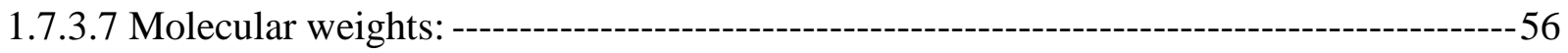

1.8 Thesis objectives: ---

2.0 Results and Discussion: ----- 59

2.1: Synthesis ---

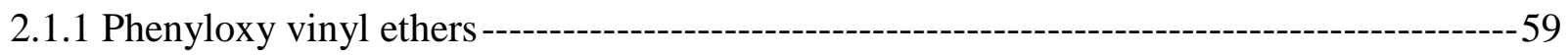

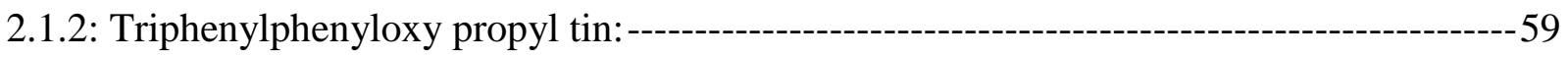

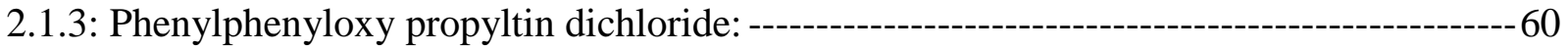

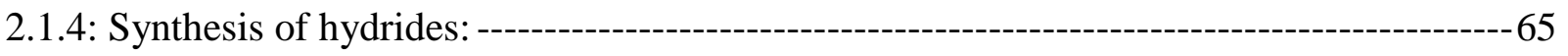

2.1.5: Dimerization of 204 and 205: ---------- 67

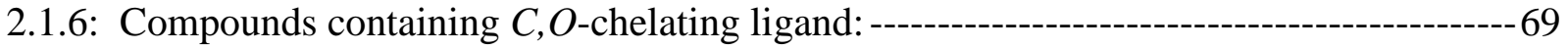

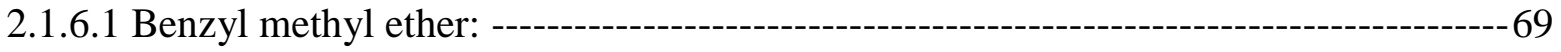

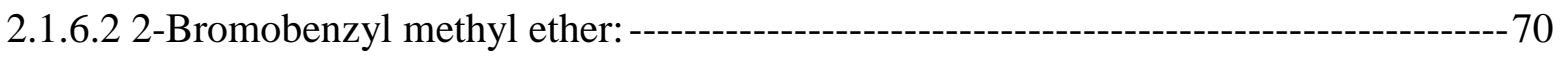

2.1.6.3 [2-( $\left.\left.\mathrm{MeOCH}_{2}\right) \mathrm{C}_{6} \mathrm{H}_{4}\right] \mathrm{Li}$------------- 70

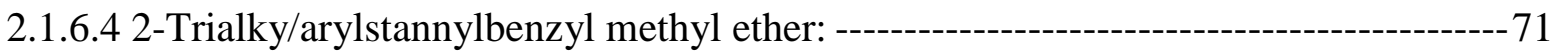


2.1.6.5 2-Chloroalkyl/arylstannylbenzyl methyl ethers: ----------------------------------71

2.1.6.6 Dihydridoalkylstannylbenzyl methyl ether:-------------------------------------74

2.1.7 Organotin compounds containing a $C, N$-chelating ligand --------------------------75

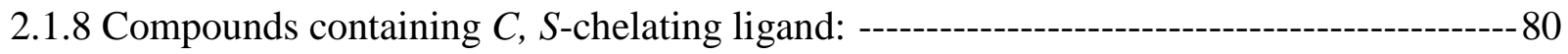

2.1.8.1 1-bromo-2-(n-propylthiomethyl) benzene -------------------------------- 80

2.1.9 Compounds containing $C, P$-chelating ligand:---

2.1.9.1 Synthesis of (o-bromobenzyl)-diphenylphosphine:---

2.1.9.2 (o-(diphenylphosphino)benzyl)stannanes: --------------------------------------- 86

2.2 Characterization and Properties:-------- 86

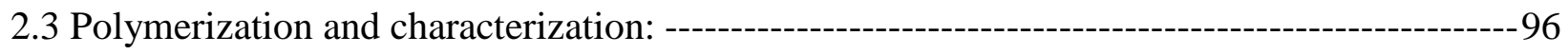

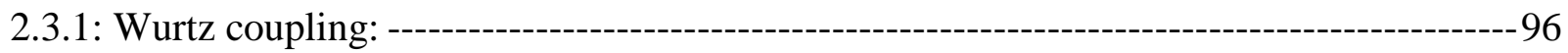

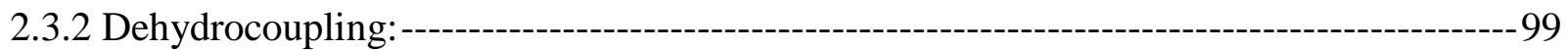

2.3.2.1: Metal catalyzed dehydrocoupling: ----------------------------------------99

2.3.2.2 Non-metal catalyzed dehydrocoupling: ----------------------------------------- 103

2.3.3 Characterization of polymers:-------- 105

2.3.3.1 GPC Characterization: --- 105

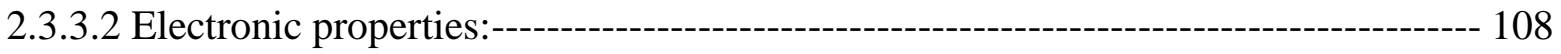

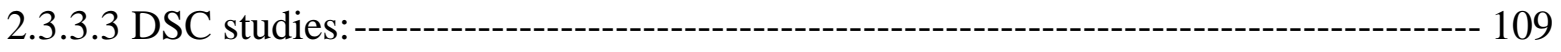

2.3.3.4 UV-Vis and NMR Stability studies: ---_- 110

3.0 Conclusion: ---------- 113

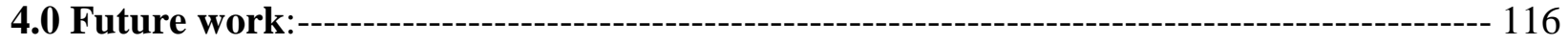

5.0 Experimental: ---

5.1 Synthesis of (allyloxy)benzene (194): -- 119

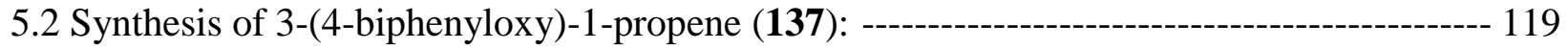

5.3 Synthesis of of 1-allyloxy-3-trifluoromethylbenzene (195): ------------ 120

5.4 Synthesis of 1-(allyloxy)-4-methoxybenzene ether (196):--- 121

5.5 Synthesis of triphenyltin hydride (254):--- 122

5.6 Synthesis of triphenyl[(3-phenyloxy)propyl]tin (197):---------------------------- 122

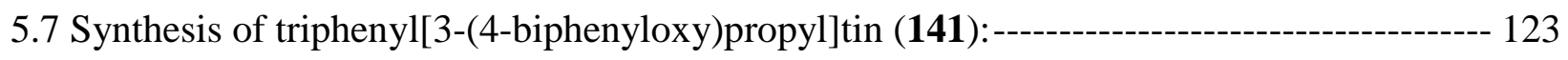

5.8 Synthesis of triphenyl [3-(3-trifluromethylphenyloxy)propyl]tin (198): -------------- 124

5.9 Synthesis of triphenyl [3-(4-methoxyphenyloxy)propyl]tin (199):------------------------ 125 
5.10 Synthesis of diphenyl[(3-phenyloxy)propyl]tin chloride (200):---------------------------- 126

5.11 Synthesis of diphenyl[3-(4-biphenyloxy)propyl]tin chloride (201):------------------------- 127

5.12 Synthesis of phenyl[3-(4-biphenyloxy)propyl]tin dibromide (146):---------------------- 128

5.13 Synthesis of phenyl[(3-phenyloxy)propyl]tin dichloride (202):------------------------- 129

5.14 Synthesis of phenyl[3-(4-biphenyloxy)propyl]tin dichloride (203):-------------------------- 130

5.15 Synthesis of diphenyl[(3-phenyloxy)propyl]tin (204): ------------------------------------- 131

5.16 Synthesis of diphenyl[3-(4-biphenyloxy)propyl]tin (205): ----------------------------- 132

5.17 Synthesis of phenyl[(3-phenyloxy)propyl]tin (206):------------------------------------ 133

5.18 Synthesis of phenyl[3-(4-biphenyloxy)propyl]tin (207): ---------------------------- 134

5.19 Dimerization of diphenyl[(3-phenyloxy)propyl]tin (208): --------------------------- 136

5.20 Dimerization of diphenyl[3-(4-biphenyloxy)propyl]tin (209): ---------------------------- 137

5.21 Polymeriztion of phenyl[(3-phenyloxy)propyl]tin (249): ------------------------------ 138

5.22 Polymerization of phenyl[3-(4-biphenyloxy)propyl]tin (250): --------------------------- 139

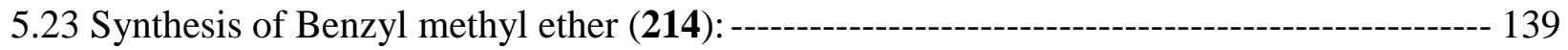

5.24 Synthesis of 2-Bromobenzyl methyl ether (215): -------------------------------------------- 140

5.25 Synthesis of [2-( $\left.\left.\mathrm{MeOCH}_{2}\right) \mathrm{C}_{6} \mathrm{H}_{4}\right] \mathrm{Li}(\mathbf{2 1 6})$ :---------------------------------------------------- 141

5.26 Synthesis of 2-Trimethylstannylbenzyl methyl ether (113): -------------------- 141

5.27 Synthesis of 2-Tributylstannylbenzyl methyl ether (217): ----------------------------------- 142

5.28 Synthesis of 2-Triphenylstannylbenzyl methyl ether (112): ----------------------------- 143

5.29 Synthesis of $\left[2-\left(\mathrm{MeOCH}_{2}\right) \mathrm{C}_{6} \mathrm{H}_{4}\right] \mathrm{MeSnCl}_{2}(\mathbf{2 1 8})$ : ---------------------- 144

5.30 Synthesis of [2-( $\left.\left.\mathrm{MeOCH}_{2}\right) \mathrm{C}_{6} \mathrm{H}_{4}\right] \mathrm{BuSnCl}_{2}$ (219):------------------------------- 144

5.31 Synthesis of [2-( $\left.\left.\mathrm{MeOCH}_{2}\right) \mathrm{C}_{6} \mathrm{H}_{4}\right] \mathrm{PhSnCl}_{2}(\mathbf{2 2 0})$ : -------------------------------- 145

5.32 Synthesis of [2-( $\left.\left.\mathrm{MeOCH}_{2}\right) \mathrm{C}_{6} \mathrm{H}_{4}\right] \mathrm{Ph}_{2} \mathrm{SnCl}(\mathbf{2 2 1})$ : ------------------------------------------- 146

5.33 Synthesis of [2-( $\left.\left.\mathrm{MeOCH}_{2}\right) \mathrm{C}_{6} \mathrm{H}_{4}\right] n-\mathrm{Bu}_{2} \mathrm{SnCl}(\mathbf{2 2 3})$ : --------------------------------------- 147

5.34 Synthesis of $\left[\left(2-\left(\mathrm{MeOCH}_{2}\right) \mathrm{C}_{6} \mathrm{H}_{4}\right)_{2}\right] n-\mathrm{BuSnCl}(\mathbf{2 2 4})$ : ---------- 148

5.35 Synthesis of $\left[\left(2-\left(\mathrm{MeOCH}_{2}\right) \mathrm{C}_{6} \mathrm{H}_{4}\right)_{2}\right] \mathrm{PhSnCl}(\mathbf{2 2 5})$ : --------- 149

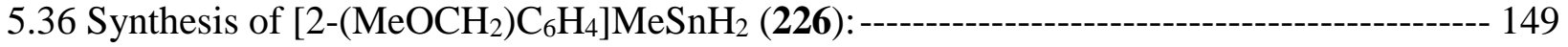

5.37 Synthesis of [2-( $\left.\left.\mathrm{MeOCH}_{2}\right) \mathrm{C}_{6} \mathrm{H}_{4}\right] n-\mathrm{BuSnH}_{2}(\mathbf{2 2 7})$ :------------------------------ 150

5.38 Polymerization of 227: -------------------------------------------------------------------------- 151

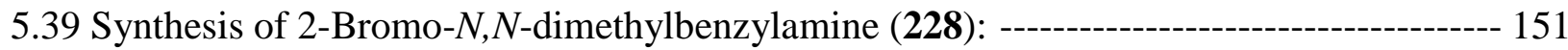

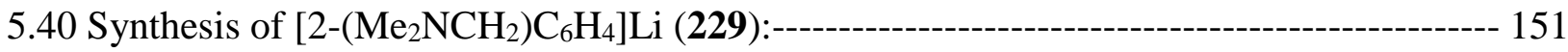


5.41 Synthesis of $\left[2-\left(\mathrm{Me}_{2} \mathrm{NCH}_{2}\right) \mathrm{C}_{6} \mathrm{H}_{4}\right] \mathrm{MeSnCl}_{2}(\mathbf{3 3})$ :-------- 152

5.42 Synthesis of $\left[2-\left(\mathrm{Me}_{2} \mathrm{NCH}_{2}\right) \mathrm{C}_{6} \mathrm{H}_{4}\right] n$ - $\mathrm{BuSnCl}_{2}(37)$ :--

5.43 Synthesis of $\left[2-\left(\mathrm{Me}_{2} \mathrm{NCH}_{2}\right) \mathrm{C}_{6} \mathrm{H}_{4}\right] \mathrm{PhSnCl}_{2}(35)$ : --- 153

5.44 Synthesis of $\left[2-\left(\mathrm{Me}_{2} \mathrm{NCH}_{2}\right) \mathrm{C}_{6} \mathrm{H}_{4}\right] \mathrm{MeSnH}_{2}$ (230):--

5.45 Synthesis of $\left[2-\left(\mathrm{Me}_{2} \mathrm{NCH}_{2}\right) \mathrm{C}_{6} \mathrm{H}_{4}\right] n-\mathrm{BuSnH}_{2}(\mathbf{2 3 1})$ :------------------------ 155

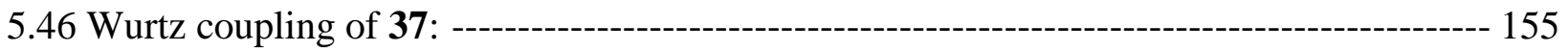

5.47 Wurtz coupling of 35: --- 156

5.48 Catalytic dehydrocoupling of $\mathbf{2 3 0}$ using $\mathrm{Cp}_{2} \mathrm{ZrMe}_{2}$ : -- 157

5.49 1-Bromo-2-(n-propylthiomethyl) benzene (233): ---

5.50 1-Bromo-2-(phenylthiomethyl) benzene (234):---------------- 158

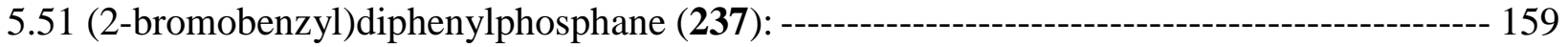

4.52 Diphenyl(2-(triphenylstannyl)benzyl)phosphine (241) ---------------------------- 160

4.53 (2-(chlordiphenylstannyl)benzyl)diphenylphosphine (242)--- 160

6.0 Appendices--------------------------- 162

List of Appendix tables --- 162

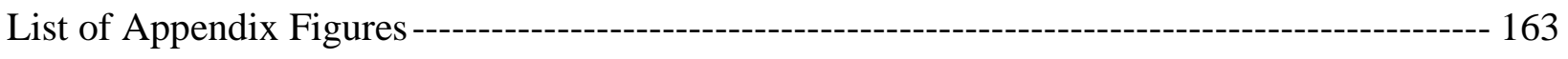

7.0 References:--- 375 


\section{List of Tables:}

Table 1: Comparison of the ${ }^{119} \mathrm{Sn}$ chemical resonances of tin compounds containing a $C, N$-ligand

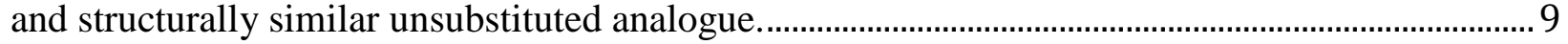

Table $2:{ }^{119} \mathrm{Sn}$ chemical shifts and $\mathrm{Sn}-\mathrm{N}$ bond distance of triorganotin halides............................ 17

Table 3: ${ }^{119} \mathrm{Sn}$ chemical shifts and Sn-N distances of diorganotin halides. ..................................... 20

Table 4: ${ }^{1} \mathrm{H}$ and ${ }^{119} \mathrm{Sn}$ NMR data of hypercoordinate triorganotin hydrides. .................................. 25

Table 5: ${ }^{1} J_{117 / 119 S n-1 H}$ coupling constants of coordinated and non-coordinated hydrides. .............. 26

Table 6: ${ }^{29} \mathrm{Si}$ chemical shift data for five- vs four-coordinate silanes ............................................. 28

Table 7: ${ }^{119} \mathrm{Sn}$ chemical shifts and $\mathrm{Sn}-\mathrm{O}$ distances of tin compounds with $C, O$-chelating ligands.

Table 8: ${ }^{119} \mathrm{Sn}$ chemical shifts and $\mathrm{Sn}-\mathrm{O}$ distances of tin compounds with $C, O$ - chelating ligands.

Table 9: ${ }^{29} \mathrm{Si} /{ }^{19} \mathrm{Sn} /{ }^{207} \mathrm{~Pb}$ chemical shifts and $\mathrm{Si} / \mathrm{Sn} / \mathrm{Pb}-\mathrm{S}$ distances of compounds with $C, S$ -

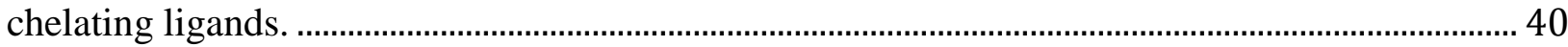

Table 10: UV-Visible spectral data and ${ }^{119}$ Sn NMR chemical shifts for polystannanes. .............. 55

Table 11: Molar weights of polystannanes. .......................................................................................... 57

Table 12: ${ }^{119} \mathrm{Sn} \mathrm{NMR}\left(\mathrm{CDCl}_{3}\right)$ chemical shift, Sn-O distances and yields of stannanes............... 64

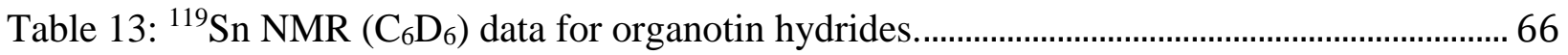

Table 14: Comparison of $J_{1195 n-119 S n}$ distannanes with and without a coordinated ligand. ............ 68

Table 15: ${ }^{119}$ Sn NMR chemical shifts of selected stannanes. ............................................................ 73

Table 16: ${ }^{119} \mathrm{Sn}$ NMR data of tin dihydrides with or without coordinating ligand......................... 75

Table $17:{ }^{119} \mathrm{Sn}$ NMR $\left(\mathrm{CDCl}_{3}\right)$ chemical shift values of tin dichlorides containing a $C, N$ -

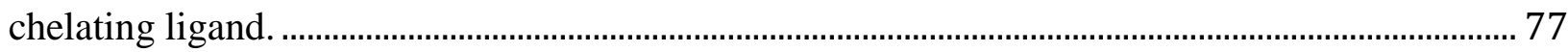

Table 18: Coupling constant values for tin dihydrides....................................................................... 79

Table 19: Crystallographic data and structural refinement for compounds 198, 200, 201, 202, 203

Table 20: Data used to obtain the transition from tetrahedral to TBP for compounds 198, 200-

203. 95

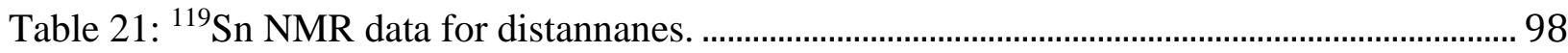

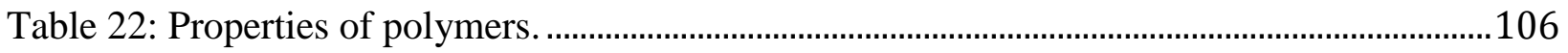




\section{List of Figures:}

Figure 1: Geometry of different Sn(IV) compounds. ..........................................................................

Figure 2: The principle coordination geometries of 5- and 6-coordinate Sn compounds.................. 3

Figure 3: The MO diagram of a (3c-4e) bond in hypervalent compounds. ........................................ 5

Figure 4: Proposed methods to make (3c-4e) bonds. ........................................................................... 5

Figure 5: Most commonly used $C, N$-chelating ligands.......................................................................

Figure 6: Tetraorganotin compounds........................................................................................... 8

Figure 7: 5-coordinate triorganotin halides................................................................................. 12

Figure 8: Two basic fluxional processes of a $C, N$-chelating ligand containing $\mathrm{R}_{3} \mathrm{SnCl}$................. 15

Figure 9: Diorganotin dihalides.......................................................................................................... 18

Figure 10: Monoorganotin halides............................................................................................... 20

Figure 11: Triorganotin hydrides. ................................................................................................ 22

Figure 12: Structure of triorganotin hydrides without chelating ligand.............................................. 24

Figure 13: Structures of hydrides containing chelating ligands.................................................... 24

Figure 14: Hypercoordinated diorganotin dihydrides...................................................................... 25

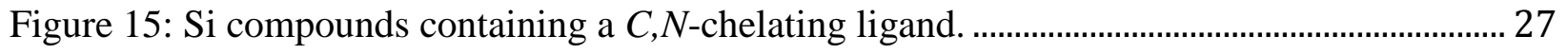

Figure 16: Organosilicon compounds containing a $C, N$-chelating ligand. ........................................ 28

Figure 17: Hypercoordinate organogermanium and organolead compounds................................... 29

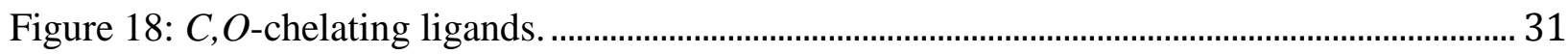

Figure 19: Hypercoordinate tin compounds containing $C, O$-chelating ligands................................ 31

Figure 20: Organotin compounds with an oxa-alkyl side chain....................................................... 34

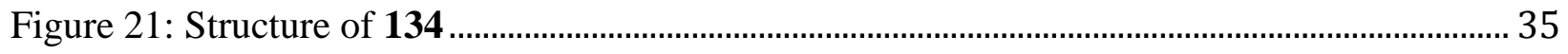

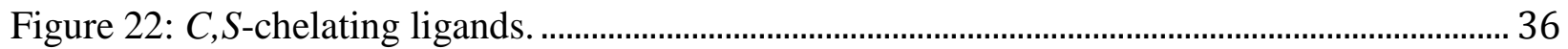

Figure 23: Group 14 compounds containing $C, S$-chelating ligands. ................................................ 37

Figure 24: Structure of $C, P$-chelating ligands................................................................................... 42

Figure 25: Organotin compounds containing $C, P$-chelating ligands................................................ 42

Figure 26: Organosilicon compounds containing $C, P$-chelating ligands........................................ 43

Figure 27: Dräger's oligostannanes with their average Sn-Sn bond lengths (pm), Sn-Sn-Sn bond angles and absorption maxima ………………………………………………………………….... 44

Figure 28: Proposed degradation mechanism of polystannanes. ....................................................... 46 
Figure 29: The electronic spectra of (A) $(n-\mathrm{Bu})_{3} \mathrm{Sn}-\left(n-\mathrm{Bu}_{2} \mathrm{Sn}\right)_{n}-\mathrm{Sn}(n-\mathrm{Bu})_{2}-\left(\mathrm{CH}_{2}\right)_{2} \mathrm{OEt}(\mathrm{n}=0-4)$,

(B) for $\mathrm{Ph}_{3} \mathrm{Sn}-\left((t-\mathrm{Bu})_{2} \mathrm{Sn}\right)_{\mathrm{n}}-\mathrm{SnPh}_{3}(\mathrm{n}=1-4)$. 54

Figure 30: ${ }^{1} \mathrm{H}$ NMR $\left(\mathrm{CDCl}_{3}\right)$ spectra showing the conversion of 197 to 200................................. 63

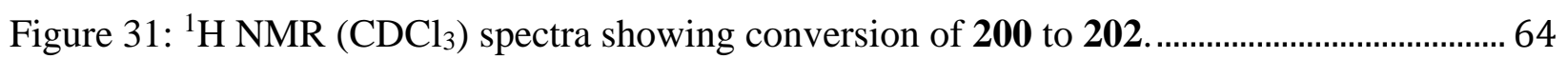

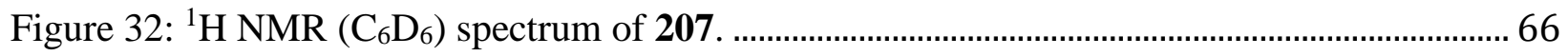

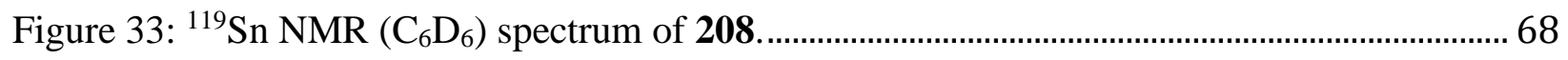

Figure 34: Flask containing the decomposed product of 216........................................................ 70

Figure 35: Possible structural isomers of 223 .............................................................................. 74

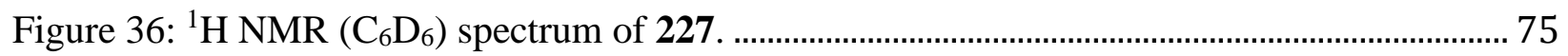

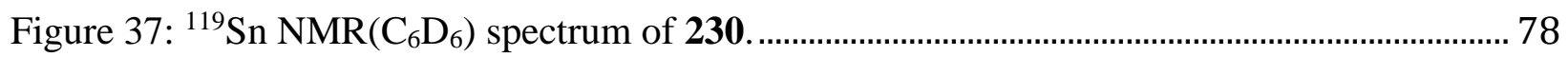

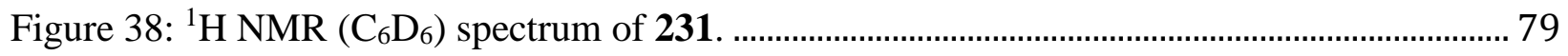

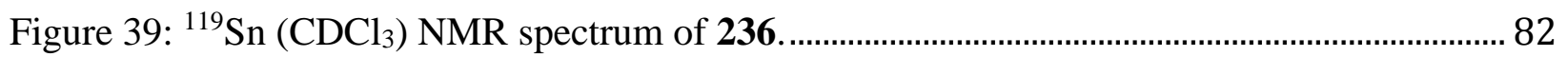

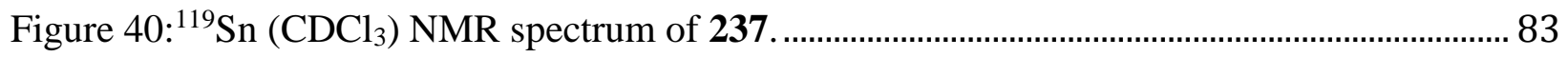

Figure 41: ${ }^{31} \mathrm{P}$ NMR $\left(\mathrm{CDCl}_{3}\right)$ spectrum of compound 238 ……................................................. 84

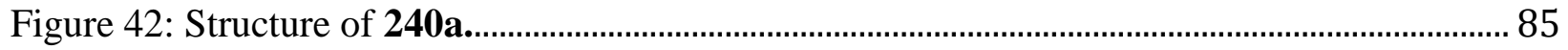

Figure 43: ${ }^{31} \mathrm{P}$ NMR $\left(\mathrm{CDCl}_{3}\right)$ spectrum of compound 239............................................................ 85

Figure 44: Transition from tetrahedral geometry to TBP................................................................ 88

Figure 45: ORTEP representation of the unit cell components for 198 ........................................... 89

Figure 46: ORTEP representation of the unit cell (A) components for 200 ....................................90

Figure 47: ORTEP representation of the unit cell components for 201.......................................... 91

Figure 48: ORTEP representation of the unit cell components for 202 ………………………...... 92

Figure 49: ORTEP representation of the unit cell components for 203.......................................... 93

Figure 50: ${ }^{119} \mathrm{Sn}$ NMR $\left(\mathrm{C}_{6} \mathrm{D}_{6}\right)$ spectrum of Wurtz coupling of $\mathbf{3 7}$ for $4 \mathrm{~h}$ at $60^{\circ} \mathrm{C}$........................ 97

Figure 51: ${ }^{119} \mathrm{Sn}$ NMR $\left(\mathrm{C}_{6} \mathrm{D}_{6}\right)$ spectrum from the Wurtz coupling of 35....................................... 98

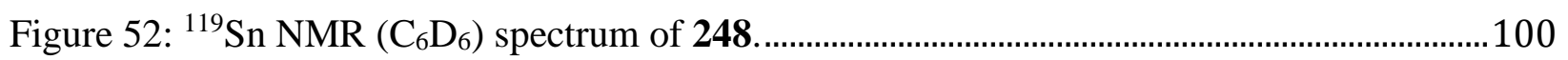

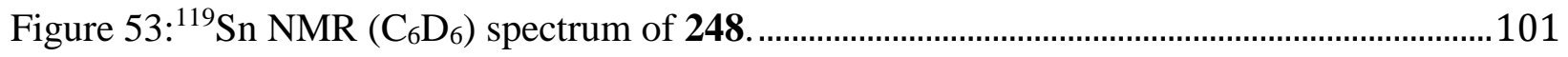

Figure 54: ${ }^{1} \mathrm{H}$ NMR $\left(\mathrm{C}_{6} \mathrm{D}_{6}\right)$ spectrum of polymer 250..............................................................102

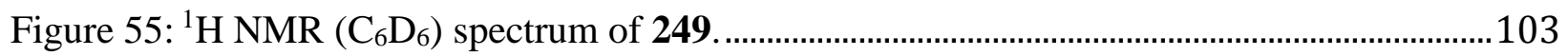

Figure 56: TMEDA catalyzed polymerization of organotin dihyrides...........................................104

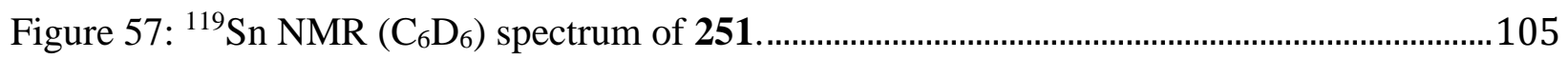

Figure 58: Structure of polymers 252 and 253 ……...................................................................106 
Figure 59: Triple detector GPC trace (THF) of polymer 248

Figure 60: UV-visible spectrum of $249 .$.

109

Figure 61: DSC heating thermograms of $\mathbf{2 4 9}$ and $\mathbf{2 5 0}$ (under $\mathrm{N}_{2}$, heating rate $5^{\circ} \mathrm{C} / \mathrm{min}$ )..........110

Figure 62: Consecutive UV-visible spectra of 250 at day 1, 10 and 20........................................112 


\section{List of Schemes:}

Scheme 1: Synthesis of tetraorganotin compounds. ……………………………………………...

Scheme 2: Synthesis of organotin halides.................................................................................. 10

Scheme 3: Routes for the synthesis of hypercoordinated organotin halides.................................. 11

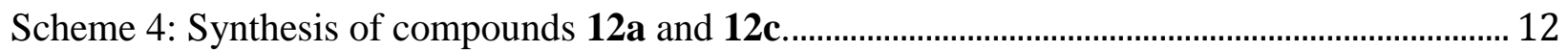

Scheme 5: Synthesis of 13(a-c) and 16(a-c) ………….............................................................. 13

Scheme 6: Diastereoisomers of 13b-16b.......................................................................................... 15

Scheme 7: The preparation of organotin hydrides. ……………………………………………...... 22

Scheme 8: Synthesis of organosilanes containing $C, N$-chelating ligands........................................ 29

Scheme 9: Synthesis of organolead compounds ............................................................................... 30

Scheme 10: Synthesis of tin complexes with phenyloxy alkyl side chains....................................... 34

Scheme 11: Synthesis of $C, O$-chelating ligand and the corresponding triarylgermanes and silane.

Scheme 12: Group 14 compounds containing a $C, S$-chelating ligand. ............................................... 37

Scheme 13: Synthesis of organosilicon compounds containing a $C, S$-chelating ligand.................. 38

Scheme 14: Synthesis of $\mathrm{SiF}_{3}, \mathrm{SiH}_{3}$ and $\mathrm{SiPhHCl}$ silanes containing a $C, S$-chelating ligand...... 39

Scheme 15: Synthesis of a $C, S$-chelating ligand and reactions to form a triarylgermane or silanes.

Scheme 16: Synthesis of $\mathrm{SiF}_{3}, \mathrm{SiH}_{3}$ and $\mathrm{SiPhHCl}$ silanes containing a $C, P$-chelating ligand. .... 43

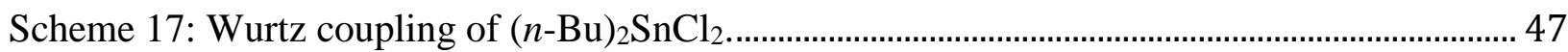

Scheme 18: Wurtz coupling reactions of perfluorinated dichlorostannanes. ................................... 48

Scheme 19: Electrochemical polymerization dialkyltin dihalides. .................................................... 49

Scheme 20: Electrochemical polymerization alkyltin trihalides...................................................... 49

Scheme 21: Catalytic dehydrocoupling of diorganostannanes........................................................ 50

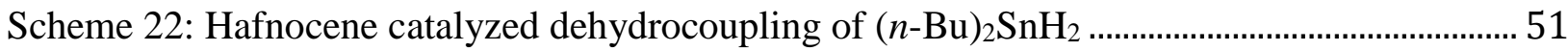

Scheme 23: Synthesis of chiral polystannanes................................................................................. 52

Scheme 24: TMEDA catalyzed dehydrocoupling ……………………………………………….... 52

Scheme 25: Williamson's ether synthesis of phenyloxy vinyl ethers. ……………………………... 59

Scheme 26: Synthesis of triphenylphenyloxy propyltin.................................................................... 60

Scheme 27: Stepwise preparation of triorganotin monochlorides and diorganotin dichlorides... 61 
Scheme 28: Attempted synthesis of a tin trihalide.......................................................................... 65

Scheme 29: Preparation of triorganotin hydrides and diorganotin dihydrides. .................................. 65

Scheme 30: Synthesis of distannanes 208 and 209...................................................................... 67

Scheme 31: 5-coordinate distannanes............................................................................................. 69

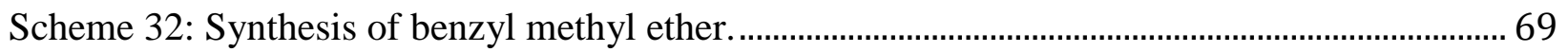

Scheme 33: Synthesis of 2-bromobenzyl methyl ether. ..................................................................... 70

Scheme 34: Lithiation of 2-bromobenzyl methyl ether.................................................................... 70

Scheme 35: Synthesis of 2-Trialky/arylstannylbenzyl methyl ether. .................................................. 71

Scheme 36: Synthesis of dichloroalkyl/arylstannylbenzyl methoxy ethers...................................... 71

Scheme 37: Synthesis of the chloro(2-(methoxymethyl)phenyl)diphenylstannane 221................. 72

Scheme 38: Alternative synthesis of chloro(2-(methoxymethyl)phenyl)arylstannane. …………... 72

Scheme 39: Attempted synthesis of chlordialkylstannyl benzyl methyl ether. ................................ 73

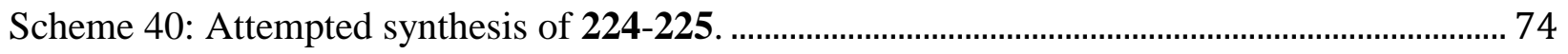

Scheme 41: Synthesis of dihydridoalkylstannyl benzyl methy ethers. .............................................. 74

Scheme 42: Preparative routes to compounds 33, 35, 37 ................................................................. 76

Scheme 43: Preparation of tin dihydrides containing a $C, N$-chelating ligand. ................................. 77

Scheme 44: Attempted synthesis of an aryltin dihydrides containing a $C, N$-ligand......................... 80

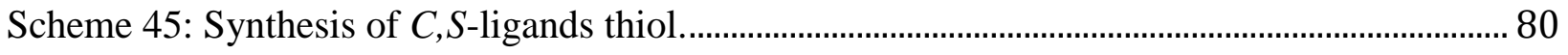

Scheme 46: Synthesis of tributylstannylbenzyl thioether................................................................. 81

Scheme 47: Synthesis of triphenylstannylbenzyl thioether................................................................ 83

Scheme 48: Attempted route for UV- light catalyzed synthesis of $o-\left(\mathrm{Ph}_{2} \mathrm{PCH}_{2}\right) \mathrm{C}_{6} \mathrm{H}_{4} \mathrm{Br}$. ............. 84

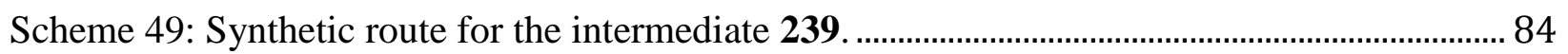

Scheme 50: Synthesis of (o-(diphenylphosphino)benzyl)stannanes.................................................. 86

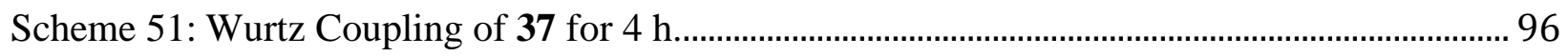

Scheme 52: Wurtz Coupling of $\mathbf{3 7}$ for 4 days................................................................................. 97

Scheme 53: Na Wurtz Coupling of 35........................................................................................ 98

Scheme 54: Polymerization of 231..............................................................................................100

Scheme 55: Transition metal catalyzed synthesis of polymers 249 and 250...............................102

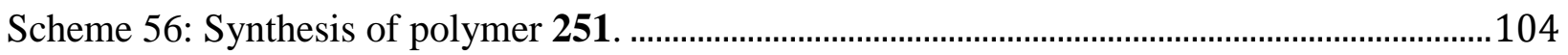




\section{List of Abbreviations:}

\begin{tabular}{|c|c|}
\hline$\AA$ & Angstrom \\
\hline AIBN & Azobisisobutyronitrile \\
\hline AOs & Atomic orbitals \\
\hline $\mathrm{BC}$ & Before Christ \\
\hline CP-MAS & Cross polarization-Magic angle spinning \\
\hline $\mathrm{d}$ & Days \\
\hline $\mathrm{Da}$ & Daltons \\
\hline DCM & Dichloromethane \\
\hline DME & Dimethylethane \\
\hline DSC & Differential Scanning Calorimetry \\
\hline Et- & Ethyl \\
\hline $\mathrm{Et}_{2} \mathrm{O}$ & Diethyl ether \\
\hline GPC & Gel Permeation Chromatography \\
\hline $\mathrm{h}$ & Hours \\
\hline Hex- & Hexyl \\
\hline HMPA & Hexamethylphosphoramide \\
\hline HOMO & Highest occupied molecular orbital \\
\hline $\mathrm{Hz}$ & Hertz \\
\hline LUMO & Lowest unoccupied molecular orbital \\
\hline Me- & Methyl \\
\hline $\mathrm{M}_{\mathrm{n}}$ & Number average molecular weight \\
\hline MO & Molecular orbital \\
\hline $\mathrm{M}_{\mathrm{w}}$ & Weight average molecular weight \\
\hline Myr & Myrtanyl \\
\hline NMR & Nuclear Magnetic Resonance \\
\hline $\mathrm{O}_{\mathrm{h}}$ & Octahedral \\
\hline PDI & Polydispersity index \\
\hline $\mathrm{Ph}-$ & Phenyl \\
\hline
\end{tabular}




$\begin{array}{ll}\text { ppm } & \text { Parts per million } \\ \text { RI } & \text { Refractive index } \\ \text { RT } & \text { Room temperature } \\ \text { SP } & \text { Square pyramidal } \\ t \text {-Bu } & \text { Tertiary butyl } \\ \text { TBP } & \text { Trigonal bipyramidal } \\ \text { TEPO } & \text { 2,2,6,6-Tetramethylpiperidinyloxy } \\ \text { THF } & \text { Tetrahydrofuran } \\ \text { TMEDA } & \text { Tetramethylethylenediamine } \\ \text { UV } & \text { Ultra Violet } \\ \text { VT } & \text { Variable temperature }\end{array}$




\subsection{Tin (Sn):}

Tin is the oldest known metal in the periodic table. It has been used to increase the hardness of copper since $3500 \mathrm{BC}$ and helped to start the Bronze Age. It was first isolated as a pure metal in 800 BC. ${ }^{1}$ Its abundance in the earth's surface is about $2 \mathrm{ppm}$, with Cassiterite, $\mathrm{SnO}_{2}$, the most important ore of tin. China and South East Asia produce 75\% of the world's production of tin and $\approx 18 \%$ from South America. ${ }^{2}$ Tin is the first metallic element of Group 14 in the periodic table with atomic number 50, atomic mass $118.90 \mathrm{~g} / \mathrm{mol}$ and a ground state valence shell electronic configuration $5 s^{2} 5 p^{2}$. It has 10 stable isotopes which is the highest number for any element of the periodic table. Tin has three NMR active isotopes $\left({ }^{115} \mathrm{Sn},{ }^{117} \mathrm{Sn}\right.$ and $\left.{ }^{119} \mathrm{Sn}\right)$ each with spin $1 / 2$. More commonly ${ }^{117} \mathrm{Sn}(7.61 \%)$ and ${ }^{119} \mathrm{Sn}(8.58 \%)$ nuclei are used for NMR experiments due to their

relatively high natural abundance. For tin chemistry, ${ }^{119} \mathrm{Sn}$ NMR spectroscopy has become a routine tool with NMR chemical shifts ranging from +4000 to $-2500 \mathrm{ppm}$ with the ${ }^{119} \mathrm{Sn}$ resonance for $\mathrm{SnMe}_{4}$ assigned to $\delta=0 \mathrm{ppm}^{2}{ }^{2}$ Tin most commonly exists in either a $\mathrm{Sn}$ (II) or $\mathrm{Sn}(\mathrm{IV}$ ) oxidation state with tetravalent structures preferred for most organotin compounds. The majority of organic and inorganic $\operatorname{Sn}(\mathrm{IV})$ compounds possess $s p^{3}$ hybridization and adopt a tetrahedral structure, e.g. $\mathrm{SnX}_{4}$ (Figure 1). The coordination number at tin can be increased through hybridization to form 5and 6-coordinated complexes. These hybridized orbitals can accept electrons from ligands to form coordination geometries which are trigonal bipyramidal, TBP $\left(\operatorname{SnX} X_{5}\right)$ or octahedral, $\mathrm{O}_{\mathrm{h}}\left(\operatorname{SnX} \mathrm{X}_{6}\right)$ respectively.

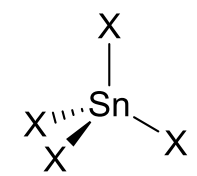

Tetrahedral

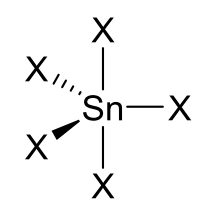

Trigonal bipyramidal

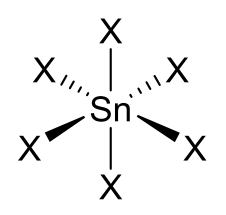

Octahedral

Figure 1: Geometry of different $\mathrm{Sn}(\mathrm{IV})$ compounds. 


\subsection{Organotin compounds:}

In 1849, the first organotin compound, diethyltin diiodide, was synthesized by Frankland ${ }^{3}$ and shortly thereafter the first oligo- or polystannane was reported by Löwig in $1852 .{ }^{4}$ Löwig studied the reaction of iodoethane with $\mathrm{Sn} / \mathrm{K}$ or $\mathrm{Sn} / \mathrm{Na}$ alloys and obtained $\mathrm{Et}_{3} \mathrm{SnH}$ and $\mathrm{Et}_{3} \mathrm{Sn}$ $\mathrm{SnEt}_{3}$. The development of organotin chemistry slowed for the next fifty years with only 37 papers published by 1900 . Organotin chemistry was revolutionized shortly thereafter with the availability of organomagnesium halides as alkylating and arylating agents. These reagents were widely used to produce $\mathrm{R}_{4} \mathrm{Sn}$ type compounds. In 1903, Pope and Peachey described the synthesis of a number of tetraalkyl- and tetraarylstannanes by reacting a suitable Grignard reagent with $\mathrm{SnCl}_{4}$ or alkyltin halide. ${ }^{5}$ Kocheshkov discovered the redistribution reaction between $\mathrm{R}_{4} \mathrm{Sn}$ and $\mathrm{SnCl}_{4}$ in 1929 which afforded tri-, di- and mono-organostannyl chloride species. ${ }^{6}$ In 1936, Yngve discovered the heat stabilizing ability of organotins on polyvinyl chloride. In 1937, a summary of the early work of organotins was published in Organometallische Chemie by Krause and von Grosse. ${ }^{7}$

\subsection{Hypervalent/ Hypercoordinate compounds:}

In recent years, research interest in compounds with non-classical chemical bonds has increased extensively. ${ }^{8}$ These include the derivatives of silicon, germanium ${ }^{9-12}$ and tin. ${ }^{13,14}$ The expansion in the coordination sphere of these elements is caused by additional intra- or intermolecular coordination interactions. In the early 1960's it was demonstrated that organotin compounds have the ability to expand their coordination spheres. ${ }^{15,16}$ In 1969 , the term "hypervalent" was introduced by Musher to explain the structure of compounds that required octet

expansion for the central atom (e.g. $\mathrm{PCl}_{5}, \mathrm{SiF}_{6}$, etc.). ${ }^{17}$ The ions or molecules of the elements of Group 15-18 that possess more electrons than the octet within a valence shell are deemed 
hypervalent. ${ }^{17}$ Compounds of elements from Groups 1,2 or 14 as the central atom are also included in the family of hypervalent compounds. Hypervalent bonds in 5- or 6-coordinate compounds of $\mathrm{Ge}, \mathrm{Sn}$ and $\mathrm{Pb}$ differ from the hypervalent bonds of $\mathrm{S}, \mathrm{P}$ and $\mathrm{Cl}$ derivatives. In this case, the donor lone pair of Group 14 elements is supplied by the ligand as the central atom has no $n s^{2}$ lone pair. The first structurally characterized pentacoordinated organotin compound was a $\mathrm{PyMe}_{3} \mathrm{SnCl}$ in 1963. ${ }^{18}$ In the last three decades, five (TBP, SP) and six $\left(\mathrm{O}_{\mathrm{h}}\right)$ coordinated tin compounds have been extensively studied. Compounds of main group elements containing $(N)$ electrons more than the octet in a valence shell associated with the central atom (E) directly bound to ligands $(L)$ are known as hypervalent compounds. Therefore, the simple designation $N$-E- $L$ is used to describe hypervalent molecules (Figure 2). ${ }^{18}$

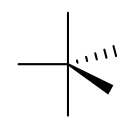

$10-\mathrm{E}-5$ TBP

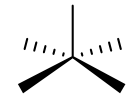

$10-E-5$ SP

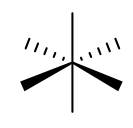

12-E-6

$\mathrm{O}_{\mathrm{h}}$

Figure 2: The principle coordination geometries of 5- and 6-coordinate Sn compounds. The formation of hypervalent bonds containing a 5- and 6-coordinated central atom can be interpreted by two concepts; the d-orbital concept or the 3c-4e bond concept.

\section{The d-orbital concept:}

According to this classical concept, filled $s$ and $p$ atomic orbitals along with vacant $5 d$ orbitals are involved in bond formation and cause an increase in the coordination number from 4 to 5 or 6 for Group 14 containing species. Two electronic configurations are envisioned to hold additional electrons that exceed the octet within the valence shell; $d s p^{3}$ or $d^{2} s p^{3} .{ }^{8}$ Sn has five vacant $5 d$ AOs which could participate in the formation of penta- and hexacoordinate structures. However, computational studies have shown that the nd AOs of Group 14 atoms (Ge, $\mathrm{Sn}$ and $\mathrm{Pb}$ ) 
are too diffuse and too high in energy to participate in bonding ${ }^{8}$ (more detail provided in the $3 \mathrm{c}-4 \mathrm{e}$ bond concept: below).

\section{3c-4e bond concept:}

This concept excludes the contribution of $5 d$ orbitals in hypervalent bonding for penta- and hexacoordinate structures. In 1951, Pimentel and Rundle proposed the idea of a 3c-4e bond using molecular orbital theory. ${ }^{20} \mathrm{~A}$ simple description of the $3 \mathrm{c}-4 \mathrm{e}$ bond model is the delocalization of one pair of bonding electrons to the two other substituents. The $3 \mathrm{c}-4 \mathrm{e}$ model suggests that $\mathrm{n} s^{2}$ orbitals of metal atom (E) could be used for bonding to equatorial ligands resulting in two-center bonds, while the $n p_{z}$ orbital could interact with an appropriate orbital of the axial substituent $(L)$ and a lone pair of the donor atom $(N)$ to form a hypervalent, $3 \mathrm{c}-4 \mathrm{e}$ bond. Initially, the concept of $3 \mathrm{c}-4 \mathrm{e}$ bond was not readily adopted by researchers, but progress in computational studies and the efforts of Kutzelnigg et $a l .{ }^{21}$ have made this idea generally acceptable today. The hypervalent bonding of molecules has also been investigated by von Schleyer et al. ${ }^{22}$ who concluded that the $d$-orbital concept is incorrect and these orbitals are not important in the acceptance of electrons beyond the octet. It has been determined that it is not possible for the $d$ orbitals to hold extra electron density because of the high energy gap between $\mathrm{n}(s p)$ and $\mathrm{n} d$, which makes the number of available metal orbitals deficient. From theoretical calculations, it has been established that in main group elements, participation of $d$ orbitals for hybridization with $s$ and $p$ orbitals of third period and heavier is negligible. Therefore, a 3c-4e bond is an electron-rich bond and the nonbonding molecular orbital becomes the highest occupied orbital. In the $3 \mathrm{c}-4 \mathrm{e}$ bond, the central atom has less than four pairs of electrons in the valence shell and does not exceed the Lewis octet due to the distribution of extra electron density on to ligands or substituents. Figure 3 represents the simplest MO diagram of the $3 \mathrm{c}-4 \mathrm{e}$ bond. 


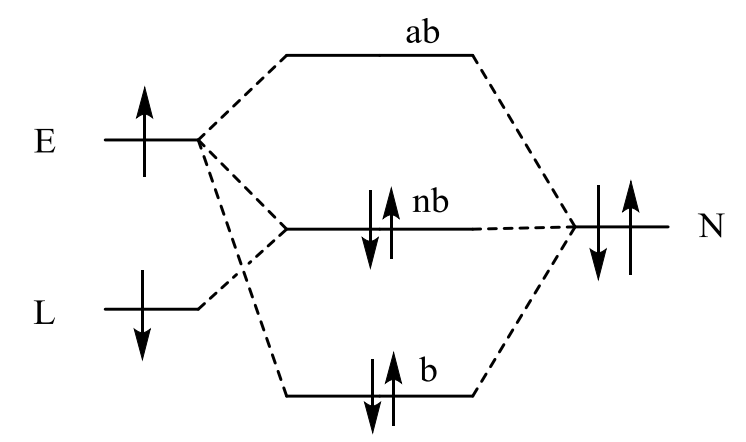

$\mathrm{b}=$ bonding, $\mathrm{nb}=$ non-bonding, $\mathrm{ab}=$ antibonding

Figure 3: The MO diagram of a (3c-4e) bond in hypervalent compounds.

In Group 14, the hypervalency of both $\mathrm{Sn}$ and $\mathrm{Si}$ has been intensively studied in comparison to Ge, with even fewer studies for compounds of $\mathrm{Pb}$. In a pentacoordinated TBP molecule, the hypervalent bond is typically seen in the axial position. Four methods were proposed by Akiba ${ }^{8}$ to construct a 3c-4e bond of a pentacoordinated molecule experimentally (Figure 4); a) add two free radicals to coordinate with a pair of unshared electrons in a $p$ orbital, b) add two pairs of unshared electrons to coordinate a vacant $p$ orbital, c) add a pair of unshared electrons to coordinate with $\sigma^{*}$ orbital of E-X bond (e.g., sulphonium, phosphonium etc.), d) add a pair of unshared electrons to coordinate with the $\sigma^{*}$ orbital of E-X bond of a neutral molecule (e.g., Si and Sn).

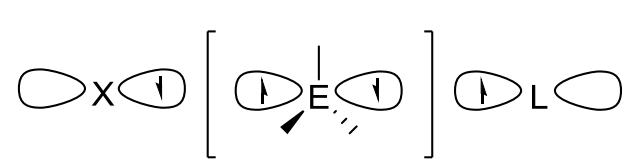

a

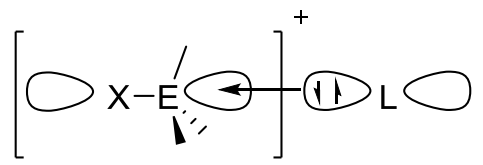

C

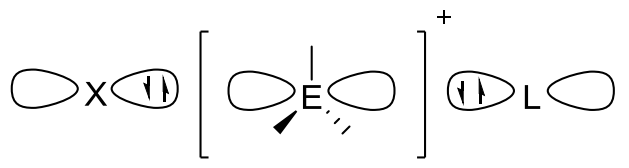

b

Figure 4: Proposed methods to make $(3 c-4 e)$ bonds. $^{8}$ 
Hypervalent bonds are favourably formed when these conditions are present:

1. The electronegativity of the ligands is typically higher than the central $\mathrm{E}$ atom.

2. For trigonal bipyramidal molecules, ligands having higher electronegativity form the hypervalent bond with central atom $\mathrm{E}$ and tend to occupy axial positions.

3. The E-L bonds are longer in penta- and hexacoordinate compounds than in tetrahedral molecules.

\subsection{Hypervalent/ Hypercoordinate compounds of Group 14:}

\subsubsection{Hypercoordinate organotin compounds containing $C, N$-chelating ligands:}

Hypervalent compounds of organotin derivatives with ligands containing an intramolecular Sn-N interaction have been known since $1960 .{ }^{23}$ These tin species have been extensively studied by single-crystal X-ray diffraction and NMR $\left({ }^{1} \mathrm{H},{ }^{13} \mathrm{C},{ }^{119} \mathrm{Sn}\right)$ spectroscopy. The strength of the Sn$\mathrm{N}$ interaction can be inferred from the bond distance; the smaller the distance, the stronger the donor-acceptor interaction. It is also observed that as the Sn-N distance decreases, the Sn-X (X = F, Cl, Br, I) bond becomes longer. This is consistent with the model shown in Figure 4(d). The

${ }^{119}$ Sn NMR spectra of hypervalent triorganotin(IV) compounds exhibit a single resonance for the central tin atom, shifted up-field with respect to a structurally similar non-hypervalent analog.

Organotin(IV) compounds possessing the 2-( $\left.\mathrm{Me}_{2} \mathrm{NCH}_{2}\right) \mathrm{C}_{6} \mathrm{H}_{4}$ - organic ligand with nitrogen as donor atom or other related potentially chelating organic ligands have been extensively investigated both in solution and in the solid state. Interest in these types of organotin compounds has been largely due to their unusual structural properties ${ }^{14,24}$ and potential biological uses. ${ }^{25}$ The different types of organotin compounds utilizing the 2-( $\left.\mathrm{Me}_{2} \mathrm{NCH}_{2}\right) \mathrm{C}_{6} \mathrm{H}_{4}$ - ligand can be classified as mono-, di-, tri- and tetraorganotin(IV) compounds. 


\subsubsection{Tetraorganotin compounds containing $C, N$-chelating ligands:}

The more commonly used $C, N$-chelating ligands are shown in Figure 5. Ligands i-iv and vii can form five membered hypervalent compounds while $\mathrm{v}$ and vi form six membered chelate rings. ${ }^{26}$
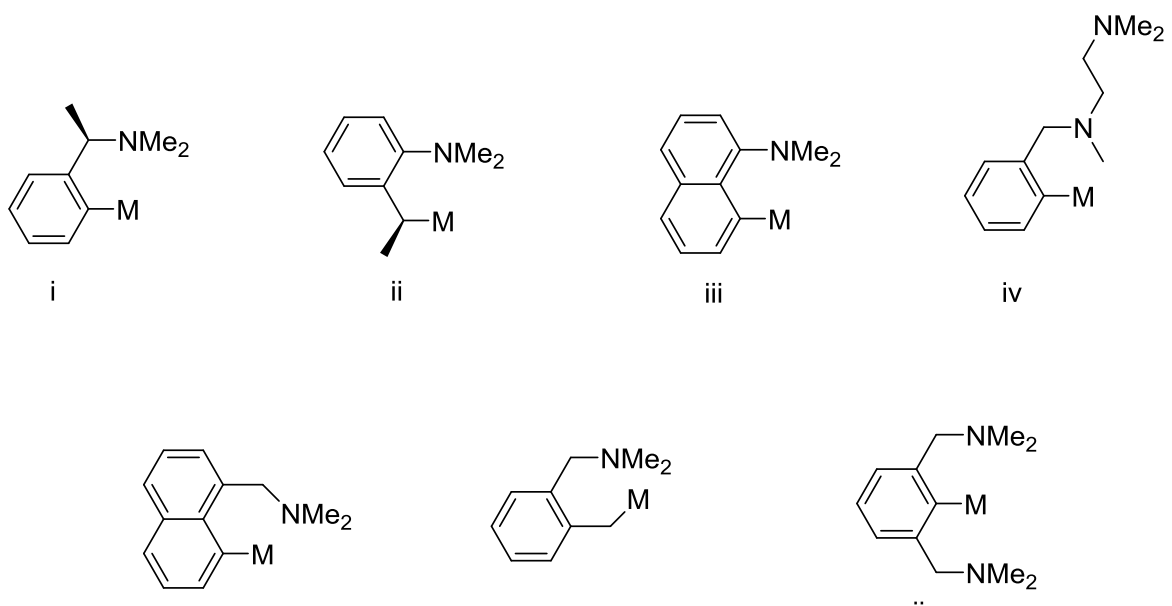

V

vi

vii

$\mathrm{M}=\mathrm{Si}, \mathrm{Ge}, \mathrm{Sn}$

Figure 5: Most commonly used $C, N$-chelating ligands.

The important structural feature of these ligands is that the Sn- $\mathrm{N}$ intramolecular coordination results in an increase of the coordination number at tin from 4 to 5 or 6 for i to iv and $\mathrm{v}$ to vi respectively.

Organotin compounds containing these ligands can be synthesized by heteroatom facilitated ortho-lithiation followed by reaction with organotin halides. Jastrzebski et $a l^{27}{ }^{27}$ reported the synthesis of 8-(dimethylamino)-1-naphthyl lithium etherate by reacting the equimolar amount of 1-(dimethylamino)naphthalene with $n$ - $\mathrm{BuLi}$ in $\mathrm{Et}_{2} \mathrm{O}$. The 8-(dimethylamino)-1-naphthyl lithium etherate was treated with $\mathrm{Me}_{3} \mathrm{MCl}(\mathrm{M}=\mathrm{Si}, \mathrm{Sn})$ to obtain the corresponding Group 14 derivatives. Reaction of 8-(dimethylamino)-1-naphthyl lithium with $\mathrm{Me}_{3} \mathrm{SnCl}$ or $\mathrm{Ph}_{3} \mathrm{SnCl}$ afforded high yields of 8-(dimethylamino)-1-naphthyltrimethyltin and 8-(dimethylamino)-1naphthyltriphenyltin, respectively (Scheme 1). ${ }^{28}$ Tetraorganotin compounds were initially 
considered as unlikely to extend their coordination number due to the poor acceptor properties of these tin centers. ${ }^{14}$
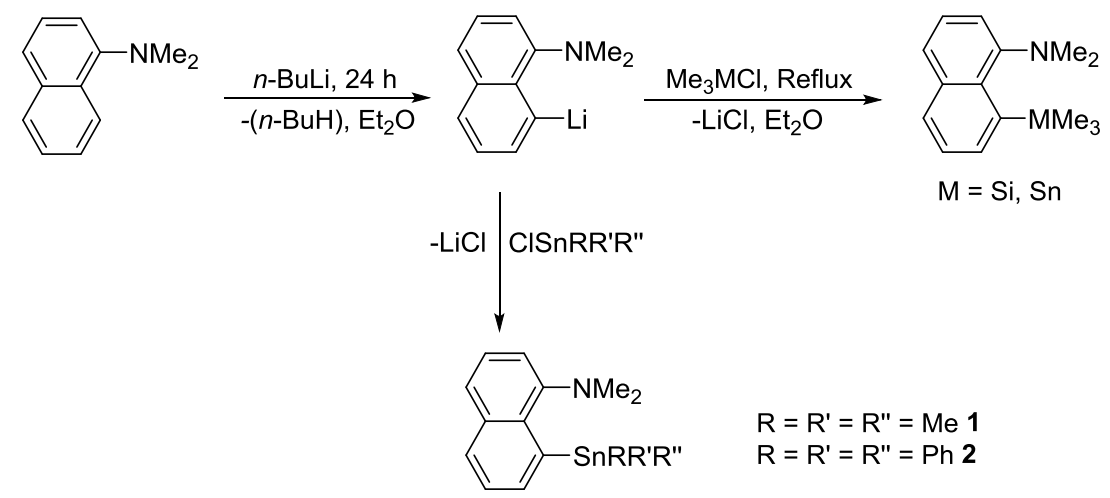

$\mathrm{M}=\mathrm{Si}, \mathrm{Sn}$

$R=R^{\prime}=R^{\prime \prime}=M e 1$

$R=R^{\prime}=R^{\prime \prime}=P h 2$

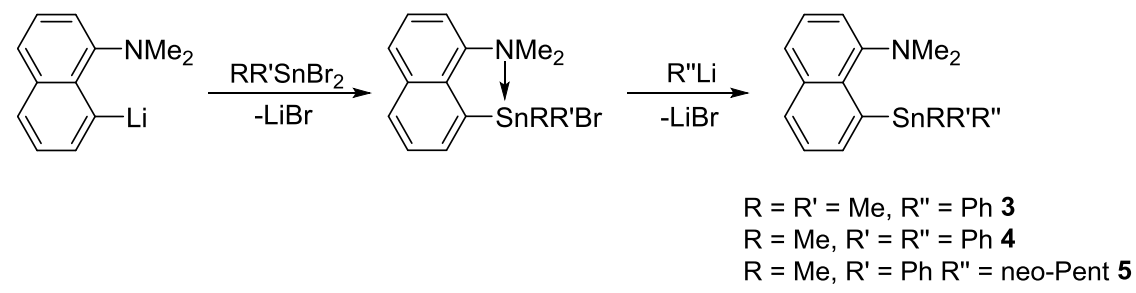

Scheme 1: Synthesis of tetraorganotin compounds. ${ }^{25,26}$

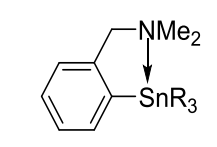

$\mathrm{R}=\mathrm{Me}$ 6, $n-\mathrm{Bu} 7, \mathrm{Ph} 8$

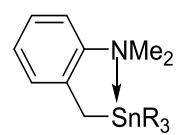

$\mathrm{R}=\mathrm{Me} 9, \mathrm{Ph} 10$

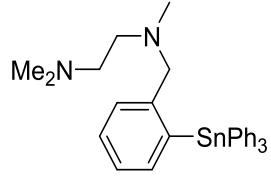

11

Figure 6: Tetraorganotin compounds.

NMR $\left({ }^{1} \mathrm{H},{ }^{13} \mathrm{C},{ }^{119} \mathrm{Sn}\right)$ spectroscopic studies have provided information concerning the structure of tin compounds with $C, N$-chelating ligands in solution. These studies indicate that the ${ }^{119} \mathrm{Sn}$ NMR chemical shift of such compounds is strongly dependent on the type of substituent and coordination number of tin. ${ }^{14}$

In compounds 1-5, the stable geometry of the 8-(dimethylamino)-1-naphthyl group ensures a Sn-N coordination bond. An X-ray structure of 2 reveals tin in TBP geometry with the $\mathrm{N}$ atom and one of the phenyl rings at axial positions. ${ }^{28}$ The ${ }^{119} \mathrm{Sn}$ chemical shifts of compounds $\mathbf{1 - 4}$ show that the replacement of methyl by phenyl groups enhances the up-field shift of Sn resonances due 
to an increasing Sn-N interaction. A significant upfield shift of the ${ }^{119} \mathrm{Sn}$ resonance for $\mathbf{6}$ compared to the unsubstituted analogue $\mathrm{Me}_{3} \mathrm{SnPh}$ is likely due to the presence of a $\mathrm{Sn}-\mathrm{N}$ intramolecular interaction.

Table 1: Comparison of the ${ }^{119} \mathrm{Sn}$ chemical resonances of tin compounds containing a $C, N$ ligand and structurally similar unsubstituted analogue.

\begin{tabular}{|c|c|c|c|}
\hline Compound & $\begin{array}{c}\text { 119} \mathbf{S n} \boldsymbol{\delta} \\
(\mathbf{p p m})\end{array}$ & $\begin{array}{c}\text { Unsubstituted } \\
\text { analogue }\end{array}$ & $\begin{array}{c}\text { 119 } \mathbf{S n} \boldsymbol{\delta} \\
(\mathbf{p p m})\end{array}$ \\
\hline $\mathbf{1}$ & $-46.0^{28}$ & $\mathrm{C}_{10} \mathrm{H}_{7} \mathrm{SnMe}_{3}$ & $-31.8^{37}$ \\
\hline $\mathbf{2}$ & $-155.3^{28}$ & $\mathrm{C}_{10} \mathrm{H}_{7} \mathrm{SnPh}_{3}$ & - \\
\hline $\mathbf{3}$ & $-81.7^{28}$ & $\mathrm{C}_{10} \mathrm{H}_{7} \mathrm{SnPhMe}_{2}$ & - \\
\hline $\mathbf{4}$ & $-110.4^{28}$ & $\mathrm{C}_{10} \mathrm{H}_{7} \mathrm{SnPh} \mathrm{Me}_{2}$ & - \\
\hline $\mathbf{5}$ & $-97.3^{28}$ & $\mathrm{C}_{10} \mathrm{H}_{7} \mathrm{SnMe}_{3}$ & $-31.8^{37}$ \\
\hline $\mathbf{6}$ & -50.0 & $\mathrm{Me}_{3} \mathrm{SnPh}$ & $-28.6^{338}$ \\
\hline $\mathbf{7}$ & $-50.0^{29 \mathrm{~b}}$ & $\mathrm{Bu}_{3} \mathrm{SnPh}$ & $-41.7^{39}$ \\
\hline $\mathbf{8}$ & $-168.5^{34}$ & $\mathrm{Ph}_{4} \mathrm{Sn}$ & -137.0 \\
\hline $\mathbf{9}$ & $-0.8^{32}$ & $\mathrm{C}_{6} \mathrm{H}_{4} \mathrm{CH}_{2} \mathrm{SnMe}_{3}$ & $3.5^{36}$ \\
\hline $\mathbf{1 0}$ & $-122.2^{32}$ & $\mathrm{C}_{6} \mathrm{H}_{4} \mathrm{CH}_{2} \mathrm{SnPh}_{3}$ & $-118.0^{37}$ \\
\hline $\mathbf{1 1}$ & $-148.0^{35}$ & - & - \\
\hline
\end{tabular}

A comparison of ${ }^{119} \mathrm{Sn}$ NMR chemical shifts of tin compounds containing a $C, N$-ligand and their unsubstituted analogues given in Table 1 indicate that compounds 9-10 (Figure 6) have no apparent intramolecular interaction between $\mathrm{Sn}$ and $\mathrm{N}$ as there is only a slight upfield shift in their resonances compared to their unsubstituted analogues. A possible reason for the absence of an intramolecular interaction, particularly in compound $\mathbf{1 0}$, is due to the energetically favourable structure having a bulky $\mathrm{SnR}_{3}$ substituent perpendicular to the plane of aryl ring, keeping the tin atom away from the potential donor atoms.

\subsubsection{1a Solution Structure of tetraorganotin compounds 1, 3-5:}

The relatively small $52 \mathrm{~Hz}^{2} J_{117 / 119 \mathrm{Sn}-1 \mathrm{H}}$ coupling values for the methyl protons present in compounds 1, 3-5 are indicative of a tetrahedral geometry at tin. ${ }^{30-33}$ The presence of two resonances for the $-\mathrm{NMe}_{2}$ group in $\mathbf{5}$ containing a stable chiral center suggest a blockage of 
pyramidal inversion at nitrogen, and may be a result of strong $\mathrm{Sn}-\mathrm{N}$ coordination or steric considerations. The ${ }^{1} \mathrm{H}$ and ${ }^{13} \mathrm{C}$ chemical shift values of the $-\mathrm{NMe}_{2}$ in compounds $\mathbf{1 - 5}$ are relatively insensitive to $\mathrm{Sn}-\mathrm{N}$ coordination. The methyl groups of $-\mathrm{NMe}_{2}$ in these compounds have ${ }^{13} \mathrm{C}$ chemical resonances at $\approx 48 \mathrm{ppm}$, very close to that of the free 1-(dimethylamino)naphthalene (46.2 ppm). A small upfield shift of $2.2 \mathrm{ppm}$ results with the substitution of phenyl for methyl at the tin center. ${ }^{32}$

\subsubsection{Synthesis of organotin halides containing $C, N$-chelating ligands:}

Organotin halides have been synthesized by a variety of methods. The most commonly used method is the conversion of alkyl or aryl halides into Grignard reagents in THF or Et $2 \mathrm{O}$ and their subsequent reaction with $\mathrm{SnCl}_{4}$ to produce tetraorganotin compounds. Tri- and diorganotin compounds can be synthesized by the comproportionation (or Kocheskov redistribution) reactions of tetraorganotin compounds with $\mathrm{SnCl}_{4}$ (Scheme 2). ${ }^{42}$

$$
\begin{gathered}
\mathrm{RBr} \underset{\mathrm{Et}_{2} \mathrm{O} \text { or THF }}{\stackrel{M g \text { turnings }}{\longrightarrow}} \mathrm{RMgBr} \stackrel{0.2 \text { eq. } \mathrm{SnCl}_{4}}{\mathrm{Et}_{2} \mathrm{O}} \mathrm{R}_{4} \mathrm{Sn} \\
2 \mathrm{R}_{2} \mathrm{SnCl}_{2} \underset{\text { neat }}{\stackrel{\text { 2 eq. SnCl}}{\longrightarrow}} \mathrm{R}_{4} \mathrm{Sn} \stackrel{3 \text { eq. } \mathrm{SnCl}_{4}}{\text { neat }} 4 \mathrm{RSnCl}_{3} \\
\mathrm{R}_{2} \mathrm{SnCl}_{2} \frac{\mathrm{R}_{4} \mathrm{Sn}}{\text { neat }} 2 \mathrm{RSnCl}_{3} \\
\mathrm{R}=\text { Alkyl or Aryl groups }
\end{gathered}
$$

Scheme 2: Synthesis of organotin halides.

The $\mathrm{R}_{2} \mathrm{SnCl}_{2}$ species are more conveniently accessed using the Kocheskov equilibrium (Scheme 2). During this reaction $\mathrm{RSnCl}_{3}$ is produced as an intermediate which immediately reacts with a tetraorganotin species to produce a diorganotin dihalide. Different techniques were reported to obtain pure tetraorganotin compounds. Caseri et al. used a $5 \% \mathrm{HCl}$ solution for tetraalkyltin compounds ${ }^{40}$ and saturated solution of $\mathrm{NH}_{4} \mathrm{Cl}$ for tetraaryltin compounds. ${ }^{41}$ Uhlig et al. purified tetraaryltin compounds by filtration of reaction mixtures through celite and extraction of the 
residues with DCM. ${ }^{42}$ Deacon et al. prepared diorganotin dihalides by reacting tetraorganotin compounds with a stoichiometric solution of $\mathrm{Br}_{2}$ or $\mathrm{I}_{2}$ in $\mathrm{CHCl}_{3}$. The halobenzene by-product was removed by prolonged drying under reduced pressure. ${ }^{43}$

Hypercoordinated diorganotin halides containing $C, N$-chelated ligands can be synthesized by reacting [2-( $\left.\left.\mathrm{Me}_{2} \mathrm{NCH}_{2}\right) \mathrm{C}_{6} \mathrm{H}_{4}\right] \mathrm{Li}$ with $\mathrm{R}_{\mathrm{n}} \mathrm{SnCl}_{4-\mathrm{n}}(\mathrm{R}=\mathrm{L}, \mathrm{Me}, n-\mathrm{Bu}, \mathrm{Ph})$ under inert atmosphere. Another method for the synthesis of diorganotin halides is the solvent-free ligand redistribution reaction between $\left[2-\left(\mathrm{Me}_{2} \mathrm{NCH}_{2}\right) \mathrm{C}_{6} \mathrm{H}_{4}\right] \mathrm{Sn}$ and $\mathrm{SnCl}_{4}$. These may also be synthesized from the direct reaction of $\left[2-\left(\mathrm{Me}_{2} \mathrm{NCH}_{2}\right) \mathrm{C}_{6} \mathrm{H}_{4}\right] \mathrm{Li}$ and $\mathrm{SnCl}_{4}(\mathrm{Scheme} 3) .{ }^{49}$

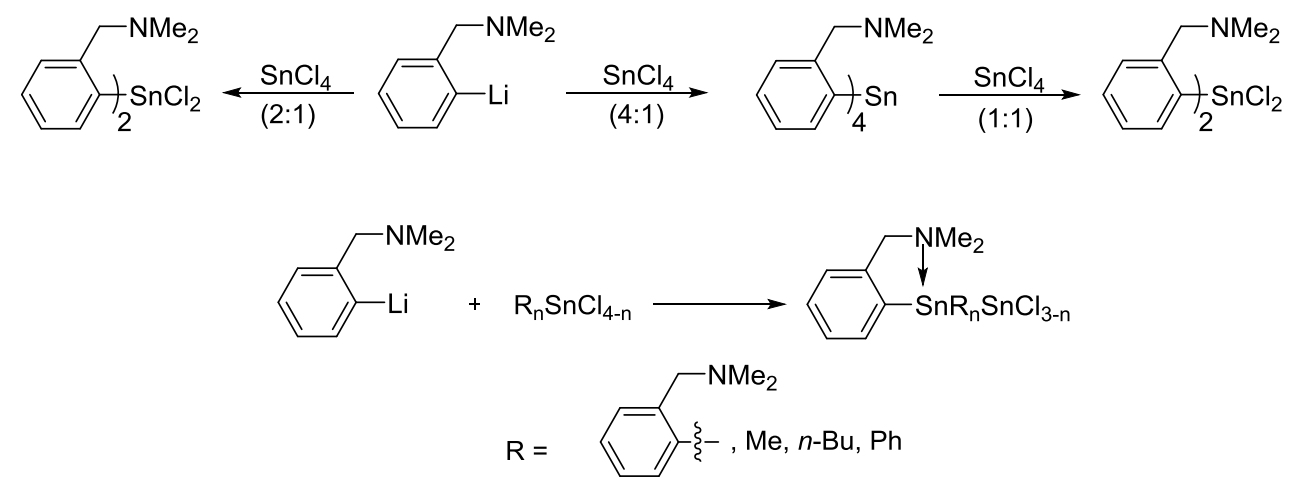

Scheme 3: Routes for the synthesis of hypercoordinated organotin halides.

\subsubsection{Triorganotin halides containing $C, N$-chelating ligands:}

There are a number of triorganotin halides (Figure 7) containing $C, N$-chelating ligands such as 2-( $\left.\mathrm{Me}_{2} \mathrm{NCH}_{2}\right) \mathrm{C}_{6} \mathrm{H}_{4-}$. The presence of a Sn-N intramolecular interaction in these species has been established by crystallography ${ }^{44}$ and ${ }^{119}$ Sn CP-MAS NMR ${ }^{45}$ (Cross Polarization-Magic Angle spinning) techniques in the solid state as well as by solution multinuclear NMR spectroscopy. ${ }^{29 b, 46}$ 


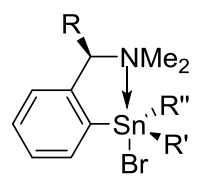

$$
\begin{array}{ll}
\mathrm{R}=\mathrm{H} 12 \mathrm{a}-\mathrm{c} & \mathrm{R}^{\prime}=\mathrm{R}^{\prime \prime}=\mathrm{Me} \mathrm{a} \\
\mathrm{R}=\mathrm{Me} 13 \mathrm{a}-\mathrm{c} & \mathrm{R}^{\prime}=\mathrm{Me}, \mathrm{R}^{\prime \prime}=\mathrm{Ph} \mathbf{b} \\
\mathrm{R}=\mathrm{Et} \mathrm{14a-c} & \mathrm{R}^{\prime}=\mathrm{R}^{\prime \prime}=\mathrm{Ph} \mathrm{c} \\
\mathrm{R}=i-\operatorname{Pr} 15 \mathrm{a}-\mathrm{c} & \\
\mathrm{R}=t-\mathrm{Bu} 16 \mathrm{a}-\mathrm{c} &
\end{array}
$$

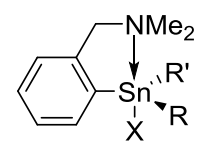

$\mathrm{R}=\mathrm{Me}, \mathrm{R}^{\prime}=\mathrm{Ph}, \mathrm{X}=\mathrm{Cl} 17$

$\mathrm{R}=\mathrm{R}^{\prime}=\mathrm{Ph}, \mathrm{X}=\mathrm{Cl} 18$

$\mathrm{R}=\mathrm{R}^{\prime}=\mathrm{Me}, \mathrm{X}=\mathrm{Cl} 19$

$\mathrm{R}=\mathrm{R}^{\prime}=n-\mathrm{Bu}, \mathrm{X}=\mathrm{Br} 20$

$\mathrm{R}=\mathrm{R}^{\prime}=n-\mathrm{Bu}, \mathrm{X}=\mathrm{Cl} 21$

$\mathrm{R}=\mathrm{R}^{\prime}=t-\mathrm{Bu}, \mathrm{X}=\mathrm{Cl} 22$

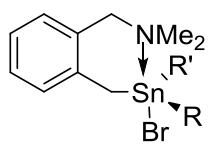

$\mathrm{R}=\mathrm{R}^{\prime}=\mathrm{Me} 25$

$\mathrm{R}=\mathrm{Me}, \mathrm{R}^{\prime}=\mathrm{Ph} 26$

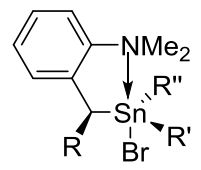

$$
\begin{array}{ll}
R=H 24 & R^{\prime}=R^{\prime \prime}=M e a \\
& R^{\prime}=M e, R^{\prime \prime}=P h \mathbf{b} \\
& R^{\prime}=R^{\prime \prime}=P h \mathbf{c}
\end{array}
$$

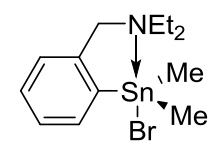

23

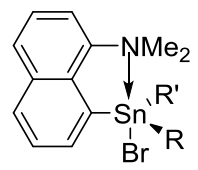

$\mathrm{R}=\mathrm{R}^{\prime}=\mathrm{Me} 27$

$\mathrm{R}=\mathrm{Me}, \mathrm{R}^{\prime}=\mathrm{Ph} 28$

$\mathrm{R}=\mathrm{R}^{\prime}=\mathrm{Ph} 29$

$\mathrm{R}=\mathrm{Me}, \mathrm{R}^{\prime}=(-)$ menthyl $\mathbf{3 0}$

$\mathrm{R}=\mathrm{Ph}, \mathrm{R}^{\prime}=(-)$ menthyl 31

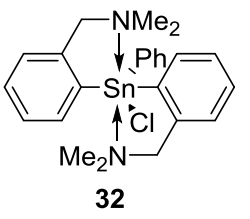

Figure 7: 5-coordinate triorganotin halides.

\subsubsection{3a Synthesis:}

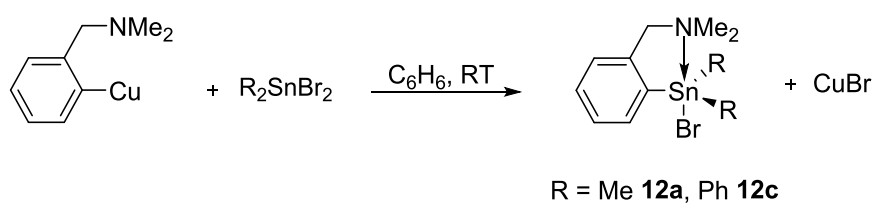

Scheme 4: Synthesis of compounds 12a and 12c. ${ }^{47}$

van Koten et al. prepared compounds $\mathbf{1 2 a}$ and $\mathbf{1 2 c}$ by treating the $\left[2-\left(\mathrm{Me}_{2} \mathrm{NCH}_{2}\right) \mathrm{C}_{6} \mathrm{H}_{4}\right] \mathrm{Cu}$ tetramer with diorganotin dihalides (Scheme 4) ${ }^{47}$ Compounds $\mathbf{1 3}$ and $\mathbf{1 4}$ were prepared by lithiation of $2-\mathrm{Me}_{2} \mathrm{NCH}(\mathrm{R}) \mathrm{C}_{6} \mathrm{H}_{5}$ with $t$-BuLi. Compounds 15 and 16 were synthesized by lithium/halogen exchange reactions of $2-\mathrm{Me}_{2} \mathrm{NCH}(\mathrm{R}) \mathrm{C}_{6} \mathrm{H}_{5}(\mathrm{R}=$ isopropyl, $t$-butyl) with $n$-BuLi because the presence of bulky substituents greatly reduces accessibility to the ortho site. The 
reaction of $\mathrm{SnBr}_{2} \mathrm{Me}_{2}, \mathrm{SnBr}_{2} \mathrm{MePh}, \mathrm{SnBr}_{2} \mathrm{Ph}_{2}$ with the lithiated species afforded hypervalent compounds $\mathbf{1 3}(\mathbf{a}-\mathbf{c})$ and $\mathbf{1 6}(\mathbf{a}-\mathbf{c})$ as white coloured crystalline solids (Scheme 5). ${ }^{56 a}$
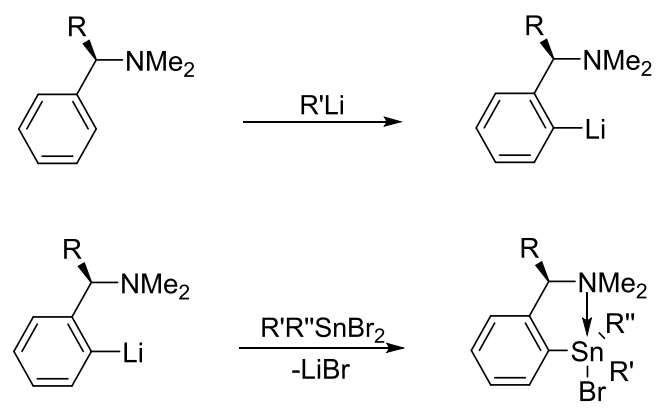

Scheme 5: Synthesis of $13(\mathbf{a}-\mathbf{c})$ and $16(\mathbf{a}-\mathbf{c}) .^{28}$

Compounds 17, 18 and 19 were previously reported by both Rippstein et al. ${ }^{48}$ and Varga et $a l .{ }^{49}$ and were synthesized by reacting $\left[2-\left(\mathrm{Me}_{2} \mathrm{NCH}_{2}\right) \mathrm{C}_{6} \mathrm{H}_{4}\right] \mathrm{Li}$ with their respective diorganotin dihalides. Alternatively, 17 was obtained by reacting $\left[2-\left(\mathrm{Me}_{2} \mathrm{NCH}_{2}\right) \mathrm{C}_{6} \mathrm{H}_{4}\right] \mathrm{Li}$ with a solution of $\mathrm{SnMePhBr}_{2}$ in $\mathrm{Et}_{2} \mathrm{O} .{ }^{50}$ Compounds $\mathbf{2 1}^{51}, \mathbf{2 2}^{52}$, and $\mathbf{3 2}{ }^{48,29 a}$ were synthesized from the reactions of a 1:1 of $n-\mathrm{Bu}_{2} \mathrm{SnCl}_{2}, t-\mathrm{Bu}_{2} \mathrm{SnCl}_{2}$ and 1:2 ratio of $\mathrm{PhSnCl}_{3}$ with [2-( $\left.\left.\mathrm{Me}_{2} \mathrm{NCH}_{2}\right) \mathrm{C}_{6} \mathrm{H}_{4}\right] \mathrm{Li}$ in $\mathrm{C}_{6} \mathrm{H}_{6}$ at room temperature. Compound $\mathbf{2 3}$ was prepared by reacting $\mathrm{Me}_{2} \mathrm{SnBr}_{2}$ with [2$\left.\left(\mathrm{Et}_{2} \mathrm{NCH}_{2}\right) \mathrm{C}_{6} \mathrm{H}_{4}\right] \mathrm{Li}^{53}$

\subsubsection{3b Structural studies:}

The x-ray crystal structure determination of [2-( $\left.\left.\mathrm{Me}_{2} \mathrm{NCH}_{2}\right) \mathrm{C}_{6} \mathrm{H}_{4}\right] \mathrm{SnPh}_{2} \mathrm{Br} \mathbf{1 2 c}$ reveals a five-coordinate distorted TBP geometry at the tin center, with three carbon ligands, including the built-in ligand and the bromine atom in equatorial and axial positions, respectively. A N-Sn-Br bond angle of $171^{\circ}$ and Sn-N bond length of $2.51 \AA$ was observed for 12c. The Sn-N bond distance may be the result of a balance between the inherent $\mathrm{Sn}-\mathrm{N}$ bond strength and the skeletal strain in the five-membered chelate ring. ${ }^{54}$ These observations provide evidence of an intramolecular interaction between Sn and N. 
The configurational stability of the triorganotin bromides having either the tin center (Figure 7) $\left(\mathrm{R}^{\prime} \neq \mathrm{R}\right.$ ', $\mathbf{1 2 b}$ and $\left.\mathbf{2 4 b}\right)$, or the benzyl carbon atom $(\mathrm{R} \neq \mathrm{H})$ or both $(\mathbf{1 2 b}-\mathbf{1 6 b})$ as chiral centers were investigated extensively by variable temperature NMR spectroscopy. ${ }^{47,50,56 a}$ Tin compounds 25 and $\mathbf{2 6}$ have a six-membered chelate ring where the tin centers have configurational stability only at low temperature (below $0{ }^{\circ} \mathrm{C}$ ), ${ }^{50}$ while triorganotin bromides having a flexible five-membered chelate ring (13b-16b, 17 and $\mathbf{2 4 b})$ are considerably more stable on the NMR time scale (up to $130{ }^{\circ} \mathrm{C}$ ). These fluxional processes were studied by the ${ }^{1} \mathrm{H}$ NMR of $\mathbf{1 2 b}$ at variable temperatures. The diastereotopicity of the methyl protons of the $-\mathrm{NMe}_{2}$ group was observed by ${ }^{1} \mathrm{H}$ NMR spectroscopy at low temperature, indicating the inertness of Sn-N coordination on the NMR time scale. At higher temperature, the $-\mathrm{NMe}_{2}$ resonances coalesce due to the process of $\mathrm{Sn}-\mathrm{N}$ dissociation/association. The process (Figure 8) by which $-\mathrm{NMe}_{2}$ methyl groups become homotopic consists of four steps including Sn-N dissociation, rotation around the $\mathrm{C}_{\text {benzyl- }} \mathrm{N}$ bond, pyramidal inversion at nitrogen and finally $\mathrm{Sn}-\mathrm{N}$ association. On the NMR time scale this process is quite fast above $0{ }^{\circ} \mathrm{C} .{ }^{32,33,54,55,56 a}$ Finally, it was found that the tin center has configurational stability up to $130{ }^{\circ} \mathrm{C}$ on the NMR time scale in triorganotin bromides having a rigid fivemembered chelate ring $(\mathbf{2 8}, \mathbf{3 0}, \mathbf{3 1}) .{ }^{27}$ The ${ }^{1} \mathrm{H}$ NMR analysis of 25 and 26 containing a sixmembered chelate ring reveal the existence of both the process of dissociation/association and inversion of configuration at tin centers. ${ }^{50}$ The ${ }^{1} \mathrm{H}$ NMR spectrum of compound 26 at $-40{ }^{\circ} \mathrm{C}$ showed an $\mathrm{AB}$ pattern for both benzylic $-\mathrm{CH}_{2}$ groups and two resonances for $-\mathrm{NMe}_{2}$ group. This indicates the stability of the Sn-N interaction and configuration of tin on the NMR time scale. At $-30{ }^{\circ} \mathrm{C}$, it was observed that $-\mathrm{NMe}_{2}$ resonances coalesce, with no change of the AB pattern up to 0 ${ }^{\circ} \mathrm{C}$. This suggests that on NMR time scale, only the $\mathrm{Sn}-\mathrm{N}$ dissociation/association process becomes 
rapid. Above $0{ }^{\circ} \mathrm{C}$, the coalescence of the two $\mathrm{AB}$ patterns to two singlets show that inversion of configuration at tin centers is rapid..$^{50}$
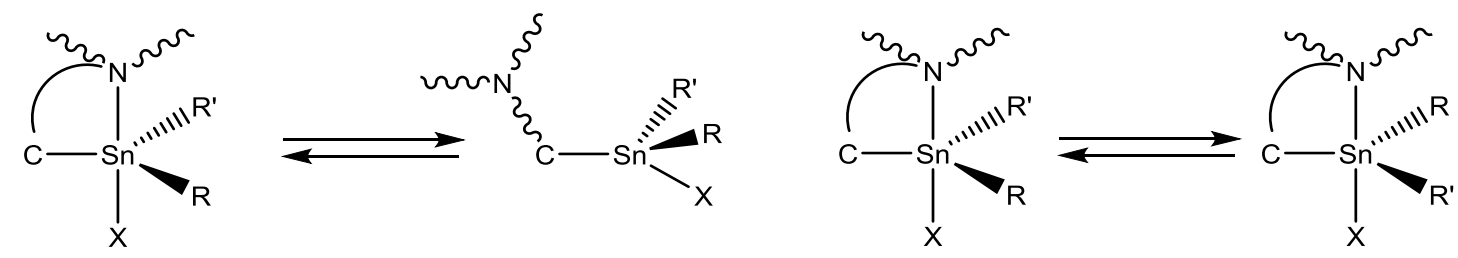

Figure 8: Two basic fluxional processes of a $C, N$-chelating ligand containing $\mathrm{R}_{3} \mathrm{SnCl}$.
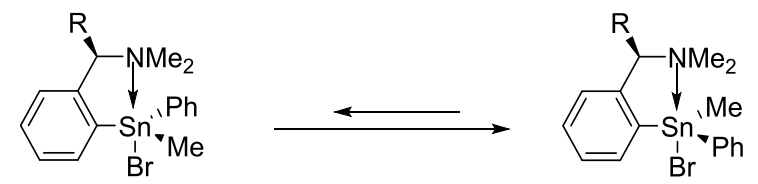

$$
\begin{aligned}
& \mathrm{R}=\mathrm{Me} 13 \mathbf{b}^{\prime} \\
& \mathrm{R}=\mathrm{Et} 14 \mathbf{b}^{\prime} \\
& \mathrm{R}=i-\operatorname{Pr} 15 \mathbf{b}^{\prime} \\
& \mathrm{R}=t-\mathrm{Bu} 16 \mathbf{b}^{\prime}
\end{aligned}
$$

$$
\begin{aligned}
& \mathrm{R}=\mathrm{Me} 13 \mathbf{b} " \\
& \mathrm{R}=\mathrm{Et} 14 \mathbf{b}^{\prime} \\
& \mathrm{R}=i-\operatorname{Pr} 15 \text { b" }
\end{aligned}
$$

Scheme 6: Diastereoisomers of 13b-16b.

Compounds 13b-16b possess two chiral centres; one at the benzylic carbon and one at the tin atom. NMR $\left({ }^{1} \mathrm{H}\right.$ and $\left.{ }^{119} \mathrm{Sn}\right)$ spectroscopy indicate the presence of two different diastereoisomers for $\mathbf{1 3 b} \mathbf{- 1 5 b}$ and a single isomer for $\mathbf{1 6 b}$ as shown in Scheme 6. The coordination geometry at the tin atoms of compounds $\mathbf{2 1}$ and $\mathbf{2 2}$ is trigonal bipyramidal having the carbon atoms and more electronegative atoms $(\mathrm{N}, \mathrm{Cl})$ at equatorial and axial positions respectively. The $\mathrm{Sn}-\mathrm{N}$ bond distance in 22 is 2.904(14) $\AA$ which is significantly longer than in 21 (2.510(5) $\AA$ ) and other known triorganotin compounds. The downfield ${ }^{119} \mathrm{Sn}$ NMR resonance of $18.5 \mathrm{ppm}$ for $\mathbf{2 2}$ is in contrast with the resonances of similar types of 5-coordinate compounds with strong Sn-N interactions such as $\operatorname{LSn}(n-\mathrm{Bu})_{2} \mathrm{Cl}(\delta=-51.7 \mathrm{ppm}), \operatorname{LSn}(n-\mathrm{Bu})_{2} \mathrm{~F}(\delta=-77.1 \mathrm{ppm}), \operatorname{LSn}(t-\mathrm{Bu})_{2} \mathrm{~F}(\delta=-92.1$ ppm), $\mathrm{LSnMe}_{2} \mathrm{~F}(\delta=-53.8 \mathrm{ppm})$ where $\mathrm{L}=2-\left(\mathrm{Me}_{2} \mathrm{NCH}_{2}\right) \mathrm{C}_{6} \mathrm{H}_{4}$. Variable temperature $(\mathrm{VT}){ }^{1} \mathrm{H}$ and ${ }^{119} \mathrm{Sn}$ NMR studies of $\mathbf{2 2}$ in different solvents and in the solid state concluded that its geometry is distorted trigonal pyramidal, but with a significantly weaker Sn-N interaction. ${ }^{52}$ 
The geometry of $\mathbf{3 2}$ can be explained in two different ways. It may be a prototypical distorted trigonal bipyramidal geometry for organotin having a $C, N$-chelating ligand located in equatorial positions where both the chlorine and nitrogen atoms are in axial positions. Here the non-coordinated nitrogen atom lies out of the tin coordination sphere and does not coordinate to tin (Sn-N1 and Sn-N2 distances at 2.4752(17) and 3.5174(19) Å respectively). Alternatively, it may be regarded as a distorted octahedron, with $\mathrm{C} 1$ and $\mathrm{C} 10, \mathrm{~N} 1$ and $\mathrm{Cl1}$, and $\mathrm{N} 2$ and $\mathrm{C} 19$ in mutual pseudo trans positions. The Sn-N1 and Sn-N2 distances are 2.5345(18) and 3.2736(19) $\AA$ for the second molecule and the bond angle between C1-Sn-C10 in these molecules is $130.66^{\circ}$ and $134.15^{\circ}$ instead of $180^{\circ}$. The asymmetric crystal unit cell of $\mathbf{3 2}$ show two independent molecules having small differences in some bond distances and angles. ${ }^{29 a}$

Triorganotin compounds tend to favour pentacoordination in the presence of donor molecules due to the increased Lewis acidity of tin. Compounds in Figure 7 contain a potentially intramolecularly coordinating $C, N$-chelating ligand capable of forming a five membered chelate ring. The tin center in these compounds has TBP geometry having the carbon ligands at equatorial sites and the more electronegative nitrogen and halogen atoms at axial positions. NMR $\left({ }^{1} \mathrm{H},{ }^{13} \mathrm{C}\right.$ and ${ }^{119} \mathrm{Sn}$ ) studies of these compounds suggest that they have same structure in both the solid state and in solution. However, fluxional processes become more frequent at higher temperature on the NMR time scale. 
Table 2: ${ }^{119} \mathrm{Sn}$ chemical shifts and $\mathrm{Sn}-\mathrm{N}$ distance of triorganotin halides.

\begin{tabular}{|c|c|c|c|c|c|}
\hline Compounds & $\mathbf{R}$ & $\mathbf{R}^{\prime}$ & $\mathbf{R}^{\prime \prime}$ & ${ }^{119}$ Sn shift $(\delta)$ ppm & Sn-N distance $(\AA)$ \\
\hline $12 \mathrm{a}$ & $\mathrm{H}$ & $\mathrm{Me}$ & $\mathrm{Me}$ & $-50.0^{26,47}$ & - \\
\hline $12 \mathrm{~b}$ & $\mathrm{H}$ & $\mathrm{Me}$ & $\mathrm{Ph}$ & $-111.0^{26,57}$ & - \\
\hline $12 \mathrm{c}$ & $\mathrm{H}$ & $\mathrm{Ph}$ & $\mathrm{Ph}$ & $-182.0^{26,47}$ & $2.511(12)^{54}$ \\
\hline $13 a$ & $\mathrm{Me}$ & $\mathrm{Me}$ & $\mathrm{Me}$ & $-55.7^{56 a}$ & - \\
\hline 13b & $\mathrm{Me}$ & $\mathrm{Me}$ & $\mathrm{Ph}$ & -117.0 and $-117.7^{56 a}$ & $2.476(7)^{56 a}$ \\
\hline $13 c$ & $\mathrm{Me}$ & $\mathrm{Ph}$ & $\mathrm{Ph}$ & $-206.9^{56 a}$ & - \\
\hline $14 a$ & $\mathrm{Et}$ & $\mathrm{Me}$ & $\mathrm{Me}$ & $-53.8^{56 \mathrm{a}}$ & - \\
\hline $14 b$ & $\mathrm{Et}$ & $\mathrm{Me}$ & $\mathrm{Ph}$ & -112.6 and $-115.1^{56 a}$ & - \\
\hline $14 c$ & $\mathrm{Et}$ & $\mathrm{Ph}$ & $\mathrm{Ph}$ & $-187.5^{56 a}$ & - \\
\hline $15 \mathbf{a}$ & $i$-Pr & $\mathrm{Me}$ & $\mathrm{Me}$ & $-46.1^{56}$ & - \\
\hline 15b & $i-\operatorname{Pr}$ & $\mathrm{Me}$ & $\mathrm{Ph}$ & -111.1 and $-113.9^{26}$ & - \\
\hline $15 c$ & $i-\operatorname{Pr}$ & $\mathrm{Ph}$ & $\mathrm{Ph}$ & $-181.6^{56 \mathrm{a}}$ & - \\
\hline $16 \mathbf{a}$ & $t-\mathrm{Bu}$ & $\mathrm{Me}$ & $\mathrm{Me}$ & $-35.4^{56 a}$ & - \\
\hline $16 b$ & $t-\mathrm{Bu}$ & $\mathrm{Me}$ & $\mathrm{Ph}$ & $-102.7^{56 a}$ & $2.552(5), 2.482(5)^{56 a}$ \\
\hline $16 \mathrm{c}$ & $t-\mathrm{Bu}$ & $\mathrm{Ph}$ & $\mathrm{Ph}$ & $-165.3^{56 a}$ & - \\
\hline 20 & $\mathrm{H}$ & $n-\mathrm{Bu}$ & $n-\mathrm{Bu}$ & $-44.9^{29 b}$ & $2.510(5)^{58}$ \\
\hline 22 & $\mathrm{H}$ & $t-\mathrm{Bu}$ & $t-\mathrm{Bu}$ & 18.5 & $2.904(14)^{52}$ \\
\hline $24 a$ & $\mathrm{H}$ & $\mathrm{Me}$ & $\mathrm{Me}$ & $22.8^{32}$ & - \\
\hline $24 b$ & $\mathrm{H}$ & $\mathrm{Me}$ & $\mathrm{Ph}$ & $-46.5^{32}$ & - \\
\hline $24 c$ & $\mathrm{H}$ & $\mathrm{Ph}$ & $\mathrm{Ph}$ & $-122.1^{32}$ & - \\
\hline 25 & - & $\mathrm{Me}$ & $\mathrm{Me}$ & $-25.0^{50}$ & - \\
\hline 26 & - & $\mathrm{Me}$ & $\mathrm{Ph}$ & $-94.8^{50}$ & - \\
\hline 27 & - & $\mathrm{Me}$ & $\mathrm{Me}$ & $-38.7^{27}$ & - \\
\hline 28 & - & $\mathrm{Me}$ & $\mathrm{Ph}$ & $-97.5^{27}$ & $2.496(6)^{28}$ \\
\hline 29 & - & $\mathrm{Ph}$ & $\mathrm{Ph}$ & $-165.1^{27}$ & - \\
\hline
\end{tabular}

${ }^{1}$ H NMR spectra of compounds 13b-13d, 14b-14d, 15b-15d and 16b-16d reveal a TBP structure for tin, where the $\mathrm{C}$-ligands occupy equatorial positions and the $\mathrm{N}$ an axial position. For compounds 13b-15b, two distinct patterns were observed in their ${ }^{1} \mathrm{H}$ NMR spectra indicative of two diastereoisomers. These observations are supported by the presence of a chiral center in these

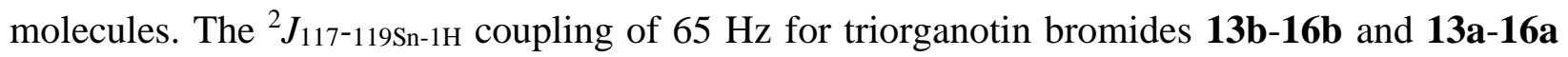
having one and two methyl groups bonded to tin also confirm the TBP geometry. ${ }^{56 a}$

The ${ }^{119} \mathrm{Sn}$ NMR data for trioganotin halides (Table 2) suggest that the formation of pentacoordinated species results in a relatively large upfield shift of ${ }^{119} \mathrm{Sn}$ NMR resonance 
compared to similar four coordinate tin species. For example, the ${ }^{119} \mathrm{Sn}$ NMR resonance for $\mathbf{1 2 c}-$ 16c range from $\delta=-105$ to $-145 \mathrm{ppm}$ compared to $\delta=-60 \mathrm{ppm}$ for $\mathrm{Ph}_{3} \mathrm{SnBr}^{56 \mathrm{~b}}$ The upfield shift of ${ }^{119} \mathrm{Sn}$ resonances is also consistent with the substitution of methyl for phenyl groups. The ${ }^{119} \mathrm{Sn}$ chemical shifts of triorganotin halides in these studies are independent of temperature (between 80 to $100{ }^{\circ} \mathrm{C}$ ) suggesting that even in the presence of fluxional process involving $\mathrm{Sn}-\mathrm{N}$ dissociation-association observable by ${ }^{1} \mathrm{H}$ and ${ }^{13} \mathrm{C}$ NMR spectroscopy, the Sn center retains its pentacoordinated geometry. Two well resolved ${ }^{119} \mathrm{Sn}$ resonances were also observed for compounds 13c-16c indicative of the existence of two diastereoisomers.

The VT ${ }^{119} \mathrm{Sn} \mathrm{NMR}$ of $\mathbf{2 5}$ and $\mathbf{2 6}$ indicate a strong temperature dependence, with an increasing downfield shift observed with increasing temperature. A significant ${ }^{119} \mathrm{Sn}$ NMR chemical shift variation was observed for $\mathbf{2 5}$ and $\mathbf{2 6}$ from $-49.4 \mathrm{ppm}$ and $-131.1 \mathrm{ppm}$ at $-85^{\circ} \mathrm{C}$ to -6.2 ppm and $-72.0 \mathrm{ppm}$ at $100{ }^{\circ} \mathrm{C}$, respectively. This downfield shift may be due to the predominance of tetrahedral coordination geometry for the tin centres at the higher temperature. The five membered ring in compounds 12-24 and 27-31 are more flexible than in 25 and 26 and this increased flexibility may promote $\mathrm{Sn}-\mathrm{N}$ dissociation, resulting in tetracoordinate tin species and a downfield shift of the ${ }^{119} \mathrm{Sn}$ NMR resonance.

\subsubsection{Diorganotin dihalides containing $C, N$-chelating ligands:}
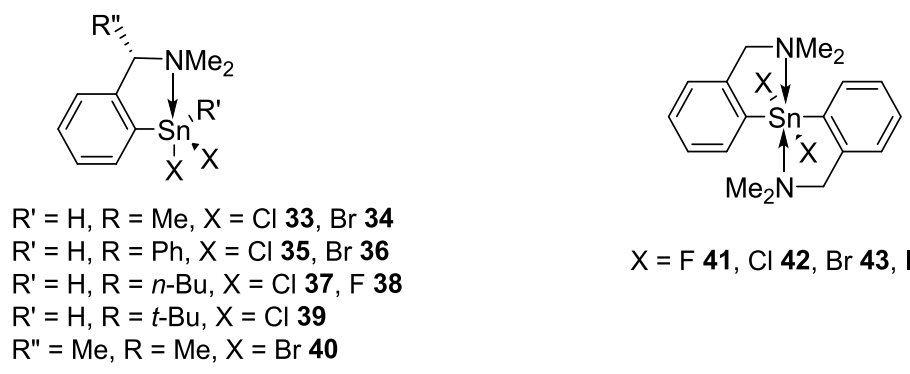

$\mathrm{X}=\mathrm{F} 41, \mathrm{Cl} 42, \mathrm{Br} 43, \mathrm{I} 44$

Figure 9: Diorganotin dihalides. 


\subsubsection{4a Synthesis:}

Diorganotin(IV) dihalides 33, $\mathbf{3 5}$ and $\mathbf{3 7}$ are compounds of interest in this thesis as they can be used directly or after modification to dihydrides as potential monomers. van Koten et al. ${ }^{59}$ synthesized compounds $\mathbf{3 3}$ and $\mathbf{3 5}$ by reacting [2-( $\left.\left.\mathrm{Me}_{2} \mathrm{NCH}_{2}\right) \mathrm{C}_{6} \mathrm{H}_{4}\right]_{4} \mathrm{Cu}_{4}$ with $\mathrm{MeSnCl}_{3}$ and $\mathrm{PhSnCl}_{3}$ respectively in $\mathrm{C}_{6} \mathrm{H}_{6}$ at room temperature. Rippstein et al. ${ }^{48}$ prepared $\mathbf{3 5}$ from the reaction of [2-( $\left.\left.\mathrm{Me}_{2} \mathrm{NCH}_{2}\right) \mathrm{C}_{6} \mathrm{H}_{4}\right] \mathrm{Li}$ with $\mathrm{PhSnCl}_{3}$ in $\mathrm{Et}_{2} \mathrm{O}$ and purified by toluene extraction. Novák et al. ${ }^{29 \mathrm{a}}$ reacted the $\left[2-\left(\mathrm{Me}_{2} \mathrm{NCH}_{2}\right) \mathrm{C}_{6} \mathrm{H}_{4}\right] \mathrm{Li}$ salt with $\mathrm{PhSnCl}_{3}$ in $\mathrm{C}_{6} \mathrm{H}_{6}$ to prepare 35. Varga et al. ${ }^{51}$ synthesized compound 37 by reacting [2-( $\left.\left.\mathrm{Me}_{2} \mathrm{NCH}_{2}\right) \mathrm{C}_{6} \mathrm{H}_{4}\right] \mathrm{Li}$ with $n-\mathrm{BuSnCl}_{3}$ in $\mathrm{C}_{6} \mathrm{H}_{6}$ and purified the product by extraction with hot petroleum ether. Compound $\mathbf{3 8}$ was obtained by reacting compound 37 with $\mathrm{KF}$ in $\mathrm{MeOH}$ at room temperature. Compounds 42 and 44 were also synthesized by reacting [2-( $\left.\left.\mathrm{Me}_{2} \mathrm{NCH}_{2}\right) \mathrm{C}_{6} \mathrm{H}_{4}\right] \mathrm{Li}$ with $\mathrm{SnX}_{4}(\mathrm{X}=\mathrm{Cl}$, I) in a 2:1 ratio.

\subsubsection{4b Structural studies:}

In compounds 35 and 37 the distance between $\mathrm{Sn}-\mathrm{N}$ is $2.444(5) \AA$ and $2.458(5) \AA$ respectively. These hypercoordinated molecules have a 3c-4e trans configuration ((N(1)-Sn(1)$\mathrm{Cl}(1)=168.15^{\circ}(12), 171.61^{\circ}(15)$ respectively) that contribute to a strong intramolecular $\mathrm{Sn}-\mathrm{N}$

interaction. The molecules possess a distorted TBP $(C, N-) \mathrm{CSnCl}_{2}$ core (hypervalent $\left.10-\mathrm{Sn}-5\right) .{ }^{29 \mathrm{a}, 51}$ Compounds 35 and 37 share two common features in their molecular structures; ${ }^{41}$ a strong coordination between the $\mathrm{Sn}$ and $\mathrm{N}$ atoms of the pendant $\mathrm{Me}_{2} \mathrm{NCH}_{2}$ arm that result in an increase in the coordination number at tin, and the $\mathrm{SnC}_{3} \mathrm{~N}$ ring folded along $\mathrm{Sn}(1)--\mathrm{CH}_{2}$ atoms.

The tin atoms in compounds 42 and 44 are bound to two $\mathrm{L}$ groups $(\mathrm{L}=2$ $\left.\left(\mathrm{Me}_{2} \mathrm{NCH}_{2}\right) \mathrm{C}_{6} \mathrm{H}_{4}\right)$ ) and two cis-bonded halides $(\mathrm{Cl}, \mathrm{I})$ with the molecules possessing pseudo octahedral geometry. The C-Sn-C bond angle for $\mathbf{4 2}$ and $\mathbf{4 4}$ are $152.47^{\circ}(7)$ and $157.97^{\circ}(11)$ respectively, whereas the ideal bond angle for octahedral geometry is $180^{\circ}$. Similarly, the N-Sn-N 
angles are $108.47^{\circ}(4)$ for $\mathbf{4 2}$ and $103.65^{\circ}(7)$ for $\mathbf{4 4}$ compared to the ideal $90^{\circ}$. Comparatively large Sn-N distances of 2.6179(13) $\AA$ for $\mathbf{4 2}$, and 2.537(2) $\AA$ and 2.648(2) $\AA$ for $\mathbf{4 4}$ were observed.

Table 3: ${ }^{119} \mathrm{Sn}$ chemical shifts and $\mathrm{Sn}-\mathrm{N}$ distances of diorganotin halides.

\begin{tabular}{|c|c|c|c|c|c|}
\hline Compounds & R" & R' & $\mathbf{X}$ & ${ }^{119}$ Sn Shift(ס) ppm & Sn-N distance (̊) \\
\hline 33 & $\mathrm{H}$ & $\mathrm{Me}$ & $\mathrm{Cl}$ & $-94.0^{26,59}$ & - \\
\hline 34 & $\mathrm{H}$ & $\mathrm{Me}$ & $\mathrm{Br}$ & $-141.4^{26,59}$ & - \\
\hline 35 & $\mathrm{H}$ & $\mathrm{Ph}$ & $\mathrm{Cl}$ & -167.6 & $2.444(5)^{57}$ \\
\hline 36 & $\mathrm{H}$ & $\mathrm{Ph}$ & $\mathrm{F}$ & - & - \\
\hline 37 & $\mathrm{H}$ & $n-\mathrm{Bu}$ & $\mathrm{Cl}$ & $-104.3,{ }^{29 b} 103.0^{51}$ & $2.428(3)^{64}, 2.458^{57}$ \\
\hline 38 & $\mathrm{H}$ & $n-\mathrm{Bu}$ & $\mathrm{F}$ & - & $2.494^{51}$ \\
\hline 39 & $\mathrm{H}$ & $t$-Bu & $\mathrm{Cl}$ & - & - \\
\hline 40 & $\mathrm{Me}$ & $\mathrm{Me}$ & $\mathrm{Br}$ & $-145.0^{26,59}$ & - \\
\hline 41 & $\mathrm{~L}$ & $\mathrm{~L}$ & $\mathrm{~F}$ & -216.1 & $2.496(2), 2.597(1)^{60}$ \\
\hline 42 & $\mathrm{~L}$ & $\mathrm{~L}$ & $\mathrm{Cl}$ & -252.8 & $2.6179(13)^{29 a}$ \\
\hline 43 & $\mathrm{~L}$ & $\mathrm{~L}$ & $\mathrm{Br}$ & $-269.6^{29 b, 59}$ & - \\
\hline 44 & $\mathrm{~L}$ & $\mathrm{~L}$ & I & -347.4 & $2.537(2), 2.648(2)^{29 a}$ \\
\hline
\end{tabular}

The ${ }^{119} \mathrm{Sn}$ NMR chemical shift data of pentacoordinated diorganotin dihalides $\mathbf{3 3}(\delta=-94.0$ $\mathrm{ppm}), 34(\delta=-141.4 \mathrm{ppm})$, and the triorganotin halide 12a $(\delta=-50.0 \mathrm{ppm})$ show an upfield shift relative to their 4-coordinate analogues. Substitution of the $\mathrm{Cl}$ in $\mathbf{3 3}$ with $\mathrm{Br}$ in $\mathbf{3 4}$ produced a downfield shift of about $50 \mathrm{ppm}$. A similar trend was observed for the diorganotin dihalide $\mathbf{3 7}$ $\left({ }^{119} \mathrm{Sn} \delta=-104.3 \mathrm{ppm}\right)$ and triorganotin halide $20(\delta=-44.9 \mathrm{ppm})$.

\subsubsection{Monoorganotin trihalides containing $C, N$-chelating ligands:}

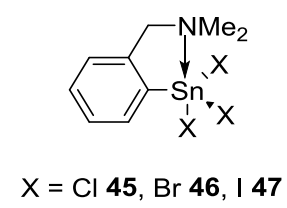

Figure 10: Monoorganotin halides. ${ }^{61}$

Compounds 45-47 were obtained in low yields from the reaction of a 1:1 ratio of LLi and $\mathrm{SnX}_{4}\left(\mathrm{~L}=\left[2-\left(\mathrm{Me}_{2} \mathrm{NCH}_{2}\right) \mathrm{C}_{6} \mathrm{H}_{4}\right] \mathrm{Li}, \mathrm{X}=\mathrm{Cl}, \mathrm{Br}, \mathrm{I}\right)$. A strong intramolecular Sn-N interaction in the hypervalent compounds 45-47 was observed from an up-field shift of the ${ }^{119}$ Sn resonances $(\delta=$ - 
227.4, -408 (broad), and $-944.4 \mathrm{ppm})^{61}$ compared to the trihalophenyl compounds, $\mathrm{PhSnX}_{3}(\delta=$ $-61.3,-227.2$ and $-699.9 \mathrm{ppm}) .{ }^{62}$ The Lewis acidity of the tin atom decreases with the lower electronegativity of $X$ which is evident from the ${ }^{3} J_{119 S n-1 \mathrm{H}}$ coupling constant for $\mathbf{4 5 - 4 7}$ (132.0, 128.6 and $126.2 \mathrm{~Hz}$ ). A distorted TBP geometry is observed in all compounds and the bond distance of the Sn-N dative interaction in $\mathbf{4 5 - 4 7}$ is $2.380(2), 2.402(3)$ and 2.436(4) $\AA$, respectively. A slight increase in the $\mathrm{Sn}-\mathrm{N}$ bond distance going from $\mathbf{4 5}$ to $\mathbf{4 7}$ is related to the relative electronegativities and sizes of $\mathrm{X}^{61}$

Data obtained for organotin compounds containing $C, N$-chelating ligands in noncoordinating apolar solvents indicate the existence of intramolecular Sn-N donor-acceptor interactions of variable strength. It increases with the decreasing number of organic substituents at the tin center. ${ }^{27 b}$ The ${ }^{119}$ Sn NMR data of $\mathbf{4 5 - 4 7}$ show the influence of additional coordination on chemical shift, which is relatively minor in the case of tetraorganotin compounds, but results in large upfield shifts for triorganotin halides and diorganotin dihalides. ${ }^{26}$

\subsubsection{Organotin hydrides containing $C, N$-chelating ligands:}

The preparation of organotin hydrides was first reported by Kraus and Geer in 1922 by reacting $\mathrm{Me}_{3} \mathrm{SnNa}$ with $\mathrm{HCl}$ in liquid ammonia. ${ }^{63}$ A revolution in the synthesis of tin hydrides occured in 1947 when Finholt et al. reported a convenient method to reduce organotin chlorides with lithium aluminum hydride $\left(\mathrm{LiAlH}_{4}\right){ }^{64}$ Afterwards, this method was successfully used for the synthesis of $\mathrm{R}_{n} \mathrm{SnH}_{4-\mathrm{n}}$. The most frequently used methods for the preparation of organotin hydrides involve reducing agents such as silicon, lithium and boron hydrides. Some other methods such as thermal decomposition of organotin formates, hydrolysis of stannyl metallic compounds and ligand exchange between organotin compounds are less commonly used. ${ }^{65}$ 


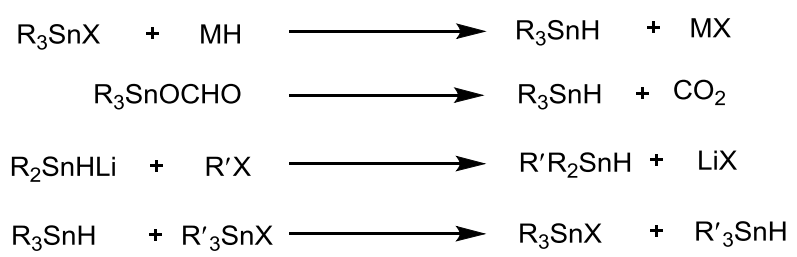

Scheme 7: The preparation of organotin hydrides. ${ }^{65}$

Organotin hydrides with chelating ligands containing donor atoms such as $\mathrm{N}, \mathrm{P}$ or $\mathrm{O}$ atom have received increasing attention in recent years due to their unusual structural properties as well as their industrial and pharmacological applications. ${ }^{74,75}$ The donor-acceptor interaction between Sn and the donor atom of the ligand is the most interesting aspect of these molecules. Surprisingly, there are few examples of hydrides having $\mathrm{Sn}-\mathrm{N}$ intramolecular interactions reported in literature. ${ }^{66-72}$ These donor-acceptor interactions have been confirmed by various techniques such as X-ray crystallogaphy ${ }^{76}$ and NMR spectroscopy. ${ }^{66-72,76}$

\subsubsection{Triorganotin hydrides containing $C, N$-chelating ligands:}
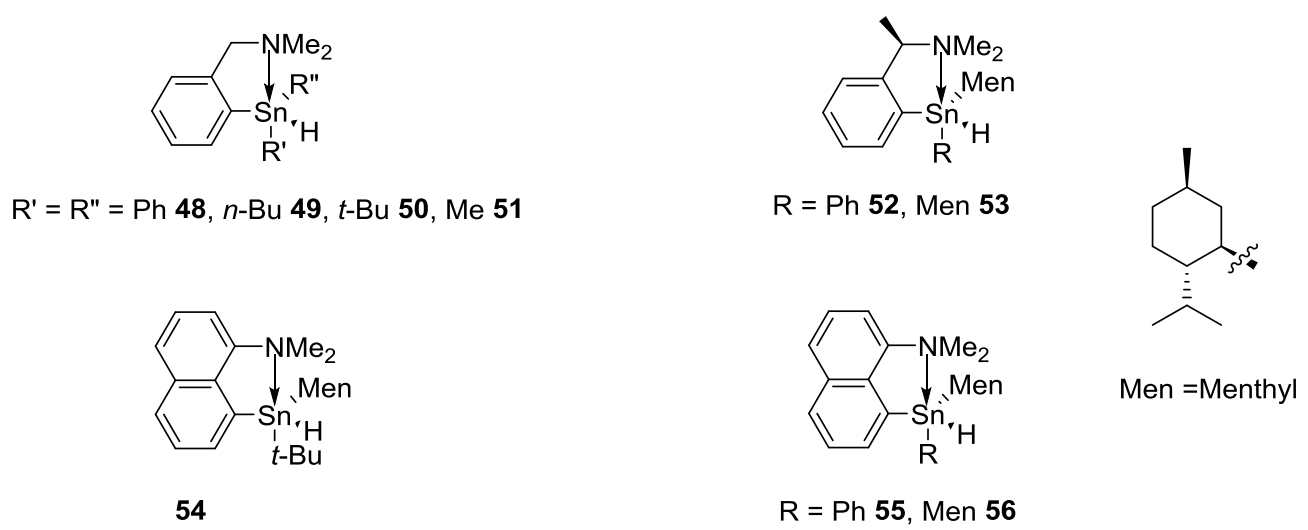

Figure 11: Triorganotin hydrides.

\subsubsection{1a Synthesis:}

Turek et al ${ }^{66}$ prepared triorganotin hydrides 48-50 by reacting [2-( $\left.\left.\mathrm{Me}_{2} \mathrm{NCH}_{2}\right) \mathrm{C}_{6} \mathrm{H}_{4}\right] \mathrm{R}_{2} \mathrm{SnX}$ $(\mathrm{R}=\mathrm{Ph}, n-\mathrm{Bu}, t-\mathrm{Bu}$ and $\mathrm{X}=\mathrm{Cl})$ with $\mathrm{K}\left(\mathrm{BEt}_{3}\right) \mathrm{H}$ in a $1: 1$ ratio in $\mathrm{THF}$ at $-20^{\circ} \mathrm{C}$ and stirring at room temperature for 5 days. These hydrides show typical ${ }^{1} \mathrm{H}$ NMR $\left(\mathrm{C}_{6} \mathrm{D}_{6}\right)$ hydride resonances at 6.98 , 5.83 and 6.01 ppm for $\mathbf{4 8}, \mathbf{4 9}$ and $\mathbf{5 0}$ respectively. Compound $\mathbf{5 1}$ was prepared from the reaction 
of $\left[2-\left[\left(\mathrm{Me}_{2} \mathrm{NCH}_{2}\right) \mathrm{C}_{6} \mathrm{H}_{4}\right] \mathrm{SnMe}_{2} \mathrm{Br}\right.$ or the analogous chloride with $\mathrm{LiAlH}_{4}$ in $\mathrm{THF} .{ }^{67} \mathrm{Compound}^{52}$ was prepared by treating the 2-[( $\left.\left.\mathrm{Me}_{2} \mathrm{NCHMe}\right) \mathrm{C}_{6} \mathrm{H}_{4}-\right]$ ligand containing triorganotin monohalides with $\mathrm{NaBH}_{4}$ in EtOH, as $\mathrm{LiAlH}_{4}$ was found to decompose the halide. Hydrides 53-56 were also obtained by treating the corresponding chlorides with $\mathrm{LiAlH}_{4}{ }^{68}$

\subsubsection{1b Structural studies:}

For triorganotin hydrides the ${ }^{1} \mathrm{H}$ NMR resonance for the proton directly bonded to the tin atom varies between 4.5-7.5 ppm. ${ }^{65}$ The presence of a ${ }^{117 / 119} \mathrm{Sn}$ couplings is a characteristic feature in ${ }^{1} \mathrm{H}$ NMR spectra of these compounds. The magnitude of this coupling is dependent on substituents bonded to tin. Tin hydrides which contain bulky alkyl groups have relatively smaller values of ${ }^{1} J_{117 / 119 \mathrm{Sn}-1 \mathrm{H}}$ whereas tin hydrides containing aromatic rings cause an increase in ${ }^{1} J_{117 / 119 S n-1 \mathrm{H}}$ coupling. The characteristic changes of the NMR chemical shifts for $\mathrm{Sn}$ and $\mathrm{N}$ and the

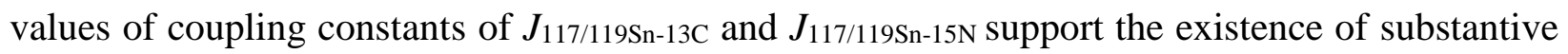
donor-acceptor interactions. Examples of tin hydrides having a donor-acceptor interaction between $\mathrm{Sn}$ and $\mathrm{N}$ include the triorganotin hydrides with 2-(N,N-dimethylaminomethyl)phenyl and 2-(4isopropyl-2-oxazolinyl)-5-phenyl substituents ${ }^{77,78}$ and diorganotin dihydrides containing 2-(4isopropyl-2-oxazolinyl)-5-phenyl as chelating ligands and phenyl, methyl, $n$-butyl and $t$-butyl substituents (Figure 13). ${ }^{66,69}$

For hypercoordinated tin hydrides 60-62 as shown in Figure 13, the $\mathrm{Sn}-\mathrm{N}$ interaction is evident from the increase in the value of coupling constants. A comparison of ${ }^{1} J_{117 / 119 S n-1 \mathrm{H}}$ coupling between tetravalent compound 57, 58, and 59 and pentavalent 60,61 and 62 show the difference in the nature of substituents bound to tin (Table 4). ${ }^{65,70}$ 


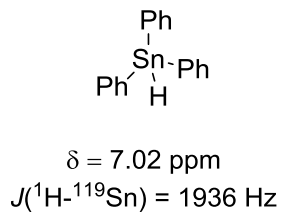

57

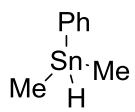

$4.66 \mathrm{ppm}$ $1744 \mathrm{~Hz}$

58

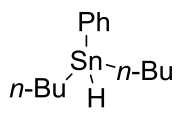

4.84 ppm $1609 \mathrm{~Hz}$

59

Figure 12: Structure of triorganotin hydrides without chelating ligand.

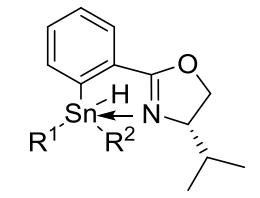

$\mathrm{R}^{1}=\mathrm{R}^{2}=\mathrm{Ph} 60$

$R^{1}=R^{2}=M e 61$

$\mathrm{R}^{1}=\mathrm{R}^{2}=n-\mathrm{Bu} 62$

$\mathrm{R}^{1}=\mathrm{Ph}, \mathrm{R}^{2}=t-\mathrm{Bu} 63$

$R^{1}=M e, R^{2}=t-B u 64$

$R^{1}=P h, R^{2}=$ Me 65

$\mathrm{R}^{1}=\mathrm{Me}, \mathrm{R}^{2}=n-\mathrm{Bu} 66$

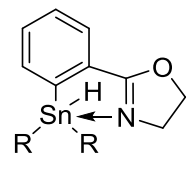

$\mathrm{R}=\mathrm{Me} 67$

$\mathrm{R}=\mathrm{Ph} 68$

Figure 13: Structures of hydrides containing chelating ligands.

Hydrides 65-72 were synthesized by Cmoch et al. ${ }^{69,71,72}$ by reacting the monohalides $(\mathrm{Br}$, I) with $\mathrm{NaBH}_{4}$ in EtOH at $0-25^{\circ} \mathrm{C}$. Unfortunately, X-ray structures of these low melting hydrides have not yet been reported. There is only one example of a triorganotin hydride, $\mathbf{5 4}$, which has been structurally determined by X-ray crystallography. The structure of $\mathbf{5 4}$ revealed the presence of both epimers in 1:1 ratio. The coordination geometry of $\mathbf{5 4}$ is a monocapped tetrahedron having only a weak Sn-N interaction; evident from the very long Sn-N distances of 2.931(3) and 2.885(3) $\AA$ in this molecule. 
Table 4: ${ }^{1} \mathrm{H}$ and ${ }^{119} \mathrm{Sn}$ NMR data of hypercoordinate triorganotin hydrides.

\begin{tabular}{|c|c|c|c|}
\hline Compound & $\delta^{1} \mathbf{H}(\mathbf{p p m})$ Sn-H & $\delta^{119} \mathrm{Sn}$ or $\delta{ }^{117} \mathrm{Sn}(\mathrm{ppm})$ & $J_{117 / 119 S n-1 \mathrm{H}}(\mathrm{Hz})$ \\
\hline 48 & 6.98 & -180.9 & $2128^{66}$ \\
\hline 49 & 5.83 & -113.2 & $1627^{66}$ \\
\hline 50 & 6.01 & -90.0 & $1638^{66}$ \\
\hline 51 & 5.64 & -30.2 & $1696 / 1777^{67}$ \\
\hline 52 & 6.46 & $-136.7,-166.8$ & $1904 / 1920^{67}$ \\
\hline 53 & 5.99 & -141.3 & $1671^{68}$ \\
\hline 54 & $6.37,6.20$ & $-91.3,-94.8$ & $1723 / 1803,1717 / 1797^{76}$ \\
\hline 55 & $6.71,6.91$ & $-140.0,-115$ & $1980,1955^{68}$ \\
\hline 56 & 6.31 & -104.6 & $1729^{68}$ \\
\hline 60 & 7.62 & -158.5 & $2167 / 2070^{70}$ \\
\hline 61 & 6.20 & -129.0 & $1753^{69}$ \\
\hline 62 & 6.42 & -92.0 & $1503^{69}$ \\
\hline 63 & $7.15,7.35$ & $-102.0,118.9$ & $1480 / 1549,1832 / 1908^{71}$ \\
\hline 64 & $6.35,6.46$ & $-60.0,-91.0$ & $1380 / 1444,1692 / 1770^{71}$ \\
\hline 65 & $6.66,6.67$ & $-160.0,-159.2$ & $1933 / 2022,1897 / 1985^{72}$ \\
\hline 66 & $6.14,6.14$ & $-112.1,-117.7$ & $1615 / 1688,1548 / 1615^{72}$ \\
\hline 67 & 6.09 & $\begin{array}{ll}-128.8 \\
\end{array}$ & $1597 / 1971^{72}$ \\
\hline 68 & 7.41 & -180.6 & $1850 / 1937^{72}$ \\
\hline
\end{tabular}

\subsubsection{Diorganotin dihydrides containing $C, N$-chelating ligands:}

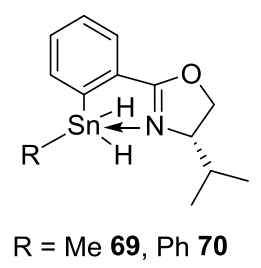

Figure 14: Hypercoordinated diorganotin dihydrides.

Dihydrides 69 and 70 (Figure 14) were prepared by Matkowska et al. ${ }^{72}$ from the reaction of dihalides (I, Br) with $\mathrm{NaBH}_{4}$ in $\mathrm{EtOH}$ at $0-25^{\circ} \mathrm{C}$. These compounds have two distinct hydrogen 
atoms directly bonded to tin. The comparison of ${ }^{1} J_{117 / 119 S n-1 \mathrm{H}}$ coupling constants of coordinated hydrides for 69 and 70 and noncoordinated analogues $\mathrm{PhMeSnH}_{2}$ and $\mathrm{Ph}_{2} \mathrm{SnH}_{2}$ provides evidence that $\mathrm{Sn}-\mathrm{N}$ interaction/coordination can cause both a strong decrease and increase of ${ }^{1} J_{117 / 119 \mathrm{Sn}-1 \mathrm{H}}$ coupling constant for 69 and negligible/strong coupling increase for 70 (Table 5). The effect of temperature ${ }^{1} J_{117 / 119 S n-1 \mathrm{H}}$ on coupling constants for these species were also investigated. It was concluded that the variation in the values of coupling constants at different temperatures may be due to the axial/equatorial positions of hydrogen atoms; one is axial and one is equatorial in 69 and both are equatorial in 70. ${ }^{117 / 119} \mathrm{Sn}$ satellites in the ${ }^{1} \mathrm{H}$ NMR spectra are doubled due to the presence of intramolecular $\mathrm{Sn}-\mathrm{N}$ coordination.

Table 5: ${ }^{1} J_{117 / 119 S n-1 \mathrm{H}}$ coupling constants of coordinated and non-coordinated hydrides.

\begin{tabular}{|c|c|c|c|c|}
\hline Compound & $\begin{array}{c}\mathbf{1} \mathbf{H}(\mathbf{p p m}) \\
\mathbf{S n - H}\end{array}$ & $\boldsymbol{J}_{117 / 119 S n-H}(\mathbf{H z})$ & $\begin{array}{c}\text { Unsubstituted } \\
\text { analogue }\end{array}$ & $\boldsymbol{J}_{117 / 119 S n-H}(\mathbf{H z})$ \\
\hline $\mathbf{6 9}$ & $6.17-6.04$ & $\begin{array}{c}1615 / 1703, \\
1888 / 1987\end{array}$ & $\mathrm{PhMeSnH}_{2}$ & $1771 / 1835^{73}$ \\
\hline $\mathbf{7 0}$ & $6.78-6.64$ & $\begin{array}{c}1853 / 1940, \\
2059 / 2155\end{array}$ & $\mathrm{Ph}_{2} \mathrm{SnH}_{2}$ & $1842 / 1928^{73}$ \\
\hline
\end{tabular}

\subsubsection{Si, Ge and Pb compounds containing a $C, N$-chelating ligand:}

Hypervalent silicon compounds (Figure 15) have been known since the beginning of $19^{\text {th }}$ century. ${ }^{77}$ Boyer et al ${ }^{78}$ prepared and characterized a number of these compounds, including $\mathbf{7 2}$, 81, 94 and 96 which were structurally studied by both X-ray crystallography and solution NMR spectroscopy. The geometry of these hypercoordinated silicon compounds was exclusively distorted TBP having the nitrogen atom at an axial position. Five- and six-membered chelate rings were formed in case of $\mathbf{8 1 , 9 4}$ and 97 respectively. Here the five-membered rings remain planar whereas the six-membered ring, such as $\mathbf{9 7}$, folds about the $\mathrm{Si}-\mathrm{CH}_{2}$ group axis. The naphthyl group in 81 and the phenyl group in 94 and 97 occupy the second axial position of the TBP complexes. 
Equatorial sites of TBP geometry were occupied by two hydrogen atoms in $\mathbf{8 1}$ and $\mathbf{9 4}$, whereas one hydrogen atom and one methyl group are found in $\mathbf{9 7}$. A comparison of the geometries of $\mathbf{9 8 \mathbf { b }}$ e (Figure 16) by X-ray diffraction revealed intramolecular coordination between the donor nitrogen atom and silicon center, resulting in five coordinate geometries.

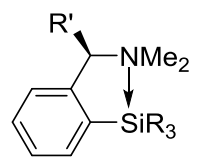

$\mathrm{R}^{\prime}=\mathrm{H}, \mathrm{R}=\mathrm{Me} 71$

$\mathrm{R}^{\prime}=\mathrm{H}, \mathrm{R}=\mathrm{F} 72$

$\mathrm{R}^{\prime}=\mathrm{Me}, \mathrm{R}=\mathrm{Me} 73$

$R^{\prime}=M e, R=F 74$

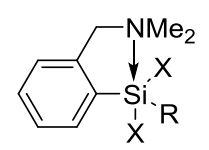

$\mathrm{R}=\mathrm{Me}, \mathrm{X}=\mathrm{F} 83$

$\mathrm{R}=\mathrm{Me}, \mathrm{X}=\mathrm{Cl} 84$

$\mathrm{R}=\mathrm{Et}, \mathrm{X}=\mathrm{Cl} 85$

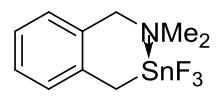

93

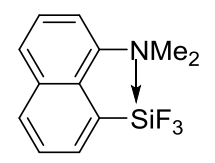

96

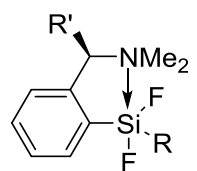

$\mathrm{R}^{\prime}=\mathrm{H}, \mathrm{R}=\mathrm{Ph} 75$

$\mathrm{R}^{\prime}=\mathrm{Me}, \mathrm{R}=\mathrm{Ph} 76$

$\mathrm{R}^{\prime}=\mathrm{H}, \mathrm{R}=\mathrm{Np} 77$

$R^{\prime}=\mathrm{Me}, \mathrm{R}=\mathrm{Np} 78$

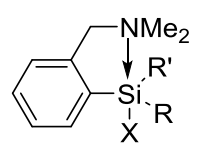

$\mathrm{R}^{\prime}=\mathrm{Me}, \mathrm{R}=\mathrm{H}, \mathrm{X}=\mathrm{F} \mathbf{8 6}$

$R^{\prime}=\mathrm{Me}, \mathrm{R}=\mathrm{H}, \mathrm{X}=\mathrm{Cl} 87$

$R^{\prime}=M e, R=H, X=B r 88$

$\mathrm{R}^{\prime}=\mathrm{Me}, \mathrm{R}=\mathrm{Np}, X=\mathrm{H} \mathbf{8 9}$

$\mathrm{R}^{\prime}=\mathrm{Me}, \mathrm{R}=\mathrm{Np}, \mathrm{X}=\mathrm{F} \mathbf{9 0}$

$\mathrm{R}^{\prime}=\mathrm{Me}, \mathrm{R}=\mathrm{Np}, \mathrm{X}=\mathrm{Cl} 91$

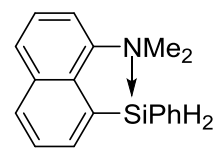

94

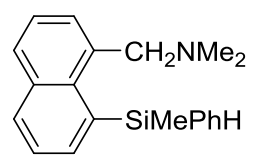

97

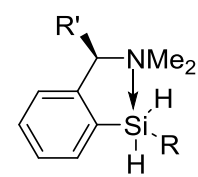

$\mathrm{R}^{\prime}=\mathrm{H}, \mathrm{R}=\mathrm{Ph} \mathbf{7 9}$

$\mathrm{R}^{\prime}=\mathrm{Me}, \mathrm{R}=\mathrm{Ph} \mathbf{8 0}$

$R^{\prime}=\mathrm{H}, \mathrm{R}=\mathrm{Np} \mathbf{8 1}$

$\mathrm{R}^{\prime}=\mathrm{Me}, \mathrm{R}=\mathrm{Np} 82$

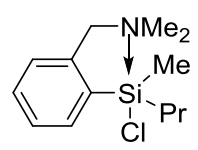

92

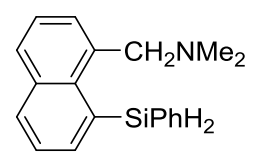

95

Figure 15: Si compounds containing a $C, N$-chelating ligand.

In most of these hypervalent compounds the axial positions are occupied by the donor atom with the halogen or hydride located in a trans configuration. The distance between donor and acceptor is significantly longer than a normal single bond distance between these two atoms. In 
the case of $98 \mathrm{e}$, the distance between the datively bonded $\mathrm{Si}$ and $\mathrm{N}$ atoms is $2.03 \AA$, whereas length of covalent bond between these atoms is $1.77 \AA$.

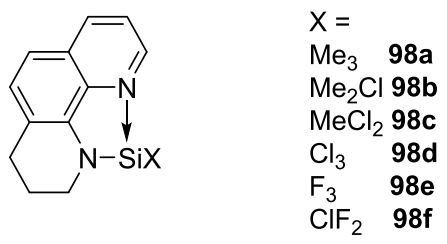

Figure 16: Organosilicon compounds containing a $C, N$-chelating ligand. ${ }^{79}$

Table 6: ${ }^{29} \mathrm{Si}$ chemical shift data for five- vs four-coordinate silanes

\begin{tabular}{|c|c|c|c|c|}
\hline Compound & $\boldsymbol{\delta}\left({ }^{\mathbf{2 9}} \mathbf{S i}\right) \mathbf{~ p p m}$ & $\mathbf{C o m p o u n d}$ & $\boldsymbol{\delta}\left({ }^{\mathbf{2 9}} \mathbf{S i}\right) \mathbf{p p m}$ & $\Delta \boldsymbol{\delta}\left({ }^{\mathbf{2 9}} \mathbf{S i}\right) \mathbf{~ p p m}$ \\
\hline $\mathbf{7 1}$ & -4.8 & $\mathrm{SiMe}_{3} \mathrm{Ph}^{78}$ & -4.1 & -0.7 \\
\hline $\mathbf{7 2}$ & -102.2 & $\mathrm{SiF}_{3} \mathrm{Ph}^{78}$ & -72.8 & -29.4 \\
\hline $\mathbf{8 1}$ & -47.2 & $\mathrm{SiH}_{2} \mathrm{Ph}_{\left(\mathrm{C}_{10} \mathrm{H}_{7}\right)^{78}}$ & -35.6 & -11.6 \\
\hline $\mathbf{8 2}$ & -52.8 & $\mathrm{SiH}_{2} \mathrm{Ph}_{\left(\mathrm{C}_{10} \mathrm{H}_{7}\right)}$ & -35.6 & -17.2 \\
\hline $\mathbf{8 3}$ & -36.1 & $\mathrm{SiMeF}_{2} \mathrm{Ph}^{78}$ & -8.5 & -44.6 \\
\hline $\mathbf{9 3}$ & -100.4 & $\mathrm{SiF}_{3}\left(\mathrm{CH}_{2} \mathrm{Ph}^{78}\right.$ & -64.0 & -36.4 \\
\hline $\mathbf{9 4}$ & -44.1 & $\mathrm{SiH}_{2} \mathrm{Ph}_{\left(\mathrm{C}_{10} \mathrm{H}_{7}\right)}$ & -35.6 & -8.5 \\
\hline $\mathbf{9 5}$ & -55.5 & $\mathrm{SiH}_{2} \mathrm{Ph}\left(\mathrm{C}_{10} \mathrm{H}_{7}\right)$ & -35.6 & -19.9 \\
\hline $\mathbf{9 7}$ & -25.8 & $\mathrm{SiHMePh}\left(\mathrm{C}_{10} \mathrm{H}_{7}\right)^{78}$ & -19.8 & -6.0 \\
\hline
\end{tabular}

${ }^{29} \mathrm{Si}$ NMR spectroscopy is a useful technique to confirm the coordination between the donor atom of the ligand and the silicon atom in solution which is evident by a substantial upfield shift of ${ }^{29} \mathrm{Si}$ resonance. Table 6 lists a comparison of ${ }^{29} \mathrm{Si}$ chemical shift of the coordinated silanes and their analogues. ${ }^{19} \mathrm{~F}$ NMR resonances of Si-F groups and the ${ }^{1} \mathrm{H}$ NMR chemical shift of groups directly bound to the donor atom of the ligand were also useful in probing these interactions. ${ }^{19} \mathrm{~F}$ NMR spectra of compounds 72 and 93 (collected at $30^{\circ} \mathrm{C}$ ) as well as 96 (collected at $80{ }^{\circ} \mathrm{C}$ ) showed sharp resonance at $\delta=-142.5,-138.0$ and -140.7 ppm respectively. On lowering temperature, these compounds showed a downfield triplet and up-field doublet which indicated that the $\mathrm{F}$ atoms occupy one axial and two equatorial positions. X-ray crystal structural analysis of 72 also confirmed its TBP geometry. ${ }^{80}$ 


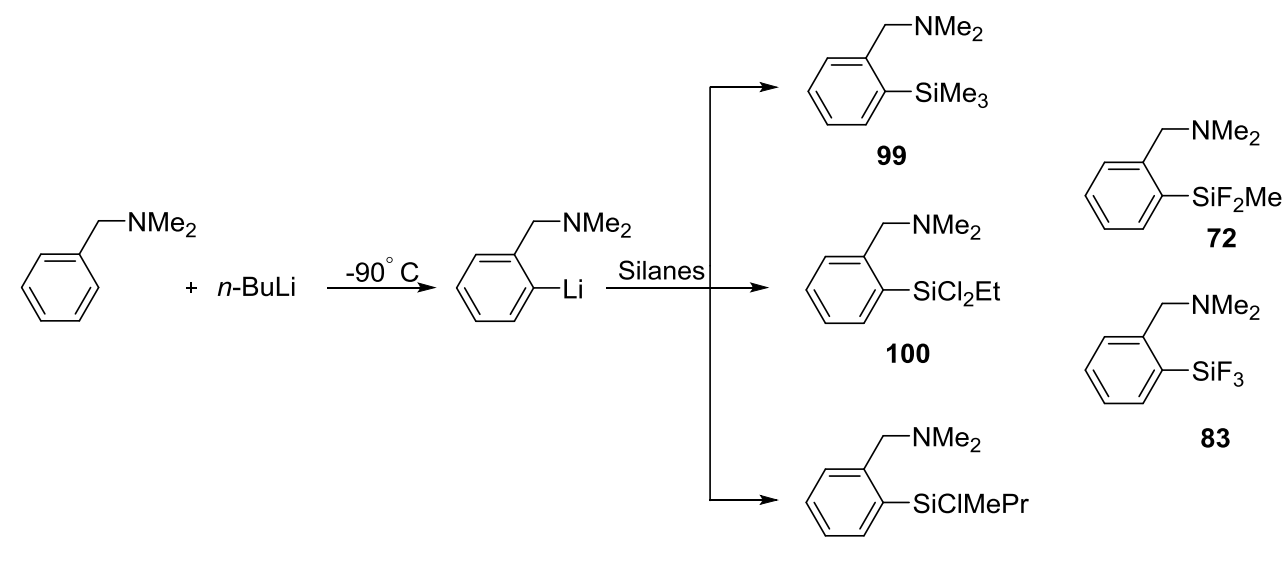

101

Scheme 8: Synthesis of organosilanes containing $C, N$-chelating ligands. ${ }^{82}$

Kelbe et al. synthesized compounds 99-101 along with the known compounds $\mathbf{7 2}$ and $\mathbf{8 3}$ according to Scheme 8 . The ${ }^{19}$ F NMR spectra of fluorosilanes $\mathbf{7 2}$ and $\mathbf{8 3}$ were obtained at low temperature in non-polar media. Compound 72 showed two peaks of equal intensity $\left(\mathrm{F}_{\mathrm{a}} \delta=-111\right.$ ppm; $\left.F_{\mathrm{e}} \delta=-154 \mathrm{ppm}\right)$, while the ${ }^{19} \mathrm{~F}$ NMR spectrum of $\mathbf{8 3}$ displayed a triplet and a doublet in a 1:2 relative intensity $\left(\mathrm{F}_{\mathrm{a}} \delta=-128 \mathrm{ppm} ; \mathrm{F}_{\mathrm{e}} \delta=-148 \mathrm{ppm}\right)$ in good agreement with a TBP geometry (having two equatorial and an apical $\mathrm{F}$ atoms). ${ }^{81}$

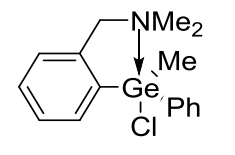

$\mathrm{R}=\mathrm{Ph}, \mathrm{X}=\mathrm{Cl} 102$

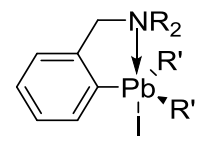

$\mathrm{R}=\mathrm{Me}, \mathrm{R}^{\prime}=\mathrm{Me} 103, \mathrm{Ph} 104$ $\mathrm{R}=\mathrm{Et}, \mathrm{R}^{\prime}=\mathrm{Ph} 105$

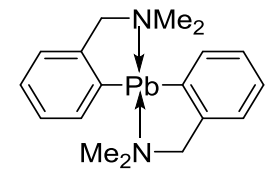

106

Figure 17: Hypercoordinate organogermanium and organolead compounds. ${ }^{81,84,85}$

Krause and Reissaus ${ }^{77}$ reported the synthesis of the first diaryl lead compounds as monomeric species in 1922, but this work could not be reproduced despite attempts by several groups. ${ }^{87-90}$ Compound 106 was synthesized by De Wit et al. ${ }^{84}$ by reacting $\mathrm{PbCl}_{2}$ with two equivalents of $\left[2-\left(\mathrm{Me}_{2} \mathrm{NCH}_{2}\right) \mathrm{C}_{6} \mathrm{H}_{4}\right] \mathrm{Li}$ in THF at $-50{ }^{\circ} \mathrm{C}$. In the ${ }^{1} \mathrm{H}$ NMR spectrum of 106, the ${ }^{3} J_{207 \mathrm{~Pb}-1 \mathrm{H}}$ and ${ }^{4} J_{207 \mathrm{~Pb}-1 \mathrm{H}}$ coupling constants for Ar-H and benzylic- $\mathrm{CH}_{2}$, respectively were detected. 
The $\mathrm{Pb}-\mathrm{N}$ interaction is suggested by the presence of a coupling $\left({ }^{2} J_{207 \mathrm{~Pb}-13 \mathrm{C}}=28 \mathrm{~Hz}\right)$ between lead and the carbon atoms of the $-\mathrm{N}\left(\mathrm{CH}_{3}\right)_{2}$ group.

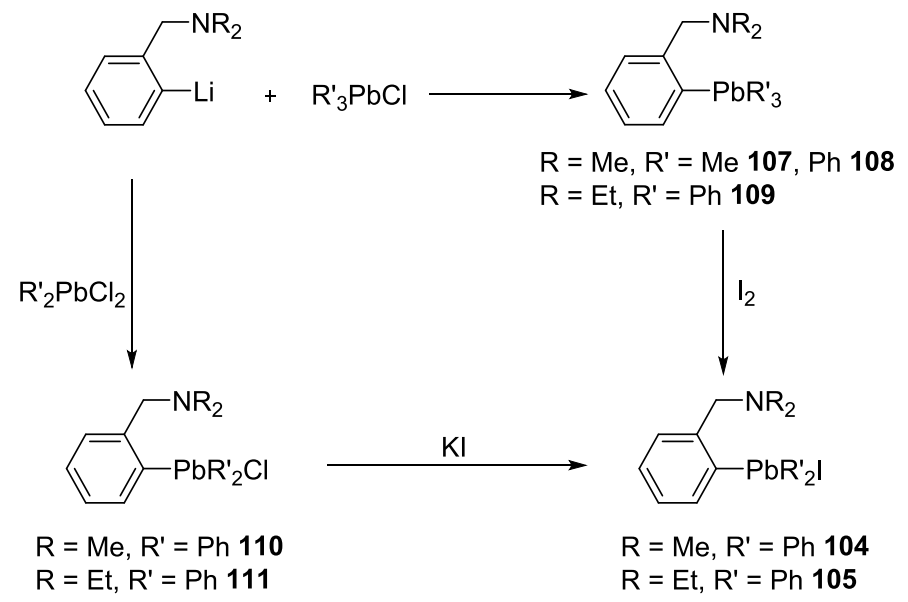

Scheme 9: Synthesis of organolead compounds. ${ }^{84}$

Compounds 104-105 and 107-111 were synthesized by Christea et al. ${ }^{85}$ by treating [2$\left.\left(\mathrm{R}_{2} \mathrm{NCH}_{2}\right) \mathrm{C}_{6} \mathrm{H}_{4}\right] \mathrm{Li}$ with tri- and diorganolead chlorides followed by reaction with $\mathrm{I}_{2}$ and $\mathrm{KI}$ respectively. These compounds were characterized by NMR $\left({ }^{1} \mathrm{H},{ }^{13} \mathrm{C}\right)$ spectroscopy, but unfortunately no ${ }^{207} \mathrm{~Pb}$ NMR data is available. A crystallographic study of $\mathbf{1 0 8}$ and $\mathbf{1 1 0}$ revealed TBP geometry around the $\mathrm{Pb}$ atom and the presence of a $\mathrm{Pb}-\mathrm{N}$ intramolecular interaction. The distance between $\mathrm{Pb}$ and $\mathrm{N}$ atoms is $3.051^{\circ}(9)$ for 108 and $2.636^{\circ}(8), 2.646^{\circ}(9) \AA$ for two distinct molecules of $\mathbf{1 1 0}$ in the unit cell. The bond angle between $\mathrm{N}-\mathrm{Pb}-\mathrm{C}$ for $\mathbf{1 0 8}$ and $\mathrm{Cl}-\mathrm{Pb}-\mathrm{N}(10-\mathrm{Pb}-5)$ for $\mathbf{1 1 0}$ is $169.1^{\circ}(3)$ and $166.11^{\circ}(19), 167.2^{\circ}(2)$ respectively. In the case of compound $\mathbf{1 0 8}$, the axial positions are occupied by $\mathrm{N}$ and the $\mathrm{C}$ atom of one of the phenyl groups while equatorial positions were occupied by carbons of the two other phenyl groups and pendant ligand. The $\mathrm{Pb}-\mathrm{N}$ interaction is much stronger in the case of compound $\mathbf{1 1 0}$ having a $\mathrm{Cl}$ atom trans to $\mathrm{N}$ with $\mathrm{Pb}$ as the central atom. In both compounds the $\mathrm{C}_{3} \mathrm{PbN}$ ring is not planar, thus creating planar chirality. The cleavage of the bond between $\mathrm{Pb}$ and phenyl carbon was preferred over cleavage of the $\mathrm{Pb}$ and pendant group $\mathrm{C}$, which provides additional evidence to support the intramolecular interaction 
between $\mathrm{Pb}$ and $\mathrm{N}$. These results suggest that in general, the geometry of pentacoordinated compounds containing later Group 14 elements ( $\mathrm{Si}, \mathrm{Ge}, \mathrm{Sn}$ and $\mathrm{Pb}$ ) is almost exclusively TBP.

\subsection{Organotin compounds containing $C, O$-chelating ligands:}

Hypervalent compounds of tin are of general interest because of their useful biological activity, reactivity and important industrial and agricultural applications. ${ }^{91-93}$ Most compounds having a Sn-O intramolecular interaction are those containing chelating ligands called "Pincer" ligands. There are several publications which report compounds containing $C, O$-chelating ligands. A variety of tin complexes with $C, O$-ligands are shown in Figure $18,19$.

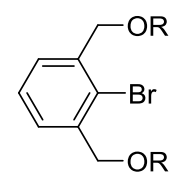

viii

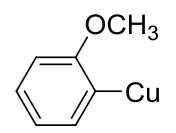

ix

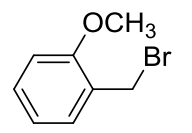

$x$

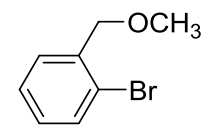

xi

Figure 18: $C, O$-chelating ligands.

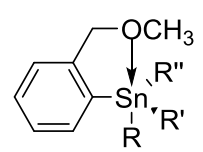

$\mathrm{R}=\mathrm{R}^{\prime}=\mathrm{R}^{\prime \prime}=\mathrm{Ph} 112$

$R=R^{\prime}=R^{\prime \prime}=$ Me 113

$\mathrm{R}=\mathrm{Br}, \mathrm{R}^{\prime}=\mathrm{R}^{\prime \prime}=\mathrm{Me} 114$

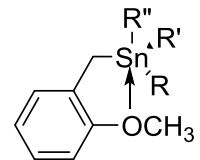

$\mathrm{R}=\mathrm{R}^{\prime}=\mathrm{R}^{\prime \prime}=\operatorname{Me} 115$

$\mathrm{R}=\mathrm{R}=\mathrm{R}^{\prime \prime}=\mathrm{Ph} 116$

$\mathrm{R}=\mathrm{R}^{\prime}=\mathrm{Ph}, \mathrm{R}^{\prime \prime}=\mathrm{Cl} 117$

$\mathrm{R}=\mathrm{Ph}, \mathrm{R}^{\prime}=\mathrm{R}^{\prime \prime}=\mathrm{Cl} 118$

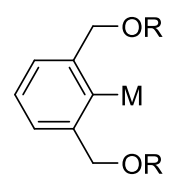

$\mathrm{R}=\mathrm{Me}, \mathrm{M}=\mathrm{SnPh}_{3} 119$

$\mathrm{R}=\mathrm{Me}, \mathrm{M}=\mathrm{SnPh}_{2} \mathrm{Cl} 120$

$\mathrm{R}=\mathrm{Me}, \mathrm{M}=\mathrm{SnPhCl}_{2} 121$

$\mathrm{R}=\mathrm{Me}, \mathrm{M}=\mathrm{SnCl}_{3} 122$

$\mathrm{R}=\mathrm{Et}, \mathrm{M}=\mathrm{SnPh}_{3} 123$

$\mathrm{R}=\mathrm{Et}, \mathrm{M}=\mathrm{SnPh}_{2} \mathrm{Cl} 124$

$\mathrm{R}=\mathrm{Et}, \mathrm{M}=\mathrm{SnPhCl}_{2} 125$

$\mathrm{R}=i-\mathrm{Pr}, \mathrm{M}=\mathrm{SnPh}_{3} 126$

$\mathrm{R}=i-\mathrm{Pr}, \mathrm{M}=\mathrm{SnPh}_{2} \mathrm{Cl} 127$

$\mathrm{R}=i-\mathrm{Pr}, \mathrm{M}=\mathrm{SnPhCl}_{2} 128$

$\mathrm{R}=i-\mathrm{Pr}, \mathrm{M}=\mathrm{SnCl}_{3} 129$

$\mathrm{R}=t-\mathrm{Bu}, \mathrm{M}=\mathrm{SnPh}_{3} 130$

$\mathrm{R}=t-\mathrm{Bu}, \mathrm{M}=\mathrm{SnPh}_{2} \mathrm{Cl} 131$

$\mathrm{R}=t-\mathrm{Bu}, \mathrm{M}=\mathrm{SnPhCl}_{2} 132$

Figure 19: Hypercoordinate tin compounds containing $C, O$-chelating ligands.

\subsubsection{Synthesis:}

In 1950 , Gilman et al. ${ }^{84}$ reported the synthesis of $\mathbf{1 1 2}$ by reacting $\left[o-\mathrm{MeOCH}_{2} \mathrm{C}_{6} \mathrm{H}_{4}\right] \mathrm{MgBr}$ with $\mathrm{Ph}_{3} \mathrm{SnCl}$ which was characterized only by melting point. Compound 113 reported by Reich 
et al. ${ }^{95}$ was prepared by treating $\left[2-\left(\mathrm{Me}_{2} \mathrm{OCH}_{2}\right) \mathrm{C}_{6} \mathrm{H}_{4}\right] \mathrm{Li}$ with $\mathrm{Me}_{3} \mathrm{SnCl}$ in THF. Compound 114 was synthesized by reacting $\left[2-\left(\mathrm{Me}_{2} \mathrm{OCH}_{2}\right) \mathrm{C}_{6} \mathrm{H}_{4}\right] \mathrm{Cu}$ with $\mathrm{Me}_{2} \mathrm{SnBr}_{2}$ while 116-118 were reported by Pannell et al. ${ }^{96}$ The synthesis of $\mathbf{1 1 5}^{96}$ and $\mathbf{1 1 6}$ was carried out by reacting the $o$ $\mathrm{MeOC}_{6} \mathrm{H}_{4} \mathrm{CH}_{2} \mathrm{MgCl}$ Grignard reagent and $\mathrm{Me}_{3} \mathrm{SnCl}$ or $\mathrm{Ph}_{3} \mathrm{SnCl}$ respectively, while 117 and 118 were obtained by successive replacement of the phenyl groups in $\mathbf{1 1 6}$ and $\mathbf{1 1 7}$ with chloride by treatment with a solution of $\mathrm{HCl} / \mathrm{Et}_{2} \mathrm{O}$ in $\mathrm{C}_{6} \mathrm{H}_{6}$. Compounds 119-132 were reported by Jambor et $a l . .^{92,98}$ and were obtained by reacting the lithiated ligands with the respective halides at low temperature in hexane.

\subsubsection{Structural studies:}

The X-ray crystallographic data for compounds 116-118 were also reported. It was found that there was a distinctive decrease in Sn-O distance with replacement of the phenyl groups bound to tin with $\mathrm{Cl}$. The substitution of one of the phenyl groups of $\mathbf{1 1 6}$ with $\mathrm{Cl}$ reduces the Sn-O bond distance from 3.07 to $2.77 \AA$. This is likely due to the increase in the Lewis acidity of the central tin atom and the transition from tetrahedral to TBP geometry. Compound $\mathbf{1 1 8}$ exhibited intermolecular interactions via $\mathrm{Sn}-\mathrm{Cl}$ resulting in dimer formation and had pseudo-Oh geometry. A comparison of chemical shift values by solid state and solution ${ }^{119} \mathrm{Sn}$ NMR spectroscopy indicated only minor difference. 
Table 7: ${ }^{119} \mathrm{Sn}$ chemical shifts and $\mathrm{Sn}-\mathrm{O}$ distances of tin compounds with $C, O$-chelating ligands.

\begin{tabular}{|c|c|c|}
\hline Compounds & $\delta\left({ }^{119} \mathrm{Sn}\right) \mathrm{ppm}$ & Sn-O distance $(\AA)$ \\
\hline 113 & $-38.2^{\mathrm{a}}$ & - \\
\hline 115 & 2.9 & - \\
\hline 116 & -144.8 & 3.07 \\
\hline 117 & -41.4 & 2.767 \\
\hline 118 & -32.7 & 2.898 \\
\hline 119 & -163.3 & $2.908(1), 2.966(1)$ \\
\hline 120 & -144.4 & - \\
\hline 121 & -208.8 & $2.619(1), 2.655(1)$ \\
\hline 122 & -270.5 & - \\
\hline 123 & -159.0 & - \\
\hline 124 & -140.0 & $2.454(1), 3.473(1)$ \\
\hline 125 & -197.0 & $2.447(1), 2.864(1)$ \\
\hline 126 & -153.8 & - \\
\hline 127 & -136.1 & - \\
\hline 128 & -177.4 & $2.475(1), 2.985(2)$ \\
\hline 129 & -238.8 & - \\
\hline 130 & -154.0 & - \\
\hline 131 & -121.7 & - \\
\hline 132 & -148.5 & $2.775(2), 2.882(2)$ \\
\hline
\end{tabular}

${ }^{\mathrm{a}}$ Solvent $=$ THF

The ${ }^{119} \mathrm{Sn}$ chemical shifts and $\mathrm{Sn}-\mathrm{O}$ bond distances of organotin compounds containing $C, O$-chelating ligands are listed in Table 7. Interestingly, one Sn-O distance in $\mathbf{1 2 4}$ was 2.454(1) $\AA$, while the second Sn-O distance is 3.473(1) $\AA$, indicating that the second interaction is out of the tin coordination sphere. ${ }^{98}$ There are only a few literature examples of tetraorganostannanes having mesogenic substituents on the tin which can expand their valence shell by additional coordination or intramolecular interactions between the electronegative $\mathrm{O}$ donor atom and tin atom. 


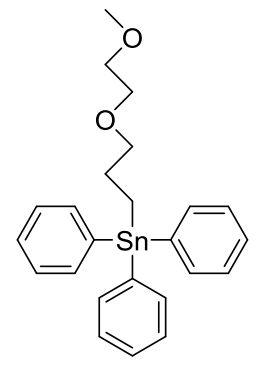

133

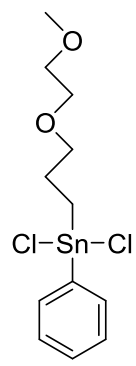

134

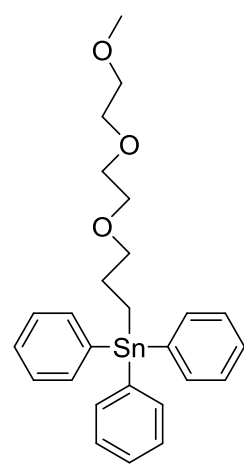

135

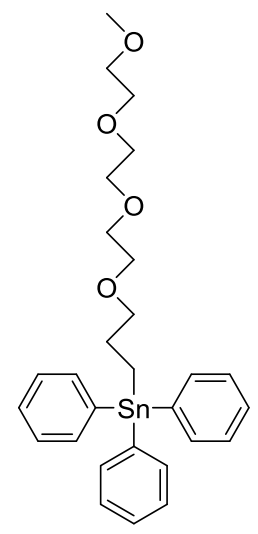

136

Figure 20: Organotin compounds with an oxa-alkyl side chain. ${ }^{99}$

Compounds 133-136 were prepared by reacting $\mathrm{Ph}_{3} \mathrm{SnH}$ with the respective oxa-alkenes in the presence of AIBN (Figure 20). Compounds 137-150 were synthesized according to the synthetic methodology shown in Scheme 10. It involves the hydrostannation of (4-biphenyloxy)1-alkenes (137-140) to produce 141-145. Compounds 141-145 were treated with molecular halogens $\left(\mathrm{Br}_{2}\right.$ and $\left.\mathrm{I}_{2}\right)$ to produce the tin dihalides 146-150. The ${ }^{119} \mathrm{Sn}$ chemical shifts and $\mathrm{Sn}-\mathrm{O}$ distance of organotin compounds $\mathbf{1 3 3 - 1 5 0}$ are listed in Table 8.

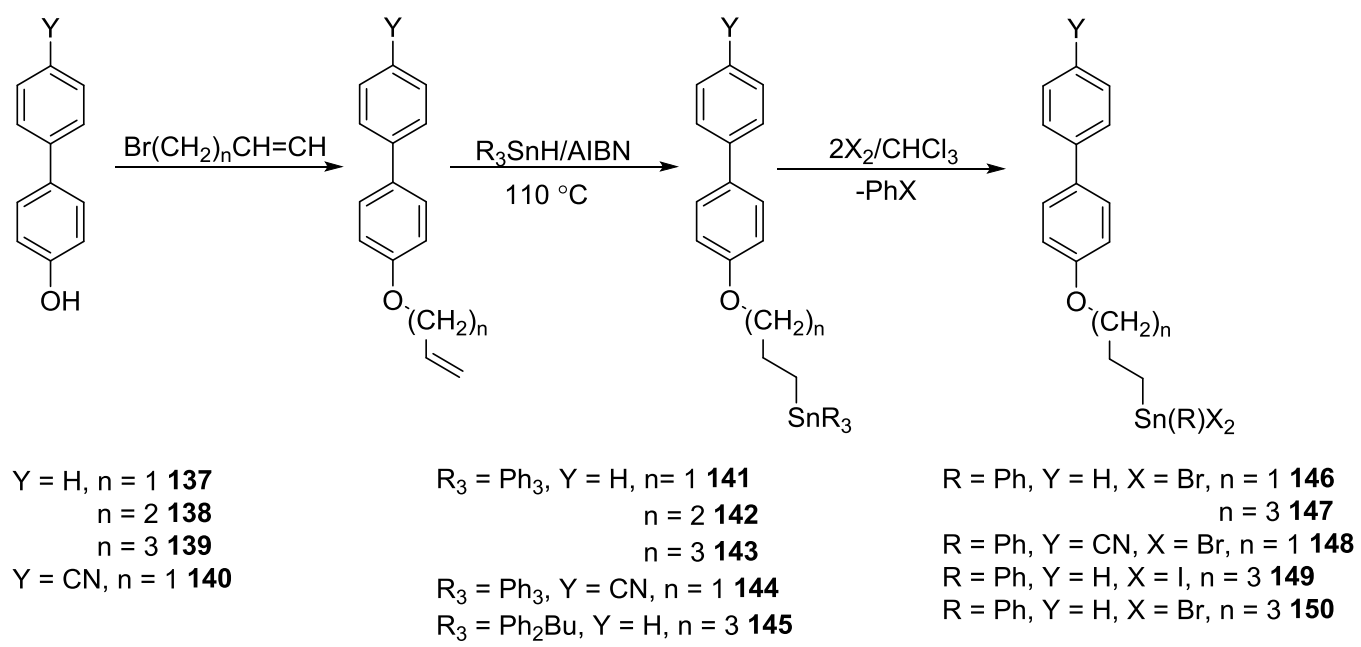

Scheme 10: Synthesis of tin complexes with phenyloxy alkyl side chains. ${ }^{43}$ 
Table 8: ${ }^{119} \mathrm{Sn}$ chemical shifts and $\mathrm{Sn}-\mathrm{O}$ distances of tin compounds with $C, O$ - chelating ligands.

\begin{tabular}{|c|c|c|}
\hline Compounds & $\boldsymbol{\delta}\left({ }^{\mathbf{1 1 9}} \mathbf{S n}\right) \mathbf{p p m}$ & Sn-O distance $(\mathbf{\AA})$ \\
\hline $\mathbf{1 3 3}^{99}$ & -100.3 & - \\
\hline $\mathbf{1 3 4}^{99}$ & -100.3 & - \\
\hline $\mathbf{1 3 5}^{99}$ & -100.4 & - \\
\hline $\mathbf{1 3 6}^{99}$ & -73.6 & - \\
\hline $\mathbf{1 4 1}^{43}$ & -100.1 & - \\
\hline $\mathbf{1 4 2}^{43}$ & -101.2 & - \\
\hline $\mathbf{1 4 3}^{43}$ & -100.4 & - \\
\hline $\mathbf{1 4 4}^{43}$ & -100.4 & $2.734(4)$ \\
\hline $\mathbf{1 4 5}^{43}$ & -72.3 & - \\
\hline $\mathbf{1 4 6}^{43}$ & -53.3 & $2.918(7)$ \\
\hline $\mathbf{1 4 7}^{43}$ & 3.5 & - \\
\hline $\mathbf{1 4 8}^{43}$ & -47.6 & - \\
\hline $\mathbf{1 4 9}^{43}$ & -157.2 & \\
\hline $\mathbf{1 5 0}^{43}$ & 88.6 & \\
\hline & & \\
\hline
\end{tabular}

The crystal structure of $\mathbf{1 3 4}$ revealed a hexacoordinated tin atom bonded to two atoms of each of $\mathrm{C}, \mathrm{Cl}$ and $\mathrm{O}$ (Figure 21). The two $\mathrm{O}$ and $\mathrm{Cl}$ atoms are cis to each other and the $\mathrm{C}$ atoms are found to occupy axial positions. The Sn-O distances in 134 are 2.553(2) and 2.540(2) $\AA$ respectively and are larger than the covalent radii of Sn and O (2.1 $\AA$ ), but shorter than sum of their van der Waal's radii $(3.7 \AA)$.

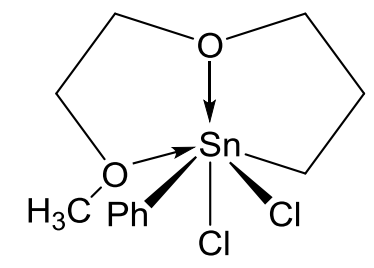

Figure 21: Structure of $134 .^{99}$

X-ray structure data for compounds 142 and 144 reveal tetrahedral geometries at tin centers having bond angles between 105-113 ${ }^{\circ}$. Compound 146 exists in a dimeric form and two molecules are linked through $\operatorname{Br}(1)----\operatorname{Sn}\left(1^{\prime}\right)$ and an octahedral sphere was completed by chelation of the ether O with a $\mathrm{Sn}(1)-\mathrm{O}(1)$ distance $2.734(4) \AA$. Generally, the Sn-O distance is longer (2.540 $2.667 \AA)^{99,100}$ for diorganotin species with $\mathrm{CN}=6$ than those with $\mathrm{CN}=5(2.382-2.448 \AA)^{101}$. 
The Sn-O distance is also affected by the presence of an additional electronegative atom which increases the Lewis acidity of tin. In the case of $\mathbf{1 4 8}$, the Sn-O distance is transoid to $\mathrm{Sn}$ - $\mathrm{Br}$ bond with an angle of $\mathrm{O}(1)-\operatorname{Sn}(1)-\operatorname{Br}(1)=169.0^{\circ}(1)$ with the molecule assuming a TBP structure..$^{43}$

Compounds 151-154 were synthesized by Takeuchi et al. using Scheme $11 .{ }^{102}$ The line width for germanium compounds were also determined and a broadening of signal was always accompanied by hypervalency. The ${ }^{73} \mathrm{Ge}$ shift for compounds $\mathbf{1 5 1 - 1 5 3}$ are $-85.0,-85.0$ and -84.0 ppm $\left(\mathrm{Ph}_{3} \mathrm{GeH} \delta=-57.0 \mathrm{ppm}\right)$ with the line widths of $350 \mathrm{~Hz}$. An X-ray crystal structure determination revealed that Ge-O distances for $\mathbf{1 5 3}$ were 3.2773(3), 3.214(3) and 3.703(3) $\mathrm{\text {A }}$. Both crystal structure and NMR data gave evidence for a dative interaction between $\mathrm{Ge}$ and $\mathrm{O}$, although it is weaker than between Ge and N.

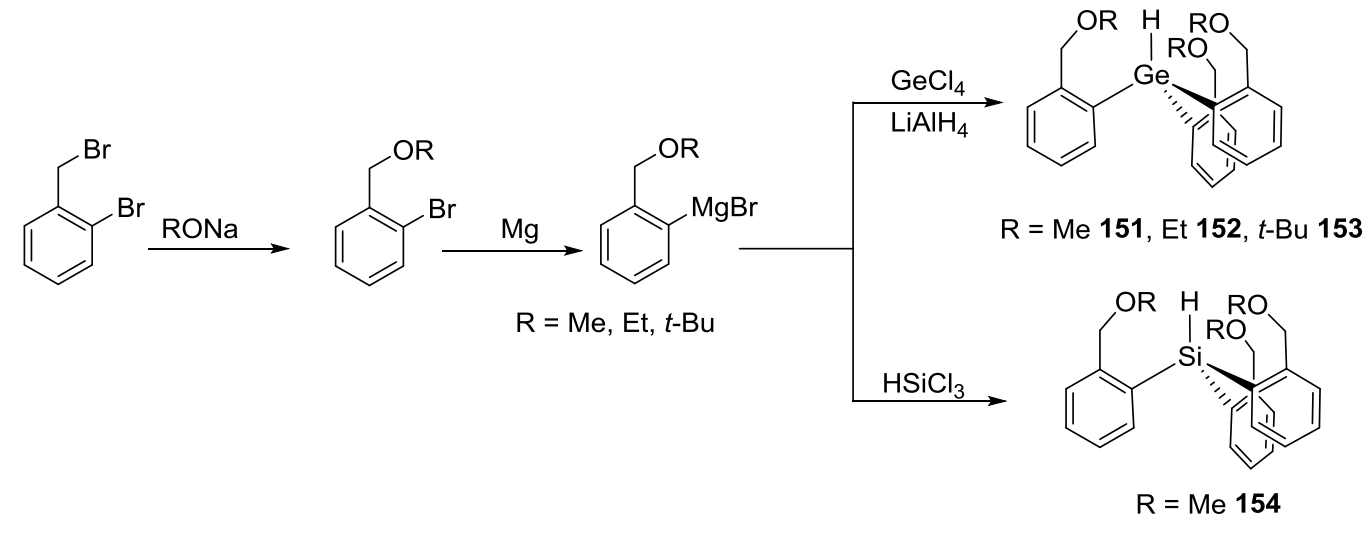

Scheme 11: Synthesis of $C, O$-chelating ligand and the corresponding triarylgermanes and silane.

\subsection{C,S-chelating ligands containing compounds of Group 14:}

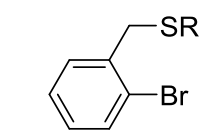

$\mathrm{R}=\mathrm{Me}, t-\mathrm{Bu}, i-\mathrm{Pr}, \mathrm{Ph}$

xii

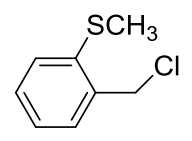

xiii

Figure 22: $C, S$-chelating ligands. 
$C, S$-chelating ligands commonly used to hypercoordinate a Group 14 element are listed in Figure 22. There are only a few reports of hypercoordinated Sn-S compounds. Ligands of type xii $(\mathrm{R}=n-\mathrm{Pr}, \mathrm{Ph})$ were synthesized using the methods reported in the literature. ${ }^{103-105}$ It is well established that Group 14 elements have the ability to expand their coordination number through dative interactions of Lewis bases of functional groups containing $\mathrm{N}, \mathrm{P}, \mathrm{S}$ and $\mathrm{O}$ donor atom) ${ }^{95}$ This strategy was used to produce a variety of hypercoordinated compounds of Sn.

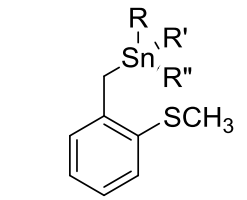

$\mathrm{R}=\mathrm{R}^{\prime}=\mathrm{R}^{\prime \prime}=\mathrm{Ph} 155$

$\mathrm{R}=\mathrm{Cl}, \mathrm{R}^{\prime}=\mathrm{R}^{\prime \prime}=\mathrm{Ph} 156$

$\mathrm{R}=\mathrm{R}^{\prime}=\mathrm{Cl}, \mathrm{R}^{\prime \prime}=\mathrm{Ph} 157$

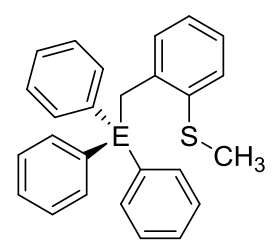

$E=S i 158, G e 159, S n 155, P b 160$

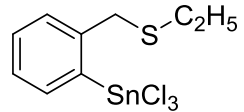

161

Figure 23: Group 14 compounds containing $C, S$-chelating ligands.

Compound 155 was successfully prepared by using methods shown in Scheme 12. An insitu synthesis of the respective Grignard reagent in the presence of the halide afforded the desired product in a good yield. ${ }^{96}$ Two phenyl groups were subsequently substituted with $\mathrm{Cl}$ by reacting 155 and 156 with of $\mathrm{HCl} / \mathrm{Et}_{2} \mathrm{O}$ in $\mathrm{C}_{6} \mathrm{H}_{6}$ to produce the dihalides 156-157 respectively. Compounds 155 and 158-160 were synthesized by reacting Group 14 halides with Li metal followed by reaction with $o-\left(\mathrm{SCH}_{3} \mathrm{C}_{6} \mathrm{H}_{4}\right) \mathrm{CH}_{2} \mathrm{Cl}$ at low temperature (Scheme 12). ${ }^{106}$

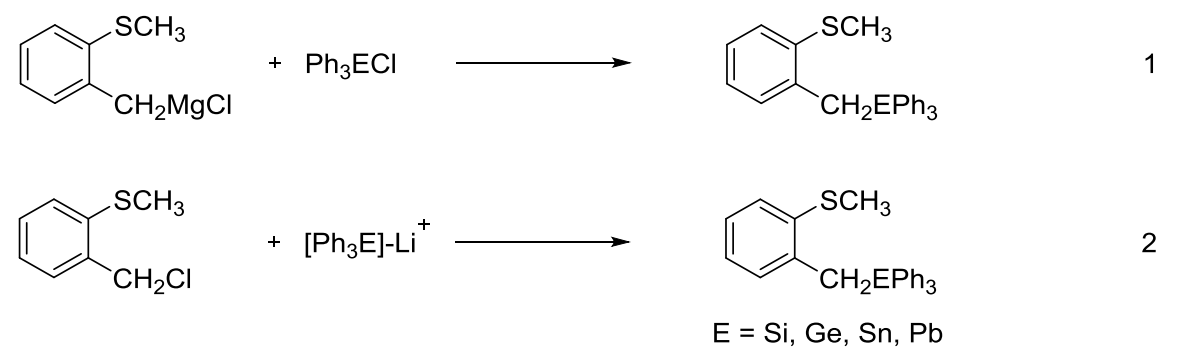

Scheme 12: Group 14 compounds containing a $C, S$-chelating ligand. 
The reaction of thiols and $\mathrm{NaH}$ followed by the reaction with 1-bromo-2bromomethylbenzene produced the corresponding sulfides 162-164 in good yields (Scheme 13). Lithiation of 162-164 with $n$ - $\mathrm{BuLi}$ in $\mathrm{Et}_{2} \mathrm{O}$ at $0{ }^{\circ} \mathrm{C}$ followed by treatment with $\mathrm{SiHCl}_{3}$ and the subsequent reduction with $\mathrm{LiAlH}_{4}$ afforded silicon dihydrides 165-167. Compounds 170-172 were reported by Berlekamp and synthesized according to Scheme $14 .{ }^{107}$ Compounds 173-174 were synthesized by Takeuchi et al. using Scheme $15{ }^{102}$

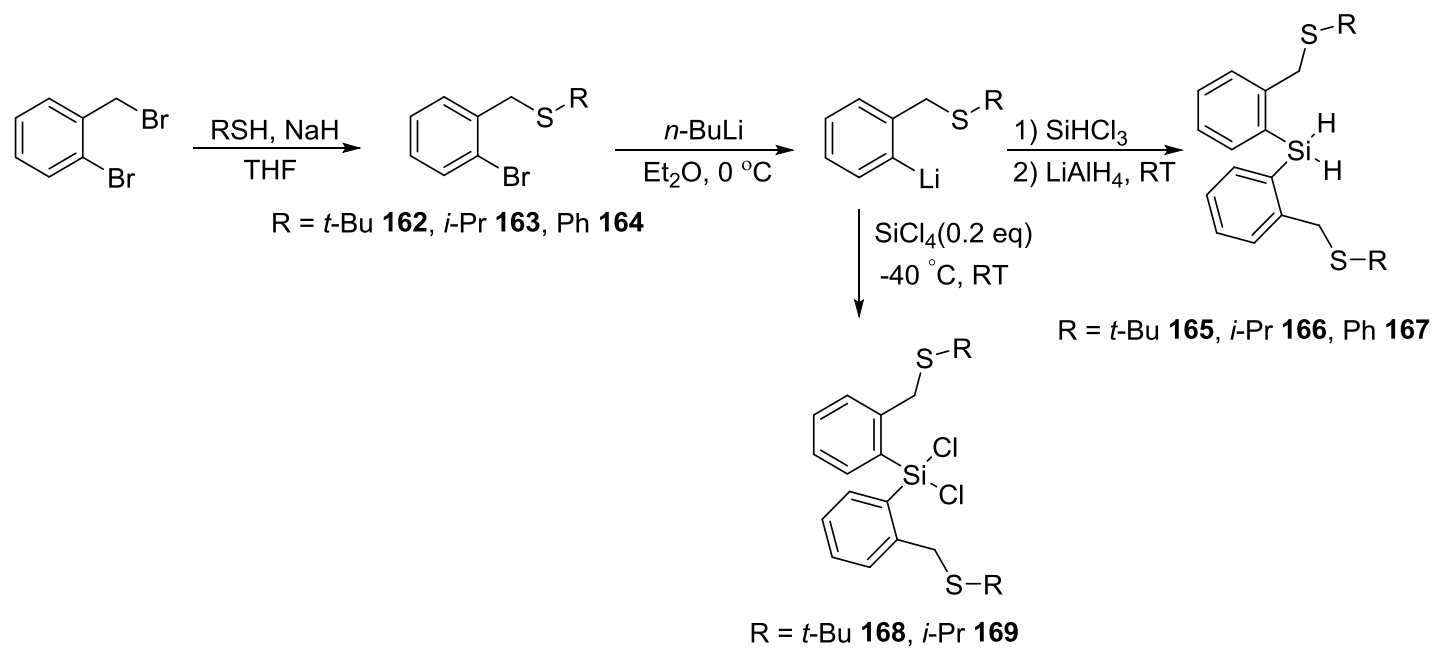

Scheme 13: Synthesis of organosilicon compounds containing a $C, S$-chelating ligand.

${ }^{119} \mathrm{Sn}$ NMR data of compound $\mathbf{1 5 5}$ showed an upfield shift of $70.5 \mathrm{ppm}$ relative to the nonchelating, structurally similar analog, suggestive of substantial S-Sn interaction. ${ }^{107}$ The ${ }^{29} \mathrm{Si}$ NMR spectra of dihydrosilanes 165-167 showed small upfield shifts compared to the closely related unsubstituted analogue $\mathrm{Ph}_{2} \mathrm{SiH}_{2}\left(\delta^{29} \mathrm{Si}=-34.5 \mathrm{ppm}\right) .{ }^{108}$ These small $\Delta \delta$ values suggest that there is only a weak to no $\mathrm{Si}-\mathrm{S}$ interaction present. 


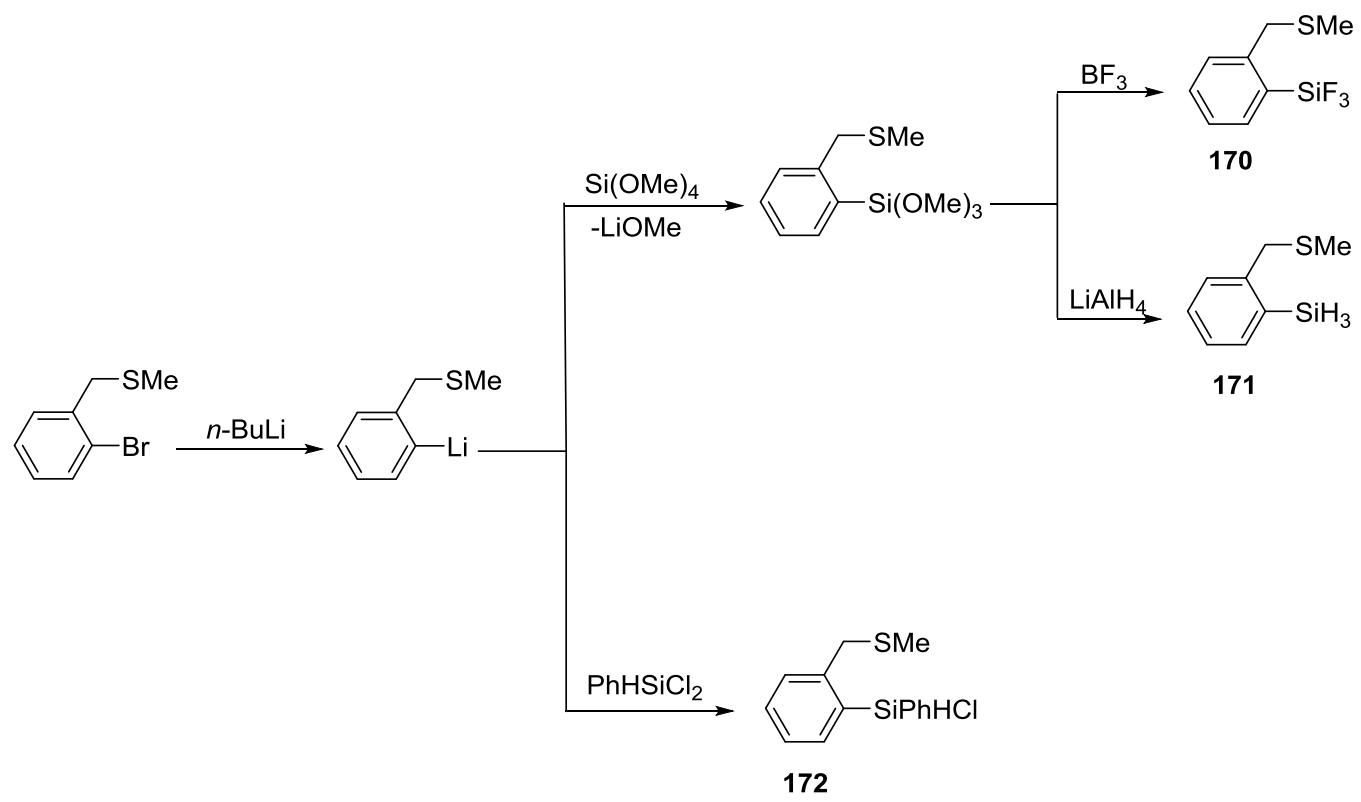

Scheme 14: Synthesis of $\mathrm{SiF}_{3}, \mathrm{SiH}_{3}$ and $\mathrm{SiPhHCl}$ silanes containing a $C, S$-chelating ligand.

The ${ }^{29} \mathrm{Si}$ NMR data of S-donor containing compounds $(\mathbf{1 5 8}, \mathbf{1 6 5}-\mathbf{1 7 4})$ give no indication of donor complexation. The observed ${ }^{29} \mathrm{Si}$ resonance for $\mathbf{1 7 0}$ is nearly identical to that of tetrahedral $\mathrm{PhSiF}_{3}$. It was also reported that the tendency of $\mathrm{S}$-donor to form an adduct with $\mathrm{SiF}_{3}$ is negligible compared to $\mathrm{N}$ - and $\mathrm{O}$-donors. These results are in accordance with recently published theoretical investigations concerning the adduct formation behaviour of $\mathrm{SiF}_{4} \cdot{ }^{109}$ Slight upfield shifts of ${ }^{29} \mathrm{Si}$ resonances of $4.9 \mathrm{ppm}$ and $7.0 \mathrm{ppm}$ for compounds $\mathbf{1 7 1}$ and $\mathbf{1 7 2}$ were observed compared to their unsubstituted analogues, again suggestive of only weak $\mathrm{Si}-\mathrm{S}$ interactions (Table 9). 
Table 9: ${ }^{29} \mathrm{Si} /{ }^{19} \mathrm{Sn} /{ }^{207} \mathrm{~Pb}$ chemical shifts and $\mathrm{Si} / \mathrm{Sn} / \mathrm{Pb}-\mathrm{S}$ distances of compounds with $C, S$ chelating ligands.

\begin{tabular}{|c|c|c|c|c|c|}
\hline Compound & $\begin{array}{c}{ }^{29} \mathrm{Si} /{ }^{119} \mathrm{Sn} /{ }^{207} \mathrm{~Pb} \\
\delta(\mathrm{ppm})\end{array}$ & $\begin{array}{c}\text { S-Si/Sn/Pb } \\
\text { distance } \\
(\AA)\end{array}$ & $\begin{array}{l}\text { Unsubstituted } \\
\text { analogue }\end{array}$ & $\begin{array}{c}{ }^{29} \mathrm{Si} /{ }^{119} \mathrm{Sn} /{ }^{207} \mathrm{~Pb} \\
\delta(\mathrm{ppm})\end{array}$ & $\begin{array}{c}\Delta \delta \\
{ }^{29} \mathrm{Si} /{ }^{119} \mathrm{Sn} /{ }^{20} \\
{ }^{7} \mathrm{~Pb}(\mathrm{ppm})\end{array}$ \\
\hline 155 & -115.5 & 3.699 & $\mathrm{Ph}_{3} \mathrm{SnCl}$ & -45.0 & 70.5 \\
\hline 156 & -46.3 & 3.062 & $\mathrm{Ph}_{2} \mathrm{SnCl}_{2}$ & -32.0 & 14.3 \\
\hline 157 & -48.5 & 2.994 & $\mathrm{PhSnCl}_{3}$ & -63.0 & -14.5 \\
\hline 158 & -11.9 & 3.985 & $\mathrm{Ph}_{3} \mathrm{SiCl}$ & 1.5 & 13.4 \\
\hline 160 & $-146.2^{a}$ & 3.953 & $\mathrm{Ph}_{3} \mathrm{PbCl}$ & 33 & 179.2 \\
\hline 165 & -41.3 & - & $\mathrm{Ph}_{2} \mathrm{SiH}_{2}$ & -34.5 & 6.8 \\
\hline 166 & -40.8 & - & $\mathrm{Ph}_{2} \mathrm{SiH}_{2}$ & -34.5 & 6.3 \\
\hline 167 & -40.1 & - & $\mathrm{Ph}_{2} \mathrm{SiH}_{2}$ & -34.5 & 5.6 \\
\hline 168 & -3.6 & - & $\mathrm{Ph}_{2} \mathrm{SiCl}_{2}$ & 6.2 & 9.8 \\
\hline 169 & -3.2 & - & $\mathrm{Ph}_{2} \mathrm{SiCl}_{2}$ & 6.2 & -3.0 \\
\hline 170 & -71.9 & - & $\mathrm{PhSiF}_{3}$ & -73.2 & -1.3 \\
\hline 171 & -65.0 & - & $\mathrm{PhSiH}_{3}$ & -60.1 & 4.9 \\
\hline 172 & -12.4 & - & $\mathrm{Ph}_{2} \mathrm{SiHCl}$ & -5.4 & 7.0 \\
\hline 174 & -33.3 & - & $\mathrm{Ph}_{3} \mathrm{SiH}$ & -21.1 & 12.2 \\
\hline
\end{tabular}

A comparison of the $\mathrm{Sn}-\mathrm{S}$ distances in compounds 155-157 showed a progressive decrease in Sn-S distances given in Table 9 which corresponds to an increase of the Lewis acidity of the central tin atom. In compounds 156 and 157 the $\mathrm{Sn}-\mathrm{S}$ bond is trans to $\mathrm{Sn}-\mathrm{Cl}$ bond with angles of $168^{\circ}$ and $167^{\circ}$ respectively. The transition from tetrahedral to TBP can also be determined from the difference between the sums of equatorial and axial angles. ${ }^{96}$ A greater difference is associated with more TBP geometry. In compounds 155 and as well as in 158-160, the $\mathrm{E}-\mathrm{S}(\mathrm{E}=\mathrm{Si}, \mathrm{Ge}, \mathrm{Sn}$, $\mathrm{Pb}$ ) distances (Table 9) indicate a progressive change in the structural features of these compounds. 


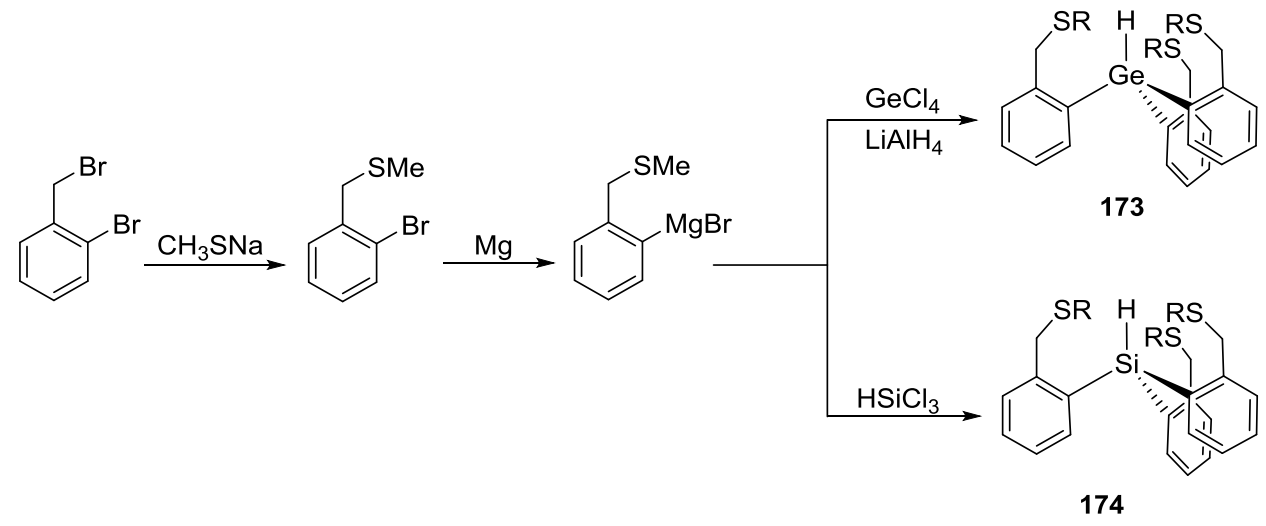

Scheme 15: Synthesis of a $C, S$-chelating ligand and reactions to form a triarylgermane or silanes. Hypervalency in the case of Ge compound $\mathbf{1 7 3}$ is associated with a large upfield ${ }^{73} \mathrm{Ge}$ NMR shift $\left(\delta=-93.0 \mathrm{ppm} v \mathrm{Ph}_{3} \mathrm{GeH} \delta=-57.0 \mathrm{ppm}\right)$ and a significant line broadening. The average distance between Ge and S was 3.778(2) A. The data obtained from X-ray crystallography and NMR spectroscopy for $\mathbf{1 7 3}$ support the presence of a Ge-S dative interaction, although it is still weaker when compared to similar Ge-N compounds.

\section{6 $C, P$-chelating ligand containing compounds of Group 14:}

The ability of Sn to expand its coordination number beyond four due to the donor-acceptor interactions with Lewis bases such as $\mathrm{N}$ and $\mathrm{O}$ has been conclusively established. The intramolecular interactions between Sn and soft phosphine donors are less studied. ${ }^{102}$ Higher coordination can be successfully induced by the integration of a donor atom into the side chain of an alkyl or aryl substituent. ${ }^{99}$ Dative interaction between Sn-P can be inferred from the upfield shifts of ${ }^{119} \mathrm{Sn}$ NMR resonance signals and short Sn-P distances and the large values of the $J_{119 \mathrm{Sn} \text { - }}$ $31 \mathrm{P}$ coupling constant compared to tetracoordinated tin compounds. ${ }^{102,103}$ The ligands used to obtain the molecules having Sn-P interaction are given in Figure 24. 


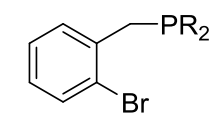

xiv

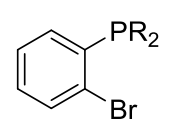

$\mathrm{xV}$

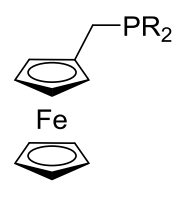

$x v i$

$\mathrm{R}=\mathrm{Ph}, \mathrm{Me}$

Figure 24: Structure of $C, P$-chelating ligands.

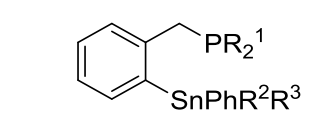

$R^{1}=R^{2}=R^{3}=P h 175$

$\mathrm{R}^{1}=\mathrm{Ph}, \mathrm{R}^{2}=\mathrm{Me}, \mathrm{R}^{3}=t$-Bu 176

$R^{1}=M e, R^{2}=P h, R^{3}=P h 177$

$R^{1}=M e, R^{2}=M e, R^{3}=t-B u 178$

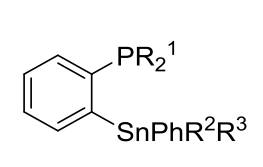

$\mathrm{R}^{1}=\mathrm{Ph}, \mathrm{R}^{2}=\mathrm{Ph}, \mathrm{R}^{3}=\mathrm{Cl} 179$

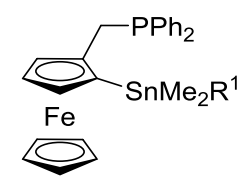

$\mathrm{R}^{1}=\mathrm{Me} 180, \mathrm{Cl} 181$

Figure 25: Organotin compounds containing $C, P$-chelating ligands. ${ }^{110,112-114}$

Compounds 175-178 and 179 (Figure 25) were synthesized by von Abicht et al. ${ }^{112}$ and Lin et al. ${ }^{110}$ by reacting $o-\left(\mathrm{C}_{6} \mathrm{H}_{4} \mathrm{CH}_{2} \mathrm{PPh}_{2}\right) \mathrm{Li}$ and $o-\left(\mathrm{Ph}_{2} \mathrm{P}\right) \mathrm{C}_{6} \mathrm{H}_{4} \mathrm{Li}$ with $\mathrm{R}_{3} \mathrm{SnCl}$ and $\mathrm{Ph}_{2} \mathrm{SnCl}_{2}$ respectively in $\mathrm{Et}_{2} \mathrm{O}$. The ${ }^{31} \mathrm{P}$ NMR resonance at $-1.0 \mathrm{ppm}$ for $\mathbf{1 7 9}$ is downfield from $-4.0 \mathrm{ppm}$ for $\mathrm{Ph}_{3} \mathrm{P}$, and the ${ }^{119} \mathrm{Sn}$ NMR chemical shift of $-101.7 \mathrm{ppm}$ for $\mathbf{1 7 9}$ is significantly upfield compared to $\mathrm{Ph}_{3} \mathrm{SnCl}(-45.0 \mathrm{ppm})$. The same observations were obtained in the case of $o-((i-$ $\left.\left.\mathrm{Pr}_{2} \mathrm{P}\right) \mathrm{C}_{6} \mathrm{H}_{4}\right)_{2} \mathrm{SnPhCl}$ 179a which showed ${ }^{119} \mathrm{Sn}$ resonance of $-126.4 \mathrm{ppm}$. X-ray crystallography of both 179 and 179a showed Sn-P distance of 3.125(4) and 3.120(1) $\AA$ which is less than the sum of van der Waals radii (4.2 $\mathrm{A}) .{ }^{110}$ Therefore, a dative interaction is likely present between Sn and P. The P-Sn-Cl bond angles of $159.76^{\circ}$ (1) and $153.92^{\circ}(1)$ clearly suggests that the coordination geometry at the tin centers is TBP.

Compound 182 was synthesized by treating $o-\left(\mathrm{C}_{6} \mathrm{H}_{4} \mathrm{CH}_{2} \mathrm{PPh}_{2}\right) \mathrm{Br}$ with $\mathrm{Me}_{2} \mathrm{SiHCl}$. Berlekamp et al. ${ }^{107}$ reported the synthesis of $\mathbf{1 8 3 - 1 8 5}$ by using the route outlined in Scheme 16. 


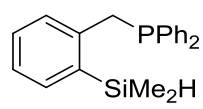

182

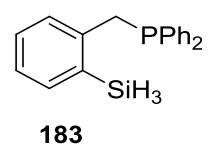

183

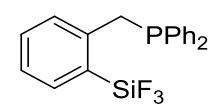

184

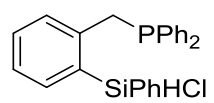

185

Figure 26: Organosilicon compounds containing $C, P$-chelating ligands. ${ }^{107,} 115$

The ${ }^{29} \mathrm{Si}$ NMR resonances for compounds 183-185 are $-62.4,-72.0$ and $-10.1 \mathrm{ppm}$ respectively, which indicate only very small upfield shifts compared to structurally similar nonchelating silanes. This data anticipates the non-coordinating behavior of $\mathrm{P}$ in compounds 183-185.

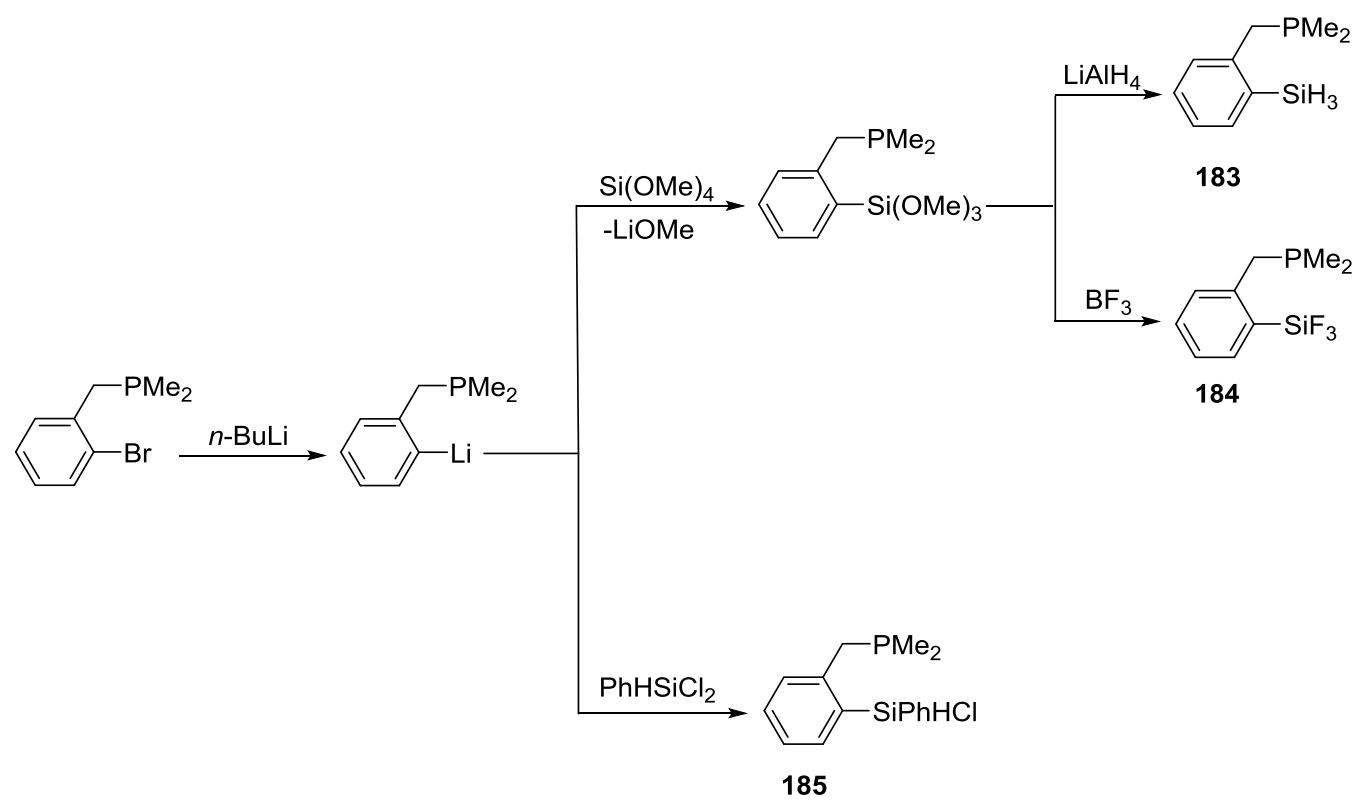

Scheme 16: Synthesis of $\mathrm{SiF}_{3}, \mathrm{SiH}_{3}$ and $\mathrm{SiPhHCl}$ silanes containing a $C, P$-chelating ligand. 


\subsection{Polystannanes:}

Group 14 elements $(\mathrm{E}=\mathrm{C}, \mathrm{Si}, \mathrm{Ge}, \mathrm{Sn}$ and $\mathrm{Pb})$ have a unique tendency towards catenation, which decreases significantly from carbon to lead. In the early 1980's, the synthesis of polysilanes introduced a new class of materials with interesting electronic and optical properties due to the presence of significant $\sigma$-delocalization ${ }^{116}$ along the polymer backbone which also stimulated a similar interest in polystannanes. Polystannanes represent a unique class of polymers having a backbone of covalently bonded metal ( $\mathrm{Sn}$ ) atoms. Polystannane possess extensive $\sigma$-delocalization compared to polysilanes because of their more diffuse orbitals, lower band gaps (>390 nm, ${ }^{116}$ some $70 \mathrm{~nm}$ or more red-shifted vs. Si) and greater metallic character. ${ }^{43}$ It was first demonstrated by Dräger et al. ${ }^{117}$ that the HOMO-LUMO energy gap of oligostannanes decreased when the number of tin atoms in the chain was increased, and a significantly red shifted absorption maxima in UV spectra was observed. A consequence of catenation in polystannane is longer central Sn-Sn bonds and flatter Sn-Sn-Sn angles. Thus, the term "molecular metal" was proposed by Dräger to describe high molecular weight analogues. ${ }^{117}$ The tri-, tetra-, penta- and hexastannanes were isolated and characterized by ${ }^{119}$ Sn NMR spectroscopy and X-ray analyses.

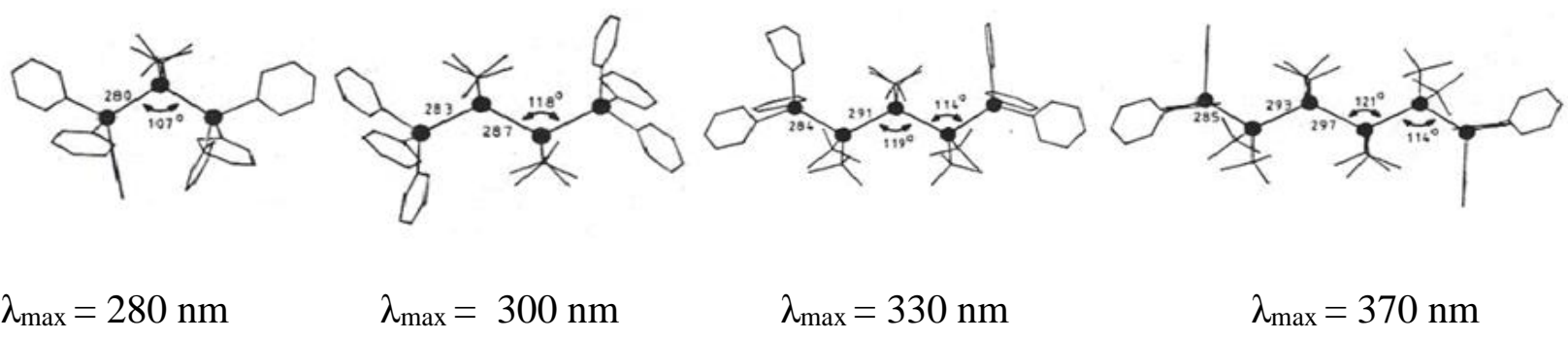

Figure 27: Dräger's oligostannanes with their average Sn-Sn bond lengths (pm), Sn-Sn-Sn bond angles and absorption maxima. ${ }^{117}$ 


\subsubsection{Challenges for polystannanes:}

Polystannanes are thermally stable up to $200^{\circ} \mathrm{C}$ in inert atmosphere as well as in air. ${ }^{108,110-}$

112 Polystannanes show sensitivity to the ambient environment, have greater stability in the solid state than in solution, and suffer a higher rate of degradation under light exposure compared to dark. ${ }^{116,118,121-123}$ Furthermore, it was found that poly(dialkylstannane)s degrade immediately upon exposure to light to five- and six-membered cyclic oligostannanes, whereas poly(diarylstannane)s were stable in the dark in air for at least one week. ${ }^{119}$

Choffat et al. ${ }^{122}$ reported degradation studies of poly(dialkylstannane)s having alkyl side groups of varying lengths, in the presence of different solvents and dyes. It was demonstrated that the length of the alkyl side chain has no significant influence on the stability of these polymers. It was found that the use of dyes such as Sudan 1, Sudan Black B, or inorganic colloidal platinum, or organic small molecules such as carotene, curcumin and TEMPO reduced the rate of degradation. The reason for the reduction of degradation may be the absorption of light by these species or the reaction with transient radicals or both. Trummer et al. ${ }^{124}$ reported that the stability of polystannanes in light is dependent on the nature of the organic side groups. The degradation behaviour of two polymers, poly[bis(4-butylphenyl)stannane] and poly(dibutylstannane) were studied, and the diarylpolystannanes were found to be more stable towards light than the dialkyl in THF as well as in DCM. The initial photochemical damage caused by laser flash photolysis is comparable for both polymers. Poly[bis(4-butylphenyl)stannane] "recovered" to $90 \%$ during a period of a few seconds after irradiation. The higher recovery of poly[bis(4-butylphenyl)stannane] degraded by photolysis may be the re-formation of polymer chains resulting in an apparent stabilization of this polymer. This type of behaviour was not observed for poly(di- $n$ butylstannane). The degradation mechanism proposed in this study is based on the random homolytic cleavage of $\mathrm{Sn}-\mathrm{Sn}$ bonds in a polymer chain resulting in two smaller chains ending with 
a radical (Figure 28). The enhanced stability of a chain ending with two aromatic groups bonded to a tin atom may be due to the radical delocalization throughout the aromatic ring which decreases the probability of the radical reacting with the polymer chains. This mechanism was further supported by the fact that in the presence of the radical scavenger 2,6-di-tert-butyl-4-methylphenol (BHT) in THF, the degradation rate of polystannanes was slow. In the absence of BHT the degradation of polystannane was rapid.

Chain scission

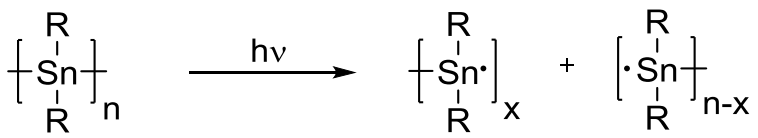

Recombination for $\mathrm{R}=$ Aryl

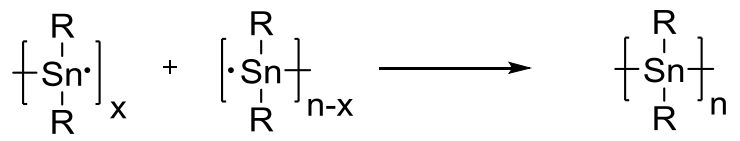

Unzipping for $\mathrm{R}=$ Alkyl

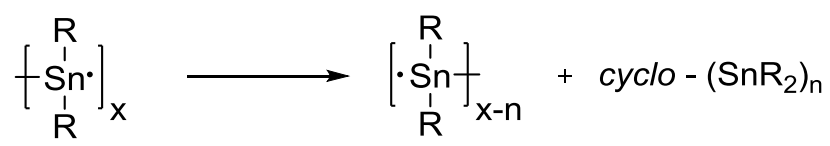

Figure 28: Proposed degradation mechanism of polystannanes.

\subsubsection{Synthesis of polystannanes:}

The common reductive coupling methods used for the synthesis of polystannanes include Wurtz type, electrochemical and catalytic dehydrogenation in the presence of a transition metal catalyst.

\subsubsection{Wurtz coupling:}

Wurtz coupling was first used to form a C-C bond from the reaction of alkyl halides with sodium metal. Later, investigations by Kipping ${ }^{125}$ showed that Wurtz coupling can be used for preparation of materials having only organosilicon units in the backbone. In these Wurtz-type reactions, a dichlorodiorganosilane is reacted with a slight excess of sodium dispersion in a high- 
boiling-point solvent such as toluene under reflux. In 1992, Zou et al. ${ }^{126}$ reported the first synthesis of high molecular weight ( $\left.10^{4} \mathrm{Da}\right)$ poly(di- $n$-butylstannane) using a Wurtz-type coupling of ( $n$ $\mathrm{Bu})_{2} \mathrm{SnCl}_{2}$ in toluene/heptane in the presence of 15-crown-5.

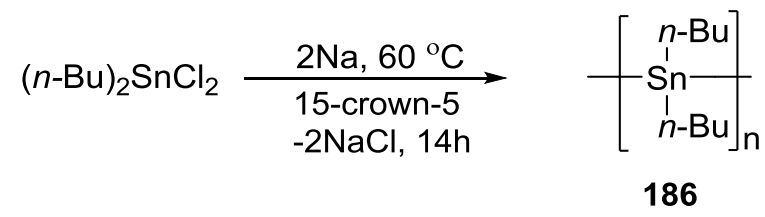

Scheme 17: Wurtz coupling of $(n-\mathrm{Bu})_{2} \mathrm{SnCl}_{2}$.

A reinvestigation of this study by Tilley failed to produce the same results and only low molecular weight oligomers were isolated under these conditions. ${ }^{118}$ This may have been due to the longer reaction times used, which caused a degradation of the polymer. In a later study by Price et al. ${ }^{127}$ high molecular weight $\left(10^{6} \mathrm{Da}\right)$ poly(di- $n$-butylstannane) was produced in toluene at 60 ${ }^{\circ} \mathrm{C}$. It was observed that the optimal yield is achieved in $4 \mathrm{~h}$; after that time, degradation of the polymer begins and cyclic oligomers such as $\left(n-\mathrm{Bu}_{2} \mathrm{Sn}\right)_{5}$ and $\left(n-\mathrm{Bu}_{2} \mathrm{Sn}\right)_{6}$ are obtained as a result of "end-biting" and "back-biting" of chains. This demonstrated that reaction time is critical for these types of reactions. In 2003, Molloy et $a l^{43}$ reported the Wurtz polymerization of $\mathrm{RBr}_{2} \mathrm{Sn}\left(\mathrm{CH}_{2}\right)_{5} \mathrm{OC}_{6} \mathrm{H}_{4} \mathrm{C}_{6} \mathrm{H}_{5}(\mathrm{R}=\mathrm{Ph}, n$-Bu $)$, yielding moderately high molecular weight polystannanes $\left(\mathrm{RR}^{\prime} \mathrm{Sn}\right)_{\mathrm{n}}\left(M_{\mathrm{w}}=3.0 \times 10^{5} \mathrm{Da} ; \mathrm{PDI}=1.30\right.$ and $M_{\mathrm{w}}=2.5 \times 10^{5} \mathrm{Da} ; \mathrm{PDI}=1.96$ respectively). Foucher et al. ${ }^{128}$ also successfully synthesized high molecular weight $\left(M_{\mathrm{w}}=1.1 \times\right.$ $10^{5} \mathrm{Da} ; \mathrm{PDI}=1.40$ and $\left.M_{\mathrm{w}}=1.47 \times 10^{5} \mathrm{Da} ; \mathrm{PDI}=1.3\right)$ fluorinated polystannanes using Wurtz coupling reactions of fluorinated dichlorostannanes. 

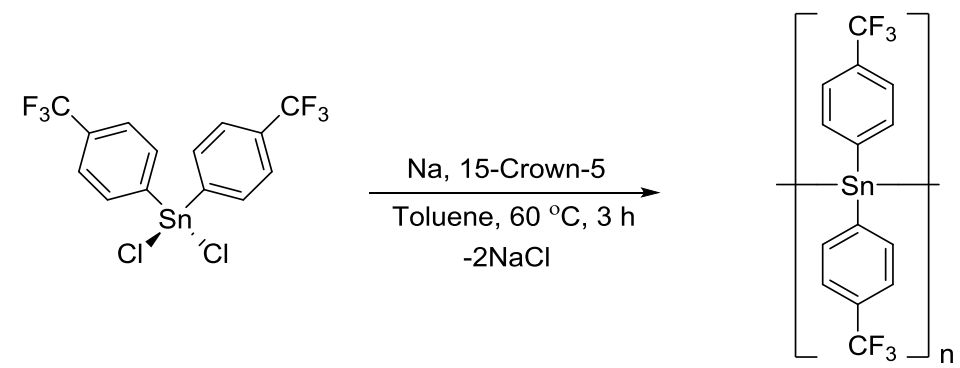

187
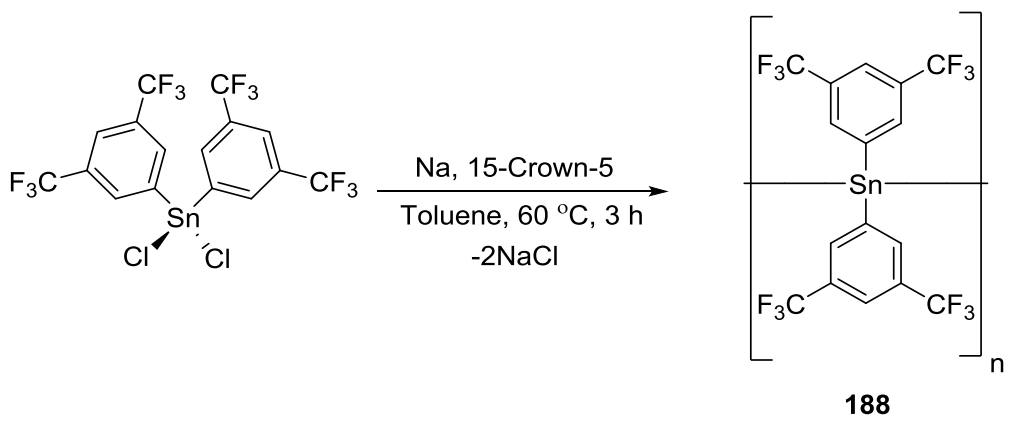

Scheme 18: Wurtz coupling reactions of fluorinated dichlorostannanes.

Recently, Caseri et al. ${ }^{129}$ reported the polymerization of dichlorodiorganostannanes with $\mathrm{Na}$ in 1:2 ratio in liquid $\mathrm{NH}_{3}$. These reaction conditions afforded the polymerization of $(n-$ $\mathrm{Bu})_{2} \mathrm{SnCl}_{2}$ and $(n-\mathrm{Oct})_{2} \mathrm{SnCl}_{2}$ to the polystannanes $\left(n-\mathrm{Bu}_{2} \mathrm{Sn}\right)_{\mathrm{n}}$ and $\left(n-\mathrm{Oct}_{2} \mathrm{Sn}\right)_{\mathrm{n}}$ having molar masses $8.0 \times 10^{3}$ and $6.0 \times 10^{3}$ Da respectively. The material produced from the reaction of $\mathrm{Ph}_{2} \mathrm{SnCl}_{2}$ under similar conditions was insoluble in organic solvents at room or elevated temperatures making molecular weight determination impossible; however the elemental composition of the isolated yellow product was in agreement with that of $\left(\mathrm{Ph}_{2} \mathrm{Sn}\right)_{\mathrm{n}} \cdot{ }^{130}$

In general, there are several disadvantages of the Wurtz synthetic method; it has limited tolerance to functional groups, the yields are moderate, the reproducibility is poor and it is dangerous due to the pyrophoric nature of Na metal and harsh reaction conditions. ${ }^{131}$ 


\subsubsection{Electrochemical synthesis:}

Electrochemical polymerization has also been successfully applied to the synthesis of linear and network polystannanes. Okano et al. ${ }^{132}$ prepared poly(di-n-butylstannane) and poly(di$n$-octylstannane) by this method. The synthesis is completed in a one compartment cell in which $20 \mathrm{~V}$ is constantly applied between the Pt cathode and Ag anode in DME or THF with tetra $n$ butylammonium perchlorate (TBAP) as the supporting electrolyte.

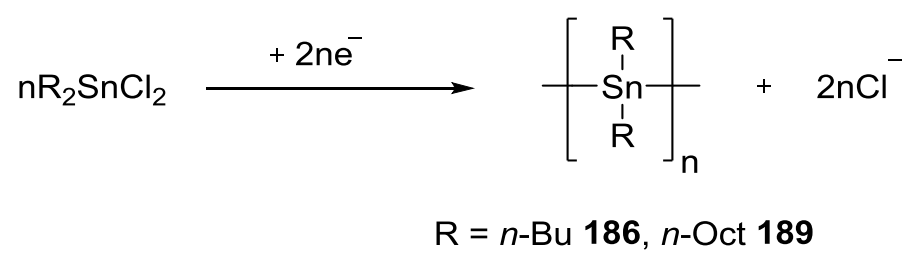

Scheme 19: Electrochemical polymerization dialkyltin dihalides.

Yields for $\left(n-\mathrm{Bu}_{2} \mathrm{Sn}\right)_{\mathrm{n}}$ and $\left(n-\mathrm{Oct} \mathrm{Sn}_{2}\right)_{\mathrm{n}}$ polymers were between $40-60 \%$ and $30-50 \%$ respectively. The highest reported molecular weight of $\left(n-\mathrm{Bu}_{2} \mathrm{Sn}\right)_{n}$ and $\left(n-\mathrm{Oct}_{2} \mathrm{Sn}\right)_{\mathrm{n}}$ from this method was $1.09 \times 10^{4}(\mathrm{PDI}=2.6)$ and $0.59 \times 10^{4} \mathrm{Da}(\mathrm{PDI}=1.7)$ respectively. ${ }^{124}$ Kulandainathan et al. ${ }^{133}$ reported the polymerization of $\mathrm{Me}_{2} \mathrm{SnCl}_{2}$ using aluminium rods as the cathode and anode and $0.4 \mathrm{M}$ tetra $n$-butylammonium tetrafluoroborate (TBATFB) in DME as the supporting electrolyte and reaction solvent.

Network polystannanes of methyl-, $n$-butyl-, $n$-octyl-, and phenyl-trichlorostannane were also synthesized by electrochemical reduction reaction. The estimated molar masses of these polymers were $4-10 \times 10^{4} \mathrm{Da}^{134}$

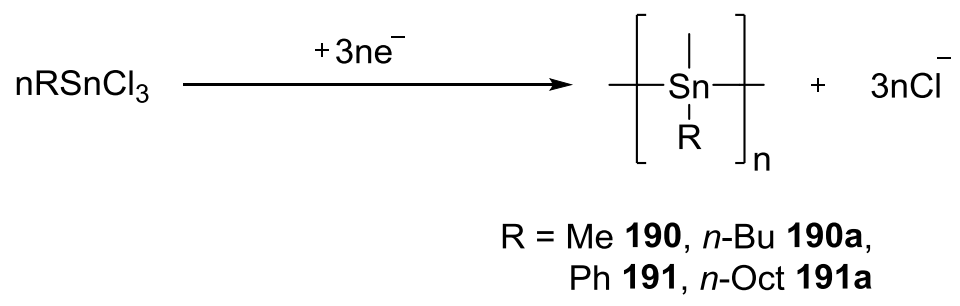

Scheme 20: Electrochemical polymerization alkyltin trihalides. 


\subsubsection{Catalytic dehydrogenation:}

Polystannanes are readily synthesized by catalytic dehydrocoupling of alkyl or aryl tin dihydrides. Harrod et al. ${ }^{135}$ had earlier reported the catalytic dehydrocoupling of silanes $\left(\mathrm{PhSiH}_{3}\right)$ and germanes $\left(\mathrm{PhGeH}_{3}\right)$ to oligosilanes and oligogermanes which is facilitated by Group IV metallocene $(\mathrm{M}=\mathrm{Ti}, \mathrm{Zr})$ catalysts $\left(\mathrm{Cp}_{2} \mathrm{MR}_{2}\left(\mathrm{Cp}=\eta^{5}-\mathrm{C}_{5} \mathrm{H}_{5}\right)\right)$. By contrast, transition metal complexes of $\mathrm{Ti}, \mathrm{Zr}, \mathrm{Hf}, \mathrm{Cr}, \mathrm{Mo}, \mathrm{W}, \mathrm{Rh}, \mathrm{Pt}$ have been employed as catalysts for the dehydropolymerization of primary and secondary stannanes. This method is most successful for the polymerization of secondary stannanes, $\mathrm{R}_{2} \mathrm{SnH}_{2}$.

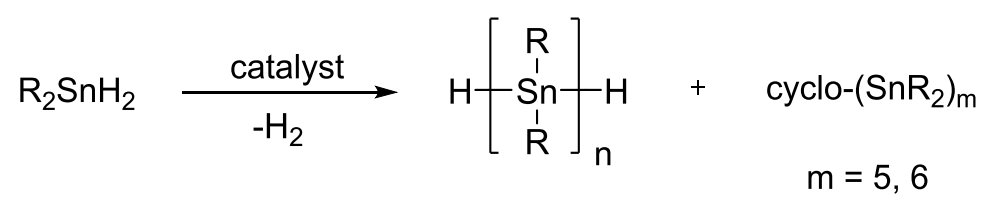

Scheme 21: Catalytic dehydrocoupling of diorganostannanes.

Tilley et al. ${ }^{136}$ reported the synthesis of high molecular weight $\left(M_{\mathrm{w}}=1.7 \times 10^{4} \mathrm{Da}\right)$ poly $(\mathrm{di}-$ $n$-butylstannane) by catalytic dehydrocoupling. Using $\left[\mathrm{Zr}\left(n-\mathrm{C}_{5} \mathrm{H}_{5}\right)\left(n-\mathrm{C}_{5} \mathrm{Me}_{5}\right)\left\{\mathrm{Si}\left(\mathrm{SiMe}_{3}\right)_{3}\right\} \mathrm{Me}\right]$. Tilley et al. ${ }^{118}$ also synthesized high molecular weight polystannanes along with cyclic products from the dehydropolymerization of secondary stannanes using the simpler organometallic zirconium catalyst $\mathrm{Cp}_{2} \mathrm{ZrMe}_{2}$. The best examples for poly(dialkylstannanes) are $\mathbf{1 8 6}\left(M_{\mathrm{w}}=4.6 \times\right.$ $\left.10^{4} \mathrm{Da}\right)$ and $\mathbf{1 8 7}\left(M_{\mathrm{w}}=9.2 \times 10^{4} \mathrm{Da}\right)$ and for poly(diarylstannanes) are $\mathrm{H}\left[\left(p-\mathrm{Bu}^{t}-\mathrm{C}_{6} \mathrm{H}_{4}\right)_{2} \mathrm{Sn}\right]_{\mathrm{n}} \mathrm{H}\left(M_{\mathrm{w}}\right.$ $\left.=5.6 \times 10^{4} \mathrm{Da}\right)$ and $\mathrm{H}\left[\left(p-\mathrm{Hex}-\mathrm{C}_{6} \mathrm{H}_{4}\right)_{2} \mathrm{Sn}\right]_{\mathrm{n}} \mathrm{H}\left(M_{\mathrm{w}}=4.8 \times 10^{4} \mathrm{Da}\right)$. Sita et al. ${ }^{121}$ catalyzed the polymerization of $(n-\mathrm{Bu})_{2} \mathrm{SnH}_{2}$ utilizing the commercially available carbonyltris(triphenylphosphine)rhodium(I) hydride, $\mathrm{HRh}(\mathrm{CO})\left(\mathrm{PPh}_{3}\right)_{3}$. The synthesis of high molecular weight $186\left(M_{\mathrm{w}}=5.0 \times 10^{4} \mathrm{Da}\right)$ was confirmed by gel permeation chromatography (GPC) along with a small amount of oligomeric or cyclic components. Kim et al. ${ }^{137}$ investigated the dehydrocoupling of the $(n-\mathrm{Bu})_{2} \mathrm{SnH}_{2}$ utilizing a series of early transition metal catalysts 
$\mathrm{Cp}_{2} \mathrm{MCl}_{2} / \operatorname{Red}-\mathrm{Al}(\mathrm{M}=\mathrm{Ti}, \mathrm{Zr}, \mathrm{Hf})$ and $\mathrm{M}(\mathrm{CO})_{6} / \operatorname{Red}-\mathrm{Al}(\mathrm{M}=\mathrm{Cr}$, Mo, W). This produced two phases of polymers, one a cross-linked insoluble material and the other a non cross-linked THF soluble solid. Tilley et al. ${ }^{138}$ has also used hafnocene stannyl complexes for the

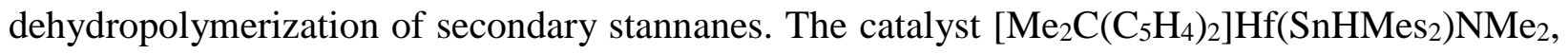
produced $\mathrm{H}\left(n-\mathrm{Bu}_{2} \mathrm{Sn}\right)_{n} \mathrm{H}$ polymer that was isolated as a yellow solid $\left(M_{\mathrm{w}}=2.0 \times 10^{4} \mathrm{Da}\right)$ along with cyclic oligomers.

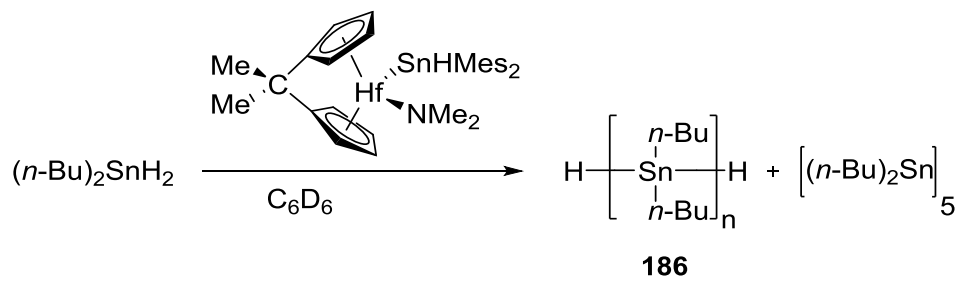

Scheme 22: Hafnocene catalyzed dehydrocoupling of $(n-\mathrm{Bu})_{2} \mathrm{SnH}_{2}$

Schubert et al. ${ }^{139}$ attempted the dehydrocoupling of stannanes $\left((n-\mathrm{Bu})_{2} \mathrm{SnH}_{2}\right.$ and $\left.\mathrm{Ph}_{2} \mathrm{SnH}_{2}\right)$ to polystannanes using $\left(\mathrm{PhMe}_{2} \mathrm{P}\right)_{2} \mathrm{PtMe}_{2}$ or $\left[\left(\kappa^{2}-\mathrm{P}, \mathrm{N}\right)-\mathrm{Ph}_{2} \mathrm{PC}_{2} \mathrm{H}_{4} \mathrm{NMe}_{2}\right] \mathrm{PtMe}$. Soluble poly $(\mathrm{di}-n-$ butylstannane) was observed by ${ }^{119} \mathrm{Sn}$ NMR analysis along with the appearance of cyclic and linear oligomers whereas only insoluble material reovered when $\mathrm{Ph}_{2} \mathrm{SnH}_{2}$ was polymerized. More recently, Caseri et al. ${ }^{19-120,140}$ reported the synthesis of high molecular weight alkylpolystannanes catalyzed by Wilkinson's catalyst, $\left[\mathrm{RhCl}\left(\mathrm{PPh}_{3}\right)_{3}\right]$. The polymers obtained were isolated without the detectable amount of cyclic oligomers. This polymerization is sensitive to the steric bulk of branched dialkylstannanes and the polymerization only proceeds if at least two methylene groups are present between the an aryl carbon and the tin atom. ${ }^{141}$

Samarium diiodide $\left(\mathrm{SmI}_{2}\right)$ was used to synthesize low molecular weight poly(dialkystannanes) under mild conditions from $\mathrm{R}_{2} \mathrm{SnCl}_{2}\left(\mathrm{R}=\mathrm{Me}-\right.$, Et-, $n$-Hex-). ${ }^{143,144} \mathrm{SmI}_{2}$ is a mild one-electron reducing agent of a homogeneous nature and has been applied to the catenation of Group 14 elements. The polymerization was carried out at room temperature using $\mathrm{SmI}_{2}(2.0$ 
eq.) in HMPA/THF and a reaction time of 24-120 h. The maximum molecular weight $4.82 \times 10^{3}$ Da was obtained for $\left(\mathrm{Et}_{2} \mathrm{Sn}\right)_{\mathrm{n}}$.

Beckmann et $a l .{ }^{142}$ prepared first example of a chiral polystannane by catalytic dehydrocoupling following Scheme 23.

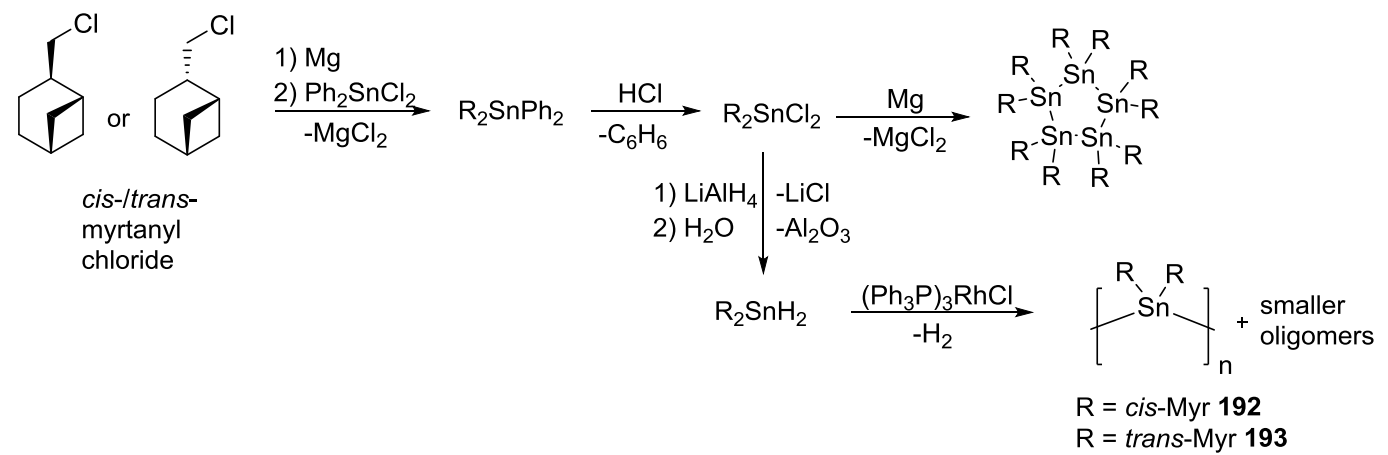

Scheme 23: Synthesis of chiral polystannanes.

Lechner et al. ${ }^{130}$ more recently reported the preparation of polystannanes by dehydrogenative coupling in the presence of TMEDA. The unsymmetrical polystannanes $[n$ $\mathrm{Bu}(\mathrm{Ph}) \mathrm{Sn}]_{\mathrm{n}}$ and $\left[(4-n-\mathrm{BuPh})_{2} \mathrm{Sn}\right]_{\mathrm{n}}$ were synthesized having molar weights of $1.3 \times 10^{4}$ and $4.6 \times$ $10^{4} \mathrm{Da}$, while PDI values were 2.0 and 3.2 , respectively.

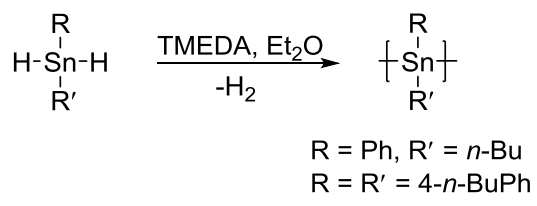

Scheme 24: TMEDA catalyzed dehydrocoupling of stannanes

\subsubsection{Properties of polystannanes:}

Polystannanes represent an interesting class of organometallic polymers due to their thermal, optical and electronic properties. These polymers are often viscous oils or solids yellow to orange in color. Polystannanes are stable in their oil/solid state but relatively less stable in different solvents, particularly in pentane and THF. However, a major drawback of these polymers is their sensitivity towards light and moisture. 


\subsubsection{Photosensitivity:}

The photobleaching of polystannanes in ambient light causes the scission of Sn-Sn bonds

resulting in the formation of cyclic oilgomers such as cyclic- $\left(\mathrm{R}_{2} \mathrm{Sn}\right)_{5}$ and $\left(\mathrm{R}_{2} \mathrm{Sn}\right)_{6}$. Recently, ${ }^{124}$ it was demonstrated that the photostability of polystannanes changes with the type of organic side groups. For instance, poly[bis(4-n-butylphenyl)stannane] was found to be more stable to light than poly(di- $n$-butyl)stannane in THF and DCM solvents.

\subsubsection{Thermal properties:}

Overall, polystannanes relatively exhibit good thermal stability. Thermogravimetric analysis showed that poly(dialkylstannanes) start decomposition at temperatures $>255-270{ }^{\circ} \mathrm{C}$ under $\mathrm{N}_{2}$ which is slightly lower than for poly(diarylstannanes) at temperature $\approx 300{ }^{\circ} \mathrm{C} .{ }^{116}$ Differential scanning calorimetry (DSC) is a technique used to measure the heat effects on the phase transitions as a function of temperature. For polystannanes it is routinely used to determine the glass transition $\left(T_{\mathrm{g}}\right)$ temperature. In the case of poly(dialkylstannanes) $T_{\mathrm{g}}$ varies between $0{ }^{\circ} \mathrm{C}$ to $91{ }^{\circ} \mathrm{C}$ depending upon the chain of the length of alkyl chain and for poly(alkylphenylstannane) ranges from $-20{ }^{\circ} \mathrm{C}$ to $-50{ }^{\circ} \mathrm{C} .{ }^{120,140}$

\subsubsection{Conductivity:}

Room temperature conductivity studies for a number of neutral polystannanes, such as poly(di(3-propylphenyl)stannanes) found values of approximately $\approx 3 \times 10^{-8} \mathrm{~S} \cdot \mathrm{cm}^{-1}$ which increase with temperature a characteristic of a semi-conducting material. ${ }^{142}$ The thin film of $\mathrm{H}(n$ $\left.\mathrm{Bu}_{2} \mathrm{Sn}\right)_{\mathrm{n}} \mathrm{H}$ and $\mathrm{H}\left(n-\mathrm{Oct}_{2} \mathrm{Sn}\right)_{\mathrm{n}} \mathrm{H}$ doped with $\mathrm{SbF}_{5}$ measured at room temperature had conductivity of $10^{-2}$ and $0.3 \mathrm{~S} \cdot \mathrm{cm}^{-1}$ respectively. ${ }^{118}$ 


\subsubsection{Electronic properties:}

A number of electronic studies were conducted on oligostannanes which showed a systematic red shift with an increase in the chain length of the molecule. Drenth et al. ${ }^{145}$ demonstrated that $\lambda_{\max }$ for $\mathrm{Et}_{3} \mathrm{Sn}-\left(\mathrm{SnEt}_{2}\right)_{\mathrm{n}}-\mathrm{SnEt}_{3}(\mathrm{n}=0-4)$ is red shifted and associated with a $\sigma$ $\sigma^{*}$ transition from $232-325 \mathrm{~nm}$. Sita at el. ${ }^{146}$ also observed similar trends for $n-\mathrm{Bu}_{3} \mathrm{Sn}-\left(n-\mathrm{Bu}_{2} \mathrm{Sn}\right)_{\mathrm{n}^{-}}$ $\mathrm{Sn}(n-\mathrm{Bu})_{2}-\mathrm{CH}_{2} \mathrm{CH}_{2} \mathrm{OEt}(\mathrm{n}=0-4)$ and Drager et al. ${ }^{117}$ for $\mathrm{Ph}_{3} \mathrm{Sn}-\left(t-\mathrm{Bu}_{2} \mathrm{Sn}\right)_{\mathrm{n}}-\mathrm{SnPh}_{3}(\mathrm{n}=1-4)$ oligomers.

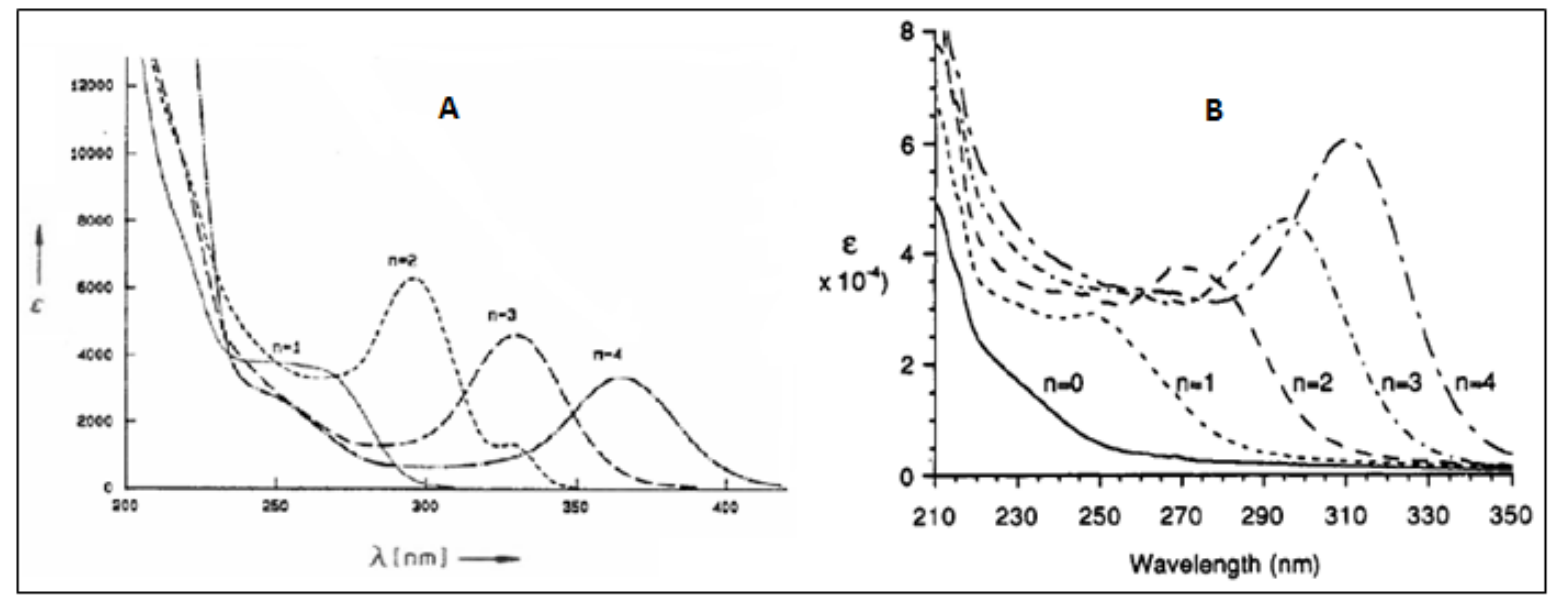

Figure 29: The electronic spectra of $(\mathrm{A})(n-\mathrm{Bu})_{3} \mathrm{Sn}-\left(n-\mathrm{Bu}{ }_{2} \mathrm{Sn}\right)_{n}-\mathrm{Sn}(n-\mathrm{Bu})_{2}-\left(\mathrm{CH}_{2}\right)_{2} \mathrm{OEt}(\mathrm{n}=0-$ $4)^{148},(\mathrm{~B})$ for $\mathrm{Ph}_{3} \mathrm{Sn}-\left((t-\mathrm{Bu})_{2} \mathrm{Sn}\right)_{\mathrm{n}}-\mathrm{SnPh}_{3}(\mathrm{n}=1-4) .{ }^{117}$

The $\lambda_{\max }$ values of hydrogen terminated poly(dialkylstannane)s are in the range of 380-480 $\mathrm{nm}$. The $\lambda_{\max }$ values depend on a number of factors such as solvent, polymer conformation, molar mass and the amount of cyclics present in the sample. The $\lambda_{\max }$ values of poly(diarylstannane)s (430-506 nm) are significantly red shifted (> $50 \mathrm{~nm}$ ) compared to poly(dialkylstannane)s, which indicate the presence of $\sigma-\pi$ conjugation between aryl substituents and the tin atoms in the backbone of the polymer. The $\lambda_{\max }$ values of polystannanes are listed in Table 10. 
Table 10: UV-Visible spectral data and ${ }^{119} \mathrm{Sn}$ NMR chemical shifts for polystannanes.

\begin{tabular}{|c|c|c|c|}
\hline Polymer & $\lambda_{\max }(\mathbf{n m})^{\mathrm{a}}$ & ${ }^{119}$ Sn NMR (ppm) & Ref. \\
\hline$\left(\mathrm{Et}_{2} \mathrm{Sn}\right)_{\mathrm{n}}$ & 368 & $-172.2^{\mathrm{c}}$ & 120 \\
\hline$\left(\operatorname{Pr}_{2} \mathrm{Sn}\right)_{n}$ & - & $-194.8^{d}$ & 120 \\
\hline$\left(n-\mathrm{Bu}_{2} \mathrm{Sn}\right)_{\mathrm{n}}$ & 390 & $-189.6^{\mathrm{e}}$ & 118,120 \\
\hline$\left(n-\mathrm{Pn}_{2} \mathrm{Sn}\right)_{\mathrm{n}}$ & - & $-192.0^{\mathrm{d}}$ & 120 \\
\hline$\left(n-\mathrm{Hex}_{2} \mathrm{Sn}\right)_{\mathrm{n}}$ & 384 & $-190.9^{\mathrm{e}}$ & 118,120 \\
\hline$\left(n-\mathrm{Oct}_{2} \mathrm{Sn}\right)_{\mathrm{n}}$ & 388 & $-190.7^{\mathrm{e}}$ & 116 \\
\hline$\left(n-\operatorname{Dod}_{2} \mathrm{Sn}\right)_{\mathrm{n}}$ & - & $-189.0^{\mathrm{d}}$ & 120 \\
\hline $\mathrm{H}\left[\left(p-t-\mathrm{Bu}-\mathrm{C}_{6} \mathrm{H}_{4}\right)_{2} \mathrm{Sn}\right]_{\mathrm{n}} \mathrm{H}$ & 432 & $-197.0^{\mathrm{d}}$ & 116,120 \\
\hline $\mathrm{H}\left[\left(p-n-\mathrm{Hex}-\mathrm{C}_{6} \mathrm{H}_{4}\right)_{2} \mathrm{Sn}\right]_{\mathrm{n}} \mathrm{H}$ & $436^{\mathrm{b}}$ & $-196.0^{\mathrm{d}}$ & 116,118 \\
\hline $\mathrm{H}\left[\left(p-n-\mathrm{BuO}-\mathrm{C}_{6} \mathrm{H}_{4}\right)_{2} \mathrm{Sn}\right]_{n} \mathrm{H}$ & $448^{\mathrm{b}}$ & $-183.0^{\mathrm{d}}$ & 116,120 \\
\hline $\mathrm{H}\left[\left(o-\mathrm{Et}-p-n-\mathrm{BuO}-\mathrm{C}_{6} \mathrm{H}_{3}\right)_{2} \mathrm{Sn}\right]_{\mathrm{n}} \mathrm{H}$ & $506^{b}$ & $-125.0^{\mathrm{d}}$ & 116,120 \\
\hline$\left.\left[\left(o-\mathrm{Et}-\mathrm{C}_{6} \mathrm{H}_{4}\right)_{2} \mathrm{Sn}\right)\right] \mathrm{n}$ & $468^{b}$ & - & 116 \\
\hline$\left[(4-n-\mathrm{BuPh})_{2} \mathrm{Sn}\right]_{\mathrm{n}}$ & $420^{\mathrm{b}}$ & - & 116 \\
\hline$[n-\mathrm{Bu}(\mathrm{Ph}) \mathrm{Sn}]_{\mathrm{n}}$ & $410^{\mathrm{b}}$ & - & - \\
\hline$\left[\left(\mathrm{PhC}_{2} \mathrm{H}_{4}\right)_{2} \mathrm{Sn}\right]_{\mathrm{n}}$ & - & $-187.0^{c}$ & 140 \\
\hline$\left[\left(\mathrm{PhC}_{3} \mathrm{H}_{6}\right)_{2} \mathrm{Sn}\right]_{\mathrm{n}}$ & - & $-192.12^{c}$ & 140 \\
\hline$\left[\left(\mathrm{PhC}_{4} \mathrm{H}_{8}\right)_{2} \mathrm{Sn}\right]_{\mathrm{n}}$ & - & $-190.4^{c}$ & 140 \\
\hline$\left[\left(p-\mathrm{CF}_{3} \mathrm{C}_{6} \mathrm{H}_{4}\right)_{2} \mathrm{Sn}\right]_{\mathrm{n}}$ & 332 & -56.7 & 128 \\
\hline$\left[\left(3,5-\mathrm{CF}_{3} \mathrm{C}_{6} \mathrm{H}_{4}\right)_{2} \mathrm{Sn}\right]_{\mathrm{n}}$ & 327 & -48.9 & 128 \\
\hline
\end{tabular}

${ }^{\mathrm{a}}$ In THF, ${ }^{\mathrm{b}}$ As film, ${ }^{\mathrm{c}}$ Measured in dichloromethane- $d_{2},{ }^{\mathrm{d}}$ benzene- $d_{6}$, and ${ }^{\mathrm{e}}$ toluene- $d_{8}$

\subsubsection{5 ${ }^{119}$ Sn NMR:}

NMR $\left({ }^{1} \mathrm{H},{ }^{13} \mathrm{C},{ }^{119} \mathrm{Sn}\right)$ spectroscopy is the key instrumental tool for the structural characterization of polystannanes. In particular, ${ }^{119} \mathrm{Sn}$ NMR can easily differentiate between linear polymer and oligomeric chains and cyclic structures. The linear poly(dialkylstannane)s in Table 10 exhibit a single resonance around -190 ppm, except for the poly(diethylstannane) which shows a signal at $-172.2 \mathrm{ppm}$. The ${ }^{119} \mathrm{Sn}$ NMR resonance for poly(diarylstannane)s range from -183 to $197 \mathrm{ppm}$ with the exception of $\left[\left(o-\mathrm{Et}-p-\mathrm{BuO}-\mathrm{C}_{6} \mathrm{H}_{3}\right)_{2} \mathrm{Sn}\right]_{n}$ which showed an unusual resonance at $125 \mathrm{ppm}$.

\subsubsection{Thermochromic properties:}

The poly(dialkylstannane)s $\quad\left(n-\mathrm{Hex}_{2} \mathrm{Sn}\right)_{\mathrm{n}}$ and $\quad\left(n-\mathrm{Oct}_{2} \mathrm{Sn}\right)_{\mathrm{n}} \quad$ showed reversible thermochromic behaviour which is evident from the discoloration of these materials upon warming 
above room temperature. UV-vis spectrometry revealed a blue shift in the absorption maximum between $384-369 \mathrm{~nm}$ for $\left(n-\mathrm{Oct}_{2} \mathrm{Sn}\right)_{\mathrm{n}}$ moving from $30-40{ }^{\circ} \mathrm{C}$ in a toluene solution and from $392-$ $382 \mathrm{~nm}$ for a solid film of $\left(n-\mathrm{Hex}_{2} \mathrm{Sn}\right)_{\mathrm{n}}$ in the same temperature range.

\subsubsection{Molecular weights:}

Gel permeation chromatography (GPC) has been used to estimate the molar masses of polystannanes. The molecular weights of polystannanes (Table 11) are determined against polystyrene standards of different known molecular weights in THF as the mobile phase using a RI detector. 
Table 11: Molar weights of polystannanes.

\begin{tabular}{|c|c|c|c|c|c|}
\hline Compound & $M_{\mathbf{w}}[\mathrm{Da}]$ & $M_{\mathbf{n}}[\mathrm{Da}]$ & PDI $\left[M_{\mathrm{w}} / M_{\mathrm{n}}\right]$ & Polymerization Method & Ref. \\
\hline$\left(\mathrm{Me}_{2} \mathrm{Sn}\right)_{\mathrm{n}}$ & 1,120 & & 1.49 & $\mathrm{SmI}_{2}$ in HMPA-THF & 145 \\
\hline$\left(\mathrm{Et}_{2} \mathrm{Sn}\right)_{\mathrm{n}}$ & $\begin{array}{c}31,000 \\
4,820 \\
4,100 \\
3,700\end{array}$ & 13,000 & $\begin{array}{l}1.47 \\
1.21 \\
1.25 \\
1.15\end{array}$ & $\begin{array}{c}\text { catalytic dehydrogenation } \\
\mathrm{SmI}_{2} \text { in HMPA-THF } \\
\mathrm{Mg} \text { in THF } \\
\mathrm{Ca} \text { in THF }\end{array}$ & $\begin{array}{l}120 \\
145 \\
145 \\
145\end{array}$ \\
\hline$\left(\operatorname{Pr}_{2} \mathrm{Sn}\right)_{\mathrm{n}}$ & 27,000 & 10,000 & 1.58 & catalytic dehydrogenation & 120 \\
\hline$\left(n-\mathrm{Bu}_{2} \mathrm{Sn}\right)_{\mathrm{n}}$ & $\begin{array}{l}91,000 \\
17,500 \\
46,000 \\
10,900\end{array}$ & $\begin{array}{c}36,000 \\
7,800 \\
13,900\end{array}$ & $\begin{array}{l}1.39 \\
2.24 \\
3.31 \\
2.26\end{array}$ & $\begin{array}{l}\text { catalytic dehydrogenation } \\
\text { catalytic dehydrogenation } \\
\text { catalytic dehydrogenation } \\
\text { Electrochemical synthesis }\end{array}$ & $\begin{array}{l}120 \\
118 \\
118 \\
132\end{array}$ \\
\hline$\left(n-\mathrm{Pn}_{2} \mathrm{Sn}\right)_{\mathrm{n}}$ & 48,000 & 19,000 & 1.30 & catalytic dehydrogenation & 120 \\
\hline$\left(n-\mathrm{Hex}_{2} \mathrm{Sn}\right)_{\mathrm{n}}$ & $\begin{array}{c}76,000 \\
36,800 \\
2,770\end{array}$ & $\begin{array}{l}31,000 \\
15,300\end{array}$ & $\begin{array}{c}1.32 \\
2.4 \\
1.18\end{array}$ & $\begin{array}{l}\text { catalytic dehydrogenation } \\
\text { catalytic dehydrogenation } \\
\mathrm{SmI}_{2} \text { in HMPA-THF }\end{array}$ & $\begin{array}{l}120 \\
118 \\
145\end{array}$ \\
\hline$\left(n-\mathrm{Oct}_{2} \mathrm{Sn}\right)_{\mathrm{n}}$ & $\begin{array}{c}97,000 \\
95,700 \\
92,600 \\
5,900 \\
\end{array}$ & $\begin{array}{l}40,000 \\
14,300 \\
21,700\end{array}$ & $\begin{array}{c}1.22 \\
6.7 \\
4.26 \\
1.7 \\
\end{array}$ & $\begin{array}{l}\text { catalytic dehydrogenation } \\
\text { catalytic dehydrogenation } \\
\text { catalytic dehydrogenation } \\
\text { Electrochemical synthesis }\end{array}$ & $\begin{array}{l}120 \\
118 \\
118 \\
132\end{array}$ \\
\hline$\left(n-\operatorname{Dod}_{2} \mathrm{Sn}\right)_{\mathrm{n}}$ & 28,000 & 19,000 & 1.16 & catalytic dehydrogenation & 120 \\
\hline $\mathrm{H}\left[\left(p-{ }^{t} \mathrm{Bu}-\mathrm{C}_{6} \mathrm{H}_{4}\right)_{2} \mathrm{Sn}\right]_{n} \mathrm{H}$ & 56,000 & 16,700 & 3.35 & catalytic dehydrogenation & 116 \\
\hline $\mathrm{H}\left[\left(p-{ }^{n} \mathrm{Hex}-\mathrm{C}_{6} \mathrm{H}_{4}\right)_{2} \mathrm{Sn}\right]_{\mathrm{n}} \mathrm{H}$ & 48,200 & 20,000 & 2.41 & catalytic dehydrogenation & 116 \\
\hline $\mathrm{H}\left[\left(p-{ }^{n} \mathrm{BuO}-\mathrm{C}_{6} \mathrm{H}_{4}\right)_{2} \mathrm{Sn}\right]_{\mathrm{n}} \mathrm{H}$ & 12,000 & 7,000 & 1.71 & catalytic dehydrogenation & 116 \\
\hline $\mathrm{H}\left[\left(o-\mathrm{Et}-p-{ }^{n} \mathrm{BuO}-\mathrm{C}_{6} \mathrm{H}_{4}\right)_{2} \mathrm{Sn}\right]_{\mathrm{n}} \mathrm{H}$ & 4,400 & 4,000 & 1.1 & catalytic dehydrogenation & 144 \\
\hline$\left[(4-n-\mathrm{BuPh})_{2} \mathrm{Sn}\right]_{\mathrm{n}}$ & 46,000 & & 3.2 & TMEDA & 147 \\
\hline$[n-\mathrm{Bu}(\mathrm{Ph}) \mathrm{Sn}]_{\mathrm{n}}$ & 13,000 & & 2.0 & TMEDA & 147 \\
\hline
\end{tabular}




\subsection{Thesis objectives:}

The main objective of this thesis is to prepare structurally stable 5-coordinate polystannanes having ligands containing different donor atoms such as $\mathrm{N}, \mathrm{S}, \mathrm{P}$, and $\mathrm{O}$.

The increase in the coordination at $\mathrm{Sn}$ is expected to achieve the following:

1) Moderate the Lewis acidity of Sn atoms in the backbone of the polystannanes.

2) Increase steric hindrance around Sn.

3) Reduce susceptibility to nucleophilic attack.

The objectives of this research can be accomplished as follows:

i) Synthesize a series of diorganotin dihalides containing $C, N-, C, P-, C, O-$ and $C, S$ chelating ligands.

ii) Prepare new diorganotin dihydrides by using $\mathrm{LiAlH}_{4}$ or $\mathrm{NaBH}_{4}$ as reducing agents.

iii) Carry out the polymerization by dehydrogenative coupling of dihydrides or by the Wurtz coupling of dihalides.

iv) Characterize all monomers and polymers by ${ }^{1} \mathrm{H},{ }^{13} \mathrm{C}$ and ${ }^{119} \mathrm{Sn}$ NMR spectroscopy, UVvis spectroscopy and X-ray crystallography where applicable.

iv) Finally, investigate the material properties and stability of these new 5-coordinate polystannanes. 


\subsection{Results and Discussion:}

\section{1: Synthesis}

\subsubsection{Phenyloxy vinyl ethers}

Vinyl ethers 137 and 194-196 were synthesized following methods reported in literature. 43,148,149 These reactions are examples of classic Williamson ether syntheses where an alcohol (4phenylphenol or phenol), in the presence of a base $\left(\mathrm{K}_{2} \mathrm{CO}_{3}\right)$ and an alkyl halide (i.e. allyl bromide) is converted to an ether. The reaction (Scheme 25) procedes by deprotonating the phenol to produce an aryloxide as an intermediate, which attacks the allyl bromide in a nucleophilic substitution $\left(\mathrm{S}_{\mathrm{N}} 2\right)$ fashion producing the desired ethers. Acetone was the preferred solvent as $\left(\mathrm{S}_{\mathrm{N}} 2\right)$ reactions work best in polar aprotic solvents. ${ }^{148}$ It was reported that a 1.1 molar equivalence of the base resulted in an optimal yield of $\mathbf{1 3 7}$ and $\mathbf{1 9 4} .^{149}$ The target ether species was purified by first redissolving the crude product in DCM, and sequentially washing the organic layer with a $1 \mathrm{M}$ $\mathrm{NaOH}$ solution, brine and water respectively to remove unreacted allyl bromide and phenol. ${ }^{148}$ After solvent removal compound $\mathbf{1 3 7}$ was obtained as a flaky white coloured solid, whereas compounds 194-196 were recovered as yellow coloured viscous oils.

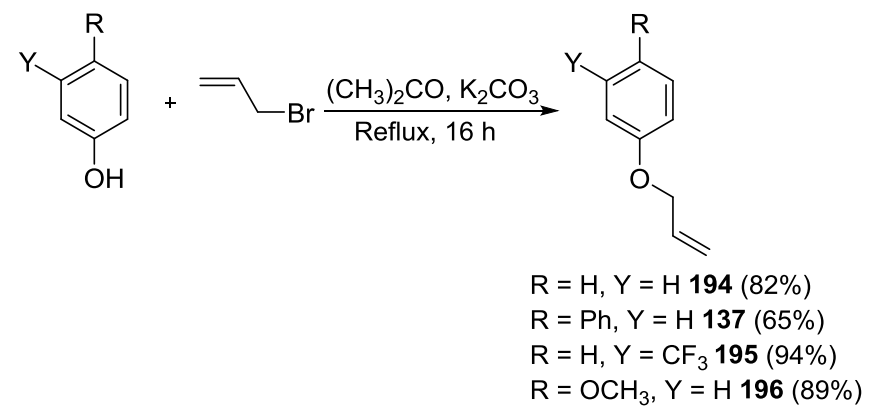

Scheme 25: Williamson's ether synthesis of phenyloxy allyl ethers.

\subsection{2: Triphenylphenyloxy propyl tin:}

Compounds 141 and 197-199 were synthesized via a radical hydrostannylation reaction (Scheme 25) utilizing azobisisobutyronitrile (AIBN) as the radical initiator. ${ }^{43}$ When an equimolar 
amount of allyl ether (137 and 194-196) and $\mathrm{Ph}_{3} \mathrm{SnH}$ were used, a significant amount (>20\%) of the distannane $\left(\mathrm{Ph}_{3} \mathrm{SnSnPh}_{3}:{ }^{119} \mathrm{Sn} \mathrm{NMR}\left(\mathrm{C}_{6} \mathrm{D}_{6}\right) \delta=-143 \mathrm{ppm}\right)$ was always observed. Compounds 141 and 197-199 are relatively air and heat stable; as a result, $\mathrm{Ph}_{3} \mathrm{SnSnPh}_{3}$ and unreacted starting material were readily separated from this mixture by vacuum sublimation. Subsequently, the dissolution of crude $\mathbf{1 4 1}$ in $\mathrm{Et}_{2} \mathrm{O}$ followed by filtration afforded a pure white coloured solid product. Compound 197 was purified by an initial wash with hexane to remove $\mathrm{Ph}_{3} \mathrm{SnSnPh}_{3}$, followed by heating under reduced pressure at $75^{\circ} \mathrm{C}$ for $12 \mathrm{~h}$ to remove unreacted 194 leaving a clear, viscous oil. Crude 198 was dissolved in $\mathrm{MeOH}$ to remove unreacted 195, filtered and finally dried under reduced pressure to afford a white coloured solid. The crude reaction mixture containing 199 was purified in two steps including sublimation to remove the $\mathrm{Ph}_{3} \mathrm{SnSnPh}_{3}$, followed by silica gel column chromatography using hexane:EtOAc $(6: 1)$. The NMR $\left({ }^{1} \mathrm{H},{ }^{13} \mathrm{C}\right.$, ${ }^{119} \mathrm{Sn}$ ) analysis of $\mathbf{1 4 1}$ was consistent with reported literature values. ${ }^{43}$ Triphenylstannanes $\mathbf{1 9 7 -}$ 199 were found to have very similar tin chemical environments $\left({ }^{119} \mathrm{Sn} \mathrm{NMR}\right)$ to $\mathbf{1 4 1}$.

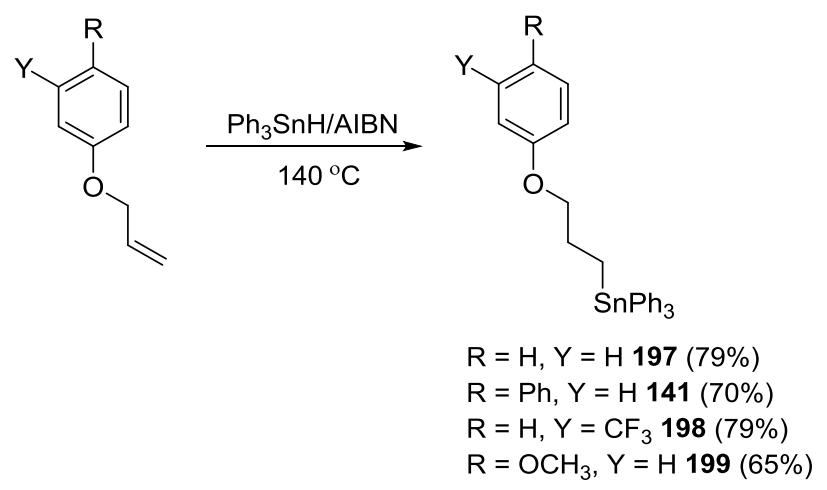

Scheme 26: Synthesis of triphenylphenyloxy propyltin.

\subsection{3: Phenylphenyloxy propyltin dichloride:}

In the original report by Molloy et al., phenyl groups attached to tin were exchanged for Br by stoichiometric bromination. ${ }^{43}$ Despite our best efforts to prepare the desired dibromide 146, most synthetic attempts resulted in the formation of a considerable amount of the mono- and 
tribromide species that proved difficult to purify. In order to make sufficient quantities of the dihalide materials for polymerization studies, an alternative route to these materials was explored. Compounds 202-203 were obtained by stepwise conversion of compounds 197 (Figure 30) and 141 initially to the triorganotin monochlorides (200-201) and then sequentially to diorganotin dichlorides (202-203). This method was first reported by Pannell et al. ${ }^{96}$ for the synthesis of diorganotin dichlorides from triphenyl starting materials. The NMR $\left({ }^{1} \mathrm{H},{ }^{13} \mathrm{C}\right.$, and $\left.{ }^{119} \mathrm{Sn}\right)$ data obtained for compound $\mathbf{2 0 3}$ showed similar chemical shifts when compared to $\mathbf{2 0 2}$.

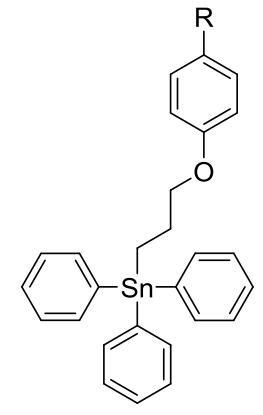

$\mathrm{R}=\mathrm{H}$ 197, Ph 141

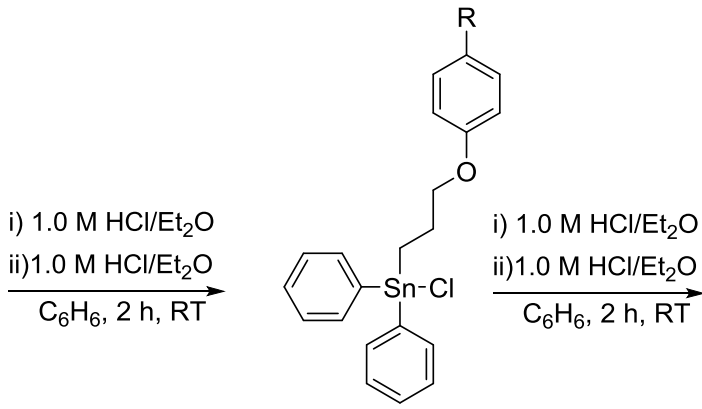

$\mathrm{R}=\mathrm{H} 200$ (81\%), Ph 201 (86\%)

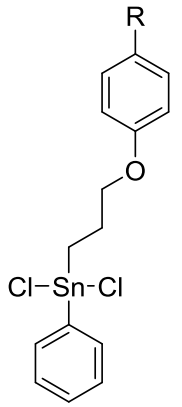

R = H 202 (91\%), Ph 203 (100\%)

Scheme 27: Stepwise preparation of triorganotin monochlorides and diorganotin dichlorides.

The initial attempt to prepare $\mathbf{2 0 0}$ resulted in a mixture of the starting material 197 and the product monochloride. An essentially pure sample of $\mathbf{2 0 0}$ was obtained by washing the crude product with hot hexane or alternatively by the addition of a stoichiometric aliquot of the $1.0 \mathrm{M}$ $\mathrm{HCl}$ solution (calculated on the basis of the amount of starting material from ${ }^{1} \mathrm{H}$ NMR, see Figure 30) required to complete conversion to chloride.

The monochlorides 200 and 201 were not completely converted to their respective dichlorides 202 and 203. The amount of the unreacted monochloride as calculated on the basis of the comparison of intensities of the ortho phenyl protons $\left(2 \mathrm{H}, o-\mathrm{C}_{6} \mathrm{H}_{4} \mathrm{O}\right)$ of the mono- and dichloride by ${ }^{1} \mathrm{H}$ NMR spectroscopy, and the required aliquot of $1.0 \mathrm{M} \mathrm{HCl} / \mathrm{Et}_{2} \mathrm{O}$ added to the solution containing the mixture of mono- and dichlorides. The progressive change from the mono- 
and dichlorides $(\mathbf{2 0 0}, \mathbf{2 0 2})$ are shown in Figure 31 respectively. The percent conversion was calculated from ${ }^{1} \mathrm{H}$ NMR by dividing the integration of the peak for the starting materials $\mathbf{2 0 0}$ and 201 by the peak for the products according to the following formula; ${ }^{150}$

$$
\% \text { Conversion }=(\mathrm{x} / 2) /((\mathrm{x} / 2+\mathrm{y} / 2))
$$

where $\mathrm{y}$ is the disappearance of peaks $(6.53 \mathrm{ppm}$ and $6.61 \mathrm{ppm}$ for 200 and 201) while $\mathrm{x}$ is the appearance of new peak (6.70 ppm and 6.75 ppm for 202 and 203) respectively. Recrystallization in DCM and hexane afforded single crystals of both 202 and 203.

The synthesis of the monochloride $\mathbf{2 0 1}$ by this method routinely resulted in the recovery of $10-15 \%$ of the unreacted starting material, 141. Compound 201 was purified by dissolving the crude product in hot hexane, letting it cool to room temperature and placing it into fridge overnight at $-20^{\circ} \mathrm{C}$. A white coloured crystalline product precipitated out of solution after $24 \mathrm{~h}$. The starting material 141 was recovered by first decanting, then removing solvent under reduced pressure. Recrystallization of compounds 200-201 using a mixture of DCM and hexane afforded clear, colourless crystals. 


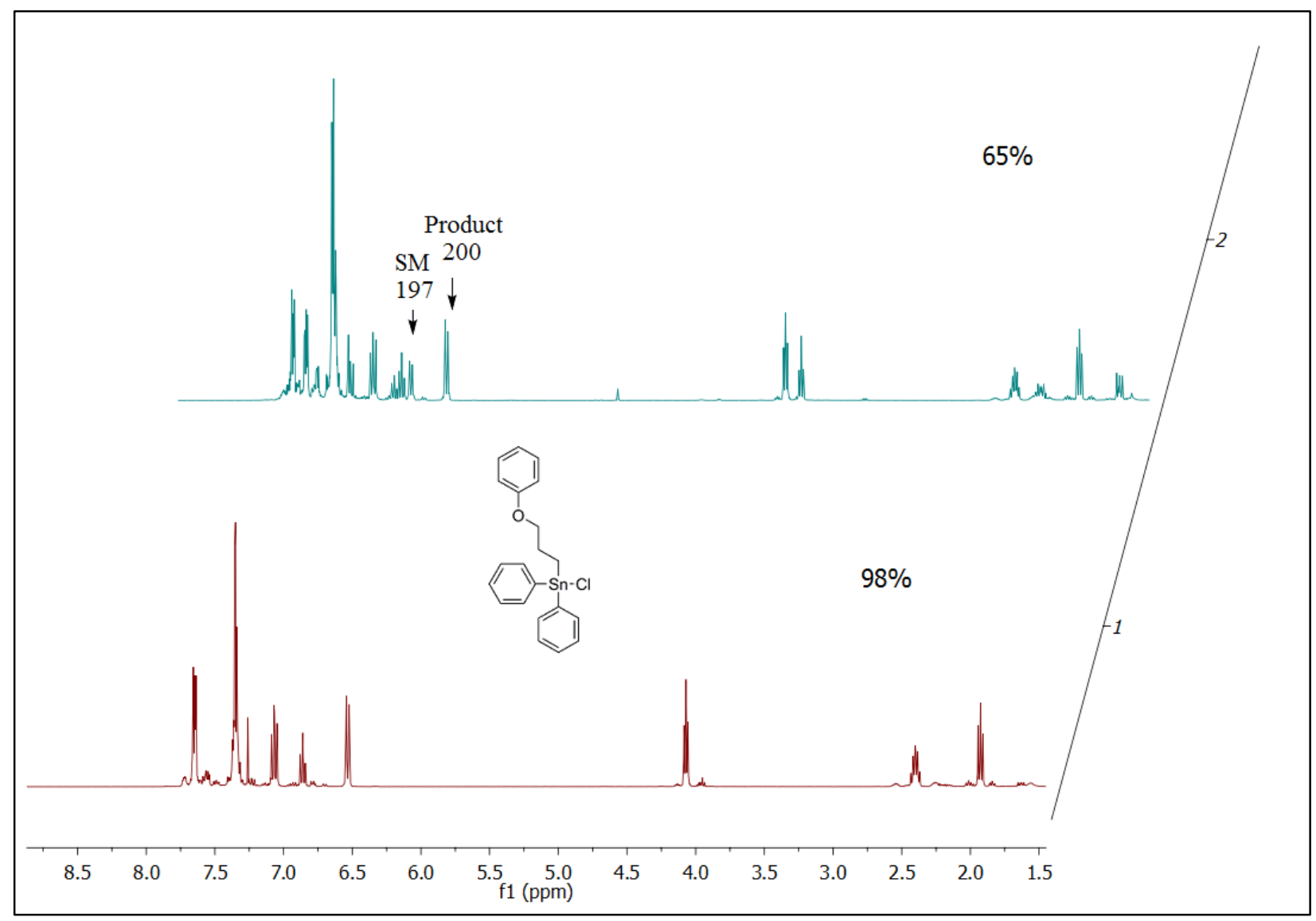

Figure 30: ${ }^{1} \mathrm{H} \mathrm{NMR}\left(\mathrm{CDCl}_{3}\right)$ spectra showing the conversion of 197 to 200. 


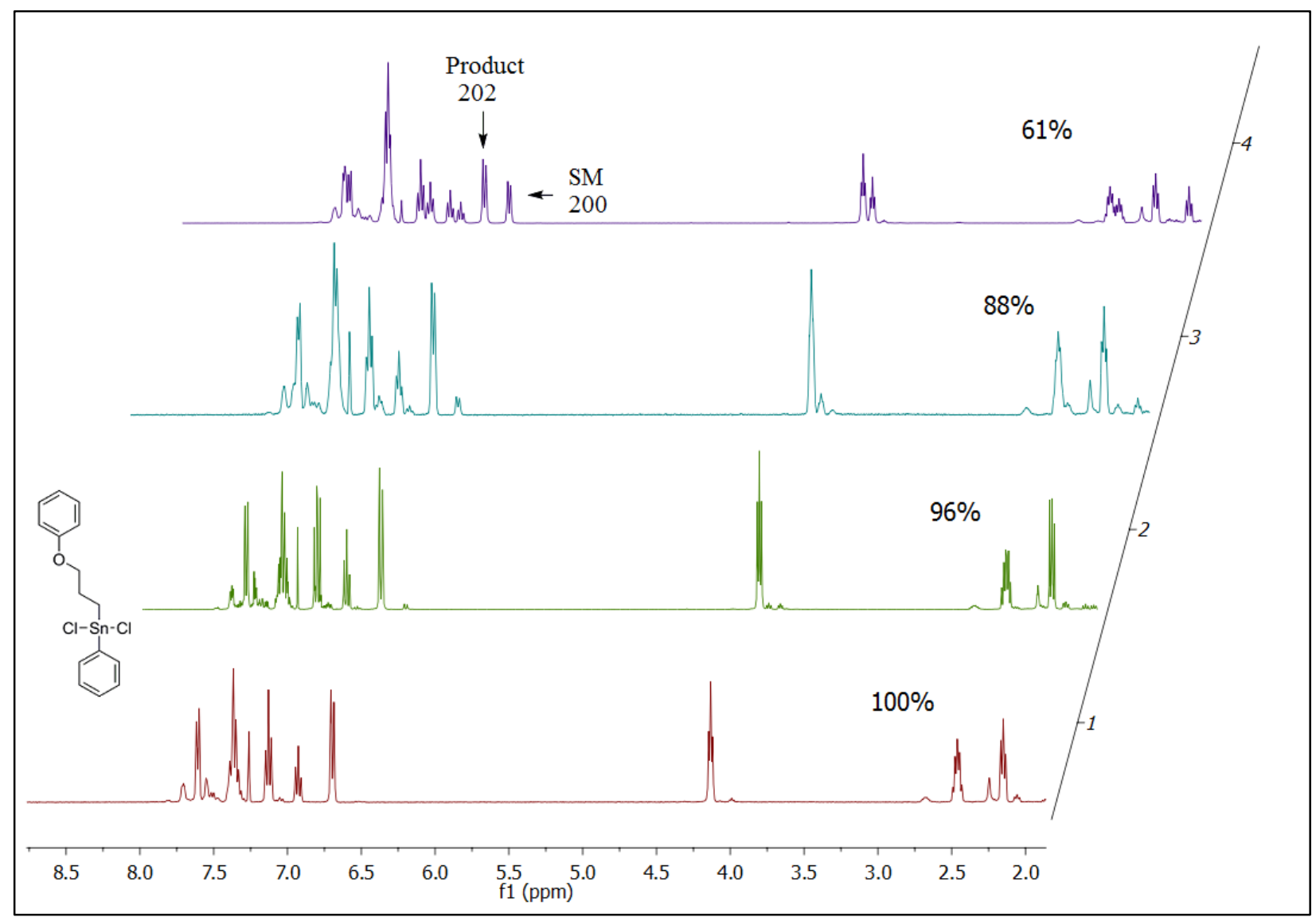

Figure 31: ${ }^{1} \mathrm{H} \mathrm{NMR}\left(\mathrm{CDCl}_{3}\right)$ spectra showing conversion of 200 to 202.

${ }^{119}$ Sn NMR chemical shifts, Sn-O distances and yields of compounds 141, 146 and 197-203 are listed in Table 12.

Table 12: ${ }^{119} \mathrm{Sn}$ NMR $\left(\mathrm{CDCl}_{3}\right)$ chemical shift, Sn-O distances and yields of stannanes.

\begin{tabular}{|c|c|c|c|}
\hline Compounds & $\begin{array}{c}\boldsymbol{\delta}^{\mathbf{1 1 9}} \mathbf{S n}(\mathbf{p p m}) \\
\text { Observed/Literature }^{\mathbf{4 3}}\end{array}$ & $\begin{array}{c}\text { Sn・OO } \\
\text { Distance } \mathbf{A}\end{array}$ & Yield (\%) \\
\hline $\mathbf{1 4 1}$ & $-99.3 /-100.1$ & - & 70 \\
\hline $\mathbf{1 4 6}$ & $-51.3 /-53.3$ & 2.73 & 81 \\
\hline $\mathbf{1 9 7}$ & -99.9 & - & 79 \\
\hline $\mathbf{1 9 8}$ & -99.3 & - & 79 \\
\hline $\mathbf{1 9 9}$ & -100.0 & - & 65 \\
\hline $\mathbf{2 0 0}$ & -26.6 & 2.80 & 81 \\
\hline $\mathbf{2 0 1}$ & -24.7 & 2.81 & 86 \\
\hline $\mathbf{2 0 2}$ & -21.9 & 2.72 & 91 \\
\hline $\mathbf{2 0 3}$ & -20.3 & 2.82 & 100 \\
\hline
\end{tabular}


An attempt to replace the third phenyl group (Scheme 27) with chlorine using Pannell's ${ }^{96}$ method for replacing the first and second phenyl groups resulted in the recovery of only clean dichloride starting material. Substitution in this case is likely hindered by the increased dative interaction between $\mathrm{Sn}-\mathrm{O}$.

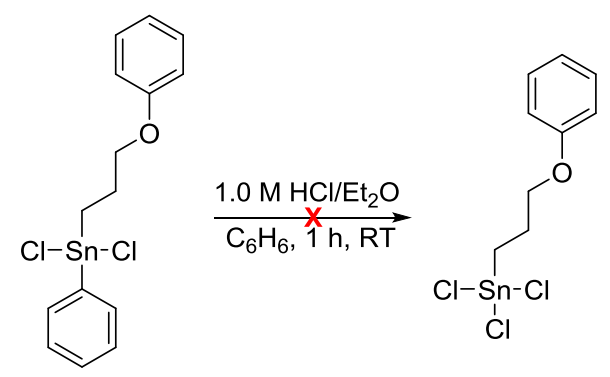

Scheme 28: Attempted synthesis of a tin trihalide.

\subsection{4: Synthesis of hydrides:}

$\mathrm{LiAlH}_{4}$ was used as a reducing agent for the synthesis of compounds 202-205 from their respective halides (Scheme 28).

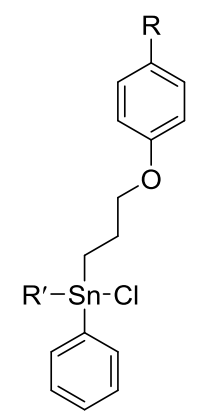

$\mathrm{R}=\mathrm{H}, \mathrm{R}^{\prime}=\mathrm{Ph} 200$

$\mathrm{R}=\mathrm{R}^{\prime}=\mathrm{Ph} 201$

$\mathrm{R}=\mathrm{H}, \mathrm{R}^{\prime}=\mathrm{Cl} 202$

$\mathrm{R}=\mathrm{Ph}, \mathrm{R}^{\prime}=\mathrm{Cl} 203$

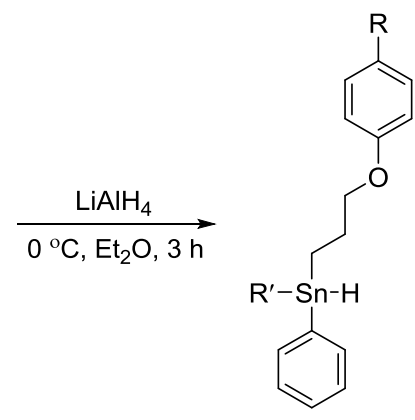

$\mathrm{R}=\mathrm{H}, \mathrm{R}^{\prime}=\mathrm{Ph} 204(70 \%)$

$\mathrm{R}=\mathrm{R}^{\prime}=\mathrm{Ph} 205(61 \%)$

$\mathrm{R}=\mathrm{H}, \mathrm{R}^{\prime}=\mathrm{H} 206(81 \%)$

$\mathrm{R}=\mathrm{Ph}, \mathrm{R}^{\prime}=\mathrm{H} 207(65 \%)$

Scheme 29: Preparation of triorganotin hydrides and diorganotin dihydrides.

The ${ }^{1} \mathrm{H}$ NMR $\left(\mathrm{C}_{6} \mathrm{D}_{6}\right)$ resonances for the hydrogen atoms attached to tin in organotin hydrides range from $4.50-7.50 \mathrm{ppm} .{ }^{65}$ The spectrum of 207 showed a large ${ }^{1} J_{117 / 119 \mathrm{Sn}-1 \mathrm{H}}$ coupling constant characteristic of most tin hydrides (Figure 32, Table 13). 


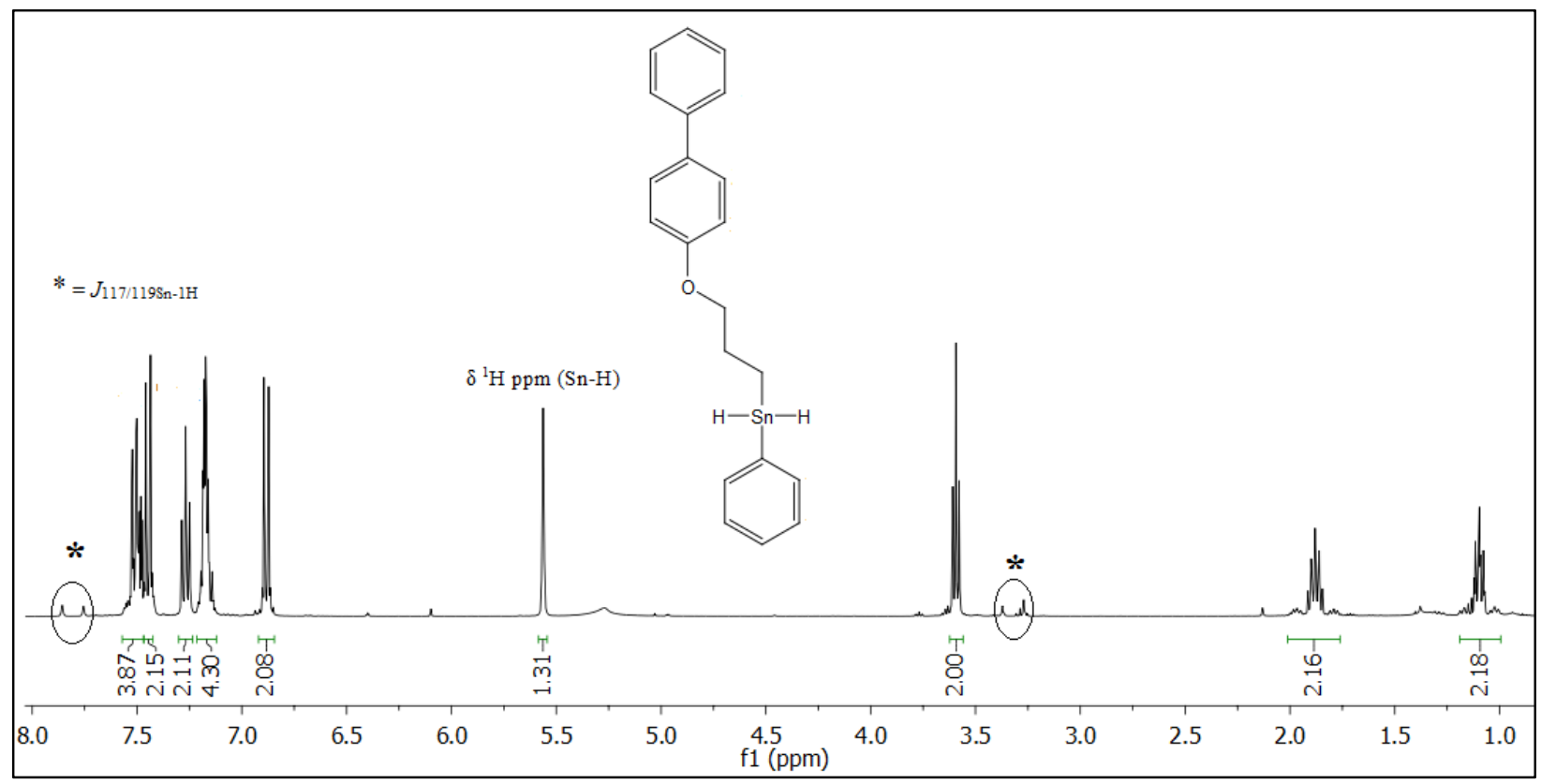

Figure 32: ${ }^{1} \mathrm{H}$ NMR $\left(\mathrm{C}_{6} \mathrm{D}_{6}\right)$ spectrum of 207.

A comparison of coupling constants in Table 13 suggest that the dative interaction between $\mathrm{Sn}$ and $\mathrm{O}$ atoms for organotin hydrides causes a slight increase in the magnitude of the coupling constants as compared to dihydrides. The ${ }^{119} \mathrm{Sn}$ and ${ }^{1} \mathrm{H}$ NMR $\left(\mathrm{C}_{6} \mathrm{D}_{6}\right)$ chemical shifts for several tin hydrides (204-207 and 69, 70) are listed in Table 13.

Table 13: ${ }^{119} \mathrm{Sn}$ NMR $\left(\mathrm{C}_{6} \mathrm{D}_{6}\right)$ data for organotin hydrides.

\begin{tabular}{|c|c|c|c|}
\hline Compound & $\boldsymbol{\delta}^{\mathbf{1 1 9}} \mathbf{S n}$ or $\boldsymbol{\delta}^{\mathbf{1 1 7}} \mathbf{S n}(\mathbf{p p m})$ & $\boldsymbol{\delta}^{\mathbf{1}} \mathbf{H}(\mathbf{p p m}) \mathbf{S n - H}$ & $\boldsymbol{J}_{\mathbf{1 1 9} / \mathbf{1 1 7} \mathbf{S n}-\mathbf{H}(\mathbf{H z})}$ \\
\hline $\mathbf{6 9}$ & $-225.9^{\mathrm{a}}$ & $6.17-6.04$ & $\begin{array}{c}1615 / 1703^{72} \\
1987 / 1883\end{array}$ \\
\hline $\mathbf{7 0}$ & $-244.5^{\mathrm{a}}$ & $6.78-6.64$ & $\begin{array}{c}1940 / 1853^{72} \\
2155 / 2059\end{array}$ \\
\hline $\mathbf{2 0 4}$ & -137.0 & 6.35 & $1856 / 1776$ \\
\hline $\mathbf{2 0 5}$ & -137.0 & 6.36 & $1862 / 1780$ \\
\hline $\mathbf{2 0 6}$ & -215.1 & 5.59 & $1837 / 1754$ \\
\hline $\mathbf{2 0 7}$ & -215.0 & 5.54 & $1835 / 1754$ \\
\hline
\end{tabular}




\subsection{5: Dimerization of 204 and 205:}

Dimerization of 204 and 205 was achieved by catalytic dehydrocoupling using $\mathrm{Pd}_{(}\left(\mathrm{PP}_{3}\right)_{4}$. The distannane 208 was purified by column chromatography using hexane:EtOAc (1:1) and 209 by extraction with petroleum ether. Similar distannanes having a hypercoordinate donor interaction were previously reported by Rupnicki et al..$^{70}$
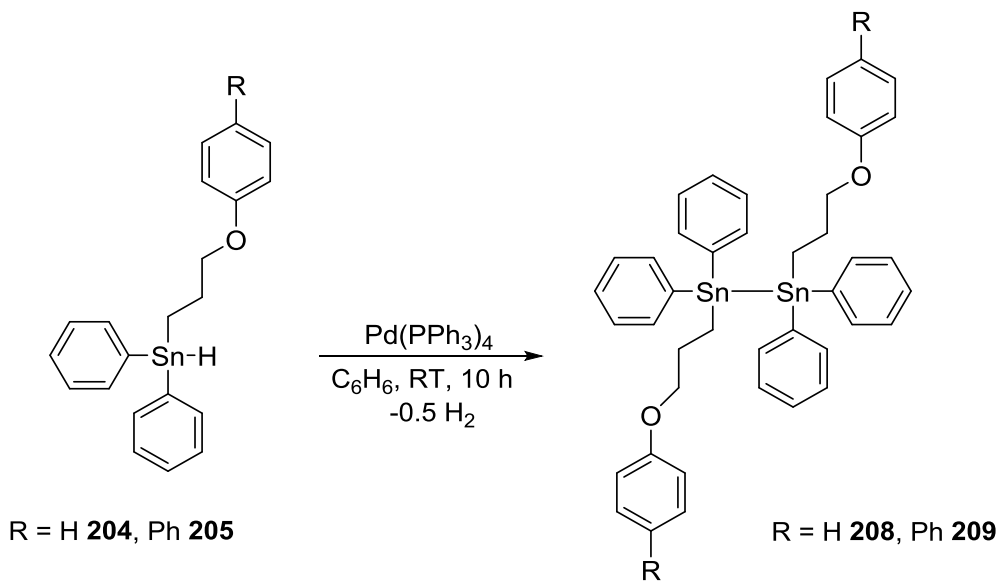

Scheme 30: Synthesis of distannanes 208 and 209. 


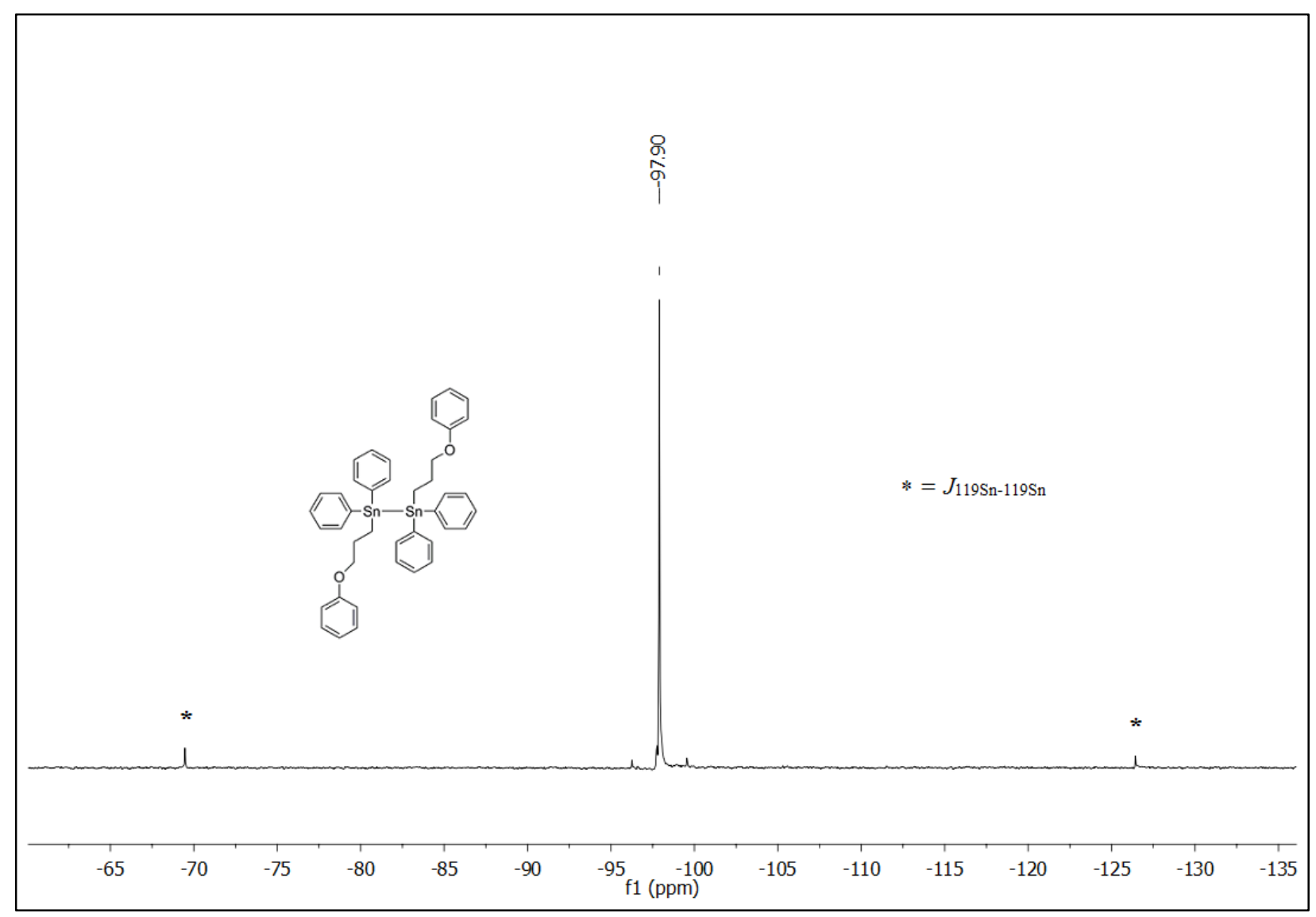

Figure 33: ${ }^{119} \mathrm{Sn}$ NMR $\left(\mathrm{C}_{6} \mathrm{D}_{6}\right)$ spectrum of 208.

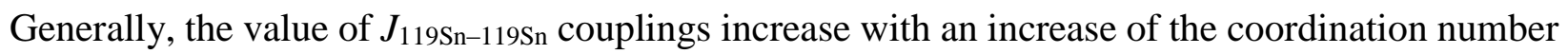

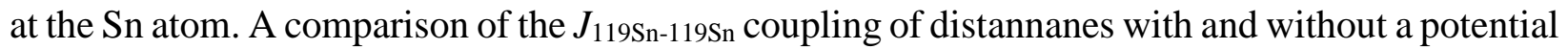
coordinating ligand is given in Table 14.

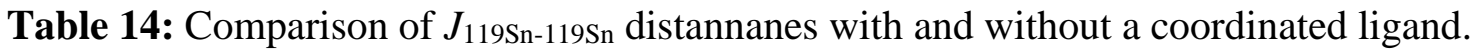

\begin{tabular}{|c|c|c|c|}
\hline Compound & $\begin{array}{c}\boldsymbol{J}^{\mathbf{1 1 9}} \mathbf{S n -}{ }^{119} \mathbf{S n} \\
(\mathbf{H z})\end{array}$ & $\begin{array}{c}\text { Non-coordinated } \\
\text { analogue }\end{array}$ & $\begin{array}{c}\boldsymbol{J}^{\mathbf{1 1 9}} \mathbf{S n - 1 1 9} \mathbf{S n} \\
\mathbf{( H z})\end{array}$ \\
\hline $\mathbf{2 0 8}$ & 8527 & $\mathrm{Ph}_{6} \mathrm{Sn}_{2}$ & $4470^{151}$ \\
\hline $\mathbf{2 0 9}$ & - & $\mathrm{Ph}_{6} \mathrm{Sn}_{2}$ & $4470^{151}$ \\
\hline $\mathbf{2 1 0}$ & $8925^{70}$ & $\mathrm{Ph}_{6} \mathrm{Sn}_{2}$ & $4470^{151}$ \\
\hline $\mathbf{2 1 1}$ & $6294^{70}$ & $(n-\mathrm{Bu})_{6} \mathrm{Sn}_{2}$ & $2748^{151}$ \\
\hline $\mathbf{2 1 2}$ & $3796^{70}$ & $(n-\mathrm{Bu})_{6} \mathrm{Sn}_{2}$ & $2748^{151}$ \\
\hline $\mathbf{2 1 3}$ & $11272^{153}$ & $(n-\mathrm{Bu})_{6} \mathrm{Sn}_{2}$ & $2748^{151}$ \\
\hline
\end{tabular}




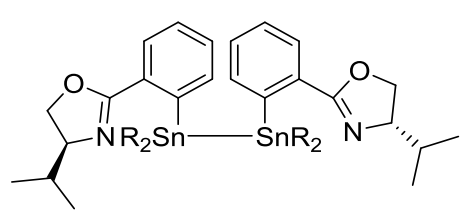

$\mathrm{R}=\mathrm{Ph} \mathbf{2 1 0}, n-\mathrm{Bu} 211$

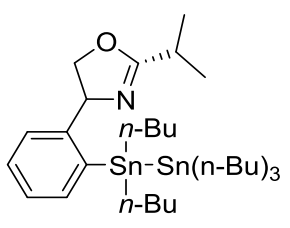

212

Scheme 31: 5-coordinate distannanes.

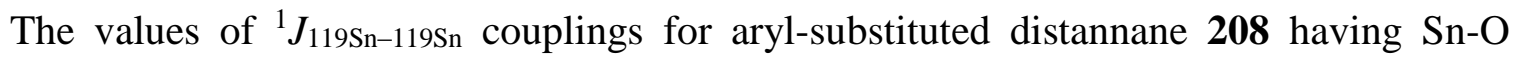
interactions are distinctly higher than $\mathrm{Ph}_{6} \mathrm{Sn}_{2}$. In the case of known distannanes 211 and 212 , replacement of one or two $n$-butyl groups in $(n-\mathrm{Bu})_{6} \mathrm{Sn}_{2}$ by the 2-(4-isopropyl-2-oxazolinyl)-5phenyl ligand results in an increase of 1048 and $3500 \mathrm{~Hz}$ for the ${ }^{1} J_{119 S n-119 S n}$ coupling compared ro $\mathrm{Ph}_{6} \mathrm{Sn}_{2}$. A remarkable increase of the ${ }^{1} J_{119 \mathrm{Sn}-119 \mathrm{Sn}}$ to $2748 \mathrm{~Hz}$ to 11272 was caused by replacement of two $n$-butyl groups in $(n-\mathrm{Bu})_{6} \mathrm{Sn}_{2}$ with electronegative acetoxy groups due to the $\mathrm{Sn}-\mathrm{O}$ interactions in $n-\mathrm{Bu}_{4} \mathrm{Sn}_{2}(\mathrm{OAc})_{2}$ 213. $^{145}$

\subsection{6: Compounds containing $C, O$-chelating ligand:}

\subsubsection{Benzyl methyl ether:}

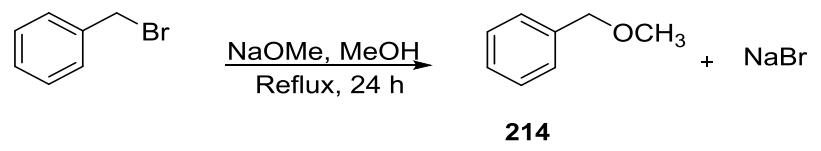

Scheme 32: Synthesis of benzyl methyl ether.

Compound 214 was prepared by reacting benzyl bromide with $\mathrm{NaOMe}$ in low yield (34\%).

This synthetic route was attempted as a means to reduce costs as it is considerably less expensive than bromobenzyl bromide. ${ }^{152}$ Due to the low yield of this intermediate, it was abandoned and bromobenzyl bromide was used exclusively for the preparation of all $C, O$-chelating ligands. 


\subsubsection{2-Bromobenzyl methyl ether:}

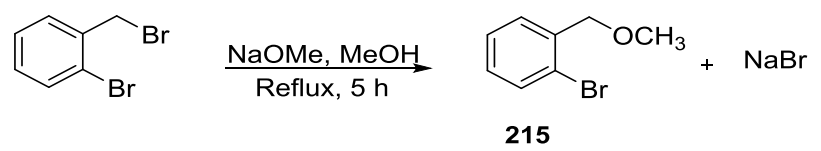

Scheme 33: Synthesis of 2-bromobenzyl methyl ether.

Compound 215 was prepared in good yield (84\%) using bromobenzyl bromide and $\mathrm{NaOMe}$ (Scheme 33). ${ }^{95,153}$ It is a stable compound and was used for further reactions.

\subsubsection{3 [2-(MeOCH 2$\left.) \mathrm{C}_{6} \mathrm{H}_{4}\right] \mathrm{Li}$ :}

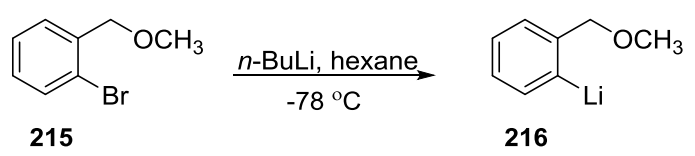

Scheme 34: Lithiation of 2-bromobenzyl methyl ether.

Compound 215 was treated with $n$ - $\mathrm{BuLi}$ at $-78^{\circ} \mathrm{C}$ in hexane and the reaction mixture stirred overnight. The solvent was decanted in the glove box and the solid washed with additional fresh hexane. The residual solvent was then removed under reduced pressure and the product stored under inert atmosphere for further use. Surprisingly, compound $\mathbf{2 1 6}$ was found to be extremely sensitive and violently decomposed while transferring to a flask (Figure 34) from the weighing dish in the glove box. Thereafter, this product was only prepared in situ for use in all further reactions.

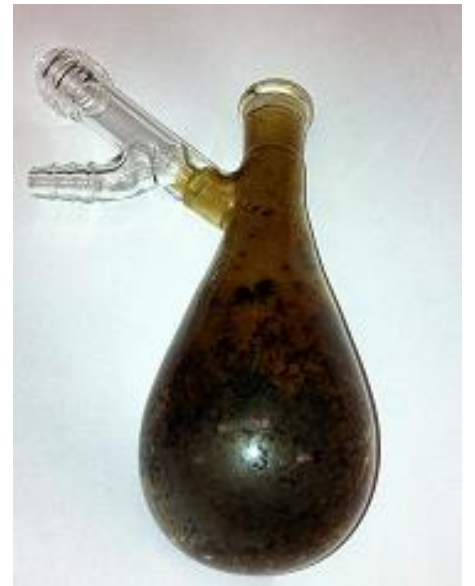

Figure 34: Flask containing the decomposed product of $\mathbf{2 1 6}$. 


\subsubsection{2-Trialky/arylstannylbenzyl methyl ether:}

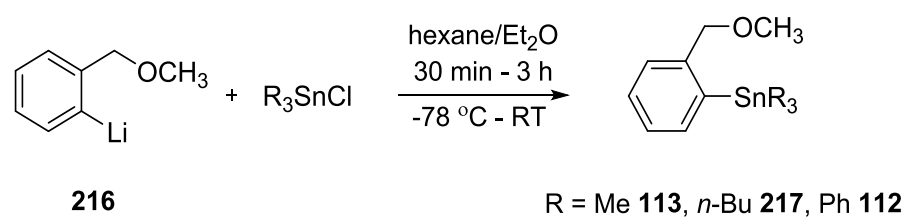

Scheme 35: Synthesis of 2-Trialky/arylstannylbenzyl methyl ether.

Compound 113 was obtained in a $70 \%$ yield and isolated as a clear oil. This yield was somewhat less than that reported for $\mathbf{1 1 3}$ in the literature $(84 \%) .{ }^{95}$ The ${ }^{119} \mathrm{Sn}$ resonance for $\mathbf{1 1 3}$ was $-32.5 \mathrm{ppm}\left(\mathrm{CDCl}_{3}\right)$ and is shifted slightly downfield from the reported value $(\delta=-38.2 \mathrm{ppm}$ obtained in $\mathrm{d}^{8}$ THF at $-78^{\circ} \mathrm{C}$ ). The stannylbenzyl methoxy ethers 112 and 217 were prepared in a manner similar to that used for 113. Compound 217 was obtained as yellow-brown coloured oil in good yield (65\%) while compound 112 was recovered as a white solid in $73 \%$ yield with a melting point of $95{ }^{\circ} \mathrm{C}$ (Scheme 35). Gilman et al. ${ }^{94}$ had previously synthesized 112 in a $35 \%$ yield by reacting $\left(o-\mathrm{MeOCH}_{2} \mathrm{C}_{6} \mathrm{H}_{4}\right) \mathrm{MgBr}$ with $\mathrm{Ph}_{3} \mathrm{SnCl}$. He reported a melting point of $94.5-95.5{ }^{\circ} \mathrm{C}$ for 112 however no NMR characterization was provided.

\subsubsection{2-Chloroalkyl/arylstannylbenzyl methoxy ethers:}

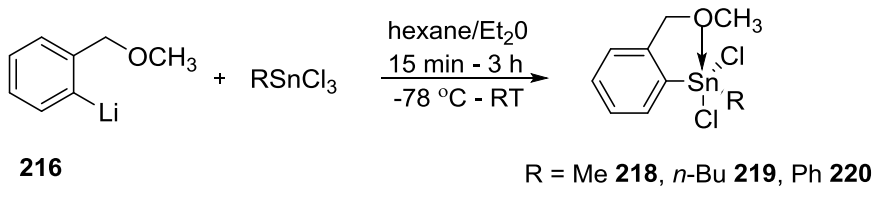

Scheme 36: Synthesis of dichloroalkyl/arylstannylbenzyl methoxy ethers.

Compounds 218-220 were prepared as brown coloured oils by addition of 216 to a precooled $\left(-78{ }^{\circ} \mathrm{C}\right)$ hexane/Et $2 \mathrm{O}$ solution of the appropriate organotin halide. Compounds 218 and 219 were isolated after extraction of their crude mixtures with toluene and hot hexane, respectively. The ${ }^{119} \mathrm{Sn}$ NMR $\left(\mathrm{CDCl}_{3}\right)$ analysis showed only a single resonance for both $\mathbf{2 1 8}(\delta=-54 \mathrm{ppm})$ and $219(\delta=-60.0 \mathrm{ppm})$. The ${ }^{119} \mathrm{Sn}$ NMR spectrum of $\mathbf{2 2 0}$ revealed two unidentified peaks of small 
intensity at $\delta=-48.0$ and $-132.0 \mathrm{ppm}$, along with the main resonance at $\delta=-28.0 \mathrm{ppm}$. Purification of compound 220 by hot hexane extraction was unsuccessful even after several attempts. The reported resonance for the closely related compound 118 prepared by Pannell et al.${ }^{96}$ was found at $\delta=-32.69 \mathrm{ppm}$ with the $\left(o-\mathrm{MeOC}_{6} \mathrm{H}_{4}\right) \mathrm{CH}_{2}$ ligand instead of $\left(o-\mathrm{MeOCH}_{2} \mathrm{C}_{6} \mathrm{H}_{4}\right)$ used for 220 .

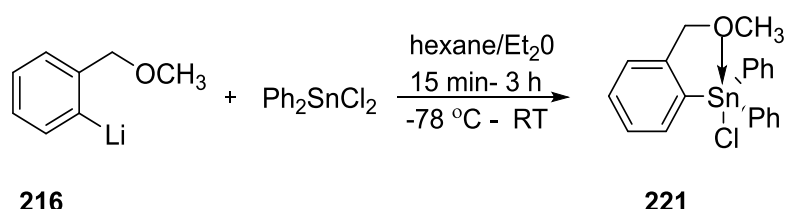

Scheme 37: Synthesis of the chloro(2-(methoxymethyl)phenyl)diphenylstannane 221.

Compound 221 was obtained as white coloured solid after precipitating the product in hexane at $-20{ }^{\circ} \mathrm{C}$. The ${ }^{119} \mathrm{Sn}$ NMR $\left(\mathrm{CDCl}_{3}\right)$ chemical shift for the recovered monochloride solid 221 was -127.0 ppm (Scheme 37). The soluble fraction from this reaction in hexane displayed a ${ }^{119} \mathrm{Sn}$ NMR chemical shift at $-136.0 \mathrm{ppm}$ which is similar to the chemical shift observed for $\mathbf{2 2 5}$ (see page 74). Compound 221 was also obtained by reacting compound 112 in $\mathrm{C}_{6} \mathrm{H}_{6}$ with $1.6 \mathrm{M}$ $\mathrm{HCl}$ solution in $\mathrm{Et}_{2} \mathrm{O}$ in a $81 \%$ yield; this is considerably higher than in Scheme 38. An attempt to convert the monochloride, $\mathbf{2 2 1}$ to the dichloride by further reaction with the $\mathrm{HCl}$ solution was undertaken (Scheme 38). This reaction resulted in only unreacted 221, which might be due to the presence of a substantial Sn-O interaction in the monochloride. ${ }^{119} \mathrm{Sn}$ NMR chemical shifts, Sn-O bond distances and yields of stannanes 112-113 and 217-221 are listed in Table 15.

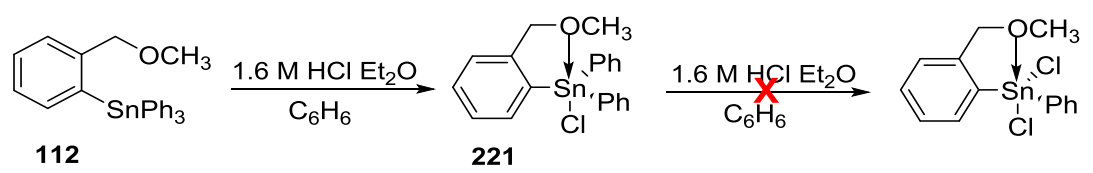

Scheme 38: Alternative route for the synthesis of chloro(2(methoxymethyl)phenyl)arylstannane. 
Table 15: ${ }^{119} \mathrm{Sn}$ NMR chemical shifts of selected stannanes.

\begin{tabular}{|c|c|c|c|c|}
\hline Compound & $\begin{array}{c}{ }^{119} \text { Sn chemical } \\
\operatorname{shift}(\delta) \text { ppm }\end{array}$ & $\begin{array}{c}\text { Unsubstituted } \\
\text { analogue }\end{array}$ & $\begin{array}{c}{ }^{119} \text { Sn chemical } \\
\operatorname{shift}(\delta) \text { ppm }\end{array}$ & $\begin{array}{c}\Delta{ }^{119} \text { Sn chemical } \\
\operatorname{shift}(\delta) \text { ppm }\end{array}$ \\
\hline 112 & -133.0 & - & - & - \\
\hline 113 & -32.5 & - & - & - \\
\hline 217 & -40.3 & - & - & - \\
\hline 218 & -54.0 & $\mathrm{PhMeSnCl}_{2}$ & $55.1^{154}$ & 99.1 \\
\hline 219 & -60.9 & $\mathrm{Ph}(n-\mathrm{Bu}) \mathrm{SnCl}_{2}$ & - & - \\
\hline 220 & -28.2 & $\mathrm{Ph}_{2} \mathrm{SnCl}_{2}$ & $-33.0^{22}$ & -3.8 \\
\hline 221 & -127.2 & $\mathrm{Ph}_{3} \mathrm{SnCl}$ & -45.0 & 82.2 \\
\hline
\end{tabular}

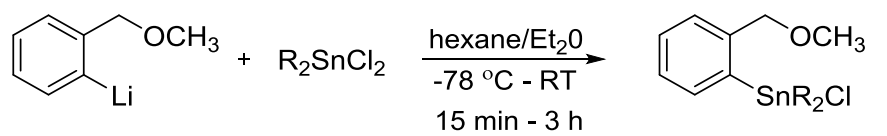

216

$\mathrm{R}=\mathrm{Me} 222, n-\mathrm{Bu} 223$

Scheme 39: Attempted synthesis of $o$-chlordialkylstannyl benzyl methyl ether.

The syntheses of $\mathbf{2 2 2}$ and $\mathbf{2 2 3}$ shown in Scheme 39 did not yield clean products. The reaction of 216 with $\mathrm{Me}_{2} \mathrm{SnCl}_{2}$ showed five resonances by ${ }^{119} \mathrm{Sn}$ NMR spectroscopy, along with a signal of higher intensity at $-71.0 \mathrm{ppm}$. No assignments of this mixture was made and no further attempts to purify this material were undertaken. Similarly, the reaction of 216 with $(n-\mathrm{Bu})_{2} \mathrm{SnCl}_{2}$ showed six unassigned ${ }^{119}$ Sn NMR signals and could not be purified by extraction with hexane. The oily reaction mixture was stored in the glove box and after 30 days, an off-white coloured solid material separated from the oil. The solid was then washed with hexane and its ${ }^{119} \mathrm{Sn}$ NMR $\left(\mathrm{CDCl}_{3}\right)$ spectrum showed two resonances at -91.0 and $-138.0 \mathrm{ppm}$ of unequal intensities. This may be due to the exchange of the axial and equatorial positions of $\mathrm{Cl}$ atom attached to the tin. When $\mathrm{Cl}$ is in axial position, it pulls the electron density from tin and promotes a strong Sn-O interaction which may be absent if the $\mathrm{Cl}$ occupies an equatorial position (Figure 35). An accurate mass determination by mass spectrometry was consistent for a structure with a formulae $\mathbf{2 2 3}$. 


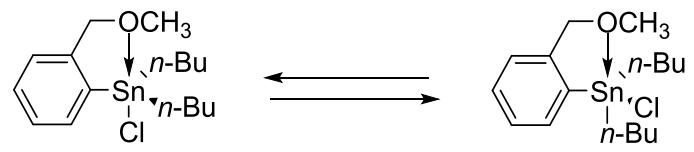

Figure 35: Possible structural isomers of 223.

Bis-chelate compounds 224-225 were synthesized according to Scheme 39. The crude products of these reactions were not successfully purified by extraction techniques, however NMR $\left({ }^{1} \mathrm{H},{ }^{13} \mathrm{C},{ }^{119} \mathrm{Sn}\right)$ and mass spectrometry of the crude products $\mathbf{2 2 4 - 2 2 5}$ were obtained. The ${ }^{119} \mathrm{Sn}$ NMR of both 224 and 225 showed three resonances, along with a signals of higher intensity at 73.0 ppm and -136 ppm respectively.

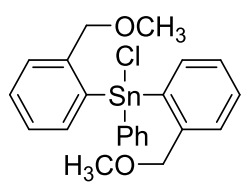

225

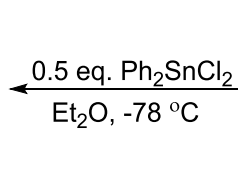

216

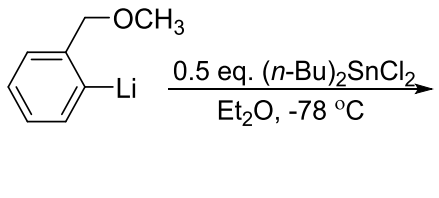

16

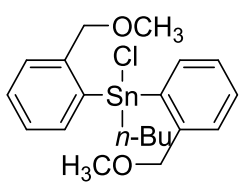

224

Scheme 40: Attempted synthesis of 224-225.

\subsubsection{Dihydridoalkylstannylbenzyl methyl ether:}

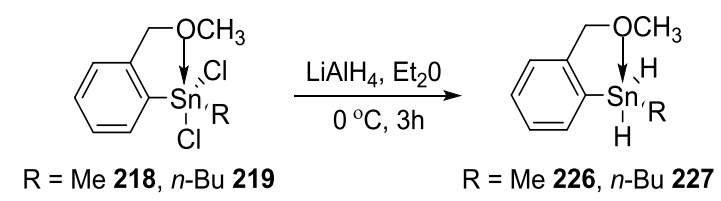

Scheme 41: Synthesis of dihydridoalkylstannyl benzyl methy ethers.

Donor-acceptor interactions between $\mathrm{Sn}$ and $\mathrm{O}$ in hypervalent compounds have been previously confirmed by small molecule X-ray diffraction techniques as well as by NMR spectroscopy. ${ }^{45,155-156}$ Dakternieks et al. obtained new triorganotin and tin hydrides containing either the chiral 2-(4-isopropyl-2-oxazolinyl)-5-phenyl or the 2-(4-isopropyl-2-oxazolinyl)-5(methyl)phenyl ligand. ${ }^{68}$ The ${ }^{1} \mathrm{H}$ NMR spectroscopy of 227 (Figure 36) show distinct ${ }^{119 / 117} \mathrm{Sn}-\mathrm{H}$ coupling constants which are larger than the structurally similar tetracoordinate tin compounds (Table 16). 
Table 16: ${ }^{119} \mathrm{Sn}$ NMR data of tin dihydrides with or without coordinating ligand.

\begin{tabular}{|c|c|c|c|c|c|}
\hline Compound & $\begin{array}{l}{ }^{119} \text { Sn NMR } \\
\text { (ppm) }\end{array}$ & $\begin{array}{c}{ }^{1} J_{119 / 117 S n-1 H} \\
(H z)\end{array}$ & $\begin{array}{c}\mathrm{R}_{2} \mathrm{SnH}_{2} \\
\text { analogue }\end{array}$ & $\begin{array}{c}{ }^{119} \text { Sn NMR } \\
\text { (ppm) }\end{array}$ & $\begin{array}{c}{ }^{1} J_{119 S n-1 H} \\
(\mathrm{~Hz})\end{array}$ \\
\hline 226 & -221.0 & 1727 & $\mathrm{PhMeSnH}_{2}$ & $-110^{157}$ & 1835 \\
\hline 227 & -210.0 & $1770 / 1677$ & $\mathrm{PhBuSnH}_{2}$ & $105^{157}$ & - \\
\hline
\end{tabular}

To the best of our knowledge, these are the first examples of the organotin dihydrides containing a $C, O$-chelating ligand (Figure 36).

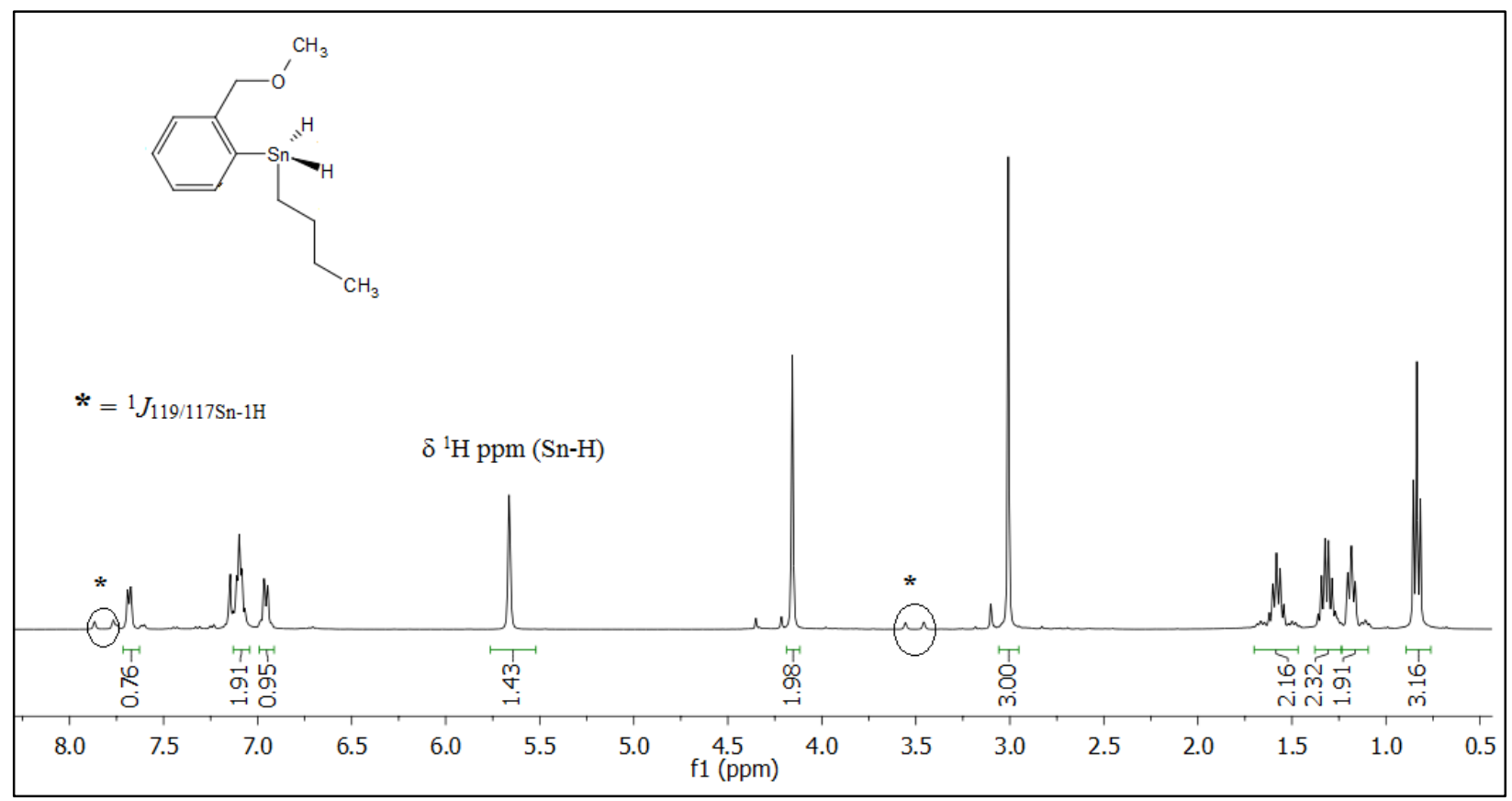

Figure 36: ${ }^{1} \mathrm{H}$ NMR $\left(\mathrm{C}_{6} \mathrm{D}_{6}\right)$ spectrum of 227.

\subsubsection{Organotin compounds containing a $C, N$-chelating ligand}

The synthesis of 2-bromo- $N, N$-dimethylbenzylamine (228) was unsuccessfully attempted using a literature preparation involving the reaction of 2-bromobenzyl bromide and dimethylamine $(1: 1.5)$ at room temperature for $6 \mathrm{~h} .{ }^{158}$ However, this $C, N$ - ligand was prepared in high yield (88\%) and purity by heating 2-bromobenzyl bromide with an excess of dimethylamine. The product was recovered by extraction with $3 \mathrm{M} \mathrm{HCl}, 20 \% \mathrm{NaOH}$ and finally with DCM. ${ }^{159}$

Compounds 33, 35 and 37 were obtained according to Scheme 42 by reacting 229 with either $\mathrm{MeSnCl}_{3}, n-\mathrm{BuSnCl}_{3}$ or $\mathrm{PhSnCl}_{3}$ respectively. Compound 33 was previously reported by 
van Koten et al. ${ }^{59}$ from the reaction of $\left[2-\left(\mathrm{Me}_{2} \mathrm{NCH}_{2}\right) \mathrm{C}_{6} \mathrm{H}_{4}\right] \mathrm{Cu}$ with $\mathrm{MeSnCl}_{3}$ in $\mathrm{C}_{6} \mathrm{H}_{6}$. Varga et $a l .{ }^{45}$ and Novák et al. ${ }^{29 \mathrm{a}}$ reported the synthesis of $\mathbf{3 7}$ and $\mathbf{3 5}$ by treating 229 with $n$ - $\mathrm{BuSnCl}_{3}$ or $\mathrm{PhSnCl}_{3}$ in $\mathrm{C}_{6} \mathrm{H}_{6}$ and the crude product purified by extraction with hot petroleum ether and hexane respectively. Compound 35 was also prepared by Rippstein et al. ${ }^{48}$ using $\mathrm{Et}_{2} \mathrm{O}$ as a solvent, followed by toluene extraction. The diorganotin dihalides $\mathbf{3 3}, \mathbf{3 5}$ and $\mathbf{3 7}$ reproduced in this study were synthesized using $\mathrm{Et}_{2} \mathrm{O}$ as a solvent and purified by extraction with hot hexane.

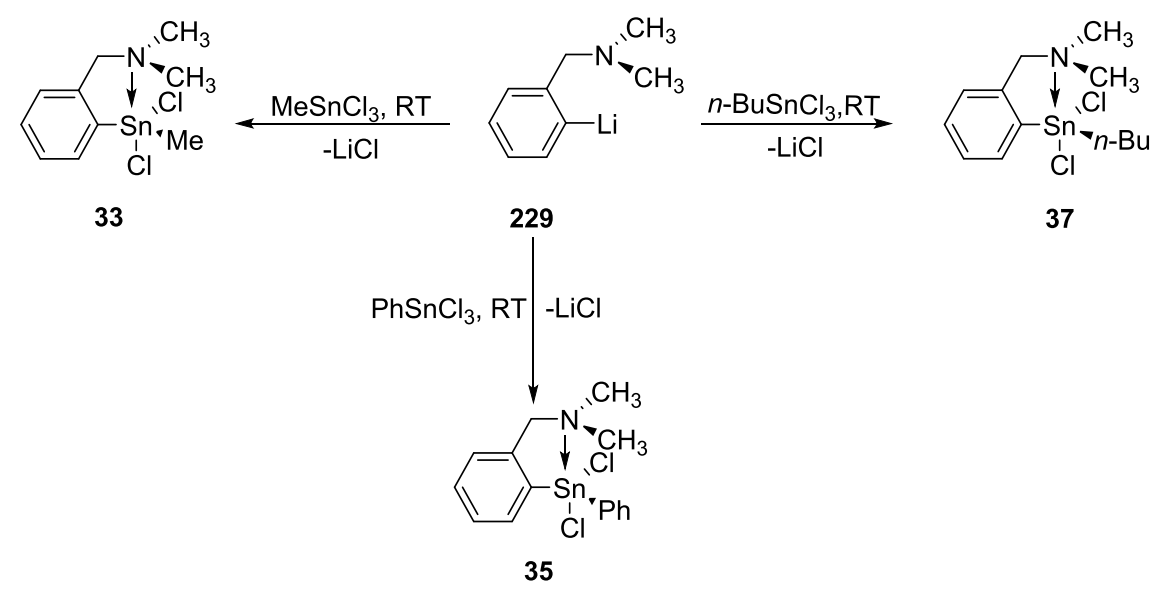

Scheme 42: Preparative routes to compounds 33, 35, 37.

The intramolecular Sn-N interaction and relative change in the Lewis acidity at the Sn center of hypervalent compounds can be monitored by ${ }^{119} \mathrm{Sn}$ and ${ }^{13} \mathrm{C}$ NMR spectroscopy. Three parameters can be used to determine the strength of Sn-N interaction: a distinct upfield shift of ${ }^{119} \mathrm{Sn}$ NMR signals of these compounds as compared to their analogues before ligand substitution, an increase in the coupling constants $\left({ }^{119} \mathrm{Sn},{ }^{13} \mathrm{C}\right)$, and the presence of $\left({ }^{2} J_{119 \mathrm{Sn}-\mathrm{CH} 3}\right)$ coupling constant between the tin and amino methyl group. A distorted TBP geometry was expected for diorganotin dihalides $(\mathbf{3 3}, \mathbf{3 5}, \mathbf{3 7})$ having an aryl and $\mathrm{R}$ group at equatorial positions, while the amino ligand occupies an axial position, leaving one axial and one equatorial position for the two halogen atoms. ${ }^{33,47,55}$ 
The ${ }^{1} \mathrm{H}$ NMR spectra of compounds $\mathbf{3 3}, \mathbf{3 5}$, and $\mathbf{3 7}$ display a downfield shift to about 8.2 ppm for the ortho protons of $\mathrm{C}_{6} \mathrm{H}_{4}$ - ring, which is similar for other known tin TBP compounds. ${ }^{59}$ The increase in ${ }^{2} J_{119 \mathrm{Sn}-1 \mathrm{H}}$ coupling constant to $80 \mathrm{~Hz}$ for $\mathbf{3 3}$ also indicates the existence of TBP geometry. The upfield shift in the ${ }^{119} \mathrm{Sn}$ NMR to $-96.4,169.4,-104.0 \mathrm{ppm}$ (Table 17) for these compounds $\mathbf{3 3}, \mathbf{3 5}$, and $\mathbf{3 7}$, as compared to their corresponding unsubstituted analogues $\left(\mathrm{MeSnCl}_{3}\right.$ : $21.0 \mathrm{ppm}, \mathrm{PhSnCl}_{3}:-63.0 \mathrm{ppm}, n-\mathrm{BuSnCl}_{3}: 6.0 \mathrm{ppm}$ respectively) provide strong evidence for a substantive Sn-N intramolecular interaction and TBP geometry.

Table $17:{ }^{119} \mathrm{Sn} \mathrm{NMR}\left(\mathrm{CDCl}_{3}\right)$ chemical shift values of tin dichlorides containing a $C, N$ chelating ligand.

\begin{tabular}{|c|c|c|c|c|}
\hline \multirow{2}{*}{ Compound } & \multicolumn{2}{|c|}{${ }^{119}$ Sn NMR (ppm) } & \multicolumn{2}{c|}{ Yield (\%) } \\
& Lit. & $-96.4^{\mathrm{a}}$ & 96 & 85 \\
\hline $\mathbf{3 3}$ & $-94^{26,59}$ & -169.4 & 68 & 69 \\
\hline $\mathbf{3 5}$ & $-170^{29 \mathrm{a}, 48}$ & & & 73 \\
\hline $\mathbf{3 7}$ & $104.3^{29 \mathrm{~b}, 51,160}$ & -104 & 86 & \\
\hline
\end{tabular}

a d-toluene

The hydrogenation of $\mathbf{3 3}$ and $\mathbf{3 7}$ was successfully attempted by reaction with $\mathrm{LiAlH}_{4}$ (Scheme 43).

Compound 228 could not be hydrogenated using the same technique.

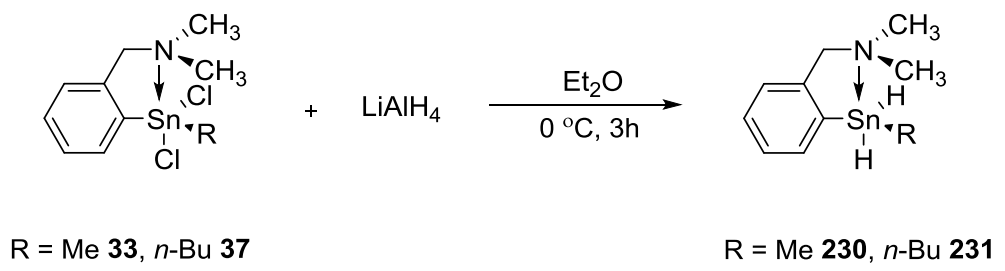

Scheme 43: Preparation of tin dihydrides containing a $C, N$-chelating ligand.

Hydrides 230 and 231 possess a typical ${ }^{1} \mathrm{H}$ NMR resonance shifted downfield $(\delta=5.73$ ppm and $\delta=5.62 \mathrm{ppm})$ compared to the stannyl dihydrides $(n-\mathrm{Bu})_{2} \mathrm{SnH}_{2}(4.78 \mathrm{ppm})^{40}$ and $\mathrm{Me}_{2} \mathrm{SnH}_{2}$ (4.46 ppm). ${ }^{161}$ Another characteristic of diorganotin hydrides is that the ${ }^{1} J_{119 \mathrm{Sn}-1 \mathrm{H}}$ coupling constants $(1760 \mathrm{~Hz}$ for $\mathbf{2 3 0}, 1860 \mathrm{~Hz}$ for 231) are larger than tetracoordinate diorganotin 
hydrides $\left(\mathrm{Me}_{2} \mathrm{SnH}_{2} 1758 \mathrm{~Hz} ;(n-\mathrm{Bu})_{2} \mathrm{SnH}_{2} 1675 \mathrm{~Hz}\right)$. The ${ }^{119} \mathrm{Sn}$ chemical shifts of $\mathbf{2 3 0}$ and 231 are $-236 \mathrm{ppm}$ (Figure 37) and $-217 \mathrm{ppm}$ respectively, and are significantly shifted upfield when compared to their structurally closest analogs $(n-\mathrm{Bu}) \mathrm{PhSnH}_{2}$ at $105 \mathrm{ppm}$ and $\mathrm{MePhSnH}_{2}-110$ ppm. ${ }^{157}$ These results suggest the presence of a stronger intramolecular $\mathrm{Sn}-\mathrm{N}$ coordination in $\mathbf{2 3 0}$ than in $\mathbf{2 3 1}$.

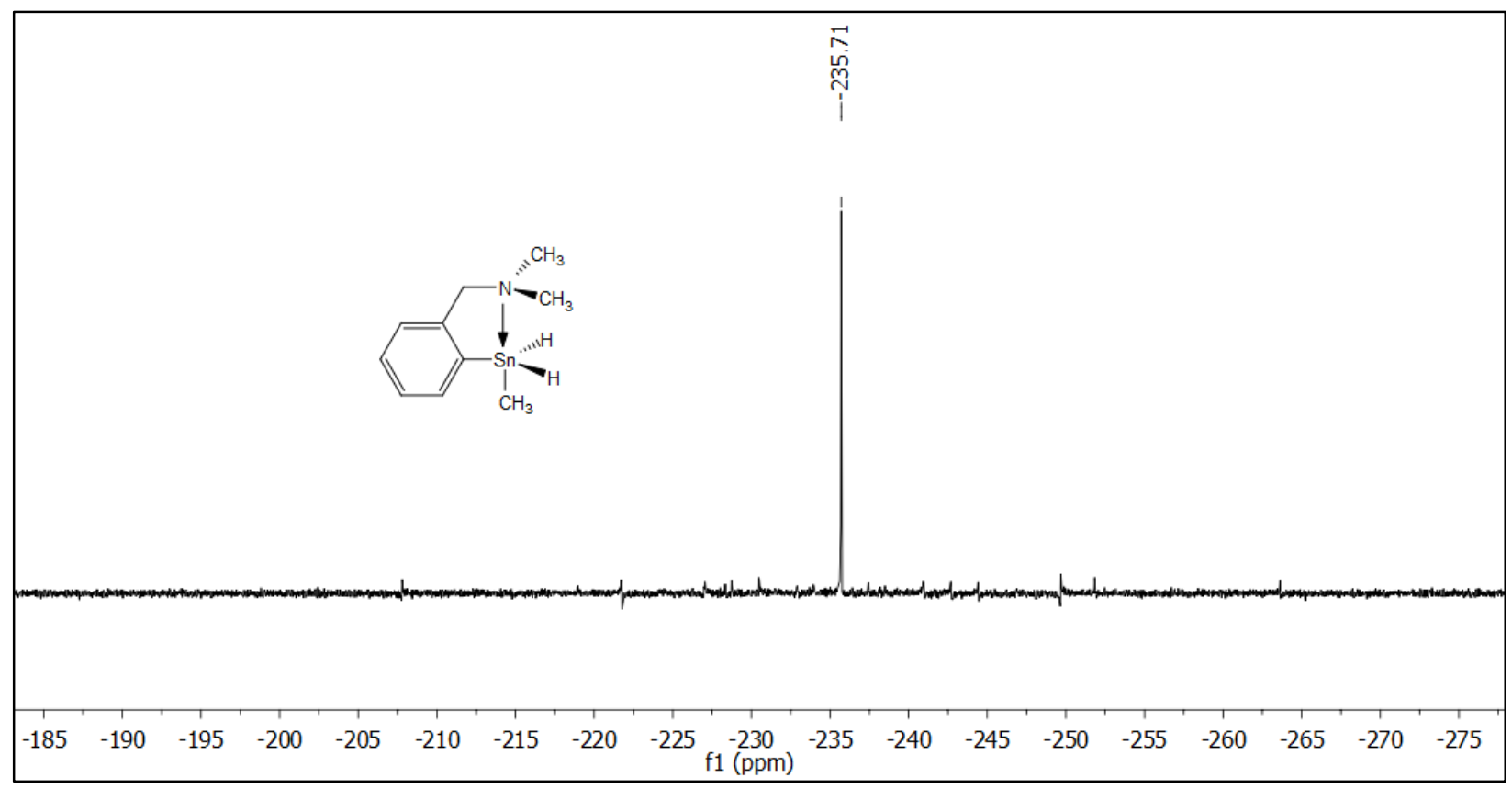

Figure 37: ${ }^{119} \mathrm{Sn} \operatorname{NMR}\left(\mathrm{C}_{6} \mathrm{D}_{6}\right)$ spectrum of $\mathbf{2 3 0}$.

The range of ${ }^{119} \mathrm{Sn}$ chemical shifts for dihydrides may be explained on the basis of the differing electron donating ability of methyl and $n$-butyl groups of $\mathbf{2 3 0}$ and $\mathbf{2 3 1}$ respectively. The hydride ligand would likely be more apicophile in $\mathbf{2 3 0}$ than in $\mathbf{2 3 1}$ for these structurally confined pentacoordinated tin centers. Unfortunately, no crystal structure determinations by X-ray crystallography are yet available for these dihydrides to compare with structural information obtained from solution NMR spectroscopy. 


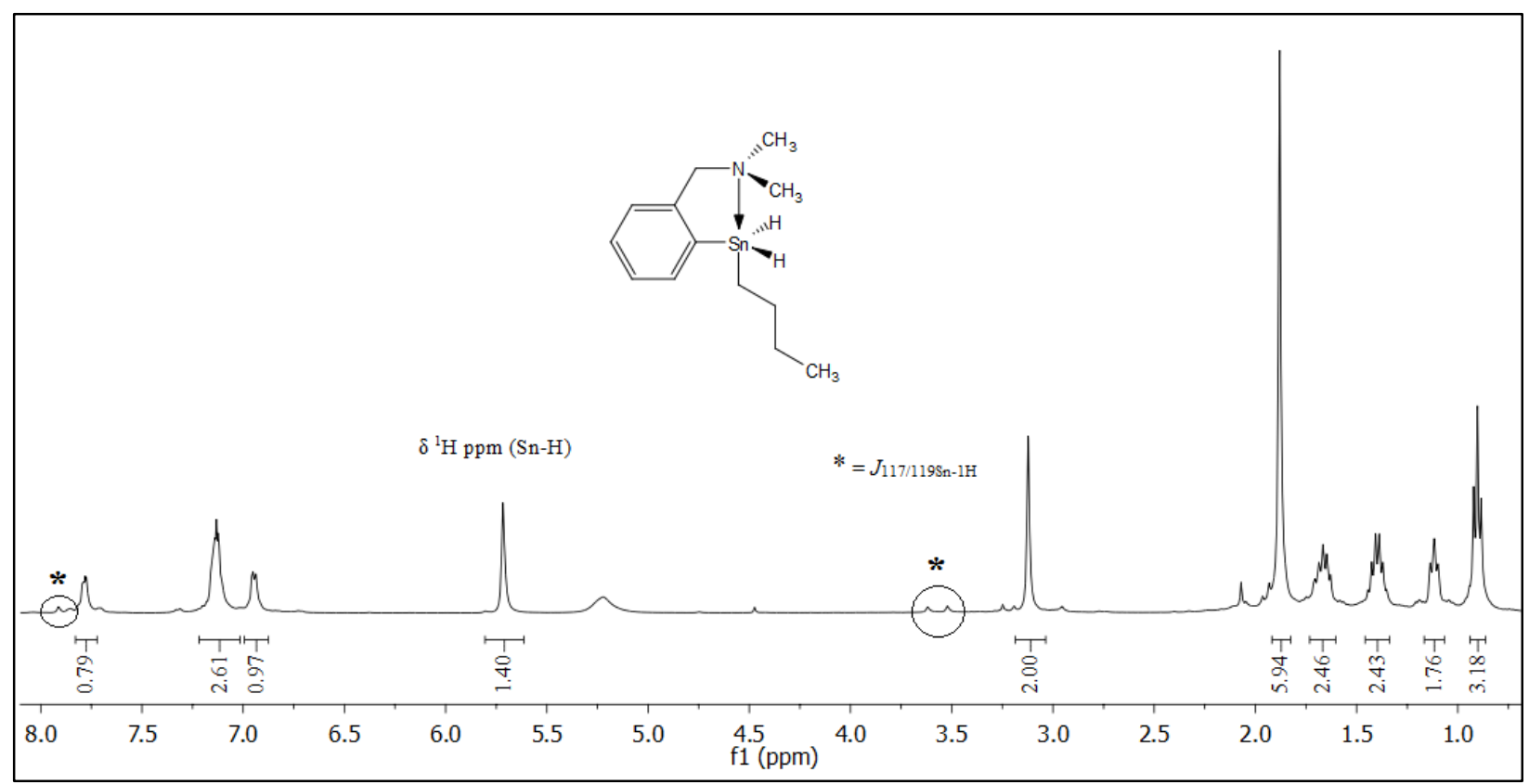

Figure 38: ${ }^{1} \mathrm{H}$ NMR $\left(\mathrm{C}_{6} \mathrm{D}_{6}\right)$ spectrum of 231.

In the literature there are only two reported examples $(\mathbf{6 9}, \mathbf{7 0})^{72}$ of stannyl dihydrides with Sn-N interactions. The presence of dative $\mathrm{Sn}-\mathrm{N}$ coordination is evident from the increase of ${ }^{1} J_{119 \mathrm{Sn}}$ $-1 \mathrm{H}$ and presence of ${ }^{3} J_{119 \mathrm{Sn}-13 \mathrm{C}}\left(\mathrm{Sn}-\mathrm{CH}_{2}\right)$ coupling values for 230 and 231 compared to four coordinate analogues.

Table 18: Coupling constant values for tin dihydrides.

\begin{tabular}{|c|c|c|c|}
\hline Compounds & ${ }^{{ }^{119} \text { Sn NMR ppm }}$ & $\begin{array}{c}\mathbf{1}_{\mathbf{J 1 9 S n}-1 \mathbf{H}} \\
(\mathbf{H z})\end{array}$ & $\begin{array}{c}\text { Sn-CH2 } \mathbf{J}_{\mathbf{1 1 9 S n}-13 C} \\
(\mathbf{H z})\end{array}$ \\
\hline$(n-\mathrm{Bu})_{2} \mathrm{SnH}_{2}$ & -203 & $1675^{40}$ & - \\
\hline $\mathrm{PhMeSnH}_{2}$ & $-110^{157}$ & $1835^{73}$ & - \\
\hline $\mathbf{6 9}^{72}$ & -225.9 & 1703,1987 & - \\
\hline $\mathbf{7 0}^{72}$ & -244.5 & 1940,2155 & 22.7 \\
\hline $\mathbf{2 3 0}$ & -217.5 & 1860 & 24.3 \\
\hline $\mathbf{2 3 1}$ & -236.0 & 1760 & \\
\hline
\end{tabular}

At temperatures above room temperature, compound $\mathbf{2 3 1}$ evolved gas (presumably $\mathrm{H}_{2}$ ) and decomposed changing from a light yellow to orange coloured semi-solid. Analysis of these residues by ${ }^{119} \mathrm{Sn}$ NMR revealed the complete absence of the resonance at $-217.5 \mathrm{ppm}$ in the orange 
product and presence of a mixture of unidentified tin containing species. The use of $\mathrm{LiAlH}_{4}$ as a reducing agent for $\mathbf{3 5}$ appeared to favour a redistribution reaction. The ${ }^{119} \mathrm{Sn}$ NMR spectrum of the product showed a resonance at $-181 \mathrm{ppm}$, similar to value reported earlier by Turek et al. ${ }^{66}$ for 232. A softer hydrogenating agent, $\mathrm{NaBH}_{4}$, was then used and a yellow coloured oil obtained (Scheme 44). Once again the ${ }^{119} \mathrm{Sn}$ NMR resonance at $-181 \mathrm{ppm}$ corresponded to the presence of the redistribution product $\mathbf{2 3 2}$ and a second unidentified resonance at -223 ppm which may be the dihydride. No further characterization of this compound was attempted at this time.

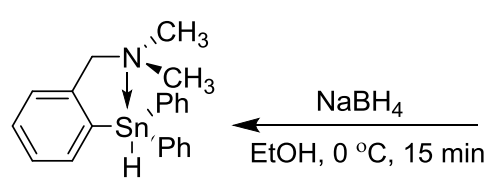

232

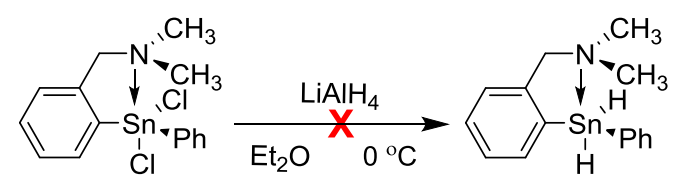

35

Scheme 44: Attempted synthesis of an aryltin dihydrides containing a $C, N$-ligand.

\subsubsection{Compounds containing $C, S$-chelating ligand:}

\subsubsection{1-bromo-2-(n-propylthiomethyl) benzene}

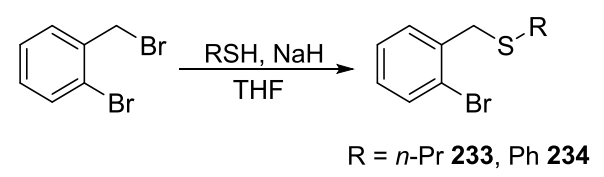

Scheme 45: Synthesis of C,S-ligands thiol.

A previously reported method was used for the synthesis of $\mathbf{2 3 3}$ and 234 . $^{105}$ Compounds

$\mathbf{2 3 3}$ and 234 were obtained after adjustment of the ratios of thiol and 2-bromobenzyl bromide (1.5:1). The use of a 1:1 ratio resulted in a substantial amount of unreacted 2-bromobenzylbromide. Similar types of thiols having $\mathrm{R}=i$-Pr, $t$-Bu substituents were also reported in literature. ${ }^{104,105}$ 


\subsubsection{Tributylstannylbenzyl thioether:}

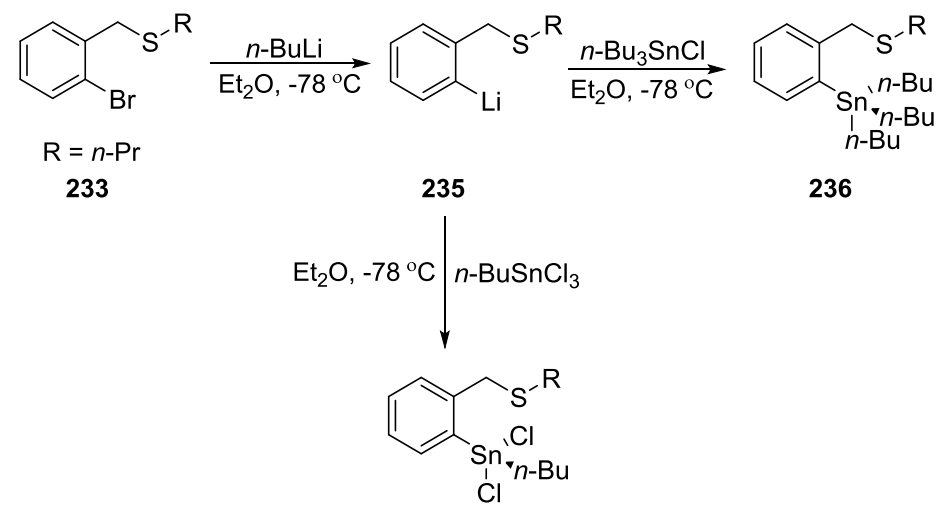

237

Scheme 46: Synthesis of tributylstannylbenzyl thioether.

An attempt to prepare compounds 236 and $\mathbf{2 3 7}$ by an initial lithiation of $\mathbf{2 3 3}$ followed by the reaction with $n-\mathrm{Bu}_{3} \mathrm{SnCl}$ and $n-\mathrm{BuSnCl}_{3}$ is shown in Scheme 45 . NMR $\left({ }^{1} \mathrm{H},{ }^{13} \mathrm{C}\right.$ and $\left.{ }^{119} \mathrm{Sn}\right)$ spectroscopy revealed the presence of multiple products. The ${ }^{119} \mathrm{Sn}$ spectrum showed three resonances; a chemical shift at $\delta=140.0$ for the starting material $n$ - $\mathrm{Bu}_{3} \mathrm{SnCl}$, and two unassigned resonances at 103.0 and $94.0 \mathrm{ppm}$ (Figure 39). This reaction was also attempted with $n$ - $\mathrm{BuSnCl}_{3}$ under the same conditions; analysis by ${ }^{119} \mathrm{Sn}$ NMR spectroscopy of the reaction mixture gave chemical shifts at -54 and -78 ppm (Figure 40). Both crude reaction mixtures could not be purified using extraction techniques. The alkyl region in both the ${ }^{1} \mathrm{H}$ NMR and ${ }^{13} \mathrm{C}$ NMR spectra of these compounds display extra resonances which have not been assigned. No further characterization of these reaction mixtures was attempted at this time. 


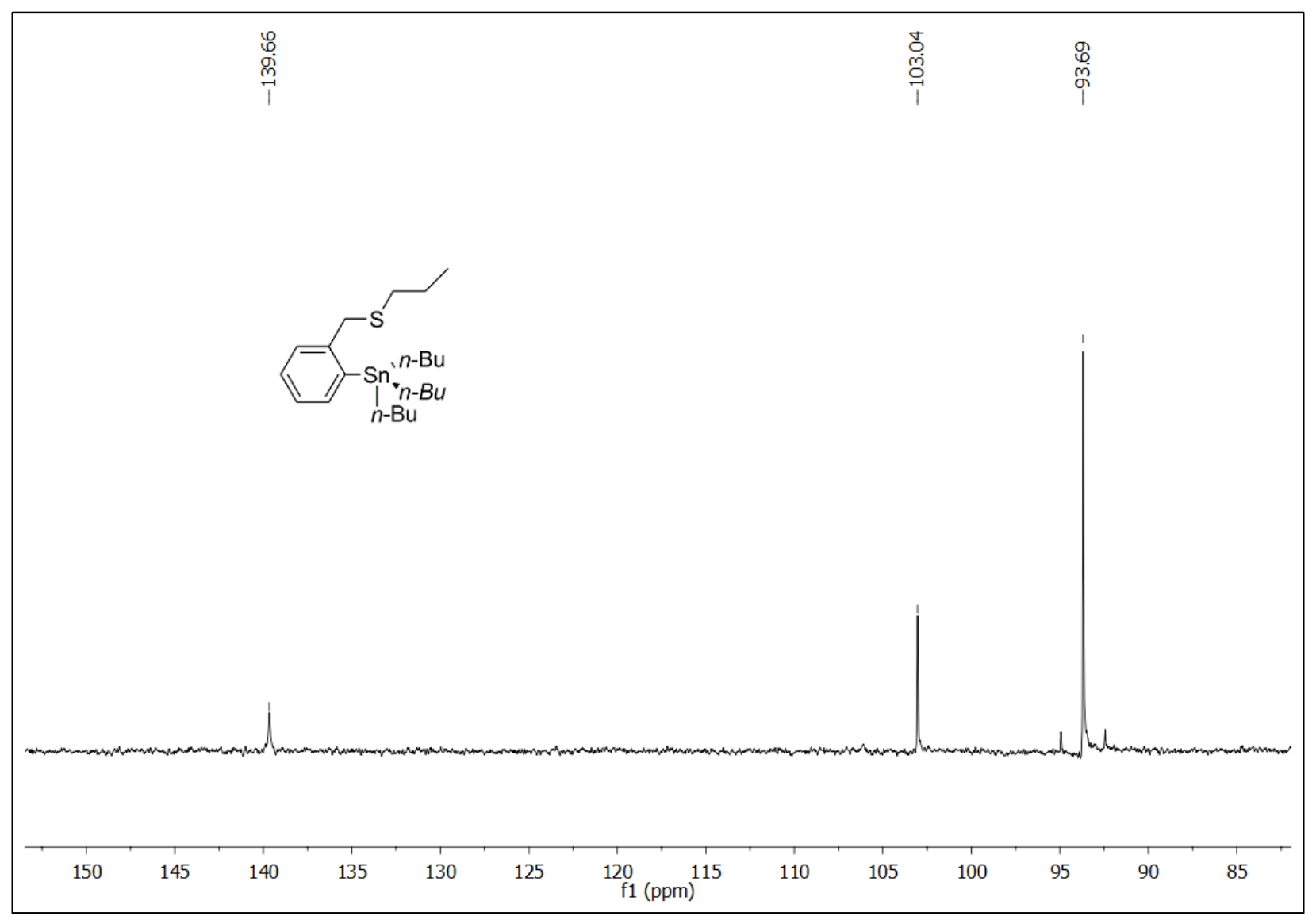

Figure 39: ${ }^{119} \mathrm{Sn}\left(\mathrm{CDCl}_{3}\right) \mathrm{NMR}$ spectrum of 236. 


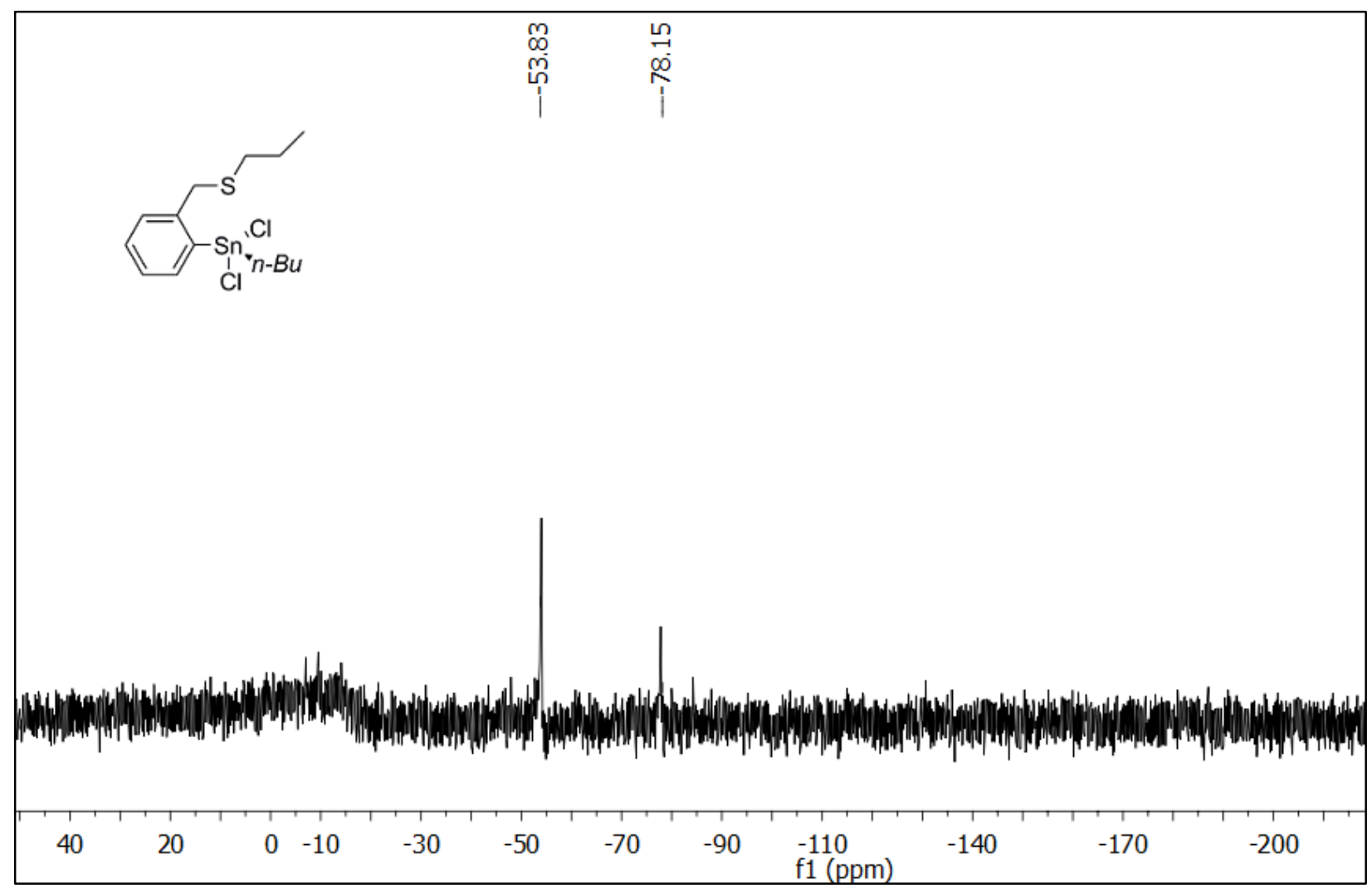

Figure 40: ${ }^{119} \mathrm{Sn}\left(\mathrm{CDCl}_{3}\right) \mathrm{NMR}$ spectrum of 237.

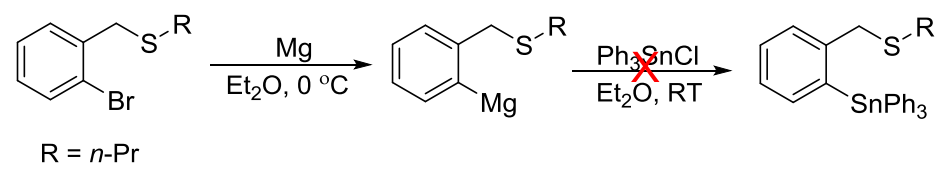

Scheme 47: Synthesis of triphenylstannylbenzyl thioether.

The reaction of $\mathrm{Ph}_{3} \mathrm{SnCl}$ with the thiobenzyl ether Grignard reagent as shown in Scheme 47 was unsuccessful. The ${ }^{119} \mathrm{Sn}$ NMR spectroscopy revealed the resonances only for the unreacted starting material, $\mathrm{Ph}_{3} \mathrm{SnCl}(\delta=-45.0 \mathrm{ppm})$.

\subsubsection{Compounds containing $C, P$-chelating ligand:}

2.1.9.1 Synthesis of (o-bromobenzyl)-diphenylphosphine:

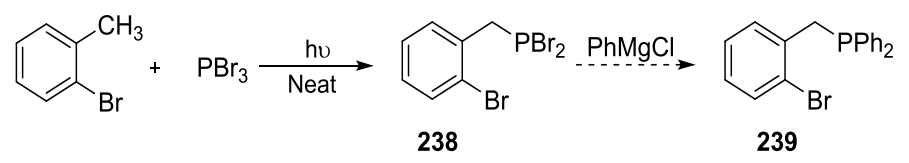


Scheme 48: Attempted route for UV- light catalyzed synthesis of $o-\left(\mathrm{Ph}_{2} \mathrm{PCH}_{2}\right) \mathrm{C}_{6} \mathrm{H}_{4} \mathrm{Br}$. The reaction mixture shown in Scheme 48 was irradiated with UV light for $4 \mathrm{~h}$. ${ }^{31} \mathrm{P}$ NMR spectroscopy revealed a resonance at 173 ppm, which was assigned to the target compound 238. Unreacted $\mathrm{PBr}_{3}$ and four other unidentified peaks (Figure 41) were also observed. The reported yield for this compound is $27 \% .{ }^{162}$ An attempt to make same product through Grignard synthesis was also tried, but was unsuccessful.

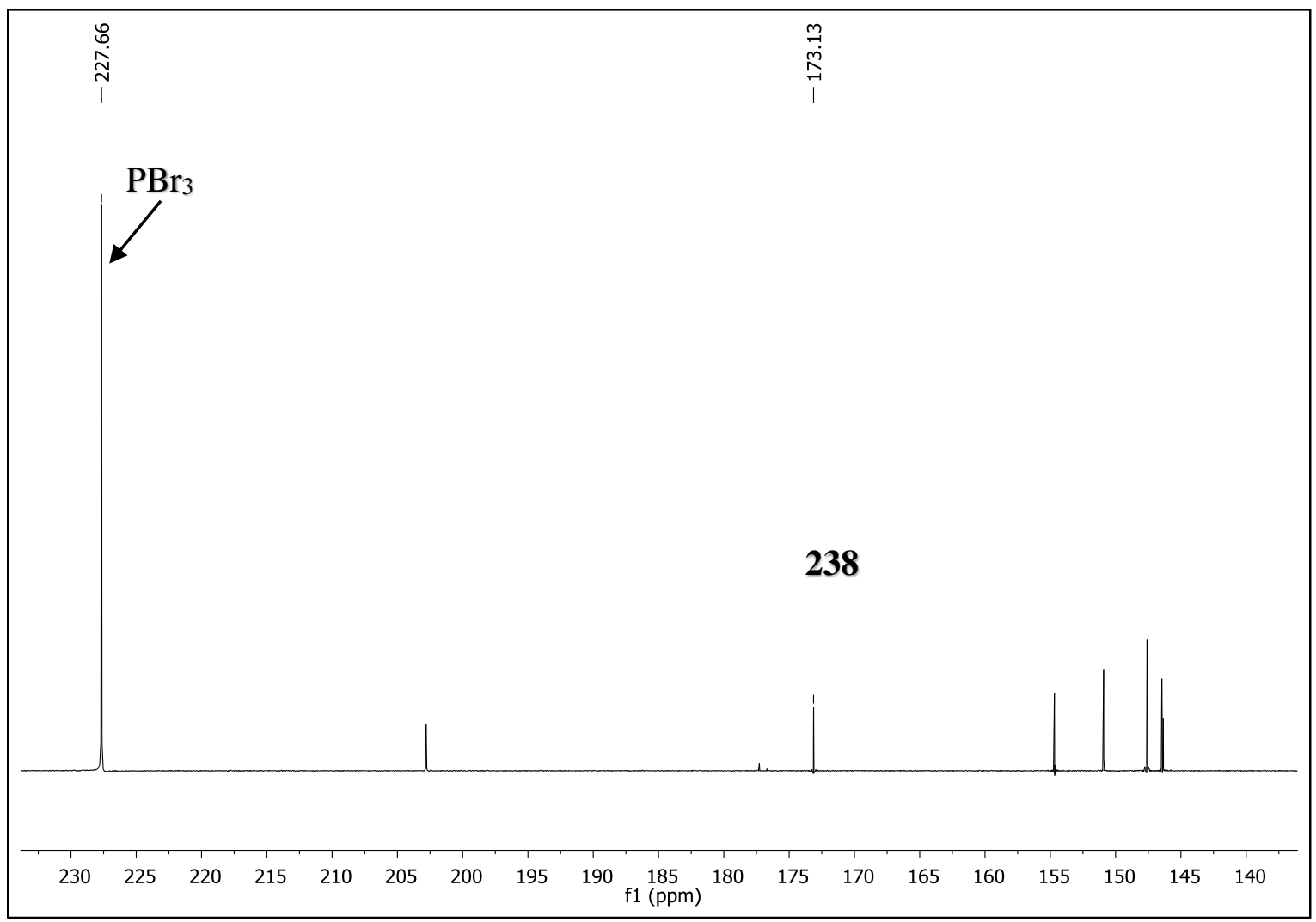

Figure 41: ${ }^{31} \mathrm{P}$ NMR $\left(\mathrm{CDCl}_{3}\right)$ spectrum of compound 238.

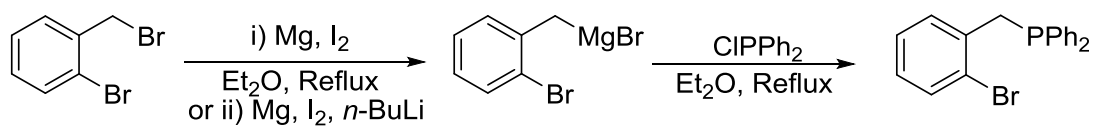

239

Scheme 49: Synthetic route for the intermediate 239. 
${ }^{31} \mathrm{P}$ NMR spectroscopy showed two resonances at $-72.0 \mathrm{ppm}$ and $-82.0 \mathrm{ppm}$ (which corresponds to $\mathrm{ClPPh}_{2}$ ). There was no indication of product formation with a reported ${ }^{31} \mathrm{P} \mathrm{NMR}$ resonance for 239 at -12.0 ppm. ${ }^{163}$ The crude product was suspended in $\mathrm{Et}_{2} \mathrm{O}$ and the insoluble component analysis of this residue removed by decantation. The residual solvent was removed under reduced pressure and a ${ }^{31} \mathrm{P}$ NMR analysis of the recovered solid showed a resonance at 14.8 ppm corresponding to the previously reported disubstituted phosphine $\mathbf{2 4 0}$ along with two other unassigned peaks at $-21.0 \mathrm{ppm}$ and $-40.0 \mathrm{ppm}$.

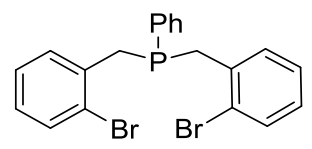

240

Figure 42: Structure of 240a.

Compound 239 was successfully prepared by using a method reported by Telleson et al. ${ }^{164}$ The addition of few drops of $n$-BuLi to activate the $\mathrm{Mg}$ is the only difference from Scheme 49. NMR data obtained agreed with that reported in literature (Figure 43). ${ }^{164}$

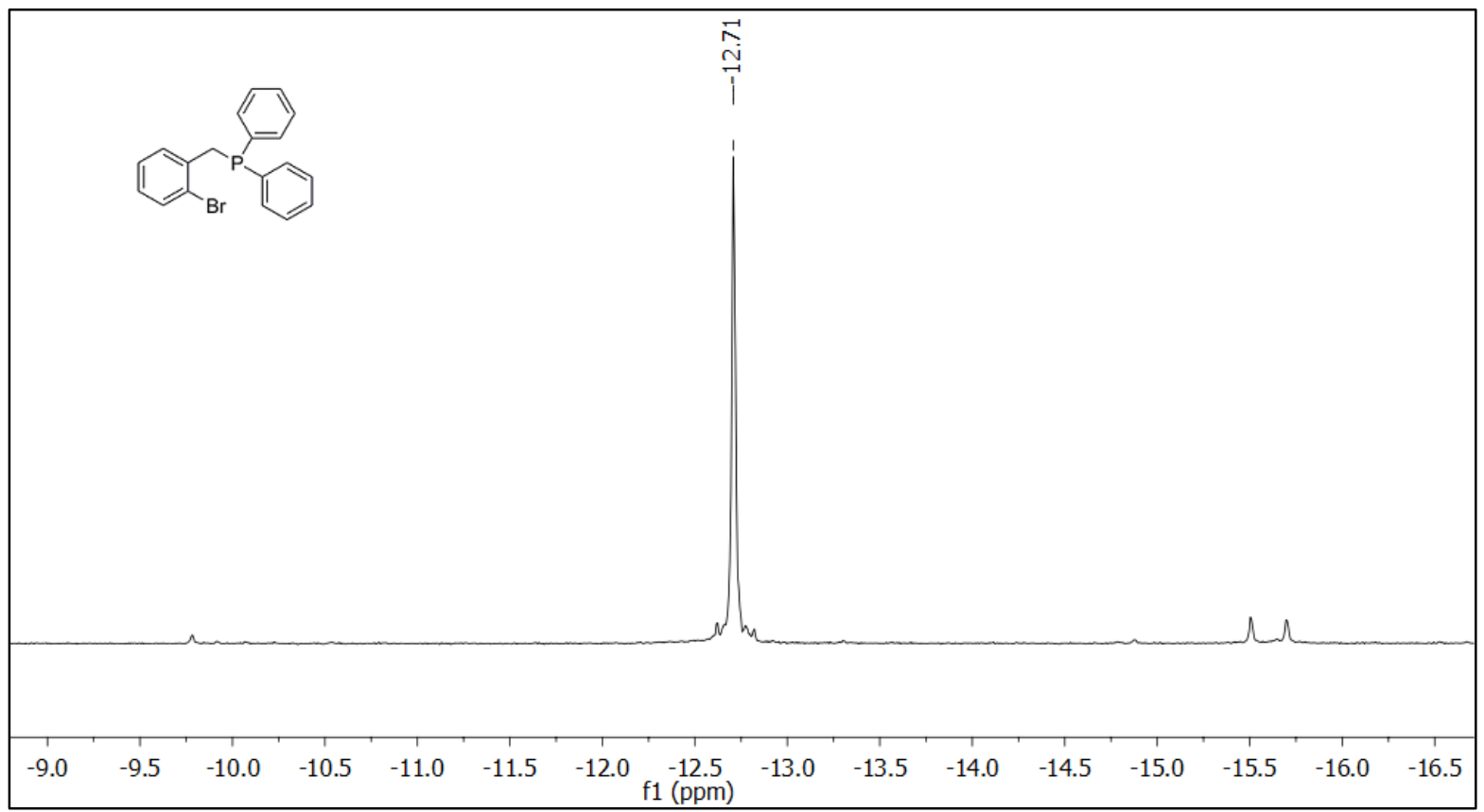

Figure 43: ${ }^{31} \mathrm{P} \mathrm{NMR}\left(\mathrm{CDCl}_{3}\right)$ spectrum of compound 239. 


\subsubsection{2 (o-(diphenylphosphino)benzyl)stannanes:}

The lithiated intermediate 240a was synthesized by following the method reported by Gossage et al., ${ }^{115}$ and isolated as orange-red powder (Scheme 50). After washing with hexane, $(o-$ (diphenylphosphino)benzyl) lithium was suspended in dry $\mathrm{C}_{6} \mathrm{H}_{6}$ and treated with solution of either $\mathrm{Ph}_{3} \mathrm{SnCl}$ or $\mathrm{PhSnCl}_{3}$ in $\mathrm{C}_{6} \mathrm{H}_{6}$ to prepare 241 and 242 respectively. The ${ }^{119} \mathrm{Sn}$ NMR spectrum of 241 showed a single resonance at $\delta=-85.0 \mathrm{ppm}$ and the ${ }^{31} \mathrm{P}$ NMR spectrum a resonance at $\delta=-9.8$ ppm. Similarly, the reaction of $\mathbf{2 4 0 a}$ with $\mathrm{PhSnCl}_{3}$ showed a ${ }^{119} \mathrm{Sn}$ resonance at $\delta=-27.0 \mathrm{ppm}$ and ${ }^{31} \mathrm{P}$ NMR resonance at $\delta=43.0 \mathrm{ppm}$ with four unidentified small impurity peaks. No further characterization of these compounds was attempted at this time.

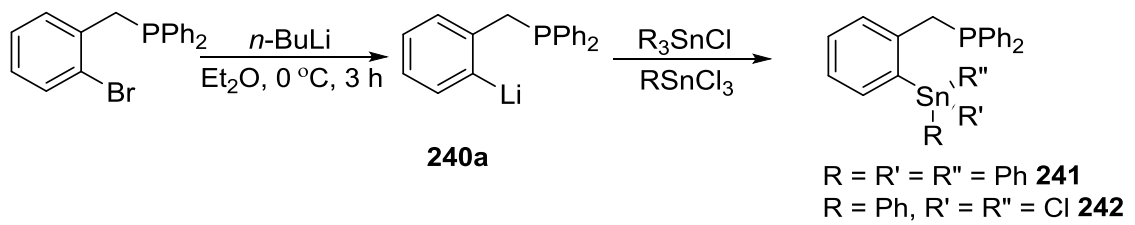

Scheme 50: Synthesis of (o-(diphenylphosphino)benzyl)stannanes.

\subsection{Characterization and Properties:}

Crystal structure data for the dibromostannane 146, similar to compounds prepared in this study $(\mathbf{2 0 0}, \mathbf{2 0 1}, \mathbf{2 0 2}, \mathbf{2 0 3})$ was previously reported by Molloy et al. ${ }^{43}$ Compound 198 was recrystallized in methanol, while 200, 201, 202 and 203 were recrystallized using a 1:1 ratio of DCM:hexane. Crystallographic data for compounds 198, 202-203 are listed in Table 19 and in Tables A1-A29 and the ORTEP representation of their unit cell components in Figure 45-48. The crystal structure determination of $\mathbf{1 9 8}$ (Figure 45) revealed a distorted tetrahedral geometry about tin with the central bond angles ranging from $103-117^{\circ}$. The absence of a Sn-O dative interaction in 198 may due to the steric bulk of three phenyl rings at tin. Compounds 200 (Figure 46), 201 (Figure 47), 202 (Figure 48) and 203 (Figure 49) all display a distorted trigonal bipyramidal 
geometry around tin with angles ranging from $69.37^{\circ}-166.3^{\circ}$ and $73.22^{\circ}-170.83^{\circ}, 97.49^{\circ}-169.78^{\circ}$, $99.53^{\circ}-171.02^{\circ}$ and $97.10^{\circ}-168.62^{\circ}$ respectively. There are two unique molecules found in the unit cell for $\mathbf{2 0 0}$ with substantially different Sn-O distances (2.72 and $2.89 \AA)$. The distance between the datively bonded $\mathrm{Sn}$ and $\mathrm{O}$ atoms is $2.80 \AA$ (average) in $\mathbf{2 0 0}, 2.81 \AA$ in $\mathbf{2 0 1}, 2.72 \AA$ in $\mathbf{2 0 2}$ and $2.82 \AA$ in $\mathbf{2 0 3}$. These values are smaller than the sum of the van der Waal's radii of oxygen and tin $(3.70 \AA)$ and larger than the sum of their covalent radii $2.066 \AA .{ }^{161}$ The values reported here represent a medium to strong Sn-O dative interaction. Molloy et al. ${ }^{43}$ observed a similar Sn-O distance of 2.734(4) $\AA$ for the hypercoordinated dibromo compound 146. The significant increase in Sn-O interaction on replacing the phenyl group by $\mathrm{Cl}$ is likely due to a moderation of the Lewis acidity at $\mathrm{Sn}$. In the structures of $\mathbf{2 0 0}$ and $\mathbf{2 0 2}$ the equatorial plane is formed by three carbon atoms while the axial positions are occupied by $\mathrm{Cl}$ and $\mathrm{O}$ atom. The values of the $\mathrm{O}-\mathrm{Sn}-\mathrm{Cl}$ bond angles are $166.30^{\circ}(1 \mathrm{~A})$ and $170.83^{\circ}(2 \mathrm{~A})$ for 200 and $171.02^{\circ}$ for $\mathbf{2 0 2}$. In $\mathbf{2 0 1}$ and $\mathbf{2 0 3}$ the equatorial plane is formed by one $\mathrm{Cl}$ and two carbon atoms while axial positions are occupied by $\mathrm{Cl}$ and $\mathrm{O}$ atoms and the values of the $\mathrm{O}-\mathrm{Sn}-\mathrm{Cl}$ bond angle are $169.78^{\circ}$ for 201 and $168.62^{\circ}$ for $\mathbf{2 0 3}$. The dative interactions between $\mathrm{Sn}$ and $\mathrm{O}$ are found trans to $\mathrm{Sn}-\mathrm{Cl}$ bond and nearly linear. For compounds 200-203 the Sn-C (phenyl) bond lengths are within the sum of covalent radii (2.15 $\AA$ ) for tin and carbon. ${ }^{165}$

The strength of Sn-O interaction can impact the geometry of these molecules. The difference between the sum of equatorial angles and sum of the axial angles can be used to monitor the transition from tetrahedral to TBP geometry (Figure 43, Table 20). ${ }^{96,101,166-168}$ This difference would be zero $\left(\Sigma\right.$ eq- $\Sigma$ ax $\left.=328.5^{\circ}-328.5^{\circ}=0\right)$ for a tetrahedral geometry while for TBP geometry it would be $90^{\circ}\left(\Sigma\right.$ eq $-\Sigma$ ax $\left.=360^{\circ}-270^{\circ}=90\right)$; the greater the value of $(\Sigma$ eq $-\Sigma$ ax $)$ more TBP the structure. 


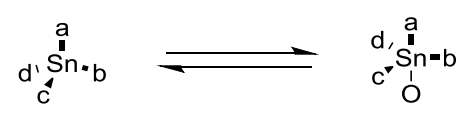

Figure 44: Transition from tetrahedral geometry to TBP.

The bond order is a measure of the strength of such interactions which is calculated using the following equation. ${ }^{101,169,170}$

$$
\mathrm{BO}=d\left(\mathrm{Sn}-\mathrm{E}_{\mathrm{ave}}\right)+1-d(\mathrm{Sn}-\mathrm{E})
$$




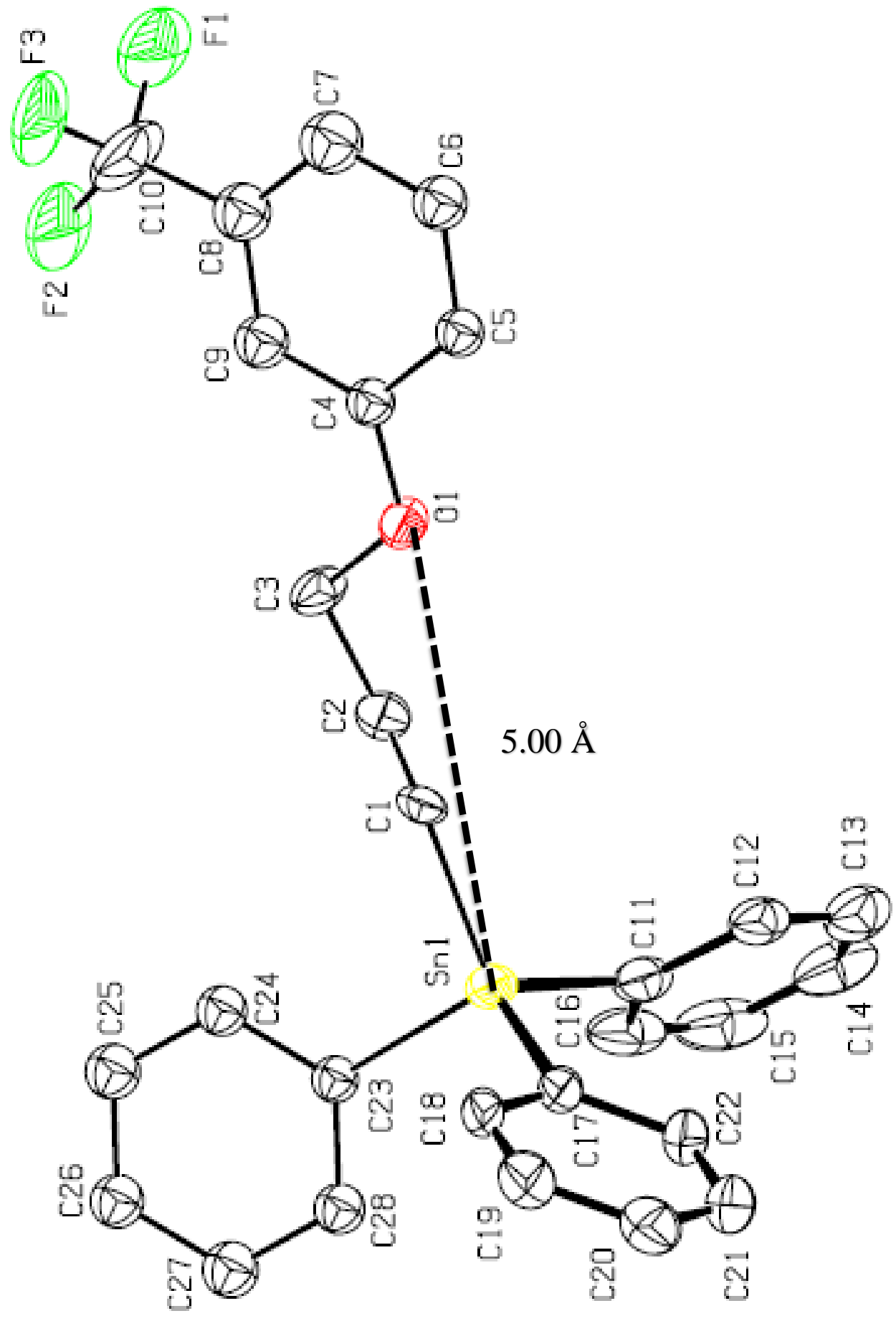

Figure 45: ORTEP representation of the unit cell components for 198. 


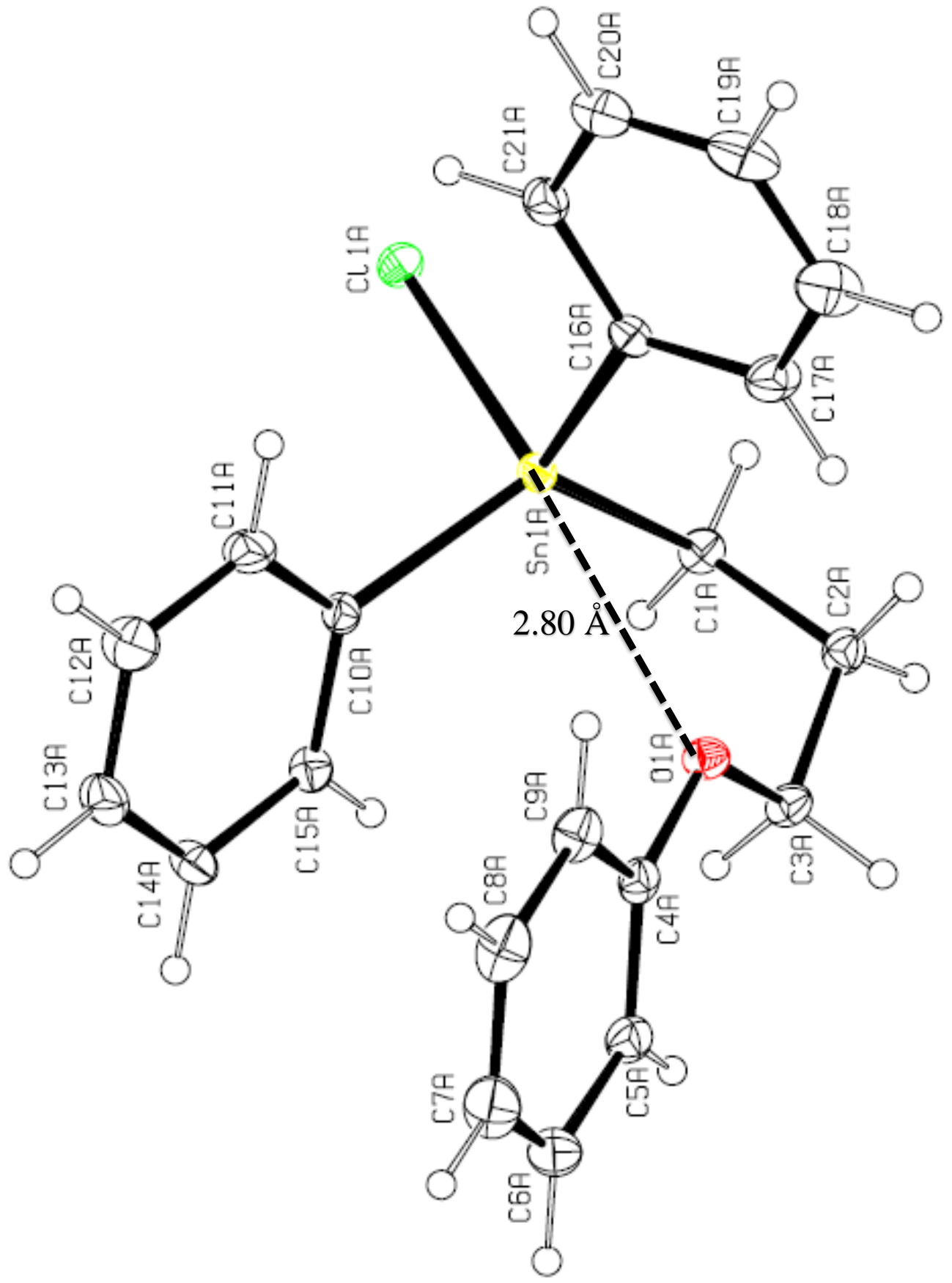

Figure 46: ORTEP representation of the unit cell (A) components for $\mathbf{2 0 0 .}$ 


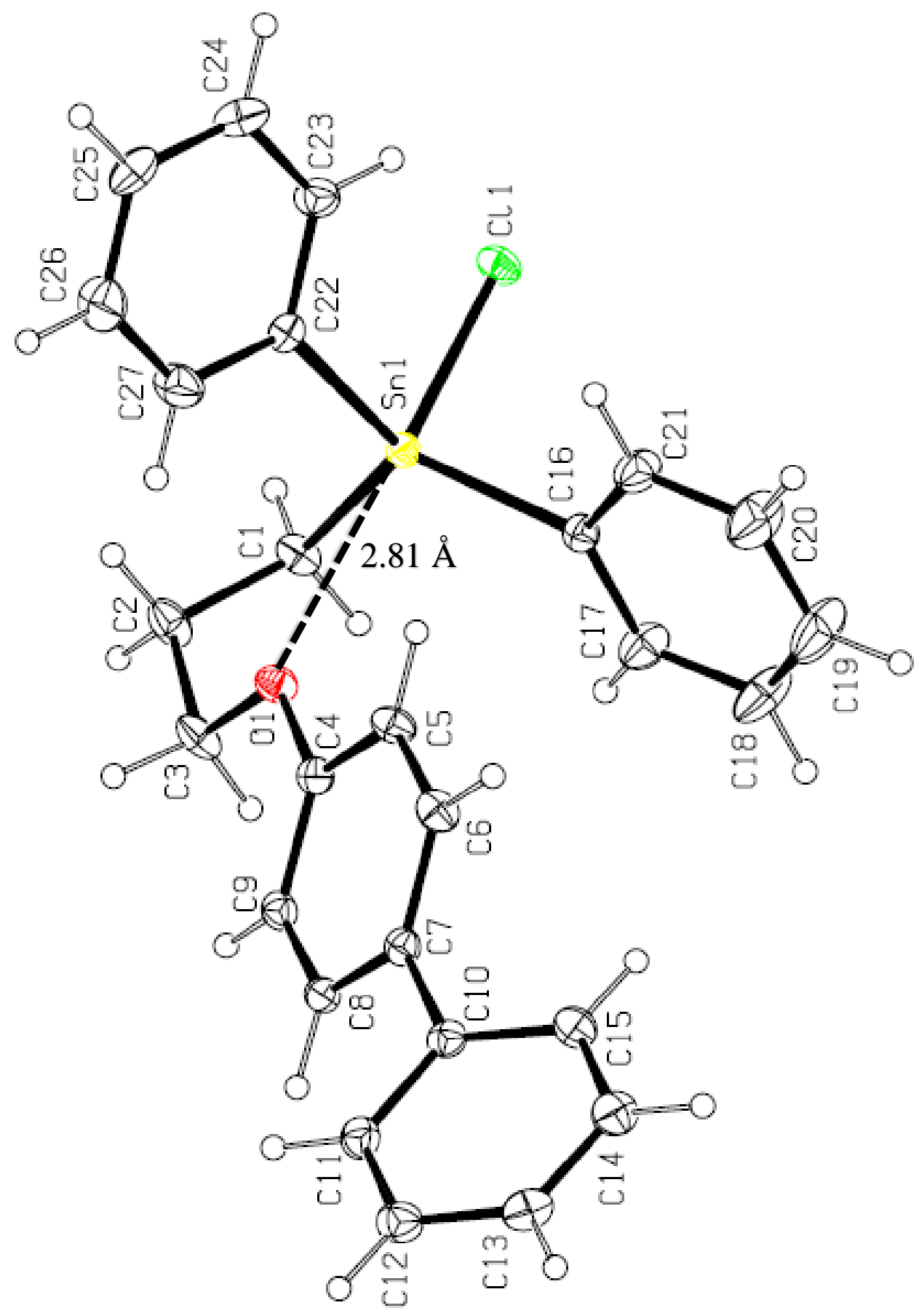

Figure 47: ORTEP representation of the unit cell components for 201. 


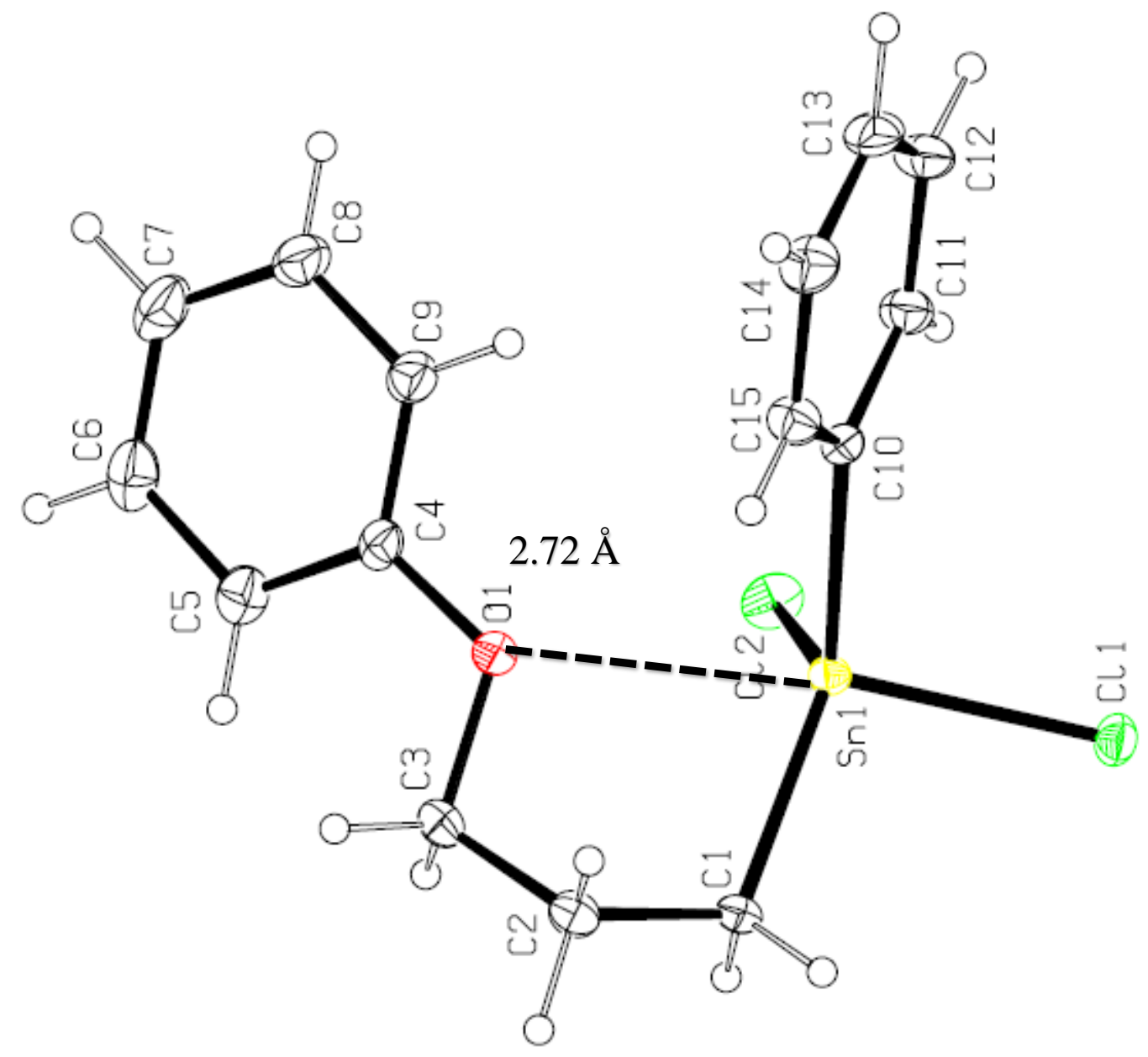

Figure 48: ORTEP representation of the unit cell components for 202. 


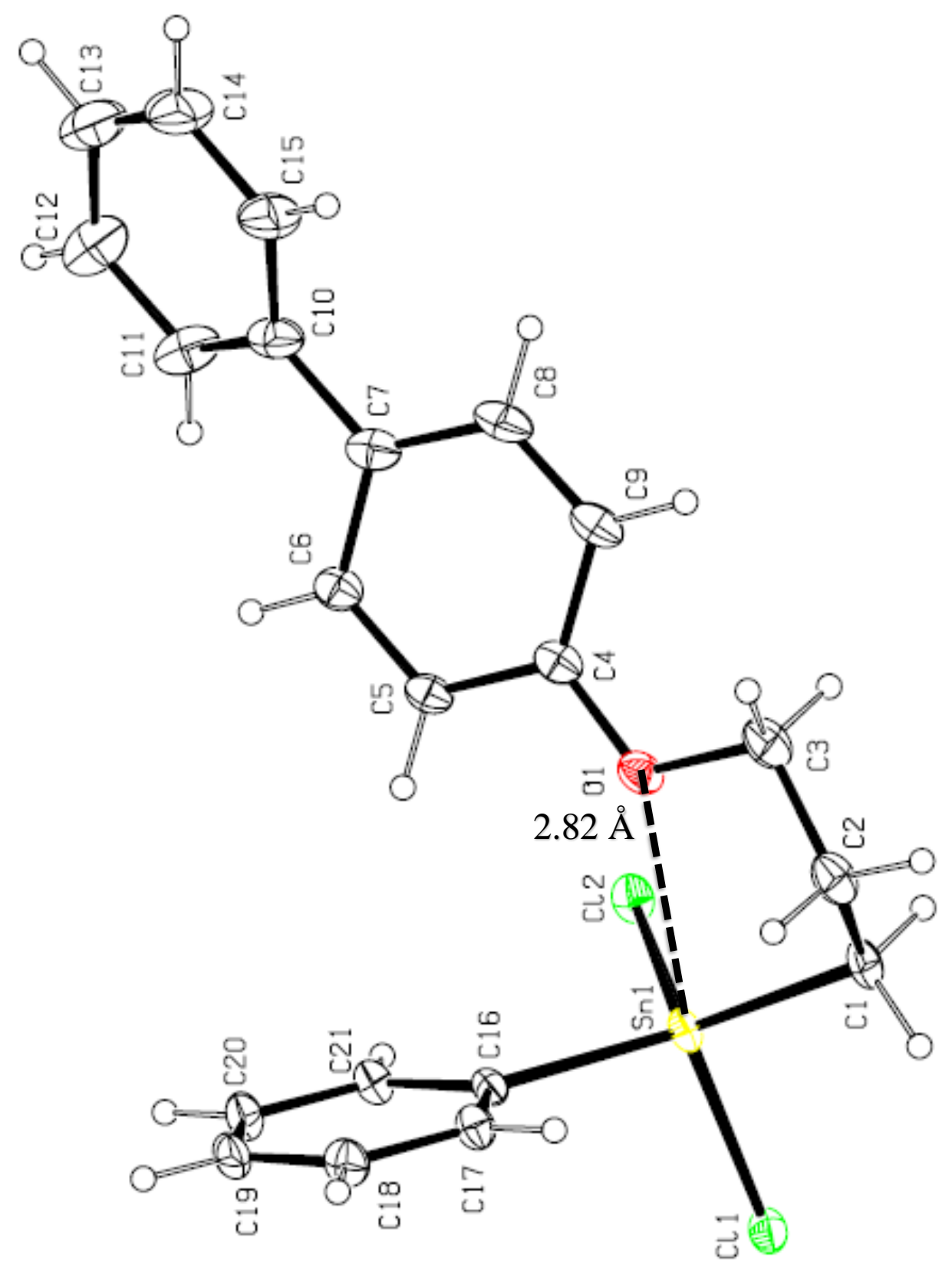

Figure 49: ORTEP representation of the unit cell components for 203. 
Table 19: Crystallographic data and structural refinement for compounds 198, 200, 201, 202, 203

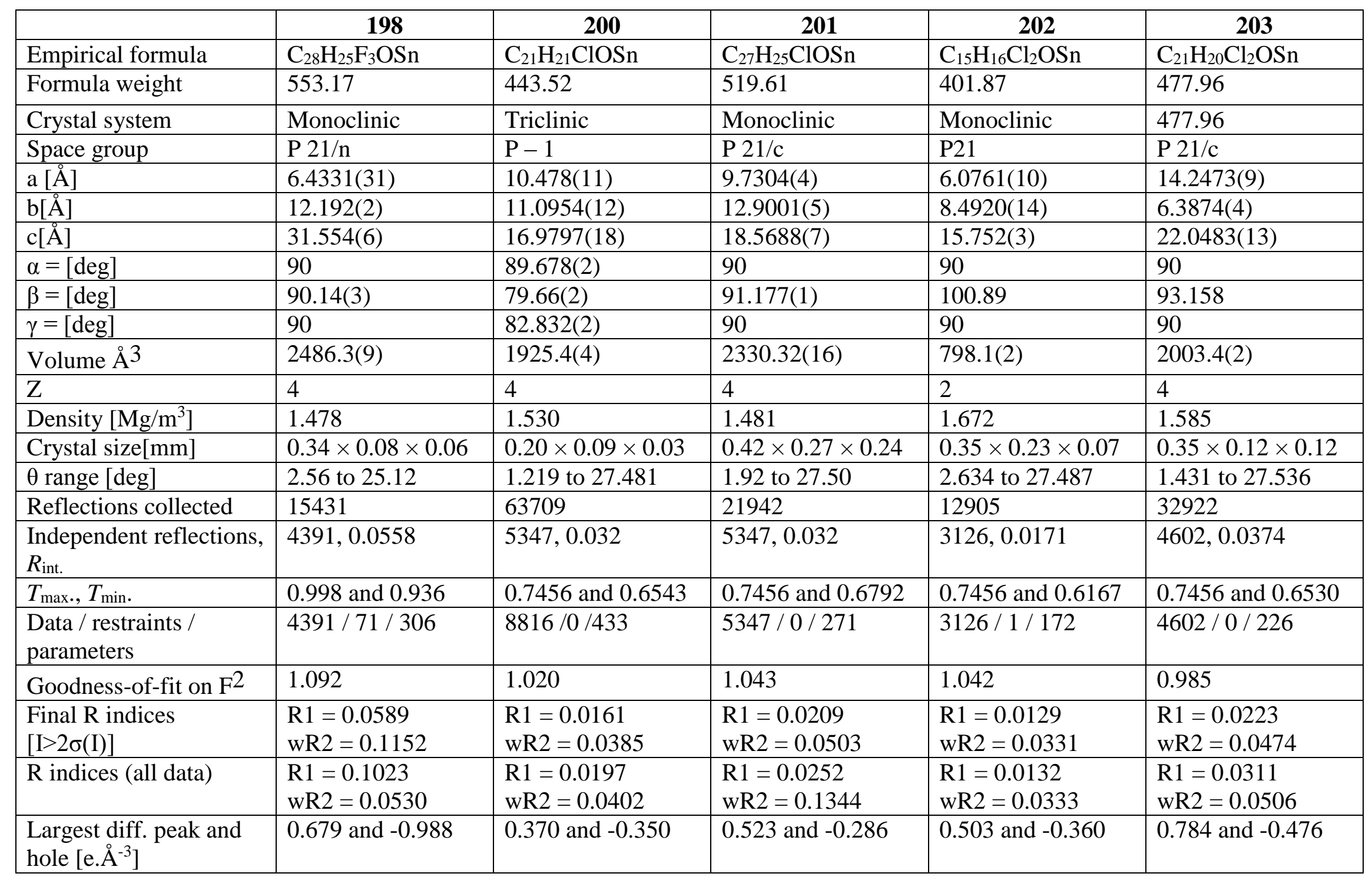


Table 20: Data used to obtain the transition from tetrahedral to TBP for compounds 198, 200203.

\begin{tabular}{|c|c|c|c|c|c|c|c|}
\hline Compound & Sn-O A & $\begin{array}{c}\text { Equatorial } \\
\text { angles }\end{array}$ & $\begin{array}{c}\begin{array}{c}\sum \text { eq } \\
(\mathrm{deg})\end{array} \\
\end{array}$ & Axial angles & $\begin{array}{c}\sum \mathrm{ax} \\
(\mathrm{deg})\end{array}$ & $\begin{array}{c}\begin{array}{c}\sum \text { eq- } \\
\text { (deg) }\end{array} \\
\end{array}$ & BO \\
\hline 198 & 5.00 & $\begin{array}{l}\text { C1-Sn-C17 } \\
\text { C1-Sn-C11 } \\
\text { C11-Sn-C17 }\end{array}$ & 330.2 & $\begin{array}{c}\mathrm{C} 1-\mathrm{Sn}-\mathrm{C} 17 \\
\mathrm{C} 1-\mathrm{Sn}-\mathrm{C} 23 \\
\mathrm{C} 11-\mathrm{Sn}-\mathrm{C} 23\end{array}$ & 328.8 & 1.4 & -1.87 \\
\hline 200 & 2.81 & $\begin{array}{l}\text { C1-Sn-Cl1 } \\
\text { C1-Sn-C10 } \\
\text { C10-Sn-Cl1 }\end{array}$ & 325.15 & $\begin{array}{c}\text { C1-Sn-C11 } \\
\text { C1-Sn-C16 } \\
\text { C10-Sn-C16 }\end{array}$ & 325.44 & -0.29 & 0.32 \\
\hline 201 & 2.81 & $\begin{array}{l}\text { C1-Sn-Cl1 } \\
\text { C1-Sn-C22 } \\
\text { C22-Sn-C11 }\end{array}$ & 315.16 & $\begin{array}{c}\text { C1-Sn-C11 } \\
\text { C1-Sn-C16 } \\
\text { C16-Sn-C22 }\end{array}$ & 334.15 & -18.99 & 0.32 \\
\hline 202 & 2.72 & $\begin{array}{l}\mathrm{C} 1-\mathrm{Sn}-\mathrm{Cl} 2 \\
\mathrm{C} 10-\mathrm{Sn}-\mathrm{C} 1 \\
\mathrm{C} 10-\mathrm{Sn}-\mathrm{Cl} 2\end{array}$ & 350.65 & $\begin{array}{l}\text { C1-Sn-Cl1 } \\
\text { C10-Sn-C1 } \\
\text { Cl1-Sn-Cl2 }\end{array}$ & 332.51 & 17.54 & 0.41 \\
\hline 203 & 2.82 & $\begin{array}{l}\text { C16-Sn-Cl2 } \\
\text { C16-Sn-C1 } \\
\text { C1-Sn-Cl2 }\end{array}$ & 347.31 & $\begin{array}{l}\text { C1-Sn-Cl1 } \\
\text { C16-Sn-C1 } \\
\text { Cl1-Sn-Cl2 }\end{array}$ & 327.78 & 19.53 & 0.31 \\
\hline
\end{tabular}

* Calculated using a Sn-O average bond distance of $2.14 \AA$ 


\subsection{Polymerization and characterization:}

\subsection{1: Wurtz coupling:}

The polymerization of $\mathbf{3 7}$ by Wurtz coupling under different conditions produced three products that were identified by ${ }^{119} \mathrm{Sn}$ NMR spectroscopy. The distannane ${ }^{171} \mathbf{2 4 3}$, stannoxane bis$\left\{[2-(N, N\right.$-dimethyaminomethyl)phenyl $] n$-butylchloro $\}$ tin oxide ${ }^{171} \mathbf{2 4 4}$ and the bis-\{[2-(N,Ndimethyaminomethyl)phenyl]di- $n$-butyltin(IV) $\mathbf{2 4 5}$ were obtained from two reaction. ${ }^{66}$ Previous work by Turek et al. ${ }^{171}$ who prepared 243 by adding a solution $\left(1: 1\right.$ hexane/ $\left.\mathrm{C}_{6} \mathrm{H}_{6}\right)$ of 37 to a $\mathrm{K}$ mirror at $-30{ }^{\circ} \mathrm{C}$ that was stirred for one week at RT produced a single ${ }^{119} \mathrm{Sn}$ NMR resonance at $\delta$ $=-88.1 \mathrm{ppm}$. Turek then bubbled oxygen through the solution containing 243 and converted it to 244 generating two ${ }^{119} \mathrm{Sn}$ NMR signals at -134.5 and $-137.5 \mathrm{ppm}$ that are presumably two possible stereoisomers 224a and 244b.

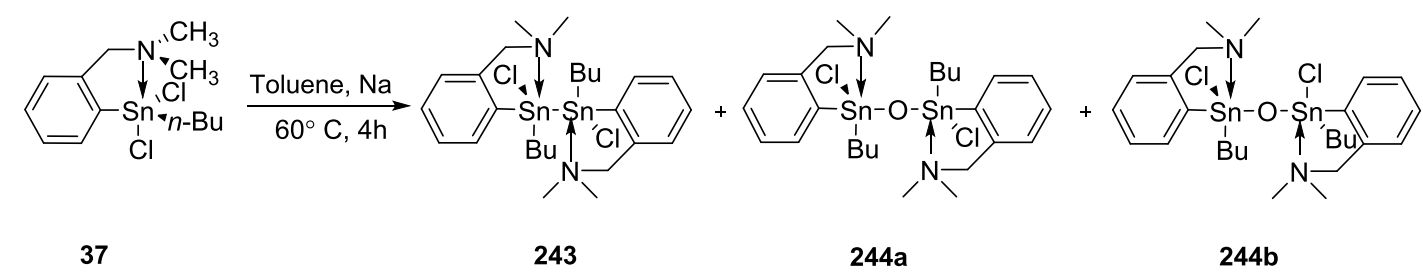

Scheme 51: Wurtz Coupling of 37 for 4 h. 


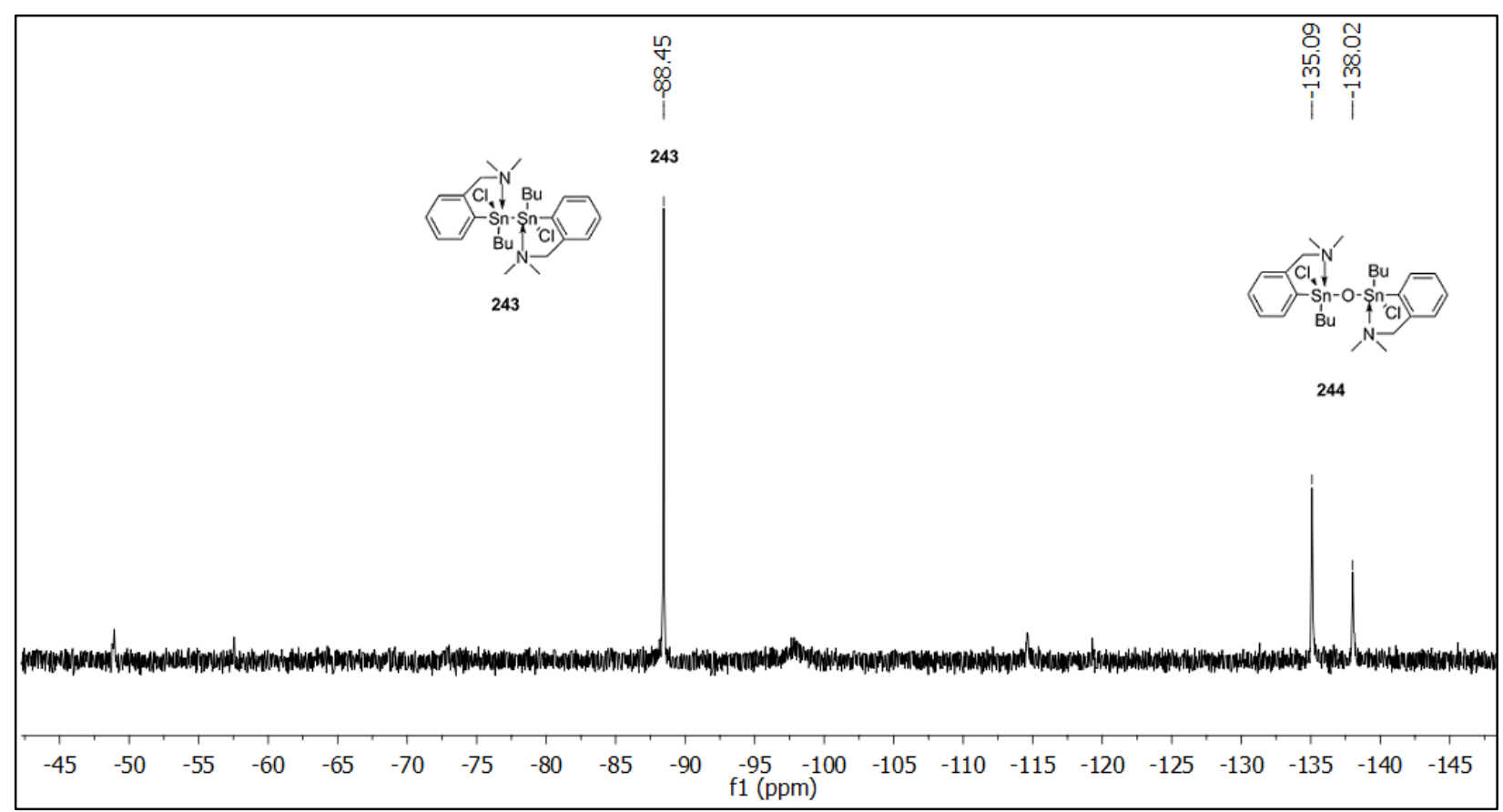

Figure 50: ${ }^{119} \mathrm{Sn}$ NMR $\left(\mathrm{C}_{6} \mathrm{D}_{6}\right)$ spectrum of Wurtz coupling of 37 for $4 \mathrm{~h}$ at $60{ }^{\circ} \mathrm{C}$.

For this study the reaction time was increased from 4 h to 4 days and 245 was produced along with other unidentified products. Turek et al. ${ }^{66}$ also prepared $\mathbf{2 4 5}$ by reacting the K-mirror and $\mathrm{L}(n-\mathrm{Bu})_{2} \mathrm{SnCl}$ in hexane for 5 days.

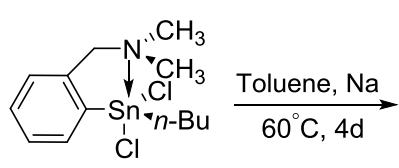

37

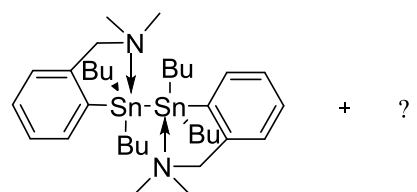

245

Scheme 52: Wurtz Coupling of $\mathbf{3 7}$ for 4 days.

The polymerization of $\mathbf{3 5}$ was attempted by the Wurtz method developed by Molloy et $a l .{ }^{127}$ for $4 \mathrm{~h}$. The ${ }^{119} \mathrm{Sn}$ NMR showed resonances at $\delta=-173.0,-210.6$ and $-212.2 \mathrm{ppm}$. The

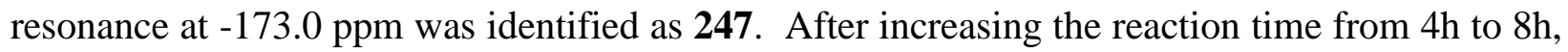
${ }^{119} \mathrm{Sn}$ NMR showed resonances at $-145.5 \mathrm{ppm}$ and $-173.0 \mathrm{ppm}$ corresponding to $\mathbf{2 4 6}$ and 247 respectively. Turek et al. ${ }^{66}$ also prepared 246 by reacting the $\mathrm{K}$-mirror and $\mathrm{L}(\mathrm{Ph})_{2} \mathrm{SnCl}$ in hexane for 5 days at $-30^{\circ} \mathrm{C}$-room temperature. 


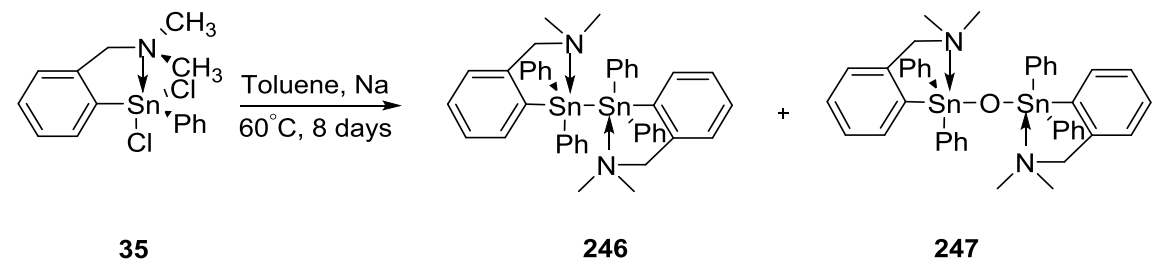

Scheme 53: Na Wurtz Coupling of 35.

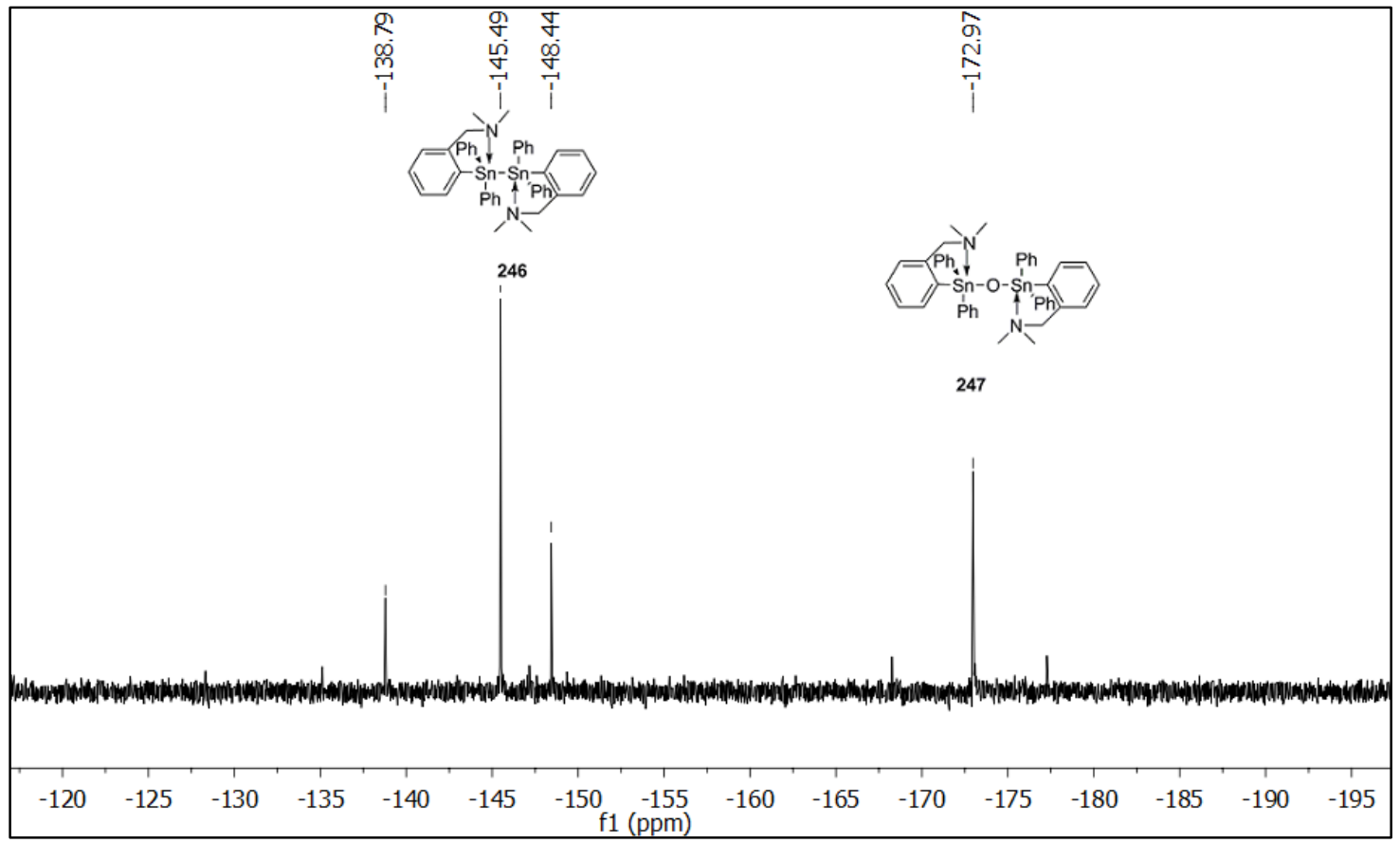

Figure 51: ${ }^{119} \mathrm{Sn}$ NMR $\left(\mathrm{C}_{6} \mathrm{D}_{6}\right)$ spectrum of the reaction mixture from the Wurtz coupling of $\mathbf{3 5}$.

Table 21: ${ }^{119}$ Sn NMR data for distannanes.

\begin{tabular}{|c|c|c|c|c|}
\hline \multirow{2}{*}{ Compounds } & \multicolumn{2}{|c|}{${ }^{119}$ Sn $\delta(\mathbf{p p m})$} & \multicolumn{1}{c|}{$\boldsymbol{1}_{1195 n-119 S n}$} & \multirow{2}{*}{ Ref. } \\
\hline $\mathbf{2 4 3}$ & -88.1 & -88.1 & $9219(9221)^{\mathrm{a}}$ & 171 \\
\hline $\mathbf{2 4 4}$ & $-134.5,-137.5$ & $-134.5,-137.5$ & - & 171 \\
\hline $\mathbf{2 4 5}$ & -105.7 & -105.7 & - & 66 \\
\hline $\mathbf{2 4 6}$ & -145.1 & -145.5 & $5520(5158)^{\mathrm{a}}$ & 66 \\
\hline $\mathbf{2 4 7}$ & -173.2 & -173.0 & - & 66 \\
\hline
\end{tabular}

${ }^{\mathrm{a}}$ Literature values 
The results obtained by the Wurtz coupling of $\mathbf{3 5}$ and $\mathbf{3 7}$ are comparable to the previously reported values. Earlier polystannanes studies showed that the reduction of $\left(n-\mathrm{Bu}_{2} \mathrm{SnCl}_{2}\right.$ using $\mathrm{Na}$ in toluene at $60{ }^{\circ} \mathrm{C}$ lead to high molecular weight polymer poly $\left(\mathrm{Bu}_{2} \mathrm{Sn}\right)_{\mathrm{n}}(n \approx 4000-5000)$ in addition to five and six membered cyclic oligomers in varying amounts. ${ }^{127}$ On the other hand, the reduction of bulkier stannanes, such as $(t-\mathrm{Bu})_{2} \mathrm{SnCl}_{2}$, mainly produced smaller oligostannane rings, such as

cyclo- $\left(t-\mathrm{Bu}_{2} \mathrm{Sn}\right)_{4}{ }^{172} \mathrm{Beckmann}$ et al. ${ }^{145}$ reported that the reduction of $c i s-\mathrm{Myr}_{2} \mathrm{SnCl}_{2}$ and trans$\mathrm{Myr}_{2} \mathrm{SnCl}_{2}(\mathrm{Myr}=$ myrtanyl $)$ under same reaction conditions resulted in a mixture of unidentified products with the ${ }^{119} \mathrm{Sn}$ NMR spectra of the crude reaction mixtures of both revealing more than 10 signals. The reduction of cis- $-\mathrm{Myr}_{2} \mathrm{SnCl}_{2}$ and trans $-\mathrm{Myr}_{2} \mathrm{SnCl}_{2}$ with $\mathrm{Mg}$ resulted in the exclusive formation of five-membered oligostannane rings cyclo-(cis-Myr2 $\mathrm{Sn})_{5}$ and cyclo-(trans- $\left.\mathrm{Myr}_{2} \mathrm{Sn}\right)_{5}$. ${ }^{119} \mathrm{Sn} \mathrm{NMR}\left(\mathrm{CDCl}_{3}\right)$ spectra of cyclo-(cis-Myr $\left.2 \mathrm{Sn}\right)_{5}$ and cyclo-(trans-Myr $\left.{ }_{2} \mathrm{Sn}\right)_{5}$ showed signals $\delta$ $=-209.9$ and $-218.1 \mathrm{ppm}$ which are close to the resonances obtained for the Wurtz coupled of $\mathbf{3 5}$ at $\delta=-210.6$ and $-212.2 \mathrm{ppm}$ which are likely the 5- and 6-membered cyclics.

\subsubsection{Dehydrocoupling:}

\subsubsection{1: Metal catalyzed dehydrocoupling:}

The synthesis of polymers $\mathbf{2 4 8 - 2 5 0}$ are based on the metal-catalyzed dehydropolymerization (Scheme 54, 55) of a diarylstannane with different chelating ligands. As the polymerization proceeded, the colour of reaction mixture changed from colourless to yellow to dark orange and finally to dark brown for the solid polymer. Dehydrocoupling of the hypercoordinated compound $\mathbf{2 3 1}$ using Wilkinson's catalyst was unsuccessful. However polymerization did occur in the presence of $\mathrm{Cp}_{2} \mathrm{ZrMe}_{2}$ in hexane. The reaction was monitored by ${ }^{1} \mathrm{H}$ NMR spectroscopy until the signal for the $\mathrm{Sn}-\mathrm{H}$ resonance completely disappeared. The solvent was removed under reduced pressure and the ${ }^{119} \mathrm{Sn}$ NMR $\left(\mathrm{C}_{6} \mathrm{D}_{6}\right)$ analysis of the crude product 
showed resonances at $\delta=-49.0,114.0,162.0 \mathrm{ppm}$. A portion of the crude product precipitated slightly in hexane and was filtered off. The solid was insoluble in common organic solvents. The ${ }^{119} \mathrm{Sn}$ NMR $\left(\mathrm{C}_{6} \mathrm{D}_{6}\right)$ of the hexane soluble fraction was obtained and it revealed a sharp single resonance at $-49 \mathrm{ppm}$ (Figure 53).
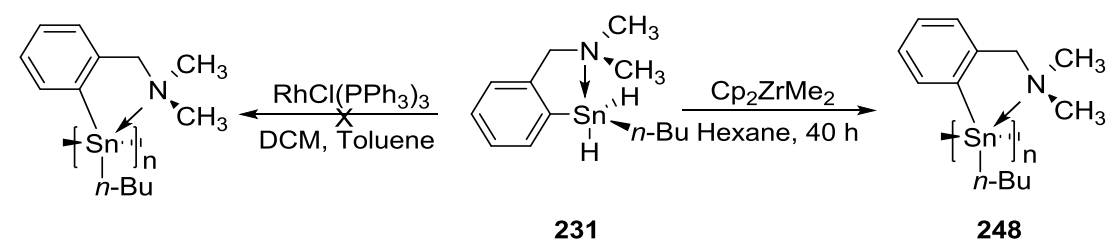

Scheme 54: Polymerization of 231.

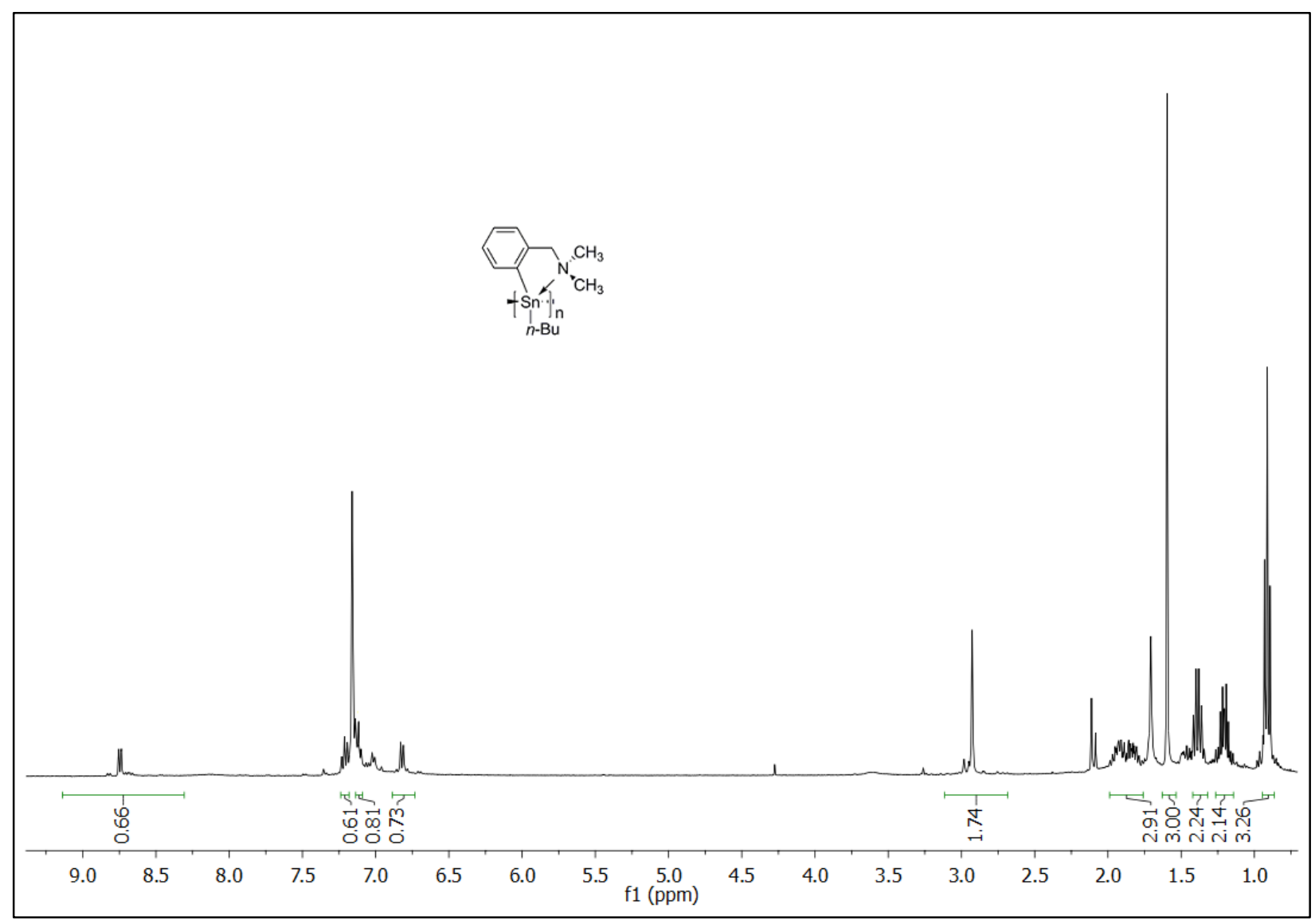

Figure 52: ${ }^{1} \mathrm{H}$ NMR $\left(\mathrm{C}_{6} \mathrm{D}_{6}\right)$ spectrum of 248. 


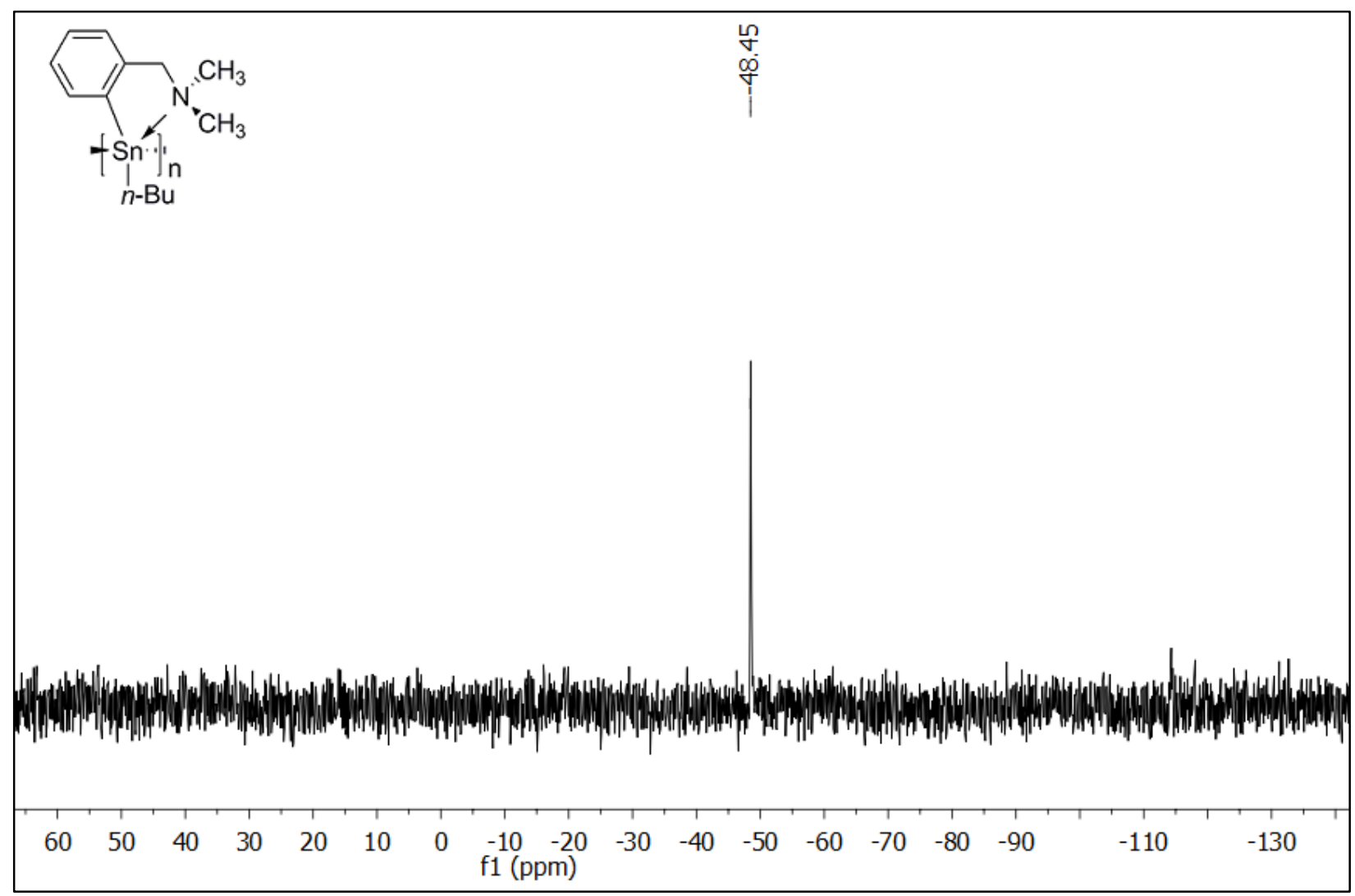

Figure 53: ${ }^{119} \mathrm{Sn} \mathrm{NMR}\left(\mathrm{C}_{6} \mathrm{D}_{6}\right)$ spectrum of $\mathbf{2 4 8}$.

Polymers 249-250 were synthesized by catalytic dehydrocoupling of monomers 206-207 using Wilkinson's catalyst in toluene. It was suggested by Molloy et al. ${ }^{43}$ that the Wurtz coupling of the dibromide $\mathbf{1 4 6}$ did not proceed as a result of the sterically hindered 5-coordinate structure at tin from the chelating propylether group. The polymerization of dihydrides $\mathbf{2 0 6}$ and $\mathbf{2 0 7}$ is an indication of the absence of 5-coordinate geometry at tin in these tin dihydrides. The polymers were purified by precipitating THF solutions of the crude mixtures twice in petroleum ether. The gummy orange/yellow coloured polymers remained attached to the walls of the flask. The residual solvent was removed from the purified product by prolonged drying under reduced pressure. The soft gummy polymers were characterized fully by NMR $\left({ }^{1} \mathrm{H},{ }^{13} \mathrm{C},{ }^{119} \mathrm{Sn}\right)$ spectroscopy. 


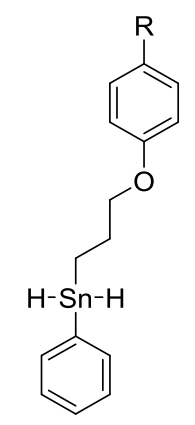

$\mathrm{R}=\mathrm{H}, 206$

$\mathrm{R}=\mathrm{Ph} 207$

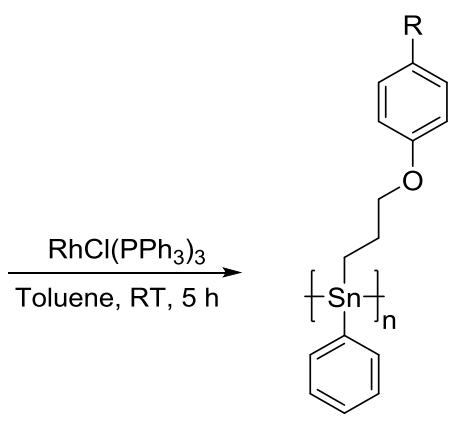

$\mathrm{R}=\mathrm{H} 249$

$\mathrm{R}=\mathrm{Ph} 250$

Scheme 55: Transition metal catalyzed synthesis of polymers 249 and 250.

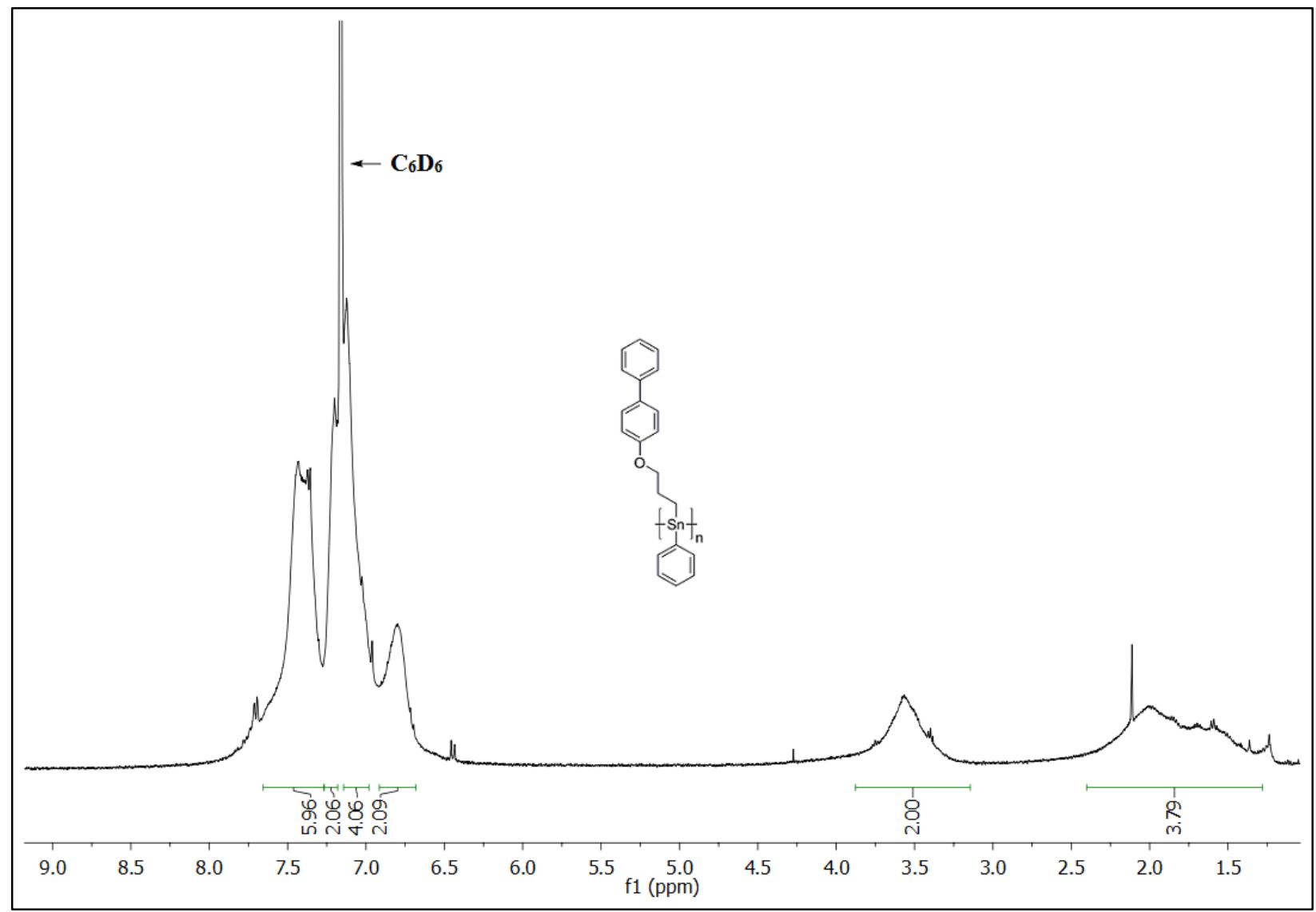

Figure 54: ${ }^{1} \mathrm{H}$ NMR $\left(\mathrm{C}_{6} \mathrm{D}_{6}\right)$ spectrum of polymer 250.

${ }^{119} \mathrm{Sn}$ NMR revealed a single broad peak at -199 ppm (Figure 55) for 249 and -195 ppm for 250 respectively, which is 16-19 ppm downfield compared to their starting monomers 206-207. The disappearance of both the monomer signal at ca. $\delta=-215 \mathrm{ppm}$ in the ${ }^{119} \mathrm{Sn}$ NMR spectrum 
and the $\mathrm{Sn}-\mathrm{H}$ signal (ca. $5.5 \mathrm{ppm}$ ) in the ${ }^{1} \mathrm{H}$ NMR spectra reflect a complete monomer conversion. ${ }^{1} \mathrm{H}$ NMR spectroscopy also showed broad peaks (Figure 54) which is typical in case of polymers.

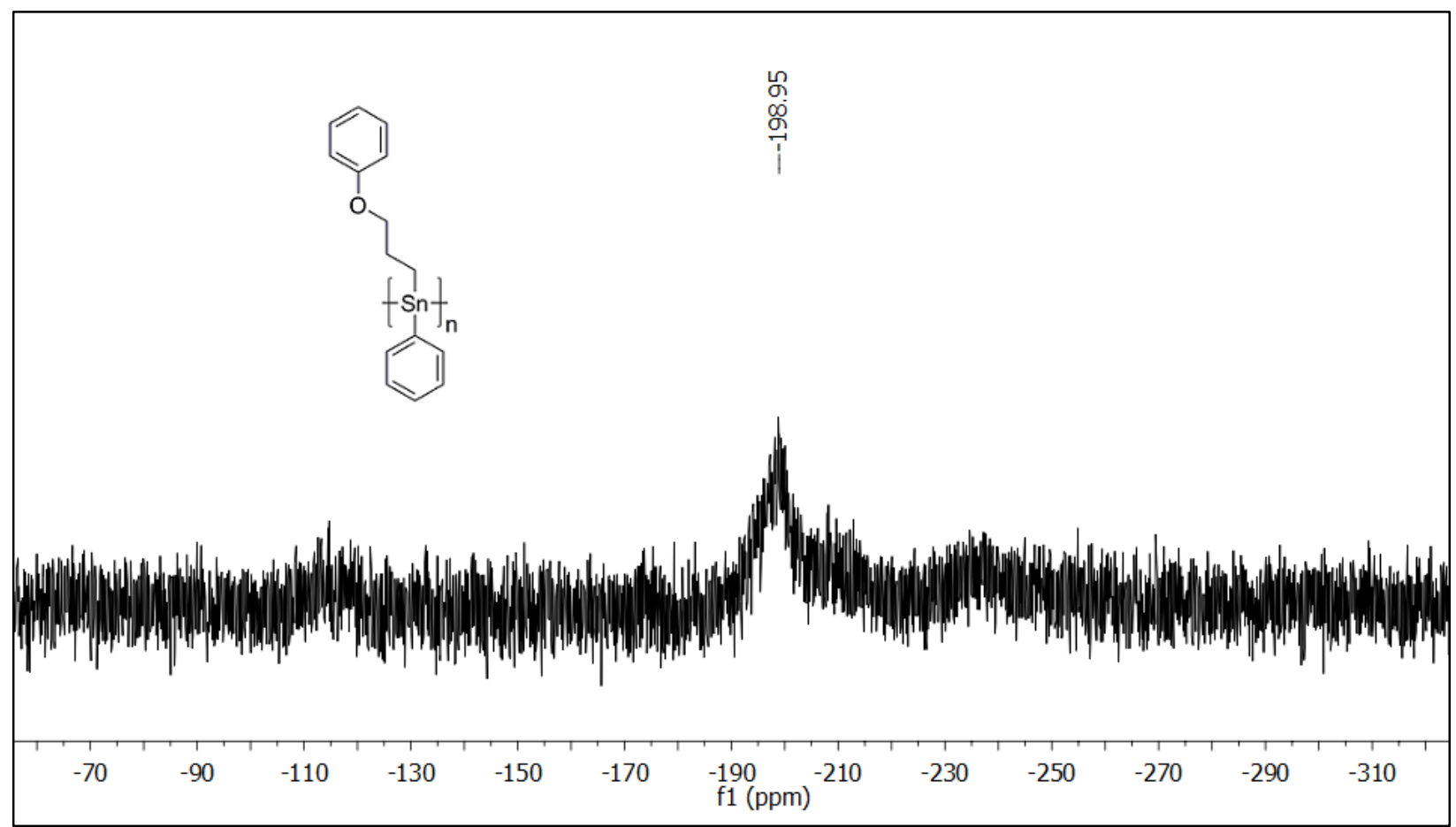

Figure 55: ${ }^{119} \mathrm{Sn}$ NMR $\left(\mathrm{C}_{6} \mathrm{D}_{6}\right)$ spectrum of 249.

\subsubsection{Non-metal catalyzed dehydrocoupling:}

Catalytic dehydropolymerization of compound $\mathbf{2 2 7}$ was unsuccessfully attempted using Wilkinson's catalyst or $\mathrm{Cp}_{2} \mathrm{ZrMe}_{2}$. This may be a result of steric crowding at tin as a result of the C, $O$-chelating ligands. Lechner et al. ${ }^{130}$ used TMEDA for the polymerization of $\mathrm{R}_{2} \mathrm{SnH}_{2}(\mathrm{R}=n$ butyl, phenyl, 4- $n$-butylphenyl) to polymers 251 and $\mathbf{2 5 2}$ which gave ${ }^{119} \mathrm{Sn}$ NMR resonance at $197 \mathrm{ppm}$, a typical region for polystannanes. 


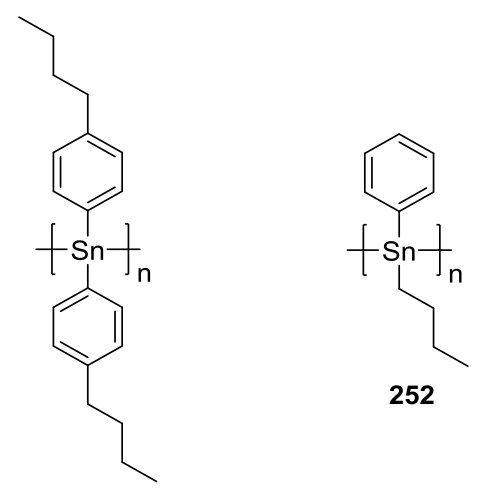

251

Figure 56: TMEDA catalyzed polymerization of organotin dihyrides. ${ }^{130}$

Dehydrocoupling of $\mathbf{2 2 7}$ in the presence of TMEDA produced the desired polymer $\mathbf{2 5 1}$ in $36 \mathrm{~h}$. This reaction proceeds most likely via a radical process as predicted by Davies et al. for the reaction of $\mathrm{R}_{2} \mathrm{SnXH}$ with pyridine. ${ }^{173}$ The ${ }^{119} \mathrm{Sn}$ NMR resonance for $\mathbf{2 5 1}$ was located at -196 ppm, shifting $13.0 \mathrm{ppm}$ downfield from the monomer chemical shift of the resonance. There are some other peaks upfield of the polymer peak at $\delta=-198 \mathrm{ppm}$ to $-200 \mathrm{ppm}$, which may be due to the cyclic oligomeric species (Figure 57).

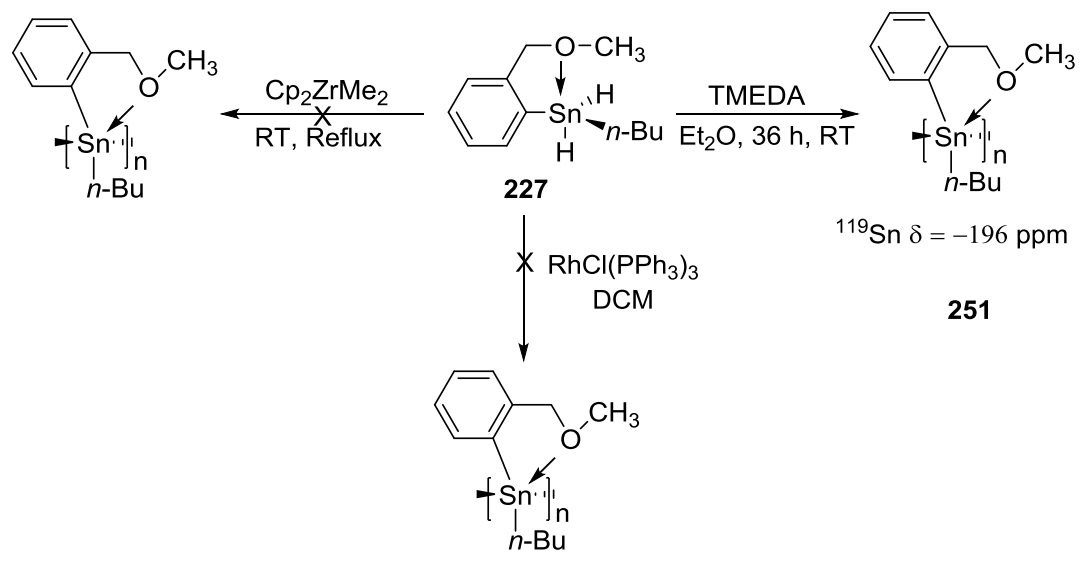

Scheme 56: Synthesis of polymer 251. 


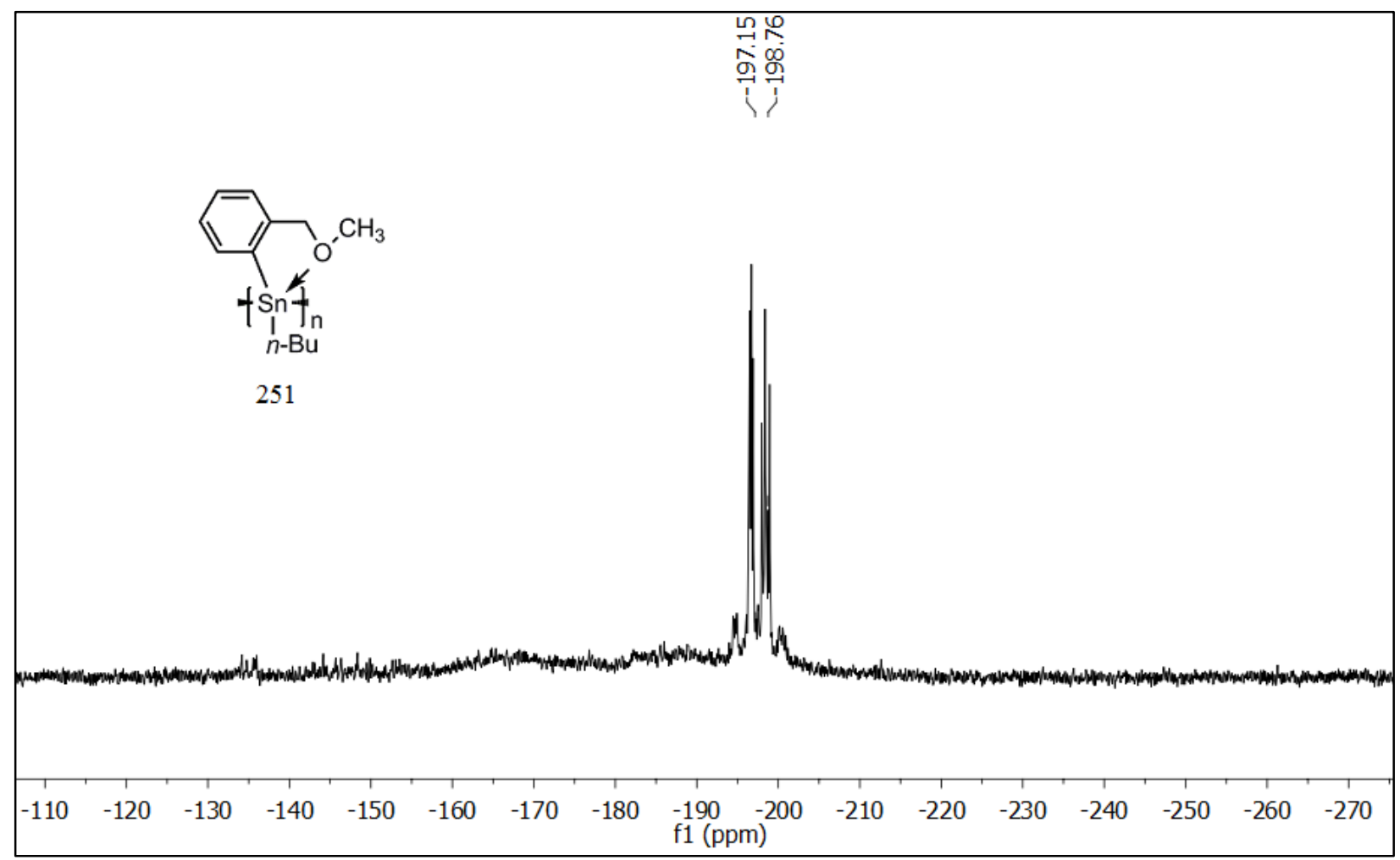

Figure 57: ${ }^{119} \mathrm{Sn}$ NMR $\left(\mathrm{C}_{6} \mathrm{D}_{6}\right)$ spectrum of 251.

\subsubsection{Characterization of polymers:}

\subsubsection{GPC Characterization:}

Gel permeation chromatography (GPC) is equipped with a triple detection system to determine the absolute molecular weight of polymers. GPC analysis of polystannanes (solution in THF) typically causes chain cleavage or clipping of polymers due to the laser $\left(\lambda_{0}=670 \mathrm{~nm}\right)$ used. The analysis of polymers $\mathbf{2 4 8 - 2 5 0}$ was attempted using solutions $\left(1 \times 10^{-4} \mathrm{M}\right)$ of these materials in THF. It was observed that the signal intensity for the right angle and low angle detectors which use the laser source would not appear in the GPC, presumably as a result of photodegradation of the polystannane. This results in the clipping of $\mathrm{Sn}-\mathrm{Sn}$ bonds in polymer backbone converting polymer to oligomers. If the laser is turned off for the light scattering detector, signal response from both the refractive index (RI) and intrinsic viscometry (IV) are observed. To mitigate this issue, a UV-A photoabsorber was introduced to the THF solutions and exposure minimized. 
Compounds 206-207 were catalytically dehydrocoupled using Wilkinson's catalyst to polymers 249 and 250 . These gummy yellow coloured materials were readily soluble in common organic solvents such as DCM, THF and $\mathrm{C}_{6} \mathrm{H}_{6}$. A solution of these polymers in THF was used for molar mass determination. The $M \mathrm{w}$ for $\mathbf{2 5 0}$ was $1.01 \times 10^{5}$ Da with a PDI 1.3 . The molar masses of these polymers are in the range of polymer material reported previously by Molloy. ${ }^{43}$ The PDI values indicate that the polymers are relatively uniform and contain chains that are essentially monomodal. Similar polymers $(\mathbf{2 5 2}, \mathbf{2 5 3})$ were synthesized by Molloy et al. ${ }^{43}$ via Wurtz coupling of dibromides $(\mathbf{1 4 7}, \mathbf{1 5 0})$ with molar masses $2.50-3.00 \times 10^{5}$ Da and with PDI of 1.3 and 2.0 respectively comparable to the values found in this effort. The properties of these polymers are given in Table 22.

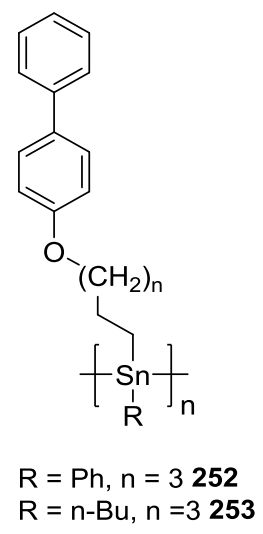

Figure 58: Structure of polymers 252 and 253

Table 22: molecular weight and ${ }^{119} \mathrm{Sn}$ chemical data for polystannanes.

\begin{tabular}{|c|c|c|c|c|}
\hline Polymer & $\boldsymbol{M}_{\mathbf{w}}(\mathbf{D a})$ & PDI & ${ }^{119}$ Sn $(\boldsymbol{\delta})$ & $\boldsymbol{\lambda}_{\max }(\mathbf{n m})$ \\
\hline $\mathbf{2 4 8}$ & $3.2 \times 10^{4}$ & 1.8 & -49.0 & - \\
\hline $\mathbf{2 4 9}$ & - & - & -199 & 263 \\
\hline $\mathbf{2 5 0}$ & $1.01 \times 10^{5}$ & 1.3 & -195 & 273 \\
\hline $\mathbf{2 5 1}$ & - & - & -196 & 380 \\
\hline $\mathbf{2 5 2}^{43}$ & $3.0 \times 10^{5}$ & 1.3 & - & 255 \\
\hline $\mathbf{2 5 3}^{43}$ & $2.5 \times 10^{5}$ & 2.0 & - & 255 \\
\hline
\end{tabular}


Polymerization of monomers $\mathbf{2 2 7}$ and $\mathbf{2 3 1}$ using Wilkinson's catalyst was unsuccessful. This may suggest that the presence of bulky groups in close proximity to the tin center such as the chelating ligand containing donor atoms like $\mathrm{N}$ and $\mathrm{O}$ hindered the polymerization with $\left[\mathrm{RhCl}\left(\mathrm{PPh}_{3}\right)_{3}\right] .{ }^{1} \mathrm{H}$ and ${ }^{119} \mathrm{Sn} \mathrm{NMR}$ spectroscopy of the polymerization attempt did not show any signals attributable to polymers.

Dehydrocoupling of 227 and 231 using TMEDA and $\mathrm{Cp}_{2} \mathrm{ZrMe}_{2}$ in $\mathrm{Et}_{2} \mathrm{O}$ and hexane respectively resulted in polymeric products. The GPC of the crude product $\mathbf{2 4 8}$ in THF produced a molecular weight $\mathrm{M} w=3.2 \times 10^{4} \mathrm{Da}$ and $\mathrm{PDI}=1.8$. An attempt to purify the polymer by precipitation of the THF solution in hexane afforded a small amount of a THF insoluble light yellow coloured solid. No further characterization of this insoluble material was undertaken. A gummy yellow coloured polymer of $\mathbf{2 4 8}$ was used for NMR and GPC analysis. 


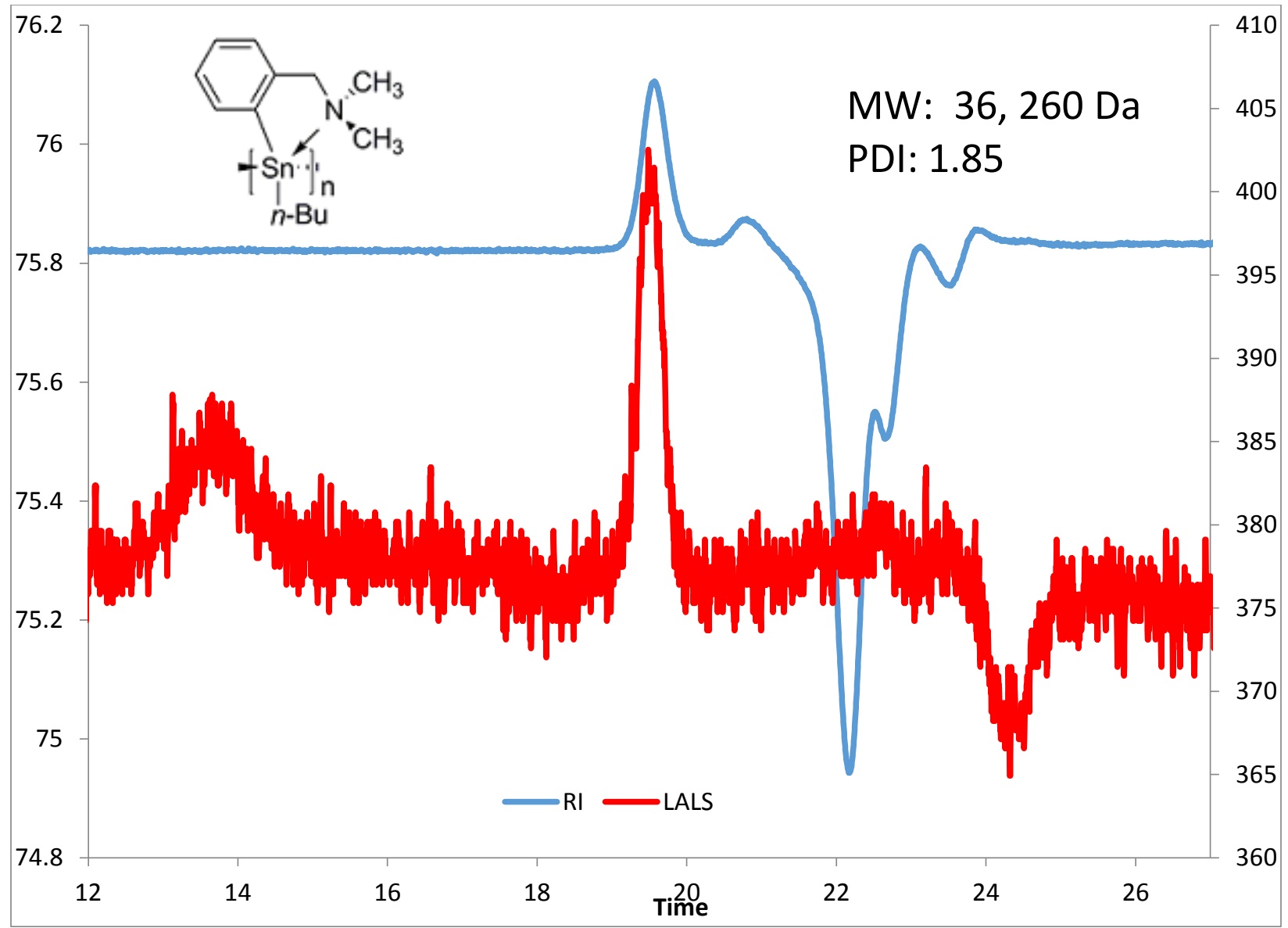

Figure 59: Triple detector GPC trace (THF) of polymer 248

\subsubsection{Electronic properties:}

The UV-Vis spectroscopy of polystananes 249 and 250 display a strong allowed $\pi-\pi^{*}$ transition at $228 \mathrm{~nm}$. A lower intensity $\sigma-\sigma^{*}$ transition is found at $\lambda_{\max }=273 \mathrm{~nm}$ for these polymers with tailing to $\lambda_{\max } 325 \mathrm{~nm}$. The tailing at $325 \mathrm{~nm}$ suggest that the tin atoms of the backbone are delocalized as a result of strong overlap. The strong $\pi-\pi^{*}$ transition at $\lambda_{\max }=255 \mathrm{~nm}$ was also reported by Molloy's et $a l .{ }^{43}$ for poly[phenyl5-(4-biphenyloxy)pentyl]tin synthesized by Wurtz coupling which does not hypercoordinate. 


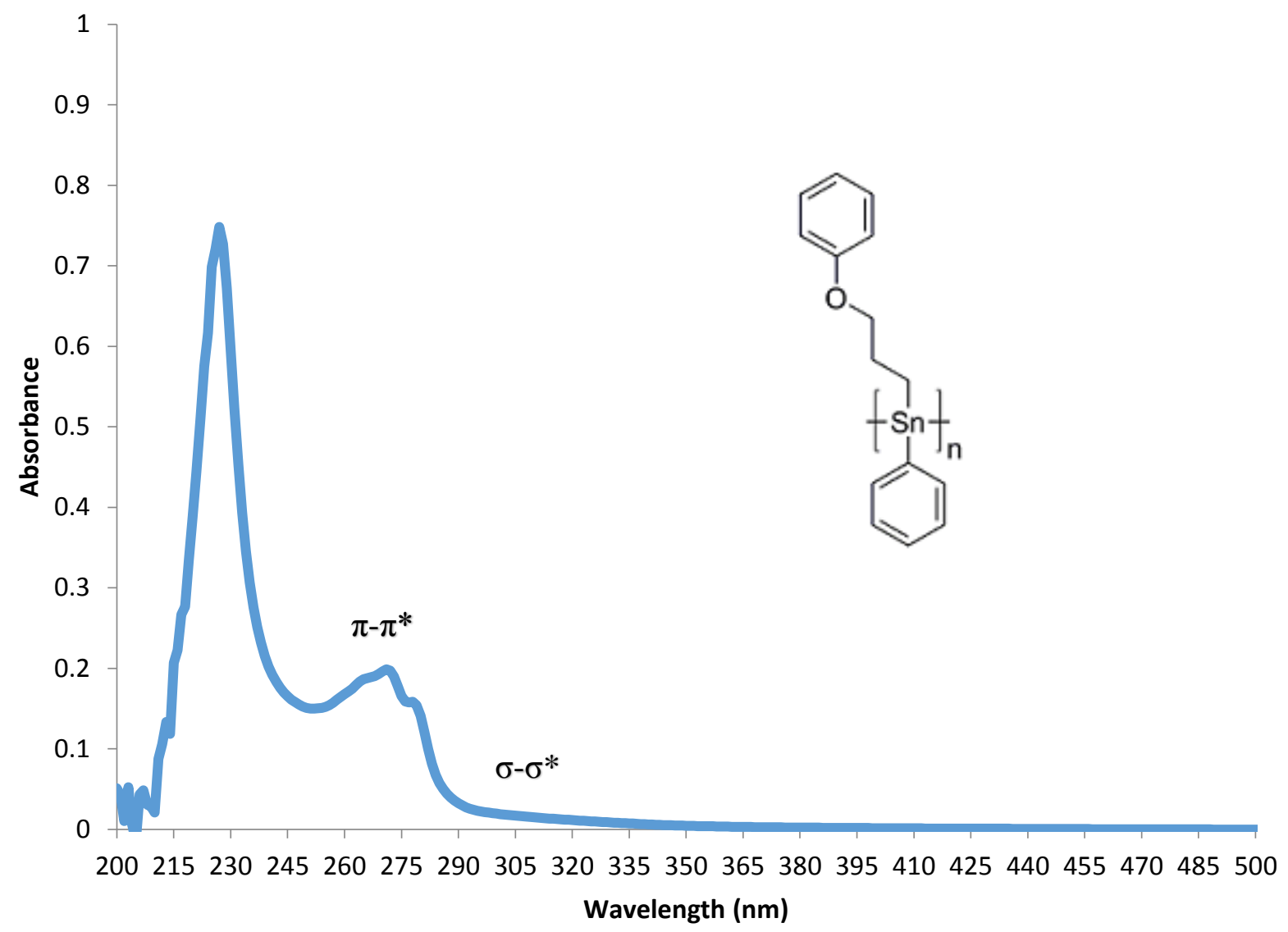

Figure 60: UV-visible spectrum of $\mathbf{2 4 9}$

\subsubsection{DSC studies:}

Thermal analysis of the polymers 249 and 250 by Differential scanning calorimetry (DSC) in the temperature range between $-5^{\circ} \mathrm{C}$ and $120^{\circ} \mathrm{C}$ revealed glass transition temperatures $\left(T_{g}\right)$ : ca. $65^{\circ} \mathrm{C}$ and $64{ }^{\circ} \mathrm{C}$ for $\mathbf{2 4 9}$ and $\mathbf{2 5 0}$ respectively. These $T_{\mathrm{g}}$ values reported for previously found for these polymers fall between for polydialkyl- $\left(0{ }^{\circ} \mathrm{C}\right.$ to $\left.91{ }^{\circ} \mathrm{C}\right)$ and polydialkyphenylstannanes $\left(-20^{\circ} \mathrm{C}\right.$ to $\left.50^{\circ} \mathrm{C}\right)$. 


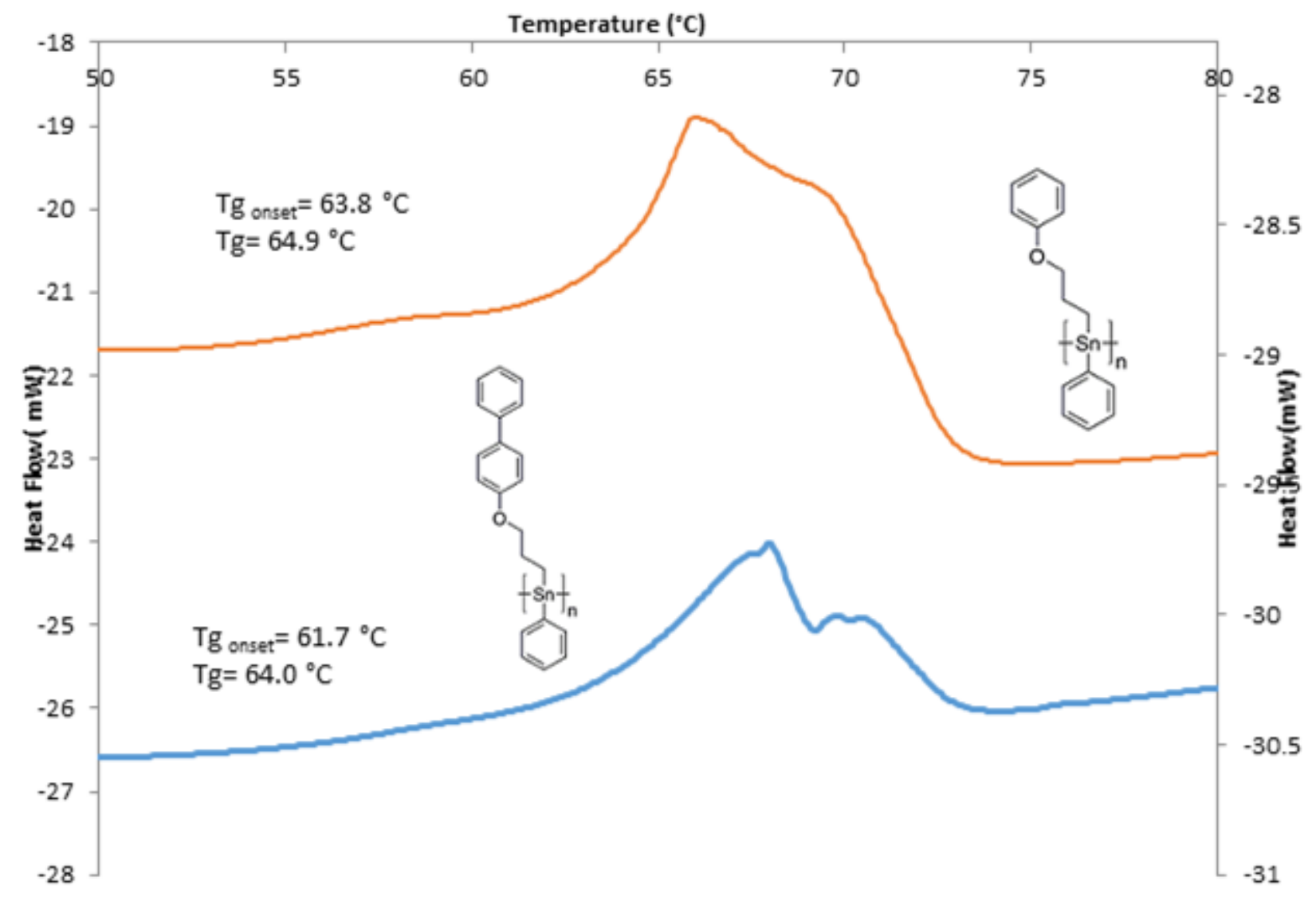

Figure 61: DSC heating thermograms of 249 and 250 (under $\mathrm{N}_{2}$, heating rate $5{ }^{\circ} \mathrm{C} / \mathrm{min}$ )

\subsubsection{UV-Vis and NMR Stability studies:}

Polystannanes $\mathbf{2 4 9}$ and $\mathbf{2 5 0}$ were found to be stable in the solid state under ambient conditions if left in a sealed flask for weeks as concluded after periodic evaluation by NMR $\left({ }^{1} \mathrm{H}\right.$ and $\left.{ }^{119} \mathrm{Sn}\right)$ spectroscopy. NMR solutions $\left(\mathrm{C}_{6} \mathrm{D}_{6}\right)$ of these polymers 249 and 250 were also stable in dark and did not show any degradation after one week. However, degradation was observed after 1 day in ambient light and the ${ }^{119} \mathrm{Sn}$ NMR signals characteristic for polymer $\mathbf{2 5 0}$ gradually disappeared with appearance of four new signals with chemical shifts $\delta=27.0,-23.0,-34.0,-67.0$ and $-207 \mathrm{ppm}$. These new signals continuously increase with continued light exposure and the ${ }^{119} \mathrm{Sn}$ NMR signals characteristic for polymer 250 completely disappeared after 10 days. ${ }^{119} \mathrm{Sn}$ 
NMR signals at $\delta=-207 \mathrm{ppm}$ are similar to that reported by Choffat et al. for five and six membered cyclo-oligo(dipropylstannane) at $\delta=-207.4$ and $-207.9 \mathrm{ppm}$ respectively.

It was previously shown that poly(dialkylstannane)s exhibit an absorption maximum in the region of $370-410 \mathrm{~nm},{ }^{118,119,}, 121,127,136,132$ which is caused by delocalization of the $\sigma$-electrons of the tin atoms along the polymer backbone. In several reports, the degree of degradation of these materials was evaluated from the UV-Vis spectroscopy. ${ }^{127,136,132}$ In this study, the extent of degradation of polymer 250 was investigated in THF solutions at a concentration $5.9 \times 10^{-5} \mathrm{~mol} / \mathrm{L}$ in the range of $200-500 \mathrm{~nm}$ for 5 consecutive scans over intervals of 10 days $(1,10,20$ days). No significant degradation was observed under these conditions as the absorption bands at different intervals are superimposable (Figure 62). The most probable reason of these conflicting solution stability results of ${ }^{119}$ Sn NMR and UV studies may be the solvent dependence. 


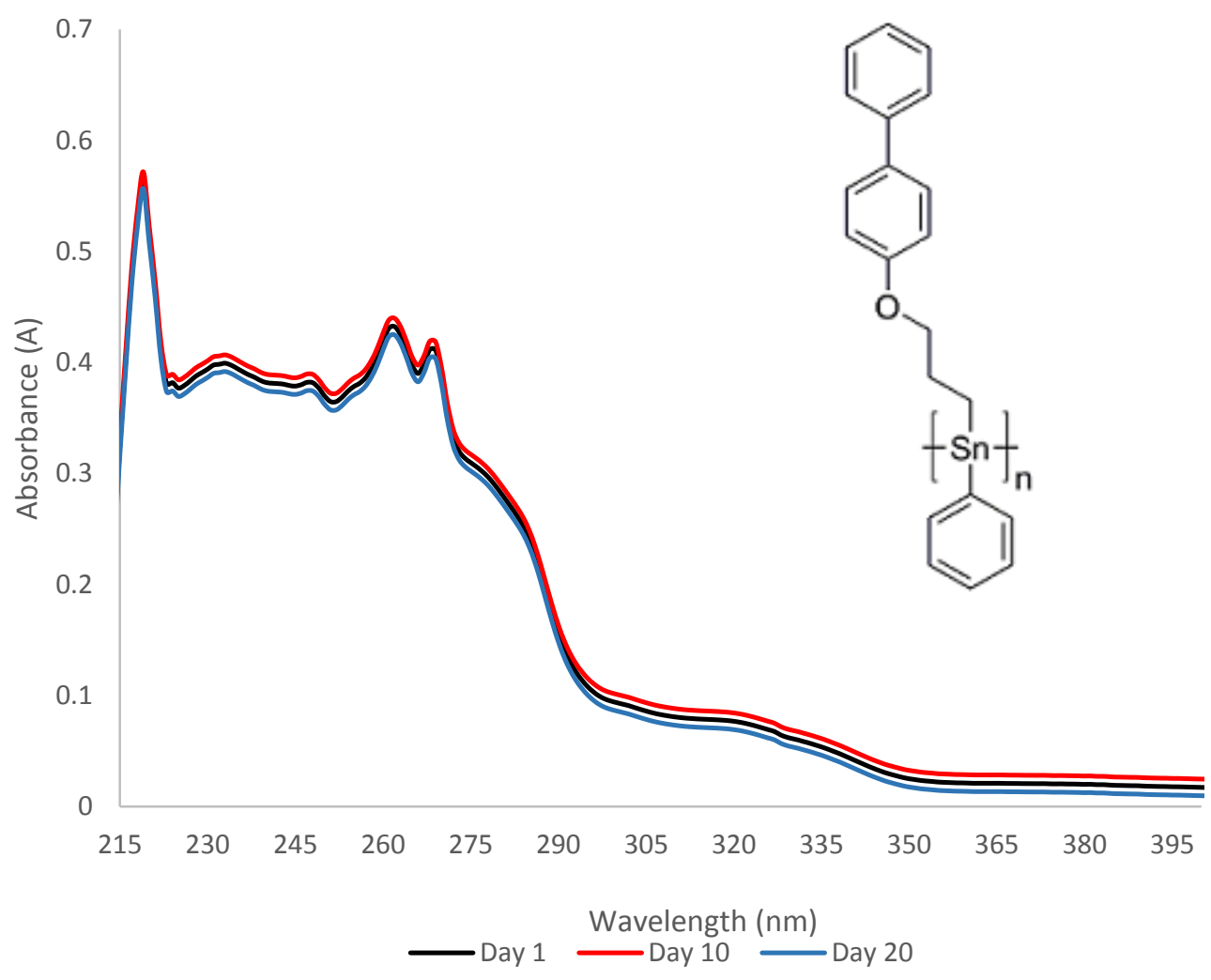

Figure 62: Consecutive UV-visible spectra of $\mathbf{2 5 0}$ at day 1, 10 and 20. 


\subsection{Conclusion:}

A variety of organotin compounds containing potential $\mathrm{Sn}-\mathrm{E}(\mathrm{E}=\mathrm{N}, \mathrm{O}, \mathrm{P}$ and $\mathrm{S})$ interactions were synthesized and characterized using different techniques. Tetraorganotin compounds 141, 198-199 with tethered phenyloxy moieties were prepared and characterized by

NMR spectroscopy $\left({ }^{1} \mathrm{H},{ }^{13} \mathrm{C},{ }^{119} \mathrm{Sn}\right)$, HRMS spectroscopy or elemental analysis and X-ray crystallography in case of $\mathbf{1 9 8}$. Triorganotin chlorides 200-201 were synthesized from a stepwise replacement of the phenyl groups with $\mathrm{HCl}$ and further converted using the same methodology to produce diorganotin dihalides 202-203. These organotin halides 200-203 were structurally characterized to reveal moderate to strong Sn-O interactions. Novel tin hydrides 204-207 were prepared by the reduction of organotin halides 200-203 with $\mathrm{LiAlH}_{4}$ and used for polymerization studies. Model hexaorganoditin 208-209 were also prepared by dehydrocoupling of triorganotin hydrides 204-205.

Organotin compounds with $C, O$ - chelating ligands were also synthesized. Tetraorganotin compounds 112 and 217 were prepared in good or improved yields. Organotin halides 218-219 containing a $C, O$ - chelating ligand were synthesized from the reaction of the lithiated salt $\mathbf{2 1 6}$ with $\mathrm{RSnCl}_{3}(\mathrm{R}=\mathrm{Me}, n-\mathrm{Bu})$. An attempt to replace the phenyl group of 112 with chlorine resulted in redistribution product 221. Reduction of tin dihalides 218 and 219 afforded two novel diorganotin dihydrides 226 and 227.

Tin dichlorides 33, 35, 37 containing a $C, N$-chelating ligand were also synthesized. The hydrogenation of $\mathbf{3 3}$ and $\mathbf{3 5}$ resulted in the formation of tin dihydrides $\mathbf{2 3 0}$ and $\mathbf{2 3 1}$. Wurtz coupling of dichlorides 35 and 37 resulted in distannanes 243-246 that were previously reported in literature by different methods. 
Sulfur containing $\mathbf{2 3 3}$ and $\mathbf{2 3 4}$ were prepared using a modified method and investigated for their suitability for organotin compounds containing a $C, S$ - chelating ligand. Reaction attempts to produce the organotin compounds with a $C, S$ - chelating ligand resulted in the formation of multiple products and the purification attempts were unsuccessful. Compound 239 with a $C, P$ functionality was synthesized and used to produce compound 241. NMR analysis of $\mathbf{2 4 1}$ revealed a single ${ }^{119} \mathrm{Sn}$ and ${ }^{31} \mathrm{P}$ resonance.

Catalytic dehydropolymerization of diorganotin dihydrides was completed using both metal and non-metal catalysts. Diorganotin dihydrides 206-207 catalytically dehydrocoupled in the presence of Wilkinson's catalyst resulted in the formation of polymers 249-250. These polymers were obtained as orange-yellow coloured gums. GPC analysis of 250 in THF yielded a moderate molecular weight polymer $\left(\mathrm{M}_{\mathrm{w}}=1.1 \times 10^{5} \mathrm{Da}, \mathrm{PDI}=1.3\right)$. A DSC thermal analysis of of polymers 249 and 250 revealed glass transition temperatures $\left(T_{\mathrm{g}}\right)$ of $65{ }^{\circ} \mathrm{C}$ and $64{ }^{\circ} \mathrm{C}$ respectively. Dehydrocoupling of compound 231 was successfully completed in the presence of $\mathrm{Cp}_{2} \mathrm{ZrMe}_{2}$ in hexane. GPC analysis of $\mathbf{2 4 8}$ in THF yielded a moderate molecular weight polymer $\left(\mathrm{M}_{\mathrm{w}}=3.2 \times 10^{4} \mathrm{Da}\right.$, PDI 1.8). Non-metal catalyzed dehydrocoupling of compound 227 in the presence of TMEDA produced the desired polymer $\mathbf{2 5 3}$ which has not been fully characterized. Stability studies of polymer $\mathbf{2 5 0}$ by ${ }^{119} \mathrm{Sn}$ NMR and UV-visible spectroscopies indicated a faster degradation in $\mathrm{C}_{6} \mathrm{D}_{6}$ than in THF. Overall, this polymer was considerably more stable than known poly(dialkyl)stannanes.

Our investigation of organotin compounds containing different chelating ligands indicates that substantial intramolecular interactions between $\operatorname{Sn}-\mathrm{E}(\mathrm{E}=\mathrm{N}, \mathrm{O})$ are present in monomers that contain at least one halide and adopt TBP geometry. The degree of the Sn-E interaction likely decreases as the halides are converted to hydrides as there is a substantial decrease of 
electronegativity at $\mathrm{Sn}$ which reduces its ability to draw electron density into a 3c-4e arrangement as well as the lack of orbitals to accommodate the extra electron density. The dative interaction in polystannanes 249-251 seems to be completely absent as is evident from their ${ }^{119} \mathrm{Sn}$ NMR chemical shifts $(\delta=-195 \mathrm{ppm}$ to $-199 \mathrm{ppm})$ which are very similar to most known polystannanes. In the case of 248, the ${ }^{119} \mathrm{Sn}$ NMR resonance at -49 ppm suggests that the dative interaction between $\mathrm{Sn}$ and $\mathrm{N}$ atom is likely still present. 


\subsection{Future work:}

In this study asymmetrical polystannanes were prepared from diorganotin dihydrides synthesized from 5-coordinate diorganotin dihalides possess $\mathrm{Sn}-\mathrm{E}(\mathrm{E}=\mathrm{N}, \mathrm{O})$ intramolecular interactions. The characterization of these new polystannanes indicated that the hypercoordination geometry is likely not preserved, except perhaps in the polymer with a $\mathrm{Sn}-\mathrm{N}$ interaction as evident by a dramatic downfield chemical shift. All polymeric materials displayed considerable stability in solid state and in solution in dark. It has been previously established that wavelengths at visible light are harmful for these type of materials and cause photodegradation. Unless the issue of photostability is sufficiently resolved, the utility of polystannanes for applications such as polymeric wires will not be realized.

To overcome this problem we propose the use of light absorbing chromophore side chains. The chromophore has the ability to absorb wavelengths of visible light and reflects or transmit the others. There are some examples of the molecule having side groups such as azobenzene that are currently being developed in our lab. These can be used as starting point to produce the dihydrides and dehydrocoupled to structurally more stable polystannanes. This will not increase the strength of Sn-Sn bonds but should reduce the light exposure of Sn-Sn bonds in the backbone of the polymers.

Further investigation of the geometry in polymers such as $\mathbf{2 4 9}$ and $\mathbf{2 5 0}$ has not been conclusively established. Analysis of the Sn environments by Mössbauer spectroscopy for these polymers would be instructive. Structural characterization by X-ray diffraction would also shed light on the geometry of the new dihydrides. Molecular modelling of monomers, oligomers and polymers could also provide insight into the extent the Lewis acidity at $\mathrm{Sn}$ is moderated by chelating ligands. This has not been established for any of the new molecules made in this study. 
Finally, a systemic investigation of the polymerization behaviour of structurally hindered tin sites to establish general rules, including solvent and catalyst choices, length of polymerizations, concentrations, to guide optimum polymerization outcomes is required. 


\subsection{Experimental:}

${ }^{1} \mathrm{H}$ NMR, ${ }^{13} \mathrm{C}\left\{{ }^{1} \mathrm{H}\right\},{ }^{119} \mathrm{Sn}\left\{{ }^{1} \mathrm{H}\right\},{ }^{19} \mathrm{~F}\left\{{ }^{1} \mathrm{H}\right\}$ and ${ }^{31} \mathrm{P}\left\{{ }^{1} \mathrm{H}\right\}$ NMR spectra were recorded on a Bruker Avance $400 \mathrm{MHz}$ NMR spectrometer. All chemical shifts are in ppm with respect to $\mathrm{Me}_{4} \mathrm{Si}\left({ }^{1} \mathrm{H}\right.$ and ${ }^{13} \mathrm{C}$ ), $\mathrm{Me}_{4} \mathrm{Sn}$ for ${ }^{119} \mathrm{Sn}, \mathrm{CFCl}_{3}$ for ${ }^{19} \mathrm{~F}$ and $85 \% \mathrm{H}_{3} \mathrm{PO}_{4}$ for ${ }^{31} \mathrm{P}$. UV-Vis measurements were carried out in THF solutions using a Perkin Elmer Lambda 40 spectrometer. Molecular weights of polymers were determined by gel permeation chromatography (GPC) using a Viscotek Triple Model 302 Detector system equipped with a Refractive Index Detector (RI), a four capillary differential viscometer (VISC), a right angle $\left(90^{\circ}\right)$ laser light scattering detector $\left(\lambda_{0}=670 \mathrm{~nm}\right)$ and a low angle $\left(7^{\circ}\right)$ laser light scattering detector. GPC columns were calibrated versus polystyrene standards (American Polymer Standards). A flow rate of $1.0 \mathrm{~mL} \mathrm{~min}^{-1}$ was used with ACS grade THF as the eluent. GPC samples were prepared using 3-10 $\mathrm{mg}$ of polymers per $\mathrm{mL}$ THF, and filtered using a $0.45 \mu \mathrm{m}$ filter. All samples were run with and without UVA (conc. $\approx$ $0.001 \mathrm{M}$ ) for comparison. All reactions were carried out under a nitrogen atmosphere using Schlenk techniques unless otherwise described. A Bruker-Nonius Kappa-CCD diffractometer at the University of Toronto was used to obtain the X-ray diffraction data for crystal structures. High resolution mass spectrometry experiments were carried out using an accuTOF DART-MS at the University of Toronto. Elemental analyses were performed by Atlantic Microlab of Norcross Georgia. 4-phenylphenol, $\mathrm{KOH}, \mathrm{KI}, \mathrm{K}_{2} \mathrm{CO}_{3}$, $\mathrm{AIBN}, \mathrm{Br}_{2}$, allyl bromide, $\mathrm{LiAlH}_{4}\left(1.0 \mathrm{M}\right.$ in $\mathrm{Et}_{2} \mathrm{O}$ ), $n$-BuLi (1.6 M in hexane), anhydrous $\mathrm{MgSO}_{4}$, anhydrous $\mathrm{CaCl}_{2}$ and Wilkinson's catalyst were purchased commercially form Sigma Aldrich and used without further purification. 1.6 M solution of $\mathrm{HCl}$ in $\mathrm{Et}_{2} \mathrm{O}$ was prepared in laboratory. Solvents were dried using $\mathrm{MBraun}$ solvent purification system or by standard procedures prior to use. 


\subsection{Synthesis of (allyloxy)benzene (194):}

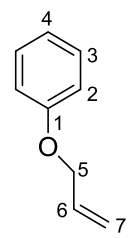

Allyl bromide (13.06 g, $108 \mathrm{mmol})$ and $\mathrm{K}_{2} \mathrm{CO}_{3}(15.0 \mathrm{~g}, 108 \mathrm{mmol})$ were added to a $250 \mathrm{~mL}$ dried two neck round bottom flask containing a solution of phenol $(10.0 \mathrm{~g}, 106 \mathrm{mmol})$ in $60 \mathrm{~mL}$ of acetone. The reaction mixture was refluxed for $16 \mathrm{~h}$ and cooled to room temperature. Removal of solvent under reduced pressure afforded a white solid. The residue was dissolved in $50 \mathrm{~mL}$ DCM and washed sequentially with $(15 \mathrm{~mL}) 1.0 \mathrm{M} \mathrm{NaOH}$, water and brine solution. The organic layer was dried over anhydrous $\mathrm{MgSO}_{4}$ and removal of solvent under reduced pressure afforded a highly viscous clear oil. NMR data $\left({ }^{1} \mathrm{H},{ }^{13} \mathrm{C}\right)$ obtained is essentially the same as reported in the literature. ${ }^{148,149}$ Yield: $9.1 \mathrm{~g}(82 \%)$.

${ }^{1} \mathbf{H}$ NMR (400 MHz, $\mathrm{CDCl}_{3}, \delta$ ): 7.36 (2H, H3), 7.0 (m, 3H, H2, H4), 6.13 (m, 1H, H6), 5.50 (dd, $\left.1 \mathrm{H}, \mathrm{H} 7, J_{\text {geminal }}=1.6 \mathrm{~Hz}, J=16 \mathrm{~Hz}\right), 5.35\left(\mathrm{dd}, 1 \mathrm{H}, \mathrm{H} 7, J_{\text {geminal }}=1.6 \mathrm{~Hz}, J=12 \mathrm{~Hz}\right), 4.60(\mathrm{~d}, 2 \mathrm{H}$, H5) ppm; ${ }^{13}$ C NMR (100 MHz, $\left.\mathrm{CDCl}_{3}, \delta\right): 158.6$ (C1), 133.4 (C), 129.4 (C), 120.8 (C), 117.6 (C), 114.7 (C), 68.7 (C5) ppm.

\subsection{Synthesis of 3-(4-biphenyloxy)-1-propene (137):}

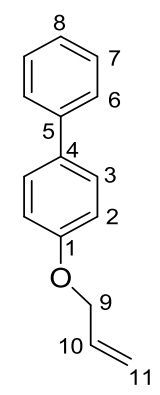


Allyl bromide ( $3.55 \mathrm{~g}, 29.4 \mathrm{mmol})$ and $\mathrm{K}_{2} \mathrm{CO}_{3}(5.1 \mathrm{~g}, 36.7 \mathrm{mmol})$ were added to a $250 \mathrm{~mL}$ dried two neck round bottom flask containing a solution of 4-phenylphenol $(5.0 \mathrm{~g}, 29.4 \mathrm{mmol})$ in 100 $\mathrm{mL}$ acetone. The reaction mixture was refluxed for $16 \mathrm{~h}$ and cooled to room temperature. Removal of solvent under reduced pressure afforded a white coloured solid. The residue was dissolved in $50 \mathrm{~mL}$ DCM, washed sequentially with $(15 \mathrm{~mL}) 1.0 \mathrm{M} \mathrm{NaOH}$, water and brine solution. The organic layer was dried over anhydrous $\mathrm{MgSO}_{4}$ and solvent removal under reduced pressure afforded a white solid coloured product. NMR data $\left({ }^{1} \mathrm{H},{ }^{13} \mathrm{C}\right)$ obtained is essentially the same as reported in the literature. ${ }^{43}$ Yield: $4.0 \mathrm{~g}(65 \%)$

${ }^{1} \mathbf{H}$ NMR (400 MHz, $\left.\mathrm{CDCl}_{3}, \delta\right): 7.63$ (m, 4H, H3, H6), 7.50 (t, 2H, H7), 7.39 (m, 1H, H8), 7.05 (d, 2H, H2), 6.17 (m, 1H, H10), 5.52 (d, 1H, cis-H11), 5.40 (d, 1H, trans-H11), 4.65 (d, 2H, H9) ppm; ${ }^{13}$ C NMR (100 MHz, $\left.\mathrm{CDCl}_{3}, \delta\right): 158.2$ (C1), 140.8 (C4), 133.9 (C5), 133.3 (C7), 128.7 (C6), 128.1 (C3), 126.7 (C8), 117.7 (C11), 115.6 (C10), 115.0 (C2), 69.2 (C9) ppm.

\subsection{Synthesis of of 1-allyloxy-3-trifluoromethylbenzene (195):}

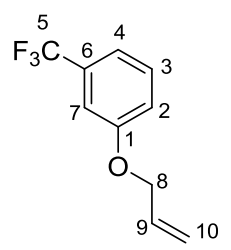

Allyl bromide (1.49 g, $12.3 \mathrm{mmol}), \mathrm{K}_{2} \mathrm{CO}_{3}(3.41 \mathrm{~g}, 24.7 \mathrm{mmol})$ was added to a solution of 3(trifluoromethyl)phenol $(2.00 \mathrm{~g}, 12.3 \mathrm{mmol})$ in $50 \mathrm{~mL}$ of acetone in a $250 \mathrm{~mL}$ round bottom flask and refluxed for $16 \mathrm{~h}$. The reaction mixture was then filtered and the solvent removed under reduced pressure. The residue was dissolved in $50 \mathrm{~mL}$ of DCM and washed sequentially with (15 mL) $1.0 \mathrm{M} \mathrm{NaOH}$, water and brine solution. The organic layer was dried over anhydrous $\mathrm{MgSO}_{4}$, and solvent removed under reduced pressure. The product was recovered as a yellow oil. NMR data $\left({ }^{1} \mathrm{H},{ }^{13} \mathrm{C},{ }^{19} \mathrm{~F}\right)$ is essentially the same as reported in the literature. ${ }^{174}$ Yield $=2.34 \mathrm{~g}(94 \%)$. 
${ }^{1} \mathrm{H}$ NMR $\left(400 \mathrm{MHz}, \mathrm{CDCl}_{3}, \delta\right): 7.40$ (t, 1H, H3), 7.22 (d, 1H, H4), 7.15 (br s, 1H, H7), 7.10 (dd, 1H, H2), 6.06 (m, 1H, H9), 5.44 (qd, 1H, cis-H10), 5.32 (qd , 1H, trans-H10), 4.59 (m, 2H, H8) ppm; ${ }^{13} \mathbf{C}$ NMR (100 MHz, $\mathrm{CDCl}_{3}, \delta$ ): 158.8 (s, C1), 132.7 (s, C9), 131.9 (q, C6, ${ }^{2} J_{13 \mathrm{C}-19 \mathrm{~F}}=30$ $\mathrm{Hz}), 130.0(\mathrm{~s}, \mathrm{C} 3), 124.1\left(\mathrm{q}, \mathrm{C} 5,{ }^{1} J_{13 \mathrm{C}-19 \mathrm{~F}}=272 \mathrm{~Hz}\right), 118.4\left(\mathrm{q}, \mathrm{C} 2,{ }^{4} J_{13 \mathrm{C}-19 \mathrm{~F}}=1.5 \mathrm{~Hz}\right), 118.3(\mathrm{~s}$, $\mathrm{C} 10), 117.6\left(\mathrm{q}, \mathrm{C} 7,{ }^{2} J_{13 \mathrm{C}-19 \mathrm{~F}}=4.0 \mathrm{~Hz}\right), 111.7\left(\mathrm{q}, \mathrm{C} 4,{ }^{2} J_{13 \mathrm{C}-19 \mathrm{~F}}=4.0 \mathrm{~Hz}\right), 69.2(\mathrm{~s}, \mathrm{C} 8), \mathrm{ppm} ;{ }^{19} \mathbf{F}$ NMR (376 MHz, $\left.\mathrm{CDCl}_{3}, \delta\right):-62.7 \mathrm{ppm}$.

\subsection{Synthesis of 1-(allyloxy)-4-methoxybenzene ether (196):}

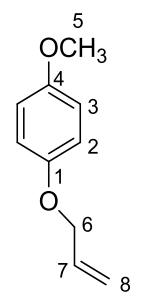

Allyl bromide (3.0 mL, $32.0 \mathrm{mmol})$ and $\mathrm{K}_{2} \mathrm{CO}_{3}(9.0 \mathrm{~g}, 64.0 \mathrm{mmol})$ were added to a solution of 4methoxyphenol $(4.0 \mathrm{~g}, 32.0 \mathrm{mmol})$ in $70 \mathrm{~mL}$ of acetone in a $250 \mathrm{~mL}$ round bottom flask and refluxed for $16 \mathrm{~h}$. After filtration, solvent was removed under reduced pressure. The residue was dissolved in $50 \mathrm{~mL}$ DCM and washed sequentially with $(15 \mathrm{~mL}) 1.0 \mathrm{M} \mathrm{NaOH}$, water and brine solution. The organic layer was dried over anhydrous $\mathrm{MgSO}_{4}$ and removal of solvent under reduced pressure afforded a white coloured solid. NMR data $\left({ }^{1} \mathrm{H},{ }^{13} \mathrm{C}\right)$ obtained is essentially the same as reported in literature. ${ }^{175}$ Yield $=4.7 \mathrm{~g}(89 \%)$.

${ }^{1} \mathbf{H}$ NMR (400 MHz, $\left.\mathrm{CDCl}_{3}, \delta\right): 6.82$ - 6.88 (m, 4H, H2, H3), 6.06 (m, 1H, H7), 5.41 (dq, 1H, cis$\left.\mathrm{H} 8,{ }^{1} J=17 \mathrm{~Hz}\right), 5.28\left(\mathrm{dq}, 1 \mathrm{H}\right.$, trans $\left.-\mathrm{H} 8,{ }^{1} J=10.5 \mathrm{~Hz},{ }^{2} J={ }^{3} J=1.5 \mathrm{~Hz}\right), 4.49\left(\mathrm{dd}, 2 \mathrm{H}, \mathrm{H} 6,{ }^{1} J=5.3\right.$ $\left.\mathrm{Hz},{ }^{2} J={ }^{3} J=1.5 \mathrm{~Hz}\right), 3.77$ (s, 3H, H5) ppm; ${ }^{13} \mathbf{C}$ NMR (100 MHz, $\left.\mathrm{CDCl}_{3}, \delta\right): 153.9(\mathrm{C} 4), 152.8$ (C1), 133.6 (C7), 117.5 (C8), 115.8 (C2), 114.6 (C3), 69.6 (C6), 55.7 (C5) ppm. 
5.5 Synthesis of triphenyltin hydride (254):

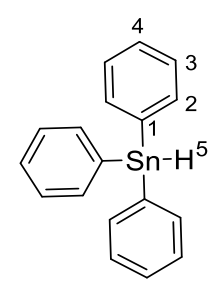

A solution $1.0 \mathrm{M} \mathrm{LiAlH}_{4}(11.0 \mathrm{~mL}, 11.0 \mathrm{mmol})$ in $20 \mathrm{~mL}$ of $\mathrm{Et}_{2} \mathrm{O}$ was added dropwise to a suspension of $\mathrm{Ph}_{3} \mathrm{SnCl}(10.51 \mathrm{~g}, 29.85 \mathrm{mmol})$ in $50 \mathrm{~mL}$ of $\mathrm{Et}_{2} \mathrm{O}$ at $0{ }^{\circ} \mathrm{C}$. The reaction mixture was stirred for $30 \mathrm{~min}$ at this temperature, allowed to warm to room temperature and stirred for an additional $2.5 \mathrm{~h}$. The reaction mixture was then placed in an ice bath and quenched with $25 \mathrm{~mL}$ of chilled degassed water. The organic layer was separated and the aqueous fraction extracted with $(3 \times 15 \mathrm{~mL}) \mathrm{Et}_{2} \mathrm{O}$. The collected organic layer was then dried over anhydrous $\mathrm{MgSO}_{4}$, filtered and the solvent removed under reduced pressure to yield a colourless viscous product. NMR data $\left({ }^{1} \mathrm{H}\right.$, $\left.{ }^{13} \mathrm{C}\right)$ obtained is essentially the same as reported in literature. ${ }^{42,64}$ Yield: $7.63 \mathrm{~g}(80 \%)$.

${ }^{1} \mathbf{H}$ NMR (400 MHz, C6 $\left.\mathrm{D}_{6}, \delta\right): 6.93\left(\mathrm{~s}, 1 \mathrm{H}, \mathrm{H} 5,{ }^{1} J_{119 \mathrm{Sn}-1 \mathrm{H}}=1935 \mathrm{~Hz},{ }^{1} J_{117 \mathrm{Sn}-1 \mathrm{H}}=1849 \mathrm{~Hz}\right), 7.13$ $(\mathrm{m}, 9 \mathrm{H}, \mathrm{H} 3, \mathrm{H} 4), 7.52\left(\mathrm{~m}, 6 \mathrm{H}, \mathrm{H} 2,{ }^{2} J_{119 \mathrm{Sn}-1 \mathrm{H}}=8.4 \mathrm{~Hz}\right) \mathrm{ppm} ;{ }^{13} \mathbf{C} \mathbf{N M R}\left(100 \mathrm{MHz}, \mathrm{C}_{6} \mathrm{D}_{6}, \delta\right): 137.6$ $\left(\mathrm{C} 2,{ }^{2} J_{119 \mathrm{Sn}-13 \mathrm{C}}=39 \mathrm{~Hz}\right), 137.3\left(\mathrm{C} 1,{ }^{1} J_{119 \mathrm{Sn}-13 \mathrm{C}}=535 \mathrm{~Hz},{ }^{1} J_{117 \mathrm{Sn}-13 \mathrm{C}}=511 \mathrm{~Hz}\right), 129.3\left(\mathrm{C} 4,{ }^{4} J_{119 \mathrm{Sn}-}\right.$ $\left.{ }_{13 \mathrm{C}}=11 \mathrm{~Hz}\right), 128.9\left(\mathrm{C} 3,{ }^{3} J_{119 \mathrm{Sn}-13 \mathrm{C}}=52 \mathrm{~Hz}\right) \operatorname{ppm} ;{ }^{119} \mathbf{S n}\left\{{ }^{1} \mathbf{H}\right\} \mathbf{N M R}\left(149 \mathrm{MHz}, \mathrm{C}_{6} \mathrm{D}_{6}, \delta\right):-163.1$ ppm.

\subsection{Synthesis of triphenyl[(3-phenyloxy)propyl]tin (197):}

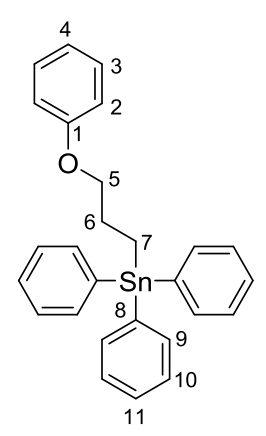


Compound 194 ( $2.0 \mathrm{~g}, 14.3 \mathrm{mmol})$ and $254(4.24 \mathrm{~g}, 7.45 \mathrm{mmol})$ were heated with AIBN (0.05 g, $0.913 \mathrm{mmol}$ ) in a dried $100 \mathrm{~mL}$ Schlenk flask at $140{ }^{\circ} \mathrm{C}$ for $1 \mathrm{~h}$. The reaction mixture was allowed to cool to room temperature, which resulted in formation of a white coloured gum. The distannane $\left(\mathrm{Ph}_{3} \mathrm{SnSnPh}_{3}\right)$ byproduct was precipitated from the mixture by adding hexane to the crude product and subsequently removed by filtration. Unreacted 194 was removed by heating under reduced pressure at $75^{\circ} \mathrm{C}$ which afforded a colourless, highly viscous oil of 197 . Yield: $5.68 \mathrm{~g} \mathrm{(79 \% )}$

${ }^{1} \mathbf{H}$ NMR (400 MHz, $\left.\mathrm{CDCl}_{3}, \delta\right): 7.64$ (m, 6H, H9), 7.43 (m, 9H, H10, H11), 7.31 (m, 2H, H3), 6.99 (t, 1H, H4), 6.88 (d, 2H, H2), 4.03 (t, 2H, H5), 2.29 (m, 2H, H6), $1.71(\mathrm{t}, 2 \mathrm{H}, \mathrm{H} 7)$ ppm; ${ }^{13} \mathbf{C}$ NMR $\left(100 \mathrm{MHz}, \mathrm{CDCl}_{3}, \delta\right): 159.0(\mathrm{C} 1), 138.7\left(\mathrm{C} 8,{ }^{1} J_{119 \mathrm{Sn}-13 \mathrm{C}}=488 \mathrm{~Hz},{ }^{1} J_{117 \mathrm{Sn}-13 \mathrm{C}}=468 \mathrm{~Hz}\right), 137.0(\mathrm{C} 9$, $\left.{ }^{2} J_{119 \mathrm{Sn}-13 \mathrm{C}}=36 \mathrm{~Hz}\right), 129.4(\mathrm{C} 3), 128.9\left(\mathrm{C} 11,{ }^{3} J_{119 \mathrm{Sn}-13 \mathrm{C}}=11 \mathrm{~Hz}\right), 128.5\left(\mathrm{C} 10,{ }^{3} J_{119 \mathrm{Sn}-13 \mathrm{C}}=49 \mathrm{~Hz}\right)$, $120.6(\mathrm{C} 4), 114.6(\mathrm{C} 2), 70.5(\mathrm{C} 5), 26.4\left(\mathrm{C} 6,{ }^{2} J_{119 \mathrm{Sn}-13 \mathrm{C}}=19 \mathrm{~Hz}\right), 6.93\left(\mathrm{C} 7,{ }^{1} J_{119 \mathrm{Sn}-13 \mathrm{C}}=388 \mathrm{~Hz}\right.$, $\left.{ }^{1} J_{117 \mathrm{Sn}-13 \mathrm{C}}=372 \mathrm{~Hz}\right) \mathrm{ppm} ;{ }^{119} \mathbf{S n}\left\{{ }^{1} \mathbf{H}\right\} \mathbf{N M R}\left(149 \mathrm{MHz}, \mathrm{C}_{6} \mathrm{D}_{6}, \delta\right):-99.9 \mathrm{ppm}$. Found: C, 66.64; H, 5.47. Calc. for $\mathrm{C}_{27} \mathrm{H}_{26} \mathrm{OSn}: \mathrm{C}, 66.84, \mathrm{H}, 5.40 \%$.

\subsection{Synthesis of triphenyl[3-(4-biphenyloxy)propyl]tin (141):}

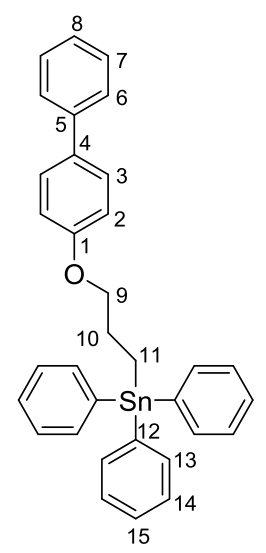

Compound 137 ( $2.78 \mathrm{~g}, 13.22 \mathrm{mmol})$ and $254(4.64 \mathrm{~g}, 13.18 \mathrm{mmol})$ were heated with AIBN (0.16 g, $0.973 \mathrm{mmol})$ in a dried $100 \mathrm{~mL}$ Schlenk flask at $140{ }^{\circ} \mathrm{C}$ under inert atmosphere. The reaction mixture was allowed to cool to room temperature. The distannane $\left(\mathrm{Ph}_{3} \mathrm{SnSnPh}_{3}\right)$ produced as a 
side-product and unreacted 137 were separated from 141 by sublimation $\left(130{ }^{\circ} \mathrm{C}\right.$ for $\left.4 \mathrm{~h}\right)$. The product (a sticky semi-solid) was further purified to remove the trace distannane $\left(\mathrm{Ph}_{3} \mathrm{SnSnPh}_{3}\right)$ by dissolving in $\mathrm{Et}_{2} \mathrm{O}$ followed by filtration. The removal of residual solvent under reduced pressure afforded a white coloured powder. NMR data $\left({ }^{1} \mathrm{H},{ }^{13} \mathrm{C},{ }^{119} \mathrm{Sn}\right)$ obtained is essentially the same as previously reported by Molloy et al. ${ }^{43}$ Yield: $5.1 \mathrm{~g}(70 \%)$.

${ }^{1} \mathbf{H}$ NMR (400 MHz, $\left.\mathrm{CDCl}_{3}, \delta\right): 7.55$ (m, 6H, H13), 7.46 (m, 2H, H6), 7.40 (m, 3H, H7, H8), 7.37 (m, 9H, H14, H15), 7.30 (d, 2H, H3), 6.85 (dd, 2H, H2), 4.02 (t, 2H, H9), 2.26 (m, 2H, H10), 1.67

(t, 2H, H11) ppm; ${ }^{13} \mathrm{C}$ NMR (100 MHz, $\left.\mathrm{CDCl}_{3}, \delta\right): 158.6$ (C1), 141.0 (C4), 138.8 (C12), 137.4 (C8), 137.2 (C13), 133.2 (C-Ar), 129.0 (C-Ar), 128.8 (C-Ar), 128.7 (C-Ar), 128.2 (C-Ar), 126.9 (C-Ar), 114.9 (C-Ar), 70.8 (C9), 26.2 (C10), 7.06 (C11) ppm; ${ }^{119}$ Sn\{ $\left.{ }^{1} \mathbf{H}\right\} \mathbf{N M R}(149.21 \mathrm{MHz}$ $\left.\mathrm{CDCl}_{3}, \delta\right):-99.3 \mathrm{ppm}$.

\subsection{Synthesis of triphenyl [3-(3-trifluromethylphenyloxy)propyl]tin (198):}

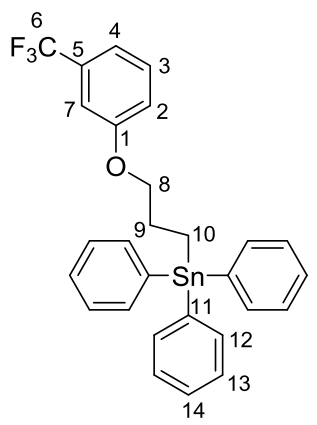

Compound 195 (1.0 g, $4.95 \mathrm{mmol}), 254$ (1.69 g, $4.83 \mathrm{mmol})$ and AIBN (0.055 g, $0.99 \mathrm{mmol})$ were heated at $120^{\circ} \mathrm{C}$ for $1 \mathrm{~h}$ in a $50 \mathrm{~mL}$ Schlenk flask. The crude product was washed with $2 \times 5 \mathrm{~mL}$ of $\mathrm{MeOH}$ to remove unreacted 195. The residue was then dissolved in $\mathrm{MeOH}$ and after decanting the solvent removed under reduced pressure. The product 198 was recovered as a white coloured powder and recrystallized in MeOH:DCM. Yield: $2.13 \mathrm{~g}(79 \%)$ m.p. $60-65^{\circ} \mathrm{C}$. 
${ }^{1} \mathbf{H}$ NMR $\left(400 \mathrm{MHz}, \mathrm{CDCl}_{3}, \delta\right):$ 7.52-7.56 (m, 6H, H12), 7.33-7.37 (m, 9H, H13, H14), 7.30 (t, $\left.J_{\mathrm{H} 9-\mathrm{H} 8}=8.0 \mathrm{~Hz}, 1 \mathrm{H}, \mathrm{H} 3\right), 7.15(\mathrm{~m}, 1 \mathrm{H}, \mathrm{H} 4), 6.98(\mathrm{~s}, 1 \mathrm{H}, \mathrm{H} 7) ; 6.90\left(\mathrm{dd}, 1 \mathrm{H}, \mathrm{H} 2, J_{\mathrm{H} 10-\mathrm{H} 9}=7.6 \mathrm{~Hz}\right.$, $\left.J_{\mathrm{H} 10-\mathrm{H} 8}=2.4 \mathrm{~Hz}\right), 3.96\left(\mathrm{t}, 2 \mathrm{H}, \mathrm{H} 8, J_{\mathrm{H} 6-\mathrm{H} 5}=6.4 \mathrm{~Hz}\right), 2.22(\mathrm{tt}, 2 \mathrm{H}, \mathrm{H} 9), 1.63\left(\mathrm{t}, 2 \mathrm{H}, \mathrm{H} 10, J_{\mathrm{H} 4-\mathrm{H} 5}=8.0\right.$ $\left.\mathrm{Hz}, J_{\mathrm{H} 4-\mathrm{Sn}}=57 \mathrm{~Hz}\right) \mathrm{ppm} ;{ }^{13} \mathrm{C} \mathbf{N M R}\left(100 \mathrm{MHz}, \mathrm{CDCl}_{3}, \delta\right): 159.1(\mathrm{C} 1), 138.7(\mathrm{C} 11), 137.7(\mathrm{C}-\mathrm{Ar})$, $137.4(\mathrm{C}-\mathrm{Ar}), 137.1\left(\mathrm{C} 13,{ }^{3} J_{119 \mathrm{Sn}-13 \mathrm{C}}=36.2\right), 129.9(\mathrm{C}-\mathrm{Ar}), 129.1\left(\mathrm{C} 14,{ }^{4} J_{119 \mathrm{Sn}-13 \mathrm{C}}=11 \mathrm{~Hz}\right), 128.7$ $\left(\mathrm{C} 12,{ }^{2} J_{119 \mathrm{Sn}-13 \mathrm{C}}=50 \mathrm{~Hz}\right), 118.1\left(\mathrm{q}, \mathrm{C} 6, J_{13 \mathrm{C}-19 \mathrm{~F}}=1.5 \mathrm{~Hz}\right), 117.3\left(\mathrm{~d}, \mathrm{C} 4, J_{13 \mathrm{C}-19 \mathrm{~F}}=4.0 \mathrm{~Hz}\right), 111.4$ $\left(\mathrm{d}, \mathrm{C} 2, J_{13 \mathrm{C}-19 \mathrm{~F}}=4.0 \mathrm{~Hz}\right), 70.8(\mathrm{C} 8), 26.3(\mathrm{C} 9), 7.06(\mathrm{C} 10) \mathrm{ppm} ;{ }^{19} \mathbf{F} \mathbf{~ N M R}\left(376 \mathrm{MHz}, \mathrm{CDCl}_{3}, \delta\right)$ : $-62.7\left(\mathrm{~s}, \mathrm{CF}_{3}\right) \mathrm{ppm} ;{ }^{119} \mathrm{Sn}\left\{{ }^{1} \mathbf{H}\right\} \mathbf{N M R}\left(149.21 \mathrm{MHz}, \mathrm{CDCl}_{3}, \delta\right):-100.0 \mathrm{ppm}$. Found: C, 60.38, H, 4.55. Calc. for $\mathrm{C}_{27} \mathrm{H}_{26} \mathrm{OSn}: \mathrm{C}, 60.79, \mathrm{H}, 4.56 \%$.

\subsection{Synthesis of triphenyl [3-(4-methoxyphenyloxy)propyl]tin (199):}

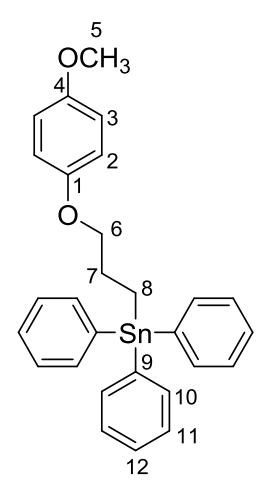

Compound 196 (0.50 g, $3.05 \mathrm{mmol}), 254(1.03 \mathrm{~g}, 2.93 \mathrm{mmol})$ and $\operatorname{AIBN}(0.02 \mathrm{~g}, 0.36 \mathrm{mmol})$ were heated at $120{ }^{\circ} \mathrm{C}$ in a sealed Schlenk flask for $1 \mathrm{~h}$ under dynamic $\mathrm{N}_{2}$. The crude product was sublimed to remove the $\mathrm{Ph}_{3} \mathrm{SnSnPh}_{3}$ side-product and further purified by silica gel column chromatograph using hexane:EtOAc (6:1). Yield $0.98 \mathrm{~g}(65 \%)$.

${ }^{1} \mathbf{H}$ NMR (400 MHz, $\left.\mathrm{CDCl}_{3}, \delta\right):$ 7.49-7.61 (m, 6H, H12), 7.36-7.38 (m, 9H, H13, H14), 6.71-80 (m, 4H, H2, H3), 3.90 (t, 2H, H8, $\left.{ }^{1} J=6.2 \mathrm{~Hz}\right), 3.76$ (s, 3H, H5), 2.18 (tt, 2H, H9), 1.64 (t, 2H, $\mathrm{H} 10, J=8.0 \mathrm{~Hz}) \mathrm{ppm} ;{ }^{13} \mathbf{C}$ NMR $\left(100 \mathrm{MHz}, \mathrm{CDCl}_{3}, \delta\right): 153.9(\mathrm{C} 4), 153.2(\mathrm{C} 1), 138.9\left(\mathrm{C} 9, J_{119 \mathrm{Sn}-}\right.$ 
$\left.13 \mathrm{C}=490 \mathrm{~Hz} J_{117 \mathrm{Sn}-13 \mathrm{C}}=468 \mathrm{~Hz}\right), 137.2\left(\mathrm{C} 11, J_{119 \mathrm{Sn}-13 \mathrm{C}}=35 \mathrm{~Hz}\right), 129.0\left(\mathrm{C} 12, J_{119 \mathrm{Sn}-13 \mathrm{C}}=11 \mathrm{~Hz}\right)$,

$128.7\left(\mathrm{C} 10, J_{119 \mathrm{Sn}-13 \mathrm{C}}=48 \mathrm{~Hz}\right) 115.6(\mathrm{C} 2), 114.7(\mathrm{C} 3), 71.4\left(\mathrm{C} 6,{ }^{3} J_{119 \mathrm{Sn}-13 \mathrm{C}}=68 \mathrm{~Hz}\right), 55.9(\mathrm{C} 5)$,

$26.5\left(\mathrm{C} 7,{ }^{2} J_{119 \mathrm{Sn}-13 \mathrm{C}}=20 \mathrm{~Hz}\right), 7.09\left(\mathrm{C} 8,{ }^{1} J_{119 \mathrm{Sn}-13 \mathrm{C}}=392 \mathrm{~Hz},{ }^{1} J_{117 \mathrm{Sn}-13 \mathrm{C}}=375 \mathrm{~Hz}\right) \mathrm{ppm}$; ${ }^{119} \mathbf{S n}\left\{{ }^{1} \mathbf{H}\right\} \mathbf{N M R}\left(149.21 \mathrm{MHz}, \mathrm{CDCl}_{3}, \delta\right):-99.3 \mathrm{ppm}$.

\subsection{Synthesis of diphenyl[(3-phenyloxy)propyl]tin chloride (200):}

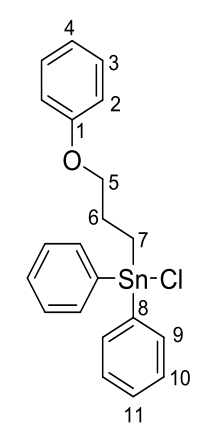

$1.0 \mathrm{M}$ solution of $\mathrm{HCl}(4.5 \mathrm{~mL}, 4.5 \mathrm{mmol})$ in $\mathrm{Et}_{2} \mathrm{O}$ was added dropwise to a solution of $\mathbf{1 9 7}$ (2.06 g, $4.24 \mathrm{mmol}$ ) dissolved in $10 \mathrm{~mL}$ of dry $\mathrm{C}_{6} \mathrm{H}_{6}$ and stirred for $1 \mathrm{~h}$. The removal of solvent under reduced pressure afforded an off-white coloured solid. The crude product was first extracted with hot hexane and filtered. The hexane solution containing the product was kept at $-20^{\circ} \mathrm{C}$ overnight, and a white coloured crystalline product recovered. The hexane was decanted and residual solvent was removed under reduced pressure. The product was recrystallized in DCM:hexane (1:1). Yield: $1.52 \mathrm{~g}(81 \%)$, m.p. $63^{\circ} \mathrm{C}$.

${ }^{1}$ H NMR (400 MHz, CDCl 3 , $)$ ): 7.64 (m, 4H, H9), 7.35 (m, 6H, H10, H11), 7.07 (m, 2H, H3), 6.88 (tt, 1H, H4), 6.53 (dd, 2H, H2), 4.07 (t, 2H, H5), 2.40 (m, 2H, H6), 1.93 (t, 2H, H7) ppm; ${ }^{13} \mathbf{C}$ NMR $\left(100 \mathrm{MHz}, \mathrm{CDCl}_{3}, \delta\right): 157.7(\mathrm{C} 1), 139.7\left(\mathrm{C} 8,{ }^{1} J_{119 \mathrm{Sn}-13 \mathrm{C}}=592 \mathrm{~Hz},{ }^{1} J_{117 \mathrm{Sn}-13 \mathrm{C}}=569 \mathrm{~Hz}\right)$, $136.1\left(\mathrm{C} 10, J_{119 \mathrm{Sn}-13 \mathrm{C}}=47 \mathrm{~Hz}\right), 129.8\left(\mathrm{C} 11, J_{119 \mathrm{Sn}-13 \mathrm{C}}=13 \mathrm{~Hz}\right), 129.2(\mathrm{C} 3), 128.8\left(\mathrm{C} 9, J_{119 \mathrm{Sn}-13 \mathrm{C}}=\right.$ $61 \mathrm{~Hz}), 121.7(\mathrm{C} 4), 115.3(\mathrm{C} 2), 70.2(\mathrm{C} 5), 25.8\left(\mathrm{C} 6,{ }^{2} J_{119 \mathrm{Sn}-13 \mathrm{C}}=27 \mathrm{~Hz}\right), 15.0\left(\mathrm{C} 7,{ }^{1} J_{119 \mathrm{Sn}-13 \mathrm{C}}=\right.$ $460 \mathrm{~Hz})$ ppm; ${ }^{119} \mathbf{S n}\left\{{ }^{1} \mathbf{H}\right\} \mathbf{N M R}\left(149.21 \mathrm{MHz}, \mathrm{CDCl}_{3}, \delta\right):-26.6 \mathrm{ppm}$. HRMS-DART (m/z): $\left[\mathrm{M}^{+}\right]+$ 
$\mathrm{H}_{2} \mathrm{O}$, Calc. for $\mathrm{C}_{21} \mathrm{H}_{23} \mathrm{ClO}_{2} \mathrm{Sn}, 462.06637$; found 462.06575. Found: C, 56.79, H, 4.78. Calc. for $\mathrm{C}_{21} \mathrm{H}_{21} \mathrm{ClOSn}: \mathrm{C}, 56.87, \mathrm{H}, 4.77 \%$.

\subsection{Synthesis of diphenyl[3-(4-biphenyloxy)propyl]tin chloride (201):}

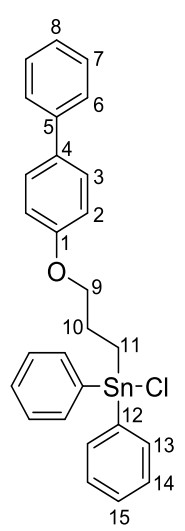

1.0 M solution of $\mathrm{HCl}(1.0 \mathrm{~mL}, 1.0 \mathrm{mmol})$ in $\mathrm{Et}_{2} \mathrm{O}$ was added dropwise to a solution of compound $141(0.561 \mathrm{~g}, 1.0 \mathrm{mmol})$ dissolved in $5 \mathrm{~mL}$ of dry $\mathrm{C}_{6} \mathrm{H}_{6}$ and stirred for $1 \mathrm{~h}$. The removal of solvent under reduced pressure afforded white coloured solid. The crude product was purified by washing with hot hexanes. The removal of solvent under reduced afforded a white coloured solid. Yield: $0.45 \mathrm{~g}(86 \%)$, m.p. $82^{\circ} \mathrm{C}$.

${ }^{1}$ H NMR (400 MHz, $\mathrm{CDCl}_{3}, \delta$ ): 7.66 (m, 4H, H13), 7.46 (m, 2H, H6), 7.40 (dd, 2H, H3), 7.35 (m, 6H, H13, H14), 7.29 (m, 3H, H7, H8), 6.58 (dd, 2H, H2), 4.11 (t, 2H, H9), 2.42 (m, 2H, H10), 1.94 (t, 2H, H11) ppm; ${ }^{13} \mathrm{C}$ NMR (100 MHz, $\left.\mathrm{CDCl}_{3}, \delta\right): 157.3$ (C, $i$-Ar), 140.5 (C- i-Ar), 139.6 $(\mathrm{C}-i$-Ar $), 136.1\left(\mathrm{C} 14,{ }^{3} J_{119 \mathrm{Sn}-13 \mathrm{C}}=47 \mathrm{~Hz}\right), 134.6(\mathrm{C}-i$-Ar $), 129.8\left(\mathrm{C} 15,{ }^{4} J_{119 \mathrm{Sn}-13 \mathrm{C}}=13 \mathrm{~Hz}\right), 128.8$ $\left(\mathrm{C} 13,{ }^{2} J_{119 \mathrm{Sn}-13 \mathrm{C}}=62 \mathrm{~Hz}\right), 128.7(\mathrm{C}-\mathrm{Ar}), 127.9(\mathrm{C}-\mathrm{Ar}), 126.8(\mathrm{C}-\mathrm{Ar}), 126.7(\mathrm{C}-\mathrm{Ar}), 115.5(\mathrm{C} 2)$, 70.4 (C9), $25.8\left(\mathrm{C} 10,{ }^{2} J_{119 \mathrm{Sn}-13 \mathrm{C}}=26 \mathrm{~Hz}\right), 15.0(\mathrm{C} 11) \mathrm{ppm} ;{ }^{119} \mathbf{S n}\left\{{ }^{1} \mathbf{H}\right\} \mathbf{N M R}\left(149.21 \mathrm{MHz}, \mathrm{CDCl}_{3}\right.$,

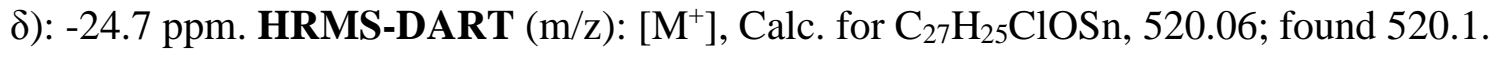




\subsection{Synthesis of phenyl[3-(4-biphenyloxy)propyl]tin dibromide (146):}

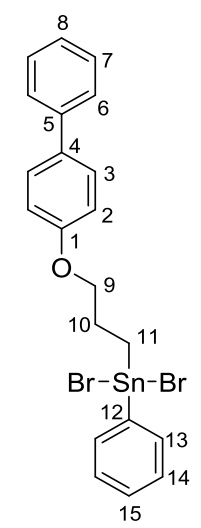

A solution of $\mathrm{Br}_{2}(2.6 \mathrm{~g}, 32.54 \mathrm{mmol})$ in $80 \mathrm{~mL}$ of DCM was added dropwise to a solution of $\mathbf{1 4 1}$ (4.54 g, $8.08 \mathrm{mmol}$ ) in $80 \mathrm{~mL}$ of $\mathrm{DCM}$ at $0{ }^{\circ} \mathrm{C}$. The resulting solution was stirred for $24 \mathrm{~h}$. The solvent was removed under reduced pressure. The crude product mixture was kept under reduced pressure at $50{ }^{\circ} \mathrm{C}$ for $12 \mathrm{~h}$ to remove the $\mathrm{C}_{6} \mathrm{H}_{5} \mathrm{Br}$ byproduct from 146. A white coloured solid was recovered. NMR data $\left({ }^{1} \mathrm{H},{ }^{13} \mathrm{C},{ }^{119} \mathrm{Sn}\right)$ obtained is essentially the same as previously reported in literature. ${ }^{43}$ Yield: $3.71 \mathrm{~g}(81 \%)$.

${ }^{1} \mathbf{H}$ NMR (400 MHz, $\left.\mathrm{CDCl}_{3}, \delta\right): 7.66$ (m, 2H, H13), 7.50 (m, 3H, H14, H15), 7.41 (m, 2H, H7), 7.35-7.39 (m, 4H, H3, H6), 7.31 (m, 1H, H8), 6.73 (d, 2H, H2), 4.16 (t, 2H, H9), 2.46 (m, 2H, H10), 2.29 (m, 2H, H11) ppm; ${ }^{13}$ C NMR (100 MHz, $\mathrm{CDCl}_{3}, \delta$ ): 157.0 (C- $i$-Ar), 140.5 (C- $i$-Ar), 140.0 (C- $i$-Ar), 134.8 (C- Ar), 134.7 (C- Ar), 130.9 (C- Ar), 129.1 (C- Ar), 128.8 (C- Ar), 127.9 (C- Ar), 126.8 (C- Ar), 126.7 (C- Ar), 116.3 (C2), 70.0 (C9), 25.7 (C10), 23.8 (C11) ppm; ${ }^{119} \mathrm{Sn}\left\{{ }^{1} \mathbf{H}\right\} \mathbf{N M R}\left(149.21 \mathrm{MHz}, \mathrm{CDCl}_{3}, \delta\right):-51.6 \mathrm{ppm}$. 


\subsection{Synthesis of phenyl[(3-phenyloxy)propyl]tin dichloride (202):}

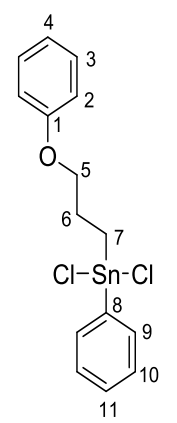

A $1.0 \mathrm{M}$ solution of $\mathrm{HCl}(5.0 \mathrm{~mL}, 5.0 \mathrm{mmol})$ in $\mathrm{Et}_{2} \mathrm{O}$ was added dropwise to a solution of 200 (2.13 g, $4.8 \mathrm{mmol}$ dissolved in $10 \mathrm{~mL}$ of dry $\mathrm{C}_{6} \mathrm{H}_{6}$ ) and stirred for $1 \mathrm{~h}$. The removal of solvent under reduced pressure afforded an off-white coloured solid. ${ }^{1} \mathrm{H}$ NMR $\left(\mathrm{CDCl}_{3}\right)$ revealed a $60 \%$ conversion of $\mathbf{2 0 0}$ to $\mathbf{2 0 2}$. The crude product was re-dissolved in $\mathrm{C}_{6} \mathrm{H}_{6}$ and an additional aliquot $(1.6 \mathrm{~mL})$ of $1.0 \mathrm{M} \mathrm{HCl}$ added. The reaction mixture was stirred for $1 \mathrm{~h}$, and removal of solvent under reduced pressure afforded a white coloured solid. Yield: $1.21 \mathrm{~g}(91 \%)$ m.p. $70{ }^{\circ} \mathrm{C}$.

${ }^{1}$ H NMR (400 MHz, $\mathrm{CDCl}_{3}, \delta$ ): 7.62 (dd, 2H, H9), 7.38 (m, 3H, H10, H11), 7.15 (tt, 2H, H3), 6.95 (dt, 1H, H4), 6.70 (dd, 2H, H2), 4.15 (t, 2H, H5), 2.49 (m, 2H, H6), 2.17 (t, 2H, H7) ppm; ${ }^{13} \mathbf{C}$ NMR (100 MHz, $\left.\mathrm{CDCl}_{3}, \delta\right): 157.2(\mathrm{C} 2), 140.2(\mathrm{C} 8), 134.9\left(\mathrm{C} 10,{ }^{3} J_{119 \mathrm{Sn}-13 \mathrm{C}}=62 \mathrm{~Hz}\right), 130.9(\mathrm{C} 11$, $\left.{ }^{4} J_{119 \mathrm{Sn}-13 \mathrm{C}}=17 \mathrm{~Hz}\right), 129.3(\mathrm{C} 3), 129.1\left(\mathrm{C} 9,{ }^{2} J_{119 \mathrm{Sn}-13 \mathrm{C}}=81 \mathrm{~Hz}\right), 122.4(\mathrm{C} 4), 116.1(\mathrm{C} 2), 69.8(\mathrm{C} 5)$, 25.2 (C6), 22.4 (C7) ppm; ${ }^{119} \mathbf{S n}\left\{{ }^{1} \mathbf{H}\right\} \mathbf{N M R}\left(149 \mathrm{MHz}, \mathrm{CDCl}_{3}, \delta\right):-21.9$ ppm. HRMS-DART (m/z): $\left[\mathrm{M}^{+}\right]+\mathrm{H}_{2} \mathrm{O}$, Calc. for $\mathrm{C}_{15} \mathrm{H}_{18} \mathrm{Cl}_{2} \mathrm{O}_{2} \mathrm{Sn}$, 419.99439; found 419.99440. Found: C, 45.01, $\mathrm{H}$, 4.14. Calc. for $\mathrm{C}_{27} \mathrm{H}_{26} \mathrm{OSn}$ : C, 44.83, H, $4.01 \%$. 
5.14 Synthesis of phenyl[3-(4-biphenyloxy)propyl]tin dichloride (203):

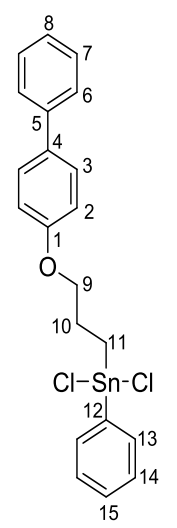

A $1.0 \mathrm{M}$ solution of $\mathrm{HCl}(3.1 \mathrm{~mL}, 3.1 \mathrm{mmol})$ in $\mathrm{Et}_{2} \mathrm{O}$ was added dropwise to a solution of 201 (1.50 g, $3.1 \mathrm{mmol}$ ) dissolved in $10 \mathrm{~mL}$ of dry $\mathrm{C}_{6} \mathrm{H}_{6}$ and stirred for $1 \mathrm{~h}$. The removal of solvent under reduced pressure afforded an off-white solid. ${ }^{1} \mathrm{H} \mathrm{NMR}\left(\mathrm{CDCl}_{3}\right)$ revealed a $95 \%$ conversion of 201 to 203. The crude mixture was re-dissolved in $\mathrm{C}_{6} \mathrm{H}_{6}$ and an additional aliquot $(0.2 \mathrm{~mL})$ of 1.0 $\mathrm{M} \mathrm{HCl}$ added, and the reaction mixture stirred for $1 \mathrm{~h}$. Removal of solvent under reduced pressure afforded a white coloured product. Yield: $1.38 \mathrm{~g}(100 \%)$, m.p. $75^{\circ} \mathrm{C}$.

${ }^{1} \mathbf{H}$ NMR (400 MHz, $\mathrm{CDCl}_{3}, \delta$ ): 7.63 (m, 2H, H13), 7.47 (m, 2H, H14), 7.36 (m, 7H, H3, H6, H7, H8), 7.30 (m, 1H, H15), 6.75 (dd, 2H, H2), 4.17 (t, 2H, H9), 2.48 (m, 2H, H10), 2.17 (t, 2H, H11) ppm; ${ }^{13}$ C NMR (100 MHz, $\left.\mathrm{CDCl}_{3}, \delta\right): 156.8$ (C- $i$-Ar), 140.4 (C- $i$-Ar), 140.3 (C, $i$-Ar), 135.0 $\left(\mathrm{C} 12,{ }^{1} J_{119 \mathrm{Sn}-13 \mathrm{C}}=40 \mathrm{~Hz}\right), 131.0(\mathrm{C}-\mathrm{Ar}), 129.2(\mathrm{C}-\mathrm{Ar}), 128.8(\mathrm{C}-\mathrm{Ar}), 128.4(\mathrm{C}-\mathrm{Ar}), 128.0(\mathrm{C}-$ Ar), 127.0 (C- Ar), 126.8 (C- Ar), 116.3 (C2), 70.0 (C9), 22.5 (C10), 25.2 (C11) ppm; ${ }^{119} \mathrm{Sn}\left\{{ }^{1} \mathbf{H}\right\} \mathbf{N M R}\left(149 \mathrm{MHz}, \mathrm{CDCl}_{3}, \delta\right):-20.3 \mathrm{ppm}$. Found: C, 52.95, H, 4.38. Calc. for $\mathrm{C}_{21} \mathrm{H}_{20} \mathrm{C}_{12} \mathrm{OSn}: \mathrm{C}, 52.77, \mathrm{H}, 4.22 \%$. 


\subsection{Synthesis of diphenyl[(3-phenyloxy)propyl]tin (204):}

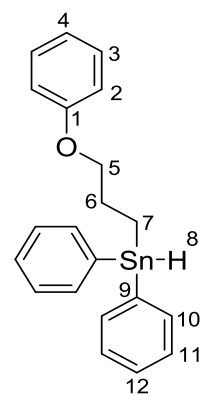

A solution of $200(0.334 \mathrm{~g}, 0.75 \mathrm{mmol})$ in $15 \mathrm{~mL}$ of $\mathrm{Et}_{2} \mathrm{O}$ was added dropwise to a Schlenk flask containing $1.0 \mathrm{M} \mathrm{LiAlH}_{4}(0.8 \mathrm{~mL}, 0.8 \mathrm{mmol})$ in $10 \mathrm{~mL}$ of $\mathrm{Et}_{2} \mathrm{O}$ over $30 \mathrm{~min}$ at $0{ }^{\circ} \mathrm{C}$. The resulting solution was allowed to stir for $3 \mathrm{~h}$ at $0{ }^{\circ} \mathrm{C}$ and the reaction mixture was then quenched with 15 $\mathrm{mL}$ of chilled degassed water. The organic layer was washed with water $(2 \times 15 \mathrm{~mL})$ and dried over anhydrous $\mathrm{MgSO}_{4}$. The dried organic layer was filtered and the solvent removed under reduced pressure to yield a colourless viscous oil. Yield: $0.22 \mathrm{~g}(70 \%)$.

${ }^{1} \mathbf{H}$ NMR $\left(400 \mathrm{MHz}, \mathrm{C}_{6} \mathrm{D}_{6}, \delta\right): 7.49\left(\mathrm{~m}, 4 \mathrm{H}, \mathrm{H} 10,{ }^{1} J_{119 \mathrm{Sn}-1 \mathrm{H}}=48 \mathrm{~Hz}\right), 7.14(\mathrm{~m}, 8 \mathrm{H}, \mathrm{H} 3, \mathrm{H} 11, \mathrm{H} 12)$, $6.81(3 \mathrm{H}, \mathrm{H} 2, \mathrm{H} 4), 6.35\left(\mathrm{t}, 1 \mathrm{H}, \mathrm{H} 8,{ }^{1} J_{119 \mathrm{Sn}-1 \mathrm{H}}=1862 \mathrm{~Hz},{ }^{1} J_{117 \mathrm{Sn}-1 \mathrm{H}}=1780 \mathrm{~Hz}\right), 3.56(\mathrm{t}, 2 \mathrm{H}, \mathrm{H} 5)$, $1.92\left(\mathrm{~m}, 2 \mathrm{H}, \mathrm{H} 6,{ }^{1} J_{119 \mathrm{Sn}-1 \mathrm{H}}=64 \mathrm{~Hz}\right), 1.23\left(\mathrm{dt}, 2 \mathrm{H}, \mathrm{H} 7,{ }^{1} J_{119 \mathrm{Sn}-1 \mathrm{H}}=56 \mathrm{~Hz}\right), \mathrm{ppm} ;{ }^{13} \mathbf{C} \mathbf{~ N M R}(100$ $\left.\mathrm{MHz}, \mathrm{C}_{6} \mathrm{D}_{6}, \delta\right): 159.6(\mathrm{C} 1), 138.3\left(\mathrm{C} 9,{ }^{1} J_{119 \mathrm{Sn}-13 \mathrm{C}}=494 \mathrm{~Hz},{ }^{1} J_{117 \mathrm{Sn}-13 \mathrm{C}}=472 \mathrm{~Hz}\right), 137.5(\mathrm{C} 10$, $\left.{ }^{2} J_{119 \mathrm{Sn}-13 \mathrm{C}}=36 \mathrm{~Hz}\right), 129.7\left(\mathrm{C} 11, J_{119 \mathrm{Sn}-13 \mathrm{C}}=29 \mathrm{~Hz}\right), 129.1\left(\mathrm{C} 12, J_{119 \mathrm{Sn}-13 \mathrm{C}}=11 \mathrm{~Hz}\right), 128.9(\mathrm{C} 3)$, $120.9(\mathrm{C} 4), 114.9(\mathrm{C} 2), 70.10\left(\mathrm{C} 5,{ }^{3} J_{119 \mathrm{Sn}-13 \mathrm{C}}=60 \mathrm{~Hz}\right), 26.9\left(\mathrm{C} 6,{ }^{2} J_{119 \mathrm{Sn}-13 \mathrm{C}}=21 \mathrm{~Hz}\right), 6.67(\mathrm{C} 7$, $\left.{ }^{1} J_{119 \mathrm{Sn}-13 \mathrm{C}}=396 \mathrm{~Hz},{ }^{1} J_{117 \mathrm{Sn}-13 \mathrm{C}}=378 \mathrm{~Hz}\right) \mathrm{ppm} ;{ }^{119} \mathbf{S n}\left\{{ }^{1} \mathbf{H}\right\} \mathbf{N M R}\left(149 \mathrm{MHz}, \mathrm{C}_{6} \mathrm{D}_{6}, \delta\right):-137.1 \mathrm{ppm}$. HRMS-DART (m/z): Calcd for $\mathrm{C}_{21} \mathrm{H}_{21} \mathrm{OSn}\left[\mathrm{M}^{+}\right]-\mathrm{H}, 409.06025$; found 409.06010. 


\subsection{Synthesis of diphenyl[3-(4-biphenyloxy)propyl]tin (205):}

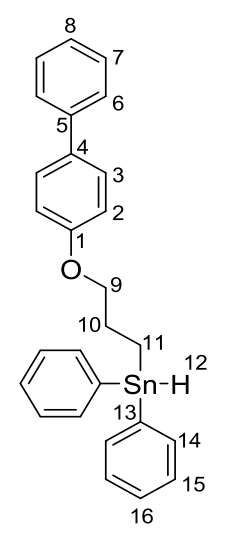

A solution of $201(0.885 \mathrm{~g}, 1.7 \mathrm{mmol})$ in $15 \mathrm{~mL}^{\text {of }} \mathrm{Et}_{2} \mathrm{O}$ was added dropwise to a solution of 1.0 $\mathrm{M} \mathrm{LiAlH}_{4}(1.0 \mathrm{~mL}, 1.0 \mathrm{mmol})$ in $10 \mathrm{~mL}$ of $\mathrm{Et}_{2} \mathrm{O}$ over $30 \mathrm{~min}$ at $0{ }^{\circ} \mathrm{C}$. The resulting solution was stirred for further $3 \mathrm{~h}$ at $0{ }^{\circ} \mathrm{C}$ and the reaction mixture was then quenched with $15 \mathrm{~mL}$ of chilled degassed water. The organic layer was washed $(2 \times 10 \mathrm{~mL})$ with water, then dried over anhydrous $\mathrm{MgSO}_{4}$. The dried organic layer was then filtered and the solvent removed under reduced pressure to afford a colourless viscous oil. Yield: $0.50 \mathrm{~g}(61 \%)$.

${ }^{1}$ H NMR (400 MHz, C 6 D $\left.6, \delta\right): 7.50$ (m, 6H, H15, H16), 7.39 (dd, 2H, H3), 7.24 (m, 2H, H6), 7.16 $(\mathrm{m}, 7 \mathrm{H}, \mathrm{H} 6, \mathrm{H} 7, \mathrm{H} 8, \mathrm{H} 14), 6.83(\mathrm{dd}, 2 \mathrm{H}, \mathrm{H} 2), 6.38\left(\mathrm{t}, 1 \mathrm{H}, \mathrm{H} 12,{ }^{1} J_{119 \mathrm{Sn}-1 \mathrm{H}}=1860 \mathrm{~Hz},{ }^{1} J_{117 \mathrm{Sn}-1 \mathrm{H}}=\right.$ $1820 \mathrm{~Hz}), 3.61\left(\mathrm{t}, 2 \mathrm{H}, \mathrm{H} 9,{ }^{3} J_{119 \mathrm{Sn}-1 \mathrm{H}}=28 \mathrm{~Hz}\right), 1.95\left(\mathrm{~m}, 2 \mathrm{H}, \mathrm{H} 10,{ }^{2} J_{119 \mathrm{Sn}-1 \mathrm{H}}=64 \mathrm{~Hz}\right), 1.27(\mathrm{dt}, 2 \mathrm{H}$, $\left.\mathrm{H} 11,{ }^{1} J_{119 \mathrm{Sn}-1 \mathrm{H}}=56 \mathrm{~Hz}\right) \mathrm{ppm} ;{ }^{13} \mathrm{C}$ NMR $\left(100 \mathrm{MHz}, \mathrm{C}_{6} \mathrm{D}_{6}, \delta\right): 159.0(\mathrm{C}-i-\mathrm{Ar}), 141.4(\mathrm{C}-i$-Ar $)$, $138.3\left(\mathrm{C} 13,{ }^{1} J_{119 \mathrm{Sn}-13 \mathrm{C}}=495 \mathrm{~Hz},{ }^{1} J_{117 \mathrm{Sn}-13 \mathrm{C}}=472 \mathrm{~Hz}\right), 137.5\left(\mathrm{C} 14,{ }^{2} J_{119 \mathrm{Sn}-13 \mathrm{C}}=36 \mathrm{~Hz}\right), 134.1(\mathrm{C}-$ i-Ar), 129.1 (C-Ar), 129.1 (C- Ar), 128.9 (C- Ar), 128.5 (C-Ar), 127.1 (C- Ar), 126.9 (C- Ar), $115.2(\mathrm{C} 2), 70.3\left(\mathrm{C} 9,{ }^{3} J_{119 \mathrm{Sn}-13 \mathrm{C}}=60 \mathrm{~Hz}\right), 26.9\left(\mathrm{C} 10,{ }^{2} J_{119 \mathrm{Sn}-13 \mathrm{C}}=21 \mathrm{~Hz}\right), 6.67\left(\mathrm{C} 11,{ }^{1} J_{119 \mathrm{Sn}-13 \mathrm{C}}=\right.$ $\left.396 \mathrm{~Hz},{ }^{1} J_{117 \mathrm{Sn}-13 \mathrm{C}}=378 \mathrm{~Hz}\right)$ ppm; ${ }^{119} \mathbf{S n}\left\{{ }^{1} \mathbf{H}\right\} \mathbf{N M R}\left(149 \mathrm{MHz}, \mathrm{C}_{6} \mathrm{D}_{6}, \delta\right):-137.0$ ppm. HRMSDART (m/z): calcd for $\mathrm{C}_{27} \mathrm{H}_{25} \mathrm{OSn}\left[\mathrm{M}^{+}\right]-\mathrm{H}, 485.09325$; found 485.09336 . 


\subsection{Synthesis of phenyl[(3-phenyloxy)propyl]tin (206):}

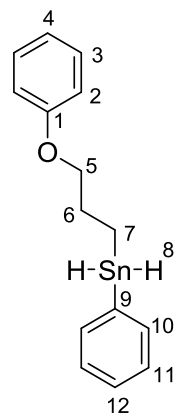

A solution of $202(1.36 \mathrm{~g}, 3.38 \mathrm{mmol})$ in $30 \mathrm{~mL}^{\text {of }} \mathrm{Et}_{2} \mathrm{O}$ was added dropwise to a solution of 1.0 $\mathrm{M} \mathrm{LiAlH}_{4}(4.0 \mathrm{~mL}, 4.0 \mathrm{mmol})$ in $15 \mathrm{~mL}^{\text {of }} \mathrm{Et}_{2} \mathrm{O}$ cooled to $0{ }^{\circ} \mathrm{C}$ over $30 \mathrm{~min}$ and the resulting mixture stirred for further $3 \mathrm{~h}$ at $0^{\circ} \mathrm{C}$. The reaction was quenched with $20 \mathrm{~mL}$ of chilled degassed water. The aqueous layer was extracted with $(3 \times 15 \mathrm{~mL}) \mathrm{Et}_{2} \mathrm{O}$. The organic layers were dried over anhydrous $\mathrm{MgSO}_{4}$, filtered and solvent removed under reduced pressure to afford a colourless, viscous oil. Yield: $0.89 \mathrm{~g}(81 \%)$.

${ }^{1}$ H NMR (400 MHz, $\left.\mathrm{C}_{6} \mathrm{D}_{6}, \delta\right): 7.48$ (m, 2H, H10), 7.19 (m, 5H, H3, H11, H12), 6.88 (3H, H2, H4), $5.57\left(\mathrm{t}, 2 \mathrm{H}, \mathrm{H} 8,{ }^{1} J_{119 \mathrm{Sn}-1 \mathrm{H}}=1836 \mathrm{~Hz},{ }^{1} J_{117 \mathrm{Sn}-1 \mathrm{H}}=1752 \mathrm{~Hz}\right), 3.59(\mathrm{t}, 2 \mathrm{H}, \mathrm{H} 5), 1.88(\mathrm{~m}, 2 \mathrm{H}$, H6, $\left.{ }^{1} J_{119 S n-1 H}=64 \mathrm{~Hz}\right), 1.10\left(\mathrm{tt}, 2 \mathrm{H}, \mathrm{H} 7,{ }^{1} J_{119 \mathrm{Sn}-1 \mathrm{H}}=56 \mathrm{~Hz}\right) \mathrm{ppm} ;{ }^{13} \mathbf{C} \mathbf{N M R}\left(100 \mathrm{MHz}, \mathrm{C}_{6} \mathrm{D}_{6}, \delta\right)$ : $159.4(\mathrm{C} 1), 137.8\left(\mathrm{C} 10,{ }^{2} J_{119 \mathrm{Sn}-\mathrm{C}}=38 \mathrm{~Hz}\right), 136.7\left(\mathrm{C} 9,{ }^{1} J_{119 \mathrm{Sn}-13 \mathrm{C}}=499 \mathrm{~Hz},{ }^{1} J_{117 \mathrm{Sn}-13 \mathrm{C}}=476 \mathrm{~Hz}\right)$, $129.7(\mathrm{C} 12), 128.9\left(\mathrm{C} 11,{ }^{3} J_{119 \mathrm{Sn}-13 \mathrm{C}}=11 \mathrm{~Hz}\right), 120.9(\mathrm{C} 4), 128.8(\mathrm{C} 3), 114.9(\mathrm{C} 2), 69.9\left(\mathrm{C} 5,{ }^{3} J_{119 \mathrm{Sn}-}\right.$ $\left.{ }_{13 \mathrm{C}}=56 \mathrm{~Hz}\right), 27.4\left(\mathrm{C} 6,{ }^{2} J_{119 \mathrm{Sn}-13 \mathrm{C}}=24 \mathrm{~Hz}\right), 5.04\left(\mathrm{C} 7,{ }^{1} J_{119 \mathrm{Sn}-13 \mathrm{C}}=406 \mathrm{~Hz},{ }^{1} J_{117 \mathrm{Sn}-13 \mathrm{C}}=387 \mathrm{~Hz}\right)$ ppm; ${ }^{119} \mathbf{S n}\left\{{ }^{1} \mathbf{H}\right\}$ NMR $\left(149 \mathrm{MHz}, \mathrm{C}_{6} \mathrm{D}_{6}, \delta\right):-215.1 \mathrm{ppm} . \mathbf{H R M S - D A R T}(\mathrm{m} / \mathrm{z}):\left[\mathrm{M}^{+}\right]$calculated for $\mathrm{C}_{15} \mathrm{H}_{17} \mathrm{OSn}, 333.02937$; found 333.03014. 


\subsection{Synthesis of phenyl[3-(4-biphenyloxy)propyl]tin (207):}

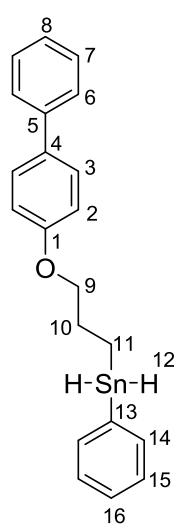

\section{Method 1:}

A solution of $203(0.912 \mathrm{~g}, 1.6 \mathrm{mmol})$ in $20 \mathrm{~mL}$ of $\mathrm{Et}_{2} \mathrm{O}$ was added dropwise to a solution of 1.0 $\mathrm{M} \mathrm{LiAlH}_{4}(4.27 \mathrm{~mL}, 4.27 \mathrm{mmol})$ in $15 \mathrm{~mL} \mathrm{Et}_{2} \mathrm{O}$ at $0{ }^{\circ} \mathrm{C}$ over $30 \mathrm{~min}$ and the mixture stirred for further $2 \mathrm{~h}$ at $0{ }^{\circ} \mathrm{C}$. The reaction was quenched with $7.0 \mathrm{~mL}$ of chilled degassed water. The organic layer was separated and the aqueous layer extracted with $(2 \times 30 \mathrm{~mL}) \mathrm{Et}_{2} \mathrm{O}$. The combined organic layers were dried over $\mathrm{MgSO}_{4}$. After filtration, the solvent was removed under reduced pressure to yield a light yellow coloured oil. Yield: $0.54 \mathrm{~g}(84 \%)$. HRMS-DART: $\left[\mathrm{M}^{+}\right]$, Calc. for $\mathrm{C}_{21} \mathrm{H}_{22} \mathrm{OSn}$ 409.0614; found 409.0613.

\section{Method 2:}

A suspension of $\mathrm{NaBH}_{4}(0.228 \mathrm{~g}, 6.16 \mathrm{mmol})$ in $20 \mathrm{~mL}$ of EtOH was added to $146(0.25 \mathrm{~g}, 0.445$ mmol) suspended in $10 \mathrm{~mL}$ of EtOH at $0{ }^{\circ} \mathrm{C}$. The resulting solution was stirred for an additional $30 \mathrm{~min}$ at same temperature. The reaction was quenched with $1.0 \mathrm{~mL}$ of chilled degassed water and product extracted with $(2 \times 15 \mathrm{~mL})$ hexane. The collected organic layers were dried over $\mathrm{MgSO}_{4}$. After filtration and solvent removal under reduced pressure, a light yellow coloured oil was recovered. Yield: $0.15 \mathrm{~g}(81 \%)$. 


\section{Method 3:}

A solution of compound $146(0.24 \mathrm{~g}, 0.586 \mathrm{mmol})$ in $15 \mathrm{~mL}$ of $\mathrm{Et}_{2} \mathrm{O}$ was added dropwise to a suspension of $1.0 \mathrm{M} \mathrm{LiAlH}_{4}(0.6 \mathrm{~mL}, 0.6 \mathrm{mmol})$ in $10 \mathrm{~mL}^{\circ} \mathrm{Et}_{2} \mathrm{O}$ at $0{ }^{\circ} \mathrm{C}$ over $30 \mathrm{~min}$ and the mixture stirred for a further $3 \mathrm{~h}$ at $0{ }^{\circ} \mathrm{C}$. The reaction mixture was then quenched with $10 \mathrm{~mL}$ of chilled degassed water. The organic layer was separated and the aqueous layer extracted with $(3 \times$ $15 \mathrm{~mL}$ ) $\mathrm{Et}_{2} \mathrm{O}$. The combined organic layers were dried over anhydrous $\mathrm{CaCl}_{2}$. The solution was filtered and solvent removed under reduced pressure to yield a colourless viscous oil. Yield: 0.13 $\mathrm{g}(65 \%)$.

${ }^{1}$ H NMR (400 MHz, C 6 D 6 , $): 7.48$ (m, 4H, H4, H10), 7.42 (m, 2H, H11), 7.23 (m, 2H, H5, H12), $7.15(\mathrm{~m}, 4 \mathrm{H}, \mathrm{H} 2, \mathrm{H} 3), 6.88(2 \mathrm{H}, \mathrm{H} 1), 5.56\left(\mathrm{t}, 2 \mathrm{H}, \mathrm{H} 9,{ }^{1} J_{119 \mathrm{Sn}-1 \mathrm{H}}=1837 \mathrm{~Hz},{ }^{1} J_{117 \mathrm{Sn}-1 \mathrm{H}}=1754 \mathrm{~Hz}\right)$, $3.57(\mathrm{t}, 2 \mathrm{H}, \mathrm{H} 6), 1.88\left(\mathrm{~m}, 2 \mathrm{H}, \mathrm{H} 7,{ }^{2} J_{119 \mathrm{Sn}-1 \mathrm{H}}=72 \mathrm{~Hz}\right), 1.10\left(\mathrm{tt}, 2 \mathrm{H}, \mathrm{H} 8,{ }^{1} J_{119 \mathrm{Sn}-1 \mathrm{H}}=60 \mathrm{~Hz}\right) \mathrm{ppm}$; ${ }^{13} \mathrm{C}$ NMR $\left(100 \mathrm{MHz}, \mathrm{C}_{6} \mathrm{D}_{6}, \delta\right): 158.9(\mathrm{C}-i$-Ar $), 141.5(\mathrm{C}-i$-Ar $), 137.80\left(\mathrm{C} 14,{ }^{2} J_{119 \mathrm{Sn}-13 \mathrm{C}}=38 \mathrm{~Hz}\right)$, $136.6\left(\mathrm{C} 13,{ }^{1} J_{119 \mathrm{Sn}-13 \mathrm{C}}=493 \mathrm{~Hz},{ }^{1} J_{117 \mathrm{Sn}-13 \mathrm{C}}=474 \mathrm{~Hz}\right), 134.1(\mathrm{C}-i-\mathrm{Ar}), 129.1(\mathrm{C}-\mathrm{Ar}), 129.0(\mathrm{C}-$ $i$-Ar), 128.8 (C- Ar ), 128.5 (C- Ar), 127.1 (C- Ar), 126.9 (C- Ar), $115.2(\mathrm{C} 2), 70.1$ (C9, ${ }^{3} J_{119 S n-}$ $\left.{ }_{13 \mathrm{C}}=57 \mathrm{~Hz}\right), 27.4\left(\mathrm{C} 10,{ }^{2} J_{119 \mathrm{Sn}-13 \mathrm{C}}=23 \mathrm{~Hz}\right), 5.01\left(\mathrm{C} 11,{ }^{1} J_{119 \mathrm{Sn}-13 \mathrm{C}}=404 \mathrm{~Hz},{ }^{1} J_{117 \mathrm{Sn}-13 \mathrm{C}}=387 \mathrm{~Hz}\right)$ ppm; ${ }^{119} \mathrm{Sn}\left\{{ }^{1} \mathbf{H}\right\} \mathbf{N M R}\left(149 \mathrm{MHz}, \mathrm{C}_{6} \mathrm{D}_{6}, \delta\right):-215.1 \mathrm{ppm}$. 


\subsection{Dimerization of diphenyl[(3-phenyloxy)propyl]tin (208):}

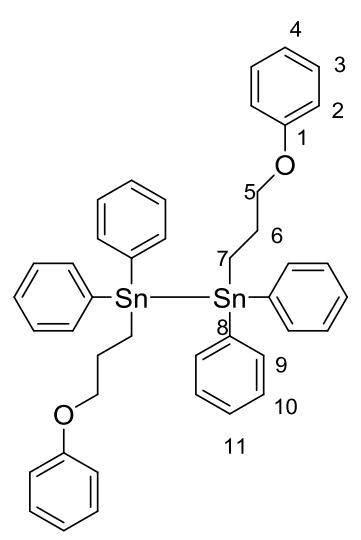

A solution of $\mathrm{Pd}\left(\mathrm{PPh}_{3}\right)_{4}(0.027 \mathrm{~g}, 0.035 \mathrm{mmol})$ in $5 \mathrm{~mL}$ of dry $\mathrm{C}_{6} \mathrm{H}_{6}$ was added slowly to a solution of $204(0.105 \mathrm{~g}, 0.26 \mathrm{mmol})$ in $5 \mathrm{~mL}$ of $\mathrm{C}_{6} \mathrm{H}_{6}$ under inert atmosphere. The mixture was stirred for $2 \mathrm{~h}$ at room temperature and then at $50{ }^{\circ} \mathrm{C}$ overnight. The product was purified by column chromatography on silica gel (hexanes:EtOAc (1:1)). Yield: $0.03 \mathrm{~g}(53 \%)$.

${ }^{1}$ H NMR (400 MHz, C6 $\left.\mathrm{D}_{6}, \delta\right): 7.55$ (m, 4H, H9), 7.36 (m, 6H, H10, H11), 7.23 (m, 2H, H3), 6.91 (m, 1H, H4), 6.79 (dd, 2H, H2), 3.94 (t, 2H, H5), 2.2 (m, 2H, H6), 1.62 (m, 2H, H7) ppm; ${ }^{13} \mathbf{C}$ NMR $\left(100 \mathrm{MHz}, \mathrm{C}_{6} \mathrm{D}_{6}, \delta\right): 159.2(\mathrm{C} 1), 138.7\left(\mathrm{C} 8,{ }^{1} J_{119 \mathrm{Sn}-13 \mathrm{C}}=488 \mathrm{~Hz}\right), 137.1\left(\mathrm{C} 10,{ }^{3} J_{119 \mathrm{Sn}-13 \mathrm{C}}=\right.$ $36 \mathrm{~Hz}), 129.30\left(\mathrm{C} 9,{ }^{2} J_{119 \mathrm{Sn}-13 \mathrm{C}}=480 \mathrm{~Hz}\right), 128.8\left(\mathrm{C} 11,{ }^{4} J_{119 \mathrm{Sn}-13 \mathrm{C}}=10 \mathrm{~Hz}\right), 128.6(\mathrm{C} 3), 120.4(\mathrm{C} 4)$, $114.5(\mathrm{C} 2), 70.0(\mathrm{C} 5), 26.3\left(\mathrm{C} 6,{ }^{2} J_{119 \mathrm{Sn}-13 \mathrm{C}}=20 \mathrm{~Hz}\right), 6.77\left(\mathrm{C} 7,{ }^{1} J_{119 \mathrm{Sn}-13 \mathrm{C}}=390 \mathrm{~Hz}\right) \mathrm{ppm}$; ${ }^{119} \mathrm{Sn}\left\{{ }^{1} \mathbf{H}\right\}$ NMR $\left(149 \mathrm{MHz}, \mathrm{C}_{6} \mathrm{D}_{6}, \delta\right):-98.0\left(J_{119 \mathrm{Sn}-117 \mathrm{Sn}}=8480 \mathrm{~Hz}\right) \mathrm{ppm}$. 


\subsection{Dimerization of diphenyl[3-(4-biphenyloxy)propyl]tin (209):}

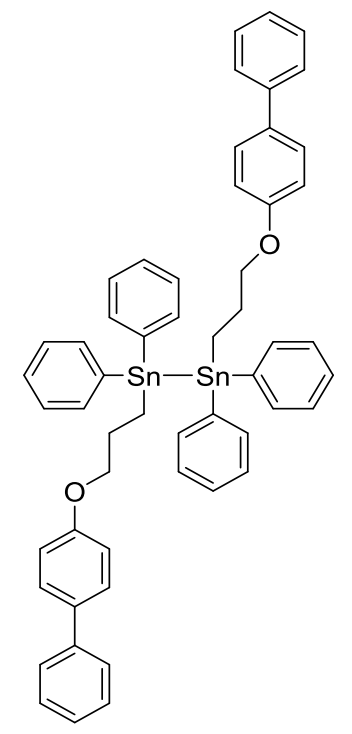

A solution of $\mathrm{Pd}\left(\mathrm{PPh}_{3}\right)_{4}(0.035 \mathrm{~g}, 0.045 \mathrm{mmol})$ in $5 \mathrm{~mL}$ of $\mathrm{C}_{6} \mathrm{H}_{6}$ was added slowly to a solution of $0.172 \mathrm{~g}(0.35 \mathrm{mmol})$ of $\mathbf{2 0 5}$ in $5 \mathrm{~mL} \mathrm{C}_{6} \mathrm{H}_{6}$ under an inert atmosphere. The mixture was stirred overnight at room temperature. The crude reaction mixture was extracted with petroleum ether. An attempt to purify the remaining reaction mixture by column chromatography on silica gel (hexanes/EtOAc) was unsuccessful. The ${ }^{1} \mathrm{H}$ NMR spectrum show unidentified resonance additional resonances.

${ }^{1} \mathbf{H}$ NMR (400 MHz, $\left.\mathrm{C}_{6} \mathrm{D}_{6}, \delta\right): 7.67-7.73(\mathrm{~m}, 10 \mathrm{H}), 7.55-7.61(\mathrm{~m}, 5 \mathrm{H}), 7.47-7.51(\mathrm{~m}, 7 \mathrm{H})$, 7.37$7.42(\mathrm{~m}, 11 \mathrm{H}), 6.92(\mathrm{~m}, 1 \mathrm{H}), 6.80(\mathrm{~m}, 1 \mathrm{H}), 3.97(\mathrm{t}, 2 \mathrm{H}),, 2.22(\mathrm{~m}, 2 \mathrm{H}),, 1.65(\mathrm{t}, 2 \mathrm{H}$,$) ppm:$

${ }^{119} \mathrm{Sn}\left\{{ }^{1} \mathbf{H}\right\} \mathbf{N M R}\left(149 \mathrm{MHz}, \mathrm{C}_{6} \mathrm{D}_{6}, \delta\right):-98.0 \mathrm{ppm}$. 


\subsection{Polymeriztion of phenyl[(3-phenyloxy)propyl]tin (249):}

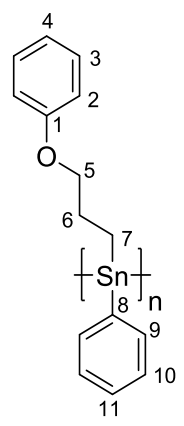

A solution of $206(0.295 \mathrm{~g}, 0.9 \mathrm{mmol})$ in $10 \mathrm{~mL}$ of dry toluene was added by syringe to a solution of Wilkinson's catalyst $(0.035 \mathrm{~g}, 0.038 \mathrm{mmol})$ in $10 \mathrm{~mL}$ of toluene in a Schlenk flask wrapped in aluminium foil. The reaction mixture was allowed to stirr for $5 \mathrm{~h}$. The solvent was removed under reduced pressure. The product was redissolved in fresh toluene, filtered and added dropwise to an excess of petroleum ether. The precipitated product was recovered as an orange/yellow coloured gum. Yield: $0.22 \mathrm{~g}(75 \%)$.

${ }^{1}$ H NMR (400 MHz, C6 $\left.\mathrm{D}_{6}, \delta\right):$ 7.29-7.76 (m, 2H, Haryl), 6.95-7.25 (bm, 5H, Haryl), 6.67-6.2 (s, 3H, H aryl), 3.12-3.97 (bs, 2H, H5), 1.04-2.44 (bs, 4H, H6, H7) ppm; ${ }^{13}$ C NMR (100 MHz, C6 6 , $\delta$ ): 159.6 (C1), 138.1 (C8), 136.6 (C9), 132.5 (C11), 129.7 (C10), 128.9 (C3), 120.7 (C4), 114.9 (C2), 70.6 (C5), 30.1 (C6), 21.9 (C7) ppm; ${ }^{119} \mathbf{S n}\left\{{ }^{1} \mathbf{H}\right\}$ NMR (149 MHz, $\mathrm{C}_{6} \mathrm{D}_{6}, \delta$ ): -199.0 ppm. Found: C, 56.68; H, 4.82. Calc. for $\mathrm{C}_{27} \mathrm{H}_{26} \mathrm{OSn}$ : C, 54.43, H, $4.87 \%$ 


\subsection{Polymerization of phenyl[3-(4-biphenyloxy)propyl]tin (250):}

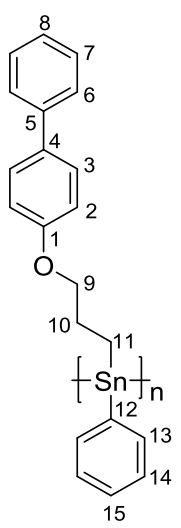

A solution of $207(0.333 \mathrm{~g}, 1.0 \mathrm{mmol}$ in $10 \mathrm{~mL}$ of toluene) was added with a syringe to a solution of Wilkinson's catalyst $(0.040 \mathrm{~g}, 0.043 \mathrm{mmol}$ in $5 \mathrm{~mL}$ of toluene $)$ in a Schlenk flask wrapped in aluminium foil stirred for $5 \mathrm{~h}$. The solvent was removed under reduced pressure. The product was dissolved in a minimum amount of fresh toluene, filtered and added dropwise into petroleum ether. The precipitated product was recovered as an orange/yellow coloured gum. Yield: $0.23 \mathrm{~g}(68 \%)$. ${ }^{1} \mathbf{H}$ NMR (400 MHz, $\mathrm{C}_{6} \mathrm{D}_{6}, \delta$ ): 7.28-7.66 (br m, 6H, $\mathrm{H}_{\text {aryl }}$ ), 7.2 (s, 2H, $\mathrm{H}_{\text {aryl }}$ ), 6.97-7.13 (br m, 4H, $\mathrm{H}_{\text {aryl }}$, 6.67-6.90 (br s, 2H, H2), 3.13-3.88 (br s, 2H, H9), 1.29-2.39 (br s, 4H, H10, H11), ppm; ${ }^{13} \mathbf{C}$ NMR (100 MHz, $\left.\mathrm{C}_{6} \mathrm{D}_{6}, \delta\right): 158.8$ (C- $i$-Ar), 140.9 (C- $i$-Ar), 138.0 (C- $i$-Ar), 137.3 (C- $i$-Ar), 133.6 (C- Ar), 129.0 (C- Ar), 128.7 (C- Ar), 128.2 (C- Ar), 126.7 (C- Ar), 126.5 (C- Ar), 125.3 (C- Ar), 114.9 (C2), 70.0 (C9), 22.4 (C10), 21.1(C11) ppm; ${ }^{119} \mathbf{S n}\left\{{ }^{1} \mathbf{H}\right\} \mathbf{N M R}\left(149 \mathrm{MHz}, \mathrm{C}_{6} \mathrm{D}_{6}, \delta\right):-195.0$ ppm. Found: C, 50.50, H, 4.75. Calc. for $\mathrm{C}_{27} \mathrm{H}_{26} \mathrm{OSn}$ : C, 61.96, H, 4.95\%.

\subsection{Synthesis of Benzyl methyl ether (214)}

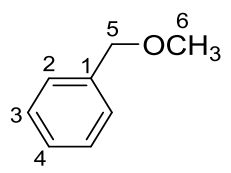

$\mathrm{NaOCH}_{3}(1.6 \mathrm{~g}, \mathrm{mmol})$ was dissolved in $50 \mathrm{~mL}$ of $\mathrm{CH}_{3} \mathrm{OH}$ and added to benzyl bromide $(5.0 \mathrm{~g}$, mmol) in a two neck round bottom flask equipped with a condenser and heated to reflux for $24 \mathrm{~h}$. 
The solution was cooled to room temperature and solvent removed under reduced pressure. $60 \mathrm{~mL}$ of EtOAc was added to the flask and the organic layer washed with $(2 \times 50 \mathrm{~mL})$ water, and dried over $\mathrm{MgSO}_{4}$. The solvent was removed under reduced pressure to obtain a colourless oil of 214. NMR data $\left({ }^{1} \mathrm{H},{ }^{13} \mathrm{C}\right)$ was comparable to that reported in the literature. ${ }^{152}$ Yield: $10.6 \mathrm{~g}(34 \%)$.

${ }^{1} \mathbf{H}$ NMR (400 MHz, $\mathrm{CDCl}_{3}, \delta$ ): 7.39-7.41 (m, 4H, H2, H3), 7.32 (m, 1H, H4), 4.51 (s, 2H, H5), 3.44 (s, 3H, H6) ppm; ${ }^{13} \mathrm{C}$ NMR (100 MHz, $\left.\mathrm{CDCl}_{3}, \delta\right): 138.3$ (C1), 128.4 (C), 127.8 (C), 127.7 (C), 74.7 (C5), 58.1 (C6) ppm.

\subsection{Synthesis of 2-Bromobenzyl methyl ether (215):}

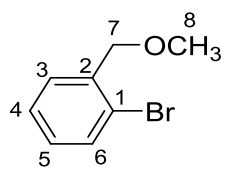

$\mathrm{NaOCH}_{3}(3.39 \mathrm{~g}, 62.75 \mathrm{mmol})$ was dissolved in $40 \mathrm{~mL}$ of $\mathrm{CH}_{3} \mathrm{OH}$ and added to 2-bromobenzyl bromide (15.6 g, $62.41 \mathrm{mmol})$ in a two neck round bottom flask equipped with a condenser and heated to reflux for $5 \mathrm{~h}$. The reaction mixture was cooled to room temperature and the solvent removed under reduced pressure. A mixture of $100 \mathrm{~mL}$ of hexane and $\mathrm{Et}_{2} \mathrm{O}$ (1:1) was added to flask. The organic layer was washed with $(2 \times 50 \mathrm{~mL})$ water and $(2 \times 50 \mathrm{~mL})$ of brine, and finally dried over $\mathrm{MgSO}_{4}$. The solvent was removed under reduced pressure to obtain a colorless oil of 1bromo-2-(methoxymethyl)benzene. NMR data $\left({ }^{1} \mathrm{H},{ }^{13} \mathrm{C}\right)$ was comparable to that reported in the literature. ${ }^{95}$ Yield: $10.6 \mathrm{~g}(84 \%)$.

${ }^{1} \mathbf{H}$ NMR $\left(400 \mathrm{MHz}, \mathrm{CDCl}_{3}, \delta\right): 7.57(\mathrm{dd}, J=8.0 \mathrm{~Hz}, 1 \mathrm{H}) 7.55(\mathrm{dd}, J=7.6 \mathrm{~Hz}, 1 \mathrm{H}), 7.33(\mathrm{td}, J$ $=7.6 \mathrm{~Hz}, 1 \mathrm{H}), 7.15(\mathrm{td}, J=8.0 \mathrm{~Hz}, 1 \mathrm{H}), 4.55(\mathrm{~s}, 2 \mathrm{H}), 3.49(\mathrm{~s}, 3 \mathrm{H}) \mathrm{ppm} ;{ }^{13} \mathrm{C} \mathbf{N M R}(100 \mathrm{MHz}$, $\mathrm{CDCl}_{3}, \delta$ ): 137.6 (C2), 132.5 (C3), 129.0 (C6), 128.9 (C4), 127.4 (C5), 122.7 (C1), 73.9 (C7), 58.6 (C8) ppm. 


\subsection{Synthesis of $\left[2-\left(\mathrm{MeOCH}_{2}\right) \mathrm{C}_{6} \mathrm{H}_{4}\right] \mathrm{Li}(216)$ :}

1.6 M solution of $n-\mathrm{BuLi}(26.0 \mathrm{~mL}, 41.6 \mathrm{mmol})$ in hexane was added dropwise to a solution of 2bromobenzyl methyl ether $(8.37 \mathrm{~g}, 41.64 \mathrm{mmol})$ in $40 \mathrm{~mL}$ of hexane at $-78{ }^{\circ} \mathrm{C}$ over $30 \mathrm{~min}$. The solution became yellow and hazy with the addition of $n$-BuLi. A yellow tinged white coloured solid began to precipitate from solution after $1 \mathrm{~h}$ and the reaction mixture allowed to stir overnight. The solid product was separated by filtration through glass frit and washed with hexane. The remaining residual solvent was removed under reduced pressure to give an off white coloured solid. Yield: $5.16 \mathrm{~g}(97 \%)$.

Warning: [2-( $\left.\left.\mathrm{MeOCH}_{2}\right) \mathrm{C}_{6} \mathrm{H}_{4}\right] \mathrm{Li}$ should be used in situ and not isolated due to its pyrophoric nature. This compound is shock sensitive and can detonate even in glove box. Therefore, avoid isolation.

\subsection{Synthesis of 2-Trimethylstannylbenzyl methyl ether (113):}

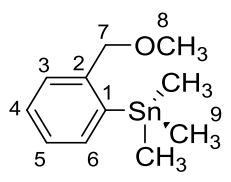

Compound 215 (2.0 g, $9.95 \mathrm{mmol})$ and $20 \mathrm{~mL}$ of hexane were added to a dry $100 \mathrm{~mL}$ Schlenk flask equipped with a magnetic stirrer and septum. $1.6 \mathrm{M} \mathrm{n}$-BuLi in hexane (6.3 mL, $10.1 \mathrm{mmol})$ was added slowly at $-78{ }^{\circ} \mathrm{C}$. The cooling bath was removed for $15 \mathrm{~min}$ and the in situ generated lithium benzyl methyl ether allowed to react with a solution of $\mathrm{Me}_{3} \mathrm{SnCl}(1.98 \mathrm{~g}, 9.94 \mathrm{mmol}$ in 10 $\mathrm{mL}$ hexane/Et $2 \mathrm{O}(1: 1))$ at $-78{ }^{\circ} \mathrm{C}$. The reaction mixture was warmed to room temperature and stirred for $3 \mathrm{~h}$. The solvent was removed under reduced pressure and the recovered crude product taken up in 1:1 $\mathrm{Et}_{2} \mathrm{O} / \mathrm{h}$ exanes $(80 \mathrm{~mL})$, washed with $(2 \times 100 \mathrm{~mL})$ water and $(2 \times 50 \mathrm{~mL})$ brine. The organic layer was dried over $\mathrm{MgSO}_{4}$, filtered, and solvent removed under reduced pressure to 
give a colourless oil. NMR data $\left({ }^{1} \mathrm{H},{ }^{13} \mathrm{C},{ }^{119} \mathrm{Sn}\right)$ is essentially the same as previously reported in the literature. ${ }^{95}$ Yield: $1.98 \mathrm{~g}(70 \%)$.

${ }^{1} \mathbf{H}$ NMR (400 MHz, $\left.\mathrm{CDCl}_{3}, \delta\right): 7.6$ (m, 1H, H6), 7.31-7.35 (m, 3H, H3, H4, H5), 4.51 (s, H7), $3.41(\mathrm{~s}, 3 \mathrm{H}, \mathrm{H} 8), 0.34\left(\mathrm{~s}, 9 \mathrm{H}, \mathrm{H} 9,{ }^{1} J_{119 \mathrm{Sn}-1 \mathrm{H}}=56 \mathrm{~Hz}\right) \mathrm{ppm} ;{ }^{13} \mathbf{C} \mathbf{N M R}\left(100 \mathrm{MHz}, \mathrm{CDCl}_{3}, \delta\right): 144.6$ $\left(\mathrm{C} 2,{ }^{2} J=27 \mathrm{~Hz}\right), 141.5\left(\mathrm{C} 1,{ }^{1} J_{119 \mathrm{Sn}-13 \mathrm{C}}=476 \mathrm{~Hz}\right), 136.6\left(\mathrm{C} 6,{ }^{2} J=44 \mathrm{~Hz}\right), 128.2\left(\mathrm{C} 3,{ }^{3} J_{119 \mathrm{Sn}-13 \mathrm{C}}=\right.$ $6.0 \mathrm{~Hz}), 127.7\left(\mathrm{C} 5,{ }^{3} J_{119 S n-13 \mathrm{C}}=11 \mathrm{~Hz}\right), 127.2(\mathrm{C} 4), 76.3\left(\mathrm{C} 7,{ }^{3} J_{119 \mathrm{Sn}-13 \mathrm{C}}=19 \mathrm{~Hz}\right), 57.8(\mathrm{C} 8), 8.06$ $\left(\mathrm{C} 9,{ }^{1} J_{119 \mathrm{Sn}-13 \mathrm{C}}=175 \mathrm{~Hz}\right) \mathrm{ppm} ;{ }^{119} \mathbf{S n}\left\{{ }^{1} \mathbf{H}\right\} \mathbf{N M R}\left(149 \mathrm{MHz}, \mathrm{CDCl}_{3}, \delta\right):-32.5 \mathrm{ppm}$.

\subsection{Synthesis of 2-Tributylstannylbenzyl methyl ether (217):}

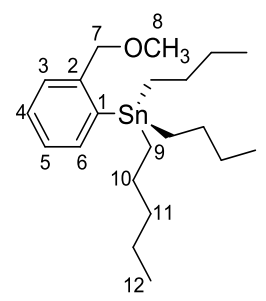

Compound 215 (2.0 g, $9.95 \mathrm{mmol})$ in $20 \mathrm{~mL}$ of hexanes was added to a $100 \mathrm{~mL}$ dry Schlenk flask equipped with a magnetic stirrer and septum. 1.6 M $n$-BuLi in hexanes $(6.2 \mathrm{~mL}, 9.92 \mathrm{mmol})$ was added at $-78{ }^{\circ} \mathrm{C}$. After addition of $n$-BuLi the cooling bath was removed for 15 minutes and the lithiated reagent allowed to react with the $n-\mathrm{Bu}_{3} \mathrm{SnCl}(3.22 \mathrm{~g}, 9.9 \mathrm{mmol})$ in $10 \mathrm{~mL}$ hexane/Et ${ }_{2} \mathrm{O}$ (1:1) at $-78{ }^{\circ} \mathrm{C}$ added slowly to the flask. The reaction mixture was stirred for $3 \mathrm{~h}$ and solvent removed under reduced pressure. The remaining solution was taken up in 1:1 $\mathrm{Et}_{2} \mathrm{O} / \mathrm{hexanes}(80$ $\mathrm{mL})$, washed with $(2 \times 100 \mathrm{~mL})$ water and $(2 \times 50 \mathrm{~mL})$ brine. The organic layer dried over $\mathrm{MgSO}_{4}$, filtered, and solvent removed under reduced pressure to give a clear, yellow-brown coloured oil. Yield: $2.64 \mathrm{~g}(65 \%)$.

${ }^{1} \mathrm{H}$ NMR (400 MHz, $\left.\mathrm{CDCl}_{3}, \delta\right): 7.55$ (d, 1H, H6), 7.31 (m, 3H, H3, H4, H5), 4.46 (s, 2H, H7), 3.41 (s, 3H, H8), 1.60 (m, 6H, H10), 1.39 (m, 6H, H11), 1.14 (m, 6H, H9), 1.11 (t, 9H, H12) ppm; ${ }^{13} \mathrm{C}$ NMR $\left(100 \mathrm{MHz}, \mathrm{CDCl}_{3}, \delta\right): 144.7\left(\mathrm{C} 2,{ }^{2} J_{119 \mathrm{Sn} 13 \mathrm{C}}=25 \mathrm{~Hz}\right), 141.6\left(\mathrm{C} 1,{ }^{1} J_{119 \mathrm{Sn}-13 \mathrm{C}}=390 \mathrm{~Hz}\right.$, 
$\left.{ }^{1} J_{119 \mathrm{Sn}-13 \mathrm{C}}=370 \mathrm{~Hz}\right), 137.2\left(\mathrm{C} 6,{ }^{2} J_{119 \mathrm{Sn} 13 \mathrm{C}}=30 \mathrm{~Hz}\right), 128.0(\mathrm{C} 4), 127.9\left(\mathrm{C} 3,{ }^{3} J_{119 \mathrm{Sn} 13 \mathrm{C}}=14 \mathrm{~Hz}\right)$,

$127.0\left(\mathrm{C}-5,{ }^{3} J_{119 \mathrm{Sn} 13 \mathrm{C}}=40 \mathrm{~Hz}\right), 76.8\left(\mathrm{C} 7,{ }^{2} J_{119 \mathrm{Sn} 13 \mathrm{C}}=18 \mathrm{~Hz}\right), 57.9(\mathrm{C} 8), 29.2\left(\mathrm{C} 11,{ }^{3} J_{\mathrm{SnC}}=20 \mathrm{~Hz}\right)$,

$27.5\left(\mathrm{C} 10,{ }^{2} J_{119 \mathrm{Sn} 13 \mathrm{C}}=60 \mathrm{~Hz}\right), 13.7(\mathrm{C} 12), 10.4\left(\mathrm{C} 9,{ }^{1} J_{119 \mathrm{Sn} 13 \mathrm{C}}=344 \mathrm{~Hz},{ }^{1} J_{119 \mathrm{Sn} 13 \mathrm{C}}=325 \mathrm{~Hz}\right) \mathrm{ppm}$;

${ }^{119} \mathrm{Sn}\left\{{ }^{1} \mathrm{H}\right\} \mathbf{N M R}\left(149 \mathrm{MHz}, \mathrm{CDCl}_{3}, \delta\right):-40.3 \mathrm{ppm}$. HRMS-DART $(\mathrm{m} / \mathrm{z}):\left[\mathrm{M}^{+}\right]$, Calc. for $\mathrm{C}_{20} \mathrm{H}_{37} \mathrm{OSn}$ 413.18664; found 413.18728.

\subsection{Synthesis of 2-Triphenylstannylbenzyl methyl ether (112):}

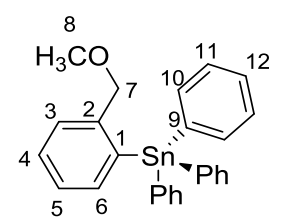

1.6 M solution of $n$ - BuLi ( $3.1 \mathrm{~mL}, 4.97 \mathrm{mmol})$ was added dropwise at $-78^{\circ} \mathrm{C}$ to a solution of 215 $(1.0 \mathrm{~g}, 4.97 \mathrm{mmol})$ in $25 \mathrm{~mL}$ hexane. The resulting yellow solution was stirred for an additional 2 $\mathrm{h}$ at RT, then added dropwise to a suspension of $\mathrm{Ph}_{3} \mathrm{SnCl}(1.87 \mathrm{~g}, 4.85 \mathrm{mmol})$ in $30 \mathrm{~mL}$ of hexane, followed by stirring for further $3 \mathrm{~h}$. The resulting solid was filtered and washed with $15 \mathrm{~mL}$ of $n$ hexane and the filtrate concentrated to $15 \mathrm{~mL}$. Cooling to $-20{ }^{\circ} \mathrm{C}$ afforded $\mathbf{1 1 2}$ as a white coloured solid. Yield: $0.80 \mathrm{~g}(73 \%)$, m.p. $95^{\circ} \mathrm{C} .{ }^{94}$

${ }^{1} \mathbf{H}$ NMR (400 MHz, $\left.\mathrm{CDCl}_{3}, \delta\right): 7.59$ (m, 6H, H10), 7.48 (d, 1H, H6), 7.36 (m, 9H, H11,12), 7.30 (m, 2H, H4, H5), $7.24(\mathrm{~m}, 1 \mathrm{H}, \mathrm{H} 3), 4.37\left(\mathrm{~s}, 2 \mathrm{H}, \mathrm{H} 7,{ }^{3} J_{119 \mathrm{Sn}-1 \mathrm{H}}=269 \mathrm{~Hz}\right), 2.80(\mathrm{~s}, 3 \mathrm{H}, \mathrm{H} 8) \mathrm{ppm}$; ${ }^{13} \mathbf{C}$ NMR $\left(100 \mathrm{MHz}, \mathrm{CDCl}_{3}, \delta\right): 145.2\left(\mathrm{C} 2,{ }^{2} J_{119 \mathrm{Sn}-13 \mathrm{C}}=33 \mathrm{~Hz}\right), 140.3\left(\mathrm{C} 9,{ }^{1} J_{119 \mathrm{Sn}-13 \mathrm{C}}=543 \mathrm{~Hz}\right)$, $138.6\left(\mathrm{C} 11,{ }^{3} J_{119 \mathrm{Sn}-13 \mathrm{C}}=38 \mathrm{~Hz}\right), 137.2\left(\mathrm{C} 10,{ }^{2} J_{119 \mathrm{Sn}-13 \mathrm{C}}=40 \mathrm{~Hz}\right), 136.5(\mathrm{C} 12), 129.0\left(\mathrm{C} 3,{ }^{3} J_{119 \mathrm{Sn}-}\right.$ $\left.{ }_{13 \mathrm{C}}=12 \mathrm{~Hz}\right), 128.5\left(\mathrm{C} 5,{ }^{3} J_{119 \mathrm{Sn}-13 \mathrm{C}}=12 \mathrm{~Hz}\right), 128.3(\mathrm{C} 4), 127.5\left(\mathrm{C} 1,{ }^{1} J_{119 \mathrm{Sn}-13 \mathrm{C}}=56 \mathrm{~Hz}\right), 127.3(\mathrm{C} 6$, $\left.{ }^{2} J_{119 S n-13 \mathrm{C}}=46 \mathrm{~Hz}\right), 75.2\left(\mathrm{C} 7,{ }^{3} J_{119 \mathrm{Sn}-13 \mathrm{C}}=20 \mathrm{~Hz}\right), 57.3(\mathrm{C} 8) \mathrm{ppm} ;{ }^{119} \mathbf{S n}\left\{{ }^{1} \mathbf{H}\right\} \mathbf{N M R}(149 \mathrm{MHz}$, $\mathrm{CDCl}_{3}, \delta$ ): -133.0 ppm. Found: C, 65.74, H, 5.11. Calc. for $\mathrm{C}_{27} \mathrm{H}_{26} \mathrm{OSn}: \mathrm{C}, 66.28, \mathrm{H}, 5.13 \%$. 


\subsection{Synthesis of [2-( $\left.\left.\mathrm{MeOCH}_{2}\right) \mathrm{C}_{6} \mathrm{H}_{4}\right] \mathrm{MeSnCl}_{2}(218)$ :}

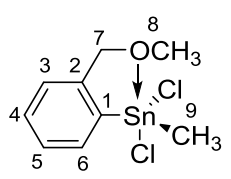

Compound $215(0.5 \mathrm{~g}, 2.48 \mathrm{mmol})$ in $20 \mathrm{~mL}$ of hexane was added to a $100 \mathrm{~mL}$ dry Schlenk flask equipped with a magnetic stirrer and septum. $1.6 \mathrm{M} n$-BuLi in hexane (1.55 mL, $2.48 \mathrm{mmol})$ was slowly added at $-78^{\circ} \mathrm{C}$. The cooling bath was removed for $30 \mathrm{~min}$, and the lithiated reagent allowed to react with a solution of $\mathrm{MeSnCl}_{3}(0.58 \mathrm{~g}, 2.08 \mathrm{mmol})$ in $10 \mathrm{~mL}$ of hexane/Et $2 \mathrm{O}(1: 1)$ at $-78^{\circ} \mathrm{C}$ added slowly to the reaction mixture. The reaction mixture was warmed to room temperature and stirred stirred for $3 \mathrm{~h}$ and solvent removed under reduced pressure. The residue was taken up in toluene $(20 \mathrm{~mL})$, decanted and the solvent removed under reduced pressure to give clear, brown oil. Yield: $0.67 \mathrm{~g}(98 \%)$.

${ }^{1}$ H NMR (400 MHz, $\left.\mathrm{CDCl}_{3}, \delta\right): 8.12$ (m, 1H, H6), 7.46 (m, 2H, H4, H5), 7.21 (m, 1H, H3), 4.77 $\left(\mathrm{s}, 2 \mathrm{H}, \mathrm{H} 7,{ }^{3} J_{119 \mathrm{Sn}-1 \mathrm{H}}=9.0 \mathrm{~Hz}\right), 3.42(\mathrm{~s}, 3 \mathrm{H}, \mathrm{H} 8), 1.26\left(\mathrm{~s}, 3 \mathrm{H}, \mathrm{H} 9,{ }^{1} J_{119 \mathrm{Sn}-1 \mathrm{H}}=83 \mathrm{~Hz},{ }^{1} J_{117 \mathrm{Sn}-1 \mathrm{H}}=\right.$ $79 \mathrm{~Hz})$ ppm; ${ }^{13} \mathbf{C}$ NMR (100 MHz, $\left.\mathrm{CDCl}_{3}, \delta\right): 141.7$ (C2), 136.3 (C4), 134.6 (C1), 131.1 (C3), 128.6 (C5), 125.2 (C6), 73.9 (C8), 58.8 (C7), 8.42 (C9) ppm; ${ }^{119} \mathbf{S n}\left\{{ }^{1} \mathbf{H}\right\} \mathbf{N M R}\left(149 \mathrm{MHz}, \mathrm{CDCl}_{3}\right.$,

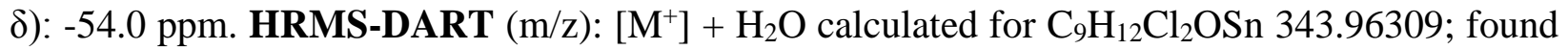
343.96336.

\subsection{Synthesis of [2-( $\left.\left.\mathrm{MeOCH}_{2}\right) \mathrm{C}_{6} \mathrm{H}_{4}\right] \mathrm{BuSnCl}_{2}$ (219):}

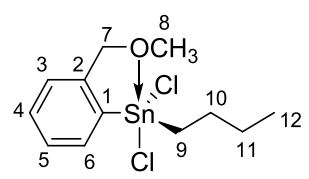

Compound 215 (2.0 g, $9.95 \mathrm{mmol})$ in $30 \mathrm{~mL}$ of hexane was added to a $100 \mathrm{~mL}$ dry Schlenk flask equipped with a magnetic stirrer and septum. The solution was cooled to $-78^{\circ} \mathrm{C}$ and $1.6 \mathrm{M} n$-BuLi in hexane $(6.2 \mathrm{~mL}, 9.92 \mathrm{mmol})$ was then added. The cooling bath was removed after $30 \mathrm{~min}$ and 
the lithiated reagent allowed to react with a solution of $n-\mathrm{BuSnCl}_{3}(2.75 \mathrm{~g}, 9.75 \mathrm{mmol})$ in $15 \mathrm{~mL}$ hexane/Et $2 \mathrm{O}(1: 1)$ at $0{ }^{\circ} \mathrm{C}$ that was slowly added to the reaction mixture and stirred for $3 \mathrm{~h}$. The solvent was removed under reduced pressure and the residue taken up in toluene $(25 \mathrm{~mL})$ and decanted. The solvent was removed under reduced pressure to give a clear, orange-brown coloured oil. The product was further purified by extraction with hot hexanes. Yield: $2.9 \mathrm{~g}(81 \%)$.

${ }^{1} \mathbf{H}$ NMR (400 MHz, $\mathrm{CDCl}_{3}, \delta$ ): 8.11 (d, 1H, H6), 7.41-7.47 (m, 2H, H4, H5), 7.22 (m, 1H, H3), 4.75 (s, 2H, H7), 3.65(s, 3H, H8), 1.85 (m, 4H, H10, H11), 1.43 (t, 2H, H9), 0.95 (t, 3H, H12) ppm; ${ }^{13} \mathrm{C}$ NMR $\left(100 \mathrm{MHz}, \mathrm{CDCl}_{3}, \delta\right): 141.8\left(\mathrm{C} 2,{ }^{2} J_{119 \mathrm{Sn}-13 \mathrm{C}}=48 \mathrm{~Hz}\right), 136.0\left(\mathrm{C} 5,{ }^{3} J_{119 \mathrm{Sn}-13 \mathrm{C}}=55\right.$ $\mathrm{Hz}), 134.9(\mathrm{C} 3), 130.8(\mathrm{C} 4), 128.3\left(\mathrm{C} 1,{ }^{1} J_{119 \mathrm{Sn}-13 \mathrm{C}}=81 \mathrm{~Hz},{ }^{1} J_{117 \mathrm{Sn}-13 \mathrm{C}}=78 \mathrm{~Hz}\right), 125.2\left(\mathrm{C} 6,{ }^{2} J_{119 \mathrm{Sn}-}\right.$ $\left.{ }_{13 \mathrm{C}}=74 \mathrm{~Hz},{ }^{2} J_{117 \mathrm{Sn}-13 \mathrm{C}}=70 \mathrm{~Hz}\right), 73.8\left(\mathrm{C} 7,{ }^{3} J_{119 \mathrm{Sn}-13 \mathrm{C}}=18 \mathrm{~Hz}\right), 59.0(\mathrm{C} 8), 27.9\left(\mathrm{C} 9,{ }^{1} J_{119 \mathrm{Sn}-13 \mathrm{C}}=\right.$ $\left.652 \mathrm{~Hz},{ }^{1} J_{117 \mathrm{Sn}-13 \mathrm{C}}=624 \mathrm{~Hz}\right), 27.1\left(\mathrm{C} 11,{ }^{3} J_{119 \mathrm{Sn}-13 \mathrm{C}}=40 \mathrm{~Hz}\right), 26.0\left(\mathrm{C} 10,{ }^{2} J_{119 \mathrm{Sn}-13 \mathrm{C}}=106 \mathrm{~Hz}\right.$, $\left.{ }^{2} J_{119 \mathrm{Sn}-13 \mathrm{C}}=102 \mathrm{~Hz}\right) 13.6(\mathrm{C} 12) \mathrm{ppm} ;{ }^{119} \mathbf{S n}\left\{{ }^{1} \mathbf{H}\right\} \mathbf{N M R}\left(149 \mathrm{MHz}, \mathrm{CDCl}_{3}, \delta\right):-61.0$ ppm. HRMSDART (m/z): $\left[\mathrm{M}^{+}\right]+\mathrm{H}_{2} \mathrm{O}$ calculated for $\mathrm{C}_{12} \mathrm{H}_{22} \mathrm{Cl}_{2} \mathrm{O}_{2} \mathrm{Sn}$ 386.00907; found 386.00918. Found: C, 39.36, H, 4.93. Calc. for $\mathrm{C}_{27} \mathrm{H}_{26} \mathrm{OSn}$ : C, 39.18, $\mathrm{H}, 4.93 \%$.

\subsection{Synthesis of [2-( $\left.\left.\mathrm{MeOCH}_{2}\right) \mathrm{C}_{6} \mathrm{H}_{4}\right] \mathrm{PhSnCl}_{2}(220)$ :}

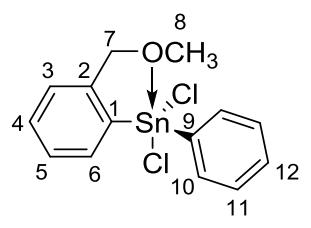

Compound $215(1.0 \mathrm{~g}, 4.98 \mathrm{mmol})$ in $30 \mathrm{~mL}$ of hexanes was added to a $100 \mathrm{~mL}$ dry Schlenk flask equipped with a magnetic stirrer and septum. The solution was cooled to $-78^{\circ} \mathrm{C}$ and $1.6 \mathrm{M} n$ - $\mathrm{BuLi}$ in hexane (3.1 mL, $4.96 \mathrm{mmol}$ ) was then added. The cooling bath was removed for $30 \mathrm{~min}$ and the lithiated $C, O$-ligand was allowed to react with a solution of $\mathrm{PhSnCl}_{3}(1.46 \mathrm{~g}, 4.83 \mathrm{mmol})$ in $15 \mathrm{~mL}$ hexane/Et $2 \mathrm{O}(1: 1)$ at $-78^{\circ} \mathrm{C}$. The reaction mixture was stirred for $3 \mathrm{~h}$ and solvent removed under reduced pressure. The product was extracted with hot hexanes and precipitated as a white coloured 
powder at $-30^{\circ} \mathrm{C}$. The yield of this product was not obtained due to the presence of a minor impurity found in the ${ }^{119}$ Sn NMR spectrum.

${ }^{1} \mathbf{H}$ NMR (400 MHz, $\left.\mathrm{CDCl}_{3}, \delta\right): 7.76$ (m, 3H, H6, H10), 7.59 (m, 3H, H11, H12), 7.52 (m, 1H, H4), 7.36 (m, 1H, H5), 7.18 (td, 1H, H3), 4.58 (s, 2H, H7), 3.45 (s, 3H, H8) ppm; ${ }^{13}$ C NMR (100 $\left.\mathrm{MHz}, \mathrm{CDCl}_{3}, \delta\right): 137.6(\mathrm{C} 2), 137.0\left(\mathrm{C} 1, J_{119 \mathrm{Sn}-13 \mathrm{C}}=72 \mathrm{~Hz}\right), 135.1\left(\mathrm{C} 10, J_{119 \mathrm{Sn}-13 \mathrm{C}}=64 \mathrm{~Hz},{ }^{1} J_{117 \mathrm{Sn}-}\right.$ $13 \mathrm{C}=60 \mathrm{~Hz}), 132.6(\mathrm{C} 5), 131.9\left(\mathrm{C} 6, J_{119 \mathrm{Sn}-13 \mathrm{C}}=20 \mathrm{~Hz}\right), 129.8\left(\mathrm{C} 9, J_{119 \mathrm{Sn}-13 \mathrm{C}}=86 \mathrm{~Hz}, J_{117 \mathrm{Sn}-13 \mathrm{C}}=\right.$ $82 \mathrm{~Hz}), 129.1$ (C4), 129.0 (C12), 127.5 (C3), 122.8 (C11), 73.9 (C7), 58.6 (C8) ppm; ${ }^{119} \mathrm{Sn}\left\{{ }^{1} \mathbf{H}\right\} \mathbf{N M R}\left(149 \mathrm{MHz}, \mathrm{CDCl}_{3}, \delta\right):-28.2 \mathrm{ppm}(-48.2 \mathrm{ppm}$, impurity)

\subsection{Synthesis of [2-( $\left.\left.\mathrm{MeOCH}_{2}\right) \mathrm{C}_{6} \mathrm{H}_{4}\right] \mathrm{Ph}_{2} \mathrm{SnCl}(221)$ :}

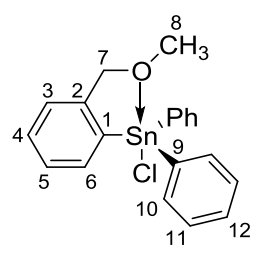

Method 1: Compound 215 (2.0 g, $9.95 \mathrm{mmol})$ in $20 \mathrm{~mL}$ of hexane was added to a $100 \mathrm{~mL}$ dry Schlenk flask equipped with a magnetic stirrer and septum. The solution was cooled to $-78^{\circ} \mathrm{C}$ and 1.6 $\mathrm{M} n$-BuLi in hexane $(1.55 \mathrm{~mL}, 9.92 \mathrm{mmol})$ was then added. The cooling bath was removed after 30 min and the lithiated $C, O$-ligand was allowed to react with a solution of $\mathrm{Ph}_{2} \mathrm{SnCl}_{2}(3.17 \mathrm{~g}$, $9.22 \mathrm{mmol})$ in $10 \mathrm{~mL}$ hexane/Et $2 \mathrm{O}(1: 1)$ at $-78^{\circ} \mathrm{C}$. The reaction mixture was stirred for further $3 \mathrm{~h}$ and solvent removed under reduced pressure. The residue was taken up in toluene ( $20 \mathrm{~mL})$. After decanting, the solvent was removed under reduced pressure to give a mixed semi-solid product. Hexane was added to the mixture of products and a solid product separated by filtration. The residual solvent was removed to obtain a white solid of (221). Yield: $1.5 \mathrm{~g}(38 \%)$ m.p. $155^{\circ} \mathrm{C}$.

Method 2: A $1.0 \mathrm{M} \mathrm{HCl}$ solution in $\mathrm{Et}_{2} \mathrm{O}(0.43 \mathrm{~mL}, 0.43 \mathrm{mmol})$ was added to a solution of 112 ( $0.21 \mathrm{~g}, 0.44 \mathrm{mmol})$ in $25 \mathrm{~mL}$ of dry $\mathrm{C}_{6} \mathrm{H}_{6}$. The resulting yellow coloured solution was stirred for 
$30 \mathrm{~min}$. The solvent was removed under reduced pressure and the crude product extracted with hot hexane. The solution was cooled to $-20^{\circ} \mathrm{C}$ and a white coloured solid precipitated overnight. Yield: $0.15 \mathrm{~g}(81 \%)$, m.p. $155^{\circ} \mathrm{C}$.

${ }^{1} \mathbf{H}$ NMR (400 MHz, $\left.\mathrm{CDCl}_{3}, \delta\right): 8.35$ (d, 1H, H6), 7.72 (m, 4H, H11), 7.50 (m, 6H, H10,12), 7.4 (m, 2H, H3, H4), 7.24 (m, 1H, H5), 4.74 (s, 2H, H7, $\left.{ }^{3} J_{119 \mathrm{Sn}-1 \mathrm{H}}=11 \mathrm{~Hz}\right), 3.13(\mathrm{~s}, 3 \mathrm{H}, \mathrm{H} 8) \mathrm{ppm} ;{ }^{13} \mathrm{C}$ NMR $\left(100 \mathrm{MHz}, \mathrm{CDCl}_{3}, \delta\right): 142.8\left(\mathrm{C} 9,{ }^{1} J_{119 \mathrm{Sn} 13 \mathrm{C}}=42 \mathrm{~Hz}\right), 141.0(\mathrm{C} 2), 137.7\left(\mathrm{C} 1,{ }^{1} J_{119 \mathrm{Sn} 13 \mathrm{C}}=41\right.$ $\mathrm{Hz}), 135.7\left(\mathrm{C} 5,{ }^{3} J_{119 \mathrm{Sn}-13 \mathrm{C}}=50 \mathrm{~Hz},{ }^{3} J_{117 \mathrm{Sn}-13 \mathrm{C}}=47 \mathrm{~Hz}\right), 133.1(\mathrm{C} 4), 129.8\left(\mathrm{C} 3,{ }^{3} J_{119 \mathrm{Sn} 13 \mathrm{C}}=14 \mathrm{~Hz}\right)$, $129.5\left(\mathrm{C} 12,{ }^{4} J_{119 \mathrm{Sn} 13 \mathrm{C}}=15 \mathrm{~Hz}\right), 128.7\left(\mathrm{C} 10,{ }^{2} J_{119 \mathrm{Sn}-13 \mathrm{C}}=70 \mathrm{~Hz}, J_{117 \mathrm{Sn}-13 \mathrm{C}}=67 \mathrm{~Hz}\right), 128.2(\mathrm{C} 6$, $\left.{ }^{2} J_{119 S n 13 \mathrm{C}}=68 \mathrm{~Hz}\right), 124.8\left(\mathrm{C} 11,{ }^{3} J_{119 \mathrm{Sn} 13 \mathrm{C}}=63 \mathrm{~Hz}\right), 74.4(\mathrm{C} 7), 58.4(\mathrm{C} 8) \mathrm{ppm} ;{ }^{119} \mathbf{S n}\left\{{ }^{1} \mathbf{H}\right\} \mathbf{N M R}$ (149 $\left.\mathrm{MHz}, \mathrm{CDCl}_{3}, \delta\right):-127.0$ ppm. HRMS-DART $(\mathrm{m} / \mathrm{z}):\left[\mathrm{M}^{+}\right]+\mathrm{H}_{2} \mathrm{O}$ calculated for $\mathrm{C}_{20} \mathrm{H}_{21} \mathrm{ClO}_{2} \mathrm{Sn} 448.05085$; found 448.05098 .

\subsection{Synthesis of $\left[2-\left(\mathrm{MeOCH}_{2}\right) \mathrm{C}_{6} \mathrm{H}_{4}\right] n-\mathrm{Bu}_{2} \mathrm{SnCl}(223)$ :}

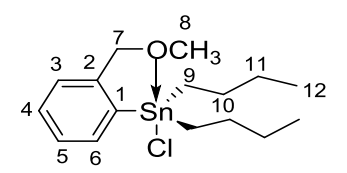

Compound 215 (1.0 g, $4.98 \mathrm{mmol})$ in $30 \mathrm{~mL}$ of hexanes was added to a $100 \mathrm{~mL}$ dry Schlenk flask equipped with a magnetic stirrer and septum. The solution was cooled to $-78^{\circ} \mathrm{C}$ and $1.6 \mathrm{M} n$-BuLi in hexane (3.1 mL, $4.96 \mathrm{mmol})$ was then added. The cooling bath was removed for $30 \mathrm{~min}$ and the lithiated $C, O$-ligand was allowed to react with a solution of $n-\mathrm{Bu}_{2} \mathrm{SnCl}_{2}(1.47 \mathrm{~g}, 4.83 \mathrm{mmol})$ in 15 $\mathrm{mL}$ hexane/Et $2 \mathrm{O}(1: 1)$ at $-78{ }^{\circ} \mathrm{C}$. The reaction mixture was stirred for $3 \mathrm{~h}$ and solvent removed under reduced pressure. The ${ }^{119} \mathrm{Sn}$ NMR of the crude product showed six resonances. An attempt to purify the crude product by extraction with hot hexanes was unsuccessful. The product was stored in glove box and solid material separated out of the oil. No yield was recorded. NMR chemical shift resonances attributable to 223 are listed below. 
${ }^{1} \mathbf{H}$ NMR $\left(400 \mathrm{MHz}, \mathrm{CDCl}_{3}, \delta\right): 8.27$ (m, 1H, H6), 7.58 (dd, 1H, H5), 7.36 (m, 1H, H4), 7.15 (m, 1H, H3), 4.71 (s, 2H, H7), 3.60 (s, 3H, H8), 1.79 (m, 8H, H10, H11), 1.43 (m, 4H, H9), 0.98 (m, 6H, H12) ppm; ${ }^{13} \mathrm{C}$ NMR (100 MHz, $\mathrm{CDCl}_{3}, \delta$ ): 141.7 (C2), 137.3 (C1), 132.6 (C6), 128.8 (C4), 127.5 (C5), 124.1 (C3), $75.1(\mathrm{C} 7), 58.5(\mathrm{C} 8), 32.9(\mathrm{C} 9$, isomer 1), 32.3 (C9, isomer 2), 27.3 (C10, isomer 1), 27.1 (C10, isomer 2), 26.6 (C11, isomer 1), 26.3 (C11, isomer 2), 13.6 (C12) ppm; ${ }^{119} \mathbf{S n}\left\{{ }^{1} \mathbf{H}\right\}$ NMR $\left(149 \mathrm{MHz}, \mathrm{CDCl}_{3}, \delta\right):-91.0,-127.0 \mathrm{ppm}$. HRMS-DART $(\mathrm{m} / \mathrm{z})$ : calcd for $\mathrm{C}_{16} \mathrm{H}_{27} \mathrm{ClOSn} 390.08$, Found $\left[\mathrm{M}+\mathrm{H}_{2} \mathrm{O}\right] 408.1$

\subsection{Synthesis of $\left[\left(2-\left(\mathrm{MeOCH}_{2}\right) \mathrm{C}_{6} \mathrm{H}_{4}\right)_{2}\right] n-\mathrm{BuSnCl}(224)$ :}

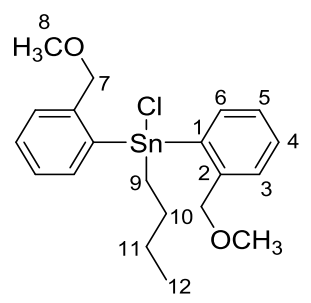

An equimolar amount of $3.1 \mathrm{~mL}$ of $n-\mathrm{BuLi}(3.1 \mathrm{~mL}, 4.97 \mathrm{mmol})$ was added dropwise at $-78{ }^{\circ} \mathrm{C}$ to a solution of $215(1.0 \mathrm{~g}, 4.97 \mathrm{mmol})$ in $15 \mathrm{~mL}$ of $\mathrm{Et}_{2} \mathrm{O}$ and stirred for an additional $2 \mathrm{~h}$. The resulting solution was added dropwise to a suspension of $n-\mathrm{BuSnCl}_{3}(0.686 \mathrm{~g}, 2.43 \mathrm{mmol})$ in 20 $\mathrm{mL}$ of hexane/Et $2 \mathrm{O}$ at $0{ }^{\circ} \mathrm{C}$, followed by stirring for further $3 \mathrm{~h}$. Purification of the crude product was unsuccessful. There are unassigned chemical shifts in ${ }^{1} \mathrm{H}$ and ${ }^{13} \mathrm{C}$ NMR spectrum indicating the presence of impurities.

${ }^{1} \mathbf{H}$ NMR (400 MHz, $\left.\mathrm{CDCl}_{3}, \delta\right): 7.74$ (d, 1H, H6), 7.43 (dd, 1H, H5), 7.28 (m, 2H, H3), 7.45 (m, 1H, H4), 3.052-3.45 (4s, 3H, H8), 4.38-4.64 (5s, 2H, H7) 2.15- 1.2 (m, 6H, H9, H10, H11), 1.1$0.8(\mathrm{~m}, 3 \mathrm{H}, \mathrm{H12}) \mathrm{ppm} ;{ }^{3} \mathbf{C}$ NMR $\left(100 \mathrm{MHz}, \mathrm{CDCl}_{3}, \delta\right): 142.9\left(\mathrm{C} 1,{ }^{1} J_{119 \mathrm{Sn}-13 \mathrm{C}}=370 \mathrm{~Hz}\right), 139.9$ $(\mathrm{C} 2), 136.3\left(\mathrm{C} 6, J_{119 \mathrm{Sn}-13 \mathrm{C}}=43 \mathrm{~Hz}\right), 132.5(\mathrm{C} 5), 129.1\left(\mathrm{C} 3, J_{119 \mathrm{Sn}-13 \mathrm{C}}=13 \mathrm{~Hz}\right), 128.9(\mathrm{C} 4),[128.4$, 127.6, 127.4, 126.6 impurities], $75.6(\mathrm{C} 7), 58.1(\mathrm{C} 8), 27.8\left(\mathrm{C} 10,{ }^{2} J_{119 \mathrm{Sn}-13 \mathrm{C}}=93 \mathrm{~Hz}\right), 26.6(\mathrm{C} 11$, 
$\left.{ }^{3} J_{119 \mathrm{Sn}-13 \mathrm{C}}=30 \mathrm{~Hz}\right), 20.8\left(\mathrm{C} 9,{ }^{1} J_{119 \mathrm{Sn}-13 \mathrm{C}}=549 \mathrm{~Hz}, J_{117 \mathrm{Sn}-13 \mathrm{C}}=525 \mathrm{~Hz}\right), 13.7(\mathrm{C} 12) \mathrm{ppm} ;$

${ }^{119} \mathbf{S n}\left\{{ }^{1} \mathbf{H}\right\} \mathbf{N M R}\left(149 \mathrm{MHz}, \mathrm{CDCl}_{3}, \delta\right):-73.0 \mathrm{ppm}$.

\subsection{Synthesis of [(2-( $\left.\left.\left.\mathrm{MeOCH}_{2}\right) \mathrm{C}_{6} \mathrm{H}_{4}\right)_{2}\right] \mathrm{PhSnCl}(225)$ :}

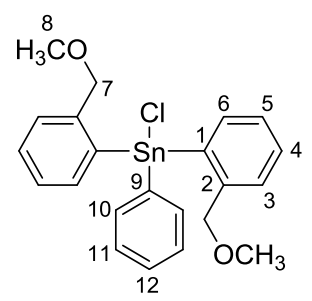

An equimolar amount of $3.1 \mathrm{~mL}$ of $n$ - $\mathrm{BuLi}(3.1 \mathrm{~mL}, 4.97 \mathrm{mmol})$ was added dropwise at $-78{ }^{\circ} \mathrm{C}$ to a solution of $215(1.0 \mathrm{~g}, 4.97 \mathrm{mmol})$ in $15 \mathrm{~mL}$ of $\mathrm{Et}_{2} \mathrm{O}$ and stirred for an additional $2 \mathrm{~h}$. The resulting solution was added dropwise to a suspension of $\mathrm{PhSnCl}_{3}(0.734 \mathrm{~g}, 2.43 \mathrm{mmol})$ in $20 \mathrm{~mL}$ of hexane/Et $2 \mathrm{O}$ at room temperature, followed by stirring for further $3 \mathrm{~h}$. An attempt to purify the crude product was attempted by washing with hexane, but was unsuccessful.

${ }^{1} \mathbf{H}$ NMR $\left(400 \mathrm{MHz}, \mathrm{CDCl}_{3}, \delta\right.$ ): 7.89 (dd, 1H, $\left.\mathrm{H}_{\text {aryl }}\right), 7.73$ (br s, $\left.1 \mathrm{H}, \mathrm{H}_{\text {aryl }}\right), 7.65$ (br s, $1 \mathrm{H}, \mathrm{H}_{\text {aryl }}$ ), 7.58 (br s, 3H, $\left.\mathrm{H}_{\text {aryl }}\right), 7.39$ (m, 7H, $\left.\mathrm{H}_{\text {aryl }}\right), 4.31$ (s, 2H, H7), 2.81 (s, 3H, H8) ppm; ${ }^{13}$ C NMR (100 $\left.\mathrm{MHz}, \mathrm{CDCl}_{3}, \delta\right): 144.9\left(\mathrm{C} 1, J_{119 \mathrm{Sn}-13 \mathrm{C}}=29 \mathrm{~Hz}\right), 140.2(\mathrm{C} 2), 138.0\left(\mathrm{C} 9, J_{119 \mathrm{Sn}-13 \mathrm{C}}=40 \mathrm{~Hz}\right), 137.2$ $(\mathrm{C} 11), 135.0(\mathrm{C} 12), 131.8\left(\mathrm{C} 10,{ }^{2} J_{119 \mathrm{Sn}-13 \mathrm{C}}=18 \mathrm{~Hz}\right), 129.7(\mathrm{C} 4), 128.6\left(\mathrm{C} 6, J_{119 \mathrm{Sn}-13 \mathrm{C}}=11 \mathrm{~Hz}\right)$, 128.1 (C3), 127.3 (C5), $75.9\left(\mathrm{C} 7,{ }^{3} J_{119 S n-13 \mathrm{C}}=23 \mathrm{~Hz}\right), 57.5(\mathrm{C} 8) \mathrm{ppm} ;{ }^{119} \mathbf{S n}\left\{{ }^{1} \mathbf{H}\right\} \mathbf{N M R}(149 \mathrm{MHz}$, $\left.\mathrm{CDCl}_{3}, \delta\right):-136.0 \mathrm{ppm}$.

\subsection{Synthesis of [2-( $\left.\left.\mathrm{MeOCH}_{2}\right) \mathrm{C}_{6} \mathrm{H}_{4}\right] \mathrm{MeSnH}_{2}$ (226):}

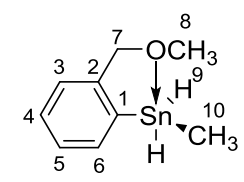

A solution of $218\left(0.52 \mathrm{~g}, 1.6 \mathrm{mmol}\right.$ in $15 \mathrm{~mL}$ of $\left.\mathrm{Et}_{2} \mathrm{O}\right)$ was added dropwise to a suspension of $\mathrm{LiAlH}_{4}\left(0.34 \mathrm{~g}, 3.0 \mathrm{mmol}\right.$ in $15 \mathrm{~mL}$ of $\left.\mathrm{Et}_{2} \mathrm{O}\right)$, and stirred at $0{ }^{\circ} \mathrm{C}$ for $3 \mathrm{~h}$. The reaction was quenched 
with $10 \mathrm{~mL}$ of degassed and chilled water. The organic layer was separated and the aqueous layer extracted with $\mathrm{Et}_{2} \mathrm{O}(3 \times 10 \mathrm{~mL})$. The combined organic layers were dried over anhydrous $\mathrm{MgSO}_{4}$. The solvent was removed under reduced pressure to yield $\mathbf{2 2 6}$ as a yellow coloured oil. Yield: 0.35 $\mathrm{g}(85 \%)$. The product start decomposing as soon as the temperature start rising after the removal of solvent. The ${ }^{1} \mathrm{H}$ and ${ }^{13} \mathrm{C}$ NMR of 226 is not included. The ${ }^{119} \mathrm{Sn}$ NMR data and mass spectrometry are listed below.

${ }^{119} \operatorname{Sn}\left\{{ }^{1}\right.$ H $\}$ NMR (149 MHz, $\left.\mathrm{C}_{6} \mathrm{D}_{6}, \delta\right):-221.0 \mathrm{ppm}$. HRMS-DART (m/z): [M+ $]$ - H calculated for $\mathrm{C}_{9} \mathrm{H}_{13} \mathrm{OSn} 256.99967$; found 256.99884.

\subsection{Synthesis of [2-( $\left.\left.\mathrm{MeOCH}_{2}\right) \mathrm{C}_{6} \mathrm{H}_{4}\right] n-\mathrm{BuSnH}_{2}(227)$ :}

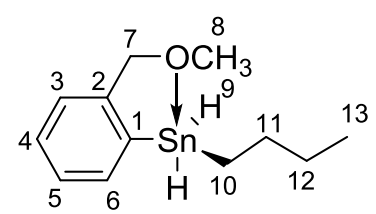

A solution of $219\left(0.5 \mathrm{~g}, 1.35 \mathrm{mmol}\right.$ in $15 \mathrm{~mL}$ of $\left.\mathrm{Et}_{2} \mathrm{O}\right)$ was added dropwise to a suspension of $\mathrm{LiAlH}_{4}\left(0.114 \mathrm{~g}, 7.0 \mathrm{mmol}\right.$ in $15 \mathrm{~mL}$ of $\left.\mathrm{Et}_{2} \mathrm{O}\right)$, and stirred at $0{ }^{\circ} \mathrm{C}$ for $3 \mathrm{~h}$. The reaction was quenched with $5 \mathrm{~mL}$ of degassed and chilled water. The organic layer was separated and the aqueous layer extracted with $\mathrm{Et}_{2} \mathrm{O}(3 \times 10 \mathrm{~mL})$. The combined organic layers were dried over anhydrous $\mathrm{MgSO}_{4}$. The solvent was removed under reduced pressure to yield $\mathbf{2 2 5}$ as a yellow coloured oil. Yield: $0.28 \mathrm{~g}(70 \%)$.

${ }^{1} \mathbf{H}$ NMR (400 MHz, $\left.\mathrm{CDCl}_{3}, \delta\right): 7.80$ (m, 1H, H6), 7.21 (m, 2H, H4, H5), 7.06 (m, 1H, H3), 5.78 $\left(\mathrm{s}, 2 \mathrm{H}, \mathrm{H} 9,{ }^{1} J_{119 \mathrm{Sn}-1 \mathrm{H}}=1727 \mathrm{~Hz},{ }^{1} J_{117 \mathrm{Sn}-1 \mathrm{H}}=1677 \mathrm{~Hz}\right), 4.27(\mathrm{~s}, 2 \mathrm{H}, \mathrm{H} 7), 3.12(\mathrm{~s}, 3 \mathrm{H}, \mathrm{H} 8), 1.70(\mathrm{~m}$, 2H, H11), 1.42 (m, 2H, H10), 1.30 (m, 2H, H12), 0.96 (t, 3H, H13) ppm; ${ }^{13}$ C NMR (100 MHz, $\left.\mathrm{CDCl}_{3}, \delta\right): 145.0\left(\mathrm{C} 2,{ }^{2} J_{119 \mathrm{Sn}-13 \mathrm{C}}=26 \mathrm{~Hz}\right), 139.3\left(\mathrm{C} 6,{ }^{2} J_{119 \mathrm{Sn}-13 \mathrm{C}}=39 \mathrm{~Hz}\right), 137.2\left(\mathrm{C} 1,{ }^{1} J_{119 \mathrm{Sn}-13 \mathrm{C}}=\right.$ $\left.522 \mathrm{~Hz},{ }^{1} J_{117 \mathrm{Sn}-13 \mathrm{C}}=498 \mathrm{~Hz}\right), 128.7\left(\mathrm{C} 5,{ }^{3} J_{119 \mathrm{Sn}-13 \mathrm{C}}=12 \mathrm{~Hz}\right), 127.7(\mathrm{C} 4), 127.4(\mathrm{C} 3), 76.1(\mathrm{C} 7$, $\left.{ }^{2} J_{119 \mathrm{Sn}-13 \mathrm{C}}=19 \mathrm{~Hz}\right), 57.1(\mathrm{C} 8), 30.6\left(\mathrm{C} 11,{ }^{2} J_{119 \mathrm{Sn}-13 \mathrm{C}}=22 \mathrm{~Hz}\right), 27.1\left(\mathrm{C} 10,{ }^{1} J_{119 \mathrm{Sn}-13 \mathrm{C}}=67 \mathrm{~Hz}\right), 13.9$ 
(C12), $10.2(\mathrm{C} 13)$ ppm; ${ }^{119} \mathbf{S n}\left\{{ }^{1} \mathbf{H}\right\} \mathbf{N M R}\left(149.21 \mathrm{MHz}, \mathrm{C}_{6} \mathrm{D}_{6}, \delta\right):-210.0$ ppm. HRMS-DART (m/z): [ $\left.\mathrm{M}^{+}\right]$calculated for ${ }^{12} \mathrm{C}_{12}{ }^{1} \mathrm{H}_{19}{ }^{16} \mathrm{O}^{116} \mathrm{Sn} 295.04534$; found 295.04595.

\subsection{Polymerization of 227:}

TMEDA (0.16 mL, $0.124 \mathrm{~g} 1.07 \mathrm{mmol})$ was added to a solution of $227(0.32 \mathrm{~g}, \mathrm{mmol})$ in $10 \mathrm{~mL}$ of $\mathrm{Et}_{2} \mathrm{O}$. The reaction mixture turned yellow in colour and was stirred for $36 \mathrm{~h}$. The product was unsuccesfuly precipitated in hexane, petroleum ether. Limited analysis was undertaken.

${ }^{119} \mathbf{S n}\left\{{ }^{1} \mathbf{H}\right\}$ NMR $\left(149 \mathrm{MHz}, \mathrm{C}_{6} \mathrm{D}_{6}, \delta\right):-196.0$.

\subsection{Synthesis of 2-Bromo- $N, N$-dimethylbenzylamine (228):}

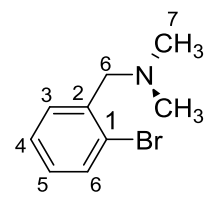

$16.0 \mathrm{~mL}$ of a $33 \%$ dimethylamine solution $(120.0 \mathrm{mmol})$ was added dropwise to $4.0 \mathrm{~g}(16.0 \mathrm{mmol})$ of 2-bromo-benzylbromide dissolved in $30 \mathrm{~mL}$ of DCM in $100 \mathrm{~mL}$ Schlenk flask. The reaction mixture was heated at $42{ }^{\circ} \mathrm{C}$ under $\mathrm{N}_{2}$ in a closed system for $7 \mathrm{~h}$. The product was extracted with $3 \mathrm{M} \mathrm{HCl}(3 \times 30 \mathrm{~mL})$ and the extract neutralized with an alkaline solution containing $20 \% \mathrm{NaOH}$. The basic product was isolated by extraction with DCM. NMR data $\left({ }^{1} \mathrm{H},{ }^{13} \mathrm{C}\right)$ agreed well with the reported literature. ${ }^{158}$ Yield: $3.0 \mathrm{~g}(87 \%)$.

${ }^{1} \mathbf{H}$ NMR (400 MHz, $\left.\mathrm{CDCl}_{3}, \delta\right): 7.53(\mathrm{~d}, 1 \mathrm{H}, \mathrm{H} 3), 7.42(\mathrm{~d}, 1 \mathrm{H}, \mathrm{H} 6), 7.27$ (m, 1H, H4), $7.11(\mathrm{~m}$, 1H, H5), 3.52 (s, 2H, H6), 2.30 (s, 6H, H7) ppm; ${ }^{13} \mathbf{C}$ NMR (100 MHz, $\left.\mathrm{CDCl}_{3}, \delta\right): 138.1(\mathrm{C} 2)$, 132.7 (C3), 130.9 (C6), 128.4 (C5), 127.2 (C4), 124.7 (C1), 63.3 (C6), 45.5 (C7) ppm.

\subsection{Synthesis of [2-( $\left.\left.\mathrm{Me}_{2} \mathrm{NCH}_{2}\right) \mathrm{C}_{6} \mathrm{H}_{4}\right] \mathrm{Li}(229):{ }^{176}$}

$63.0 \mathrm{~mL}(100.0 \mathrm{mmol})$ of $1.6 \mathrm{M}$ solution of $n-\mathrm{BuLi}$ in hexane was added dropwise to a solution of $13.52 \mathrm{~g}$ (100.0 mmol) of $\mathbf{2 2 8}$ in $150 \mathrm{~mL} \mathrm{Et} 2 \mathrm{O}$. The solution became yellow and hazy during the 
addition of $n$-BuLi. The white solid started to precipitate after $1 \mathrm{~h}$. The reaction mixture was stirred overnight. The solid product was separated by decantation and the remaining residual solvent was removed under reduced pressure. Yield: $12.1 \mathrm{~g}(87 \%)$.

\subsection{Synthesis of [2-( $\left.\left.\mathrm{Me}_{2} \mathrm{NCH}_{2}\right) \mathrm{C}_{6} \mathrm{H}_{4}\right] \mathrm{MeSnCl}_{2}(33)$ :}

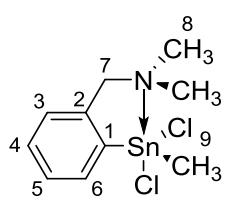

A suspension of $229(0.294 \mathrm{~g}, 2.08 \mathrm{mmol})$ in $30 \mathrm{~mL} \mathrm{Et}_{2} \mathrm{O}$ was added dropwise over $30 \mathrm{~min}$ to a solution of $\mathrm{MeSnCl}_{3}(0.500 \mathrm{~g}, 2.08 \mathrm{mmol})$ in $20 \mathrm{~mL} \mathrm{Et}{ }_{2} \mathrm{O}$ at $-78{ }^{\circ} \mathrm{C}$. The reaction mixture was stirred for $3 \mathrm{~h}$ at room temperature. The salt was removed by decantation in the glove box, and the solvent removed under reduced pressure. The product was purified by extraction with toluene. The product was white coloured solid. NMR data $\left({ }^{1} \mathrm{H},{ }^{13} \mathrm{C},{ }^{119} \mathrm{Sn}\right)$ agreed with the reported literature. ${ }^{26}$ Yield: $0.35 \mathrm{~g}(85 \%)$

${ }^{1} \mathbf{H}$ NMR $\left(400 \mathrm{MHz}, \mathrm{CDCl}_{3}, \delta\right): 8.18\left(\mathrm{~m}, 1 \mathrm{H}, \mathrm{H} 6,{ }^{2} J_{119 \mathrm{Sn}-1 \mathrm{H}}=100 \mathrm{~Hz},{ }^{2} J_{117 \mathrm{Sn}-1 \mathrm{H}}=98.0 \mathrm{~Hz}\right), 7.44$ (m, 2H, H4, H5), 7.20 (m, 1H, H3), 3.76 (s, 2H, H7), 2.43 (s, 6H, H8), 1.26 (s, 3H, H9, ${ }^{1} J_{119 S n-1 H}$ $\left.=84 \mathrm{~Hz},{ }^{1} J_{117 \mathrm{Sn}-1 \mathrm{H}}=76 \mathrm{~Hz}\right) \mathrm{ppm} ;{ }^{13} \mathbf{C} \mathbf{N M R}\left(100 \mathrm{MHz}, \mathrm{CDCl}_{3}, \delta\right): 141.9\left(\mathrm{C} 2,{ }^{2} J_{119 \mathrm{Sn}-13 \mathrm{C}}=52 \mathrm{~Hz}\right.$, $\left.{ }^{2} J_{117 \mathrm{Sn}-13 \mathrm{C}}=49 \mathrm{~Hz}\right), 138.5(\mathrm{C} 1), 137.0\left(\mathrm{C} 3,{ }^{3} J_{119 \mathrm{Sn}-13 \mathrm{C}}=67 \mathrm{~Hz},{ }^{2} J_{117 \mathrm{Sn}-13 \mathrm{C}}=65 \mathrm{~Hz}\right), 131.2(\mathrm{C} 4)$, $128.7\left(\mathrm{C} 6,{ }^{2} J_{119 \mathrm{Sn}-13 \mathrm{C}}=94 \mathrm{~Hz}\right), 127.4\left(\mathrm{C} 5,{ }^{3} J_{119 \mathrm{Sn}-13 \mathrm{C}}=79 \mathrm{~Hz},{ }^{3} J_{117 \mathrm{Sn}-13 \mathrm{C}}=76 \mathrm{~Hz}\right), 63.2\left(\mathrm{C} 7,{ }^{3} J_{119 \mathrm{Sn}-}\right.$ $\left.{ }_{13 \mathrm{C}}=39 \mathrm{~Hz}\right), 44.9(\mathrm{C} 8), 8.0\left(\mathrm{C} 9,{ }^{1} J_{119 \mathrm{Sn}-13 \mathrm{C}}=696 \mathrm{~Hz},{ }^{1} J_{117 \mathrm{Sn}-13 \mathrm{C}}=669 \mathrm{~Hz}\right) \mathrm{ppm} ;{ }^{119} \mathbf{S n}\left\{{ }^{1} \mathbf{H}\right\} \mathbf{N M R}$ (149 $\left.\mathrm{MHz}, \mathrm{CDCl}_{3}, \delta\right):-96.4 \mathrm{ppm}$.

\subsection{Synthesis of $\left[2-\left(\mathrm{Me}_{2} \mathrm{NCH}_{2}\right) \mathrm{C}_{6} \mathrm{H}_{4}\right] n-\mathrm{BuSnCl}_{2}(37)$ :}

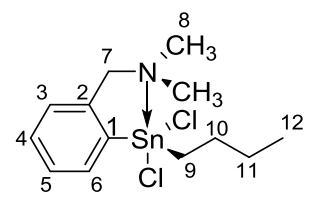


A suspension of $229(2.0 \mathrm{~g}, 14.2 \mathrm{mmol})$ in $30 \mathrm{~mL} \mathrm{Et}_{2} \mathrm{O}$ was added dropwise over $30 \mathrm{~min}$ to solution of $n$ - $\mathrm{BuSnCl}_{3}(4.0 \mathrm{~g}, 14.2 \mathrm{mmol})$ in $20 \mathrm{mLEt}_{2} \mathrm{O}$ at $0{ }^{\circ} \mathrm{C}$. The reaction mixture was stirred overnight. The crude product was separated by decantation in the glove box. The solvent was removed under reduced pressure. The product was extracted with hot hexane and a white solid product obtained. NMR data $\left({ }^{1} \mathrm{H},{ }^{13} \mathrm{C},{ }^{119} \mathrm{Sn}\right)$ agreed with the reported literature. ${ }^{29 b, 51}$ Yield: $3.9 \mathrm{~g}(73 \%)$.

${ }^{1} \mathbf{H}$ NMR (400 MHz, $\left.\mathrm{CDCl}_{3}, \delta\right): 8.18$ (m, 1H, H6), 7.41 (m, 2H, H4, H5), 7.20 (m, 1H, H3), 3.74 (s, 2H, H7), 2.43 (s, 6H, H8), 1.92 (m, 2H, H10), 1.80 (t, 2H, H9), 1.45 (sex, 2H, H11), 0.95 (t, 3H, H12) ppm; ${ }^{13} \mathrm{C}$ NMR (100 MHz, $\left.\mathrm{CDCl}_{3}, \delta\right): 141.1\left(\mathrm{C} 2,{ }^{2} J_{119 \mathrm{Sn}-13 \mathrm{C}}=48 \mathrm{~Hz}\right), 139.6(\mathrm{C} 1), 137.0$ $\left(\mathrm{C} 6,{ }^{2} J_{119 \mathrm{Sn}-13 \mathrm{C}}=65 \mathrm{~Hz},{ }^{2} J_{117 \mathrm{Sn}-13 \mathrm{C}}=63 \mathrm{~Hz}\right), 130.9\left(\mathrm{C} 4,{ }^{4} J_{119 \mathrm{Sn}-13 \mathrm{C}}=16 \mathrm{~Hz}\right), 128.6\left(\mathrm{C} 5,{ }^{3} J_{119 \mathrm{Sn}-13 \mathrm{C}}\right.$ $\left.=89 \mathrm{~Hz},{ }^{3} J_{119 \mathrm{Sn}-13 \mathrm{C}}=85 \mathrm{~Hz}\right), 127.4\left(\mathrm{C} 3,{ }^{3} J_{119 \mathrm{Sn}-13 \mathrm{C}}=74 \mathrm{~Hz},{ }^{3} J_{117 \mathrm{Sn}-13 \mathrm{C}}=72 \mathrm{~Hz}\right), 63.4\left(\mathrm{C} 7,{ }^{3} J_{119 \mathrm{Sn}-}\right.$ $13 \mathrm{C}=34 \mathrm{~Hz}), 45.1(\mathrm{C} 8), 27.4\left(\mathrm{C} 11,{ }^{3} J_{119 \mathrm{Sn}-13 \mathrm{C}}=44 \mathrm{~Hz},{ }^{3} J_{117 \mathrm{Sn}-13 \mathrm{C}}=42 \mathrm{~Hz}\right), 27.2\left(\mathrm{C} 9,{ }^{1} J_{119 \mathrm{Sn}-13 \mathrm{C}}=\right.$ $\left.694 \mathrm{~Hz},{ }^{1} J_{117 \mathrm{Sn}-13 \mathrm{C}}=664 \mathrm{~Hz}\right), 26.3\left(\mathrm{C} 10,{ }^{2} J_{119 \mathrm{Sn}-13 \mathrm{C}}=120 \mathrm{~Hz},{ }^{2} J_{117 \mathrm{Sn}-13 \mathrm{C}}=115 \mathrm{~Hz}\right), 13.7(\mathrm{C} 12)$ ppm; ${ }^{119} \mathbf{S n}\left\{{ }^{1} \mathbf{H}\right\} \mathbf{N M R}\left(149 \mathrm{MHz}, \mathrm{CDCl}_{3}, \delta\right):-104.0 \mathrm{ppm}$.

\subsection{Synthesis of [2-( $\left.\left.\mathrm{Me}_{2} \mathrm{NCH}_{2}\right) \mathrm{C}_{6} \mathrm{H}_{4}\right] \mathrm{PhSnCl}_{2}(35)$ :}

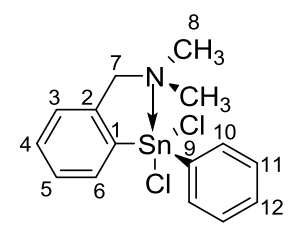

A suspension of $229(0.467 \mathrm{~g}, 3.1 \mathrm{mmol})$ in $30 \mathrm{~mL} \mathrm{Et}_{2} \mathrm{O}$ was added dropwise over 30 min to a solution of $\mathrm{PhSnCl}_{3}(1.0 \mathrm{~g}, 3.1 \mathrm{mmol})$ in $20 \mathrm{~mL} \mathrm{Et} 2 \mathrm{O}$ at $0{ }^{\circ} \mathrm{C}$. With the addition of 229 a white precipitate formed. The reaction mixture was stirred for $3 \mathrm{~h}$ at room temperature. The solid byproduct was separated by decantation in the glove box. The solvent was removed under reduced pressure. The crude product was purified by extraction with toluene and the solvent was removed 
under reduced pressure, and a white coloured powder recovered. NMR data $\left({ }^{1} \mathrm{H},{ }^{13} \mathrm{C},{ }^{119} \mathrm{Sn}\right)$ agreed with the reported literature. ${ }^{26,29 a, 35}$ Yield: $1.1 \mathrm{~g}(69 \%)$.

${ }^{1} \mathbf{H}$ NMR (400 MHz, $\left.\mathrm{CDCl}_{3}, \delta\right): 8.36\left(\mathrm{~d}, 1 \mathrm{H}, \mathrm{H} 6,{ }^{3} \mathrm{~J}_{119 \mathrm{Sn}-1 \mathrm{H}}=101 \mathrm{~Hz}\right), 7.68(\mathrm{~d}, 2 \mathrm{H}, \mathrm{H} 10), 7.50(\mathrm{~m}$, 2H, H4, H5), 7.46 (m, 3H, H1, H12), 7.26 (d, 1H, H3), 3.72 (s, 2H, H7), 2.19 (s, 6H, H8) ppm; ${ }^{13} \mathrm{C}$ NMR $\left(100 \mathrm{MHz}, \mathrm{CDCl}_{3}, \delta\right): 141.6(\mathrm{C} 2), 141.5\left(\mathrm{C} 1,{ }^{1} J_{119 \mathrm{Sn}-13 \mathrm{C}}=56 \mathrm{~Hz}\right), 137.9\left(\mathrm{C} 11,{ }^{1} J_{119 \mathrm{Sn}-}\right.$ $\left.{ }_{13 \mathrm{C}}=66 \mathrm{~Hz}\right), 136.8(\mathrm{C} 4), 133.8\left(\mathrm{C} 6,{ }^{2} J_{119 \mathrm{Sn}-13 \mathrm{C}}=65 \mathrm{~Hz}\right), 131.6\left(\mathrm{C} 3,{ }^{3} J_{119 \mathrm{Sn}-13 \mathrm{C}}=17 \mathrm{~Hz}\right), 130.5$ $\left(\mathrm{C} 5,{ }^{3} J_{119 \mathrm{Sn}-13 \mathrm{C}}=20 \mathrm{~Hz}\right), 129.4\left(\mathrm{C} 9,{ }^{1} J_{119 \mathrm{Sn}-13 \mathrm{C}}=95 \mathrm{~Hz}\right), 129.0\left(\mathrm{C} 10,{ }^{1} J_{119 \mathrm{Sn}-13 \mathrm{C}}=94 \mathrm{~Hz}\right), 127.8$ $\left(\mathrm{C} 10,{ }^{2} J_{119 \mathrm{Sn}-13 \mathrm{C}}=83 \mathrm{~Hz}\right), 63.2\left(\mathrm{C} 7,{ }^{3} J_{119 \mathrm{Sn}-13 \mathrm{C}}=44 \mathrm{~Hz}\right), 45.4(\mathrm{C} 8) \mathrm{ppm} ;{ }^{119} \mathbf{S n}\left\{{ }^{1} \mathbf{H}\right\} \mathbf{N M R}(149$ $\left.\mathrm{MHz}, \mathrm{CDCl}_{3}, \delta\right):-168.0 \mathrm{ppm}$.

\subsection{Synthesis of [2-( $\left.\left.\mathrm{Me}_{2} \mathrm{NCH}_{2}\right) \mathrm{C}_{6} \mathrm{H}_{4}\right] \mathrm{MeSnH}_{2}(230)$ :}

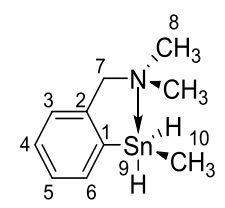

A suspension of $33(0.240 \mathrm{~g}, 0.71 \mathrm{mmol})$ in $20 \mathrm{~mL} \mathrm{Et}_{2} \mathrm{O}$ was added dropwise to $1.0 \mathrm{M} \mathrm{LiAlH}_{4}(1.5$ $\mathrm{mL}, 1.5 \mathrm{mmol})$ in $30 \mathrm{~mL}$ of $\mathrm{Et}_{2} \mathrm{O}$ at $0{ }^{\circ} \mathrm{C}$. The reaction mixture was stirred for $3 \mathrm{~h}$ at $0{ }^{\circ} \mathrm{C}$. The reaction was quenched with $10 \mathrm{~mL}$ of degassed and chilled water. The organic layer was separated and dried over anhydrous $\mathrm{MgSO}_{4}$, and the solvent removed under reduced pressure to give a yellow coloured oil was obtained. It is relatively stable at low temperature. Yield $=0.15 \mathrm{~g}(76 \%)$.

${ }^{1}$ H NMR (400 MHz, C6 $\left.\mathrm{D}_{6}, \delta\right): 7.78$ (m, 1H, H6), 7.15 (m, 2H, H4, H5), 6.95 (m, 1H, H3), 5.63 (q, $\left.2 \mathrm{H}, \mathrm{H} 9,{ }^{1} J_{119 \mathrm{Sn}-1 \mathrm{H}}=1824 \mathrm{~Hz},{ }^{1} J_{117 \mathrm{Sn}-1 \mathrm{H}}=1756 \mathrm{~Hz}\right), 3.13(\mathrm{~s}, 2 \mathrm{H}, \mathrm{H} 7), 1.87(\mathrm{~s}, 6 \mathrm{H}, \mathrm{H} 8), 0.35(\mathrm{t}, 3 \mathrm{H}$, $\left.\mathrm{H} 10,{ }^{1} J_{119 \mathrm{Sn}-1 \mathrm{H}}=60 \mathrm{~Hz}\right) \mathrm{ppm} ;{ }^{13} \mathbf{C}$ NMR $\left(100 \mathrm{MHz}, \mathrm{C}_{6} \mathrm{D}_{6}, \delta\right): 145.0\left(\mathrm{C} 2,{ }^{2} J_{119 \mathrm{Sn}-13 \mathrm{C}}=27 \mathrm{~Hz}\right), 138.85$ $(\mathrm{C} 1), 138.5\left(\mathrm{C} 6,{ }^{2} J_{119 \mathrm{Sn}-13 \mathrm{C}}=47 \mathrm{~Hz}\right), 128.6\left(\mathrm{C} 5,{ }^{3} J_{119 \mathrm{Sn}-13 \mathrm{C}}=12 \mathrm{~Hz}\right), 127.8(\mathrm{C} 4), 127.2(\mathrm{C} 3), 64.9$ $\left(\mathrm{C} 7,{ }^{3} J_{119 \mathrm{Sn}-13 \mathrm{C}}=24 \mathrm{~Hz}\right), 43.6(\mathrm{C} 8),-11.3\left(\mathrm{C} 9,{ }^{1} J_{119 \mathrm{Sn}-13 \mathrm{C}}=392 \mathrm{~Hz},{ }^{1} J_{117 \mathrm{Sn}-13 \mathrm{C}}=382 \mathrm{~Hz}\right) \mathrm{ppm}$; 
${ }^{119} \mathrm{Sn}\left\{{ }^{1} \mathrm{H}\right\}$ NMR $\left(149.21 \mathrm{MHz}, \mathrm{C}_{6} \mathrm{D}_{6}, \delta\right):-236.0 \mathrm{ppm}$. HRMS-DART (m/z): $\left[\mathrm{M}^{+}\right]-\mathrm{H}$ calculated for $\mathrm{C}_{10} \mathrm{H}_{16} \mathrm{NSn}=270.03 ;$ found $=270.03$.

\subsection{Synthesis of [2-( $\left.\left.\mathrm{Me}_{2} \mathrm{NCH}_{2}\right) \mathrm{C}_{6} \mathrm{H}_{4}\right] n-\mathrm{BuSnH}_{2}(231)$ :}

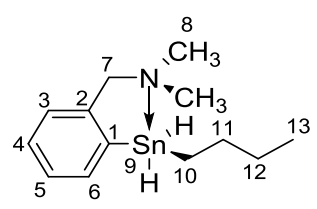

A solution of $37(1.59 \mathrm{~g}, 4.16 \mathrm{mmol})$ in $30 \mathrm{~mL} \mathrm{Et}_{2} \mathrm{O}$ was added dropwise to $1.0 \mathrm{M} \mathrm{LiAlH}_{4}(5.0$ $\mathrm{mL}, 5.0 \mathrm{mmol})$ in $50 \mathrm{~mL}$ of $\mathrm{Et}_{2} \mathrm{O}$ at $0{ }^{\circ} \mathrm{C}$. The reaction mixture was stirred for $3 \mathrm{~h}$ at $0{ }^{\circ} \mathrm{C}$. The reaction was quenched with $15.0 \mathrm{~mL}$ of degassed and chilled water. The organic layer was separated and dried over anhydrous $\mathrm{MgSO}_{4}$. After filtration, the solvent was removed under reduced pressure. A yellow coloured oil was recovered. The product $\mathbf{2 3 0}$ is relatively stable at low temperature. Yield: $1.05 \mathrm{~g}(81 \%)$.

${ }^{1}$ H NMR (400 MHz, C $\left.6 \mathrm{D}_{6}, \delta\right): 7.78$ (m, 1H, H6), 7.13 (m, 2H, H4, H5), 6.94 (m, 1H, H3), 5.72 (s, $\left.2 \mathrm{H}, \mathrm{H} 9,{ }^{1} J_{119 \mathrm{Sn}-1 \mathrm{H}}=1760 \mathrm{~Hz},{ }^{1} J_{117 \mathrm{Sn}-1 \mathrm{H}}=1680 \mathrm{~Hz}\right), 3.12(\mathrm{~s}, 2 \mathrm{H}, \mathrm{H} 7), 1.88(\mathrm{~s}, 6 \mathrm{H}, \mathrm{H} 8), 1.70(\mathrm{~m}$, 2H, H11), 1.62 (m, 2H, H10), 1.14 (m, 2H, H12), 0.90 (t, 3H, H13) ppm; ${ }^{13}$ C NMR (100 MHz, $\left.\mathrm{C}_{6} \mathrm{D}_{6}, \delta\right): 145.4\left(\mathrm{C} 2,{ }^{2} J_{119 \mathrm{Sn}-13 \mathrm{C}}=24 \mathrm{~Hz}\right), 139.5(\mathrm{C} 1), 139.2\left(\mathrm{C} 6,{ }^{2} J_{119 \mathrm{Sn}-13 \mathrm{C}}=44 \mathrm{~Hz}\right), 128.8(\mathrm{C} 3$, $\left.{ }^{3} J_{119 \mathrm{Sn}-13 \mathrm{C}}=11 \mathrm{~Hz}\right), 128.1\left(\mathrm{C} 5,{ }^{3} J_{119 \mathrm{Sn}-13 \mathrm{C}}=69 \mathrm{~Hz}\right), 127.5(\mathrm{C} 4), 65.4\left(\mathrm{C} 7,{ }^{3} J_{119 \mathrm{Sn}-13 \mathrm{C}}=33 \mathrm{~Hz}\right), 44.0$ $(\mathrm{C} 8), 30.9\left(\mathrm{C} 12,{ }^{3} J_{119 \mathrm{Sn}-13 \mathrm{C}}=21 \mathrm{~Hz}\right), 27.3\left(\mathrm{C} 11,{ }^{2} J_{119 \mathrm{Sn}-13 \mathrm{C}}=65 \mathrm{~Hz}\right), 14.0(\mathrm{C} 13), 10.4\left(\mathrm{C} 10,{ }^{1} J_{119 \mathrm{Sn}-}\right.$ $\left.{ }_{13 \mathrm{C}}=434 \mathrm{~Hz},{ }^{1} J_{117 \mathrm{Sn}-13 \mathrm{C}}=415 \mathrm{~Hz}\right) \mathrm{ppm} ;{ }^{119} \mathbf{S n}\left\{{ }^{1} \mathbf{H}\right\} \mathbf{N M R}\left(149 \mathrm{MHz}, \mathrm{C}_{6} \mathrm{D}_{6}, \delta\right)-217.0$. HRMSDART (m/z): [M+H] $]^{+}$calculated for $\mathrm{C}_{13} \mathrm{H}_{22} \mathrm{NSn}, 314.09346$; found 314.09338.

\subsection{Wurtz coupling of 37 :}

Compound $37(1.0 \mathrm{~g}, 2.61 \mathrm{mmol})$ was dissolved in $5 \mathrm{~mL}$ of toluene in a Schlenk flask and 15crown-5-ether (12 mg, $0.054 \mathrm{mmol})$ added. In a separate 3-neck flask equipped with reflux condenser, a dispersion of $\mathrm{Na}(0.130 \mathrm{~g}, 5.65 \mathrm{mmol})$ in $5 \mathrm{~mL}$ toluene was prepared by reflux at 110 
${ }^{\circ} \mathrm{C}$ for $1 \mathrm{~h}$. The Na dispersion was allowed to cool to room temperature and the flask wrapped in aluminium foil to protect the reaction from light. The solution of $\mathbf{3 7}$ was added dropwise to the stirring sodium dispersion with a syringe. The reaction mixture was heated for $4 \mathrm{~h}$ at $60{ }^{\circ} \mathrm{C}$. The reaction mixture filtered through a frit into aluminium foil wrapped Schlenk flask. The solvent was removed under reduced pressure to produce a light yellow coloured viscous product. ${ }^{119} \mathbf{S n}\left\{{ }^{1} \mathbf{H}\right\}$ NMR $\left(149 \mathrm{MHz}, \mathrm{C}_{6} \mathrm{D}_{6}, \delta\right):-88.4$ ppm (243), -135.0 and -138.0 ppm (244). By NMR spectra the percentages of (243) and (244) are 60\% and 40\% respectively.

Following the above procedure the reaction time was increased from $4 \mathrm{~h}$ to 4 days. ${ }^{119} \mathbf{S n}\left\{{ }^{1} \mathbf{H}\right\} \mathbf{N M R}$ (149 MHz, $\left.\mathrm{C}_{6} \mathrm{D}_{6}, \delta\right):-105.7 \mathrm{ppm}(\mathbf{2 4 5})$.

\subsection{Wurtz coupling of 35 :}

Compound 35 (0.50 g, $1.27 \mathrm{mmol})$ was dissolved in $5 \mathrm{~mL}$ of toluene in a Schlenk flask and 15crown-5-ether $(0.012 \mathrm{mg}, 0.054 \mathrm{mmol})$ added. In a separate 3-neck flask equipped with reflux condenser, $\mathrm{Na}(0.060 \mathrm{~g}, 2.61 \mathrm{mmol})$ dispersion was prepared in $5 \mathrm{~mL}$ of toluene by reflux at 110 ${ }^{\circ} \mathrm{C}$ for $1 \mathrm{~h}$. The Na dispersion was allowed to cool to room temperature and flask was then wrapped in aluminium foil to protect the reaction products from light. The solution of $\mathbf{3 5}$ added dropwise to the stirring sodium dispersion with a syringe and the reaction mixture was heated for $8 \mathrm{~h}$ at 60 ${ }^{\circ} \mathrm{C}$. The reaction mixture filtered through a frit into aluminium foil wrapped Schlenk flask. The solvent was removed under reduced pressure to produce a yellow coloured product.

${ }^{119} \mathrm{Sn}\left\{{ }^{1} \mathrm{H}\right\}$ NMR $\left(149 \mathrm{MHz}, \mathrm{C}_{6} \mathrm{D}_{6}, \delta\right):-145.5$ (246), -173.0 (247), -210.6, -212.2 ppm (cyclooligostannane). 
5.48 Catalytic dehydrocoupling of 230 using $\mathrm{Cp}_{2} \mathrm{ZrMe}_{2}$ :

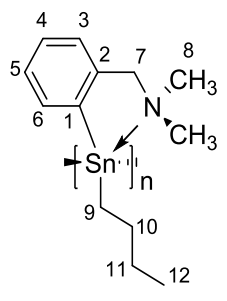

$\mathrm{Cp}_{2} \mathrm{ZrMe}_{2}(0.005 \mathrm{~g}, 0.019 \mathrm{mmol})$ was added to a solution of $230(0.30 \mathrm{~g}, \mathrm{mmol})$ in $10 \mathrm{~mL}$ of hexane. The reaction mixture was stirred for $44 \mathrm{~h}$. The solvent was removed under reduced pressure. The product was isolated as brown gummy solid. The crude product was unsuccessfully purified using hexane and $\mathrm{MeOH}$. Some insoluble precipitate was filtered off. The hexane soluble product was used to obtain the NMR data.

${ }^{1} \mathbf{H}$ NMR (400 MHz, $\mathrm{CDCl}_{3}, \delta$ ): 8.74 (1H, H6), 7.21 (1H, H4), 7.0 (1H, H5), 6.83 (1H, H3), 2.92 (2H, H7), 1.60 (3H, H8), 1.88 (m, 2H, H10), 1.40 (m, 2H, H11), 1.19 (m, 2H, H9), 0.91 (t, 3H, H12) ppm; ${ }^{13} \mathrm{C}$ NMR (100 MHz, $\left.\mathrm{CDCl}_{3}, \delta\right): 142.3\left(\mathrm{C} 2,{ }^{2} J_{119 \mathrm{Sn}-13 \mathrm{C}}=16 \mathrm{~Hz}\right), 141.4(\mathrm{C} 1), 138.6$ $\left(\mathrm{C} 6,{ }^{2} J_{119 \mathrm{Sn}-13 \mathrm{C}}=19 \mathrm{~Hz}\right), 128.9\left(\mathrm{C} 3 .{ }^{3} J_{119 \mathrm{Sn}-13 \mathrm{C}}=15 \mathrm{~Hz}\right), 128.1(\mathrm{C} 5), 126.4(\mathrm{C} 4), 64.9\left(\mathrm{C} 7,{ }^{3} J_{119 \mathrm{Sn}-}\right.$ $13 \mathrm{C}=18 \mathrm{~Hz}), 44.6(\mathrm{C} 8), 28.3\left(\mathrm{C} 11,{ }^{3} J_{119 \mathrm{Sn}-13 \mathrm{C}}=30 \mathrm{~Hz}\right), 26.9\left(\mathrm{C} 10,{ }^{2} J_{119 \mathrm{Sn}-13 \mathrm{C}}=82 \mathrm{~Hz}\right), 18.1(\mathrm{C} 9$, $\left.{ }^{1} J_{119 \mathrm{Sn}-13 \mathrm{C}}=494 \mathrm{~Hz},{ }^{1} J_{117 \mathrm{Sn}-13 \mathrm{C}}=472 \mathrm{~Hz}\right), 13.5(\mathrm{C} 12) \mathrm{ppm} ;{ }^{119} \mathbf{S n}\left\{{ }^{1} \mathbf{H}\right\} \mathbf{N M R}\left(149 \mathrm{MHz}, \mathrm{C}_{6} \mathrm{D}_{6}, \delta\right)$ : -49.0 ppm.

5.49 1-Bromo-2-(n-propylthiomethyl) benzene (233):

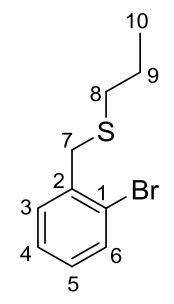


A solution of $\mathrm{NaH}(0.33 \mathrm{~g}, 13.13 \mathrm{mmol})$ in $10 \mathrm{~mL}$ of THF was slowly added at $0{ }^{\circ} \mathrm{C}$ to a solution of 1-propanethiol (1.0 g, $13.13 \mathrm{mmol})$ in $60 \mathrm{~mL}$ of THF. The reaction mixture was stirred for an additional $2 \mathrm{~h}$ and a solution of 2-bromobenzylbromide $(2.18 \mathrm{~g}, 8.73 \mathrm{mmol})$ in $10 \mathrm{~mL}$ of THF added. The resulting mixture was refluxed for $16 \mathrm{~h}$ overnight. After the solution was cooled to room temperature, a saturated $\mathrm{NH}_{4} \mathrm{Cl}$ solution $(100 \mathrm{~mL})$ was added and the organic phase separated. The water phase was extracted with $\mathrm{Et}_{2} \mathrm{O}(3 \times 30 \mathrm{~mL})$. The combined organic layers were dried over anhydrous $\mathrm{MgSO}_{4}$. A clear yellow coloured oil was obtained after the removal of solvent under reduced pressure. Yield: $1.8 \mathrm{~g}(84 \%)$.

${ }^{1} \mathbf{H}$ NMR (400 MHz, $\mathrm{CDCl}_{3}, \delta$ ): 7.56 (dd, 1H, H6), 7.40 (dd, 1H, H3), 7.28 (dt, 1H, H5), 7.12 (dt, 1H, H6), 3.85 (s, H7), 2.49 (t, 2H, H8), 1.64 (sex, 2H, H9), 1.0 (t, 3H, H10) ppm; ${ }^{13}$ C NMR (100 MHz, $\mathrm{CDCl}_{3}, \delta$ ): 138.2 (C2), 133.1 (C6), 130.8 (C3), 128.5 (C5), 127.4 (C4), 124.5 (C1), 36.8 (C7), 33.9 (C8), 22.8 (C9), 13.6 (C10) ppm.

\subsection{1-Bromo-2-(phenylthiomethyl) benzene (234):}

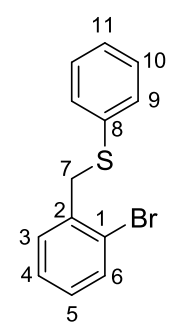

A solution of $\mathrm{NaH}(0.22 \mathrm{~g}, 9.07 \mathrm{mmol})$ in $10 \mathrm{~mL}$ of THF was slowly added at $0{ }^{\circ} \mathrm{C}$ to a solution of thiophenol $(1.0 \mathrm{~g}, 9.07 \mathrm{mmol})$ in $60 \mathrm{~mL}$ of THF and the reaction mixture stirred for $1 \mathrm{~h}$. A solution of 2-bromobenzylbromide (1.51 g, $6.04 \mathrm{mmol})$ in $10 \mathrm{~mL}$ of THF was then added and the resulting mixture refluxed for $16 \mathrm{~h}$. The solution was cooled to room temperature, a saturated $\mathrm{NH}_{4} \mathrm{Cl}$ solution $(100 \mathrm{~mL})$ added, and the organic phase separated. The aqueous phase was extracted with $\mathrm{Et}_{2} \mathrm{O}(3 \times 30 \mathrm{~mL})$. The combined organic layers were dried over anhydrous $\mathrm{MgSO}_{4}$. 
A clear yellow coloured oil was obtained after the removal of solvent under reduced pressure. The NMR data agreed with the literature. ${ }^{105}$ Yield: $1.05 \mathrm{~g}(63 \%)$.

${ }^{1} \mathbf{H}$ NMR (400 MHz, $\mathrm{CDCl}_{3}, \delta$ ): 7.62 (dd, 1H, H3), 7.41 (dd, 2H, H4, H5), 7.32 (m, 5H, H9, H10, H11), 7.15(dd, 1H, H6), 4.30 (s, 2H, H7), ppm; ${ }^{13} \mathbf{C}$ NMR (100 MHz, $\left.\mathrm{CDCl}_{3}, \delta\right): 136.9$ (s, C2) 135.9 (C8), 133.0 (C6), 130.7 (d, C3), 130.5 (C9), 129.2 (d, C10), 128.9 (d, C5), 127.5 (d, C11), $126.8(\mathrm{C} 1), 125.7(\mathrm{C} 4), 35.8(\mathrm{C} 7) \mathrm{ppm}$.

\subsection{1 (2-bromobenzyl)diphenylphosphane (237):}

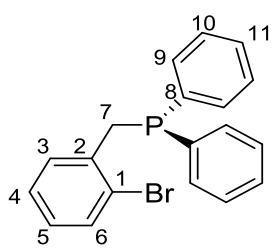

To 3-neck $250 \mathrm{~mL}$ round bottom flask containing magnesium turnings $(0.5 \mathrm{~g}, 20.6 \mathrm{mmol})$ in 30 $\mathrm{mL}$ of $\mathrm{Et}_{2} \mathrm{O}$, a few crystals of $\mathrm{I}_{2}$ and a few drops of $n$-BuLi (1.6 M) were added. A solution of 1bromo-2-(bromomethyl)benzene $(4.68 \mathrm{~g}, 18.72 \mathrm{mmol})$ in $20 \mathrm{~mL}$ of $\mathrm{Et}_{2} \mathrm{O}$ was added dropwise. After the addition the reaction was refluxed for an additional $30 \mathrm{~min} . \mathrm{ClPPh}_{2}(3.0 \mathrm{~mL}, 16.24 \mathrm{mmol})$ in $20 \mathrm{~mL}$ of $\mathrm{Et}_{2} \mathrm{O}$ was added dropwise to the stirred Grignard solution. The reaction mixture was refluxed for an additional hour and then cooled to room temperature and quenched with aqueous $10 \% \mathrm{NH}_{4} \mathrm{Cl}$ under nitrogen. The organic layer was washed with water and dried using $\mathrm{MgSO} 4$. After filtration, the solvent was removed under reduced pressure to obtain white solid. NMR data agreed with literature. ${ }^{163,164}$ Yield: $3.55 \mathrm{~g}(57 \%)$.

${ }^{1} \mathbf{H}$ NMR $\left(400 \mathrm{MHz}, \mathrm{CDCl}_{3}, \delta\right): 7.57(\mathrm{~m}, 1 \mathrm{H}), 7.48(\mathrm{~m}, 4 \mathrm{H}), 7.39(\mathrm{~m}, 6 \mathrm{H}), 7.07(\mathrm{~s}, 2 \mathrm{H}), 6.86(\mathrm{~s}$, 1H), $3.6\left(\mathrm{~s}, 2 \mathrm{H}, \mathrm{CH}_{2} \mathrm{PPh}_{2}\right) \mathrm{ppm} ;{ }^{13} \mathbf{C} \mathbf{N M R}\left(100 \mathrm{MHz}, \mathrm{CDCl}_{3}, \delta\right): 138.0\left(\mathrm{~d}, \mathrm{C} 9,{ }^{2} J_{31 \mathrm{P}-13 \mathrm{C}}=15 \mathrm{~Hz}\right)$, $137.0\left(\mathrm{~d}, \mathrm{C} 10,{ }^{2} J_{31 \mathrm{P}-13 \mathrm{C}}=8.0 \mathrm{~Hz}\right), 133.2\left(\mathrm{~d}, \mathrm{C} 8,{ }^{1} J_{31 \mathrm{P}-13 \mathrm{C}}=19 \mathrm{~Hz}\right), 132.9\left(\mathrm{C} 5,{ }^{5} J_{31 \mathrm{P}-13 \mathrm{C}}=2.0 \mathrm{~Hz}\right)$, $131.2\left(\mathrm{C} 2,{ }^{2} J_{31 \mathrm{P}-13 \mathrm{C}}=8.0 \mathrm{~Hz}\right), 128.9(\mathrm{C} 11), 128.5\left(\mathrm{C} 3,{ }^{3} J_{31 \mathrm{P}-13 \mathrm{C}}=6.0 \mathrm{~Hz}\right), 127.7\left(\mathrm{C} 6,{ }^{4} J_{31 \mathrm{P}-13 \mathrm{C}}=\right.$ 
$2.0 \mathrm{~Hz}), 127.2(\mathrm{C} 4), 125.1\left(\mathrm{~d}, \mathrm{C} 1,{ }^{3} J_{31 \mathrm{P}-13 \mathrm{C}}=5.0 \mathrm{~Hz}\right), 36.5\left(\mathrm{~d}, \mathrm{C} 7,{ }^{1} J_{31 \mathrm{P}-13 \mathrm{C}}=17 \mathrm{~Hz}\right) \mathrm{ppm} ;{ }^{31} \mathbf{P}$ $\operatorname{NMR}\left(161.9 \mathrm{MHz}, \mathrm{CDCl}_{3}, \delta\right):-12.7 \mathrm{ppm}$.

4.52 diphenyl(2-(triphenylstannyl)benzyl)phosphine (241):

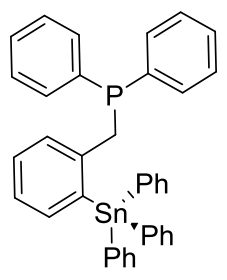

1.6 M solution of $n-\mathrm{BuLi}(1.46 \mathrm{~mL}, 2.73 \mathrm{mmol})$ in hexane was added dropwise to a solution $\mathbf{2 3 7}$ $(0.97 \mathrm{~g}, 2.73 \mathrm{mmo})$ in $\mathrm{Et}_{2} \mathrm{O}$ at $0{ }^{\circ} \mathrm{C}$. Reaction mixture was stirred for $3 \mathrm{~h}$ and removal of solvent under reduced pressure afforded an orange-red coloured powder which was washed with hexane and the residual solvent removed under reduced pressure. This lithiated species $(0.153 \mathrm{~g}, 5.42$ mmol) was suspended in dry $\mathrm{C}_{6} \mathrm{H}_{6}$ and a solution of $\mathrm{Ph}_{3} \mathrm{SnCl}(0.209 \mathrm{~g}, 5.42 \mathrm{mmol})$ in $\mathrm{C}_{6} \mathrm{H}_{6}$ added at room temperature. The reaction mixture was stirred for $3 \mathrm{~h}$ and after filtration in glove box, the solvent was removed under reduced pressure. The crude product was dissolved in benzene and filtered. The removal of solvent under reduced pressure afforded a light yellow coloured semisolid. No yield was recorded.

${ }^{119} \mathbf{S n}\left\{{ }^{1} \mathbf{H}\right\}$ NMR $\left(149 \mathrm{MHz}, \mathrm{CDCl}_{3}, \delta\right):-85.0 \mathrm{ppm} ;{ }^{31} \mathbf{P}$ NMR (161.9 MHz, $\left.\mathrm{CDCl}_{3}, \delta\right):-9.8 \mathrm{ppm}$.

\subsection{3 (2-(chlordiphenylstannyl)benzyl)diphenylphosphine (242):}

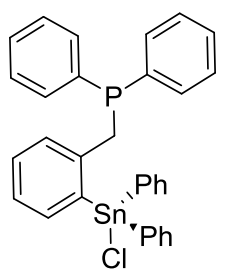

To a suspension of lithiated $241(0.619 \mathrm{~g}, \mathrm{mmol})$ in dry $\mathrm{C}_{6} \mathrm{H}_{6}$, a solution $\mathrm{PhSnCl}_{3}(0.662 \mathrm{~g}, \mathrm{mmol})$ in dry $\mathrm{C}_{6} \mathrm{H}_{6}$ added at room temperature. The reaction mixture was stirred for $3 \mathrm{~h}$ and solvent was 
removed under reduced pressure after filtration in glove box. The crude product was dissolved in benzene and filtered. The removal of solvent under reduced pressure afforded a yellow brown coloured semisolid. No yield was recorded.

${ }^{119} \mathrm{Sn}\left\{{ }^{1} \mathbf{H}\right\} \mathbf{N M R}\left(149 \mathrm{MHz}, \mathrm{CDCl}_{3}, \delta\right):-27.0$ ppm; ${ }^{31} \mathbf{P}$ NMR $\left(161.9 \mathrm{MHz}, \mathrm{CDCl}_{3}, \delta\right): 43.2 \mathrm{ppm}$. 


\subsection{Appendices}

\section{List of Appendix tables}

Table A 1. Crystal data and structure refinement for 198.............................................................168

Table A 2. Atomic coordinates and equivalent isotropic displacement parameters for 198.....169

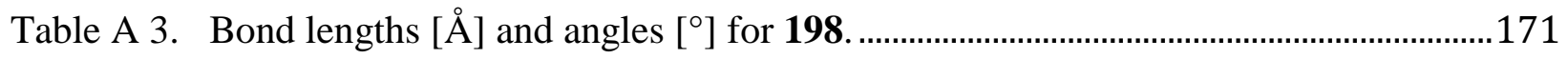

Table A 4. Anisotropic displacement parameters for 198. ...........................................................179

Table A 5. Hydrogen coordinates and isotropic displacement parameters for 198.......................179

Table A 6. Crystal data and structure refinement for 200 .............................................................182

Table A 7. Atomic coordinates and equivalent isotropic displacement parameters for $\mathbf{2 0 0}$.....183

Table A 8. Bond lengths $[\AA]$ and angles $\left[{ }^{\circ}\right]$ for 200 ...............................................................184

Table A 9. Anisotropic displacement parameters for 200 .............................................................193

Table A 10. Hydrogen coordinates and isotropic displacement parameters for 200 ...................194

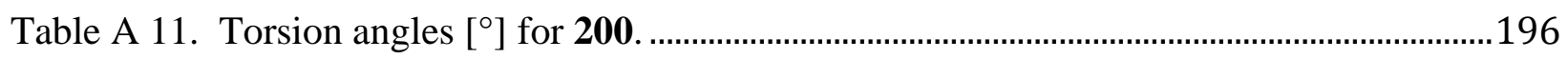

Table A 12. Crystal data and structure refinement for 201..........................................................199

Table A 13. Atomic coordinates and equivalent isotropic displacement parameters for $201 \ldots 200$

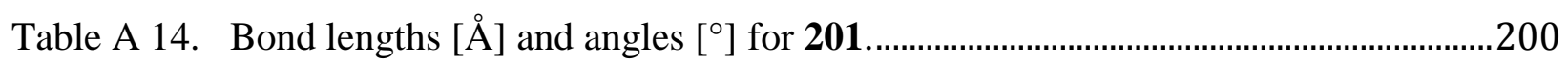

Table A 15. Anisotropic displacement parameters for 201..........................................................206

Table A 16. Hydrogen coordinates and isotropic displacement parameters for 201..................206

Table A 17. Torsion angles $\left[^{\circ}\right]$ for 201.......................................................................................20

Table A 18. Crystal data and structure refinement for 202 .........................................................2.

Table A 19. Atomic coordinates $\left(\times 10^{4}\right)$ and equivalent isotropic displacement for $\mathbf{2 0 2} \ldots \ldots . . .211$

Table A 20. Bond lengths $[\AA]$ and angles $\left[{ }^{\circ}\right]$ for 202 ..............................................................2

Table A 21. Anisotropic displacement parameters for 202........................................................215

Table A 22. Hydrogen coordinates and isotropic displacement parameters for 202 ...................215

Table A 23. Torsion angles $\left[^{\circ}\right]$ for 202.

Table A 24. Crystal data and structure refinement for 203 …....................................................218

Table A 25. Atomic coordinates and equivalent isotropic displacement parameters for 203....219

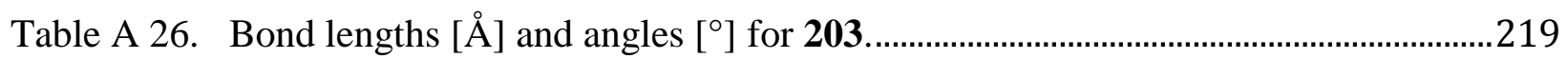

Table A 27. Anisotropic displacement parameters for 203........................................................224 
Table A 28. Hydrogen coordinates and isotropic displacement parameters 203 ........................224

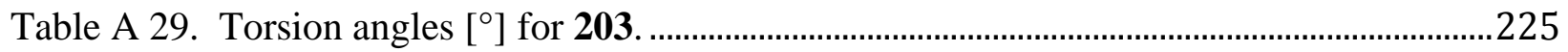

\section{List of Appendix Figures}

Figure A 1: ${ }^{1} \mathrm{H}$ NMR $\left(\mathrm{CDCl}_{3}\right)$ spectrum of compound 194......................................................228

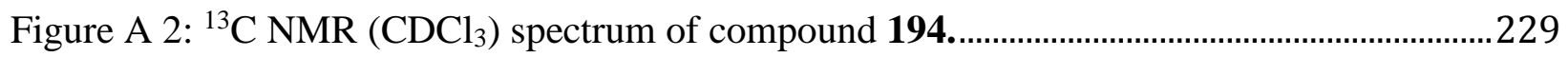

Figure A 3: ${ }^{1} \mathrm{H}$ NMR $\left(\mathrm{CDCl}_{3}\right)$ spectrum of compound 137.....................................................230

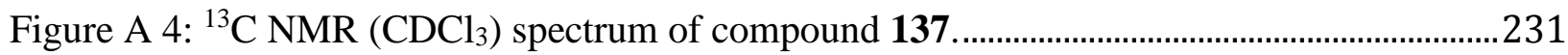

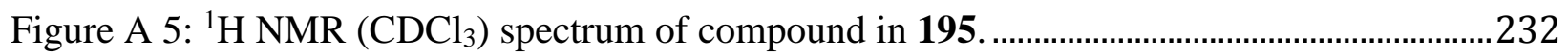

Figure A 6: ${ }^{13} \mathrm{C}$ NMR $\left(\mathrm{CDCl}_{3}\right)$ spectrum of compound 195........................................................233

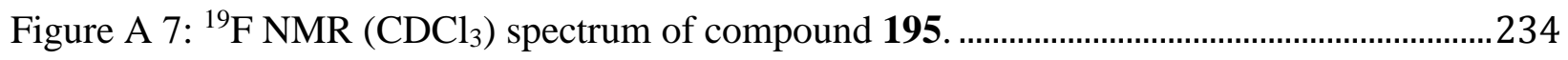

Figure A 8: ${ }^{1} \mathrm{H}$ NMR $\left(\mathrm{CDCl}_{3}\right)$ spectrum of compound 196.........................................................235

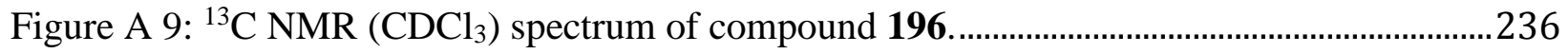

Figure A 10: ${ }^{1} \mathrm{H}$ NMR $\left(\mathrm{CDCl}_{3}\right)$ spectrum of compound 254.......................................................237

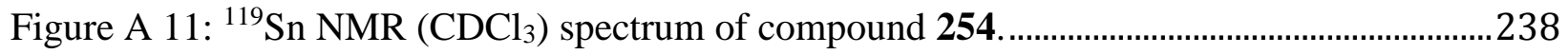

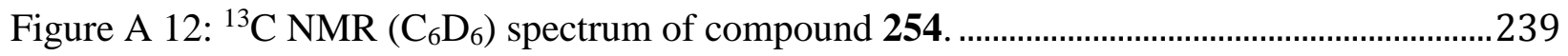

Figure A 13: ${ }^{1} \mathrm{H}$ NMR $\left(\mathrm{C}_{6} \mathrm{D}_{6}\right)$ spectrum of compound 197.......................................................240

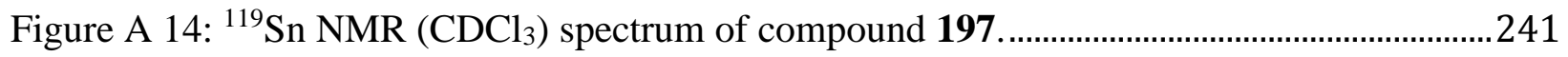

Figure A 15: ${ }^{13} \mathrm{C}$ NMR $\left(\mathrm{CDCl}_{3}\right)$ spectrum of compound 197.................................................242

Figure A 16: ${ }^{1} \mathrm{H}$ NMR $\left(\mathrm{CDCl}_{3}\right)$ spectrum of compound 141 .........................................................243

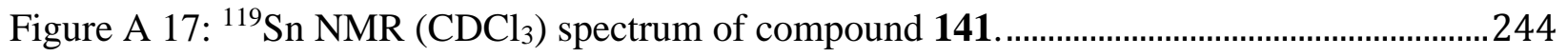

Figure A 18: ${ }^{13} \mathrm{C}$ NMR $\left(\mathrm{CDCl}_{3}\right)$ spectrum of compound 141.....................................................245

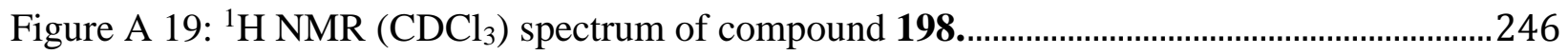

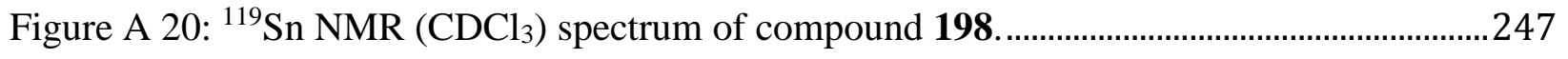

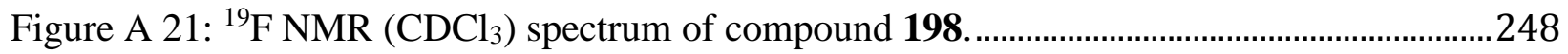

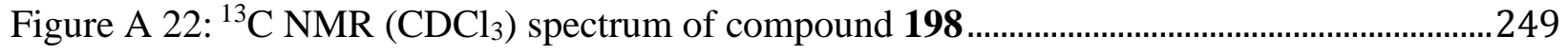

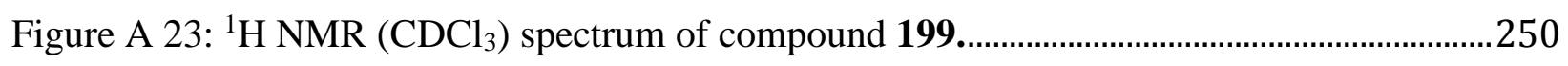

Figure A 24: ${ }^{119} \mathrm{Sn} \mathrm{NMR}\left(\mathrm{CDCl}_{3}\right)$ spectrum of compound 199....................................................251

Figure A 25: ${ }^{13} \mathrm{C}$ NMR $\left(\mathrm{CDCl}_{3}\right)$ spectrum of compound 199..................................................252

Figure A 26: ${ }^{1} \mathrm{H}$ NMR $\left(\mathrm{CDCl}_{3}\right)$ spectrum of compound 200......................................................253

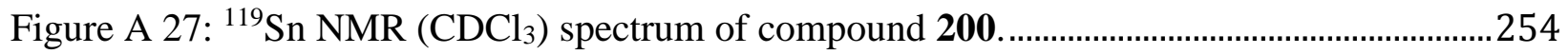




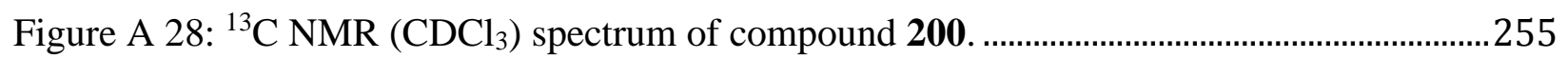

Figure A 29: ${ }^{1} \mathrm{H}$ NMR $\left(\mathrm{CDCl}_{3}\right)$ spectrum of compound 201....................................................256

Figure A 30: ${ }^{119} \mathrm{Sn}$ NMR $\left(\mathrm{CDCl}_{3}\right)$ spectrum of compound 201..................................................2.

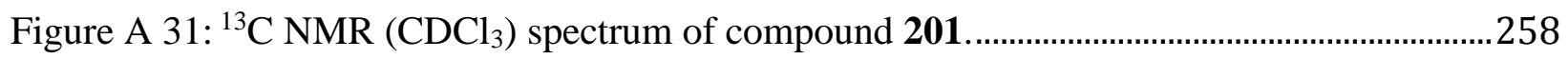

Figure A 32: ${ }^{1} \mathrm{H}$ NMR $\left(\mathrm{CDCl}_{3}\right)$ spectrum of compound 202 .......................................................259

Figure A 33: ${ }^{119} \mathrm{Sn} \mathrm{NMR}\left(\mathrm{CDCl}_{3}\right)$ spectrum of compound 202 ...................................................26

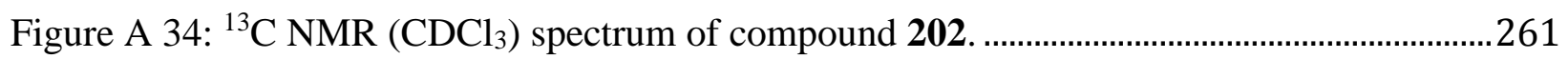

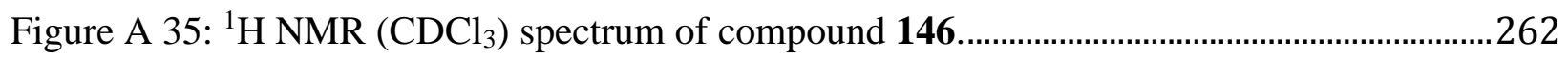

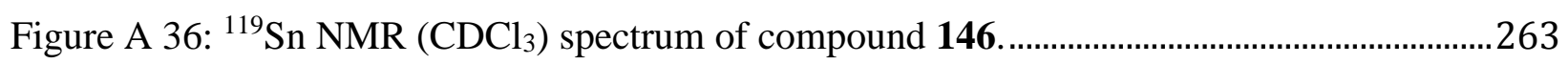

Figure A 37: ${ }^{1} \mathrm{H}$ NMR $\left(\mathrm{CDCl}_{3}\right)$ spectrum of compound 203 ......................................................264

Figure A 38: ${ }^{119} \mathrm{Sn}$ NMR $\left(\mathrm{CDCl}_{3}\right)$ spectrum of compound 203 ..................................................265

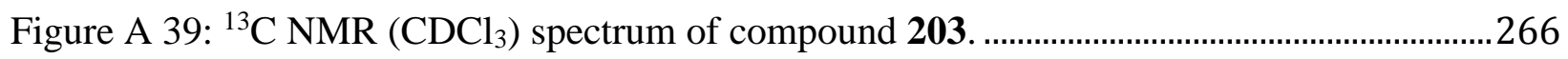

Figure A 40: ${ }^{1} \mathrm{H}$ NMR $\left(\mathrm{C}_{6} \mathrm{D}_{6}\right)$ spectrum of compound 204.......................................................267

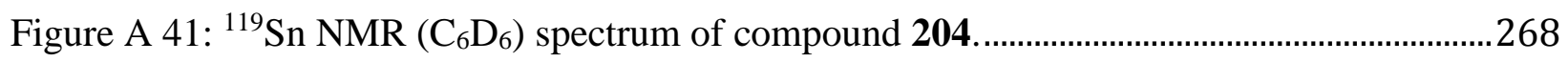

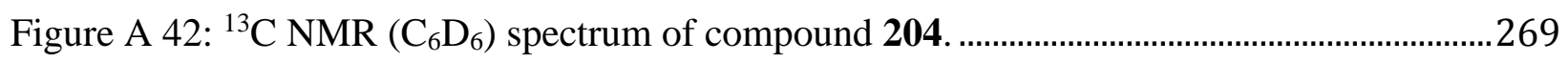

Figure A 43: ${ }^{1} \mathrm{H}$ NMR $\left(\mathrm{C}_{6} \mathrm{D}_{6}\right)$ spectrum of compound 205......................................................270

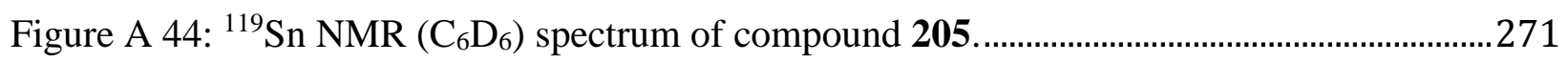

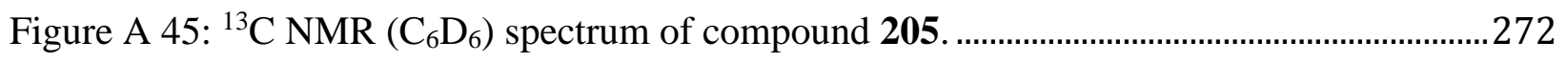

Figure A 46: ${ }^{1} \mathrm{H}$ NMR $\left(\mathrm{C}_{6} \mathrm{D}_{6}\right)$ spectrum of compound 206......................................................2.

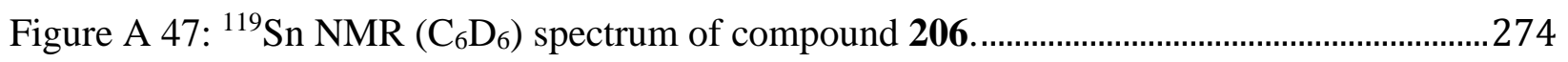

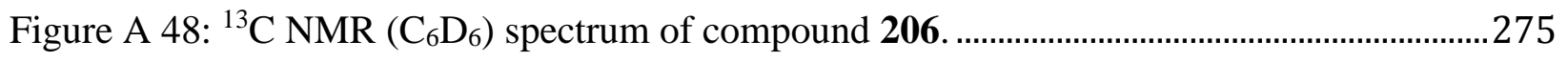

Figure A 49: ${ }^{1} \mathrm{H}$ NMR $\left(\mathrm{C}_{6} \mathrm{D}_{6}\right)$ spectrum of compound 207 ………….......................................2.

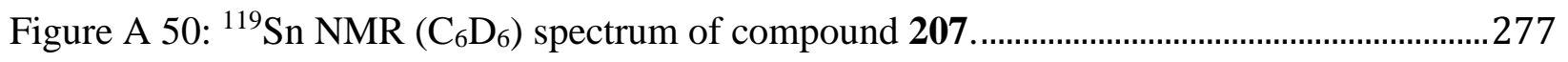

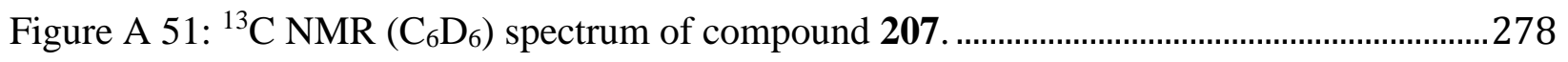

Figure A 52: ${ }^{1} \mathrm{H}$ NMR $\left(\mathrm{C}_{6} \mathrm{D}_{6}\right)$ spectrum of compound 208......................................................2.

Figure A 53: ${ }^{119} \mathrm{Sn}$ NMR $\left(\mathrm{C}_{6} \mathrm{D}_{6}\right)$ spectrum of compound 208..................................................2. 280

Figure A 54: ${ }^{13} \mathrm{C}$ NMR $\left(\mathrm{C}_{6} \mathrm{D}_{6}\right)$ spectrum of compound 208 .....................................................281

Figure A 55: ${ }^{1} \mathrm{H}$ NMR $\left(\mathrm{C}_{6} \mathrm{D}_{6}\right)$ spectrum of compound 249.......................................................282

Figure A 56: ${ }^{119} \mathrm{Sn}$ NMR $\left(\mathrm{C}_{6} \mathrm{D}_{6}\right)$ spectrum of compound 249.....................................................283

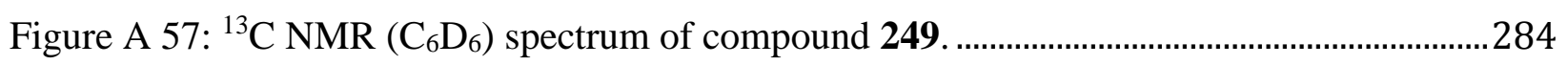

Figure A 58: ${ }^{1} \mathrm{H}$ NMR $\left(\mathrm{C}_{6} \mathrm{D}_{6}\right)$ spectrum of compound 250......................................................28 


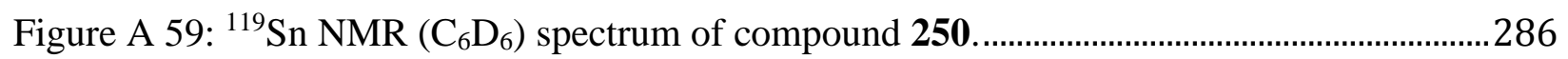

Figure A 60: ${ }^{1} \mathrm{H}$ NMR $\left(\mathrm{CDCl}_{3}\right)$ spectrum of compound 214 .........................................................28

Figure A 61: ${ }^{13} \mathrm{C}$ NMR $\left(\mathrm{CDCl}_{3}\right)$ spectrum of compound 214 .....................................................28

Figure A 62: ${ }^{1} \mathrm{H}$ NMR $\left(\mathrm{CDCl}_{3}\right)$ spectrum of compound 215....................................................289

Figure A 63: ${ }^{13} \mathrm{C} \mathrm{NMR}\left(\mathrm{CDCl}_{3}\right)$ spectrum of compound 215 ....................................................2. 290

Figure A 64: ${ }^{1} \mathrm{H}$ NMR $\left(\mathrm{CDCl}_{3}\right)$ spectrum of compound 113......................................................291

Figure A 65: ${ }^{119} \mathrm{Sn}$ NMR $\left(\mathrm{CDCl}_{3}\right)$ spectrum of compound 113 .................................................292

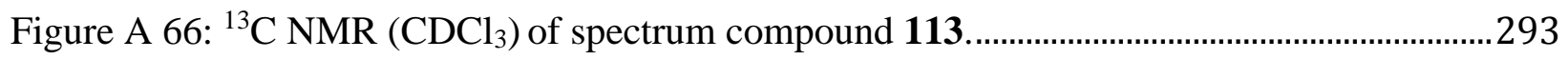

Figure A 67: ${ }^{1} \mathrm{H}$ NMR $\left(\mathrm{CDCl}_{3}\right)$ spectrum of compound 217......................................................29

Figure A 68: ${ }^{119} \mathrm{Sn}$ NMR $\left(\mathrm{CDCl}_{3}\right)$ spectrum of compound 217....................................................2.

Figure A 69: ${ }^{13} \mathrm{C}$ NMR $\left(\mathrm{CDCl}_{3}\right)$ spectrum of compound 217.....................................................296

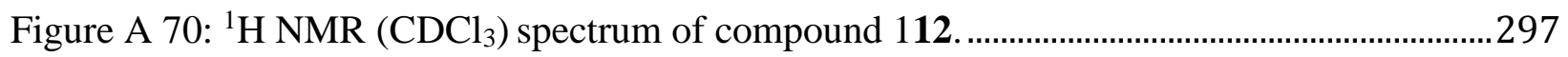

Figure A 71: ${ }^{119} \mathrm{Sn}$ NMR $\left(\mathrm{CDCl}_{3}\right)$ spectrum of compound 112 ..................................................298

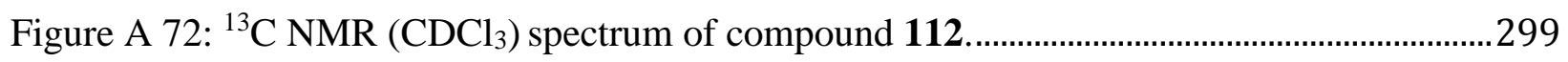

Figure A 73: ${ }^{1} \mathrm{H}$ NMR $\left(\mathrm{CDCl}_{3}\right)$ spectrum of compound 219........................................................300

Figure A 74: ${ }^{119} \mathrm{Sn}$ NMR $\left(\mathrm{CDCl}_{3}\right)$ spectrum of compound 219................................................... 301

Figure A 75: ${ }^{13} \mathrm{C}$ NMR $\left(\mathrm{CDCl}_{3}\right)$ spectrum of compound 219..................................................302

Figure A 76: ${ }^{1} \mathrm{H}$ NMR $\left(\mathrm{CDCl}_{3}\right)$ spectrum of compound 218.........................................................303

Figure A 77: ${ }^{119} \mathrm{Sn}$ NMR $\left(\mathrm{CDCl}_{3}\right)$ spectrum of compound 218 ...................................................304

Figure A 78: ${ }^{13} \mathrm{C}$ NMR $\left(\mathrm{CDCl}_{3}\right)$ spectrum of compound 218 ………………………....................305

Figure A 79: ${ }^{1} \mathrm{H}$ NMR $\left(\mathrm{CDCl}_{3}\right)$ spectrum of compound 220 ........................................................306

Figure A 80: ${ }^{119} \mathrm{Sn} \mathrm{NMR}\left(\mathrm{CDCl}_{3}\right)$ spectrum of compound 220 ......................................................307

Figure A 81: ${ }^{13} \mathrm{C} \mathrm{NMR}\left(\mathrm{CDCl}_{3}\right)$ spectrum of compound 220...................................................... 308

Figure A 82: ${ }^{1} \mathrm{H} \mathrm{NMR}\left(\mathrm{CDCl}_{3}\right)$ spectrum of compound 221......................................................309

Figure A 83: ${ }^{119} \mathrm{Sn}$ NMR $\left(\mathrm{CDCl}_{3}\right)$ spectrum of compound 221.......................................................

Figure A 84: ${ }^{13} \mathrm{C}$ NMR $\left(\mathrm{CDCl}_{3}\right)$ spectrum of compound 221....................................................311

Figure A 85: ${ }^{1} \mathrm{H}$ NMR $\left(\mathrm{C}_{6} \mathrm{D}_{6}\right)$ spectrum of compound 227.......................................................312

Figure A 86: ${ }^{119} \mathrm{Sn}$ NMR $\left(\mathrm{C}_{6} \mathrm{D}_{6}\right)$ spectrum of compound 227....................................................313

Figure A 87: ${ }^{13} \mathrm{C}$ NMR $\left(\mathrm{C}_{6} \mathrm{D}_{6}\right)$ spectrum of compound 227.................................................... 314

Figure A 88: ${ }^{119}$ Sn NMR $\left(\mathrm{C}_{6} \mathrm{D}_{6}\right)$ spectrum of compound 226......................................................

Figure A 89: ${ }^{119} \mathrm{Sn}$ NMR $\left(\mathrm{CDCl}_{3}\right)$ spectrum of compound 224 …................................................316 


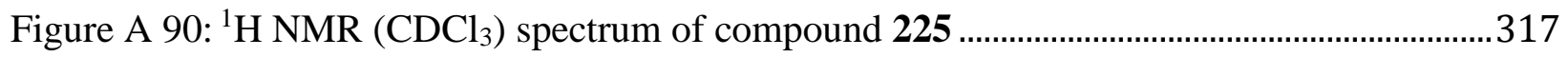

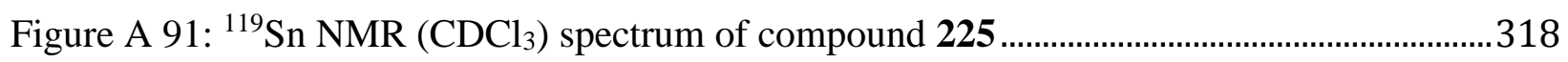

Figure A 92: ${ }^{13} \mathrm{C}$ NMR $\left(\mathrm{CDCl}_{3}\right)$ spectrum of compound 225 .....................................................319

Figure A 93: ${ }^{1} \mathrm{H}$ NMR $\mathrm{CDCl}_{3}$ spectrum of compound 228 ..........................................................320

Figure A 94: ${ }^{13} \mathrm{C}\left(\mathrm{CDCl}_{3}\right)$ NMR spectrum of compound 228 ...................................................... 321

Figure A 95: ${ }^{1} \mathrm{H}$ NMR $\left(\mathrm{CDCl}_{3}\right)$ spectrum of compound 37.........................................................322

Figure A 96: ${ }^{119} \mathrm{Sn}$ NMR $\left(\mathrm{CDCl}_{3}\right)$ spectrum of compound 37 .....................................................323

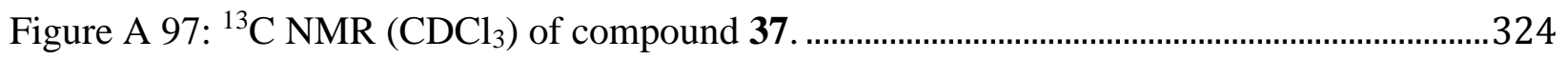

Figure A 98: ${ }^{1} \mathrm{H}$ NMR $\left(\mathrm{CDCl}_{3}\right)$ spectrum of compound 33 .......................................................325

Figure A 99: ${ }^{119} \mathrm{Sn} \mathrm{NMR}\left(\mathrm{CDCl}_{3}\right)$ spectrum of compound 33 ....................................................326

Figure A 100: ${ }^{13} \mathrm{C}$ NMR $\left(\mathrm{CDCl}_{3}\right)$ spectrum of compound 33 ....................................................327

Figure A 101: ${ }^{1} \mathrm{H}$ NMR $\left(\mathrm{CDCl}_{3}\right)$ spectrum of compound 35.......................................................328

Figure A 102: ${ }^{119} \mathrm{Sn}$ NMR $\left(\mathrm{CDCl}_{3}\right)$ spectrum of compound 35...................................................329

Figure A 103: ${ }^{1} \mathrm{H}$ NMR $\left(\mathrm{C}_{6} \mathrm{D}_{6}\right)$ spectrum of compound 231 ......................................................330

Figure A 104: ${ }^{119}$ Sn NMR $\left(\mathrm{C}_{6} \mathrm{D}_{6}\right)$ spectrum of compound 231................................................... 331

Figure A 105: ${ }^{13} \mathrm{C}$ NMR $\left(\mathrm{C}_{6} \mathrm{D}_{6}\right)$ spectrum of compound 231......................................................332

Figure A 106: ${ }^{1} \mathrm{H}$ NMR $\left(\mathrm{C}_{6} \mathrm{D}_{6}\right)$ spectrum of compound 230 .........................................................333

Figure A 107: ${ }^{119} \mathrm{Sn}$ NMR $\left(\mathrm{C}_{6} \mathrm{D}_{6}\right)$ spectrum of compound 230..................................................334

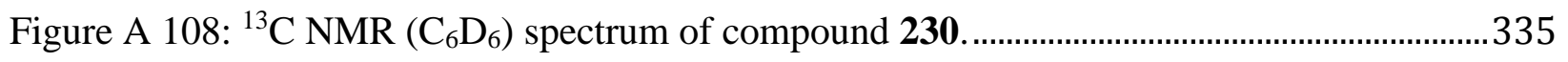

Figure A 109: ${ }^{1} \mathrm{H}$ NMR $\left(\mathrm{CDCl}_{3}\right)$ spectrum of compound 233 .....................................................336

Figure A 110: ${ }^{13} \mathrm{C} \mathrm{NMR}\left(\mathrm{CDCl}_{3}\right)$ spectrum of compound 233 ………………………...............337

Figure A 111: ${ }^{1} \mathrm{H}$ NMR $\left(\mathrm{CDCl}_{3}\right)$ spectrum of compound 234 ....................................................338

Figure A 112: ${ }^{1} \mathrm{H}$ NMR $\left(\mathrm{CDCl}_{3}\right)$ spectrum of compound 237 ......................................................339

Figure A 113: ${ }^{31} \mathrm{P}$ NMR $\left(\mathrm{CDCl}_{3}\right)$ spectrum of compound 237. ...................................................340

Figure A 114: ${ }^{13} \mathrm{C}$ NMR $\left(\mathrm{CDCl}_{3}\right)$ spectrum of compound 237..................................................... 341

Figure A 115: ${ }^{119} \mathrm{Sn} \mathrm{NMR}\left(\mathrm{CDCl}_{3}\right)$ spectrum of compound 241.................................................342

Figure A 116: ${ }^{31} \mathrm{P}$ NMR $\left(\mathrm{CDCl}_{3}\right)$ spectrum of compound 241 .....................................................343

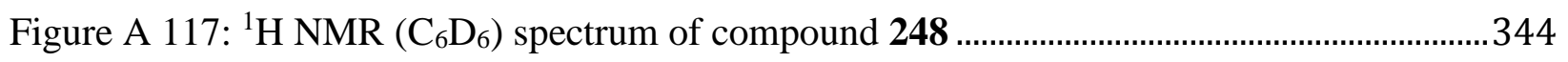

Figure A 118: ${ }^{119} \mathrm{Sn}$ NMR $\left(\mathrm{C}_{6} \mathrm{D}_{6}\right)$ spectrum of compound 248...................................................345

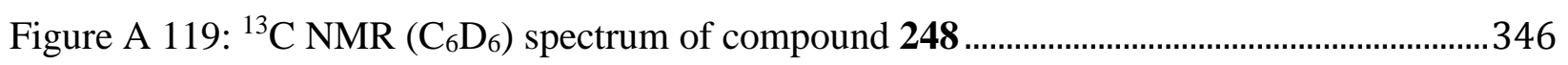

Figure A 120: DART spectrum of compound 197 .........................................................................347 


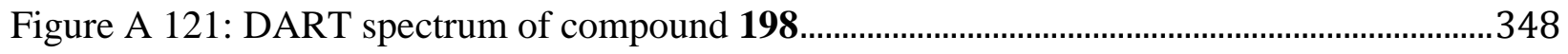

Figure A 122: HRMS-DART spectrum of compound 200.........................................................349

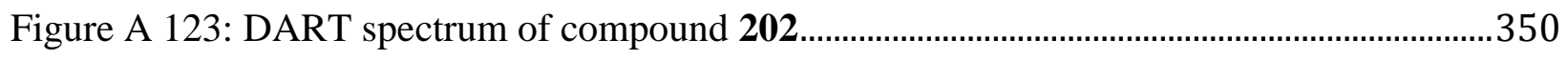

Figure A 124: HRMS-DART spectrum of compound 202 ……………………………................351

Figure A 125: DART spectrum of compound 204 ......................................................................352

Figure A 126: HRMS-DART spectrum of compound 204............................................................353

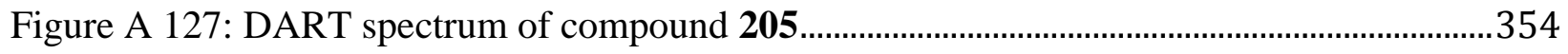

Figure A 128: HRMS-DART spectrum of compound 205 ............................................................355

Figure A 129: DART spectrum of compound 206.......................................................................356

Figure A 130: HRMS-DART spectrum of compound 206.............................................................357

Figure A 131: DART spectrum of compound 217 ………………………………………….....358

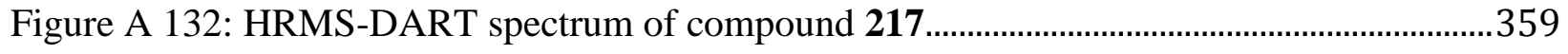

Figure A 133: DART spectrum of compound 112 ........................................................................360

Figure A 134: DART spectrum of compound 112 ........................................................................361

Figure A 135: HRMS-DART spectrum of compound 112............................................................362

Figure A 136: DART spectrum of compound 219 ………………………………………….......363

Figure A 137: HRMS-DART spectrum of compound 219.............................................................364

Figure A 138: DART spectrum of compound 218 ………………………………………….....365

Figure A 139: HRMS-DART spectrum of compound 218 ……………………………………....366

Figure A 140: DART spectrum of compound 226.......................................................................367

Figure A 141: DART spectrum of compound 227 ……………………………………………....368

Figure A 142: HRMS-DART spectrum of compound 227..............................................................369

Figure A 143: DART spectrum of compound 224 ........................................................................370

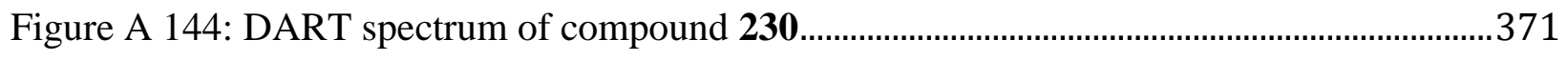

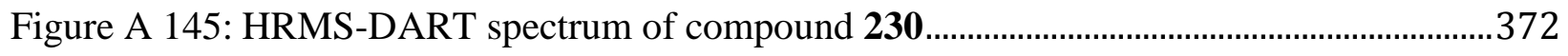

Figure A 146: DART spectrum of compound 231 ………………………………………….......373

Figure A 147: HRMS-DART spectrum of compound 231..........................................................374 
Table A 1. Crystal data and structure refinement for 198.

Identification code

Empirical formula

Formula weight

Temperature

Wavelength

Crystal system

Space group

Unit cell dimensions

Volume

$\mathrm{Z}$

Density (calculated)

Absorption coefficient

$\mathrm{F}(000)$

Crystal size

Theta range for data collection

Index ranges

Reflections collected

Independent reflections

Completeness to theta $=25.12^{\circ}$

Absorption correction

Max. and min. transmission

Refinement method

Data / restraints / parameters

Goodness-of-fit on $\mathrm{F}^{2}$

Final R indices [I $>2 \operatorname{sigma}(\mathrm{I})]$

$\mathrm{R}$ indices (all data)

Largest diff. peak and hole k11250a

$\mathrm{C}_{28} \mathrm{H}_{25} \mathrm{~F}_{3} \mathrm{OSn}$

553.17

200(1) K

$0.71073 \AA$

Monoclinic

P 21/n

$\mathrm{a}=6.4631(13) \AA \quad \alpha=90^{\circ}$.

$\mathrm{b}=12.192(2) \AA$

$\beta=90.14(3)^{\circ}$.

$\mathrm{c}=31.554(6) \AA$

2486.3(9) $\AA^{3}$

4

$1.478 \mathrm{Mg} / \mathrm{m}^{3}$

$1.067 \mathrm{~mm}^{-1}$

1112

$0.34 \times 0.08 \times 0.06 \mathrm{~mm}^{3}$

2.56 to $25.12^{\circ}$.

$-7<=\mathrm{h}<=7,-14<=\mathrm{k}<=14,-37<=\mathrm{l}<=36$

15431

$4391[\mathrm{R}(\mathrm{int})=0.0558]$

$98.6 \%$

Semi-empirical from equivalents

0.998 and 0.936

Full-matrix least-squares on $\mathrm{F}^{2}$

4391 / 71 / 306

1.092

$\mathrm{R} 1=0.0589, \mathrm{wR} 2=0.1152$

$\mathrm{R} 1=0.1023, \mathrm{wR} 2=0.1344$

0.679 and -0.988 e. $\AA^{-3}$ 
Table A 2. Atomic coordinates $\left(x \mathbf{1 0}^{4}\right)$ and equivalent isotropic displacement parameters $\left(\AA^{2} \times 10^{3}\right)$ for 198.

\begin{tabular}{|c|c|c|c|c|}
\hline & $\mathrm{X}$ & $\mathrm{y}$ & $\mathrm{z}$ & $\mathrm{U}(\mathrm{eq})$ \\
\hline $\operatorname{Sn}(1)$ & $60(1)$ & $8785(1)$ & $1562(1)$ & $73(1)$ \\
\hline $\mathrm{F}(1)$ & $10730(13)$ & $5463(8)$ & $-541(3)$ & $135(3)$ \\
\hline $\mathrm{F}(2)$ & $7737(16)$ & $4942(7)$ & $-354(3)$ & $138(3)$ \\
\hline $\mathrm{F}(3)$ & $8194(17)$ & $5626(8)$ & $-965(3)$ & $146(3)$ \\
\hline $\mathrm{F}(1 \mathrm{~A})$ & $9470(30)$ & $5134(12)$ & $-463(5)$ & $139(3)$ \\
\hline $\mathrm{F}(2 \mathrm{~A})$ & $6471(18)$ & $5357(10)$ & $-725(5)$ & $141(3)$ \\
\hline$F(3 A)$ & $9090(30)$ & $6011(11)$ & $-1038(4)$ & $143(3)$ \\
\hline $\mathrm{O}(1)$ & $5369(15)$ & $8166(8)$ & 436(3) & $58(3)$ \\
\hline $\mathrm{C}(1)$ & $1690(20)$ & $8535(11)$ & $967(4)$ & $57(5)$ \\
\hline$C(2)$ & $3222(15)$ & $7595(9)$ & $1006(3)$ & $56(3)$ \\
\hline $\mathrm{C}(3)$ & 4292(19) & $7242(8)$ & 601(3) & $59(3)$ \\
\hline $\mathrm{O}(1 \mathrm{~A})$ & $4540(20)$ & $8471(10)$ & 293(5) & $61(5)$ \\
\hline$C(1 \mathrm{~A})$ & $2490(18)$ & $8340(20)$ & $1116(5)$ & $54(7)$ \\
\hline$C(2 A)$ & $1410(20)$ & $8137(15)$ & $696(4)$ & $44(4)$ \\
\hline$C(3 A)$ & $2970(20)$ & $7686(13)$ & $382(6)$ & $56(5)$ \\
\hline $\mathrm{C}(4)$ & $6632(11)$ & $7989(6)$ & $103(2)$ & $43(3)$ \\
\hline$C(5)$ & $7811(12)$ & $8875(5)$ & $-32(2)$ & $43(3)$ \\
\hline$C(6)$ & $9337(11)$ & $8725(6)$ & $-336(3)$ & $55(3)$ \\
\hline$C(7)$ & $9685(12)$ & $7689(7)$ & $-505(2)$ & $72(4)$ \\
\hline $\mathrm{C}(8)$ & $8505(13)$ & $6803(5)$ & $-370(3)$ & $62(4)$ \\
\hline $\mathrm{C}(9)$ & $6979(12)$ & $6952(5)$ & $-66(2)$ & $54(3)$ \\
\hline $\mathrm{C}(4 \mathrm{~A})$ & $6055(15)$ & $8167(8)$ & $24(3)$ & $43(4)$ \\
\hline$C(5 A)$ & $7268(17)$ & $9043(6)$ & $-109(3)$ & $32(4)$ \\
\hline$C(6 A)$ & $8773(16)$ & $8885(7)$ & $-417(3)$ & $38(4)$ \\
\hline$C(7 A)$ & $9065(16)$ & $7851(9)$ & $-592(3)$ & $51(5)$ \\
\hline$C(8 A)$ & $7851(17)$ & $6975(7)$ & $-460(3)$ & $36(4)$ \\
\hline $\mathrm{C}(9 \mathrm{~A})$ & $6347(16)$ & $7133(7)$ & $-152(3)$ & $37(4)$ \\
\hline$C(10)$ & $8725(12)$ & $5760(8)$ & $-556(3)$ & $132(6)$ \\
\hline$C(10 A)$ & $8260(20)$ & 5901(10) & $-648(4)$ & $132(6)$ \\
\hline $\mathrm{C}(11)$ & $1894(11)$ & $9015(5)$ & $2121(2)$ & $66(2)$ \\
\hline$C(12)$ & $3340(11)$ & $9854(5)$ & 2151(3) & $65(2)$ \\
\hline
\end{tabular}




$\begin{array}{lrrrr}\mathrm{C}(13) & 4564(13) & 9997(6) & 2509(3) & 77(2) \\ \mathrm{C}(14) & 4322(15) & 9264(8) & 2842(3) & 91(3) \\ \mathrm{C}(15) & 2921(17) & 8423(8) & 2819(4) & 103(4) \\ \mathrm{C}(16) & 1720(14) & 8305(7) & 2466(4) & 89(3) \\ \mathrm{C}(17) & -1741(11) & 10240(6) & 1467(2) & 63(2) \\ \mathrm{C}(18) & -3139(12) & 10337(7) & 1130(2) & 69(2) \\ \mathrm{C}(19) & -4313(13) & 11264(8) & 1072(3) & 81(2) \\ \mathrm{C}(20) & -4123(12) & 12132(7) & 1349(3) & 77(2) \\ \mathrm{C}(21) & -2754(12) & 12068(6) & 1685(3) & 71(2) \\ \mathrm{C}(22) & -1588(11) & 11141(6) & 1739(2) & 65(2) \\ \mathrm{C}(23) & -1701(12) & 7295(4) & 1708(3) & 41(3) \\ \mathrm{C}(24) & -1440(12) & 6394(6) & 1444(3) & 48(3) \\ \mathrm{C}(25) & -2756(13) & 5497(5) & 1477(3) & 57(4) \\ \mathrm{C}(26) & -4335(12) & 5500(5) & 1776(3) & 54(3) \\ \mathrm{C}(27) & -4597(12) & 6401(6) & 2040(3) & 59(5) \\ \mathrm{C}(28) & -3280(13) & 7298(5) & 2007(3) & 48(4) \\ \mathrm{C}(23 \mathrm{~A}) & -2252(11) & 7464(6) & 1549(3) & 38(3) \\ \mathrm{C}(24 \mathrm{~A}) & -2273(13) & 6689(7) & 1226(3) & 50(4) \\ \mathrm{C}(25 \mathrm{~A}) & -3553(15) & 5776(7) & 1255(3) & 62(5) \\ \mathrm{C}(26 \mathrm{~A}) & -4812(13) & 5638(5) & 1608(4) & 53(4) \\ \mathrm{C}(28 \mathrm{~A}) & -4790(13) & 6412(7) & 1931(3) & 41(4) \\ \mathrm{C}(27 \mathrm{~A}) & -3510(13) & 7325(6) & 1902(3) & 41(4)\end{array}$


Table A 3. Bond lengths $[\AA ̊ \AA]$ and angles $\left[{ }^{\circ}\right]$ for 198.

\begin{tabular}{|c|c|}
\hline $\operatorname{Sn}(1)-C(11)$ & $2.141(8)$ \\
\hline $\operatorname{Sn}(1)-C(17)$ & $2.143(8)$ \\
\hline $\operatorname{Sn}(1)-C(1 A)$ & $2.178(9)$ \\
\hline $\operatorname{Sn}(1)-C(1)$ & $2.179(9)$ \\
\hline $\operatorname{Sn}(1)-C(23)$ & $2.193(4)$ \\
\hline $\operatorname{Sn}(1)-C(23 A)$ & $2.197(4)$ \\
\hline $\mathrm{F}(1)-\mathrm{C}(10)$ & $1.346(5)$ \\
\hline $\mathrm{F}(2)-\mathrm{C}(10)$ & $1.346(5)$ \\
\hline $\mathrm{F}(3)-\mathrm{C}(10)$ & $1.345(5)$ \\
\hline $\mathrm{F}(1 \mathrm{~A})-\mathrm{C}(10 \mathrm{~A})$ & $1.351(5)$ \\
\hline $\mathrm{F}(2 \mathrm{~A})-\mathrm{C}(10 \mathrm{~A})$ & $1.352(5)$ \\
\hline $\mathrm{F}(3 \mathrm{~A})-\mathrm{C}(10 \mathrm{~A})$ & $1.352(5)$ \\
\hline $\mathrm{O}(1)-\mathrm{C}(4)$ & $1.350(6)$ \\
\hline $\mathrm{O}(1)-\mathrm{C}(3)$ & $1.423(9)$ \\
\hline $\mathrm{C}(1)-\mathrm{C}(2)$ & $1.518(7)$ \\
\hline $\mathrm{C}(1)-\mathrm{H}(1 \mathrm{~A})$ & 0.9900 \\
\hline $\mathrm{C}(1)-\mathrm{H}(1 \mathrm{~B})$ & 0.9900 \\
\hline$C(2)-C(3)$ & $1.517(7)$ \\
\hline $\mathrm{C}(2)-\mathrm{H}(2 \mathrm{~A})$ & 0.9900 \\
\hline $\mathrm{C}(2)-\mathrm{H}(2 \mathrm{~B})$ & 0.9900 \\
\hline $\mathrm{C}(3)-\mathrm{H}(3 \mathrm{~A})$ & 0.9900 \\
\hline $\mathrm{C}(3)-\mathrm{H}(3 \mathrm{~B})$ & 0.9900 \\
\hline $\mathrm{O}(1 \mathrm{~A})-\mathrm{C}(4 \mathrm{~A})$ & $1.351(6)$ \\
\hline $\mathrm{O}(1 \mathrm{~A})-\mathrm{C}(3 \mathrm{~A})$ & $1.424(9)$ \\
\hline $\mathrm{C}(1 \mathrm{~A})-\mathrm{C}(2 \mathrm{~A})$ & $1.517(7)$ \\
\hline $\mathrm{C}(1 \mathrm{~A})-\mathrm{H}(1 \mathrm{AA})$ & 0.9900 \\
\hline $\mathrm{C}(1 \mathrm{~A})-\mathrm{H}(1 \mathrm{AB})$ & 0.9900 \\
\hline$C(2 A)-C(3 A)$ & $1.517(7)$ \\
\hline $\mathrm{C}(2 \mathrm{~A})-\mathrm{H}(2 \mathrm{AA})$ & 0.9900 \\
\hline $\mathrm{C}(2 \mathrm{~A})-\mathrm{H}(2 \mathrm{AB})$ & 0.9900 \\
\hline $\mathrm{C}(3 \mathrm{~A})-\mathrm{H}(3 \mathrm{AA})$ & 0.9900 \\
\hline $\mathrm{C}(3 \mathrm{~A})-\mathrm{H}(3 \mathrm{AB})$ & 0.9900 \\
\hline$C(4)-C(5)$ & 1.3900 \\
\hline
\end{tabular}




\begin{tabular}{|c|c|}
\hline$C(4)-C(9)$ & 1.3900 \\
\hline$C(5)-C(6)$ & 1.3900 \\
\hline $\mathrm{C}(5)-\mathrm{H}(5 \mathrm{~A})$ & 0.9500 \\
\hline$C(6)-C(7)$ & 1.3900 \\
\hline $\mathrm{C}(6)-\mathrm{H}(6 \mathrm{~A})$ & 0.9500 \\
\hline$C(7)-C(8)$ & 1.3900 \\
\hline $\mathrm{C}(7)-\mathrm{H}(7 \mathrm{~A})$ & 0.9500 \\
\hline $\mathrm{C}(8)-\mathrm{C}(9)$ & 1.3900 \\
\hline$C(8)-C(10)$ & $1.407(11)$ \\
\hline $\mathrm{C}(9)-\mathrm{H}(9 \mathrm{~A})$ & 0.9500 \\
\hline$C(4 A)-C(5 A)$ & 1.3900 \\
\hline$C(4 A)-C(9 A)$ & 1.3900 \\
\hline$C(5 A)-C(6 A)$ & 1.3900 \\
\hline $\mathrm{C}(5 \mathrm{~A})-\mathrm{H}(5 \mathrm{AA})$ & 0.9500 \\
\hline $\mathrm{C}(6 \mathrm{~A})-\mathrm{C}(7 \mathrm{~A})$ & 1.3900 \\
\hline $\mathrm{C}(6 \mathrm{~A})-\mathrm{H}(6 \mathrm{AA})$ & 0.9500 \\
\hline $\mathrm{C}(7 \mathrm{~A})-\mathrm{C}(8 \mathrm{~A})$ & 1.3900 \\
\hline C(7A)-H(7AA) & 0.9500 \\
\hline $\mathrm{C}(8 \mathrm{~A})-\mathrm{C}(9 \mathrm{~A})$ & 1.3900 \\
\hline$C(8 A)-C(10 A)$ & $1.462(13)$ \\
\hline $\mathrm{C}(9 \mathrm{~A})-\mathrm{H}(9 \mathrm{AA})$ & 0.9500 \\
\hline $\mathrm{C}(11)-\mathrm{C}(12)$ & $1.389(9)$ \\
\hline$C(11)-C(16)$ & $1.395(11)$ \\
\hline $\mathrm{C}(12)-\mathrm{C}(13)$ & $1.389(11)$ \\
\hline $\mathrm{C}(12)-\mathrm{H}(12 \mathrm{~A})$ & 0.9500 \\
\hline $\mathrm{C}(13)-\mathrm{C}(14)$ & $1.388(11)$ \\
\hline $\mathrm{C}(13)-\mathrm{H}(13 \mathrm{~A})$ & 0.9500 \\
\hline$C(14)-C(15)$ & $1.369(13)$ \\
\hline $\mathrm{C}(14)-\mathrm{H}(14 \mathrm{~A})$ & 0.9500 \\
\hline$C(15)-C(16)$ & $1.364(13)$ \\
\hline $\mathrm{C}(15)-\mathrm{H}(15 \mathrm{~A})$ & 0.9500 \\
\hline $\mathrm{C}(16)-\mathrm{H}(16 \mathrm{~A})$ & 0.9500 \\
\hline$C(17)-C(22)$ & $1.396(9)$ \\
\hline $\mathrm{C}(17)-\mathrm{C}(18)$ & $1.399(10)$ \\
\hline$C(18)-C(19)$ & $1.373(11)$ \\
\hline $\mathrm{C}(18)-\mathrm{H}(18 \mathrm{~A})$ & 0.9500 \\
\hline
\end{tabular}




\begin{tabular}{|c|c|}
\hline $\mathrm{C}(19)-\mathrm{C}(20)$ & $1.379(11)$ \\
\hline C(19)-H(19A) & 0.9500 \\
\hline $\mathrm{C}(20)-\mathrm{C}(21)$ & $1.380(10)$ \\
\hline $\mathrm{C}(20)-\mathrm{H}(20 \mathrm{~A})$ & 0.9500 \\
\hline $\mathrm{C}(21)-\mathrm{C}(22)$ & $1.369(10)$ \\
\hline $\mathrm{C}(21)-\mathrm{H}(21 \mathrm{~A})$ & 0.9500 \\
\hline $\mathrm{C}(22)-\mathrm{H}(22 \mathrm{~A})$ & 0.9500 \\
\hline $\mathrm{C}(23)-\mathrm{C}(24)$ & 1.3900 \\
\hline $\mathrm{C}(23)-\mathrm{C}(28)$ & 1.3900 \\
\hline$C(24)-C(25)$ & 1.3900 \\
\hline $\mathrm{C}(24)-\mathrm{H}(24 \mathrm{~A})$ & 0.9500 \\
\hline$C(25)-C(26)$ & 1.3900 \\
\hline $\mathrm{C}(25)-\mathrm{H}(25 \mathrm{~A})$ & 0.9500 \\
\hline$C(26)-C(27)$ & 1.3900 \\
\hline $\mathrm{C}(26)-\mathrm{H}(26 \mathrm{~A})$ & 0.9500 \\
\hline $\mathrm{C}(27)-\mathrm{C}(28)$ & 1.3900 \\
\hline $\mathrm{C}(27)-\mathrm{H}(27 \mathrm{~A})$ & 0.9500 \\
\hline $\mathrm{C}(28)-\mathrm{H}(28 \mathrm{~A})$ & 0.9500 \\
\hline $\mathrm{C}(23 \mathrm{~A})-\mathrm{C}(24 \mathrm{~A})$ & 1.3900 \\
\hline $\mathrm{C}(23 \mathrm{~A})-\mathrm{C}(27 \mathrm{~A})$ & 1.3900 \\
\hline $\mathrm{C}(24 \mathrm{~A})-\mathrm{C}(25 \mathrm{~A})$ & 1.3900 \\
\hline $\mathrm{C}(24 \mathrm{~A})-\mathrm{H}(24 \mathrm{~B})$ & 0.9500 \\
\hline$C(25 A)-C(26 A)$ & 1.3900 \\
\hline $\mathrm{C}(25 \mathrm{~A})-\mathrm{H}(25 \mathrm{~B})$ & 0.9500 \\
\hline $\mathrm{C}(26 \mathrm{~A})-\mathrm{C}(28 \mathrm{~A})$ & 1.3900 \\
\hline $\mathrm{C}(26 \mathrm{~A})-\mathrm{H}(26 \mathrm{~B})$ & 0.9500 \\
\hline $\mathrm{C}(28 \mathrm{~A})-\mathrm{C}(27 \mathrm{~A})$ & 1.3900 \\
\hline $\mathrm{C}(28 \mathrm{~A})-\mathrm{H}(28 \mathrm{~B})$ & 0.9500 \\
\hline $\mathrm{C}(27 \mathrm{~A})-\mathrm{H}(27 \mathrm{~B})$ & 0.9500 \\
\hline $\mathrm{C}(11)-\mathrm{Sn}(1)-\mathrm{C}(17)$ & $107.8(3)$ \\
\hline$C(11)-\operatorname{Sn}(1)-C(1 A)$ & $99.6(4)$ \\
\hline$C(17)-S n(1)-C(1 A)$ & $120.4(7)$ \\
\hline $\mathrm{C}(11)-\mathrm{Sn}(1)-\mathrm{C}(1)$ & $117.4(4)$ \\
\hline $\mathrm{C}(17)-\mathrm{Sn}(1)-\mathrm{C}(1)$ & $105.0(4)$ \\
\hline$C(1 \mathrm{~A})-\operatorname{Sn}(1)-C(1)$ & $19.4(5)$ \\
\hline
\end{tabular}




\begin{tabular}{|c|c|}
\hline$C(11)-\operatorname{Sn}(1)-C(23)$ & $102.8(3)$ \\
\hline$C(17)-\operatorname{Sn}(1)-C(23)$ & $115.7(3)$ \\
\hline$C(1 \mathrm{~A})-\operatorname{Sn}(1)-\mathrm{C}(23)$ & $107.9(7)$ \\
\hline$C(1)-\operatorname{Sn}(1)-C(23)$ & $108.6(5)$ \\
\hline$C(11)-\operatorname{Sn}(1)-C(23 A)$ & 119.1(3) \\
\hline$C(17)-\operatorname{Sn}(1)-C(23 A)$ & $103.6(3)$ \\
\hline$C(1 \mathrm{~A})-\operatorname{Sn}(1)-C(23 \mathrm{~A})$ & $107.4(6)$ \\
\hline$C(1)-S n(1)-C(23 A)$ & $102.3(5)$ \\
\hline$C(23)-\operatorname{Sn}(1)-C(23 A)$ & $17.0(3)$ \\
\hline $\mathrm{C}(4)-\mathrm{O}(1)-\mathrm{C}(3)$ & $117.1(8)$ \\
\hline$C(2)-C(1)-\operatorname{Sn}(1)$ & $110.6(7)$ \\
\hline $\mathrm{C}(2)-\mathrm{C}(1)-\mathrm{H}(1 \mathrm{~A})$ & 109.5 \\
\hline $\operatorname{Sn}(1)-C(1)-H(1 A)$ & 109.5 \\
\hline $\mathrm{C}(2)-\mathrm{C}(1)-\mathrm{H}(1 \mathrm{~B})$ & 109.5 \\
\hline $\operatorname{Sn}(1)-C(1)-H(1 B)$ & 109.5 \\
\hline $\mathrm{H}(1 \mathrm{~A})-\mathrm{C}(1)-\mathrm{H}(1 \mathrm{~B})$ & 108.1 \\
\hline$C(3)-C(2)-C(1)$ & 116.3(9) \\
\hline $\mathrm{C}(3)-\mathrm{C}(2)-\mathrm{H}(2 \mathrm{~A})$ & 108.2 \\
\hline $\mathrm{C}(1)-\mathrm{C}(2)-\mathrm{H}(2 \mathrm{~A})$ & 108.2 \\
\hline $\mathrm{C}(3)-\mathrm{C}(2)-\mathrm{H}(2 \mathrm{~B})$ & 108.2 \\
\hline $\mathrm{C}(1)-\mathrm{C}(2)-\mathrm{H}(2 \mathrm{~B})$ & 108.2 \\
\hline $\mathrm{H}(2 \mathrm{~A})-\mathrm{C}(2)-\mathrm{H}(2 \mathrm{~B})$ & 107.4 \\
\hline $\mathrm{O}(1)-\mathrm{C}(3)-\mathrm{C}(2)$ & $107.9(7)$ \\
\hline $\mathrm{O}(1)-\mathrm{C}(3)-\mathrm{H}(3 \mathrm{~A})$ & 110.1 \\
\hline $\mathrm{C}(2)-\mathrm{C}(3)-\mathrm{H}(3 \mathrm{~A})$ & 110.1 \\
\hline $\mathrm{O}(1)-\mathrm{C}(3)-\mathrm{H}(3 \mathrm{~B})$ & 110.1 \\
\hline $\mathrm{C}(2)-\mathrm{C}(3)-\mathrm{H}(3 \mathrm{~B})$ & 110.1 \\
\hline $\mathrm{H}(3 \mathrm{~A})-\mathrm{C}(3)-\mathrm{H}(3 \mathrm{~B})$ & 108.4 \\
\hline $\mathrm{C}(4 \mathrm{~A})-\mathrm{O}(1 \mathrm{~A})-\mathrm{C}(3 \mathrm{~A})$ & $117.4(11)$ \\
\hline$C(2 A)-C(1 A)-S n(1)$ & $105.9(8)$ \\
\hline $\mathrm{C}(2 \mathrm{~A})-\mathrm{C}(1 \mathrm{~A})-\mathrm{H}(1 \mathrm{AA})$ & 110.6 \\
\hline $\operatorname{Sn}(1)-C(1 A)-H(1 A A)$ & 110.6 \\
\hline $\mathrm{C}(2 \mathrm{~A})-\mathrm{C}(1 \mathrm{~A})-\mathrm{H}(1 \mathrm{AB})$ & 110.6 \\
\hline $\operatorname{Sn}(1)-C(1 A)-H(1 A B)$ & 110.6 \\
\hline $\mathrm{H}(1 \mathrm{AA})-\mathrm{C}(1 \mathrm{~A})-\mathrm{H}(1 \mathrm{AB})$ & 108.7 \\
\hline$C(1 \mathrm{~A})-\mathrm{C}(2 \mathrm{~A})-\mathrm{C}(3 \mathrm{~A})$ & $109.1(12)$ \\
\hline
\end{tabular}




\begin{tabular}{|c|c|}
\hline $\mathrm{C}(1 \mathrm{~A})-\mathrm{C}(2 \mathrm{~A})-\mathrm{H}(2 \mathrm{AA})$ & 109.9 \\
\hline $\mathrm{C}(3 \mathrm{~A})-\mathrm{C}(2 \mathrm{~A})-\mathrm{H}(2 \mathrm{AA})$ & 109.9 \\
\hline $\mathrm{C}(1 \mathrm{~A})-\mathrm{C}(2 \mathrm{~A})-\mathrm{H}(2 \mathrm{AB})$ & 109.9 \\
\hline $\mathrm{C}(3 \mathrm{~A})-\mathrm{C}(2 \mathrm{~A})-\mathrm{H}(2 \mathrm{AB})$ & 109.9 \\
\hline $\mathrm{H}(2 \mathrm{AA})-\mathrm{C}(2 \mathrm{~A})-\mathrm{H}(2 \mathrm{AB})$ & 108.3 \\
\hline $\mathrm{O}(1 \mathrm{~A})-\mathrm{C}(3 \mathrm{~A})-\mathrm{C}(2 \mathrm{~A})$ & 111.1(11) \\
\hline $\mathrm{O}(1 \mathrm{~A})-\mathrm{C}(3 \mathrm{~A})-\mathrm{H}(3 \mathrm{AA})$ & 109.4 \\
\hline $\mathrm{C}(2 \mathrm{~A})-\mathrm{C}(3 \mathrm{~A})-\mathrm{H}(3 \mathrm{AA})$ & 109.4 \\
\hline $\mathrm{O}(1 \mathrm{~A})-\mathrm{C}(3 \mathrm{~A})-\mathrm{H}(3 \mathrm{AB})$ & 109.4 \\
\hline $\mathrm{C}(2 \mathrm{~A})-\mathrm{C}(3 \mathrm{~A})-\mathrm{H}(3 \mathrm{AB})$ & 109.4 \\
\hline $\mathrm{H}(3 \mathrm{AA})-\mathrm{C}(3 \mathrm{~A})-\mathrm{H}(3 \mathrm{AB})$ & 108.0 \\
\hline $\mathrm{O}(1)-\mathrm{C}(4)-\mathrm{C}(5)$ & $116.6(6)$ \\
\hline $\mathrm{O}(1)-\mathrm{C}(4)-\mathrm{C}(9)$ & $122.9(6)$ \\
\hline $\mathrm{C}(5)-\mathrm{C}(4)-\mathrm{C}(9)$ & 120.0 \\
\hline$C(4)-C(5)-C(6)$ & 120.0 \\
\hline $\mathrm{C}(4)-\mathrm{C}(5)-\mathrm{H}(5 \mathrm{~A})$ & 120.0 \\
\hline $\mathrm{C}(6)-\mathrm{C}(5)-\mathrm{H}(5 \mathrm{~A})$ & 120.0 \\
\hline$C(5)-C(6)-C(7)$ & 120.0 \\
\hline$C(5)-C(6)-H(6 A)$ & 120.0 \\
\hline$C(7)-C(6)-H(6 A)$ & 120.0 \\
\hline$C(8)-C(7)-C(6)$ & 120.0 \\
\hline $\mathrm{C}(8)-\mathrm{C}(7)-\mathrm{H}(7 \mathrm{~A})$ & 120.0 \\
\hline $\mathrm{C}(6)-\mathrm{C}(7)-\mathrm{H}(7 \mathrm{~A})$ & 120.0 \\
\hline $\mathrm{C}(7)-\mathrm{C}(8)-\mathrm{C}(9)$ & 120.0 \\
\hline$C(7)-C(8)-C(10)$ & $121.3(6)$ \\
\hline $\mathrm{C}(9)-\mathrm{C}(8)-\mathrm{C}(10)$ & $118.6(6)$ \\
\hline $\mathrm{C}(8)-\mathrm{C}(9)-\mathrm{C}(4)$ & 120.0 \\
\hline $\mathrm{C}(8)-\mathrm{C}(9)-\mathrm{H}(9 \mathrm{~A})$ & 120.0 \\
\hline $\mathrm{C}(4)-\mathrm{C}(9)-\mathrm{H}(9 \mathrm{~A})$ & 120.0 \\
\hline $\mathrm{O}(1 \mathrm{~A})-\mathrm{C}(4 \mathrm{~A})-\mathrm{C}(5 \mathrm{~A})$ & $113.0(8)$ \\
\hline $\mathrm{O}(1 \mathrm{~A})-\mathrm{C}(4 \mathrm{~A})-\mathrm{C}(9 \mathrm{~A})$ & $126.7(8)$ \\
\hline$C(5 A)-C(4 A)-C(9 A)$ & 120.0 \\
\hline$C(6 A)-C(5 A)-C(4 A)$ & 120.0 \\
\hline $\mathrm{C}(6 \mathrm{~A})-\mathrm{C}(5 \mathrm{~A})-\mathrm{H}(5 \mathrm{AA})$ & 120.0 \\
\hline $\mathrm{C}(4 \mathrm{~A})-\mathrm{C}(5 \mathrm{~A})-\mathrm{H}(5 \mathrm{AA})$ & 120.0 \\
\hline$C(7 A)-C(6 A)-C(5 A)$ & 120.0 \\
\hline
\end{tabular}




\begin{tabular}{|c|c|}
\hline$C(7 A)-C(6 A)-H(6 A A)$ & 120.0 \\
\hline $\mathrm{C}(5 \mathrm{~A})-\mathrm{C}(6 \mathrm{~A})-\mathrm{H}(6 \mathrm{AA})$ & 120.0 \\
\hline$C(6 A)-C(7 A)-C(8 A)$ & 120.0 \\
\hline $\mathrm{C}(6 \mathrm{~A})-\mathrm{C}(7 \mathrm{~A})-\mathrm{H}(7 \mathrm{AA})$ & 120.0 \\
\hline $\mathrm{C}(8 \mathrm{~A})-\mathrm{C}(7 \mathrm{~A})-\mathrm{H}(7 \mathrm{AA})$ & 120.0 \\
\hline $\mathrm{C}(9 \mathrm{~A})-\mathrm{C}(8 \mathrm{~A})-\mathrm{C}(7 \mathrm{~A})$ & 120.0 \\
\hline$C(9 A)-C(8 A)-C(10 A)$ & $122.4(8)$ \\
\hline$C(7 A)-C(8 A)-C(10 A)$ & $117.6(8)$ \\
\hline $\mathrm{C}(8 \mathrm{~A})-\mathrm{C}(9 \mathrm{~A})-\mathrm{C}(4 \mathrm{~A})$ & 120.0 \\
\hline $\mathrm{C}(8 \mathrm{~A})-\mathrm{C}(9 \mathrm{~A})-\mathrm{H}(9 \mathrm{AA})$ & 120.0 \\
\hline $\mathrm{C}(4 \mathrm{~A})-\mathrm{C}(9 \mathrm{~A})-\mathrm{H}(9 \mathrm{AA})$ & 120.0 \\
\hline $\mathrm{F}(3)-\mathrm{C}(10)-\mathrm{F}(2)$ & $104.3(6)$ \\
\hline $\mathrm{F}(3)-\mathrm{C}(10)-\mathrm{F}(1)$ & $104.1(6)$ \\
\hline $\mathrm{F}(2)-\mathrm{C}(10)-\mathrm{F}(1)$ & $104.0(6)$ \\
\hline $\mathrm{F}(3)-\mathrm{C}(10)-\mathrm{C}(8)$ & $118.9(8)$ \\
\hline $\mathrm{F}(2)-\mathrm{C}(10)-\mathrm{C}(8)$ & $115.0(8)$ \\
\hline $\mathrm{F}(1)-\mathrm{C}(10)-\mathrm{C}(8)$ & $109.0(8)$ \\
\hline $\mathrm{F}(1 \mathrm{~A})-\mathrm{C}(10 \mathrm{~A})-\mathrm{F}(3 \mathrm{~A})$ & $103.3(6)$ \\
\hline $\mathrm{F}(1 \mathrm{~A})-\mathrm{C}(10 \mathrm{~A})-\mathrm{F}(2 \mathrm{~A})$ & $103.3(6)$ \\
\hline $\mathrm{F}(3 \mathrm{~A})-\mathrm{C}(10 \mathrm{~A})-\mathrm{F}(2 \mathrm{~A})$ & $103.2(6)$ \\
\hline $\mathrm{F}(1 \mathrm{~A})-\mathrm{C}(10 \mathrm{~A})-\mathrm{C}(8 \mathrm{~A})$ & $123.2(11)$ \\
\hline $\mathrm{F}(3 \mathrm{~A})-\mathrm{C}(10 \mathrm{~A})-\mathrm{C}(8 \mathrm{~A})$ & $110.8(11)$ \\
\hline $\mathrm{F}(2 \mathrm{~A})-\mathrm{C}(10 \mathrm{~A})-\mathrm{C}(8 \mathrm{~A})$ & $111.0(11)$ \\
\hline $\mathrm{C}(12)-\mathrm{C}(11)-\mathrm{C}(16)$ & $117.4(8)$ \\
\hline $\mathrm{C}(12)-\mathrm{C}(11)-\mathrm{Sn}(1)$ & $121.6(6)$ \\
\hline $\mathrm{C}(16)-\mathrm{C}(11)-\mathrm{Sn}(1)$ & $121.0(6)$ \\
\hline $\mathrm{C}(13)-\mathrm{C}(12)-\mathrm{C}(11)$ & $122.0(8)$ \\
\hline $\mathrm{C}(13)-\mathrm{C}(12)-\mathrm{H}(12 \mathrm{~A})$ & 119.0 \\
\hline $\mathrm{C}(11)-\mathrm{C}(12)-\mathrm{H}(12 \mathrm{~A})$ & 119.0 \\
\hline $\mathrm{C}(14)-\mathrm{C}(13)-\mathrm{C}(12)$ & $118.0(9)$ \\
\hline $\mathrm{C}(14)-\mathrm{C}(13)-\mathrm{H}(13 \mathrm{~A})$ & 121.0 \\
\hline $\mathrm{C}(12)-\mathrm{C}(13)-\mathrm{H}(13 \mathrm{~A})$ & 121.0 \\
\hline C(15)-C(14)-C(13) & $121.2(10)$ \\
\hline $\mathrm{C}(15)-\mathrm{C}(14)-\mathrm{H}(14 \mathrm{~A})$ & 119.4 \\
\hline $\mathrm{C}(13)-\mathrm{C}(14)-\mathrm{H}(14 \mathrm{~A})$ & 119.4 \\
\hline $\mathrm{C}(16)-\mathrm{C}(15)-\mathrm{C}(14)$ & $119.8(10)$ \\
\hline
\end{tabular}




\begin{tabular}{|c|c|}
\hline $\mathrm{C}(16)-\mathrm{C}(15)-\mathrm{H}(15 \mathrm{~A})$ & 120.1 \\
\hline $\mathrm{C}(14)-\mathrm{C}(15)-\mathrm{H}(15 \mathrm{~A})$ & 120.1 \\
\hline$C(15)-C(16)-C(11)$ & $121.6(9)$ \\
\hline $\mathrm{C}(15)-\mathrm{C}(16)-\mathrm{H}(16 \mathrm{~A})$ & 119.2 \\
\hline $\mathrm{C}(11)-\mathrm{C}(16)-\mathrm{H}(16 \mathrm{~A})$ & 119.2 \\
\hline $\mathrm{C}(22)-\mathrm{C}(17)-\mathrm{C}(18)$ & $116.4(7)$ \\
\hline $\mathrm{C}(22)-\mathrm{C}(17)-\mathrm{Sn}(1)$ & $121.9(6)$ \\
\hline $\mathrm{C}(18)-\mathrm{C}(17)-\mathrm{Sn}(1)$ & $121.7(5)$ \\
\hline C(19)-C(18)-C(17) & $121.7(7)$ \\
\hline $\mathrm{C}(19)-\mathrm{C}(18)-\mathrm{H}(18 \mathrm{~A})$ & 119.1 \\
\hline $\mathrm{C}(17)-\mathrm{C}(18)-\mathrm{H}(18 \mathrm{~A})$ & 119.1 \\
\hline $\mathrm{C}(18)-\mathrm{C}(19)-\mathrm{C}(20)$ & $120.0(8)$ \\
\hline $\mathrm{C}(18)-\mathrm{C}(19)-\mathrm{H}(19 \mathrm{~A})$ & 120.0 \\
\hline $\mathrm{C}(20)-\mathrm{C}(19)-\mathrm{H}(19 \mathrm{~A})$ & 120.0 \\
\hline C(19)-C(20)-C(21) & $119.9(8)$ \\
\hline $\mathrm{C}(19)-\mathrm{C}(20)-\mathrm{H}(20 \mathrm{~A})$ & 120.0 \\
\hline $\mathrm{C}(21)-\mathrm{C}(20)-\mathrm{H}(20 \mathrm{~A})$ & 120.0 \\
\hline $\mathrm{C}(22)-\mathrm{C}(21)-\mathrm{C}(20)$ & $119.6(7)$ \\
\hline $\mathrm{C}(22)-\mathrm{C}(21)-\mathrm{H}(21 \mathrm{~A})$ & 120.2 \\
\hline $\mathrm{C}(20)-\mathrm{C}(21)-\mathrm{H}(21 \mathrm{~A})$ & 120.2 \\
\hline $\mathrm{C}(21)-\mathrm{C}(22)-\mathrm{C}(17)$ & $122.3(8)$ \\
\hline $\mathrm{C}(21)-\mathrm{C}(22)-\mathrm{H}(22 \mathrm{~A})$ & 118.8 \\
\hline $\mathrm{C}(17)-\mathrm{C}(22)-\mathrm{H}(22 \mathrm{~A})$ & 118.8 \\
\hline $\mathrm{C}(24)-\mathrm{C}(23)-\mathrm{C}(28)$ & 120.0 \\
\hline $\mathrm{C}(24)-\mathrm{C}(23)-\mathrm{Sn}(1)$ & $117.6(4)$ \\
\hline $\mathrm{C}(28)-\mathrm{C}(23)-\mathrm{Sn}(1)$ & $121.5(4)$ \\
\hline $\mathrm{C}(25)-\mathrm{C}(24)-\mathrm{C}(23)$ & 120.0 \\
\hline $\mathrm{C}(25)-\mathrm{C}(24)-\mathrm{H}(24 \mathrm{~A})$ & 120.0 \\
\hline $\mathrm{C}(23)-\mathrm{C}(24)-\mathrm{H}(24 \mathrm{~A})$ & 120.0 \\
\hline $\mathrm{C}(24)-\mathrm{C}(25)-\mathrm{C}(26)$ & 120.0 \\
\hline $\mathrm{C}(24)-\mathrm{C}(25)-\mathrm{H}(25 \mathrm{~A})$ & 120.0 \\
\hline $\mathrm{C}(26)-\mathrm{C}(25)-\mathrm{H}(25 \mathrm{~A})$ & 120.0 \\
\hline $\mathrm{C}(27)-\mathrm{C}(26)-\mathrm{C}(25)$ & 120.0 \\
\hline $\mathrm{C}(27)-\mathrm{C}(26)-\mathrm{H}(26 \mathrm{~A})$ & 120.0 \\
\hline $\mathrm{C}(25)-\mathrm{C}(26)-\mathrm{H}(26 \mathrm{~A})$ & 120.0 \\
\hline $\mathrm{C}(26)-\mathrm{C}(27)-\mathrm{C}(28)$ & 120.0 \\
\hline
\end{tabular}




$\begin{array}{ll}\mathrm{C}(26)-\mathrm{C}(27)-\mathrm{H}(27 \mathrm{~A}) & 120.0 \\ \mathrm{C}(28)-\mathrm{C}(27)-\mathrm{H}(27 \mathrm{~A}) & 120.0 \\ \mathrm{C}(27)-\mathrm{C}(28)-\mathrm{C}(23) & 120.0 \\ \mathrm{C}(27)-\mathrm{C}(28)-\mathrm{H}(28 \mathrm{~A}) & 120.0 \\ \mathrm{C}(23)-\mathrm{C}(28)-\mathrm{H}(28 \mathrm{~A}) & 120.0 \\ \mathrm{C}(24 \mathrm{~A})-\mathrm{C}(23 \mathrm{~A})-\mathrm{C}(27 \mathrm{~A}) & 120.0 \\ \mathrm{C}(24 \mathrm{~A})-\mathrm{C}(23 \mathrm{~A})-\mathrm{Sn}(1) & 121.1(5) \\ \mathrm{C}(27 \mathrm{~A})-\mathrm{C}(23 \mathrm{~A})-\mathrm{Sn}(1) & 118.3(5) \\ \mathrm{C}(23 \mathrm{~A})-\mathrm{C}(24 \mathrm{~A})-\mathrm{C}(25 \mathrm{~A}) & 120.0 \\ \mathrm{C}(23 \mathrm{~A})-\mathrm{C}(24 \mathrm{~A})-\mathrm{H}(24 \mathrm{~B}) & 120.0 \\ \mathrm{C}(25 \mathrm{~A})-\mathrm{C}(24 \mathrm{~A})-\mathrm{H}(24 \mathrm{~B}) & 120.0 \\ \mathrm{C}(26 \mathrm{~A})-\mathrm{C}(25 \mathrm{~A})-\mathrm{C}(24 \mathrm{~A}) & 120.0 \\ \mathrm{C}(26 \mathrm{~A})-\mathrm{C}(25 \mathrm{~A})-\mathrm{H}(25 \mathrm{~B}) & 120.0 \\ \mathrm{C}(24 \mathrm{~A})-\mathrm{C}(25 \mathrm{~A})-\mathrm{H}(25 \mathrm{~B}) & 120.0 \\ \mathrm{C}(28 \mathrm{~A})-\mathrm{C}(26 \mathrm{~A})-\mathrm{C}(25 \mathrm{~A}) & 120.0 \\ \mathrm{C}(28 \mathrm{~A})-\mathrm{C}(26 \mathrm{~A})-\mathrm{H}(26 \mathrm{~B}) & 120.0 \\ \mathrm{C}(25 \mathrm{~A})-\mathrm{C}(26 \mathrm{~A})-\mathrm{H}(26 \mathrm{~B}) & 120.0 \\ \mathrm{C}(26 \mathrm{~A})-\mathrm{C}(28 \mathrm{~A})-\mathrm{C}(27 \mathrm{~A}) & 120.0 \\ \mathrm{C}(26 \mathrm{~A})-\mathrm{C}(28 \mathrm{~A})-\mathrm{H}(28 \mathrm{~B}) & 120.0 \\ \mathrm{C}(27 \mathrm{~A})-\mathrm{C}(28 \mathrm{~A})-\mathrm{H}(28 \mathrm{~B}) & 120.0 \\ \mathrm{C}(28 \mathrm{~A})-\mathrm{C}(27 \mathrm{~A})-\mathrm{C}(23 \mathrm{~A}) & 120.0 \\ \mathrm{C}(28 \mathrm{~A})-\mathrm{C}(27 \mathrm{~A})-\mathrm{H}(27 \mathrm{~B}) & 120.0 \\ \mathrm{C}(23 \mathrm{~A})-\mathrm{C}(27 \mathrm{~A})-\mathrm{H}(27 \mathrm{~B}) & 120.0\end{array}$

Symmetry transformations used to generate equivalent atoms: 
Table A 4. Anisotropic displacement parameters $\left(\AA^{2} \times 1^{3}\right)$ for 198 . The anisotropic displacement factor exponent takes the form: $-2 p^{2}\left[h^{2} a^{* 2} U^{11}+\ldots+2 h k a^{*} b^{*} U^{12}\right]$

\begin{tabular}{|c|c|c|c|c|c|c|}
\hline & $\mathrm{U}^{11}$ & $\mathrm{U}^{22}$ & $\mathrm{U}^{33}$ & $\mathrm{U}^{23}$ & $\mathrm{U}^{13}$ & $\mathrm{U}^{12}$ \\
\hline $\operatorname{Sn}(1)$ & $70(1)$ & $44(1)$ & $105(1)$ & $-27(1)$ & $50(1)$ & $-22(1)$ \\
\hline $\mathrm{F}(1)$ & $165(8)$ & $60(5)$ & $180(7)$ & $-33(5)$ & $54(6)$ & $-8(5)$ \\
\hline $\mathrm{F}(2)$ & $180(7)$ & $52(4)$ & $182(6)$ & $-36(4)$ & 41(5) & $-27(4)$ \\
\hline $\mathrm{F}(3)$ & $201(8)$ & $62(5)$ & $173(6)$ & $-58(5)$ & $29(5)$ & $-37(5)$ \\
\hline $\mathrm{F}(1 \mathrm{~A})$ & $177(8)$ & $58(4)$ & $182(6)$ & $-33(5)$ & $44(6)$ & $-14(5)$ \\
\hline $\mathrm{F}(2 \mathrm{~A})$ & $189(8)$ & $57(4)$ & $177(6)$ & $-51(5)$ & $34(5)$ & $-34(4)$ \\
\hline $\mathrm{F}(3 \mathrm{~A})$ & 202(9) & $59(6)$ & $168(7)$ & $-57(5)$ & $36(6)$ & $-43(6)$ \\
\hline $\mathrm{O}(1)$ & $79(6)$ & $45(6)$ & $51(6)$ & $-10(4)$ & $30(5)$ & $-22(5)$ \\
\hline $\mathrm{C}(1)$ & $29(8)$ & $46(9)$ & $95(13)$ & $-16(8)$ & $20(8)$ & $-5(6)$ \\
\hline $\mathrm{C}(2)$ & $57(7)$ & $58(8)$ & $54(8)$ & $1(6)$ & $17(5)$ & $-12(6)$ \\
\hline $\mathrm{C}(3)$ & $81(8)$ & $35(6)$ & $62(8)$ & $-3(5)$ & $36(7)$ & $-4(6)$ \\
\hline $\mathrm{O}(1 \mathrm{~A})$ & 101(12) & $30(8)$ & $52(10)$ & $-7(6)$ & $37(9)$ & $-20(7)$ \\
\hline$C(1 \mathrm{~A})$ & $26(12)$ & $46(13)$ & $89(16)$ & $-18(12)$ & $14(11)$ & $2(10)$ \\
\hline $\mathrm{C}(2 \mathrm{~A})$ & $44(10)$ & $49(11)$ & $40(11)$ & $8(8)$ & $11(8)$ & $1(8)$ \\
\hline$C(3 A)$ & $69(12)$ & $37(10)$ & $63(13)$ & $1(8)$ & $10(9)$ & $0(8)$ \\
\hline$C(10)$ & 179(13) & $62(8)$ & $158(13)$ & $-49(8)$ & $86(11)$ & $-8(8)$ \\
\hline$C(10 A)$ & $179(13)$ & $62(8)$ & $158(13)$ & $-49(8)$ & $86(11)$ & $-8(8)$ \\
\hline $\mathrm{C}(11)$ & $68(4)$ & $42(4)$ & $88(6)$ & $-7(4)$ & $39(4)$ & $-2(3)$ \\
\hline $\mathrm{C}(12)$ & $67(5)$ & $40(4)$ & $87(6)$ & $1(4)$ & $34(4)$ & 2(3) \\
\hline $\mathrm{C}(13)$ & $84(6)$ & $56(5)$ & $92(7)$ & $-5(5)$ & $24(5)$ & $13(4)$ \\
\hline $\mathrm{C}(14)$ & $105(7)$ & $85(7)$ & $82(7)$ & $8(5)$ & $34(5)$ & $41(6)$ \\
\hline $\mathrm{C}(15)$ & $105(8)$ & 72(7) & $131(10)$ & $35(6)$ & $67(7)$ & $36(6)$ \\
\hline$C(16)$ & $83(6)$ & $51(5)$ & $133(9)$ & $18(6)$ & $62(6)$ & $9(4)$ \\
\hline $\mathrm{C}(17)$ & $65(4)$ & $57(4)$ & $67(5)$ & $-23(4)$ & $32(4)$ & $-30(3)$ \\
\hline $\mathrm{C}(18)$ & 71(5) & $74(6)$ & $61(5)$ & $-32(4)$ & $31(4)$ & $-40(4)$ \\
\hline$C(19)$ & $75(5)$ & $85(6)$ & $82(6)$ & $-16(5)$ & 19(4) & $-28(5)$ \\
\hline$C(20)$ & $68(5)$ & $69(6)$ & $93(7)$ & $-4(5)$ & $18(5)$ & $-13(4)$ \\
\hline $\mathrm{C}(21)$ & 71(5) & $52(5)$ & $89(6)$ & $-21(4)$ & $13(5)$ & $-12(4)$ \\
\hline$C(22)$ & $69(5)$ & $54(4)$ & $71(5)$ & $-22(4)$ & $13(4)$ & $-18(4)$ \\
\hline
\end{tabular}


Table A 5. Hydrogen coordinates $\left(x 10^{4}\right)$ and isotropic displacement parameters $\left(\AA^{2} \times 10\right.$ 3) for 198 .

\begin{tabular}{|c|c|c|c|c|}
\hline & $\mathrm{x}$ & $\mathrm{y}$ & $\mathrm{z}$ & $\mathrm{U}(\mathrm{eq})$ \\
\hline $\mathrm{H}(1 \mathrm{~A})$ & 685 & 8372 & 739 & 68 \\
\hline $\mathrm{H}(1 \mathrm{~B})$ & 2443 & 9215 & 889 & 68 \\
\hline $\mathrm{H}(2 \mathrm{~A})$ & 4297 & 7807 & 1215 & 67 \\
\hline $\mathrm{H}(2 \mathrm{~B})$ & 2480 & 6953 & 1123 & 67 \\
\hline $\mathrm{H}(3 \mathrm{~A})$ & 5275 & 6638 & 661 & 71 \\
\hline $\mathrm{H}(3 \mathrm{~B})$ & 3259 & 6978 & 393 & 71 \\
\hline $\mathrm{H}(1 \mathrm{AA})$ & 3504 & 8948 & 1089 & 65 \\
\hline $\mathrm{H}(1 \mathrm{AB})$ & 3225 & 7676 & 1213 & 65 \\
\hline $\mathrm{H}(2 \mathrm{AA})$ & 814 & 8830 & 587 & 53 \\
\hline $\mathrm{H}(2 \mathrm{AB})$ & 269 & 7605 & 736 & 53 \\
\hline $\mathrm{H}(3 \mathrm{AA})$ & 3606 & 7014 & 500 & 68 \\
\hline $\mathrm{H}(3 \mathrm{AB})$ & 2244 & 7485 & 116 & 68 \\
\hline $\mathrm{H}(5 \mathrm{~A})$ & 7574 & 9583 & 84 & 51 \\
\hline $\mathrm{H}(6 \mathrm{~A})$ & 10143 & 9331 & -428 & 65 \\
\hline $\mathrm{H}(7 \mathrm{~A})$ & 10728 & 7587 & -713 & 87 \\
\hline $\mathrm{H}(9 \mathrm{~A})$ & 6173 & 6346 & 26 & 65 \\
\hline $\mathrm{H}(5 \mathrm{AA})$ & 7069 & 9750 & 11 & 38 \\
\hline $\mathrm{H}(6 \mathrm{AA})$ & 9602 & 9484 & -507 & 46 \\
\hline $\mathrm{H}(7 \mathrm{AA})$ & 10093 & 7743 & -803 & 61 \\
\hline $\mathrm{H}(9 \mathrm{AA})$ & 5517 & 6534 & -61 & 44 \\
\hline $\mathrm{H}(12 \mathrm{~A})$ & 3496 & 10345 & 1920 & 78 \\
\hline $\mathrm{H}(13 \mathrm{~A})$ & 5538 & 10579 & 2526 & 92 \\
\hline $\mathrm{H}(14 \mathrm{~A})$ & 5143 & 9346 & 3090 & 109 \\
\hline $\mathrm{H}(15 \mathrm{~A})$ & 2787 & 7923 & 3048 & 123 \\
\hline $\mathrm{H}(16 \mathrm{~A})$ & 740 & 7725 & 2454 & 107 \\
\hline $\mathrm{H}(18 \mathrm{~A})$ & -3280 & 9745 & 936 & 83 \\
\hline $\mathrm{H}(19 \mathrm{~A})$ & -5256 & 11306 & 841 & 97 \\
\hline $\mathrm{H}(20 \mathrm{~A})$ & -4934 & 12774 & 1310 & 92 \\
\hline $\mathrm{H}(21 \mathrm{~A})$ & -2621 & 12664 & 1877 & 85 \\
\hline $\mathrm{H}(22 \mathrm{~A})$ & -640 & 11110 & 1969 & 78 \\
\hline $\mathrm{H}(24 \mathrm{~A})$ & -361 & 6392 & 1240 & 57 \\
\hline
\end{tabular}




$\begin{array}{lrrrr}\mathrm{H}(25 \mathrm{~A}) & -2577 & 4882 & 1296 & 68 \\ \mathrm{H}(26 \mathrm{~A}) & -5235 & 4887 & 1799 & 65 \\ \mathrm{H}(27 \mathrm{~A}) & -5675 & 6403 & 2244 & 71 \\ \mathrm{H}(28 \mathrm{~A}) & -3459 & 7913 & 2188 & 57 \\ \mathrm{H}(24 \mathrm{~B}) & -1413 & 6784 & 985 & 60 \\ \mathrm{H}(25 \mathrm{~B}) & -3568 & 5247 & 1034 & 75 \\ \mathrm{H}(26 \mathrm{~B}) & -5686 & 5014 & 1628 & 63 \\ \mathrm{H}(28 \mathrm{~B}) & -5650 & 6317 & 2172 & 49 \\ \mathrm{H}(27 \mathrm{~B}) & -3496 & 7854 & 2123 & 50\end{array}$


Table A 6. Crystal data and structure refinement for 200.

Identification code

Empirical formula

Formula weight

Temperature

Wavelength

Crystal system

Space group

Unit cell dimensions

Volume

$\mathrm{Z}$

Density (calculated)

Absorption coefficient

$\mathrm{F}(000)$

Crystal size

Theta range for data collection

Index ranges

Reflections collected

Independent reflections

Completeness to theta $=25.242^{\circ}$

Absorption correction

Max. and min. transmission

Refinement method

Data / restraints / parameters

Goodness-of-fit on $\mathrm{F}^{2}$

Final R indices [I $>2 \operatorname{sigma}(\mathrm{I})]$

$\mathrm{R}$ indices (all data)

Extinction coefficient

Largest diff. peak and hole d13211

$\mathrm{C}_{21} \mathrm{H}_{21} \mathrm{ClOSn}$

443.52

147(2) K

$0.71073 \AA$

Triclinic

$\mathrm{P}-1$

$\mathrm{a}=10.4728(11) \AA \quad \alpha=89.678(2)^{\circ}$.

$\mathrm{b}=11.0954(12) \AA \quad \beta=79.636(2)^{\circ}$.

$\mathrm{c}=16.9797(18) \AA \quad \gamma=82.832(2)^{\circ}$.

1925.4(4) $\AA^{3}$

4

$1.530 \mathrm{Mg} / \mathrm{m}^{3}$

$1.470 \mathrm{~mm}^{-1}$

888

$0.200 \times 0.090 \times 0.030 \mathrm{~mm}^{3}$

1.219 to $27.481^{\circ}$.

$-13<=\mathrm{h}<=13,-14<=\mathrm{k}<=14,-21<=\mathrm{l}<=22$

63709

$8816[\mathrm{R}(\mathrm{int})=0.0236]$

$99.8 \%$

Semi-empirical from equivalents

0.7456 and 0.6543

Full-matrix least-squares on $\mathrm{F}^{2}$

8816 / 0 / 433

1.020

$\mathrm{R} 1=0.0161, \mathrm{wR} 2=0.0385$

$\mathrm{R} 1=0.0197, \mathrm{wR} 2=0.0402$

$\mathrm{n} / \mathrm{a}$

0.370 and -0.350 e. $\AA^{-3}$ 
Table A 7. Atomic coordinates $\left(\times 1^{4}\right)$ and equivalent isotropic displacement parameters $\left(\AA^{2} \times 10^{3}\right)$ for 200.

\begin{tabular}{|c|c|c|c|c|}
\hline & $\mathrm{X}$ & $\mathrm{y}$ & $\mathrm{z}$ & $\mathrm{U}(\mathrm{eq})$ \\
\hline $\operatorname{Sn}(1 \mathrm{~A})$ & $3605(1)$ & $5501(1)$ & $2673(1)$ & $18(1)$ \\
\hline $\mathrm{Cl}(1 \mathrm{~A})$ & $3448(1)$ & $5998(1)$ & 1311(1) & $25(1)$ \\
\hline $\mathrm{O}(1 \mathrm{~A})$ & $4348(1)$ & $4535(1)$ & $4132(1)$ & $23(1)$ \\
\hline $\mathrm{C}(1 \mathrm{~A})$ & $5688(1)$ & $5211(2)$ & $2558(1)$ & $23(1)$ \\
\hline $\mathrm{C}(2 \mathrm{~A})$ & $6212(2)$ & $5218(2)$ & $3340(1)$ & $25(1)$ \\
\hline $\mathrm{C}(3 \mathrm{~A})$ & $5744(1)$ & $4227(2)$ & $3890(1)$ & $24(1)$ \\
\hline $\mathrm{C}(4 \mathrm{~A})$ & $3690(2)$ & $3722(2)$ & $4620(1)$ & $24(1)$ \\
\hline $\mathrm{C}(5 \mathrm{~A})$ & $4261(2)$ & $2579(2)$ & $4796(1)$ & $28(1)$ \\
\hline$C(6 A)$ & $3497(2)$ & $1817(2)$ & $5276(1)$ & $38(1)$ \\
\hline $\mathrm{C}(7 \mathrm{~A})$ & $2195(2)$ & 2192(2) & $5573(1)$ & $44(1)$ \\
\hline $\mathrm{C}(8 \mathrm{~A})$ & $1637(2)$ & $3336(2)$ & $5396(1)$ & $41(1)$ \\
\hline $\mathrm{C}(9 \mathrm{~A})$ & $2377(2)$ & $4111(2)$ & 4923(1) & $31(1)$ \\
\hline$C(10 A)$ & $2382(1)$ & $4100(1)$ & 2901(1) & $21(1)$ \\
\hline$C(11 A)$ & $1028(2)$ & $4435(2)$ & $3015(1)$ & $33(1)$ \\
\hline $\mathrm{C}(12 \mathrm{~A})$ & $176(2)$ & $3581(2)$ & $3244(1)$ & $41(1)$ \\
\hline$C(13 A)$ & $656(2)$ & 2399(2) & $3380(1)$ & $34(1)$ \\
\hline $\mathrm{C}(14 \mathrm{~A})$ & 1993(2) & $2055(1)$ & $3268(1)$ & $27(1)$ \\
\hline$C(15 A)$ & $2850(2)$ & 2901(1) & $3024(1)$ & $23(1)$ \\
\hline$C(16 A)$ & 2591(1) & $7116(1)$ & $3275(1)$ & $22(1)$ \\
\hline $\mathrm{C}(17 \mathrm{~A})$ & 2891(2) & $7528(2)$ & $3987(1)$ & $33(1)$ \\
\hline $\mathrm{C}(18 \mathrm{~A})$ & 2192(2) & $8576(2)$ & $4373(1)$ & $44(1)$ \\
\hline $\mathrm{C}(19 \mathrm{~A})$ & $1185(2)$ & $9211(2)$ & $4058(1)$ & $38(1)$ \\
\hline$C(20 A)$ & $874(2)$ & $8816(2)$ & $3357(1)$ & $33(1)$ \\
\hline$C(21 A)$ & $1569(2)$ & $7779(2)$ & $2966(1)$ & $28(1)$ \\
\hline $\operatorname{Sn}(1 B)$ & $3075(1)$ & $-288(1)$ & $7735(1)$ & $18(1)$ \\
\hline $\mathrm{Cl}(1 \mathrm{~B})$ & $2376(1)$ & $-923(1)$ & $6528(1)$ & $24(1)$ \\
\hline $\mathrm{O}(1 \mathrm{~B})$ & $4184(1)$ & $549(1)$ & $8925(1)$ & $25(1)$ \\
\hline$C(1 B)$ & 4961(1) & $143(2)$ & $7187(1)$ & $26(1)$ \\
\hline $\mathrm{C}(2 \mathrm{~B})$ & $5867(2)$ & $286(2)$ & $7784(1)$ & $28(1)$ \\
\hline $\mathrm{C}(3 \mathrm{~B})$ & $5232(2)$ & $1134(2)$ & $8472(1)$ & $28(1)$ \\
\hline
\end{tabular}




$\begin{array}{lrrrr}\mathrm{C}(4 \mathrm{~B}) & 3515(2) & 1122(2) & 9630(1) & 25(1) \\ \mathrm{C}(5 \mathrm{~B}) & 3592(2) & 2319(2) & 9824(1) & 32(1) \\ \mathrm{C}(6 \mathrm{~B}) & 2867(2) & 2817(2) & 10545(1) & 37(1) \\ \mathrm{C}(7 \mathrm{~B}) & 2089(2) & 2136(2) & 11060(1) & 37(1) \\ \mathrm{C}(8 \mathrm{~B}) & 2026(2) & 935(2) & 10862(1) & 38(1) \\ \mathrm{C}(9 B) & 2738(2) & 426(2) & 10147(1) & 32(1) \\ \mathrm{C}(10 B) & 2835(2) & -1890(1) & 8413(1) & 21(1) \\ \mathrm{C}(11 B) & 1711(2) & -2446(1) & 8414(1) & 26(1) \\ \mathrm{C}(12 B) & 1512(2) & -3487(2) & 8855(1) & 33(1) \\ \mathrm{C}(13 B) & 2445(2) & -3990(2) & 9287(1) & 35(1) \\ \mathrm{C}(14 B) & 3565(2) & -3452(2) & 9287(1) & 32(1) \\ \mathrm{C}(15 B) & 3762(2) & -2403(2) & 8856(1) & 26(1) \\ \mathrm{C}(16 B) & 1682(1) & 1233(1) & 8152(1) & 20(1) \\ \mathrm{C}(17 \mathrm{~B}) & 1844(2) & 2378(1) & 7832(1) & 25(1) \\ \mathrm{C}(18 B) & 1030(2) & 3405(2) & 8158(1) & 31(1) \\ \mathrm{C}(19 B) & 37(2) & 3302(2) & 8803(1) & 34(1) \\ \mathrm{C}(20 B) & 2172(2) & 9121(1) & 35(1) \\ \mathrm{C}(21 B) & 678(2) & 1144(2) & 8802(1) & 27(1) \\ \end{array}$


Table A 8. Bond lengths $[\AA ̊]$ and angles $\left[{ }^{\circ}\right]$ for 200.

\begin{tabular}{|c|c|}
\hline $\operatorname{Sn}(1 \mathrm{~A})-\mathrm{C}(10 \mathrm{~A})$ & $2.1249(15)$ \\
\hline $\operatorname{Sn}(1 \mathrm{~A})-\mathrm{C}(16 \mathrm{~A})$ & $2.1304(15)$ \\
\hline $\operatorname{Sn}(1 \mathrm{~A})-\mathrm{C}(1 \mathrm{~A})$ & $2.1383(15)$ \\
\hline $\operatorname{Sn}(1 \mathrm{~A})-\mathrm{Cl}(1 \mathrm{~A})$ & $2.4047(4)$ \\
\hline $\operatorname{Sn}(1 \mathrm{~A})-\mathrm{O}(1 \mathrm{~A})$ & $2.8894(11)$ \\
\hline $\mathrm{O}(1 \mathrm{~A})-\mathrm{C}(4 \mathrm{~A})$ & $1.3842(18)$ \\
\hline $\mathrm{O}(1 \mathrm{~A})-\mathrm{C}(3 \mathrm{~A})$ & $1.4432(18)$ \\
\hline$C(1 A)-C(2 A)$ & $1.526(2)$ \\
\hline $\mathrm{C}(1 \mathrm{~A})-\mathrm{H}(1 \mathrm{AA})$ & 0.9900 \\
\hline $\mathrm{C}(1 \mathrm{~A})-\mathrm{H}(1 \mathrm{AB})$ & 0.9900 \\
\hline$C(2 A)-C(3 A)$ & $1.510(2)$ \\
\hline $\mathrm{C}(2 \mathrm{~A})-\mathrm{H}(2 \mathrm{AA})$ & 0.9900 \\
\hline $\mathrm{C}(2 \mathrm{~A})-\mathrm{H}(2 \mathrm{AB})$ & 0.9900 \\
\hline $\mathrm{C}(3 \mathrm{~A})-\mathrm{H}(3 \mathrm{AA})$ & 0.9900 \\
\hline $\mathrm{C}(3 \mathrm{~A})-\mathrm{H}(3 \mathrm{AB})$ & 0.9900 \\
\hline $\mathrm{C}(4 \mathrm{~A})-\mathrm{C}(5 \mathrm{~A})$ & $1.388(2)$ \\
\hline $\mathrm{C}(4 \mathrm{~A})-\mathrm{C}(9 \mathrm{~A})$ & $1.393(2)$ \\
\hline$C(5 A)-C(6 A)$ & $1.395(2)$ \\
\hline $\mathrm{C}(5 \mathrm{~A})-\mathrm{H}(5 \mathrm{AA})$ & 0.9500 \\
\hline$C(6 A)-C(7 A)$ & $1.378(3)$ \\
\hline $\mathrm{C}(6 \mathrm{~A})-\mathrm{H}(6 \mathrm{AA})$ & 0.9500 \\
\hline $\mathrm{C}(7 \mathrm{~A})-\mathrm{C}(8 \mathrm{~A})$ & $1.384(3)$ \\
\hline $\mathrm{C}(7 \mathrm{~A})-\mathrm{H}(7 \mathrm{AA})$ & 0.9500 \\
\hline$C(8 A)-C(9 A)$ & $1.386(3)$ \\
\hline $\mathrm{C}(8 \mathrm{~A})-\mathrm{H}(8 \mathrm{AA})$ & 0.9500 \\
\hline $\mathrm{C}(9 \mathrm{~A})-\mathrm{H}(9 \mathrm{AA})$ & 0.9500 \\
\hline$C(10 A)-C(15 A)$ & $1.388(2)$ \\
\hline$C(10 A)-C(11 A)$ & $1.400(2)$ \\
\hline $\mathrm{C}(11 \mathrm{~A})-\mathrm{C}(12 \mathrm{~A})$ & $1.388(2)$ \\
\hline $\mathrm{C}(11 \mathrm{~A})-\mathrm{H}(11 \mathrm{~A})$ & 0.9500 \\
\hline $\mathrm{C}(12 \mathrm{~A})-\mathrm{C}(13 \mathrm{~A})$ & $1.378(3)$ \\
\hline $\mathrm{C}(12 \mathrm{~A})-\mathrm{H}(12 \mathrm{~A})$ & 0.9500 \\
\hline $\mathrm{C}(13 \mathrm{~A})-\mathrm{C}(14 \mathrm{~A})$ & $1.383(2)$ \\
\hline $\mathrm{C}(13 \mathrm{~A})-\mathrm{H}(13 \mathrm{~A})$ & 0.9500 \\
\hline
\end{tabular}




\begin{tabular}{|c|c|}
\hline $\mathrm{C}(14 \mathrm{~A})-\mathrm{C}(15 \mathrm{~A})$ & $1.390(2)$ \\
\hline $\mathrm{C}(14 \mathrm{~A})-\mathrm{H}(14 \mathrm{~A})$ & 0.9500 \\
\hline $\mathrm{C}(15 \mathrm{~A})-\mathrm{H}(15 \mathrm{~A})$ & 0.9500 \\
\hline$C(16 A)-C(17 A)$ & $1.396(2)$ \\
\hline$C(16 A)-C(21 A)$ & $1.402(2)$ \\
\hline $\mathrm{C}(17 \mathrm{~A})-\mathrm{C}(18 \mathrm{~A})$ & $1.392(2)$ \\
\hline $\mathrm{C}(17 \mathrm{~A})-\mathrm{H}(17 \mathrm{~A})$ & 0.9500 \\
\hline $\mathrm{C}(18 \mathrm{~A})-\mathrm{C}(19 \mathrm{~A})$ & $1.381(3)$ \\
\hline $\mathrm{C}(18 \mathrm{~A})-\mathrm{H}(18 \mathrm{~A})$ & 0.9500 \\
\hline$C(19 A)-C(20 A)$ & $1.376(3)$ \\
\hline C(19A)-H(19A) & 0.9500 \\
\hline$C(20 A)-C(21 A)$ & $1.386(2)$ \\
\hline $\mathrm{C}(20 \mathrm{~A})-\mathrm{H}(20 \mathrm{~A})$ & 0.9500 \\
\hline $\mathrm{C}(21 \mathrm{~A})-\mathrm{H}(21 \mathrm{~A})$ & 0.9500 \\
\hline $\operatorname{Sn}(1 B)-C(16 B)$ & $2.1216(15)$ \\
\hline $\mathrm{Sn}(1 \mathrm{~B})-\mathrm{C}(10 \mathrm{~B})$ & $2.1294(15)$ \\
\hline $\operatorname{Sn}(1 \mathrm{~B})-\mathrm{C}(1 \mathrm{~B})$ & $2.1365(15)$ \\
\hline $\operatorname{Sn}(1 \mathrm{~B})-\mathrm{Cl}(1 \mathrm{~B})$ & $2.4322(4)$ \\
\hline $\operatorname{Sn}(1 \mathrm{~B})-\mathrm{O}(1 \mathrm{~B})$ & $2.7254(11)$ \\
\hline $\mathrm{O}(1 \mathrm{~B})-\mathrm{C}(4 \mathrm{~B})$ & $1.3866(19)$ \\
\hline $\mathrm{O}(1 \mathrm{~B})-\mathrm{C}(3 \mathrm{~B})$ & $1.4439(19)$ \\
\hline $\mathrm{C}(1 \mathrm{~B})-\mathrm{C}(2 \mathrm{~B})$ & $1.528(2)$ \\
\hline $\mathrm{C}(1 \mathrm{~B})-\mathrm{H}(1 \mathrm{BA})$ & 0.9900 \\
\hline $\mathrm{C}(1 \mathrm{~B})-\mathrm{H}(1 \mathrm{BB})$ & 0.9900 \\
\hline $\mathrm{C}(2 \mathrm{~B})-\mathrm{C}(3 \mathrm{~B})$ & $1.506(2)$ \\
\hline $\mathrm{C}(2 \mathrm{~B})-\mathrm{H}(2 \mathrm{BA})$ & 0.9900 \\
\hline $\mathrm{C}(2 \mathrm{~B})-\mathrm{H}(2 \mathrm{BB})$ & 0.9900 \\
\hline $\mathrm{C}(3 \mathrm{~B})-\mathrm{H}(3 \mathrm{BA})$ & 0.9900 \\
\hline $\mathrm{C}(3 \mathrm{~B})-\mathrm{H}(3 \mathrm{BB})$ & 0.9900 \\
\hline $\mathrm{C}(4 \mathrm{~B})-\mathrm{C}(5 \mathrm{~B})$ & $1.386(2)$ \\
\hline $\mathrm{C}(4 \mathrm{~B})-\mathrm{C}(9 \mathrm{~B})$ & $1.387(2)$ \\
\hline$C(5 B)-C(6 B)$ & $1.396(3)$ \\
\hline $\mathrm{C}(5 \mathrm{~B})-\mathrm{H}(5 \mathrm{BA})$ & 0.9500 \\
\hline$C(6 B)-C(7 B)$ & $1.374(3)$ \\
\hline $\mathrm{C}(6 \mathrm{~B})-\mathrm{H}(6 \mathrm{BA})$ & 0.9500 \\
\hline $\mathrm{C}(7 \mathrm{~B})-\mathrm{C}(8 \mathrm{~B})$ & $1.388(3)$ \\
\hline
\end{tabular}




\begin{tabular}{|c|c|}
\hline $\mathrm{C}(7 \mathrm{~B})-\mathrm{H}(7 \mathrm{BA})$ & 0.9500 \\
\hline $\mathrm{C}(8 \mathrm{~B})-\mathrm{C}(9 \mathrm{~B})$ & $1.387(3)$ \\
\hline $\mathrm{C}(8 \mathrm{~B})-\mathrm{H}(8 \mathrm{BA})$ & 0.9500 \\
\hline $\mathrm{C}(9 \mathrm{~B})-\mathrm{H}(9 \mathrm{BA})$ & 0.9500 \\
\hline$C(10 B)-C(11 B)$ & $1.395(2)$ \\
\hline$C(10 B)-C(15 B)$ & $1.396(2)$ \\
\hline$C(11 B)-C(12 B)$ & $1.390(2)$ \\
\hline $\mathrm{C}(11 \mathrm{~B})-\mathrm{H}(11 \mathrm{~B})$ & 0.9500 \\
\hline$C(12 B)-C(13 B)$ & $1.386(3)$ \\
\hline $\mathrm{C}(12 \mathrm{~B})-\mathrm{H}(12 \mathrm{~B})$ & 0.9500 \\
\hline$C(13 B)-C(14 B)$ & $1.380(3)$ \\
\hline $\mathrm{C}(13 \mathrm{~B})-\mathrm{H}(13 \mathrm{~B})$ & 0.9500 \\
\hline$C(14 B)-C(15 B)$ & $1.390(2)$ \\
\hline $\mathrm{C}(14 \mathrm{~B})-\mathrm{H}(14 \mathrm{~B})$ & 0.9500 \\
\hline $\mathrm{C}(15 \mathrm{~B})-\mathrm{H}(15 \mathrm{~B})$ & 0.9500 \\
\hline$C(16 B)-C(21 B)$ & $1.393(2)$ \\
\hline$C(16 B)-C(17 B)$ & $1.397(2)$ \\
\hline $\mathrm{C}(17 \mathrm{~B})-\mathrm{C}(18 \mathrm{~B})$ & $1.388(2)$ \\
\hline $\mathrm{C}(17 \mathrm{~B})-\mathrm{H}(17 \mathrm{~B})$ & 0.9500 \\
\hline $\mathrm{C}(18 \mathrm{~B})-\mathrm{C}(19 \mathrm{~B})$ & $1.382(3)$ \\
\hline $\mathrm{C}(18 \mathrm{~B})-\mathrm{H}(18 \mathrm{~B})$ & 0.9500 \\
\hline$C(19 B)-C(20 B)$ & $1.384(3)$ \\
\hline $\mathrm{C}(19 \mathrm{~B})-\mathrm{H}(19 \mathrm{~B})$ & 0.9500 \\
\hline $\mathrm{C}(20 \mathrm{~B})-\mathrm{C}(21 \mathrm{~B})$ & $1.391(2)$ \\
\hline $\mathrm{C}(20 \mathrm{~B})-\mathrm{H}(20 \mathrm{~B})$ & 0.9500 \\
\hline $\mathrm{C}(21 \mathrm{~B})-\mathrm{H}(21 \mathrm{~B})$ & 0.9500 \\
\hline$C(10 A)-S n(1 A)-C(16 A)$ & $108.37(6)$ \\
\hline$C(10 A)-S n(1 A)-C(1 A)$ & $124.01(6)$ \\
\hline$C(16 A)-S n(1 A)-C(1 A)$ & $117.83(6)$ \\
\hline$C(10 \mathrm{~A})-\operatorname{Sn}(1 \mathrm{~A})-\mathrm{Cl}(1 \mathrm{~A})$ & $101.90(4)$ \\
\hline$C(16 A)-S n(1 A)-C l(1 A)$ & $100.34(4)$ \\
\hline $\mathrm{C}(1 \mathrm{~A})-\mathrm{Sn}(1 \mathrm{~A})-\mathrm{Cl}(1 \mathrm{~A})$ & $99.24(4)$ \\
\hline$C(10 A)-S n(1 A)-O(1 A)$ & $79.62(4)$ \\
\hline$C(16 A)-S n(1 A)-O(1 A)$ & $91.96(5)$ \\
\hline $\mathrm{C}(1 \mathrm{~A})-\mathrm{Sn}(1 \mathrm{~A})-\mathrm{O}(1 \mathrm{~A})$ & $69.37(4)$ \\
\hline
\end{tabular}




\begin{tabular}{|c|c|}
\hline $\mathrm{Cl}(1 \mathrm{~A})-\mathrm{Sn}(1 \mathrm{~A})-\mathrm{O}(1 \mathrm{~A})$ & $166.30(2)$ \\
\hline $\mathrm{C}(4 \mathrm{~A})-\mathrm{O}(1 \mathrm{~A})-\mathrm{C}(3 \mathrm{~A})$ & $116.94(12)$ \\
\hline$C(4 A)-O(1 A)-S n(1 A)$ & $124.73(8)$ \\
\hline$C(3 A)-O(1 A)-S n(1 A)$ & $01.75(8)$ \\
\hline$C(2 A)-C(1 A)-S n(1 A)$ & $115.69(10)$ \\
\hline $\mathrm{C}(2 \mathrm{~A})-\mathrm{C}(1 \mathrm{~A})-\mathrm{H}(1 \mathrm{AA})$ & \\
\hline$n(1 \mathrm{~A})-\mathrm{C}(1 \mathrm{~A})-\mathrm{H}(1 \mathrm{AA})$ & \\
\hline$(2 \mathrm{~A})-\mathrm{C}(1 \mathrm{~A})-\mathrm{H}(1 \mathrm{AB})$ & \\
\hline $\operatorname{Sn}(1 \mathrm{~A})-\mathrm{C}(1 \mathrm{~A})-\mathrm{H}(1 \mathrm{AB})$ & \\
\hline $\mathrm{H}(1 \mathrm{AA})-\mathrm{C}(1 \mathrm{~A})-\mathrm{H}(1 \mathrm{AB})$ & \\
\hline$C(3 \mathrm{~A})-\mathrm{C}(2 \mathrm{~A})-\mathrm{C}(1 \mathrm{~A})$ & \\
\hline $\mathrm{C}(3 \mathrm{~A})-\mathrm{C}(2 \mathrm{~A})-\mathrm{H}(2 \mathrm{AA})$ & 109.3 \\
\hline $\mathrm{C}(1 \mathrm{~A})-\mathrm{C}(2 \mathrm{~A})-\mathrm{H}(2 \mathrm{AA})$ & \\
\hline $\mathrm{C}(3 \mathrm{~A})-\mathrm{C}(2 \mathrm{~A})-\mathrm{H}(2 \mathrm{AB})$ & \\
\hline $\mathrm{C}(1 \mathrm{~A})-\mathrm{C}(2 \mathrm{~A})-\mathrm{H}(2 \mathrm{AB})$ & \\
\hline $\mathrm{H}(2 \mathrm{AA})-\mathrm{C}(2 \mathrm{~A})-\mathrm{H}(2 \mathrm{AB})$ & 107.9 \\
\hline $\mathrm{O}(1 \mathrm{~A})-\mathrm{C}(3 \mathrm{~A})-\mathrm{C}(2 \mathrm{~A})$ & $106.56(12)$ \\
\hline $\mathrm{O}(1 \mathrm{~A})-\mathrm{C}(3 \mathrm{~A})-\mathrm{H}(3 \mathrm{AA})$ & \\
\hline $\mathrm{C}(2 \mathrm{~A})-\mathrm{C}(3 \mathrm{~A})-\mathrm{H}(3 \mathrm{AA})$ & 110.4 \\
\hline $\mathrm{O}(1 \mathrm{~A})-\mathrm{C}(3 \mathrm{~A})-\mathrm{H}(3 \mathrm{AB})$ & \\
\hline $\mathrm{C}(2 \mathrm{~A})-\mathrm{C}(3 \mathrm{~A})-\mathrm{H}(3 \mathrm{AB})$ & 110.4 \\
\hline $\mathrm{H}(3 \mathrm{AA})-\mathrm{C}(3 \mathrm{~A})-\mathrm{H}(3 \mathrm{AB})$ & \\
\hline $\mathrm{O}(1 \mathrm{~A})-\mathrm{C}(4 \mathrm{~A})-\mathrm{C}(5 \mathrm{~A})$ & $123.69(14)$ \\
\hline $\mathrm{O}(1 \mathrm{~A})-\mathrm{C}(4 \mathrm{~A})-\mathrm{C}$ & $6(14)$ \\
\hline$C(5 A)-C(4 A)-C(9 A)$ & $120.74(15)$ \\
\hline$C(4 A)-C(5 A)-C(6 A)$ & $118.98(16)$ \\
\hline $\mathrm{C}(4 \mathrm{~A})-\mathrm{C}(5 \mathrm{~A})-\mathrm{H}(5 \mathrm{AA})$ & 120.5 \\
\hline$C(6 A)-C(5 A)-H(5 A A)$ & 120.5 \\
\hline$C(7 A)-C(6 A)-C(5 A)$ & $120.61(18)$ \\
\hline$C(7 A)-C(6 A)-H(6 A A)$ & 119.7 \\
\hline$C(5 A)-C(6 A)-H(6 A A)$ & 119.7 \\
\hline$C(6 A)-C(7 A)-C(8 A)$ & $119.91(17)$ \\
\hline $\mathrm{C}(6 \mathrm{~A})-\mathrm{C}(7 \mathrm{~A})-\mathrm{H}(7 \mathrm{AA})$ & 120.0 \\
\hline $\mathrm{C}(8 \mathrm{~A})-\mathrm{C}(7 \mathrm{~A})-\mathrm{H}(7 \mathrm{AA})$ & 120.0 \\
\hline $\mathrm{C}(7 \mathrm{~A})-\mathrm{C}(8 \mathrm{~A})-\mathrm{C}(9 \mathrm{~A})$ & $120.55(18)$ \\
\hline $\mathrm{C}(7 \mathrm{~A})-\mathrm{C}(8 \mathrm{~A})-\mathrm{H}(8 \mathrm{AA})$ & 119.7 \\
\hline
\end{tabular}




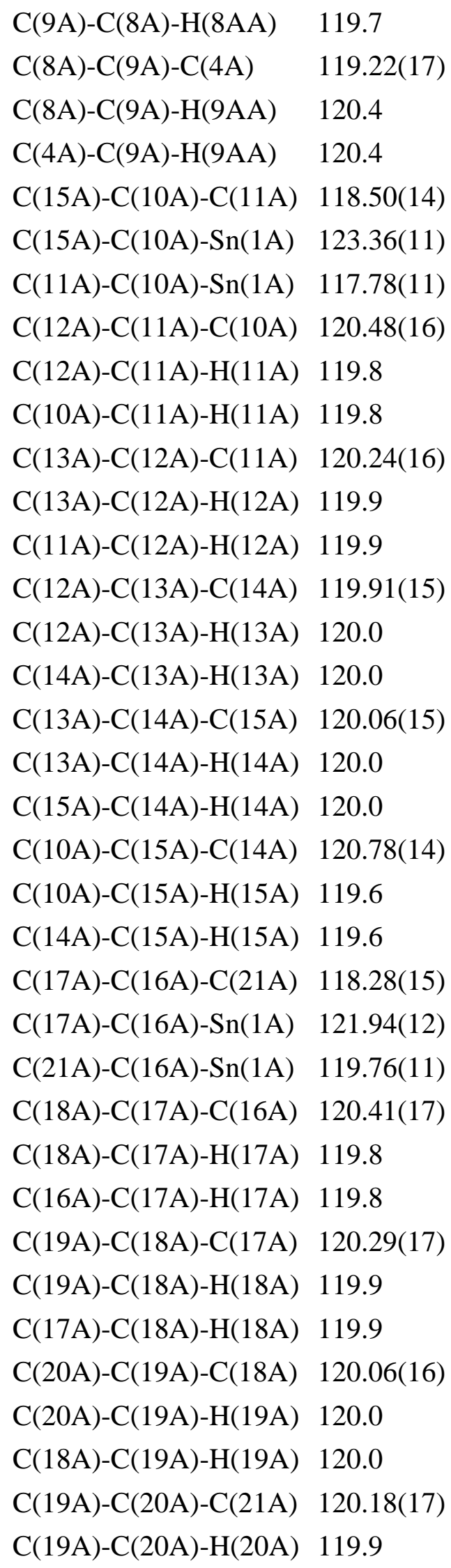




$$
\begin{aligned}
& \text { C(21A)-C(20A)-H(20A) } 119.9 \\
& \text { C(20A)-C(21A)-C(16A) 120.78(16) } \\
& \text { C(20A)-C(21A)-H(21A) } 119.6 \\
& \text { C(16A)-C(21A)-H(21A) } 119.6 \\
& \mathrm{C}(16 \mathrm{~B})-\mathrm{Sn}(1 \mathrm{~B})-\mathrm{C}(10 \mathrm{~B}) \quad 114.56(6) \\
& \mathrm{C}(16 \mathrm{~B})-\mathrm{Sn}(1 \mathrm{~B})-\mathrm{C}(1 \mathrm{~B}) \quad 115.02(6) \\
& \text { C(10B)-Sn(1B)-C(1B) 121.92(6) } \\
& \mathrm{C}(16 \mathrm{~B})-\mathrm{Sn}(1 \mathrm{~B})-\mathrm{Cl}(1 \mathrm{~B}) \quad \text { 103.19(4) } \\
& \mathrm{C}(10 \mathrm{~B})-\mathrm{Sn}(1 \mathrm{~B})-\mathrm{Cl}(1 \mathrm{~B}) \quad 98.65(4) \\
& \mathrm{C}(1 \mathrm{~B})-\mathrm{Sn}(1 \mathrm{~B})-\mathrm{Cl}(1 \mathrm{~B}) \quad 97.74(5) \\
& \mathrm{C}(16 \mathrm{~B})-\mathrm{Sn}(1 \mathrm{~B})-\mathrm{O}(1 \mathrm{~B}) \quad 79.85(4) \\
& \mathrm{C}(10 \mathrm{~B})-\mathrm{Sn}(1 \mathrm{~B})-\mathrm{O}(1 \mathrm{~B}) \quad 87.73(5) \\
& \mathrm{C}(1 \mathrm{~B})-\mathrm{Sn}(1 \mathrm{~B})-\mathrm{O}(1 \mathrm{~B}) \quad 73.22(5) \\
& \mathrm{Cl}(1 \mathrm{~B})-\mathrm{Sn}(1 \mathrm{~B})-\mathrm{O}(1 \mathrm{~B}) \quad 170.83(3) \\
& \text { C(4B)-O(1B)-C(3B) 117.13(12) } \\
& \mathrm{C}(4 \mathrm{~B})-\mathrm{O}(1 \mathrm{~B})-\mathrm{Sn}(1 \mathrm{~B}) \quad \text { 125.83(9) } \\
& \mathrm{C}(3 \mathrm{~B})-\mathrm{O}(1 \mathrm{~B})-\mathrm{Sn}(1 \mathrm{~B}) \quad \text { 101.51(8) } \\
& \mathrm{C}(2 \mathrm{~B})-\mathrm{C}(1 \mathrm{~B})-\mathrm{Sn}(1 \mathrm{~B}) \quad 113.60(11) \\
& \text { C(2B)-C(1B)-H(1BA) } 108.8 \\
& \mathrm{Sn}(1 \mathrm{~B})-\mathrm{C}(1 \mathrm{~B})-\mathrm{H}(1 \mathrm{BA}) \quad 108.8 \\
& \mathrm{C}(2 \mathrm{~B})-\mathrm{C}(1 \mathrm{~B})-\mathrm{H}(1 \mathrm{BB}) \quad 108.8 \\
& \mathrm{Sn}(1 \mathrm{~B})-\mathrm{C}(1 \mathrm{~B})-\mathrm{H}(1 \mathrm{BB}) \quad 108.8 \\
& \text { H(1BA)-C(1B)-H(1BB) } 107.7 \\
& \mathrm{C}(3 \mathrm{~B})-\mathrm{C}(2 \mathrm{~B})-\mathrm{C}(1 \mathrm{~B}) \quad 112.62(13) \\
& \mathrm{C}(3 \mathrm{~B})-\mathrm{C}(2 \mathrm{~B})-\mathrm{H}(2 \mathrm{BA}) \quad 109.1 \\
& \mathrm{C}(1 \mathrm{~B})-\mathrm{C}(2 \mathrm{~B})-\mathrm{H}(2 \mathrm{BA}) \quad 109.1 \\
& \mathrm{C}(3 \mathrm{~B})-\mathrm{C}(2 \mathrm{~B})-\mathrm{H}(2 \mathrm{BB}) \quad 109.1 \\
& \mathrm{C}(1 \mathrm{~B})-\mathrm{C}(2 \mathrm{~B})-\mathrm{H}(2 \mathrm{BB}) \quad 109.1 \\
& \text { H(2BA)-C(2B)-H(2BB) } 107.8 \\
& \mathrm{O}(1 \mathrm{~B})-\mathrm{C}(3 \mathrm{~B})-\mathrm{C}(2 \mathrm{~B}) \quad 106.60(12) \\
& \mathrm{O}(1 \mathrm{~B})-\mathrm{C}(3 \mathrm{~B})-\mathrm{H}(3 \mathrm{BA}) \quad 110.4 \\
& \mathrm{C}(2 \mathrm{~B})-\mathrm{C}(3 \mathrm{~B})-\mathrm{H}(3 \mathrm{BA}) \quad 110.4 \\
& \mathrm{O}(1 \mathrm{~B})-\mathrm{C}(3 \mathrm{~B})-\mathrm{H}(3 \mathrm{BB}) \quad 110.4 \\
& \mathrm{C}(2 \mathrm{~B})-\mathrm{C}(3 \mathrm{~B})-\mathrm{H}(3 \mathrm{BB}) \quad 110.4 \\
& \text { H(3BA)-C(3B)-H(3BB) } 108.6 \\
& \mathrm{C}(5 \mathrm{~B})-\mathrm{C}(4 \mathrm{~B})-\mathrm{C}(9 \mathrm{~B}) \quad 120.52(16)
\end{aligned}
$$




$$
\begin{aligned}
& \mathrm{C}(5 \mathrm{~B})-\mathrm{C}(4 \mathrm{~B})-\mathrm{O}(1 \mathrm{~B}) \quad 123.39(15) \\
& \mathrm{C}(9 \mathrm{~B})-\mathrm{C}(4 \mathrm{~B})-\mathrm{O}(1 \mathrm{~B}) \quad 116.09(14) \\
& \mathrm{C}(4 \mathrm{~B})-\mathrm{C}(5 \mathrm{~B})-\mathrm{C}(6 \mathrm{~B}) \quad 118.96(17) \\
& \text { C(4B)-C(5B)-H(5BA) } 120.5 \\
& \text { C(6B)-C(5B)-H(5BA) } 120.5 \\
& \mathrm{C}(7 \mathrm{~B})-\mathrm{C}(6 \mathrm{~B})-\mathrm{C}(5 \mathrm{~B}) \quad \text { 120.94(18) } \\
& \mathrm{C}(7 \mathrm{~B})-\mathrm{C}(6 \mathrm{~B})-\mathrm{H}(6 \mathrm{BA}) \quad 119.5 \\
& \mathrm{C}(5 \mathrm{~B})-\mathrm{C}(6 \mathrm{~B})-\mathrm{H}(6 \mathrm{BA}) \quad 119.5 \\
& \mathrm{C}(6 \mathrm{~B})-\mathrm{C}(7 \mathrm{~B})-\mathrm{C}(8 \mathrm{~B}) \quad 119.61(17) \\
& \mathrm{C}(6 \mathrm{~B})-\mathrm{C}(7 \mathrm{~B})-\mathrm{H}(7 \mathrm{BA}) \quad 120.2 \\
& \text { C(8B)-C(7B)-H(7BA) } 120.2 \\
& \mathrm{C}(9 \mathrm{~B})-\mathrm{C}(8 \mathrm{~B})-\mathrm{C}(7 \mathrm{~B}) \quad 120.31(18) \\
& \mathrm{C}(9 \mathrm{~B})-\mathrm{C}(8 \mathrm{~B})-\mathrm{H}(8 \mathrm{BA}) \quad 119.8 \\
& \mathrm{C}(7 \mathrm{~B})-\mathrm{C}(8 \mathrm{~B})-\mathrm{H}(8 \mathrm{BA}) \quad 119.8 \\
& \text { C(4B)-C(9B)-C(8B) 119.67(17) } \\
& \text { C(4B)-C(9B)-H(9BA) } 120.2 \\
& \text { C(8B)-C(9B)-H(9BA) } 120.2 \\
& \text { C(11B)-C(10B)-C(15B) 118.93(14) } \\
& \text { C(11B)-C(10B)-Sn(1B) 118.74(11) } \\
& \text { C(15B)-C(10B)-Sn(1B) 122.33(12) } \\
& \text { C(12B)-C(11B)-C(10B) 120.49(15) } \\
& \text { C(12B)-C(11B)-H(11B) } 119.8 \\
& \text { C(10B)-C(11B)-H(11B) } 119.8 \\
& \text { C(13B)-C(12B)-C(11B) 119.88(16) } \\
& \text { C(13B)-C(12B)-H(12B) } 120.1 \\
& \text { C(11B)-C(12B)-H(12B) } 120.1 \\
& \text { C(14B)-C(13B)-C(12B) 120.21(16) } \\
& \text { C(14B)-C(13B)-H(13B) } 119.9 \\
& \text { C(12B)-C(13B)-H(13B) } 119.9 \\
& \text { C(13B)-C(14B)-C(15B) 120.20(16) } \\
& \text { C(13B)-C(14B)-H(14B) } 119.9 \\
& \text { C(15B)-C(14B)-H(14B) } 119.9 \\
& \text { C(14B)-C(15B)-C(10B) 120.28(16) } \\
& \text { C(14B)-C(15B)-H(15B) } 119.9 \\
& \text { C(10B)-C(15B)-H(15B) } 119.9 \\
& \text { C(21B)-C(16B)-C(17B) 118.48(14) }
\end{aligned}
$$




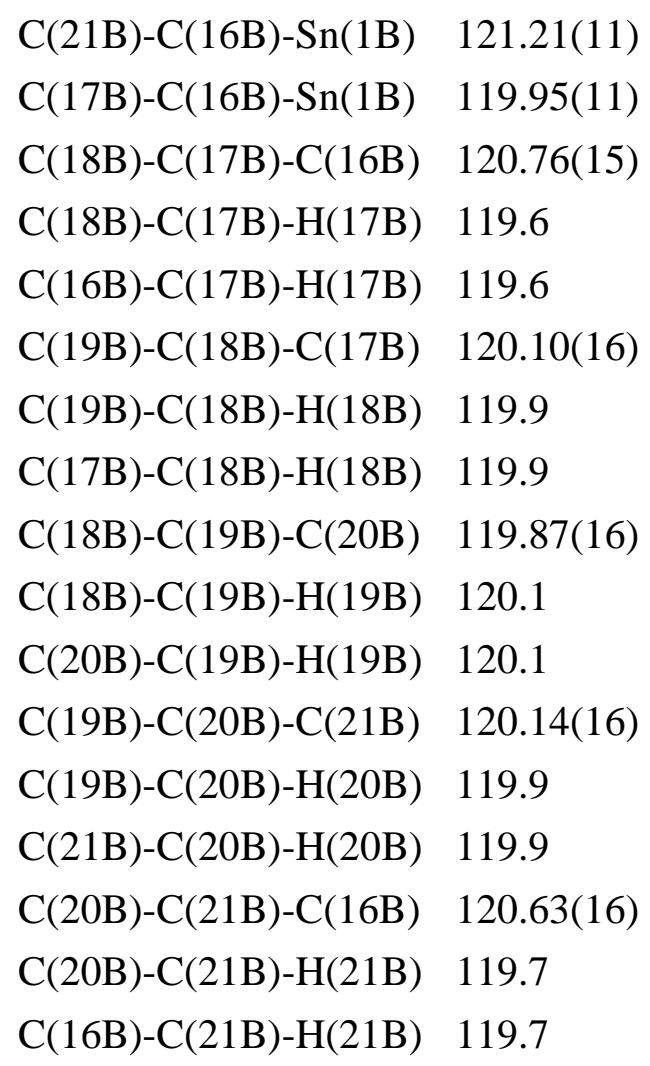

Symmetry transformations used to generate equivalent atoms: 
Table A 9. Anisotropic displacement parameters $\left(\AA^{2} \times 1^{3}\right)$ for 200. The anisotropic displacement factor exponent takes the form: $-2 \mathrm{p}^{2}\left[\mathrm{~h}^{2} \mathrm{a}^{* 2} \mathrm{U}^{11}+\ldots+2 \mathrm{hk} \mathrm{a}^{*} \mathrm{~b}^{*} \mathrm{U}^{12}\right]$

\begin{tabular}{|c|c|c|c|c|c|c|}
\hline & $\mathrm{U}^{11}$ & $\mathrm{U}^{22}$ & U33 & $\mathrm{U}^{23}$ & $\mathrm{U}^{13}$ & $\mathrm{U}^{12}$ \\
\hline $\operatorname{Sn}(1 \mathrm{~A})$ & $21(1)$ & $17(1)$ & $17(1)$ & $0(1)$ & $-3(1)$ & $-4(1)$ \\
\hline $\mathrm{Cl}(1 \mathrm{~A})$ & $33(1)$ & $28(1)$ & $16(1)$ & $3(1)$ & $-7(1)$ & $-7(1)$ \\
\hline $\mathrm{O}(1 \mathrm{~A})$ & 21(1) & $24(1)$ & $22(1)$ & $4(1)$ & $-2(1)$ & $-2(1)$ \\
\hline $\mathrm{C}(1 \mathrm{~A})$ & $22(1)$ & $28(1)$ & $20(1)$ & $2(1)$ & $-1(1)$ & $-3(1)$ \\
\hline $\mathrm{C}(2 \mathrm{~A})$ & 21(1) & $29(1)$ & $25(1)$ & 2(1) & $-5(1)$ & $-6(1)$ \\
\hline $\mathrm{C}(3 \mathrm{~A})$ & $20(1)$ & $28(1)$ & $24(1)$ & $3(1)$ & $-5(1)$ & $-1(1)$ \\
\hline $\mathrm{C}(4 \mathrm{~A})$ & $27(1)$ & $30(1)$ & $15(1)$ & 2(1) & $-5(1)$ & $-9(1)$ \\
\hline $\mathrm{C}(5 \mathrm{~A})$ & $31(1)$ & $32(1)$ & $23(1)$ & $4(1)$ & $-8(1)$ & $-7(1)$ \\
\hline$C(6 \mathrm{~A})$ & $50(1)$ & $37(1)$ & $32(1)$ & $13(1)$ & $-14(1)$ & $-16(1)$ \\
\hline$C(7 A)$ & $49(1)$ & $58(1)$ & $30(1)$ & $13(1)$ & $-5(1)$ & $-27(1)$ \\
\hline $\mathrm{C}(8 \mathrm{~A})$ & $29(1)$ & $65(1)$ & $28(1)$ & $2(1)$ & $0(1)$ & $-15(1)$ \\
\hline $\mathrm{C}(9 \mathrm{~A})$ & $28(1)$ & 41(1) & $22(1)$ & $-1(1)$ & $-2(1)$ & $-5(1)$ \\
\hline$C(10 A)$ & $23(1)$ & $22(1)$ & $18(1)$ & 1(1) & $-6(1)$ & $-6(1)$ \\
\hline $\mathrm{C}(11 \mathrm{~A})$ & $25(1)$ & $28(1)$ & $48(1)$ & $10(1)$ & $-12(1)$ & $-2(1)$ \\
\hline $\mathrm{C}(12 \mathrm{~A})$ & $18(1)$ & $38(1)$ & $66(1)$ & $10(1)$ & $-10(1)$ & $-4(1)$ \\
\hline$C(13 \mathrm{~A})$ & $26(1)$ & $31(1)$ & $45(1)$ & $7(1)$ & $-6(1)$ & $-13(1)$ \\
\hline $\mathrm{C}(14 \mathrm{~A})$ & $29(1)$ & 19(1) & $33(1)$ & 1(1) & $-4(1)$ & $-4(1)$ \\
\hline$C(15 A)$ & $20(1)$ & $24(1)$ & $25(1)$ & $-2(1)$ & $-3(1)$ & $-2(1)$ \\
\hline$C(16 A)$ & $23(1)$ & 19(1) & $22(1)$ & $1(1)$ & $-1(1)$ & $-5(1)$ \\
\hline $\mathrm{C}(17 \mathrm{~A})$ & $46(1)$ & $28(1)$ & $23(1)$ & $-1(1)$ & $-9(1)$ & $2(1)$ \\
\hline $\mathrm{C}(18 \mathrm{~A})$ & $72(1)$ & $33(1)$ & $22(1)$ & $-6(1)$ & $-3(1)$ & $0(1)$ \\
\hline $\mathrm{C}(19 \mathrm{~A})$ & $47(1)$ & $23(1)$ & $35(1)$ & $0(1)$ & $15(1)$ & $4(1)$ \\
\hline$C(20 A)$ & $22(1)$ & $28(1)$ & $45(1)$ & $7(1)$ & $3(1)$ & $0(1)$ \\
\hline$C(21 \mathrm{~A})$ & $24(1)$ & $26(1)$ & $33(1)$ & $2(1)$ & $-5(1)$ & $-6(1)$ \\
\hline $\operatorname{Sn}(1 \mathrm{~B})$ & $17(1)$ & $17(1)$ & 21(1) & $0(1)$ & $-4(1)$ & $-2(1)$ \\
\hline $\mathrm{Cl}(1 \mathrm{~B})$ & $26(1)$ & $28(1)$ & $21(1)$ & $-1(1)$ & $-8(1)$ & $-2(1)$ \\
\hline $\mathrm{O}(1 \mathrm{~B})$ & $25(1)$ & $24(1)$ & $28(1)$ & $-3(1)$ & $-6(1)$ & $-7(1)$ \\
\hline $\mathrm{C}(1 \mathrm{~B})$ & $20(1)$ & $27(1)$ & $29(1)$ & $1(1)$ & $-1(1)$ & $-3(1)$ \\
\hline$C(2 B)$ & $18(1)$ & $28(1)$ & $38(1)$ & $-3(1)$ & $-3(1)$ & $-5(1)$ \\
\hline $\mathrm{C}(3 \mathrm{~B})$ & $22(1)$ & $26(1)$ & $38(1)$ & $-2(1)$ & $-7(1)$ & $-9(1)$ \\
\hline $\mathrm{C}(4 \mathrm{~B})$ & $24(1)$ & $29(1)$ & $25(1)$ & $-1(1)$ & $-12(1)$ & $-3(1)$ \\
\hline
\end{tabular}




$\begin{array}{lllllll}\mathrm{C}(5 \mathrm{~B}) & 36(1) & 28(1) & 33(1) & -3(1) & -12(1) & -4(1) \\ \mathrm{C}(6 \mathrm{~B}) & 44(1) & 32(1) & 37(1) & -9(1) & -16(1) & 1(1) \\ \mathrm{C}(7 \mathrm{~B}) & 34(1) & 51(1) & 28(1) & -10(1) & -13(1) & 2(1) \\ \mathrm{C}(8 \mathrm{~B}) & 34(1) & 52(1) & 29(1) & -1(1) & -8(1) & -13(1) \\ \mathrm{C}(9 \mathrm{~B}) & 34(1) & 34(1) & 32(1) & -3(1) & -10(1) & -11(1) \\ \mathrm{C}(10 \mathrm{~B}) & 27(1) & 18(1) & 19(1) & -2(1) & -4(1) & -1(1) \\ \mathrm{C}(11 \mathrm{~B}) & 27(1) & 23(1) & 28(1) & 1(1) & -3(1) & -2(1) \\ \mathrm{C}(12 \mathrm{~B}) & 34(1) & 25(1) & 35(1) & 0(1) & 7(1) & -7(1) \\ \mathrm{C}(13 \mathrm{~B}) & 53(1) & 21(1) & 22(1) & 3(1) & 9(1) & 2(1) \\ \mathrm{C}(14 \mathrm{~B}) & 48(1) & 28(1) & 18(1) & 1(1) & -6(1) & 8(1) \\ \mathrm{C}(15 \mathrm{~B}) & 32(1) & 25(1) & 22(1) & -2(1) & -9(1) & -1(1) \\ \mathrm{C}(16 \mathrm{~B}) & 18(1) & 21(1) & 22(1) & -2(1) & -7(1) & -1(1) \\ \mathrm{C}(17 \mathrm{~B}) & 24(1) & 25(1) & 26(1) & 1(1) & -4(1) & -1(1) \\ \mathrm{C}(18 \mathrm{~B}) & 33(1) & 22(1) & 38(1) & -1(1) & -9(1) & 0(1) \\ \mathrm{C}(19 \mathrm{~B}) & 29(1) & 29(1) & 43(1) & -12(1) & -5(1) & 5(1) \\ \mathrm{C}(20 \mathrm{~B}) & 27(1) & 38(1) & 35(1) & -9(1) & 5(1) & -3(1) \\ \mathrm{C}(21 \mathrm{~B}) & 26(1) & 27(1) & 29(1) & 0(1) & -2(1) & -5(1)\end{array}$


Table A 10. Hydrogen coordinates $\left(x 1^{4}\right)$ and isotropic displacement parameters $\left(\AA^{2} \times\right.$ $10^{3}$ ) for 200.

\begin{tabular}{|c|c|c|c|c|}
\hline & $\mathrm{x}$ & $\mathrm{y}$ & $\mathrm{z}$ & $\mathrm{U}(\mathrm{eq})$ \\
\hline $\mathrm{H}(1 \mathrm{AA})$ & 6011 & 4420 & 2282 & 28 \\
\hline $\mathrm{H}(1 \mathrm{AB})$ & 6058 & 5849 & 2211 & 28 \\
\hline $\mathrm{H}(2 \mathrm{AA})$ & 7180 & 5105 & 3220 & 29 \\
\hline $\mathrm{H}(2 \mathrm{AB})$ & 5922 & 6016 & 3615 & 29 \\
\hline $\mathrm{H}(3 \mathrm{AA})$ & 5944 & 3429 & 3607 & 29 \\
\hline $\mathrm{H}(3 \mathrm{AB})$ & 6177 & 4183 & 4364 & 29 \\
\hline $\mathrm{H}(5 \mathrm{AA})$ & 5159 & 2320 & 4593 & 34 \\
\hline $\mathrm{H}(6 \mathrm{AA})$ & 3877 & 1033 & 5400 & 46 \\
\hline $\mathrm{H}(7 \mathrm{AA})$ & 1681 & 1666 & 5898 & 53 \\
\hline $\mathrm{H}(8 \mathrm{AA})$ & 739 & 3593 & 5601 & 49 \\
\hline $\mathrm{H}(9 \mathrm{AA})$ & 1994 & 4899 & 4807 & 37 \\
\hline $\mathrm{H}(11 \mathrm{~A})$ & 688 & 5252 & 2935 & 40 \\
\hline $\mathrm{H}(12 \mathrm{~A})$ & -742 & 3812 & 3308 & 49 \\
\hline $\mathrm{H}(13 \mathrm{~A})$ & 71 & 1821 & 3550 & 40 \\
\hline $\mathrm{H}(14 \mathrm{~A})$ & 2325 & 1239 & 3358 & 33 \\
\hline $\mathrm{H}(15 \mathrm{~A})$ & 3767 & 2655 & 2940 & 27 \\
\hline $\mathrm{H}(17 \mathrm{~A})$ & 3576 & 7091 & 4211 & 39 \\
\hline $\mathrm{H}(18 \mathrm{~A})$ & 2409 & 8855 & 4854 & 52 \\
\hline $\mathrm{H}(19 \mathrm{~A})$ & 706 & 9922 & 4326 & 46 \\
\hline $\mathrm{H}(20 \mathrm{~A})$ & 182 & 9255 & 3142 & 40 \\
\hline $\mathrm{H}(21 \mathrm{~A})$ & 1349 & 7514 & 2481 & 33 \\
\hline $\mathrm{H}(1 \mathrm{BA})$ & 5380 & -508 & 6795 & 31 \\
\hline $\mathrm{H}(1 \mathrm{BB})$ & 4851 & 908 & 6891 & 31 \\
\hline $\mathrm{H}(2 \mathrm{BA})$ & 6133 & -521 & 7996 & 34 \\
\hline $\mathrm{H}(2 \mathrm{BB})$ & 6667 & 601 & 7501 & 34 \\
\hline $\mathrm{H}(3 \mathrm{BA})$ & 5875 & 1279 & 8812 & 33 \\
\hline $\mathrm{H}(3 \mathrm{BB})$ & 4883 & 1923 & 8271 & 33 \\
\hline $\mathrm{H}(5 \mathrm{BA})$ & 4131 & 2794 & 9472 & 38 \\
\hline $\mathrm{H}(6 \mathrm{BA})$ & 2912 & 3637 & 10682 & 45 \\
\hline $\mathrm{H}(7 \mathrm{BA})$ & 1598 & 2485 & 11548 & 45 \\
\hline $\mathrm{H}(8 \mathrm{BA})$ & 1492 & 461 & 11217 & 45 \\
\hline
\end{tabular}




\begin{tabular}{lrrrr}
$\mathrm{H}(9 \mathrm{BA})$ & 2695 & -397 & 10013 & 39 \\
$\mathrm{H}(11 \mathrm{~B})$ & 1077 & -2111 & 8112 & 32 \\
$\mathrm{H}(12 B)$ & 738 & -3854 & 8861 & 39 \\
$\mathrm{H}(13 \mathrm{~B})$ & 2314 & -4706 & 9584 & 41 \\
$\mathrm{H}(14 \mathrm{~B})$ & 4203 & -3801 & 9583 & 39 \\
$\mathrm{H}(15 \mathrm{~B})$ & 4530 & -2032 & 8862 & 31 \\
$\mathrm{H}(17 \mathrm{~B})$ & 2518 & 2454 & 7385 & 30 \\
$\mathrm{H}(18 \mathrm{~B})$ & 1157 & 4180 & 7939 & 37 \\
$\mathrm{H}(19 B)$ & -518 & 4006 & 9027 & 41 \\
$\mathrm{H}(20 B)$ & -838 & 2099 & 9558 & 42 \\
$\mathrm{H}(21 \mathrm{~B})$ & 556 & 373 & 9030 & 33 \\
\hline
\end{tabular}


Table A 11. Torsion angles $\left[{ }^{\circ}\right]$ for 200.

\begin{tabular}{lc}
\hline $\mathrm{Sn}(1 \mathrm{~A})-\mathrm{C}(1 \mathrm{~A})-\mathrm{C}(2 \mathrm{~A})-\mathrm{C}(3 \mathrm{~A})$ & $60.90(16)$ \\
$\mathrm{C}(4 \mathrm{~A})-\mathrm{O}(1 \mathrm{~A})-\mathrm{C}(3 \mathrm{~A})-\mathrm{C}(2 \mathrm{~A})$ & $177.59(12)$ \\
$\mathrm{Sn}(1 \mathrm{~A})-\mathrm{O}(1 \mathrm{~A})-\mathrm{C}(3 \mathrm{~A})-\mathrm{C}(2 \mathrm{~A})$ & $38.27(12)$ \\
$\mathrm{C}(1 \mathrm{~A})-\mathrm{C}(2 \mathrm{~A})-\mathrm{C}(3 \mathrm{~A})-\mathrm{O}(1 \mathrm{~A})$ & $-66.84(16)$ \\
$\mathrm{C}(3 \mathrm{~A})-\mathrm{O}(1 \mathrm{~A})-\mathrm{C}(4 \mathrm{~A})-\mathrm{C}(5 \mathrm{~A})$ & $-8.7(2)$ \\
$\mathrm{Sn}(1 \mathrm{~A})-\mathrm{O}(1 \mathrm{~A})-\mathrm{C}(4 \mathrm{~A})-\mathrm{C}(5 \mathrm{~A})$ & $120.36(14)$ \\
$\mathrm{C}(3 \mathrm{~A})-\mathrm{O}(1 \mathrm{~A})-\mathrm{C}(4 \mathrm{~A})-\mathrm{C}(9 \mathrm{~A})$ & $172.45(13)$ \\
$\mathrm{Sn}(1 \mathrm{~A})-\mathrm{O}(1 \mathrm{~A})-\mathrm{C}(4 \mathrm{~A})-\mathrm{C}(9 \mathrm{~A})$ & $-58.49(16)$ \\
$\mathrm{O}(1 \mathrm{~A})-\mathrm{C}(4 \mathrm{~A})-\mathrm{C}(5 \mathrm{~A})-\mathrm{C}(6 \mathrm{~A})$ & $-178.12(14)$ \\
$\mathrm{C}(9 \mathrm{~A})-\mathrm{C}(4 \mathrm{~A})-\mathrm{C}(5 \mathrm{~A})-\mathrm{C}(6 \mathrm{~A})$ & $0.7(2)$ \\
$\mathrm{C}(4 \mathrm{~A})-\mathrm{C}(5 \mathrm{~A})-\mathrm{C}(6 \mathrm{~A})-\mathrm{C}(7 \mathrm{~A})$ & $-0.1(3)$ \\
$\mathrm{C}(5 \mathrm{~A})-\mathrm{C}(6 \mathrm{~A})-\mathrm{C}(7 \mathrm{~A})-\mathrm{C}(8 \mathrm{~A})$ & $-0.2(3)$ \\
$\mathrm{C}(6 \mathrm{~A})-\mathrm{C}(7 \mathrm{~A})-\mathrm{C}(8 \mathrm{~A})-\mathrm{C}(9 \mathrm{~A})$ & $0.0(3)$ \\
$\mathrm{C}(7 \mathrm{~A})-\mathrm{C}(8 \mathrm{~A})-\mathrm{C}(9 \mathrm{~A})-\mathrm{C}(4 \mathrm{~A})$ & $0.6(3)$ \\
$\mathrm{O}(1 \mathrm{~A})-\mathrm{C}(4 \mathrm{~A})-\mathrm{C}(9 \mathrm{~A})-\mathrm{C}(8 \mathrm{~A})$ & $177.99(14)$ \\
$\mathrm{C}(5 \mathrm{~A})-\mathrm{C}(4 \mathrm{~A})-\mathrm{C}(9 \mathrm{~A})-\mathrm{C}(8 \mathrm{~A})$ & $-0.9(2)$ \\
$\mathrm{C}(15 \mathrm{~A})-\mathrm{C}(10 \mathrm{~A})-\mathrm{C}(11 \mathrm{~A})-\mathrm{C}(12 \mathrm{~A})$ & $-0.2(3)$ \\
$\mathrm{Sn}(1 \mathrm{~A})-\mathrm{C}(10 \mathrm{~A})-\mathrm{C}(11 \mathrm{~A})-\mathrm{C}(12 \mathrm{~A})$ & $-173.52(15)$ \\
$\mathrm{C}(10 \mathrm{~A})-\mathrm{C}(11 \mathrm{~A})-\mathrm{C}(12 \mathrm{~A})-\mathrm{C}(13 \mathrm{~A})$ & $1.5(3)$ \\
$\mathrm{C}(11 \mathrm{~A})-\mathrm{C}(12 \mathrm{~A})-\mathrm{C}(13 \mathrm{~A})-\mathrm{C}(14 \mathrm{~A})$ & $-1.6(3)$ \\
$\mathrm{C}(12 \mathrm{~A})-\mathrm{C}(13 \mathrm{~A})-\mathrm{C}(14 \mathrm{~A})-\mathrm{C}(15 \mathrm{~A})$ & $0.4(3)$ \\
$\mathrm{C}(11 \mathrm{~A})-\mathrm{C}(10 \mathrm{~A})-\mathrm{C}(15 \mathrm{~A})-\mathrm{C}(14 \mathrm{~A})$ & $-1.0(2)$ \\
$\mathrm{Sn}(1 \mathrm{~A})-\mathrm{C}(10 \mathrm{~A})-\mathrm{C}(15 \mathrm{~A})-\mathrm{C}(14 \mathrm{~A})$ & $171.94(12)$ \\
$\mathrm{C}(13 \mathrm{~A})-\mathrm{C}(14 \mathrm{~A})-\mathrm{C}(15 \mathrm{~A})-\mathrm{C}(10 \mathrm{~A})$ & $0.9(2)$ \\
$\mathrm{C}(21 \mathrm{~A})-\mathrm{C}(16 \mathrm{~A})-\mathrm{C}(17 \mathrm{~A})-\mathrm{C}(18 \mathrm{~A})$ & $-0.4(3)$ \\
$\mathrm{Sn}(1 \mathrm{~A})-\mathrm{C}(16 \mathrm{~A})-\mathrm{C}(17 \mathrm{~A})-\mathrm{C}(18 \mathrm{~A})$ & $-178.81(14)$ \\
$\mathrm{C}(16 \mathrm{~A})-\mathrm{C}(17 \mathrm{~A})-\mathrm{C}(18 \mathrm{~A})-\mathrm{C}(19 \mathrm{~A})$ & $0.7(3)$ \\
$\mathrm{C}(17 \mathrm{~A})-\mathrm{C}(18 \mathrm{~A})-\mathrm{C}(19 \mathrm{~A})-\mathrm{C}(20 \mathrm{~A})$ & $-0.5(3)$ \\
$\mathrm{C}(18 \mathrm{~A})-\mathrm{C}(19 \mathrm{~A})-\mathrm{C}(20 \mathrm{~A})-\mathrm{C}(21 \mathrm{~A})$ & $0.1(3)$ \\
$\mathrm{C}(19 \mathrm{~A})-\mathrm{C}(20 \mathrm{~A})-\mathrm{C}(21 \mathrm{~A})-\mathrm{C}(16 \mathrm{~A})$ & $0.2(2)$ \\
$\mathrm{C}(17 \mathrm{~A})-\mathrm{C}(16 \mathrm{~A})-\mathrm{C}(21 \mathrm{~A})-\mathrm{C}(20 \mathrm{~A})$ & $0.0(2)$ \\
$\mathrm{Sn}(1 \mathrm{~A})-\mathrm{C}(16 \mathrm{~A})-\mathrm{C}(21 \mathrm{~A})-\mathrm{C}(20 \mathrm{~A})$ & $-50.53(17)$ \\
$\mathrm{Sn}(1 \mathrm{~B})-\mathrm{C}(1 \mathrm{~B})-\mathrm{C}(2 \mathrm{~B})-\mathrm{C}(3 \mathrm{~B})$ & \\
$\mathrm{C}(4 \mathrm{~B})-\mathrm{O}(1 \mathrm{~B})-\mathrm{C}(3 \mathrm{~B})-\mathrm{C}(2 \mathrm{~B})$ & \\
& \\
& \\
&
\end{tabular}




$$
\begin{array}{lc}
\mathrm{Sn}(1 \mathrm{~B})-\mathrm{O}(1 \mathrm{~B})-\mathrm{C}(3 \mathrm{~B})-\mathrm{C}(2 \mathrm{~B}) & -43.97(13) \\
\mathrm{C}(1 \mathrm{~B})-\mathrm{C}(2 \mathrm{~B})-\mathrm{C}(3 \mathrm{~B})-\mathrm{O}(1 \mathrm{~B}) & 66.51(17) \\
\mathrm{C}(3 \mathrm{~B})-\mathrm{O}(1 \mathrm{~B})-\mathrm{C}(4 \mathrm{~B})-\mathrm{C}(5 \mathrm{~B}) & 14.9(2) \\
\mathrm{Sn}(1 \mathrm{~B})-\mathrm{O}(1 \mathrm{~B})-\mathrm{C}(4 \mathrm{~B})-\mathrm{C}(5 \mathrm{~B}) & -115.33(14) \\
\mathrm{C}(3 \mathrm{~B})-\mathrm{O}(1 \mathrm{~B})-\mathrm{C}(4 \mathrm{~B})-\mathrm{C}(9 \mathrm{~B}) & -165.17(14) \\
\mathrm{Sn}(1 \mathrm{~B})-\mathrm{O}(1 \mathrm{~B})-\mathrm{C}(4 \mathrm{~B})-\mathrm{C}(9 \mathrm{~B}) & 64.56(17) \\
\mathrm{C}(9 \mathrm{~B})-\mathrm{C}(4 \mathrm{~B})-\mathrm{C}(5 \mathrm{~B})-\mathrm{C}(6 \mathrm{~B}) & -0.6(2) \\
\mathrm{O}(1 \mathrm{~B})-\mathrm{C}(4 \mathrm{~B})-\mathrm{C}(5 \mathrm{~B})-\mathrm{C}(6 \mathrm{~B}) & 179.28(15) \\
\mathrm{C}(4 \mathrm{~B})-\mathrm{C}(5 \mathrm{~B})-\mathrm{C}(6 \mathrm{~B})-\mathrm{C}(7 \mathrm{~B}) & 0.2(3) \\
\mathrm{C}(5 \mathrm{~B})-\mathrm{C}(6 \mathrm{~B})-\mathrm{C}(7 \mathrm{~B})-\mathrm{C}(8 \mathrm{~B}) & 0.2(3) \\
\mathrm{C}(6 \mathrm{~B})-\mathrm{C}(7 \mathrm{~B})-\mathrm{C}(8 \mathrm{~B})-\mathrm{C}(9 \mathrm{~B}) & -0.3(3) \\
\mathrm{C}(5 \mathrm{~B})-\mathrm{C}(4 \mathrm{~B})-\mathrm{C}(9 \mathrm{~B})-\mathrm{C}(8 \mathrm{~B}) & 0.6(2) \\
\mathrm{O}(1 \mathrm{~B})-\mathrm{C}(4 \mathrm{~B})-\mathrm{C}(9 \mathrm{~B})-\mathrm{C}(8 \mathrm{~B}) & -179.34(15) \\
\mathrm{C}(7 \mathrm{~B})-\mathrm{C}(8 \mathrm{~B})-\mathrm{C}(9 \mathrm{~B})-\mathrm{C}(4 \mathrm{~B}) & -0.1(3) \\
\mathrm{C}(15 \mathrm{~B})-\mathrm{C}(10 \mathrm{~B})-\mathrm{C}(11 \mathrm{~B})-\mathrm{C}(12 \mathrm{~B}) & 0.6(2) \\
\mathrm{Sn}(1 \mathrm{~B})-\mathrm{C}(10 \mathrm{~B})-\mathrm{C}(11 \mathrm{~B})-\mathrm{C}(12 \mathrm{~B}) & -179.40(12) \\
\mathrm{C}(10 \mathrm{~B})-\mathrm{C}(11 \mathrm{~B})-\mathrm{C}(12 \mathrm{~B})-\mathrm{C}(13 \mathrm{~B}) & -1.0(2) \\
\mathrm{C}(11 \mathrm{~B})-\mathrm{C}(12 \mathrm{~B})-\mathrm{C}(13 \mathrm{~B})-\mathrm{C}(14 \mathrm{~B}) & 0.7(3) \\
\mathrm{C}(12 \mathrm{~B})-\mathrm{C}(13 \mathrm{~B})-\mathrm{C}(14 \mathrm{~B})-\mathrm{C}(15 \mathrm{~B}) & 0.2(2) \\
\mathrm{C}(13 \mathrm{~B})-\mathrm{C}(14 \mathrm{~B})-\mathrm{C}(15 \mathrm{~B})-\mathrm{C}(10 \mathrm{~B}) & -0.6(2) \\
\mathrm{C}(11 \mathrm{~B})-\mathrm{C}(10 \mathrm{~B})-\mathrm{C}(15 \mathrm{~B})-\mathrm{C}(14 \mathrm{~B}) & 0.3(2) \\
\mathrm{Sn}(1 \mathrm{~B})-\mathrm{C}(10 \mathrm{~B})-\mathrm{C}(15 \mathrm{~B})-\mathrm{C}(14 \mathrm{~B}) & -179.77(12) \\
\mathrm{C}(21 \mathrm{~B})-\mathrm{C}(16 \mathrm{~B})-\mathrm{C}(17 \mathrm{~B})-\mathrm{C}(18 \mathrm{~B}) & 0.6(2) \\
\mathrm{Sn}(1 \mathrm{~B})-\mathrm{C}(16 \mathrm{~B})-\mathrm{C}(17 \mathrm{~B})-\mathrm{C}(18 \mathrm{~B}) & -172.59(12) \\
\mathrm{C}(16 \mathrm{~B})-\mathrm{C}(17 \mathrm{~B})-\mathrm{C}(18 \mathrm{~B})-\mathrm{C}(19 \mathrm{~B}) & -0.7(3) \\
\mathrm{C}(17 \mathrm{~B})-\mathrm{C}(18 \mathrm{~B})-\mathrm{C}(19 \mathrm{~B})-\mathrm{C}(20 \mathrm{~B}) & -0.1(3) \\
\mathrm{C}(18 \mathrm{~B})-\mathrm{C}(19 \mathrm{~B})-\mathrm{C}(20 \mathrm{~B})-\mathrm{C}(21 \mathrm{~B}) & 1.0(3) \\
\mathrm{C}(19 \mathrm{~B})-\mathrm{C}(20 \mathrm{~B})-\mathrm{C}(21 \mathrm{~B})-\mathrm{C}(16 \mathrm{~B}) & 0.3(2) \\
\mathrm{C}(17 \mathrm{~B})-\mathrm{C}(16 \mathrm{~B})-\mathrm{C}(21 \mathrm{~B})-\mathrm{C}(20 \mathrm{~B}) & 173.41(13) \\
\mathrm{Sn}(1 \mathrm{~B})-\mathrm{C}(16 \mathrm{~B})-\mathrm{C}(21 \mathrm{~B})-\mathrm{C}(20 \mathrm{~B}) & \\
& \\
& \\
\end{array}
$$

Symmetry transformations used to generate equivalent atoms: 
Table A 12. Crystal data and structure refinement for 201.

Identification code

Empirical formula

Formula weight

Temperature

Wavelength

Crystal system

Space group

Unit cell dimensions

Volume

$\mathrm{Z}$

Density (calculated)

Absorption coefficient

$\mathrm{F}(000)$

Crystal size

Theta range for data collection

Index ranges

Reflections collected

Independent reflections

Completeness to theta $=27.50^{\circ}$

Absorption correction

Max. and min. transmission

Refinement method

Data / restraints / parameters

Goodness-of-fit on $\mathrm{F}^{2}$

Final R indices [I $>2 \operatorname{sigma}(\mathrm{I})]$

$\mathrm{R}$ indices (all data)

Largest diff. peak and hole d1362

$\mathrm{C}_{27} \mathrm{H}_{25} \mathrm{ClOSn}$

519.61

147(2) K

$0.71073 \AA$

Monoclinic

P 21/c

$\mathrm{a}=9.7304(4) \AA$

$\alpha=90^{\circ}$.

$\mathrm{b}=12.9001(5) \AA$

$\beta=91.177(1)^{\circ}$.

$\mathrm{c}=18.5688(7) \AA$

2330.32(16) $\AA^{3}$

4

$1.481 \mathrm{Mg} / \mathrm{m}^{3}$

$1.227 \mathrm{~mm}^{-1}$

1048

$0.42 \times 0.27 \times 0.24 \mathrm{~mm}^{3}$

1.92 to $27.50^{\circ}$.

$-12<=\mathrm{h}<=12,-14<=\mathrm{k}<=16,-24<=\mathrm{l}<=24$

21942

$5347[\mathrm{R}(\mathrm{int})=0.0321]$

$99.9 \%$

Semi-empirical from equivalents

0.7456 and 0.6792

Full-matrix least-squares on $\mathrm{F}^{2}$

5347 / 0 / 271

1.043

$\mathrm{R} 1=0.0209, \mathrm{wR} 2=0.0503$

$\mathrm{R} 1=0.0252, \mathrm{wR} 2=0.0530$

0.523 and -0.286 e. $\AA^{-3}$ 
Table A 13. Atomic coordinates $\left(x \mathbf{1 0}^{4}\right)$ and equivalent isotropic displacement parameters $\left(\AA^{2} \times 10^{3}\right)$ for 201.

\begin{tabular}{|c|c|c|c|c|}
\hline & $\mathrm{x}$ & $\mathrm{y}$ & z & $\mathrm{U}(\mathrm{eq})$ \\
\hline $\operatorname{Sn}(1)$ & $1969(1)$ & $4361(1)$ & $1534(1)$ & $20(1)$ \\
\hline $\mathrm{Cl}(1)$ & $2120(1)$ & $6230(1)$ & $1516(1)$ & $29(1)$ \\
\hline $\mathrm{O}(1)$ & 1309(1) & $2238(1)$ & $1634(1)$ & $26(1)$ \\
\hline $\mathrm{C}(1)$ & $-1(2)$ & $4157(2)$ & 1999(1) & $28(1)$ \\
\hline $\mathrm{C}(2)$ & $-71(2)$ & $3178(2)$ & 2461(1) & $35(1)$ \\
\hline$C(3)$ & $37(2)$ & $2201(2)$ & $2020(1)$ & $31(1)$ \\
\hline $\mathrm{C}(4)$ & $1574(2)$ & $1405(1)$ & 1184(1) & $23(1)$ \\
\hline$C(5)$ & $2780(2)$ & $1459(2)$ & 799(1) & $27(1)$ \\
\hline$C(6)$ & $3110(2)$ & $662(2)$ & $332(1)$ & $28(1)$ \\
\hline$C(7)$ & $2271(2)$ & $-209(1)$ & $251(1)$ & $23(1)$ \\
\hline$C(8)$ & $1076(2)$ & $-250(1)$ & $652(1)$ & $24(1)$ \\
\hline $\mathrm{C}(9)$ & $717(2)$ & $552(1)$ & 1113(1) & $25(1)$ \\
\hline$C(10)$ & $2640(2)$ & $-1078(2)$ & $-238(1)$ & $25(1)$ \\
\hline $\mathrm{C}(11)$ & $2397(2)$ & $-2102(2)$ & $-39(1)$ & $29(1)$ \\
\hline$C(12)$ & $2759(2)$ & $-2921(2)$ & $-483(1)$ & $33(1)$ \\
\hline$C(13)$ & $3360(2)$ & $-2722(2)$ & $-1141(1)$ & $34(1)$ \\
\hline$C(14)$ & $3591(2)$ & $-1713(2)$ & $-1348(1)$ & $35(1)$ \\
\hline$C(15)$ & $3246(2)$ & $-892(2)$ & $-902(1)$ & $30(1)$ \\
\hline$C(16)$ & $2246(2)$ & $4046(1)$ & $427(1)$ & $23(1)$ \\
\hline$C(17)$ & $1160(2)$ & $3829(2)$ & $-42(1)$ & $36(1)$ \\
\hline$C(18)$ & $1394(2)$ & $3655(2)$ & $-766(1)$ & $52(1)$ \\
\hline$C(19)$ & $2705(3)$ & $3694(3)$ & $-1022(1)$ & $61(1)$ \\
\hline$C(20)$ & $3803(3)$ & $3886(3)$ & $-558(1)$ & $56(1)$ \\
\hline$C(21)$ & $3575(2)$ & $4065(2)$ & 162(1) & $37(1)$ \\
\hline$C(22)$ & $3749(2)$ & $4077(1)$ & $2200(1)$ & $23(1)$ \\
\hline $\mathrm{C}(23)$ & $4742(2)$ & $4844(2)$ & $2282(1)$ & $31(1)$ \\
\hline$C(24)$ & $5892(2)$ & $4696(2)$ & $2724(1)$ & $38(1)$ \\
\hline$C(25)$ & $6057(2)$ & $3784(2)$ & $3097(1)$ & $37(1)$ \\
\hline$C(26)$ & $5095(2)$ & $3022(2)$ & $3019(1)$ & $46(1)$ \\
\hline $\mathrm{C}(27)$ & $3948(2)$ & $3158(2)$ & $2570(1)$ & $39(1)$ \\
\hline
\end{tabular}


Table A 14. Bond lengths $[\AA \AA]$ and angles $\left[^{\circ}\right]$ for 201.

\begin{tabular}{|c|c|}
\hline $\operatorname{Sn}(1)-C(16)$ & $2.1171(17)$ \\
\hline $\operatorname{Sn}(1)-C(1)$ & $2.1348(18)$ \\
\hline $\operatorname{Sn}(1)-C(22)$ & 2.1391(17) \\
\hline $\mathrm{Sn}(1)-\mathrm{Cl}(1)$ & $2.4155(5)$ \\
\hline $\mathrm{O}(1)-\mathrm{C}(4)$ & $1.389(2)$ \\
\hline $\mathrm{O}(1)-\mathrm{C}(3)$ & $1.443(2)$ \\
\hline$C(1)-C(2)$ & $1.530(3)$ \\
\hline $\mathrm{C}(1)-\mathrm{H}(1 \mathrm{~A})$ & 0.9900 \\
\hline $\mathrm{C}(1)-\mathrm{H}(1 \mathrm{~B})$ & 0.9900 \\
\hline$C(2)-C(3)$ & $1.508(3)$ \\
\hline $\mathrm{C}(2)-\mathrm{H}(2 \mathrm{~A})$ & 0.9900 \\
\hline $\mathrm{C}(2)-\mathrm{H}(2 \mathrm{~B})$ & 0.9900 \\
\hline $\mathrm{C}(3)-\mathrm{H}(3 \mathrm{~A})$ & 0.9900 \\
\hline $\mathrm{C}(3)-\mathrm{H}(3 \mathrm{~B})$ & 0.9900 \\
\hline$C(4)-C(9)$ & $1.385(3)$ \\
\hline$C(4)-C(5)$ & $1.388(2)$ \\
\hline$C(5)-C(6)$ & $1.387(3)$ \\
\hline $\mathrm{C}(5)-\mathrm{H}(5 \mathrm{~A})$ & 0.9500 \\
\hline$C(6)-C(7)$ & $1.394(3)$ \\
\hline $\mathrm{C}(6)-\mathrm{H}(6 \mathrm{~A})$ & 0.9500 \\
\hline$C(7)-C(8)$ & $1.394(2)$ \\
\hline$C(7)-C(10)$ & 1.491(3) \\
\hline $\mathrm{C}(8)-\mathrm{C}(9)$ & $1.392(3)$ \\
\hline $\mathrm{C}(8)-\mathrm{H}(8 \mathrm{~A})$ & 0.9500 \\
\hline $\mathrm{C}(9)-\mathrm{H}(9 \mathrm{~A})$ & 0.9500 \\
\hline $\mathrm{C}(10)-\mathrm{C}(11)$ & $1.394(3)$ \\
\hline$C(10)-C(15)$ & $1.398(2)$ \\
\hline $\mathrm{C}(11)-\mathrm{C}(12)$ & $1.390(3)$ \\
\hline $\mathrm{C}(11)-\mathrm{H}(11 \mathrm{~A})$ & 0.9500 \\
\hline$C(12)-C(13)$ & $1.389(3)$ \\
\hline $\mathrm{C}(12)-\mathrm{H}(12 \mathrm{~A})$ & 0.9500 \\
\hline$C(13)-C(14)$ & $1.376(3)$ \\
\hline C(13)-H(13A) & 0.9500 \\
\hline$C(14)-C(15)$ & $1.390(3)$ \\
\hline
\end{tabular}




\begin{tabular}{|c|c|}
\hline $\mathrm{C}(14)-\mathrm{H}(14 \mathrm{~A})$ & 0.9500 \\
\hline $\mathrm{C}(15)-\mathrm{H}(15 \mathrm{~A})$ & 0.9500 \\
\hline$C(16)-C(17)$ & $1.384(3)$ \\
\hline$C(16)-C(21)$ & $1.393(3)$ \\
\hline$C(17)-C(18)$ & $1.388(3)$ \\
\hline $\mathrm{C}(17)-\mathrm{H}(17 \mathrm{~A})$ & 0.9500 \\
\hline $\mathrm{C}(18)-\mathrm{C}(19)$ & $1.371(3)$ \\
\hline $\mathrm{C}(18)-\mathrm{H}(18 \mathrm{~A})$ & 0.9500 \\
\hline$C(19)-C(20)$ & $1.380(3)$ \\
\hline $\mathrm{C}(19)-\mathrm{H}(19 \mathrm{~A})$ & 0.9500 \\
\hline$C(20)-C(21)$ & $1.380(3)$ \\
\hline $\mathrm{C}(20)-\mathrm{H}(20 \mathrm{~A})$ & 0.9500 \\
\hline $\mathrm{C}(21)-\mathrm{H}(21 \mathrm{~A})$ & 0.9500 \\
\hline$C(22)-C(27)$ & $1.382(3)$ \\
\hline$C(22)-C(23)$ & $1.389(3)$ \\
\hline$C(23)-C(24)$ & $1.388(3)$ \\
\hline $\mathrm{C}(23)-\mathrm{H}(23 \mathrm{~A})$ & 0.9500 \\
\hline$C(24)-C(25)$ & $1.372(3)$ \\
\hline $\mathrm{C}(24)-\mathrm{H}(24 \mathrm{~A})$ & 0.9500 \\
\hline$C(25)-C(26)$ & $1.363(3)$ \\
\hline $\mathrm{C}(25)-\mathrm{H}(25 \mathrm{~A})$ & 0.9500 \\
\hline$C(26)-C(27)$ & $1.390(3)$ \\
\hline $\mathrm{C}(26)-\mathrm{H}(26 \mathrm{~A})$ & 0.9500 \\
\hline $\mathrm{C}(27)-\mathrm{H}(27 \mathrm{~A})$ & 0.9500 \\
\hline $\mathrm{C}(16)-\mathrm{Sn}(1)-\mathrm{C}(1)$ & $120.18(7)$ \\
\hline$C(16)-\operatorname{Sn}(1)-C(22)$ & $114.20(6)$ \\
\hline $\mathrm{C}(1)-\mathrm{Sn}(1)-\mathrm{C}(22)$ & $117.90(7)$ \\
\hline $\mathrm{C}(16)-\mathrm{Sn}(1)-\mathrm{Cl}(1)$ & $99.77(5)$ \\
\hline $\mathrm{C}(1)-\mathrm{Sn}(1)-\mathrm{Cl}(1)$ & $100.60(5)$ \\
\hline $\mathrm{C}(22)-\mathrm{Sn}(1)-\mathrm{Cl}(1)$ & $97.49(5)$ \\
\hline $\mathrm{C}(4)-\mathrm{O}(1)-\mathrm{C}(3)$ & $116.43(14)$ \\
\hline$C(2)-C(1)-S n(1)$ & $112.34(13)$ \\
\hline $\mathrm{C}(2)-\mathrm{C}(1)-\mathrm{H}(1 \mathrm{~A})$ & 109.1 \\
\hline $\operatorname{Sn}(1)-C(1)-H(1 A)$ & 109.1 \\
\hline $\mathrm{C}(2)-\mathrm{C}(1)-\mathrm{H}(1 \mathrm{~B})$ & 109.1 \\
\hline
\end{tabular}




\begin{tabular}{|c|c|}
\hline $\mathrm{Sn}(1)-\mathrm{C}(1)-\mathrm{H}(1 \mathrm{~B})$ & 109.1 \\
\hline $\mathrm{H}(1 \mathrm{~A})-\mathrm{C}(1)-\mathrm{H}(1 \mathrm{~B})$ & 107.9 \\
\hline $\mathrm{C}(3)-\mathrm{C}(2)-\mathrm{C}(1)$ & $112.40(16)$ \\
\hline $\mathrm{C}(3)-\mathrm{C}(2)-\mathrm{H}(2 \mathrm{~A})$ & 109.1 \\
\hline $\mathrm{C}(1)-\mathrm{C}(2)-\mathrm{H}(2 \mathrm{~A})$ & 109.1 \\
\hline $\mathrm{C}(3)-\mathrm{C}(2)-\mathrm{H}(2 \mathrm{~B})$ & 109.1 \\
\hline $\mathrm{C}(1)-\mathrm{C}(2)-\mathrm{H}(2 \mathrm{~B})$ & 109.1 \\
\hline $\mathrm{H}(2 \mathrm{~A})-\mathrm{C}(2)-\mathrm{H}(2 \mathrm{~B})$ & 107.9 \\
\hline $\mathrm{O}(1)-\mathrm{C}(3)-\mathrm{C}(2)$ & $108.21(15)$ \\
\hline $\mathrm{O}(1)-\mathrm{C}(3)-\mathrm{H}(3 \mathrm{~A})$ & 110.1 \\
\hline $\mathrm{C}(2)-\mathrm{C}(3)-\mathrm{H}(3 \mathrm{~A})$ & 110.1 \\
\hline $\mathrm{O}(1)-\mathrm{C}(3)-\mathrm{H}(3 \mathrm{~B})$ & 110.1 \\
\hline $\mathrm{C}(2)-\mathrm{C}(3)-\mathrm{H}(3 \mathrm{~B})$ & 110.1 \\
\hline $\mathrm{H}(3 \mathrm{~A})-\mathrm{C}(3)-\mathrm{H}(3 \mathrm{~B})$ & 108.4 \\
\hline$C(9)-C(4)-C(5)$ & $120.33(17)$ \\
\hline $\mathrm{C}(9)-\mathrm{C}(4)-\mathrm{O}(1)$ & $123.52(15)$ \\
\hline $\mathrm{C}(5)-\mathrm{C}(4)-\mathrm{O}(1)$ & $116.15(16)$ \\
\hline$C(6)-C(5)-C(4)$ & $119.57(17)$ \\
\hline $\mathrm{C}(6)-\mathrm{C}(5)-\mathrm{H}(5 \mathrm{~A})$ & 120.2 \\
\hline $\mathrm{C}(4)-\mathrm{C}(5)-\mathrm{H}(5 \mathrm{~A})$ & 120.2 \\
\hline$C(5)-C(6)-C(7)$ & $121.43(17)$ \\
\hline$C(5)-C(6)-H(6 A)$ & 119.3 \\
\hline$C(7)-C(6)-H(6 A)$ & 119.3 \\
\hline$C(8)-C(7)-C(6)$ & $117.79(17)$ \\
\hline $\mathrm{C}(8)-\mathrm{C}(7)-\mathrm{C}(10)$ & $120.68(16)$ \\
\hline$C(6)-C(7)-C(10)$ & $121.53(16)$ \\
\hline $\mathrm{C}(9)-\mathrm{C}(8)-\mathrm{C}(7)$ & $121.50(17)$ \\
\hline $\mathrm{C}(9)-\mathrm{C}(8)-\mathrm{H}(8 \mathrm{~A})$ & 119.2 \\
\hline $\mathrm{C}(7)-\mathrm{C}(8)-\mathrm{H}(8 \mathrm{~A})$ & 119.2 \\
\hline $\mathrm{C}(4)-\mathrm{C}(9)-\mathrm{C}(8)$ & $119.36(16)$ \\
\hline $\mathrm{C}(4)-\mathrm{C}(9)-\mathrm{H}(9 \mathrm{~A})$ & 120.3 \\
\hline $\mathrm{C}(8)-\mathrm{C}(9)-\mathrm{H}(9 \mathrm{~A})$ & 120.3 \\
\hline $\mathrm{C}(11)-\mathrm{C}(10)-\mathrm{C}(15)$ & $118.19(17)$ \\
\hline$C(11)-C(10)-C(7)$ & $120.51(16)$ \\
\hline $\mathrm{C}(15)-\mathrm{C}(10)-\mathrm{C}(7)$ & $121.30(17)$ \\
\hline $\mathrm{C}(12)-\mathrm{C}(11)-\mathrm{C}(10)$ & $121.14(17)$ \\
\hline
\end{tabular}




\begin{tabular}{|c|c|}
\hline $\mathrm{C}(12)-\mathrm{C}(11)-\mathrm{H}(11 \mathrm{~A})$ & 119.4 \\
\hline $\mathrm{C}(10)-\mathrm{C}(11)-\mathrm{H}(11 \mathrm{~A})$ & 119.4 \\
\hline $\mathrm{C}(13)-\mathrm{C}(12)-\mathrm{C}(11)$ & $119.85(19)$ \\
\hline $\mathrm{C}(13)-\mathrm{C}(12)-\mathrm{H}(12 \mathrm{~A})$ & 120.1 \\
\hline $\mathrm{C}(11)-\mathrm{C}(12)-\mathrm{H}(12 \mathrm{~A})$ & 120.1 \\
\hline C(14)-C(13)-C(12) & 119.61(18) \\
\hline $\mathrm{C}(14)-\mathrm{C}(13)-\mathrm{H}(13 \mathrm{~A})$ & 120.2 \\
\hline $\mathrm{C}(12)-\mathrm{C}(13)-\mathrm{H}(13 \mathrm{~A})$ & 120.2 \\
\hline $\mathrm{C}(13)-\mathrm{C}(14)-\mathrm{C}(15)$ & $120.76(18)$ \\
\hline $\mathrm{C}(13)-\mathrm{C}(14)-\mathrm{H}(14 \mathrm{~A})$ & 119.6 \\
\hline $\mathrm{C}(15)-\mathrm{C}(14)-\mathrm{H}(14 \mathrm{~A})$ & 119.6 \\
\hline $\mathrm{C}(14)-\mathrm{C}(15)-\mathrm{C}(10)$ & $120.42(19)$ \\
\hline $\mathrm{C}(14)-\mathrm{C}(15)-\mathrm{H}(15 \mathrm{~A})$ & 119.8 \\
\hline $\mathrm{C}(10)-\mathrm{C}(15)-\mathrm{H}(15 \mathrm{~A})$ & 119.8 \\
\hline $\mathrm{C}(17)-\mathrm{C}(16)-\mathrm{C}(21)$ & $118.92(17)$ \\
\hline$C(17)-C(16)-\operatorname{Sn}(1)$ & $122.57(13)$ \\
\hline$C(21)-C(16)-S n(1)$ & $118.51(13)$ \\
\hline $\mathrm{C}(16)-\mathrm{C}(17)-\mathrm{C}(18)$ & $120.27(19)$ \\
\hline$C(16)-C(17)-H(17 A)$ & 119.9 \\
\hline $\mathrm{C}(18)-\mathrm{C}(17)-\mathrm{H}(17 \mathrm{~A})$ & 119.9 \\
\hline C(19)-C(18)-C(17) & $120.2(2)$ \\
\hline $\mathrm{C}(19)-\mathrm{C}(18)-\mathrm{H}(18 \mathrm{~A})$ & 119.9 \\
\hline $\mathrm{C}(17)-\mathrm{C}(18)-\mathrm{H}(18 \mathrm{~A})$ & 119.9 \\
\hline$C(18)-C(19)-C(20)$ & $120.3(2)$ \\
\hline $\mathrm{C}(18)-\mathrm{C}(19)-\mathrm{H}(19 \mathrm{~A})$ & 119.8 \\
\hline $\mathrm{C}(20)-\mathrm{C}(19)-\mathrm{H}(19 \mathrm{~A})$ & 119.8 \\
\hline $\mathrm{C}(19)-\mathrm{C}(20)-\mathrm{C}(21)$ & $119.8(2)$ \\
\hline$C(19)-C(20)-H(20 A)$ & 120.1 \\
\hline $\mathrm{C}(21)-\mathrm{C}(20)-\mathrm{H}(20 \mathrm{~A})$ & 120.1 \\
\hline $\mathrm{C}(20)-\mathrm{C}(21)-\mathrm{C}(16)$ & $120.56(19)$ \\
\hline $\mathrm{C}(20)-\mathrm{C}(21)-\mathrm{H}(21 \mathrm{~A})$ & 119.7 \\
\hline $\mathrm{C}(16)-\mathrm{C}(21)-\mathrm{H}(21 \mathrm{~A})$ & 119.7 \\
\hline $\mathrm{C}(27)-\mathrm{C}(22)-\mathrm{C}(23)$ & $117.77(17)$ \\
\hline $\mathrm{C}(27)-\mathrm{C}(22)-\mathrm{Sn}(1)$ & $122.58(14)$ \\
\hline $\mathrm{C}(23)-\mathrm{C}(22)-\mathrm{Sn}(1)$ & $119.63(14)$ \\
\hline $\mathrm{C}(24)-\mathrm{C}(23)-\mathrm{C}(22)$ & $121.19(19)$ \\
\hline
\end{tabular}




$\begin{array}{ll}\mathrm{C}(24)-\mathrm{C}(23)-\mathrm{H}(23 \mathrm{~A}) & 119.4 \\ \mathrm{C}(22)-\mathrm{C}(23)-\mathrm{H}(23 \mathrm{~A}) & 119.4 \\ \mathrm{C}(25)-\mathrm{C}(24)-\mathrm{C}(23) & 120.0(2) \\ \mathrm{C}(25)-\mathrm{C}(24)-\mathrm{H}(24 \mathrm{~A}) & 120.0 \\ \mathrm{C}(23)-\mathrm{C}(24)-\mathrm{H}(24 \mathrm{~A}) & 120.0 \\ \mathrm{C}(26)-\mathrm{C}(25)-\mathrm{C}(24) & 119.46(19) \\ \mathrm{C}(26)-\mathrm{C}(25)-\mathrm{H}(25 \mathrm{~A}) & 120.3 \\ \mathrm{C}(24)-\mathrm{C}(25)-\mathrm{H}(25 \mathrm{~A}) & 120.3 \\ \mathrm{C}(25)-\mathrm{C}(26)-\mathrm{C}(27) & 120.9(2) \\ \mathrm{C}(25)-\mathrm{C}(26)-\mathrm{H}(26 \mathrm{~A}) & 119.6 \\ \mathrm{C}(27)-\mathrm{C}(26)-\mathrm{H}(26 \mathrm{~A}) & 119.6 \\ \mathrm{C}(22)-\mathrm{C}(27)-\mathrm{C}(26) & 120.6(2) \\ \mathrm{C}(22)-\mathrm{C}(27)-\mathrm{H}(27 \mathrm{~A}) & 119.7 \\ \mathrm{C}(26)-\mathrm{C}(27)-\mathrm{H}(27 \mathrm{~A}) & 119.7\end{array}$

Symmetry transformations used to generate equivalent atoms: 
Table A 15. Anisotropic displacement parameters $\left(\AA^{2} \times 10^{3}\right)$ for 201. The anisotropic displacement factor exponent takes the form: $-2 p^{2}\left[h^{2} a^{* 2} U^{11}+\ldots+2 h k a^{*} b^{*} U^{12}\right]$

\begin{tabular}{|c|c|c|c|c|c|c|}
\hline & $\mathrm{U}^{11}$ & $\mathrm{U}^{22}$ & $\mathrm{U}^{33}$ & $\mathrm{U}^{23}$ & $\mathrm{U}^{13}$ & $\mathrm{U}^{12}$ \\
\hline $\operatorname{Sn}(1)$ & $19(1)$ & $19(1)$ & $21(1)$ & 1(1) & $1(1)$ & $1(1)$ \\
\hline $\mathrm{Cl}(1)$ & $29(1)$ & $19(1)$ & $38(1)$ & $4(1)$ & $0(1)$ & $0(1)$ \\
\hline $\mathrm{O}(1)$ & $22(1)$ & $25(1)$ & $31(1)$ & $-2(1)$ & $4(1)$ & $-2(1)$ \\
\hline $\mathrm{C}(1)$ & $26(1)$ & $22(1)$ & $37(1)$ & $-1(1)$ & $9(1)$ & $1(1)$ \\
\hline $\mathrm{C}(2)$ & $40(1)$ & $27(1)$ & $39(1)$ & $2(1)$ & $18(1)$ & $0(1)$ \\
\hline$C(3)$ & $30(1)$ & $24(1)$ & $42(1)$ & $4(1)$ & $15(1)$ & $-1(1)$ \\
\hline$C(4)$ & $23(1)$ & $21(1)$ & $25(1)$ & $2(1)$ & $-2(1)$ & $3(1)$ \\
\hline$C(5)$ & $20(1)$ & $23(1)$ & $38(1)$ & $2(1)$ & $0(1)$ & $-1(1)$ \\
\hline$C(6)$ & $20(1)$ & $30(1)$ & $35(1)$ & $3(1)$ & $4(1)$ & $2(1)$ \\
\hline$C(7)$ & $22(1)$ & $23(1)$ & $24(1)$ & $3(1)$ & $-2(1)$ & $2(1)$ \\
\hline $\mathrm{C}(8)$ & $24(1)$ & $24(1)$ & $25(1)$ & $4(1)$ & $-4(1)$ & $-4(1)$ \\
\hline $\mathrm{C}(9)$ & $20(1)$ & $29(1)$ & $25(1)$ & $5(1)$ & $0(1)$ & $-2(1)$ \\
\hline$C(10)$ & $21(1)$ & $27(1)$ & $26(1)$ & $1(1)$ & $-4(1)$ & $2(1)$ \\
\hline $\mathrm{C}(11)$ & $32(1)$ & $29(1)$ & $27(1)$ & $3(1)$ & $-2(1)$ & $1(1)$ \\
\hline$C(12)$ & $34(1)$ & $27(1)$ & $39(1)$ & $0(1)$ & $-4(1)$ & $0(1)$ \\
\hline$C(13)$ & $27(1)$ & $36(1)$ & $38(1)$ & $-11(1)$ & $-1(1)$ & $3(1)$ \\
\hline$C(14)$ & $27(1)$ & $43(1)$ & $34(1)$ & $-4(1)$ & $6(1)$ & $-3(1)$ \\
\hline$C(15)$ & $26(1)$ & $30(1)$ & $34(1)$ & $1(1)$ & $4(1)$ & $-2(1)$ \\
\hline$C(16)$ & $24(1)$ & $22(1)$ & $24(1)$ & $1(1)$ & $0(1)$ & $2(1)$ \\
\hline$C(17)$ & $22(1)$ & $50(1)$ & $35(1)$ & $-7(1)$ & $-3(1)$ & $5(1)$ \\
\hline$C(18)$ & $38(1)$ & $80(2)$ & $36(1)$ & $-18(1)$ & $-14(1)$ & 11(1) \\
\hline C(19) & $54(2)$ & $102(2)$ & $27(1)$ & $-19(1)$ & $4(1)$ & $5(2)$ \\
\hline$C(20)$ & $37(1)$ & $95(2)$ & $36(1)$ & $-17(1)$ & $12(1)$ & $-6(1)$ \\
\hline$C(21)$ & $27(1)$ & $52(1)$ & $30(1)$ & $-7(1)$ & $1(1)$ & $-7(1)$ \\
\hline $\mathrm{C}(22)$ & $22(1)$ & $25(1)$ & $21(1)$ & $-2(1)$ & $0(1)$ & $5(1)$ \\
\hline$C(23)$ & $33(1)$ & $24(1)$ & $37(1)$ & $0(1)$ & $-6(1)$ & $-1(1)$ \\
\hline $\mathrm{C}(24)$ & $33(1)$ & $37(1)$ & $44(1)$ & $-11(1)$ & $-10(1)$ & $-2(1)$ \\
\hline$C(25)$ & $31(1)$ & $48(1)$ & $30(1)$ & $-8(1)$ & $-10(1)$ & $9(1)$ \\
\hline$C(26)$ & $43(1)$ & $44(1)$ & $52(1)$ & $17(1)$ & $-14(1)$ & $6(1)$ \\
\hline $\mathrm{C}(27)$ & $35(1)$ & $31(1)$ & $52(1)$ & $10(1)$ & $-10(1)$ & $-3(1)$ \\
\hline
\end{tabular}


Table A 16. Hydrogen coordinates $\left(\times 10^{4}\right)$ and isotropic displacement parameters $\left(\AA^{2} \times\right.$ $10^{3}$ ) for 201.

\begin{tabular}{|c|c|c|c|c|}
\hline & $\mathrm{x}$ & $\mathrm{y}$ & $\mathrm{Z}$ & $\mathrm{U}(\mathrm{eq})$ \\
\hline $\mathrm{H}(1 \mathrm{~A})$ & -210 & 4768 & 2301 & 34 \\
\hline $\mathrm{H}(1 \mathrm{~B})$ & -709 & 4117 & 1610 & 34 \\
\hline $\mathrm{H}(2 \mathrm{~A})$ & 685 & 3191 & 2826 & 42 \\
\hline $\mathrm{H}(2 \mathrm{~B})$ & -951 & 3171 & 2720 & 42 \\
\hline $\mathrm{H}(3 \mathrm{~A})$ & 26 & 1586 & 2338 & 38 \\
\hline $\mathrm{H}(3 \mathrm{~B})$ & -751 & 2151 & 1676 & 38 \\
\hline $\mathrm{H}(5 \mathrm{~A})$ & 3376 & 2038 & 855 & 33 \\
\hline $\mathrm{H}(6 \mathrm{~A})$ & 3925 & 710 & 61 & 34 \\
\hline $\mathrm{H}(8 \mathrm{~A})$ & 493 & -838 & 609 & 29 \\
\hline $\mathrm{H}(9 \mathrm{~A})$ & -108 & 515 & 1377 & 30 \\
\hline $\mathrm{H}(11 \mathrm{~A})$ & 1976 & -2243 & 407 & 35 \\
\hline $\mathrm{H}(12 \mathrm{~A})$ & 2597 & -3615 & -337 & 40 \\
\hline $\mathrm{H}(13 \mathrm{~A})$ & 3609 & -3278 & -1447 & 40 \\
\hline $\mathrm{H}(14 \mathrm{~A})$ & 3991 & -1577 & -1800 & 42 \\
\hline $\mathrm{H}(15 \mathrm{~A})$ & 3423 & -201 & -1049 & 36 \\
\hline $\mathrm{H}(17 \mathrm{~A})$ & 250 & 3798 & 134 & 43 \\
\hline $\mathrm{H}(18 \mathrm{~A})$ & 645 & 3509 & -1086 & 62 \\
\hline $\mathrm{H}(19 \mathrm{~A})$ & 2858 & 3589 & -1520 & 73 \\
\hline $\mathrm{H}(20 \mathrm{~A})$ & 4713 & 3894 & -735 & 67 \\
\hline $\mathrm{H}(21 \mathrm{~A})$ & 4331 & 4202 & 480 & 44 \\
\hline $\mathrm{H}(23 \mathrm{~A})$ & 4632 & 5480 & 2030 & 38 \\
\hline $\mathrm{H}(24 \mathrm{~A})$ & 6564 & 5227 & 2770 & 46 \\
\hline $\mathrm{H}(25 \mathrm{~A})$ & 6836 & 3685 & 3406 & 44 \\
\hline $\mathrm{H}(26 \mathrm{~A})$ & 5209 & 2389 & 3275 & 56 \\
\hline $\mathrm{H}(27 \mathrm{~A})$ & 3295 & 2615 & 2518 & 47 \\
\hline
\end{tabular}


Table A 17. Torsion angles $\left[{ }^{\circ}\right]$ for 201.

\begin{tabular}{lc}
\hline $\mathrm{C}(16)-\mathrm{Sn}(1)-\mathrm{C}(1)-\mathrm{C}(2)$ & $104.65(15)$ \\
$\mathrm{C}(22)-\mathrm{Sn}(1)-\mathrm{C}(1)-\mathrm{C}(2)$ & $-42.96(16)$ \\
$\mathrm{Cl}(1)-\mathrm{Sn}(1)-\mathrm{C}(1)-\mathrm{C}(2)$ & $-147.38(13)$ \\
$\mathrm{Sn}(1)-\mathrm{C}(1)-\mathrm{C}(2)-\mathrm{C}(3)$ & $-66.9(2)$ \\
$\mathrm{C}(4)-\mathrm{O}(1)-\mathrm{C}(3)-\mathrm{C}(2)$ & $-178.78(15)$ \\
$\mathrm{C}(1)-\mathrm{C}(2)-\mathrm{C}(3)-\mathrm{O}(1)$ & $57.4(2)$ \\
$\mathrm{C}(3)-\mathrm{O}(1)-\mathrm{C}(4)-\mathrm{C}(9)$ & $-2.3(2)$ \\
$\mathrm{C}(3)-\mathrm{O}(1)-\mathrm{C}(4)-\mathrm{C}(5)$ & $178.24(16)$ \\
$\mathrm{C}(9)-\mathrm{C}(4)-\mathrm{C}(5)-\mathrm{C}(6)$ & $1.2(3)$ \\
$\mathrm{O}(1)-\mathrm{C}(4)-\mathrm{C}(5)-\mathrm{C}(6)$ & $-179.29(16)$ \\
$\mathrm{C}(4)-\mathrm{C}(5)-\mathrm{C}(6)-\mathrm{C}(7)$ & $-1.5(3)$ \\
$\mathrm{C}(5)-\mathrm{C}(6)-\mathrm{C}(7)-\mathrm{C}(8)$ & $0.6(3)$ \\
$\mathrm{C}(5)-\mathrm{C}(6)-\mathrm{C}(7)-\mathrm{C}(10)$ & $-178.55(17)$ \\
$\mathrm{C}(6)-\mathrm{C}(7)-\mathrm{C}(8)-\mathrm{C}(9)$ & $0.6(3)$ \\
$\mathrm{C}(10)-\mathrm{C}(7)-\mathrm{C}(8)-\mathrm{C}(9)$ & $179.76(16)$ \\
$\mathrm{C}(5)-\mathrm{C}(4)-\mathrm{C}(9)-\mathrm{C}(8)$ & $-0.1(3)$ \\
$\mathrm{O}(1)-\mathrm{C}(4)-\mathrm{C}(9)-\mathrm{C}(8)$ & $-179.50(16)$ \\
$\mathrm{C}(7)-\mathrm{C}(8)-\mathrm{C}(9)-\mathrm{C}(4)$ & $-0.9(3)$ \\
$\mathrm{C}(8)-\mathrm{C}(7)-\mathrm{C}(10)-\mathrm{C}(11)$ & $-38.0(3)$ \\
$\mathrm{C}(6)-\mathrm{C}(7)-\mathrm{C}(10)-\mathrm{C}(11)$ & $141.14(19)$ \\
$\mathrm{C}(8)-\mathrm{C}(7)-\mathrm{C}(10)-\mathrm{C}(15)$ & $142.55(18)$ \\
$\mathrm{C}(6)-\mathrm{C}(7)-\mathrm{C}(10)-\mathrm{C}(15)$ & $-38.3(3)$ \\
$\mathrm{C}(15)-\mathrm{C}(10)-\mathrm{C}(11)-\mathrm{C}(12)$ & $0.7(3)$ \\
$\mathrm{C}(7)-\mathrm{C}(10)-\mathrm{C}(11)-\mathrm{C}(12)$ & $-178.74(17)$ \\
$\mathrm{C}(10)-\mathrm{C}(11)-\mathrm{C}(12)-\mathrm{C}(13)$ & $-0.8(3)$ \\
$\mathrm{C}(11)-\mathrm{C}(12)-\mathrm{C}(13)-\mathrm{C}(14)$ & $0.0(3)$ \\
$\mathrm{C}(12)-\mathrm{C}(13)-\mathrm{C}(14)-\mathrm{C}(15)$ & $0.8(3)$ \\
$\mathrm{C}(13)-\mathrm{C}(14)-\mathrm{C}(15)-\mathrm{C}(10)$ & $-0.8(3)$ \\
$\mathrm{C}(11)-\mathrm{C}(10)-\mathrm{C}(15)-\mathrm{C}(14)$ & $0.1(3)$ \\
$\mathrm{C}(7)-\mathrm{C}(10)-\mathrm{C}(15)-\mathrm{C}(14)$ & $179.55(18)$ \\
$\mathrm{C}(1)-\mathrm{Sn}(1)-\mathrm{C}(16)-\mathrm{C}(17)$ & $8.1(2)$ \\
$\mathrm{C}(22)-\mathrm{Sn}(1)-\mathrm{C}(16)-\mathrm{C}(17)$ & $156.88(16)$ \\
$\mathrm{Cl}(1)-\mathrm{Sn}(1)-\mathrm{C}(16)-\mathrm{C}(17)$ & $-100.28(17)$ \\
$\mathrm{C}(1)-\mathrm{Sn}(1)-\mathrm{C}(16)-\mathrm{C}(21)$ & $-172.22(15)$ \\
& \\
& \\
& \\
&
\end{tabular}




$\begin{array}{lc}\mathrm{C}(22)-\mathrm{Sn}(1)-\mathrm{C}(16)-\mathrm{C}(21) & -23.49(19) \\ \mathrm{Cl}(1)-\mathrm{Sn}(1)-\mathrm{C}(16)-\mathrm{C}(21) & 79.36(16) \\ \mathrm{C}(21)-\mathrm{C}(16)-\mathrm{C}(17)-\mathrm{C}(18) & -1.4(3) \\ \mathrm{Sn}(1)-\mathrm{C}(16)-\mathrm{C}(17)-\mathrm{C}(18) & 178.24(19) \\ \mathrm{C}(16)-\mathrm{C}(17)-\mathrm{C}(18)-\mathrm{C}(19) & 0.2(4) \\ \mathrm{C}(17)-\mathrm{C}(18)-\mathrm{C}(19)-\mathrm{C}(20) & 1.3(5) \\ \mathrm{C}(18)-\mathrm{C}(19)-\mathrm{C}(20)-\mathrm{C}(21) & -1.7(5) \\ \mathrm{C}(19)-\mathrm{C}(20)-\mathrm{C}(21)-\mathrm{C}(16) & 0.5(4) \\ \mathrm{C}(17)-\mathrm{C}(16)-\mathrm{C}(21)-\mathrm{C}(20) & 1.0(4) \\ \mathrm{Sn}(1)-\mathrm{C}(16)-\mathrm{C}(21)-\mathrm{C}(20) & -178.6(2) \\ \mathrm{C}(16)-\mathrm{Sn}(1)-\mathrm{C}(22)-\mathrm{C}(27) & -95.88(17) \\ \mathrm{C}(1)-\mathrm{Sn}(1)-\mathrm{C}(22)-\mathrm{C}(27) & 53.61(18) \\ \mathrm{Cl}(1)-\mathrm{Sn}(1)-\mathrm{C}(22)-\mathrm{C}(27) & 159.84(16) \\ \mathrm{C}(16)-\mathrm{Sn}(1)-\mathrm{C}(22)-\mathrm{C}(23) & 85.54(15) \\ \mathrm{C}(1)-\mathrm{Sn}(1)-\mathrm{C}(22)-\mathrm{C}(23) & -124.97(14) \\ \mathrm{Cl}(1)-\mathrm{Sn}(1)-\mathrm{C}(22)-\mathrm{C}(23) & -18.74(14) \\ \mathrm{C}(27)-\mathrm{C}(22)-\mathrm{C}(23)-\mathrm{C}(24) & -0.4(3) \\ \mathrm{Sn}(1)-\mathrm{C}(22)-\mathrm{C}(23)-\mathrm{C}(24) & 178.25(15) \\ \mathrm{C}(22)-\mathrm{C}(23)-\mathrm{C}(24)-\mathrm{C}(25) & -0.6(3) \\ \mathrm{C}(23)-\mathrm{C}(24)-\mathrm{C}(25)-\mathrm{C}(26) & 0.9(3) \\ \mathrm{C}(24)-\mathrm{C}(25)-\mathrm{C}(26)-\mathrm{C}(27) & -0.2(4) \\ \mathrm{C}(23)-\mathrm{C}(22)-\mathrm{C}(27)-\mathrm{C}(26) & 1.1(3) \\ \mathrm{Sn}(1)-\mathrm{C}(22)-\mathrm{C}(27)-\mathrm{C}(26) & -0.9(4) \\ \mathrm{C}(25)-\mathrm{C}(26)-\mathrm{C}(27)-\mathrm{C}(22) & \\ & \\ & \\ & \end{array}$

Symmetry transformations used to generate equivalent atoms: 
Table A 18. Crystal data and structure refinement for 202.

Identification code

Empirical formula

Formula weight

Temperature

Wavelength

Crystal system

Space group

Unit cell dimensions

Volume

$\mathrm{Z}$

Density (calculated)

Absorption coefficient

$\mathrm{F}(000)$

Crystal size

Theta range for data collection

Index ranges

Reflections collected

Independent reflections

Completeness to theta $=25.242^{\circ}$

Absorption correction

Max. and min. transmission

Refinement method

Data / restraints / parameters

Goodness-of-fit on $\mathrm{F}^{2}$

Final R indices [I $>2 \operatorname{sigma}(\mathrm{I})]$

$\mathrm{R}$ indices (all data)

Absolute structure parameter

Extinction coefficient

Largest diff. peak and hole d13230

$\mathrm{C}_{15} \mathrm{H}_{16} \mathrm{Cl}_{2} \mathrm{OSn}$

401.87

147(2) K

$0.71073 \AA$

Monoclinic

P 21

$\mathrm{a}=6.0761(10) \AA \quad \alpha=90^{\circ}$.

$\mathrm{b}=8.4920(14) \AA$

$\beta=100.891(3)^{\circ}$.

$\mathrm{c}=15.752(3) \AA$

798.1(2) $\AA^{3}$

2

$1.672 \mathrm{Mg} / \mathrm{m}^{3}$

$1.925 \mathrm{~mm}^{-1}$

396

$0.350 \times 0.230 \times 0.070 \mathrm{~mm}^{3}$

2.634 to $27.487^{\circ}$.

$-7<=\mathrm{h}<=7,-9<=\mathrm{k}<=11,-20<=\mathrm{l}<=20$

12905

$3126[\mathrm{R}(\mathrm{int})=0.0171]$

$99.9 \%$

Semi-empirical from equivalents

0.7456 and 0.6167

Full-matrix least-squares on $\mathrm{F}^{2}$

3126 / 1 / 172

1.042

$\mathrm{R} 1=0.0129, \mathrm{wR} 2=0.0331$

$\mathrm{R} 1=0.0132, \mathrm{wR} 2=0.0333$

$0.031(7)$

n/a

0.503 and -0.360 e. $\AA^{-3}$ 
Table A 19. Atomic coordinates $\left(\times 10^{4}\right)$ and equivalent isotropic displacement parameters $\left(\AA^{2} \times 10^{3}\right)$ for 202.

\begin{tabular}{lrrrr}
\hline & $\mathrm{x}$ & $\mathrm{y}$ & $\mathrm{z}$ & $\mathrm{U}(\mathrm{eq})$ \\
\hline $\mathrm{Sn}(1)$ & & & & \\
$\mathrm{Cl}(1)$ & $4179(1)$ & $2458(1)$ & $1450(1)$ & $18(1)$ \\
$\mathrm{Cl}(2)$ & $3012(1)$ & $130(1)$ & $638(1)$ & $28(1)$ \\
$\mathrm{O}(1)$ & $8006(1)$ & $2309(1)$ & $1357(1)$ & $33(1)$ \\
$\mathrm{C}(1)$ & $5332(3)$ & $5358(2)$ & $2137(1)$ & $27(1)$ \\
$\mathrm{C}(2)$ & $2457(4)$ & $4164(4)$ & $597(2)$ & $23(1)$ \\
$\mathrm{C}(3)$ & $2128(5)$ & $5729(4)$ & $1023(2)$ & $32(1)$ \\
$\mathrm{C}(4)$ & $4326(5)$ & $6443(4)$ & $1466(2)$ & $34(1)$ \\
$\mathrm{C}(5)$ & $7240(4)$ & $5878(3)$ & $2688(2)$ & $26(1)$ \\
$\mathrm{C}(6)$ & $8516(4)$ & $7139(4)$ & $2500(2)$ & $35(1)$ \\
$\mathrm{C}(7)$ & $10407(4)$ & $7589(6)$ & $3106(2)$ & $43(1)$ \\
$\mathrm{C}(8)$ & $10994(5)$ & $6791(5)$ & $3884(2)$ & $45(1)$ \\
$\mathrm{C}(9)$ & $9709(5)$ & $5531(4)$ & $4055(2)$ & $41(1)$ \\
$\mathrm{C}(10)$ & $7835(4)$ & $5055(4)$ & $3461(2)$ & $33(1)$ \\
$\mathrm{C}(11)$ & $4162(4)$ & $1706(3)$ & $2728(2)$ & $23(1)$ \\
$\mathrm{C}(12)$ & $5717(5)$ & $559(4)$ & $3075(2)$ & $30(1)$ \\
$\mathrm{C}(13)$ & $5770(5)$ & $-35(5)$ & $3898(2)$ & $38(1)$ \\
$\mathrm{C}(14)$ & $4284(5)$ & $552(4)$ & $4389(2)$ & $40(1)$ \\
$\mathrm{C}(15)$ & $2738(5)$ & $1700(4)$ & $4057(2)$ & $39(1)$ \\
\hline & $2663(4)$ & $2277(5)$ & $3224(2)$ & $29(1)$ \\
\hline
\end{tabular}


Table A 20. Bond lengths $[\AA \AA]$ and angles $\left[^{\circ}\right]$ for 202.

\begin{tabular}{|c|c|}
\hline $\operatorname{Sn}(1)-C(1)$ & $2.114(3)$ \\
\hline $\operatorname{Sn}(1)-C(10)$ & $2.114(2)$ \\
\hline $\mathrm{Sn}(1)-\mathrm{Cl}(2)$ & $2.3610(6)$ \\
\hline $\mathrm{Sn}(1)-\mathrm{Cl}(1)$ & $2.3887(8)$ \\
\hline $\mathrm{O}(1)-\mathrm{C}(4)$ & $1.383(3)$ \\
\hline $\mathrm{O}(1)-\mathrm{C}(3)$ & $1.448(3)$ \\
\hline $\mathrm{C}(1)-\mathrm{C}(2)$ & $1.519(4)$ \\
\hline $\mathrm{C}(1)-\mathrm{H}(1 \mathrm{~A})$ & 0.9900 \\
\hline $\mathrm{C}(1)-\mathrm{H}(1 \mathrm{~B})$ & 0.9900 \\
\hline$C(2)-C(3)$ & $1.512(4)$ \\
\hline $\mathrm{C}(2)-\mathrm{H}(2 \mathrm{~A})$ & 0.9900 \\
\hline $\mathrm{C}(2)-\mathrm{H}(2 \mathrm{~B})$ & 0.9900 \\
\hline $\mathrm{C}(3)-\mathrm{H}(3 \mathrm{~A})$ & 0.9900 \\
\hline $\mathrm{C}(3)-\mathrm{H}(3 \mathrm{~B})$ & 0.9900 \\
\hline$C(4)-C(5)$ & $1.386(4)$ \\
\hline$C(4)-C(9)$ & $1.392(4)$ \\
\hline$C(5)-C(6)$ & $1.401(4)$ \\
\hline $\mathrm{C}(5)-\mathrm{H}(5 \mathrm{~A})$ & 0.9500 \\
\hline$C(6)-C(7)$ & $1.387(6)$ \\
\hline $\mathrm{C}(6)-\mathrm{H}(6 \mathrm{~A})$ & 0.9500 \\
\hline$C(7)-C(8)$ & $1.380(5)$ \\
\hline $\mathrm{C}(7)-\mathrm{H}(7 \mathrm{~A})$ & 0.9500 \\
\hline $\mathrm{C}(8)-\mathrm{C}(9)$ & $1.390(4)$ \\
\hline $\mathrm{C}(8)-\mathrm{H}(8 \mathrm{~A})$ & 0.9500 \\
\hline $\mathrm{C}(9)-\mathrm{H}(9 \mathrm{~A})$ & 0.9500 \\
\hline$C(10)-C(15)$ & $1.393(4)$ \\
\hline $\mathrm{C}(10)-\mathrm{C}(11)$ & $1.394(4)$ \\
\hline $\mathrm{C}(11)-\mathrm{C}(12)$ & $1.385(4)$ \\
\hline $\mathrm{C}(11)-\mathrm{H}(11 \mathrm{~A})$ & 0.9500 \\
\hline $\mathrm{C}(12)-\mathrm{C}(13)$ & $1.389(5)$ \\
\hline $\mathrm{C}(12)-\mathrm{H}(12 \mathrm{~A})$ & 0.9500 \\
\hline $\mathrm{C}(13)-\mathrm{C}(14)$ & $1.386(5)$ \\
\hline $\mathrm{C}(13)-\mathrm{H}(13 \mathrm{~A})$ & 0.9500 \\
\hline$C(14)-C(15)$ & $1.394(4)$ \\
\hline
\end{tabular}




\begin{tabular}{|c|c|}
\hline $\mathrm{C}(14)-\mathrm{H}(14 \mathrm{~A})$ & 0.9500 \\
\hline $\mathrm{C}(15)-\mathrm{H}(15 \mathrm{~A})$ & 0.9500 \\
\hline$C(1)-\operatorname{Sn}(1)-C(10)$ & $136.18(10)$ \\
\hline $\mathrm{C}(1)-\mathrm{Sn}(1)-\mathrm{Cl}(2)$ & $111.85(8)$ \\
\hline $\mathrm{C}(10)-\mathrm{Sn}(1)-\mathrm{Cl}(2)$ & $103.02(7)$ \\
\hline $\mathrm{C}(1)-\mathrm{Sn}(1)-\mathrm{Cl}(1)$ & 99.53(9) \\
\hline $\mathrm{C}(10)-\mathrm{Sn}(1)-\mathrm{Cl}(1)$ & $101.87(8)$ \\
\hline $\mathrm{Cl}(2)-\mathrm{Sn}(1)-\mathrm{Cl}(1)$ & $96.80(3)$ \\
\hline $\mathrm{C}(4)-\mathrm{O}(1)-\mathrm{C}(3)$ & $116.3(2)$ \\
\hline$C(2)-C(1)-\operatorname{Sn}(1)$ & $114.17(18)$ \\
\hline $\mathrm{C}(2)-\mathrm{C}(1)-\mathrm{H}(1 \mathrm{~A})$ & 108.7 \\
\hline $\mathrm{Sn}(1)-\mathrm{C}(1)-\mathrm{H}(1 \mathrm{~A})$ & 108.7 \\
\hline $\mathrm{C}(2)-\mathrm{C}(1)-\mathrm{H}(1 \mathrm{~B})$ & 108.7 \\
\hline $\operatorname{Sn}(1)-C(1)-H(1 B)$ & 108.7 \\
\hline $\mathrm{H}(1 \mathrm{~A})-\mathrm{C}(1)-\mathrm{H}(1 \mathrm{~B})$ & 107.6 \\
\hline $\mathrm{C}(3)-\mathrm{C}(2)-\mathrm{C}(1)$ & $112.1(2)$ \\
\hline $\mathrm{C}(3)-\mathrm{C}(2)-\mathrm{H}(2 \mathrm{~A})$ & 109.2 \\
\hline $\mathrm{C}(1)-\mathrm{C}(2)-\mathrm{H}(2 \mathrm{~A})$ & 109.2 \\
\hline $\mathrm{C}(3)-\mathrm{C}(2)-\mathrm{H}(2 \mathrm{~B})$ & 109.2 \\
\hline $\mathrm{C}(1)-\mathrm{C}(2)-\mathrm{H}(2 \mathrm{~B})$ & 109.2 \\
\hline $\mathrm{H}(2 \mathrm{~A})-\mathrm{C}(2)-\mathrm{H}(2 \mathrm{~B})$ & 107.9 \\
\hline $\mathrm{O}(1)-\mathrm{C}(3)-\mathrm{C}(2)$ & $107.2(3)$ \\
\hline $\mathrm{O}(1)-\mathrm{C}(3)-\mathrm{H}(3 \mathrm{~A})$ & 110.3 \\
\hline $\mathrm{C}(2)-\mathrm{C}(3)-\mathrm{H}(3 \mathrm{~A})$ & 110.3 \\
\hline $\mathrm{O}(1)-\mathrm{C}(3)-\mathrm{H}(3 \mathrm{~B})$ & 110.3 \\
\hline $\mathrm{C}(2)-\mathrm{C}(3)-\mathrm{H}(3 \mathrm{~B})$ & 110.3 \\
\hline $\mathrm{H}(3 \mathrm{~A})-\mathrm{C}(3)-\mathrm{H}(3 \mathrm{~B})$ & 108.5 \\
\hline $\mathrm{O}(1)-\mathrm{C}(4)-\mathrm{C}(5)$ & $123.3(2)$ \\
\hline $\mathrm{O}(1)-\mathrm{C}(4)-\mathrm{C}(9)$ & $115.9(3)$ \\
\hline $\mathrm{C}(5)-\mathrm{C}(4)-\mathrm{C}(9)$ & $120.8(3)$ \\
\hline$C(4)-C(5)-C(6)$ & $119.0(3)$ \\
\hline $\mathrm{C}(4)-\mathrm{C}(5)-\mathrm{H}(5 \mathrm{~A})$ & 120.5 \\
\hline $\mathrm{C}(6)-\mathrm{C}(5)-\mathrm{H}(5 \mathrm{~A})$ & 120.5 \\
\hline$C(7)-C(6)-C(5)$ & $120.6(4)$ \\
\hline $\mathrm{C}(7)-\mathrm{C}(6)-\mathrm{H}(6 \mathrm{~A})$ & 119.7 \\
\hline
\end{tabular}




\begin{tabular}{|c|c|}
\hline $\mathrm{C}(5)-\mathrm{C}(6)-\mathrm{H}(6 \mathrm{~A})$ & 119.7 \\
\hline$C(8)-C(7)-C(6)$ & $119.4(3)$ \\
\hline $\mathrm{C}(8)-\mathrm{C}(7)-\mathrm{H}(7 \mathrm{~A})$ & 120.3 \\
\hline $\mathrm{C}(6)-\mathrm{C}(7)-\mathrm{H}(7 \mathrm{~A})$ & 120.3 \\
\hline $\mathrm{C}(7)-\mathrm{C}(8)-\mathrm{C}(9)$ & 121.1(3) \\
\hline $\mathrm{C}(7)-\mathrm{C}(8)-\mathrm{H}(8 \mathrm{~A})$ & 119.5 \\
\hline $\mathrm{C}(9)-\mathrm{C}(8)-\mathrm{H}(8 \mathrm{~A})$ & 119.5 \\
\hline $\mathrm{C}(8)-\mathrm{C}(9)-\mathrm{C}(4)$ & 119.2(3) \\
\hline $\mathrm{C}(8)-\mathrm{C}(9)-\mathrm{H}(9 \mathrm{~A})$ & 120.4 \\
\hline $\mathrm{C}(4)-\mathrm{C}(9)-\mathrm{H}(9 \mathrm{~A})$ & 120.4 \\
\hline$C(15)-C(10)-C(11)$ & $119.4(3)$ \\
\hline$C(15)-C(10)-\operatorname{Sn}(1)$ & $123.3(2)$ \\
\hline$C(11)-C(10)-\operatorname{Sn}(1)$ & 117.30(19) \\
\hline$C(12)-C(11)-C(10)$ & $121.0(3)$ \\
\hline $\mathrm{C}(12)-\mathrm{C}(11)-\mathrm{H}(11 \mathrm{~A})$ & 119.5 \\
\hline $\mathrm{C}(10)-\mathrm{C}(11)-\mathrm{H}(11 \mathrm{~A})$ & 119.5 \\
\hline $\mathrm{C}(11)-\mathrm{C}(12)-\mathrm{C}(13)$ & 119.2(3) \\
\hline $\mathrm{C}(11)-\mathrm{C}(12)-\mathrm{H}(12 \mathrm{~A})$ & 120.4 \\
\hline $\mathrm{C}(13)-\mathrm{C}(12)-\mathrm{H}(12 \mathrm{~A})$ & 120.4 \\
\hline $\mathrm{C}(14)-\mathrm{C}(13)-\mathrm{C}(12)$ & $120.5(3)$ \\
\hline $\mathrm{C}(14)-\mathrm{C}(13)-\mathrm{H}(13 \mathrm{~A})$ & 119.7 \\
\hline $\mathrm{C}(12)-\mathrm{C}(13)-\mathrm{H}(13 \mathrm{~A})$ & 119.7 \\
\hline $\mathrm{C}(13)-\mathrm{C}(14)-\mathrm{C}(15)$ & $120.1(3)$ \\
\hline $\mathrm{C}(13)-\mathrm{C}(14)-\mathrm{H}(14 \mathrm{~A})$ & 119.9 \\
\hline $\mathrm{C}(15)-\mathrm{C}(14)-\mathrm{H}(14 \mathrm{~A})$ & 119.9 \\
\hline $\mathrm{C}(10)-\mathrm{C}(15)-\mathrm{C}(14)$ & $119.8(3)$ \\
\hline $\mathrm{C}(10)-\mathrm{C}(15)-\mathrm{H}(15 \mathrm{~A})$ & 120.1 \\
\hline $\mathrm{C}(14)-\mathrm{C}(15)-\mathrm{H}(15 \mathrm{~A})$ & 120.1 \\
\hline
\end{tabular}

Symmetry transformations used to generate equivalent atoms: 
Table A 21. Anisotropic displacement parameters $\left(\AA^{2} \times 10^{3}\right)$ for 202. The anisotropic displacement factor exponent takes the form: $-2 \pi^{2}\left[h^{2} a^{* 2} U^{11}+\ldots+2 h a^{*} b^{*} U^{12}\right]$

\begin{tabular}{lllllll}
\hline & $\mathrm{U} 11$ & $\mathrm{U}^{22}$ & $\mathrm{U}^{33}$ & $\mathrm{U} 23$ & $\mathrm{U} 13$ & $\mathrm{U} 12$ \\
\hline $\mathrm{Sn}(1)$ & $17(1)$ & $20(1)$ & $18(1)$ & $1(1)$ & $3(1)$ & $2(1)$ \\
$\mathrm{Cl}(1)$ & $30(1)$ & $22(1)$ & $31(1)$ & $-6(1)$ & $1(1)$ & $1(1)$ \\
$\mathrm{Cl}(2)$ & $17(1)$ & $46(1)$ & $36(1)$ & $-1(1)$ & $7(1)$ & $1(1)$ \\
$\mathrm{O}(1)$ & $31(1)$ & $22(1)$ & $25(1)$ & $1(1)$ & $-1(1)$ & $2(1)$ \\
$\mathrm{C}(1)$ & $25(1)$ & $21(2)$ & $21(1)$ & $4(1)$ & $1(1)$ & $3(1)$ \\
$\mathrm{C}(2)$ & $32(1)$ & $25(2)$ & $34(2)$ & $2(1)$ & $-3(1)$ & $7(1)$ \\
$\mathrm{C}(3)$ & $42(2)$ & $20(2)$ & $36(2)$ & $3(1)$ & $-4(1)$ & $4(1)$ \\
$\mathrm{C}(4)$ & $25(1)$ & $23(1)$ & $29(1)$ & $-8(1)$ & $3(1)$ & $6(1)$ \\
$\mathrm{C}(5)$ & $34(1)$ & $34(3)$ & $37(1)$ & $-5(1)$ & $6(1)$ & $-1(1)$ \\
$\mathrm{C}(6)$ & $33(1)$ & $35(2)$ & $60(2)$ & $-11(2)$ & $7(1)$ & $-3(2)$ \\
$\mathrm{C}(7)$ & $34(2)$ & $44(2)$ & $53(2)$ & $-17(2)$ & $-8(1)$ & $4(1)$ \\
$\mathrm{C}(8)$ & $44(2)$ & $38(2)$ & $35(2)$ & $-6(1)$ & $-7(1)$ & $11(1)$ \\
$\mathrm{C}(9)$ & $35(1)$ & $30(2)$ & $31(1)$ & $-5(1)$ & $1(1)$ & $3(1)$ \\
$\mathrm{C}(10)$ & $25(1)$ & $23(1)$ & $19(1)$ & $0(1)$ & $1(1)$ & $-3(1)$ \\
$\mathrm{C}(11)$ & $30(1)$ & $31(2)$ & $27(1)$ & $3(1)$ & $2(1)$ & $4(1)$ \\
$\mathrm{C}(12)$ & $40(1)$ & $38(2)$ & $32(1)$ & $11(2)$ & $-5(1)$ & $1(1)$ \\
$\mathrm{C}(13)$ & $51(2)$ & $46(2)$ & $20(1)$ & $8(1)$ & $2(1)$ & $-12(1)$ \\
$\mathrm{C}(14)$ & $47(2)$ & $44(2)$ & $28(1)$ & $-3(1)$ & $15(1)$ & $-5(1)$ \\
$\mathrm{C}(15)$ & $32(1)$ & $31(2)$ & $27(1)$ & $2(1)$ & $9(1)$ & $4(1)$ \\
\hline
\end{tabular}


Table A 22. Hydrogen coordinates $\left(\times 10^{4}\right)$ and isotropic displacement parameters $\left(\AA^{2} \times\right.$ $10^{3}$ ) for 202.

\begin{tabular}{lcccc}
\hline & $\mathrm{x}$ & $\mathrm{y}$ & $\mathrm{z}$ & $\mathrm{U}(\mathrm{eq})$ \\
\hline $\mathrm{H}(1 \mathrm{~A})$ & 3301 & 4350 & 127 & 27 \\
$\mathrm{H}(1 \mathrm{~B})$ & 969 & 3738 & 331 & 27 \\
$\mathrm{H}(2 \mathrm{~A})$ & 1359 & 6471 & 579 & 38 \\
$\mathrm{H}(2 \mathrm{~B})$ & 1155 & 5570 & 1453 & 38 \\
$\mathrm{H}(3 \mathrm{~A})$ & 5328 & 6601 & 1045 & 41 \\
$\mathrm{H}(3 \mathrm{~B})$ & 4064 & 7477 & 1720 & 41 \\
$\mathrm{H}(5 \mathrm{~A})$ & 8114 & 7689 & 1968 & 42 \\
$\mathrm{H}(6 \mathrm{~A})$ & 11295 & 8449 & 2983 & 51 \\
$\mathrm{H}(7 \mathrm{~A})$ & 12268 & 7108 & 4296 & 55 \\
$\mathrm{H}(8 \mathrm{~A})$ & 10111 & 4981 & 4587 & 49 \\
$\mathrm{H}(9 \mathrm{~A})$ & 6972 & 4179 & 3582 & 39 \\
$\mathrm{H}(11 \mathrm{~A})$ & 6756 & 180 & 2742 & 36 \\
$\mathrm{H}(12 \mathrm{~A})$ & 6811 & -835 & 4123 & 46 \\
$\mathrm{H}(13 \mathrm{~A})$ & 4328 & 164 & 4958 & 47 \\
$\mathrm{H}(14 \mathrm{~A})$ & 1727 & 2094 & 4398 & 46 \\
$\mathrm{H}(15 \mathrm{~A})$ & 1594 & 3057 & 2994 & 35 \\
\hline & & & & \\
\hline
\end{tabular}


Table A 23. Torsion angles $\left[{ }^{\circ}\right]$ for 202.

\begin{tabular}{lc}
\hline $\mathrm{Sn}(1)-\mathrm{C}(1)-\mathrm{C}(2)-\mathrm{C}(3)$ & $57.0(3)$ \\
$\mathrm{C}(4)-\mathrm{O}(1)-\mathrm{C}(3)-\mathrm{C}(2)$ & $-172.7(2)$ \\
$\mathrm{C}(1)-\mathrm{C}(2)-\mathrm{C}(3)-\mathrm{O}(1)$ & $-61.8(3)$ \\
$\mathrm{C}(3)-\mathrm{O}(1)-\mathrm{C}(4)-\mathrm{C}(5)$ & $-17.4(4)$ \\
$\mathrm{C}(3)-\mathrm{O}(1)-\mathrm{C}(4)-\mathrm{C}(9)$ & $162.5(2)$ \\
$\mathrm{O}(1)-\mathrm{C}(4)-\mathrm{C}(5)-\mathrm{C}(6)$ & $179.2(3)$ \\
$\mathrm{C}(9)-\mathrm{C}(4)-\mathrm{C}(5)-\mathrm{C}(6)$ & $-0.8(4)$ \\
$\mathrm{C}(4)-\mathrm{C}(5)-\mathrm{C}(6)-\mathrm{C}(7)$ & $-0.2(5)$ \\
$\mathrm{C}(5)-\mathrm{C}(6)-\mathrm{C}(7)-\mathrm{C}(8)$ & $0.7(6)$ \\
$\mathrm{C}(6)-\mathrm{C}(7)-\mathrm{C}(8)-\mathrm{C}(9)$ & $-0.2(5)$ \\
$\mathrm{C}(7)-\mathrm{C}(8)-\mathrm{C}(9)-\mathrm{C}(4)$ & $-0.8(5)$ \\
$\mathrm{O}(1)-\mathrm{C}(4)-\mathrm{C}(9)-\mathrm{C}(8)$ & $-178.7(3)$ \\
$\mathrm{C}(5)-\mathrm{C}(4)-\mathrm{C}(9)-\mathrm{C}(8)$ & $1.3(4)$ \\
$\mathrm{C}(15)-\mathrm{C}(10)-\mathrm{C}(11)-\mathrm{C}(12)$ & $1.0(5)$ \\
$\mathrm{Sn}(1)-\mathrm{C}(10)-\mathrm{C}(11)-\mathrm{C}(12)$ & $-177.9(3)$ \\
$\mathrm{C}(10)-\mathrm{C}(11)-\mathrm{C}(12)-\mathrm{C}(13)$ & $-1.6(5)$ \\
$\mathrm{C}(11)-\mathrm{C}(12)-\mathrm{C}(13)-\mathrm{C}(14)$ & $1.1(5)$ \\
$\mathrm{C}(12)-\mathrm{C}(13)-\mathrm{C}(14)-\mathrm{C}(15)$ & $-0.1(5)$ \\
$\mathrm{C}(11)-\mathrm{C}(10)-\mathrm{C}(15)-\mathrm{C}(14)$ & $0.1(5)$ \\
$\mathrm{Sn}(1)-\mathrm{C}(10)-\mathrm{C}(15)-\mathrm{C}(14)$ & $178.9(2)$ \\
$\mathrm{C}(13)-\mathrm{C}(14)-\mathrm{C}(15)-\mathrm{C}(10)$ & $-0.5(5)$ \\
& \\
\hline
\end{tabular}

Symmetry transformations used to generate equivalent atoms: 
Table A 24. Crystal data and structure refinement for 203.

Identification code

Empirical formula

Formula weight

Temperature

Wavelength

Crystal system

Space group

Unit cell dimensions

Volume

$\mathrm{Z}$

Density (calculated)

Absorption coefficient

$\mathrm{F}(000)$

Crystal size

Theta range for data collection

Index ranges

Reflections collected

Independent reflections

Completeness to theta $=25.242^{\circ}$

Absorption correction

Max. and min. transmission

Refinement method

Data / restraints / parameters

Goodness-of-fit on $\mathrm{F}^{2}$

Final R indices [I $>2 \operatorname{sigma}(\mathrm{I})]$

$\mathrm{R}$ indices (all data)

Extinction coefficient

Largest diff. peak and hole d1409

$\mathrm{C}_{21} \mathrm{H}_{20} \mathrm{C}_{12} \mathrm{OSn}$

477.96

147(2) K

$0.71073 \AA$

Monoclinic

P 21/c

$\mathrm{a}=14.2473(9) \AA \quad \alpha=90^{\circ}$.

$\mathrm{b}=6.3874(4) \AA$

$\beta=93.158(2)^{\circ}$.

$\mathrm{c}=22.0483(13) \AA$

$\gamma=90^{\circ}$.
4

$1.585 \mathrm{Mg} / \mathrm{m}^{3}$

$1.548 \mathrm{~mm}^{-1}$

952

$0.350 \times 0.120 \times 0.120 \mathrm{~mm}^{3}$

1.431 to $27.536^{\circ}$.

$-18<=\mathrm{h}<=18,-8<=\mathrm{k}<=8,-28<=\mathrm{l}<=28$

32922

$4602[\mathrm{R}(\mathrm{int})=0.0374]$

$99.9 \%$

Semi-empirical from equivalents

0.7456 and 0.6530

Full-matrix least-squares on $\mathrm{F}^{2}$

4602 / 0 / 226

0.985

$\mathrm{R} 1=0.0223, \mathrm{wR} 2=0.0474$

$\mathrm{R} 1=0.0311, \mathrm{wR} 2=0.0506$

$\mathrm{n} / \mathrm{a}$

0.784 and -0.476 e. $\AA^{-3}$ 
Table A 25. Atomic coordinates $\left(\times 10^{4}\right)$ and equivalent isotropic displacement parameters $\left(\AA^{2} \times 10^{3}\right)$ for 203.

\begin{tabular}{|c|c|c|c|c|}
\hline & $\mathrm{x}$ & $\mathrm{y}$ & $\mathrm{z}$ & $\mathrm{U}(\mathrm{eq})$ \\
\hline $\operatorname{Sn}(1)$ & $4277(1)$ & $11560(1)$ & $907(1)$ & $23(1)$ \\
\hline $\mathrm{Cl}(1)$ & 5931(1) & $12098(1)$ & $870(1)$ & $30(1)$ \\
\hline $\mathrm{Cl}(2)$ & 4299(1) & 7894(1) & $828(1)$ & $31(1)$ \\
\hline $\mathrm{O}(1)$ & $2327(1)$ & $10875(2)$ & $697(1)$ & $32(1)$ \\
\hline $\mathrm{C}(1)$ & $3752(2)$ & 13117(3) & 104(1) & $30(1)$ \\
\hline$C(2)$ & $2742(2)$ & $13849(4)$ & $128(1)$ & $36(1)$ \\
\hline$C(3)$ & 2063(2) & $12066(4)$ & $160(1)$ & $38(1)$ \\
\hline $\mathrm{C}(4)$ & $1767(2)$ & $9208(4)$ & $832(1)$ & $30(1)$ \\
\hline$C(5)$ & $1965(2)$ & $8229(3)$ & 1388(1) & $30(1)$ \\
\hline$C(6)$ & $1426(2)$ & $6557(4)$ & $1565(1)$ & $32(1)$ \\
\hline$C(7)$ & $671(2)$ & $5808(4)$ & 1195(1) & $34(1)$ \\
\hline$C(8)$ & 493(2) & $6808(4)$ & $640(1)$ & $41(1)$ \\
\hline$C(9)$ & $1028(2)$ & $8482(4)$ & $450(1)$ & $38(1)$ \\
\hline$C(10)$ & $89(2)$ & $4026(4)$ & 1389(1) & $40(1)$ \\
\hline$C(11)$ & $479(2)$ & 2384(4) & 1728(1) & $46(1)$ \\
\hline$C(12)$ & $-65(2)$ & $718(5)$ & $1912(2)$ & $57(1)$ \\
\hline$C(13)$ & $-1016(2)$ & 673(6) & 1743(2) & $66(1)$ \\
\hline$C(14)$ & $-1413(2)$ & $2282(7)$ & $1412(2)$ & $74(1)$ \\
\hline$C(15)$ & $-876(2)$ & $3970(6)$ & 1237(1) & $60(1)$ \\
\hline$C(16)$ & 3964(1) & $12137(3)$ & $1820(1)$ & $22(1)$ \\
\hline$C(17)$ & $3602(2)$ & $14058(4)$ & 1993(1) & $28(1)$ \\
\hline$C(18)$ & $3393(2)$ & $14410(4)$ & 2591(1) & $34(1)$ \\
\hline$C(19)$ & $3530(2)$ & $12836(4)$ & $3016(1)$ & $35(1)$ \\
\hline$C(20)$ & $3889(2)$ & $10922(4)$ & $2850(1)$ & $35(1)$ \\
\hline $\mathrm{C}(21)$ & $4113(2)$ & $10564(4)$ & $2253(1)$ & $28(1)$ \\
\hline
\end{tabular}


Table A 26. Bond lengths $[\AA \AA]$ and angles $\left[^{\circ}\right]$ for 203.

\begin{tabular}{|c|c|}
\hline $\operatorname{Sn}(1)-C(16)$ & 2.1184(19) \\
\hline $\operatorname{Sn}(1)-C(1)$ & $2.1289(19)$ \\
\hline $\mathrm{Sn}(1)-\mathrm{Cl}(2)$ & $2.3483(6)$ \\
\hline $\operatorname{Sn}(1)-C l(1)$ & $2.3868(6)$ \\
\hline $\mathrm{O}(1)-\mathrm{C}(4)$ & $1.373(3)$ \\
\hline $\mathrm{O}(1)-\mathrm{C}(3)$ & $1.441(3)$ \\
\hline $\mathrm{C}(1)-\mathrm{C}(2)$ & $1.517(3)$ \\
\hline $\mathrm{C}(1)-\mathrm{H}(1 \mathrm{~A})$ & 0.9900 \\
\hline $\mathrm{C}(1)-\mathrm{H}(1 \mathrm{~B})$ & 0.9900 \\
\hline$C(2)-C(3)$ & $1.498(3)$ \\
\hline $\mathrm{C}(2)-\mathrm{H}(2 \mathrm{~A})$ & 0.9900 \\
\hline $\mathrm{C}(2)-\mathrm{H}(2 \mathrm{~B})$ & 0.9900 \\
\hline $\mathrm{C}(3)-\mathrm{H}(3 \mathrm{~A})$ & 0.9900 \\
\hline $\mathrm{C}(3)-\mathrm{H}(3 \mathrm{~B})$ & 0.9900 \\
\hline C(4)-C(9) & $1.390(3)$ \\
\hline$C(4)-C(5)$ & 1.391(3) \\
\hline$C(5)-C(6)$ & $1.383(3)$ \\
\hline $\mathrm{C}(5)-\mathrm{H}(5 \mathrm{~A})$ & 0.9500 \\
\hline$C(6)-C(7)$ & $1.399(3)$ \\
\hline $\mathrm{C}(6)-\mathrm{H}(6 \mathrm{~A})$ & 0.9500 \\
\hline $\mathrm{C}(7)-\mathrm{C}(8)$ & $1.390(4)$ \\
\hline C(7)-C(10) & $1.485(4)$ \\
\hline $\mathrm{C}(8)-\mathrm{C}(9)$ & $1.391(4)$ \\
\hline C(8)-H(8A) & 0.9500 \\
\hline $\mathrm{C}(9)-\mathrm{H}(9 \mathrm{~A})$ & 0.9500 \\
\hline$C(10)-C(11)$ & $1.387(4)$ \\
\hline$C(10)-C(15)$ & $1.398(3)$ \\
\hline $\mathrm{C}(11)-\mathrm{C}(12)$ & $1.390(4)$ \\
\hline $\mathrm{C}(11)-\mathrm{H}(11 \mathrm{~A})$ & 0.9500 \\
\hline $\mathrm{C}(12)-\mathrm{C}(13)$ & $1.385(4)$ \\
\hline $\mathrm{C}(12)-\mathrm{H}(12 \mathrm{~A})$ & 0.9500 \\
\hline C(13)-C(14) & $1.366(5)$ \\
\hline $\mathrm{C}(13)-\mathrm{H}(13 \mathrm{~A})$ & 0.9500 \\
\hline$C(14)-C(15)$ & $1.388(5)$ \\
\hline
\end{tabular}




\begin{tabular}{|c|c|}
\hline $\mathrm{C}(14)-\mathrm{H}(14 \mathrm{~A})$ & 0.9500 \\
\hline C(15)-H(15A) & 0.9500 \\
\hline$C(16)-C(17)$ & $1.392(3)$ \\
\hline$C(16)-C(21)$ & $1.395(3)$ \\
\hline $\mathrm{C}(17)-\mathrm{C}(18)$ & $1.386(3)$ \\
\hline C(17)-H(17A) & 0.9500 \\
\hline $\mathrm{C}(18)-\mathrm{C}(19)$ & $1.379(3)$ \\
\hline $\mathrm{C}(18)-\mathrm{H}(18 \mathrm{~A})$ & 0.9500 \\
\hline$C(19)-C(20)$ & $1.382(3)$ \\
\hline $\mathrm{C}(19)-\mathrm{H}(19 \mathrm{~A})$ & 0.9500 \\
\hline $\mathrm{C}(20)-\mathrm{C}(21)$ & $1.391(3)$ \\
\hline $\mathrm{C}(20)-\mathrm{H}(20 \mathrm{~A})$ & 0.9500 \\
\hline $\mathrm{C}(21)-\mathrm{H}(21 \mathrm{~A})$ & 0.9500 \\
\hline$C(16)-S n(1)-C(1)$ & $128.74(8)$ \\
\hline $\mathrm{C}(16)-\mathrm{Sn}(1)-\mathrm{Cl}(2)$ & $104.36(6)$ \\
\hline $\mathrm{C}(1)-\mathrm{Sn}(1)-\mathrm{Cl}(2)$ & $114.21(6)$ \\
\hline $\mathrm{C}(16)-\mathrm{Sn}(1)-\mathrm{Cl}(1)$ & $105.49(5)$ \\
\hline $\mathrm{C}(1)-\mathrm{Sn}(1)-\mathrm{Cl}(1)$ & $101.94(6)$ \\
\hline $\mathrm{Cl}(2)-\mathrm{Sn}(1)-\mathrm{Cl}(1)$ & $97.10(2)$ \\
\hline $\mathrm{C}(4)-\mathrm{O}(1)-\mathrm{C}(3)$ & $117.35(17)$ \\
\hline $\mathrm{C}(2)-\mathrm{C}(1)-\mathrm{Sn}(1)$ & $113.95(14)$ \\
\hline $\mathrm{C}(2)-\mathrm{C}(1)-\mathrm{H}(1 \mathrm{~A})$ & 108.8 \\
\hline $\mathrm{Sn}(1)-\mathrm{C}(1)-\mathrm{H}(1 \mathrm{~A})$ & 108.8 \\
\hline $\mathrm{C}(2)-\mathrm{C}(1)-\mathrm{H}(1 \mathrm{~B})$ & 108.8 \\
\hline $\operatorname{Sn}(1)-\mathrm{C}(1)-\mathrm{H}(1 \mathrm{~B})$ & 108.8 \\
\hline $\mathrm{H}(1 \mathrm{~A})-\mathrm{C}(1)-\mathrm{H}(1 \mathrm{~B})$ & 107.7 \\
\hline$C(3)-C(2)-C(1)$ & $112.5(2)$ \\
\hline $\mathrm{C}(3)-\mathrm{C}(2)-\mathrm{H}(2 \mathrm{~A})$ & 109.1 \\
\hline $\mathrm{C}(1)-\mathrm{C}(2)-\mathrm{H}(2 \mathrm{~A})$ & 109.1 \\
\hline $\mathrm{C}(3)-\mathrm{C}(2)-\mathrm{H}(2 \mathrm{~B})$ & 109.1 \\
\hline $\mathrm{C}(1)-\mathrm{C}(2)-\mathrm{H}(2 \mathrm{~B})$ & 109.1 \\
\hline $\mathrm{H}(2 \mathrm{~A})-\mathrm{C}(2)-\mathrm{H}(2 \mathrm{~B})$ & 107.8 \\
\hline $\mathrm{O}(1)-\mathrm{C}(3)-\mathrm{C}(2)$ & $107.46(18)$ \\
\hline $\mathrm{O}(1)-\mathrm{C}(3)-\mathrm{H}(3 \mathrm{~A})$ & 110.2 \\
\hline $\mathrm{C}(2)-\mathrm{C}(3)-\mathrm{H}(3 \mathrm{~A})$ & 110.2 \\
\hline
\end{tabular}




\begin{tabular}{|c|c|}
\hline $\mathrm{O}(1)-\mathrm{C}(3)-\mathrm{H}(3 \mathrm{~B})$ & 110.2 \\
\hline $\mathrm{C}(2)-\mathrm{C}(3)-\mathrm{H}(3 \mathrm{~B})$ & 110.2 \\
\hline $\mathrm{H}(3 \mathrm{~A})-\mathrm{C}(3)-\mathrm{H}(3 \mathrm{~B})$ & 108.5 \\
\hline $\mathrm{O}(1)-\mathrm{C}(4)-\mathrm{C}(9)$ & $123.9(2)$ \\
\hline $\mathrm{O}(1)-\mathrm{C}(4)-\mathrm{C}(5)$ & $116.57(18)$ \\
\hline $\mathrm{C}(9)-\mathrm{C}(4)-\mathrm{C}(5)$ & $119.5(2)$ \\
\hline$C(6)-C(5)-C(4)$ & $120.5(2)$ \\
\hline $\mathrm{C}(6)-\mathrm{C}(5)-\mathrm{H}(5 \mathrm{~A})$ & 119.8 \\
\hline $\mathrm{C}(4)-\mathrm{C}(5)-\mathrm{H}(5 \mathrm{~A})$ & 119.8 \\
\hline$C(5)-C(6)-C(7)$ & $121.4(2)$ \\
\hline $\mathrm{C}(5)-\mathrm{C}(6)-\mathrm{H}(6 \mathrm{~A})$ & 119.3 \\
\hline$C(7)-C(6)-H(6 A)$ & 119.3 \\
\hline$C(8)-C(7)-C(6)$ & $116.9(2)$ \\
\hline$C(8)-C(7)-C(10)$ & $121.9(2)$ \\
\hline$C(6)-C(7)-C(10)$ & $121.2(2)$ \\
\hline $\mathrm{C}(7)-\mathrm{C}(8)-\mathrm{C}(9)$ & $122.8(2)$ \\
\hline $\mathrm{C}(7)-\mathrm{C}(8)-\mathrm{H}(8 \mathrm{~A})$ & 118.6 \\
\hline $\mathrm{C}(9)-\mathrm{C}(8)-\mathrm{H}(8 \mathrm{~A})$ & 118.6 \\
\hline$C(4)-C(9)-C(8)$ & $118.9(2)$ \\
\hline $\mathrm{C}(4)-\mathrm{C}(9)-\mathrm{H}(9 \mathrm{~A})$ & 120.6 \\
\hline $\mathrm{C}(8)-\mathrm{C}(9)-\mathrm{H}(9 \mathrm{~A})$ & 120.6 \\
\hline $\mathrm{C}(11)-\mathrm{C}(10)-\mathrm{C}(15)$ & $118.0(3)$ \\
\hline$C(11)-C(10)-C(7)$ & $121.5(2)$ \\
\hline$C(15)-C(10)-C(7)$ & $120.5(3)$ \\
\hline $\mathrm{C}(10)-\mathrm{C}(11)-\mathrm{C}(12)$ & $121.5(3)$ \\
\hline $\mathrm{C}(10)-\mathrm{C}(11)-\mathrm{H}(11 \mathrm{~A})$ & 119.2 \\
\hline $\mathrm{C}(12)-\mathrm{C}(11)-\mathrm{H}(11 \mathrm{~A})$ & 119.2 \\
\hline $\mathrm{C}(13)-\mathrm{C}(12)-\mathrm{C}(11)$ & $119.4(3)$ \\
\hline $\mathrm{C}(13)-\mathrm{C}(12)-\mathrm{H}(12 \mathrm{~A})$ & 120.3 \\
\hline $\mathrm{C}(11)-\mathrm{C}(12)-\mathrm{H}(12 \mathrm{~A})$ & 120.3 \\
\hline C(14)-C(13)-C(12) & $119.9(3)$ \\
\hline $\mathrm{C}(14)-\mathrm{C}(13)-\mathrm{H}(13 \mathrm{~A})$ & 120.1 \\
\hline $\mathrm{C}(12)-\mathrm{C}(13)-\mathrm{H}(13 \mathrm{~A})$ & 120.1 \\
\hline C(13)-C(14)-C(15) & $121.0(3)$ \\
\hline $\mathrm{C}(13)-\mathrm{C}(14)-\mathrm{H}(14 \mathrm{~A})$ & 119.5 \\
\hline $\mathrm{C}(15)-\mathrm{C}(14)-\mathrm{H}(14 \mathrm{~A})$ & 119.5 \\
\hline
\end{tabular}




$\begin{array}{ll}\mathrm{C}(14)-\mathrm{C}(15)-\mathrm{C}(10) & 120.2(3) \\ \mathrm{C}(14)-\mathrm{C}(15)-\mathrm{H}(15 \mathrm{~A}) & 119.9 \\ \mathrm{C}(10)-\mathrm{C}(15)-\mathrm{H}(15 \mathrm{~A}) & 119.9 \\ \mathrm{C}(17)-\mathrm{C}(16)-\mathrm{C}(21) & 119.48(18) \\ \mathrm{C}(17)-\mathrm{C}(16)-\mathrm{Sn}(1) & 120.97(15) \\ \mathrm{C}(21)-\mathrm{C}(16)-\mathrm{Sn}(1) & 119.54(15) \\ \mathrm{C}(18)-\mathrm{C}(17)-\mathrm{C}(16) & 120.4(2) \\ \mathrm{C}(18)-\mathrm{C}(17)-\mathrm{H}(17 \mathrm{~A}) & 119.8 \\ \mathrm{C}(16)-\mathrm{C}(17)-\mathrm{H}(17 \mathrm{~A}) & 119.8 \\ \mathrm{C}(19)-\mathrm{C}(18)-\mathrm{C}(17) & 119.8(2) \\ \mathrm{C}(19)-\mathrm{C}(18)-\mathrm{H}(18 \mathrm{~A}) & 120.1 \\ \mathrm{C}(17)-\mathrm{C}(18)-\mathrm{H}(18 \mathrm{~A}) & 120.1 \\ \mathrm{C}(18)-\mathrm{C}(19)-\mathrm{C}(20) & 120.4(2) \\ \mathrm{C}(18)-\mathrm{C}(19)-\mathrm{H}(19 \mathrm{~A}) & 119.8 \\ \mathrm{C}(20)-\mathrm{C}(19)-\mathrm{H}(19 \mathrm{~A}) & 119.8 \\ \mathrm{C}(19)-\mathrm{C}(20)-\mathrm{C}(21) & 120.2(2) \\ \mathrm{C}(19)-\mathrm{C}(20)-\mathrm{H}(20 \mathrm{~A}) & 119.9 \\ \mathrm{C}(21)-\mathrm{C}(20)-\mathrm{H}(20 \mathrm{~A}) & 119.9 \\ \mathrm{C}(20)-\mathrm{C}(21)-\mathrm{C}(16) & 119.7(2) \\ \mathrm{C}(20)-\mathrm{C}(21)-\mathrm{H}(21 \mathrm{~A}) & 120.2 \\ \mathrm{C}(16)-\mathrm{C}(21)-\mathrm{H}(21 \mathrm{~A}) & 120.2 \\ & \\ & \end{array}$

Symmetry transformations used to generate equivalent atoms: 
Table A 27. Anisotropic displacement parameters $\left(\AA^{2} \times 10^{3}\right)$ for 203. The anisotropic displacement factor exponent takes the form: $-2 \pi^{2}\left[h^{2} a^{* 2} U^{11}+\ldots+2 h k a^{*} b^{*} U^{12}\right]$

\begin{tabular}{|c|c|c|c|c|c|c|}
\hline & $\mathrm{U}^{11}$ & $\mathrm{U}^{22}$ & $\mathrm{U}^{33}$ & $\mathrm{U}^{23}$ & $\mathrm{U}^{13}$ & $\mathrm{U}^{12}$ \\
\hline $\operatorname{Sn}(1)$ & $31(1)$ & $23(1)$ & $16(1)$ & $-1(1)$ & $0(1)$ & $5(1)$ \\
\hline $\mathrm{Cl}(1)$ & $31(1)$ & $36(1)$ & $23(1)$ & 1(1) & $3(1)$ & $2(1)$ \\
\hline $\mathrm{Cl}(2)$ & $42(1)$ & $23(1)$ & $28(1)$ & $-3(1)$ & $4(1)$ & $5(1)$ \\
\hline $\mathrm{O}(1)$ & $33(1)$ & $32(1)$ & $30(1)$ & $3(1)$ & $-8(1)$ & $2(1)$ \\
\hline $\mathrm{C}(1)$ & $44(1)$ & $26(1)$ & $18(1)$ & $3(1)$ & $-1(1)$ & $7(1)$ \\
\hline$C(2)$ & $46(1)$ & $32(1)$ & $30(1)$ & $5(1)$ & $-8(1)$ & $10(1)$ \\
\hline$C(3)$ & $41(1)$ & $40(1)$ & $33(1)$ & $6(1)$ & $-12(1)$ & $10(1)$ \\
\hline $\mathrm{C}(4)$ & $26(1)$ & $32(1)$ & $32(1)$ & $-5(1)$ & $-3(1)$ & $8(1)$ \\
\hline$C(5)$ & $24(1)$ & $33(1)$ & $32(1)$ & $-4(1)$ & $-6(1)$ & $4(1)$ \\
\hline$C(6)$ & $25(1)$ & $36(1)$ & $35(1)$ & $-3(1)$ & $-2(1)$ & $5(1)$ \\
\hline$C(7)$ & $24(1)$ & $35(1)$ & $43(1)$ & $-12(1)$ & $0(1)$ & $5(1)$ \\
\hline $\mathrm{C}(8)$ & $30(1)$ & $49(2)$ & $42(1)$ & $-13(1)$ & $-12(1)$ & $3(1)$ \\
\hline C(9) & $36(1)$ & $42(1)$ & $34(1)$ & $-5(1)$ & $-12(1)$ & $6(1)$ \\
\hline$C(10)$ & $27(1)$ & $46(2)$ & $46(1)$ & $-16(1)$ & $3(1)$ & $-3(1)$ \\
\hline $\mathrm{C}(11)$ & $32(1)$ & $34(1)$ & $74(2)$ & $-14(1)$ & 15(1) & $0(1)$ \\
\hline$C(12)$ & $54(2)$ & $40(2)$ & $79(2)$ & $-15(2)$ & $29(2)$ & $-3(1)$ \\
\hline $\mathrm{C}(13)$ & $57(2)$ & $68(2)$ & $75(2)$ & $-28(2)$ & $26(2)$ & $-30(2)$ \\
\hline$C(14)$ & $37(2)$ & $113(3)$ & $72(2)$ & $-13(2)$ & $0(2)$ & $-28(2)$ \\
\hline$C(15)$ & $32(1)$ & $86(2)$ & $60(2)$ & $-3(2)$ & $-6(1)$ & $-13(2)$ \\
\hline$C(16)$ & $22(1)$ & $27(1)$ & $18(1)$ & $-2(1)$ & $0(1)$ & $2(1)$ \\
\hline $\mathrm{C}(17)$ & $30(1)$ & $30(1)$ & $24(1)$ & $1(1)$ & $1(1)$ & $5(1)$ \\
\hline$C(18)$ & $35(1)$ & $37(1)$ & $30(1)$ & $-9(1)$ & $7(1)$ & $4(1)$ \\
\hline C(19) & $34(1)$ & $52(2)$ & 19(1) & $-6(1)$ & $5(1)$ & $-3(1)$ \\
\hline$C(20)$ & $39(1)$ & $43(1)$ & $22(1)$ & $5(1)$ & $-1(1)$ & $0(1)$ \\
\hline $\mathrm{C}(21)$ & $33(1)$ & $30(1)$ & $22(1)$ & $0(1)$ & $-1(1)$ & $3(1)$ \\
\hline
\end{tabular}


Table A 28. Hydrogen coordinates $\left(\times 10^{4}\right)$ and isotropic displacement parameters $\left(\AA^{2} \times\right.$ $10^{3}$ ) for 203.

\begin{tabular}{|c|c|c|c|c|}
\hline & $\mathrm{x}$ & $\mathrm{y}$ & $\mathrm{Z}$ & $\mathrm{U}(\mathrm{eq})$ \\
\hline $\mathrm{H}(1 \mathrm{~A})$ & 4156 & 14344 & 33 & 36 \\
\hline $\mathrm{H}(1 \mathrm{~B})$ & 3798 & 12156 & -245 & 36 \\
\hline $\mathrm{H}(2 \mathrm{~A})$ & 2685 & 14756 & 488 & 44 \\
\hline $\mathrm{H}(2 \mathrm{~B})$ & 2574 & 14698 & -237 & 44 \\
\hline $\mathrm{H}(3 \mathrm{~A})$ & 1414 & 12603 & 181 & 46 \\
\hline $\mathrm{H}(3 \mathrm{~B})$ & 2091 & 11174 & -206 & 46 \\
\hline $\mathrm{H}(5 \mathrm{~A})$ & 2473 & 8712 & 1648 & 36 \\
\hline $\mathrm{H}(6 \mathrm{~A})$ & 1573 & 5905 & 1945 & 39 \\
\hline $\mathrm{H}(8 \mathrm{~A})$ & -15 & 6327 & 380 & 49 \\
\hline $\mathrm{H}(9 \mathrm{~A})$ & 890 & 9119 & 66 & 46 \\
\hline $\mathrm{H}(11 \mathrm{~A})$ & 1133 & 2398 & 1838 & 55 \\
\hline $\mathrm{H}(12 \mathrm{~A})$ & 212 & -379 & 2151 & 68 \\
\hline $\mathrm{H}(13 \mathrm{~A})$ & -1391 & -476 & 1858 & 79 \\
\hline $\mathrm{H}(14 \mathrm{~A})$ & -2066 & 2247 & 1299 & 89 \\
\hline $\mathrm{H}(15 \mathrm{~A})$ & -1166 & 5090 & 1013 & 72 \\
\hline $\mathrm{H}(17 \mathrm{~A})$ & 3498 & 15134 & 1700 & 34 \\
\hline $\mathrm{H}(18 \mathrm{~A})$ & 3157 & 15731 & 2709 & 41 \\
\hline $\mathrm{H}(19 \mathrm{~A})$ & 3375 & 13069 & 3424 & 42 \\
\hline $\mathrm{H}(20 \mathrm{~A})$ & 3983 & 9849 & 3146 & 42 \\
\hline$H(21 A)$ & 4367 & 9253 & 2141 & 34 \\
\hline
\end{tabular}


Table A 29. Torsion angles $\left[{ }^{\circ}\right]$ for 203.

\begin{tabular}{|c|c|}
\hline $\mathrm{Sn}(1)-\mathrm{C}(1)-\mathrm{C}(2)-\mathrm{C}(3)$ & $-65.4(2)$ \\
\hline $\mathrm{C}(4)-\mathrm{O}(1)-\mathrm{C}(3)-\mathrm{C}(2)$ & $176.96(18)$ \\
\hline $\mathrm{C}(1)-\mathrm{C}(2)-\mathrm{C}(3)-\mathrm{O}(1)$ & $59.0(2)$ \\
\hline $\mathrm{C}(3)-\mathrm{O}(1)-\mathrm{C}(4)-\mathrm{C}(9)$ & $6.9(3)$ \\
\hline $\mathrm{C}(3)-\mathrm{O}(1)-\mathrm{C}(4)-\mathrm{C}(5)$ & $-172.61(19)$ \\
\hline $\mathrm{O}(1)-\mathrm{C}(4)-\mathrm{C}(5)-\mathrm{C}(6)$ & 178.96(19) \\
\hline$C(9)-C(4)-C(5)-C(6)$ & $-0.6(3)$ \\
\hline$C(4)-C(5)-C(6)-C(7)$ & $-0.2(3)$ \\
\hline$C(5)-C(6)-C(7)-C(8)$ & $0.6(3)$ \\
\hline$C(5)-C(6)-C(7)-C(10)$ & $-179.4(2)$ \\
\hline $\mathrm{C}(6)-\mathrm{C}(7)-\mathrm{C}(8)-\mathrm{C}(9)$ & $-0.1(4)$ \\
\hline $\mathrm{C}(10)-\mathrm{C}(7)-\mathrm{C}(8)-\mathrm{C}(9)$ & $179.9(2)$ \\
\hline $\mathrm{O}(1)-\mathrm{C}(4)-\mathrm{C}(9)-\mathrm{C}(8)$ & $-178.5(2)$ \\
\hline$C(5)-C(4)-C(9)-C(8)$ & $1.0(3)$ \\
\hline$C(7)-C(8)-C(9)-C(4)$ & $-0.7(4)$ \\
\hline $\mathrm{C}(8)-\mathrm{C}(7)-\mathrm{C}(10)-\mathrm{C}(11)$ & $145.2(3)$ \\
\hline$C(6)-C(7)-C(10)-C(11)$ & $-34.8(3)$ \\
\hline $\mathrm{C}(8)-\mathrm{C}(7)-\mathrm{C}(10)-\mathrm{C}(15)$ & $-35.4(4)$ \\
\hline$C(6)-C(7)-C(10)-C(15)$ & $144.6(3)$ \\
\hline $\mathrm{C}(15)-\mathrm{C}(10)-\mathrm{C}(11)-\mathrm{C}(12)$ & $0.4(4)$ \\
\hline$C(7)-C(10)-C(11)-C(12)$ & $179.8(2)$ \\
\hline $\mathrm{C}(10)-\mathrm{C}(11)-\mathrm{C}(12)-\mathrm{C}(13)$ & $1.2(4)$ \\
\hline $\mathrm{C}(11)-\mathrm{C}(12)-\mathrm{C}(13)-\mathrm{C}(14)$ & $-1.5(5)$ \\
\hline $\mathrm{C}(12)-\mathrm{C}(13)-\mathrm{C}(14)-\mathrm{C}(15)$ & $0.3(5)$ \\
\hline $\mathrm{C}(13)-\mathrm{C}(14)-\mathrm{C}(15)-\mathrm{C}(10)$ & $1.3(5)$ \\
\hline $\mathrm{C}(11)-\mathrm{C}(10)-\mathrm{C}(15)-\mathrm{C}(14)$ & $-1.6(4)$ \\
\hline$C(7)-C(10)-C(15)-C(14)$ & $179.0(3)$ \\
\hline $\mathrm{C}(21)-\mathrm{C}(16)-\mathrm{C}(17)-\mathrm{C}(18)$ & $0.1(3)$ \\
\hline $\operatorname{Sn}(1)-C(16)-C(17)-C(18)$ & $179.46(16)$ \\
\hline$C(16)-C(17)-C(18)-C(19)$ & $-1.1(3)$ \\
\hline $\mathrm{C}(17)-\mathrm{C}(18)-\mathrm{C}(19)-\mathrm{C}(20)$ & $1.1(4)$ \\
\hline $\mathrm{C}(18)-\mathrm{C}(19)-\mathrm{C}(20)-\mathrm{C}(21)$ & $-0.3(4)$ \\
\hline$C(19)-C(20)-C(21)-C(16)$ & $-0.7(3)$ \\
\hline$C(17)-C(16)-C(21)-C(20)$ & $0.8(3)$ \\
\hline
\end{tabular}


Symmetry transformations used to generate equivalent atoms: 


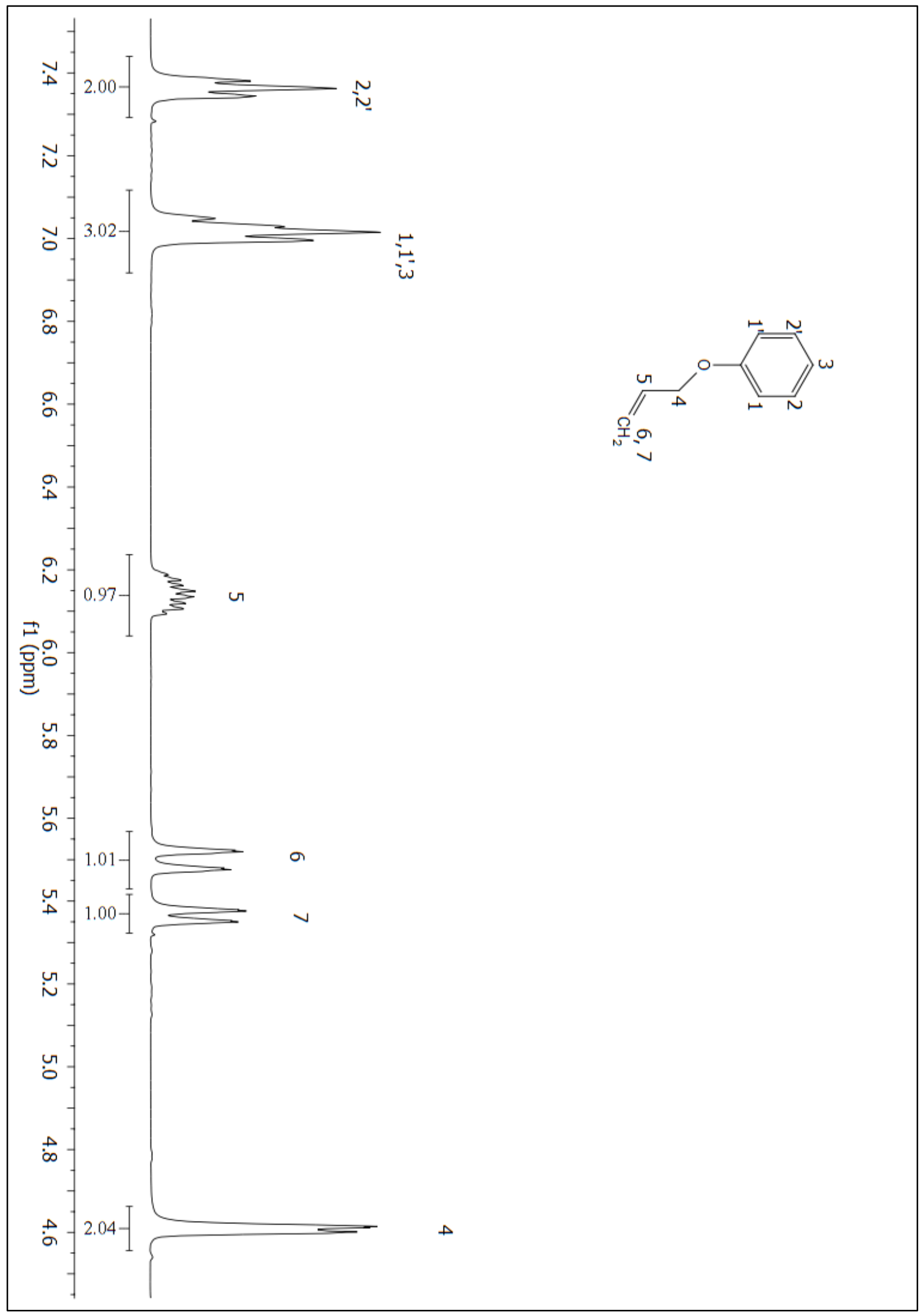

Figure A 1: ${ }^{1} \mathrm{H}$ NMR $\left(\mathrm{CDCl}_{3}\right)$ spectrum of compound 194. 


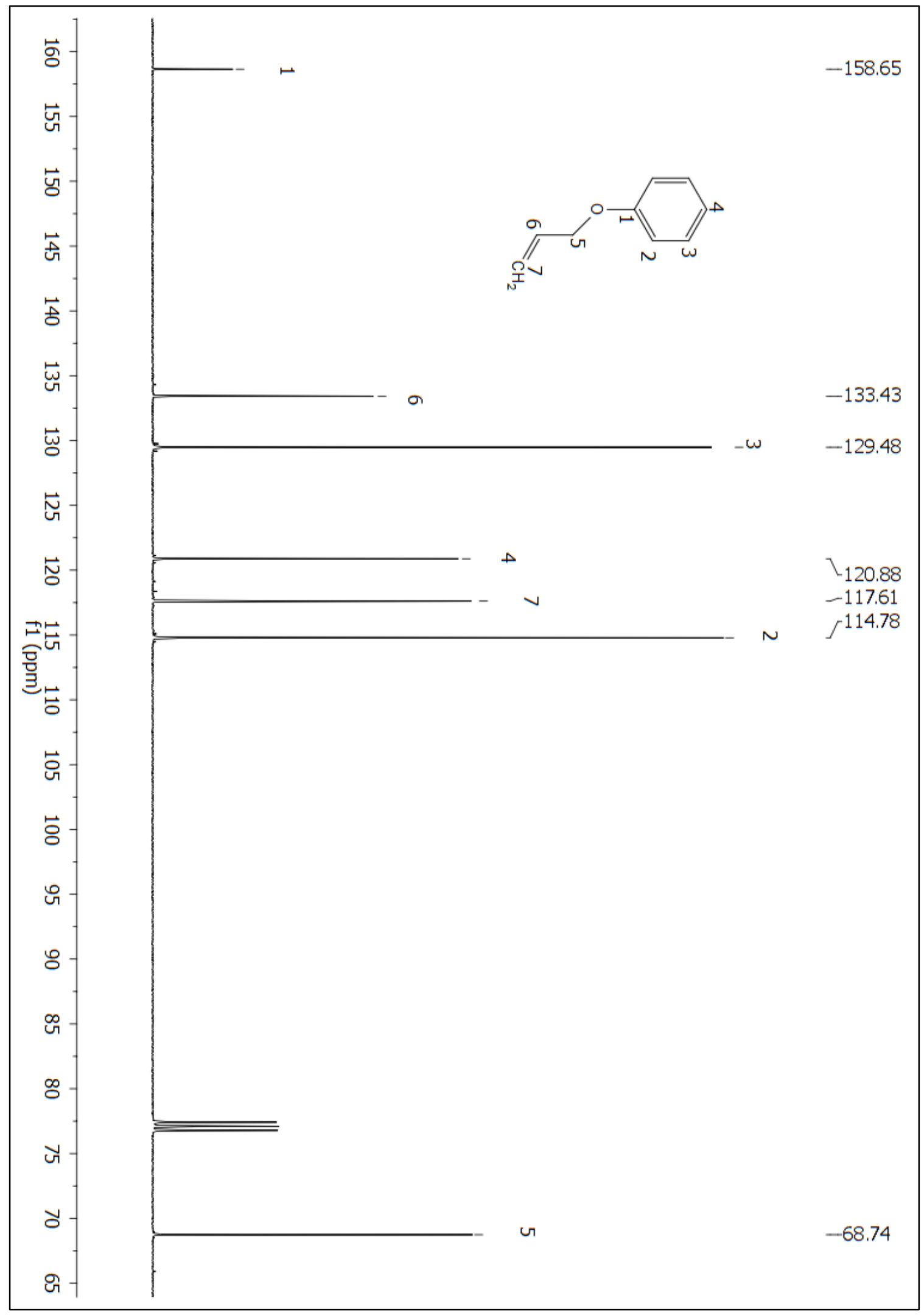

Figure A 2: ${ }^{13} \mathrm{C}$ NMR $\left(\mathrm{CDCl}_{3}\right)$ spectrum of compound 194. 


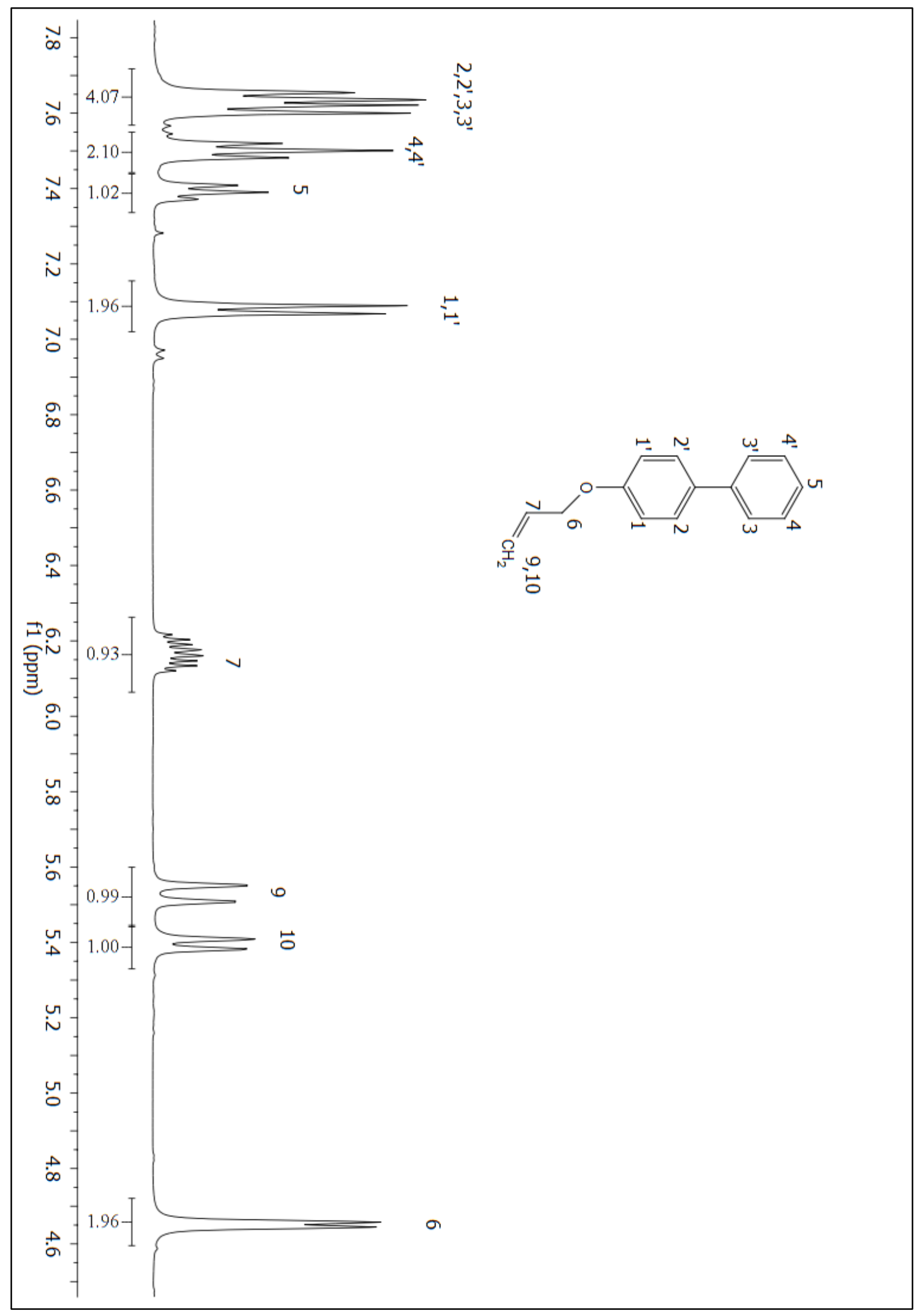

Figure A 3: ${ }^{1} \mathrm{H}$ NMR $\left(\mathrm{CDCl}_{3}\right)$ spectrum of compound 137. 


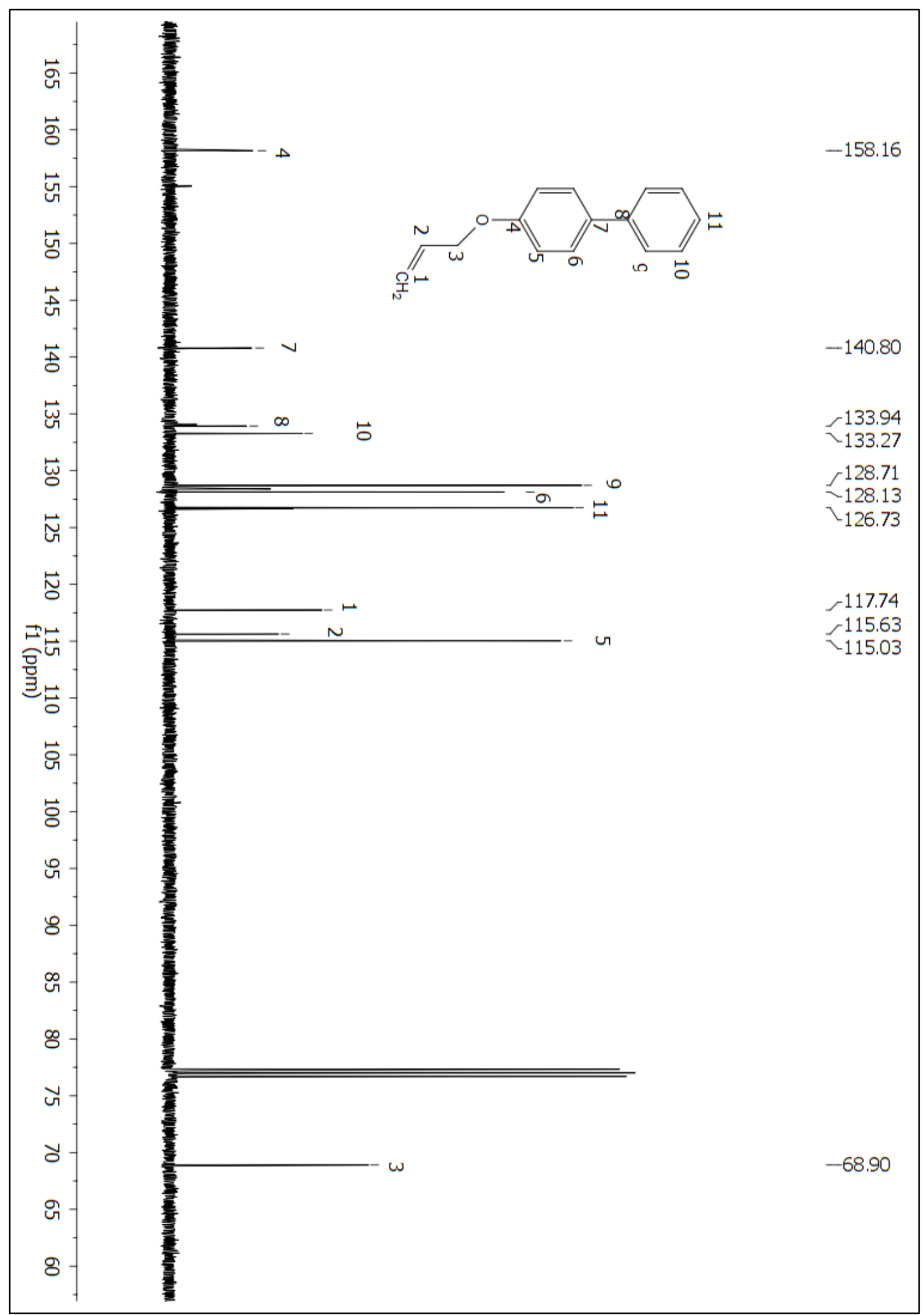

Figure A 4: ${ }^{13} \mathrm{C} \mathrm{NMR}\left(\mathrm{CDCl}_{3}\right)$ spectrum of compound 137. 


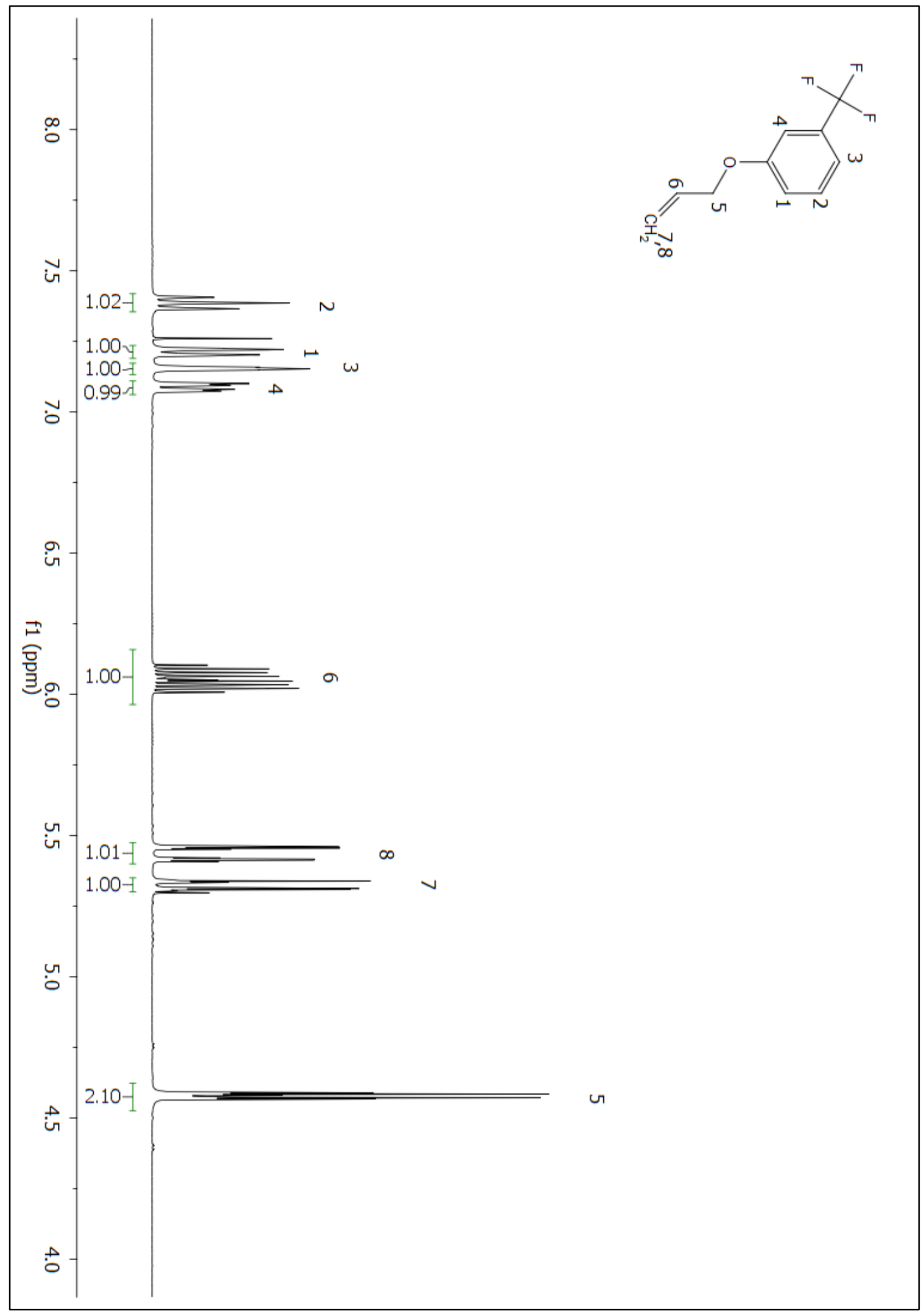

Figure A 5: ${ }^{1} \mathrm{H}$ NMR $\left(\mathrm{CDCl}_{3}\right)$ spectrum of compound in 195. 


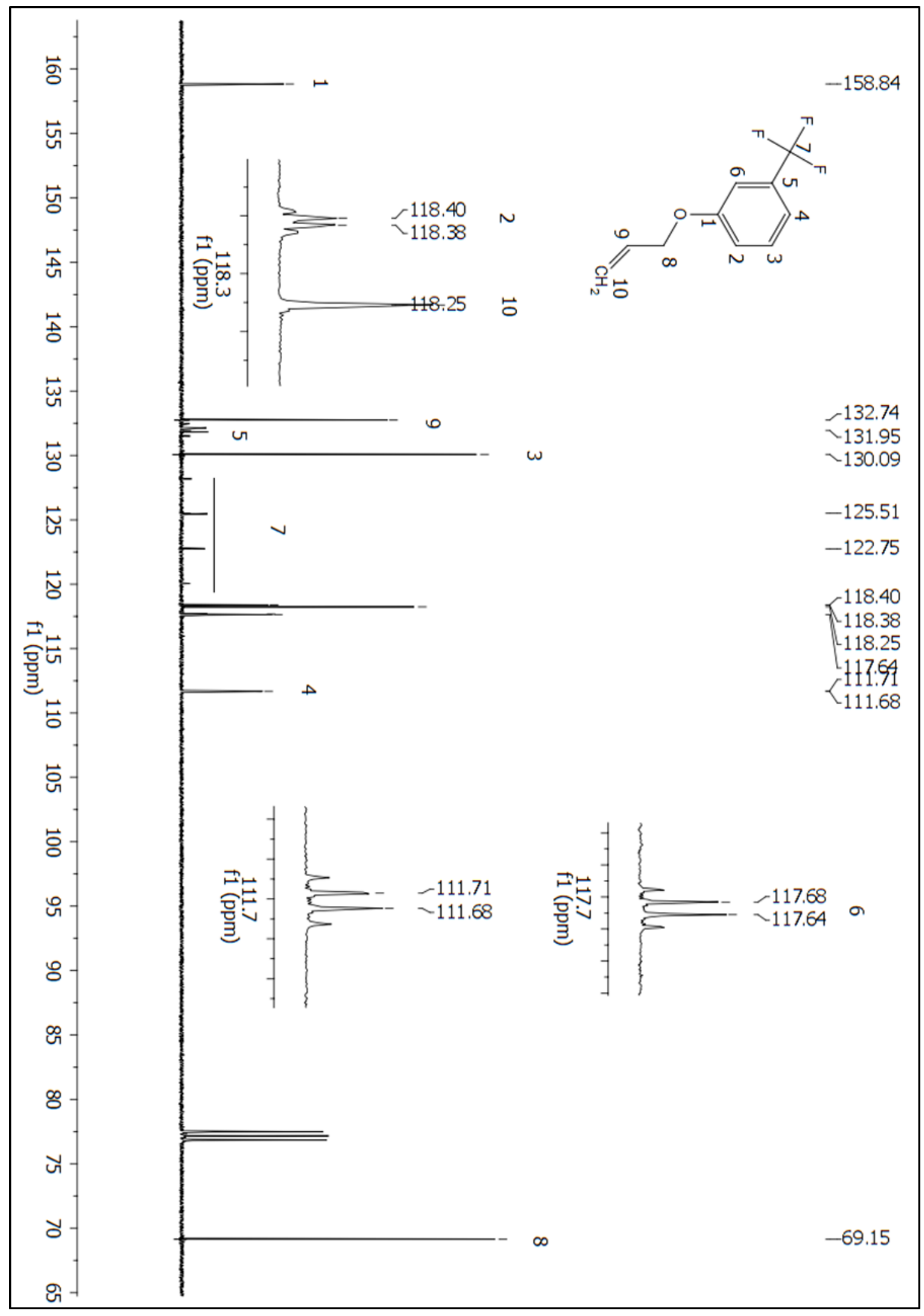

Figure A 6: ${ }^{13} \mathrm{C}$ NMR $\left(\mathrm{CDCl}_{3}\right)$ spectrum of compound 195. 


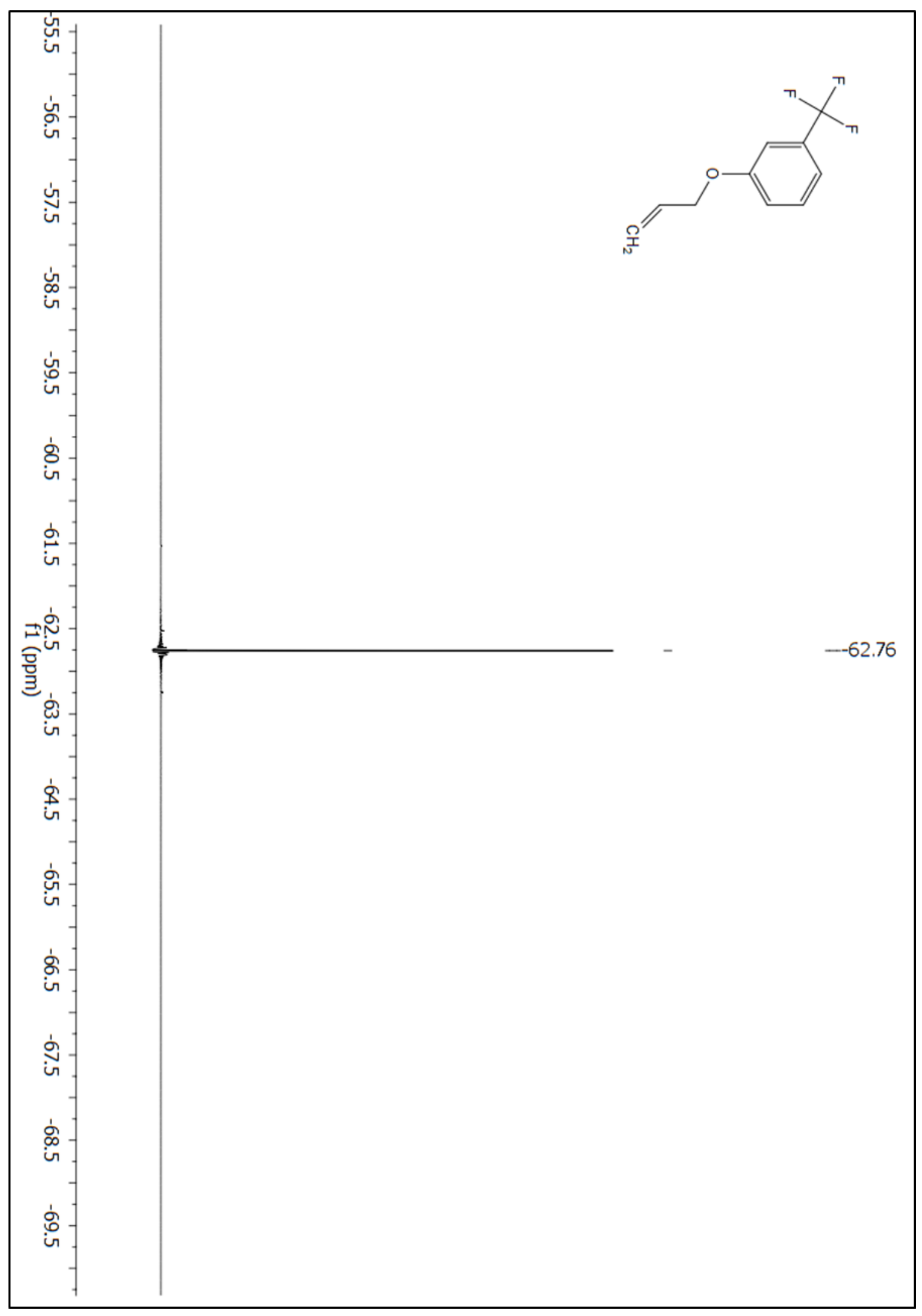

Figure A 7: ${ }^{19} \mathrm{~F}$ NMR $\left(\mathrm{CDCl}_{3}\right)$ spectrum of compound 195. 


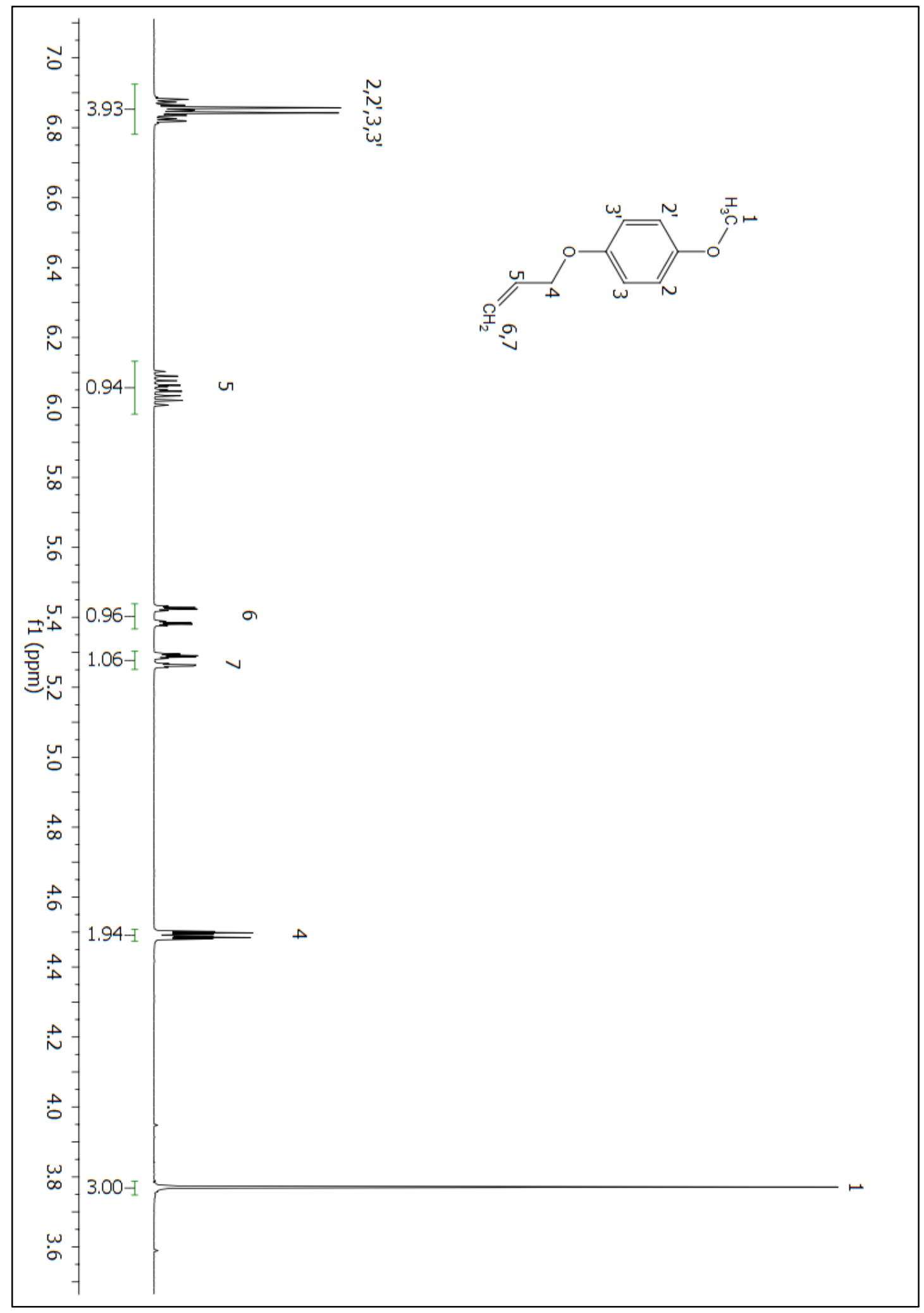

Figure A 8: ${ }^{1} \mathrm{H}$ NMR $\left(\mathrm{CDCl}_{3}\right)$ spectrum of compound 196. 


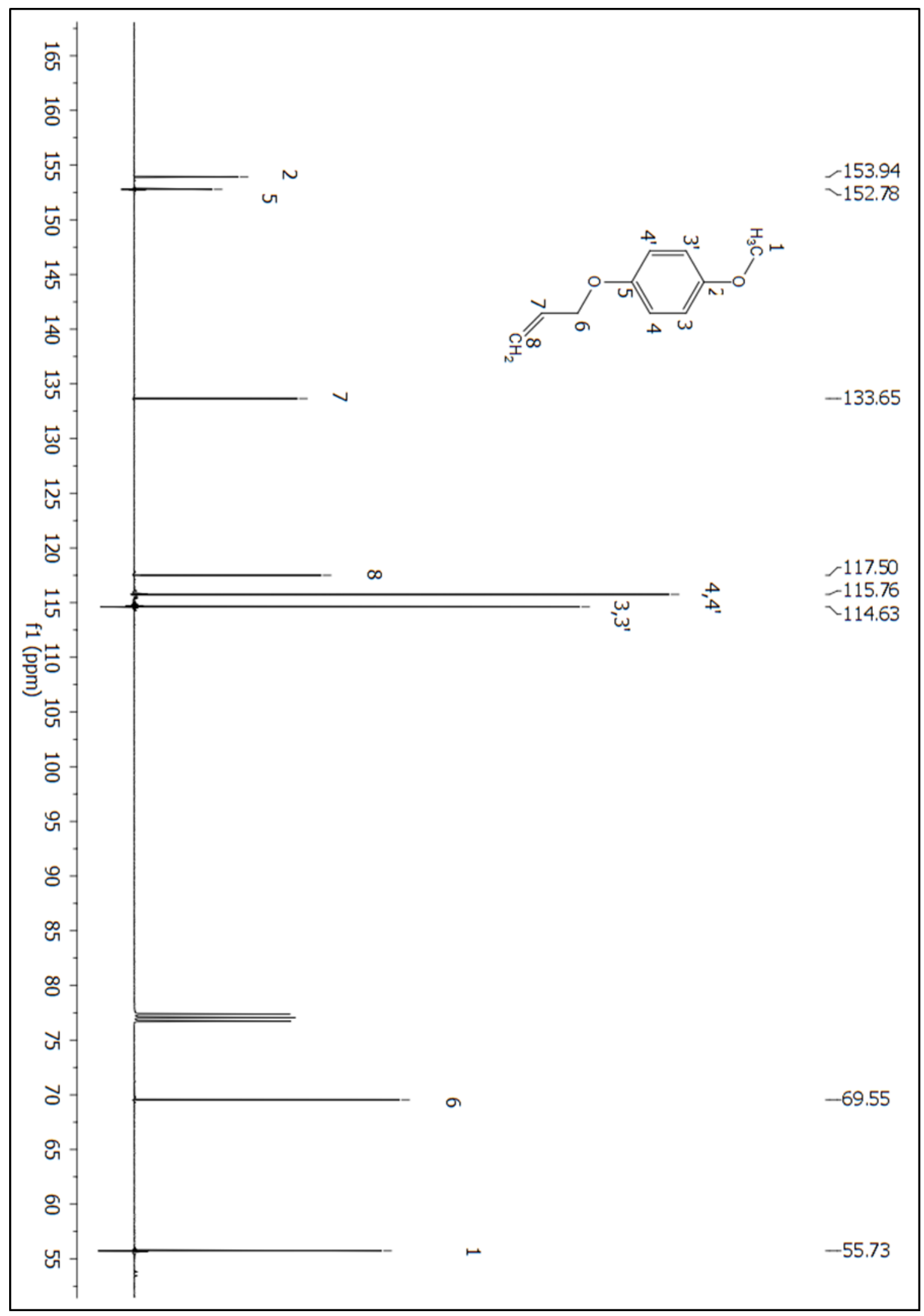

Figure A 9: ${ }^{13} \mathrm{C}$ NMR $\left(\mathrm{CDCl}_{3}\right)$ spectrum of compound 196. 


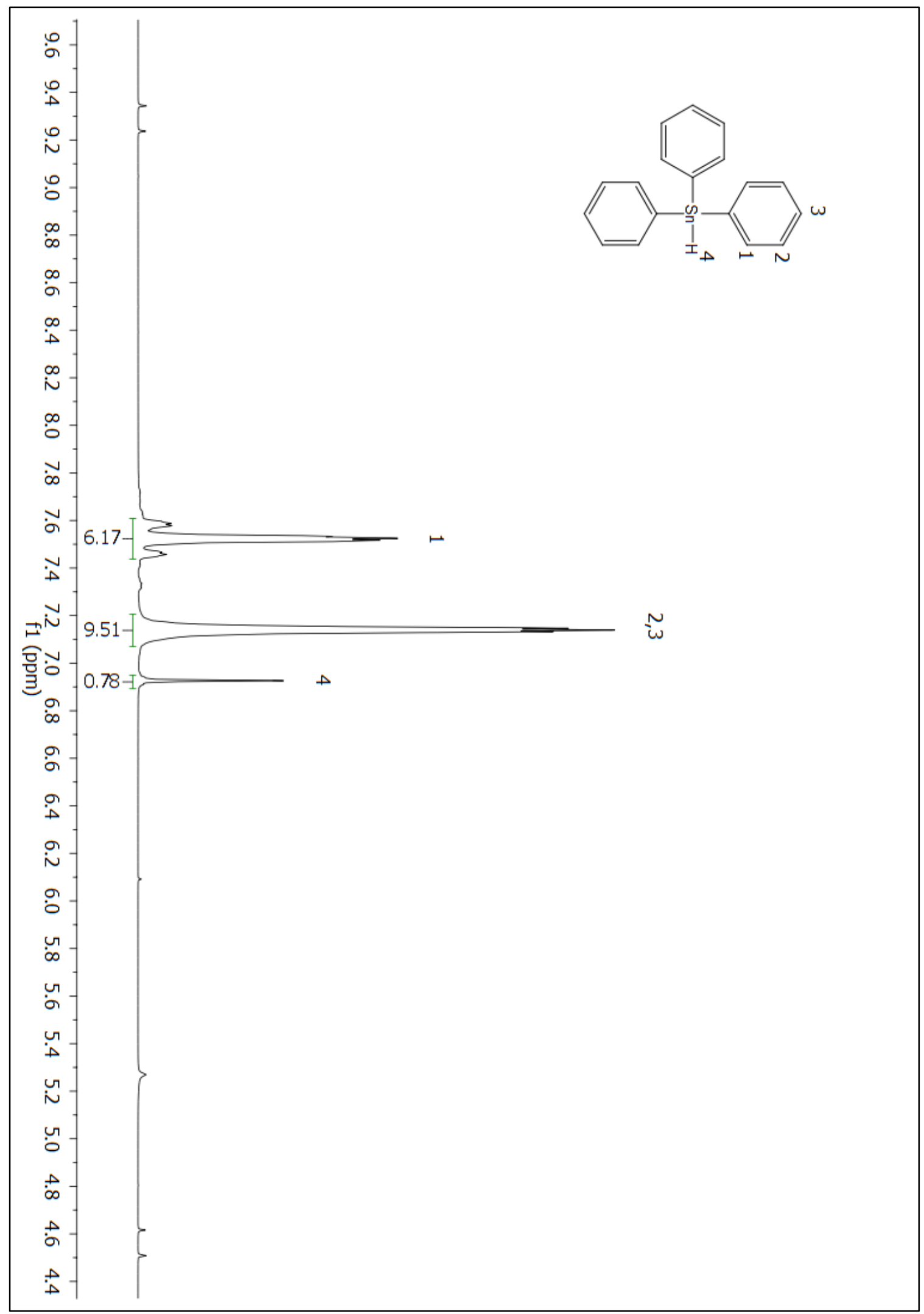

Figure A 10: ${ }^{1} \mathrm{H}$ NMR $\left(\mathrm{CDCl}_{3}\right)$ spectrum of compound 254. 


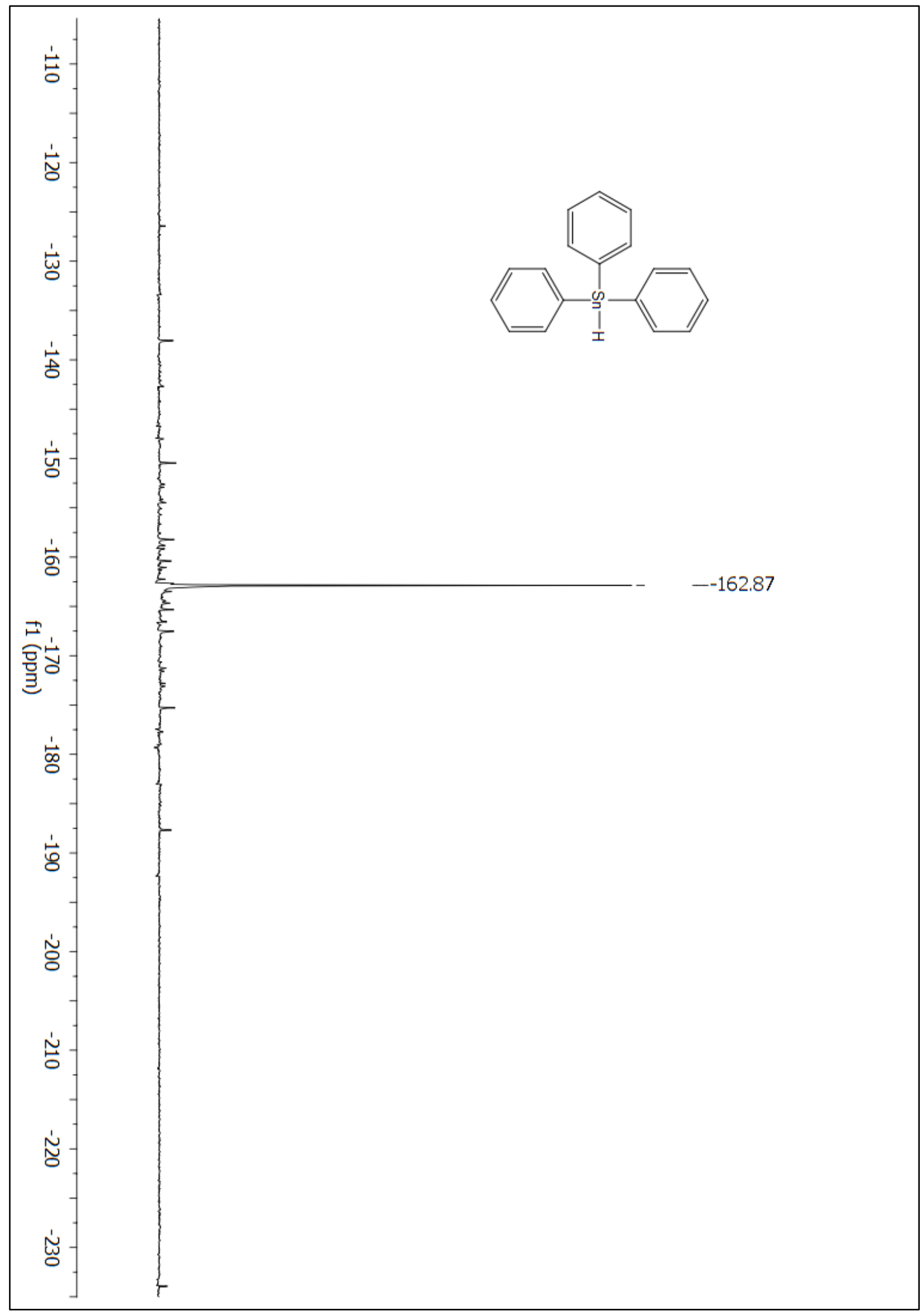

Figure A 11: ${ }^{119} \mathrm{Sn}$ NMR $\left(\mathrm{CDCl}_{3}\right)$ spectrum of compound 254. 


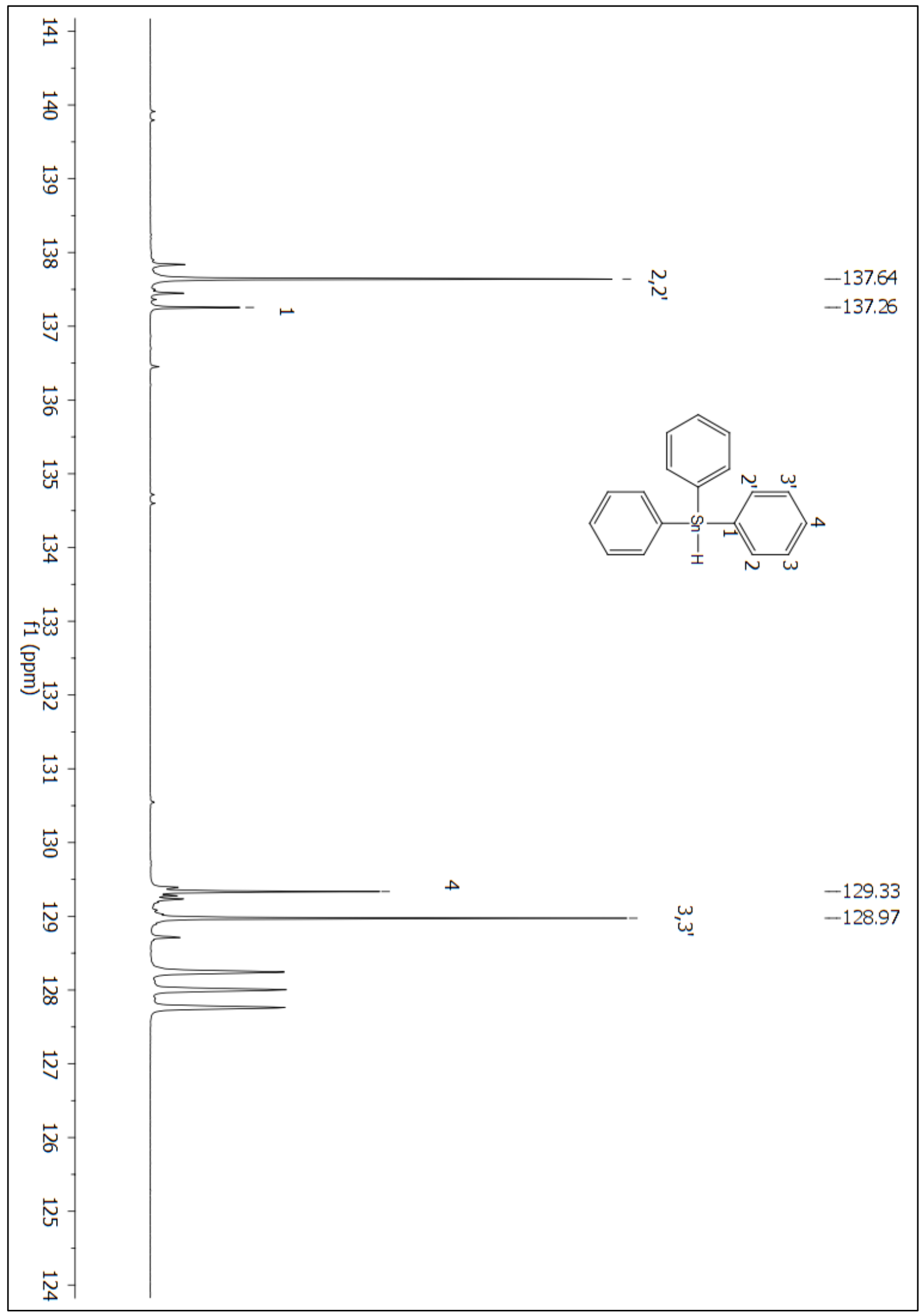

Figure A 12: ${ }^{13} \mathrm{C}$ NMR ( $\left.\mathrm{C}_{6} \mathrm{D}_{6}\right)$ spectrum of compound 254. 


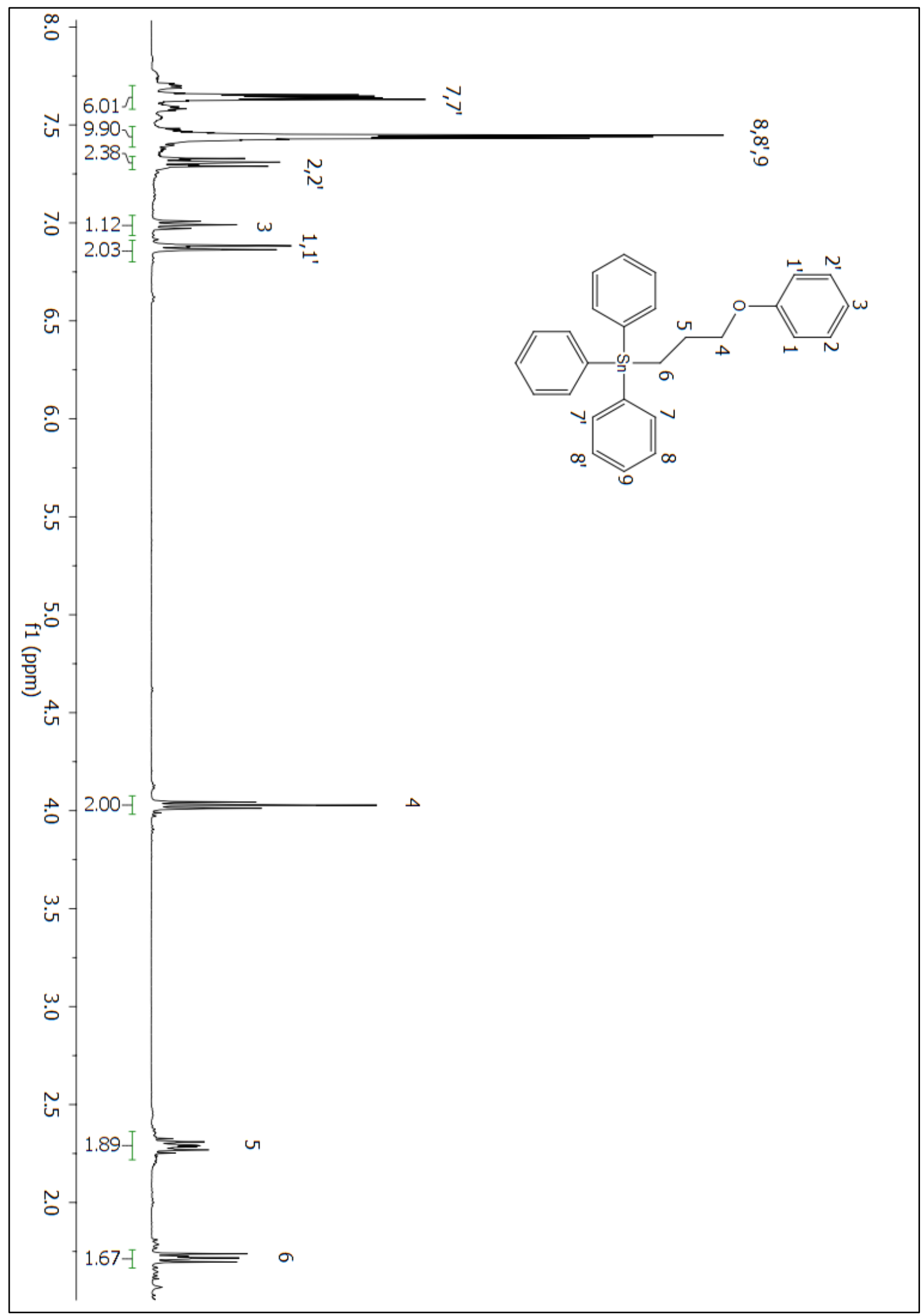

Figure A 13: ${ }^{1} \mathrm{H}$ NMR (C6 $\left.\mathrm{D}_{6}\right)$ spectrum of compound 197. 


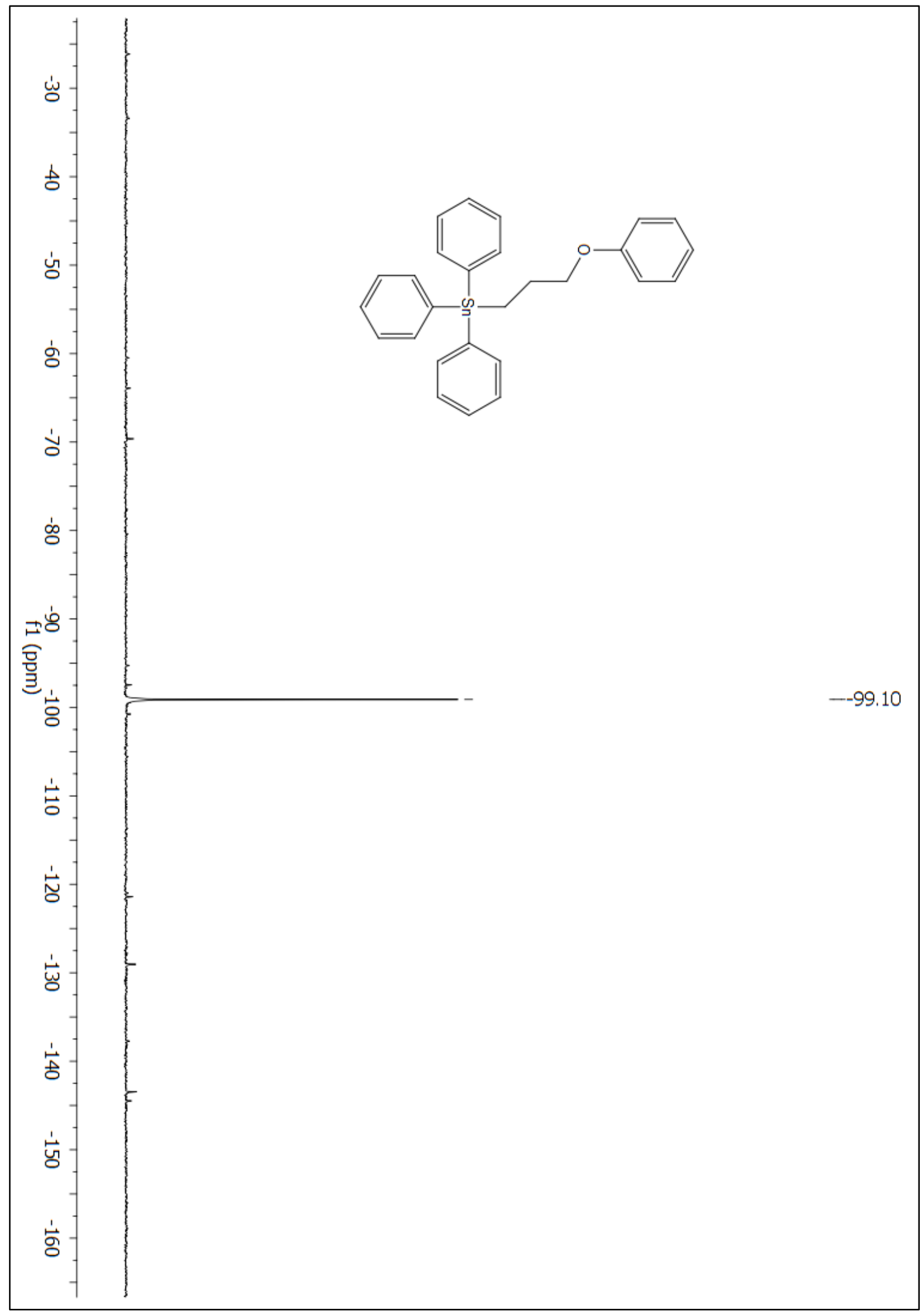

Figure A 14: ${ }^{119} \mathrm{Sn}$ NMR $\left(\mathrm{CDCl}_{3}\right)$ spectrum of compound 197. 


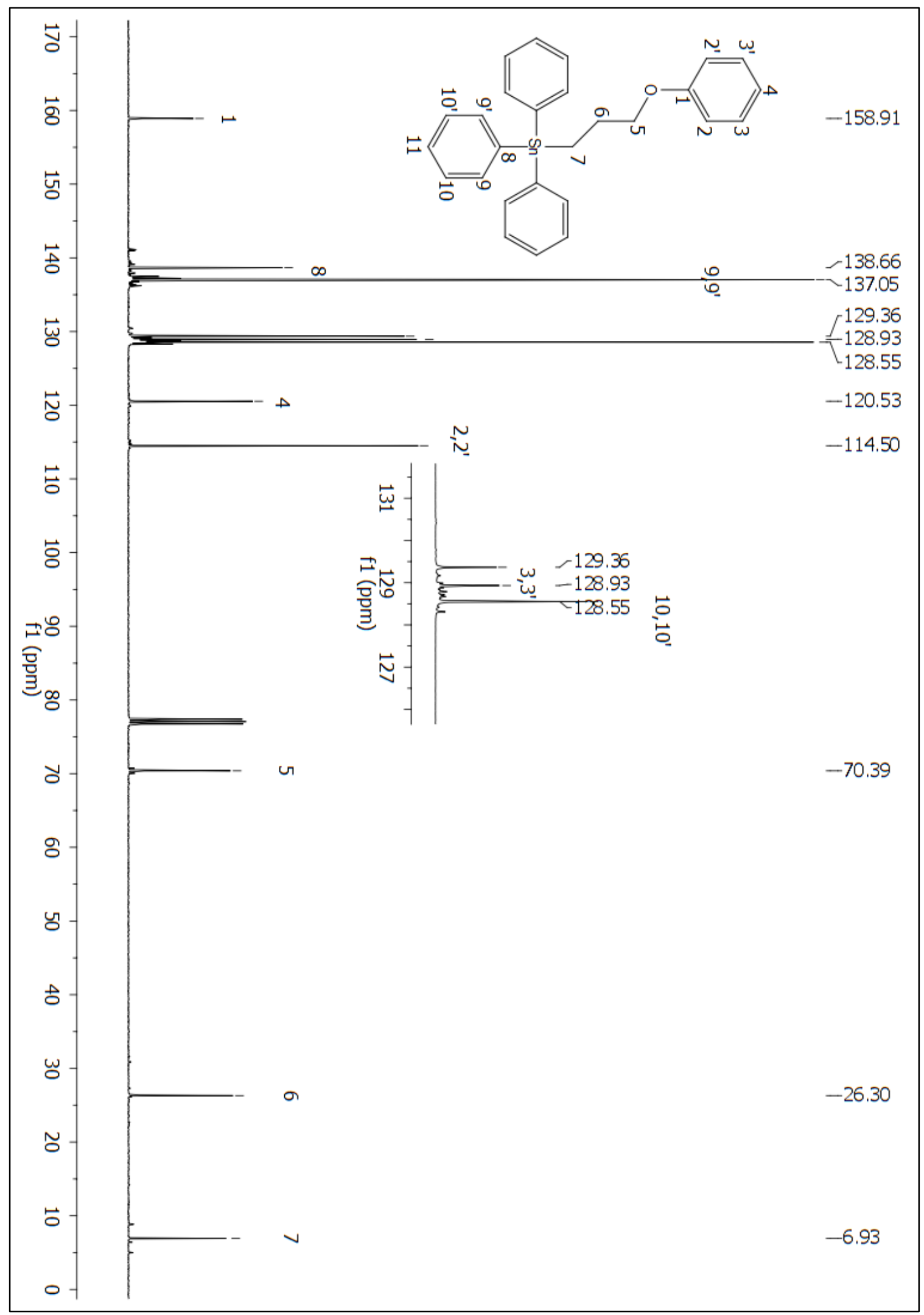

Figure A 15: ${ }^{13} \mathrm{C}$ NMR $\left(\mathrm{CDCl}_{3}\right)$ spectrum of compound 197. 


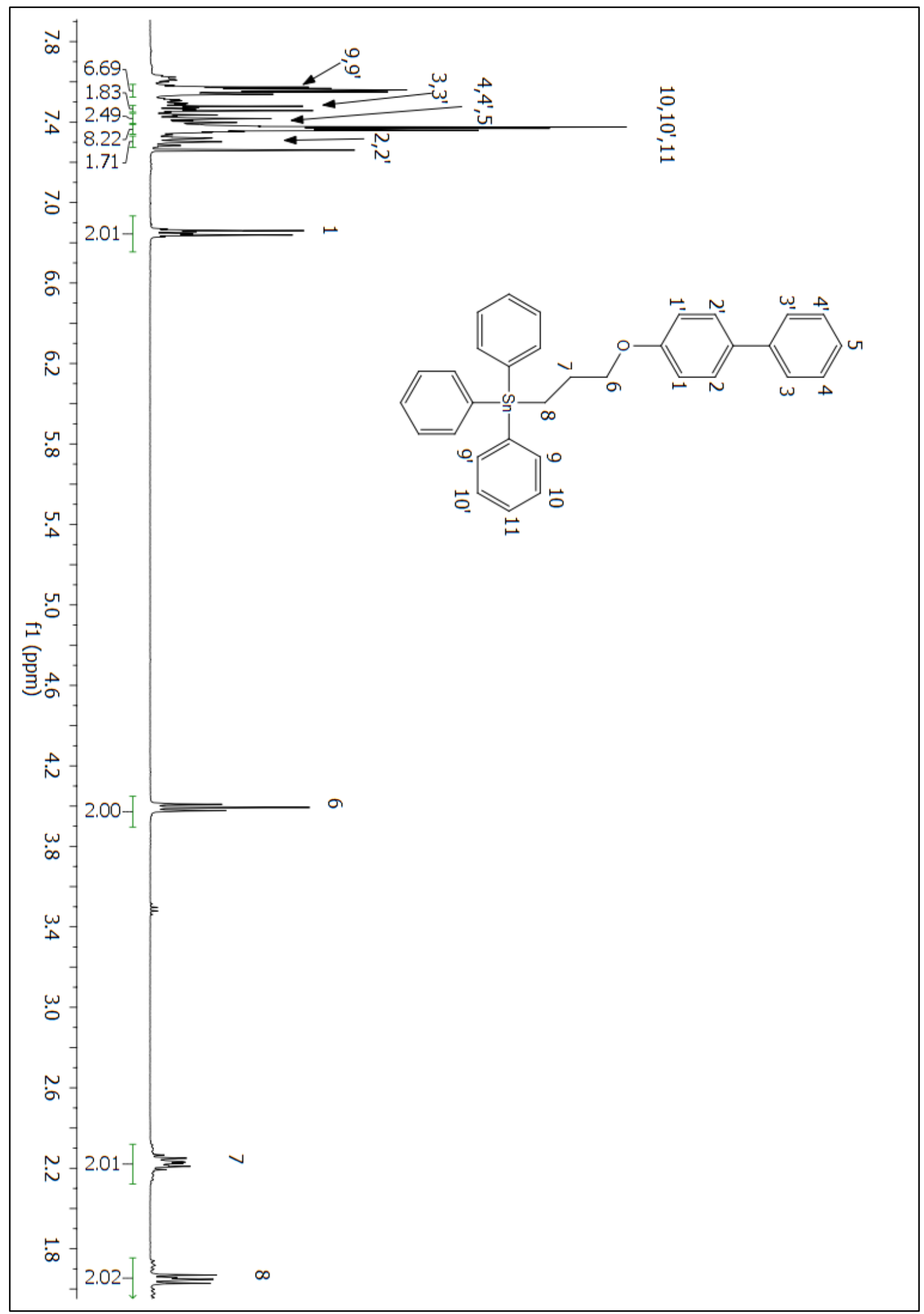

Figure A 16: ${ }^{1} \mathrm{H}$ NMR $\left(\mathrm{CDCl}_{3}\right)$ spectrum of compound 141. 


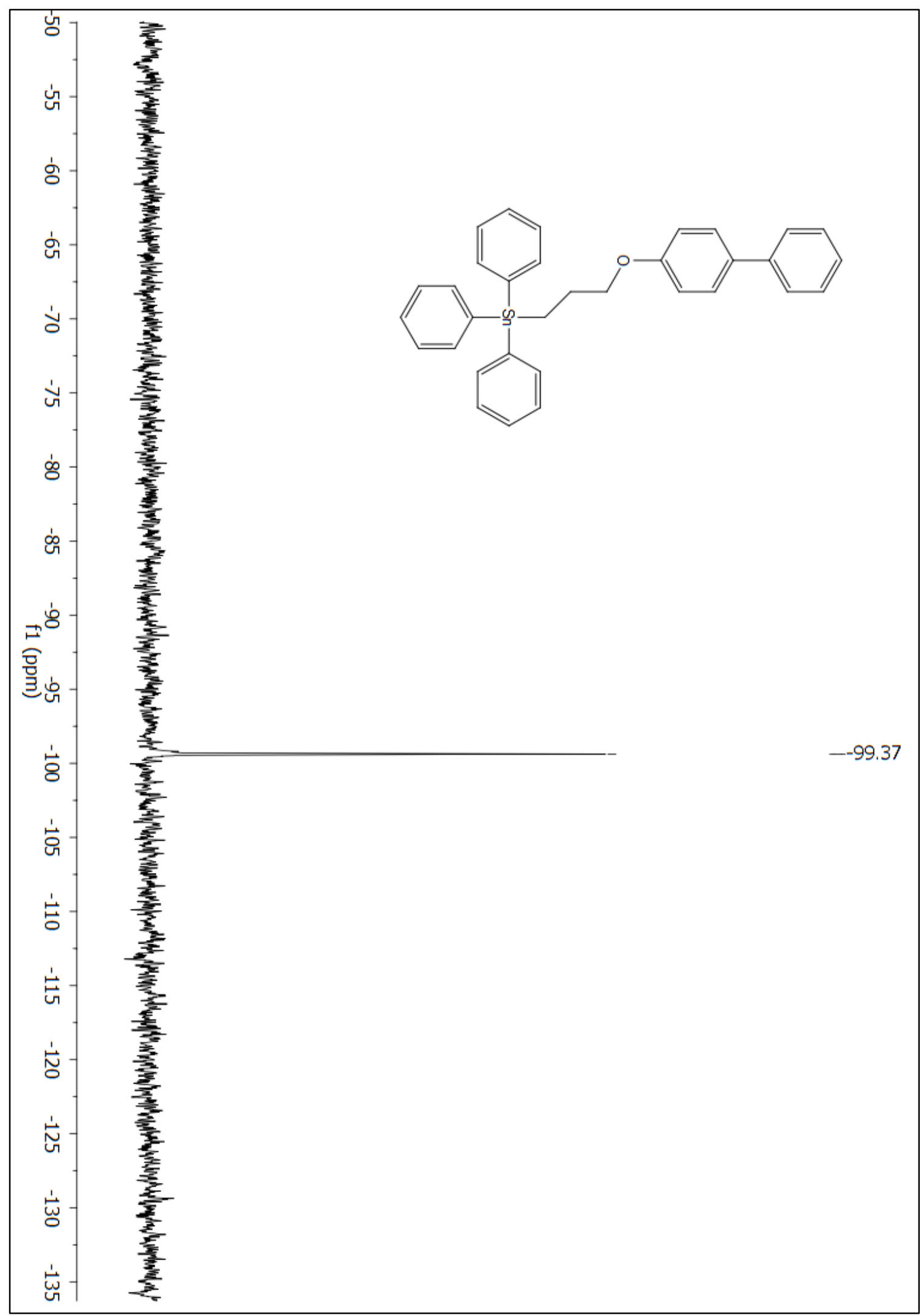

Figure A 17: ${ }^{119} \mathrm{Sn}$ NMR $\left(\mathrm{CDCl}_{3}\right)$ spectrum of compound 141. 


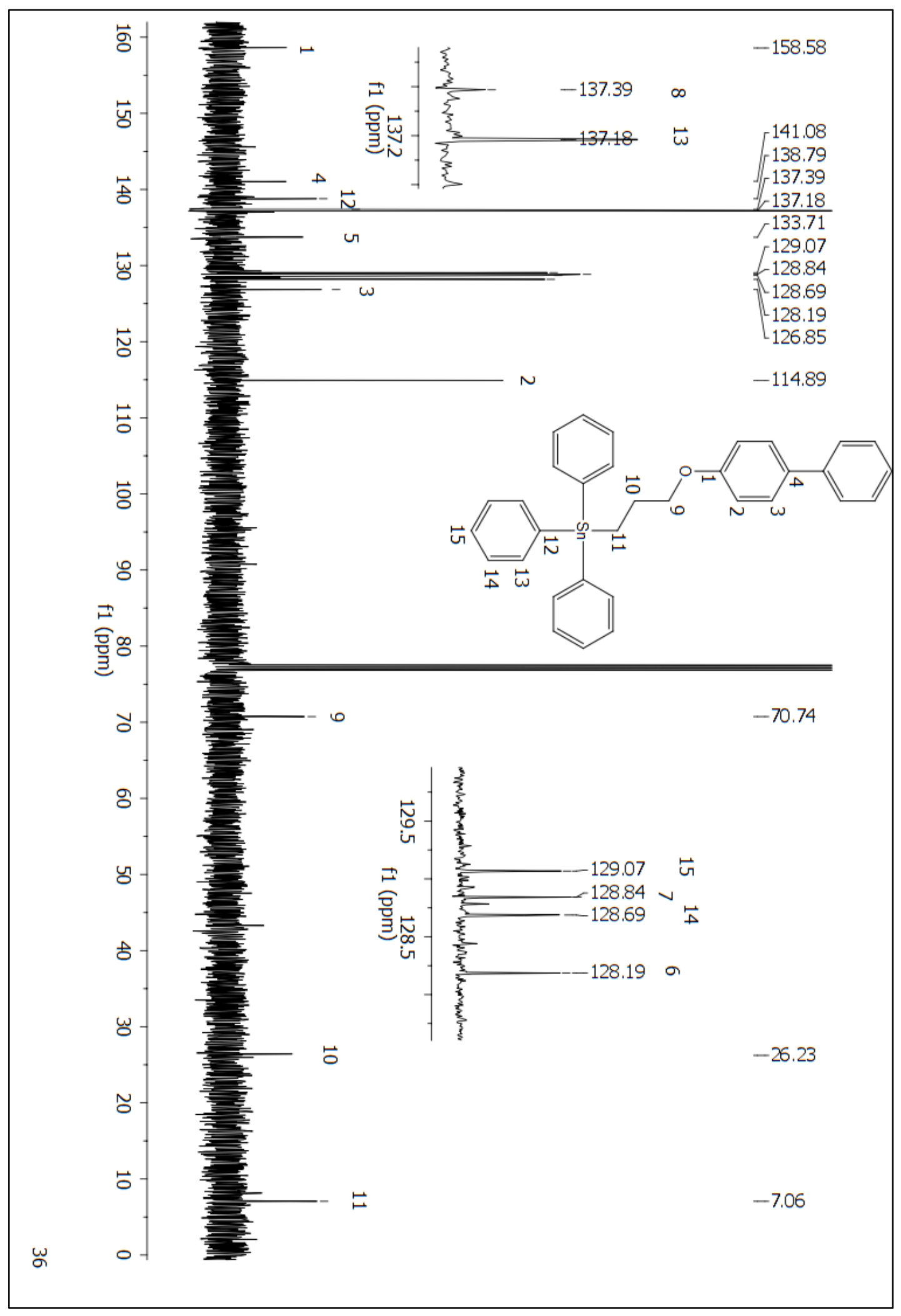

Figure A 18: ${ }^{13} \mathrm{C}$ NMR $\left(\mathrm{CDCl}_{3}\right)$ spectrum of compound 141 


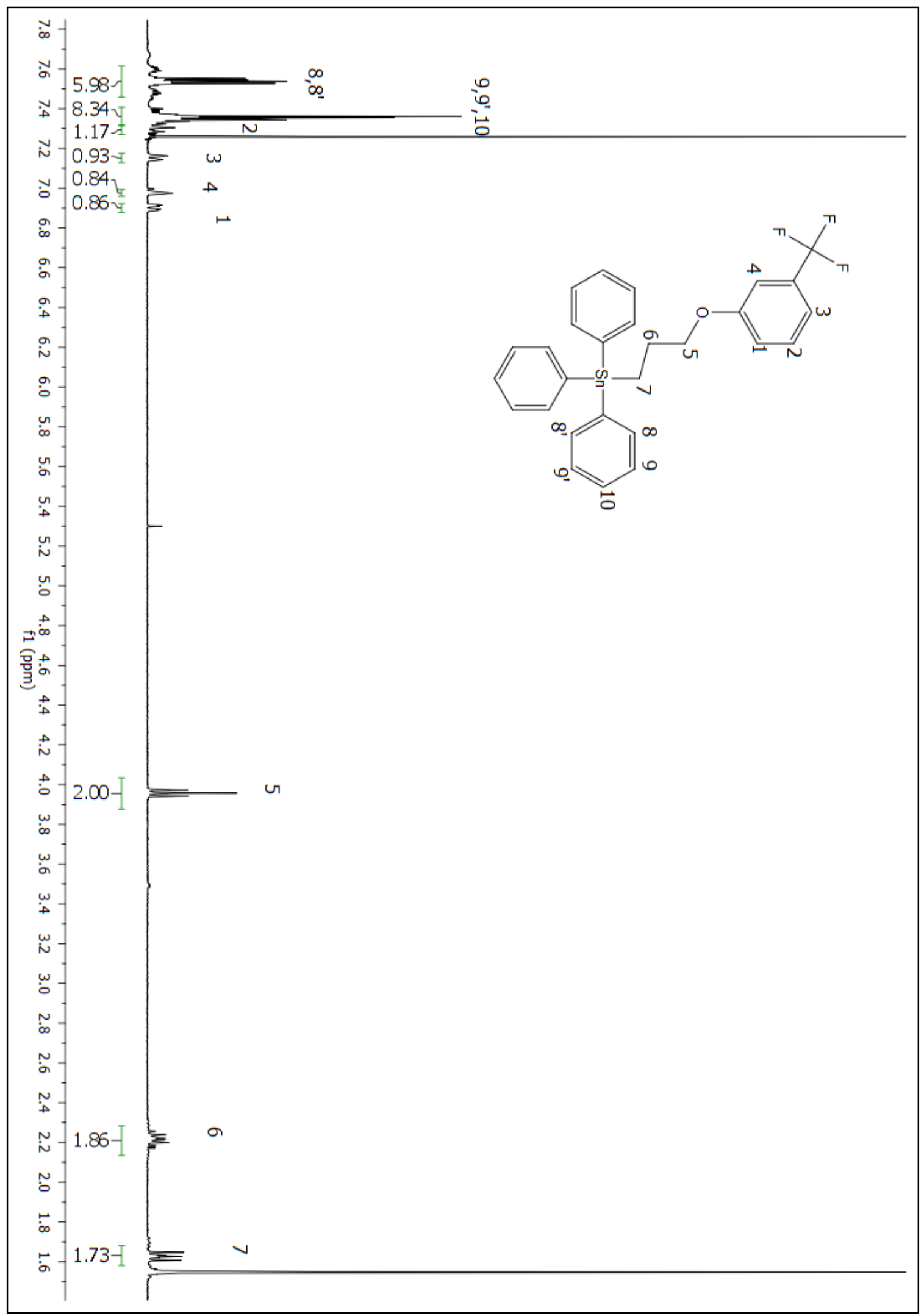

Figure A 19: ${ }^{1} \mathrm{H}$ NMR $\left(\mathrm{CDCl}_{3}\right)$ spectrum of compound 198. 


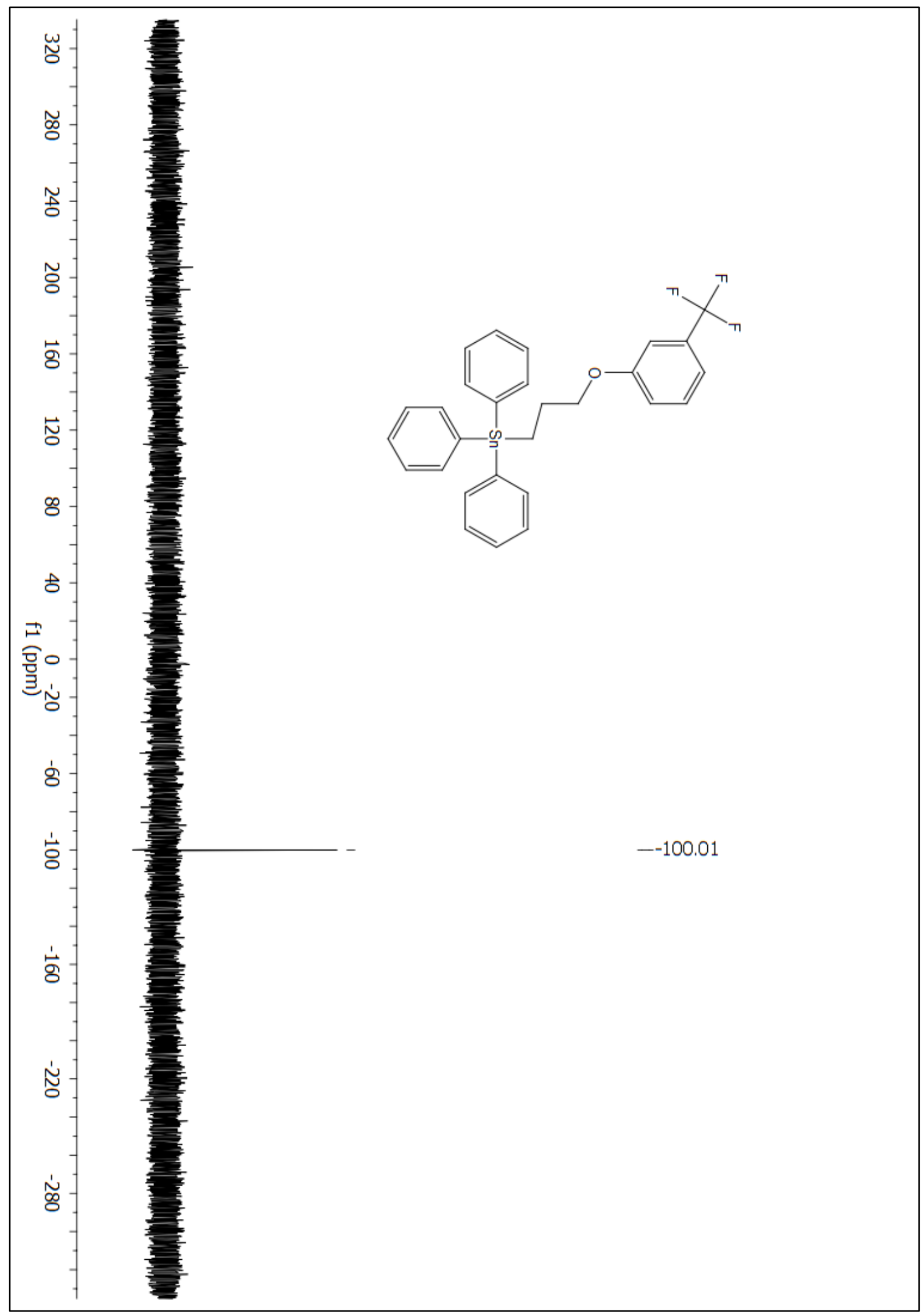

Figure A 20: ${ }^{119} \mathrm{Sn}$ NMR $\left(\mathrm{CDCl}_{3}\right)$ spectrum of compound 198. 


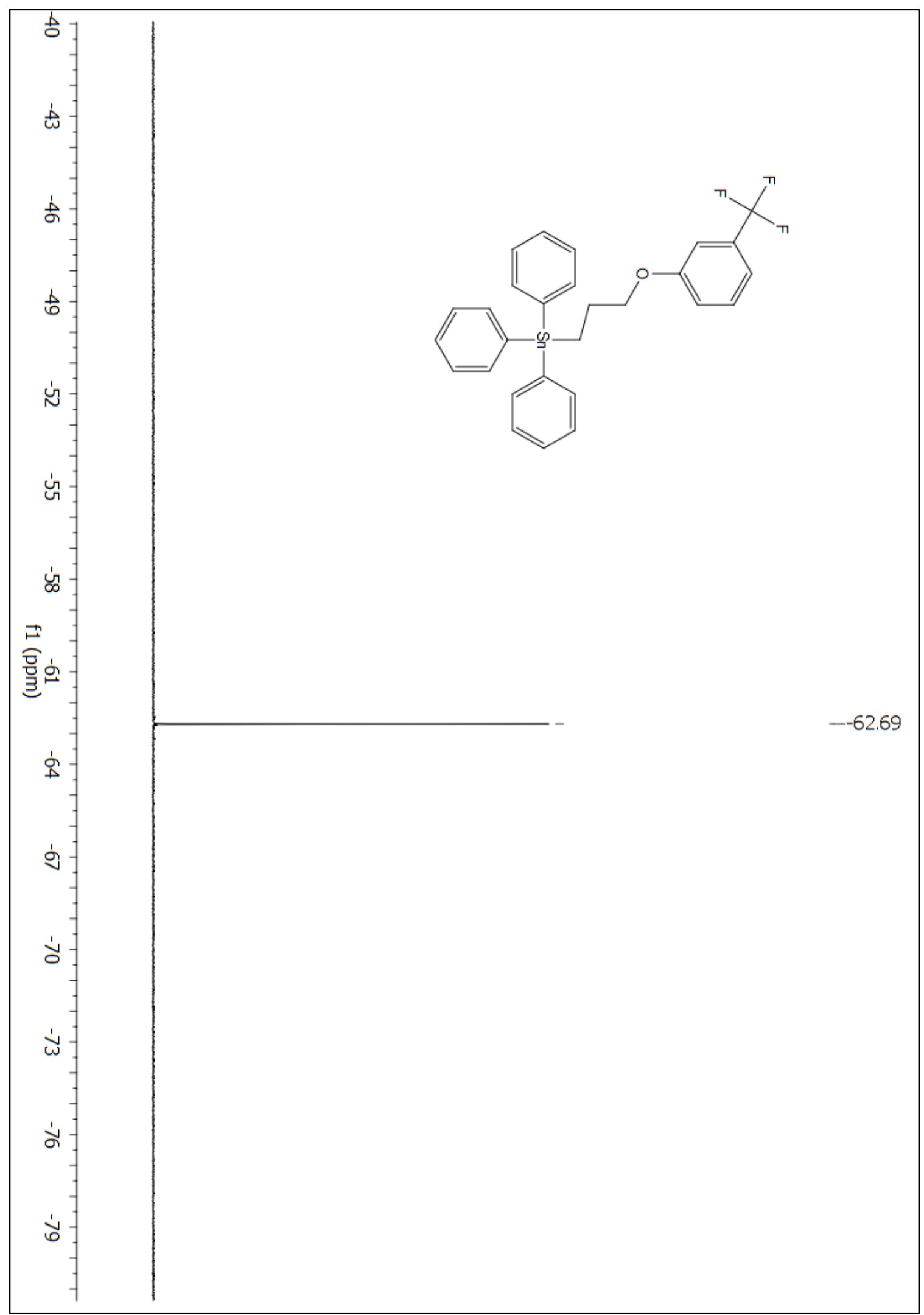

Figure A 21: ${ }^{19} \mathrm{~F}$ NMR $\left(\mathrm{CDCl}_{3}\right)$ spectrum of compound 198. 


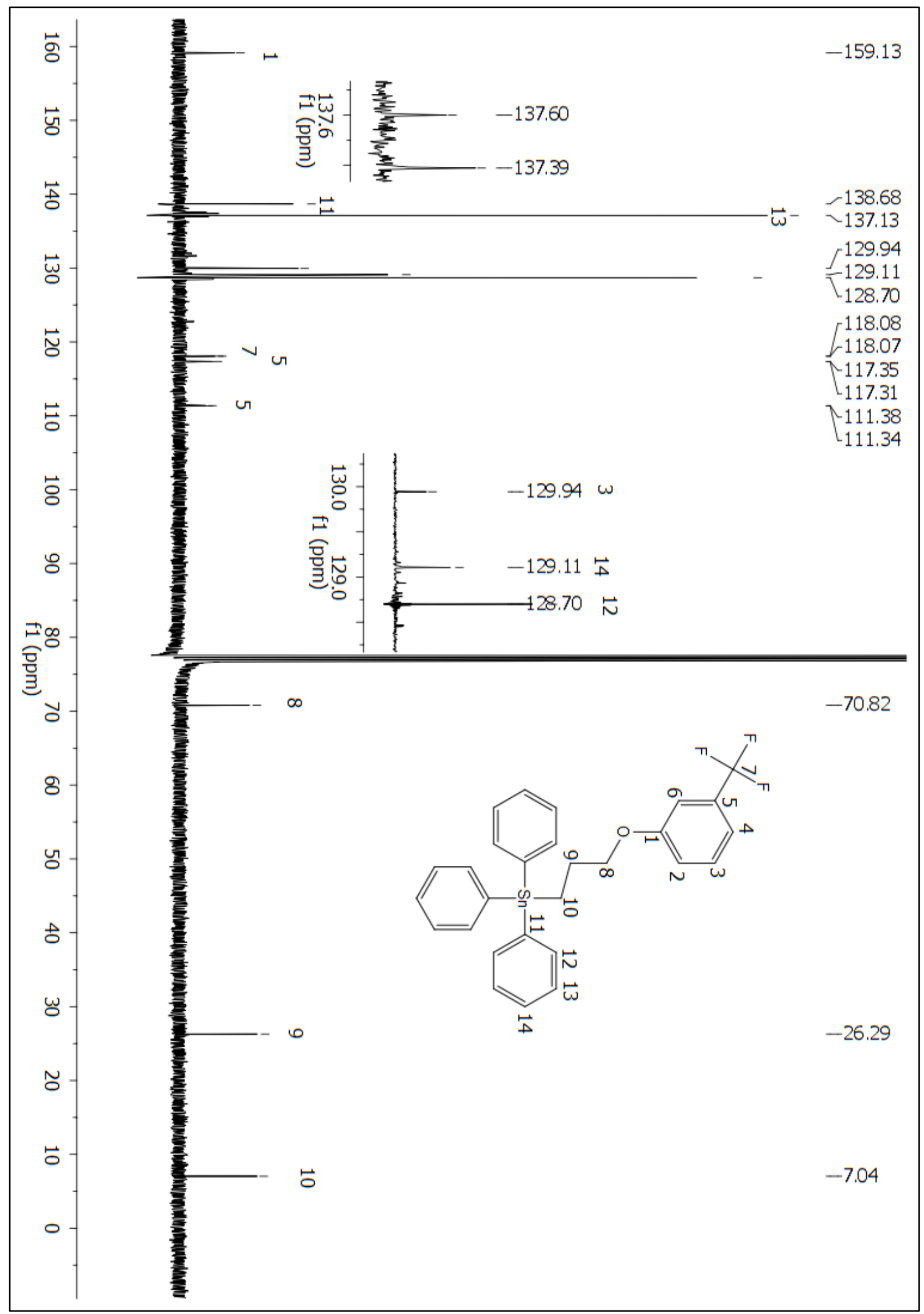

Figure A 22: ${ }^{13} \mathrm{C}$ NMR $\left(\mathrm{CDCl}_{3}\right)$ spectrum of compound 198 


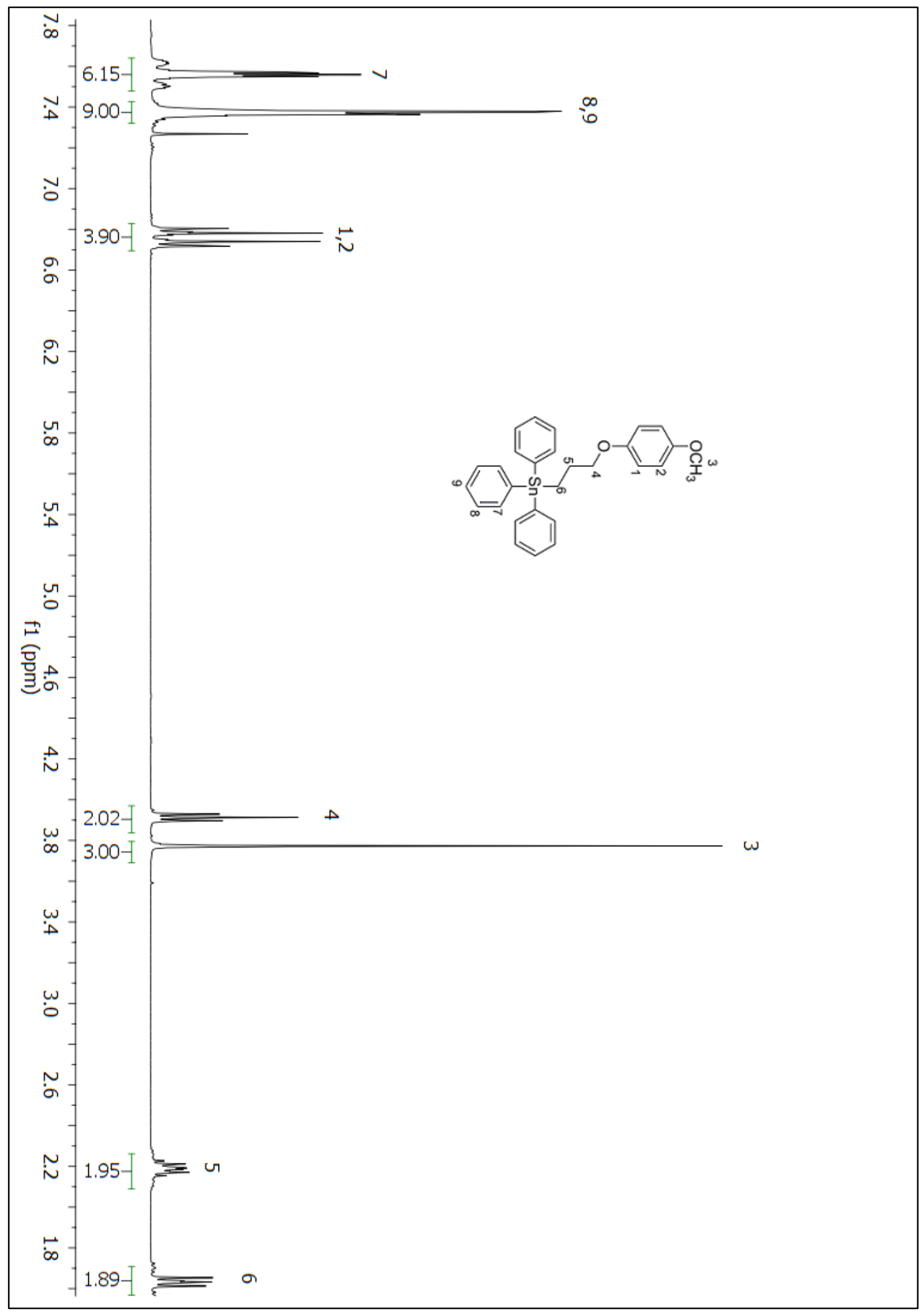

Figure A 23: ${ }^{1} \mathrm{H}$ NMR $\left(\mathrm{CDCl}_{3}\right)$ spectrum of compound 199. 


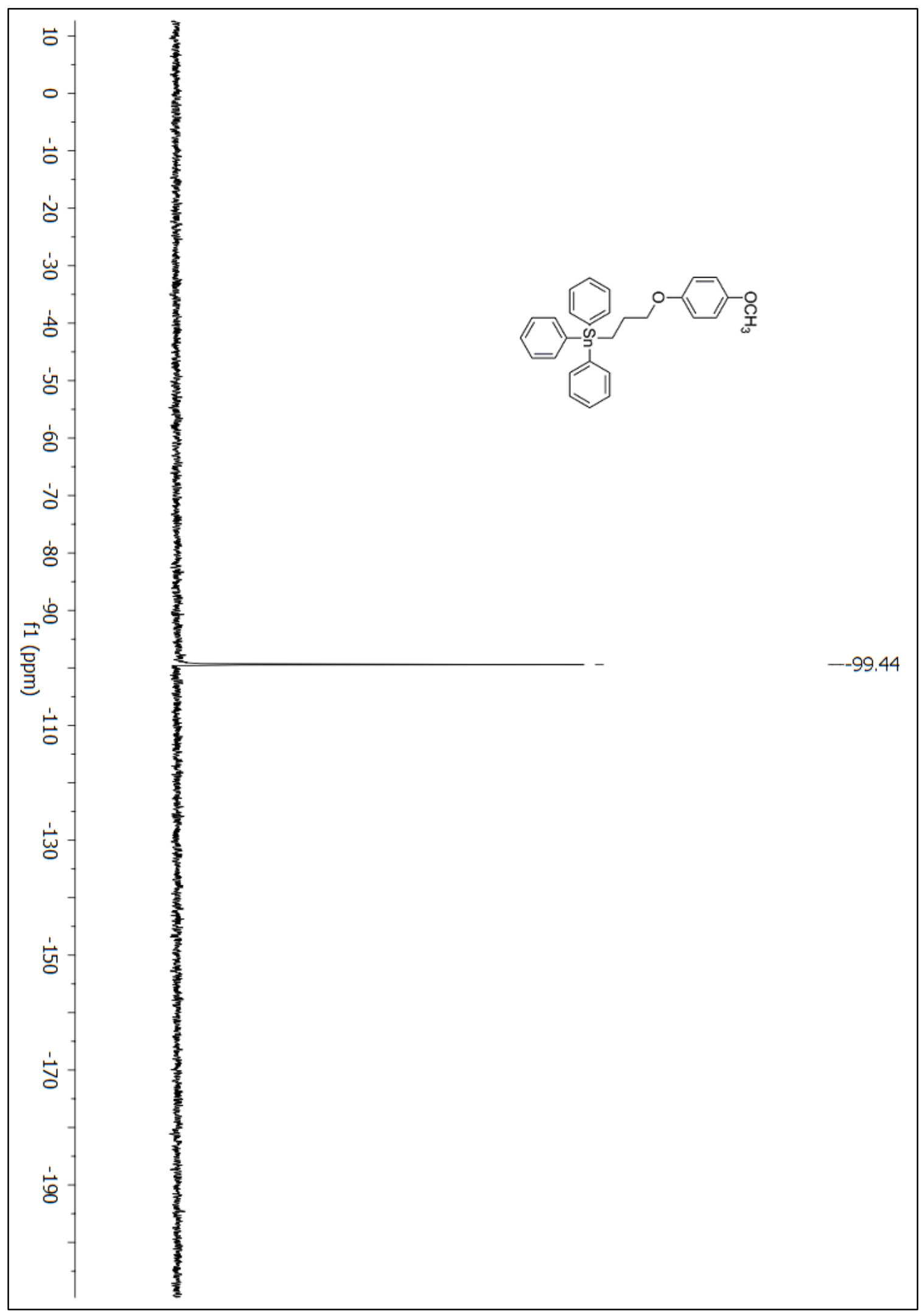

Figure A 24: ${ }^{119} \mathrm{Sn}$ NMR $\left(\mathrm{CDCl}_{3}\right)$ spectrum of compound 199. 


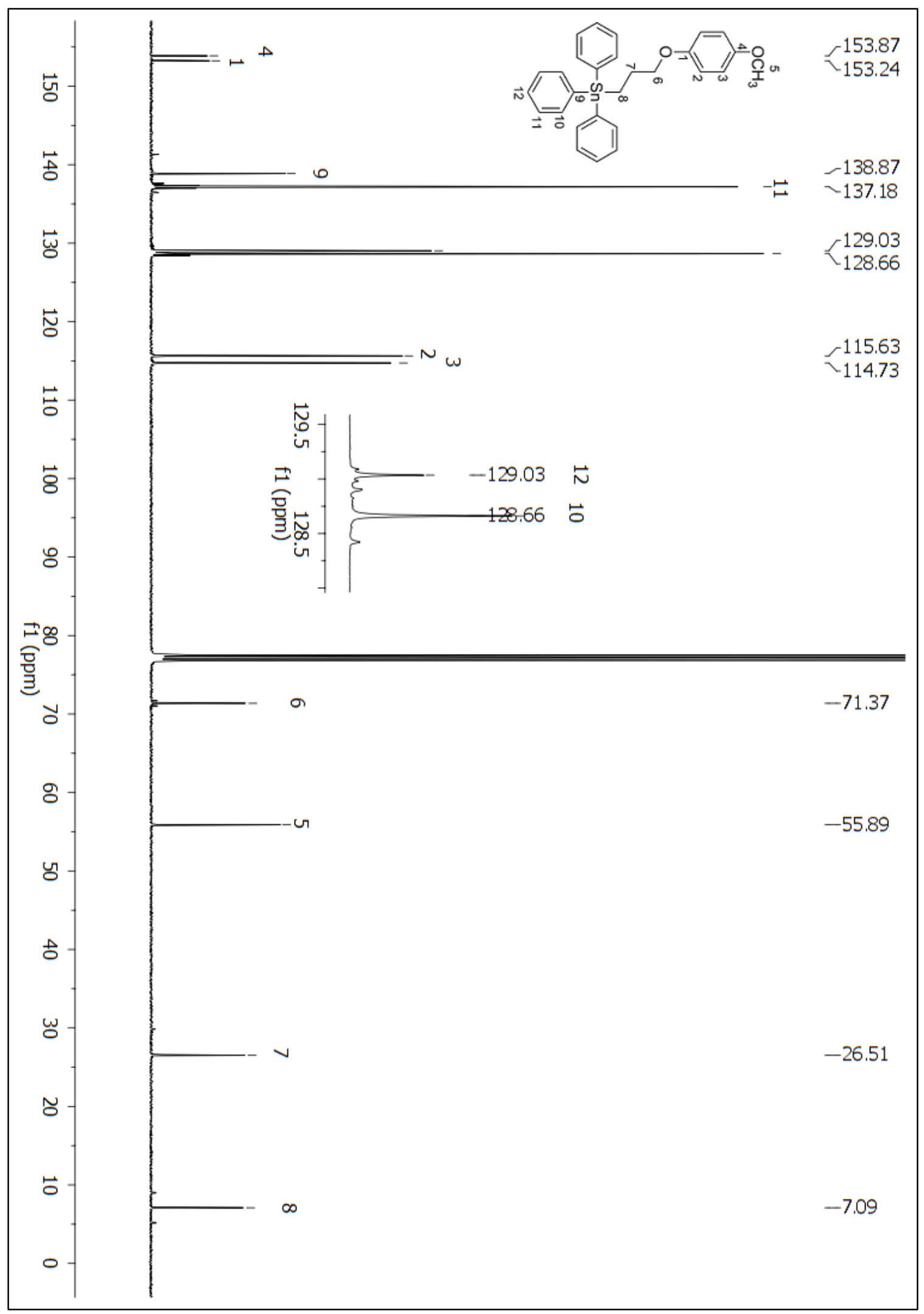

Figure A 25: ${ }^{13} \mathrm{C}$ NMR $\left(\mathrm{CDCl}_{3}\right)$ spectrum of compound 199. 


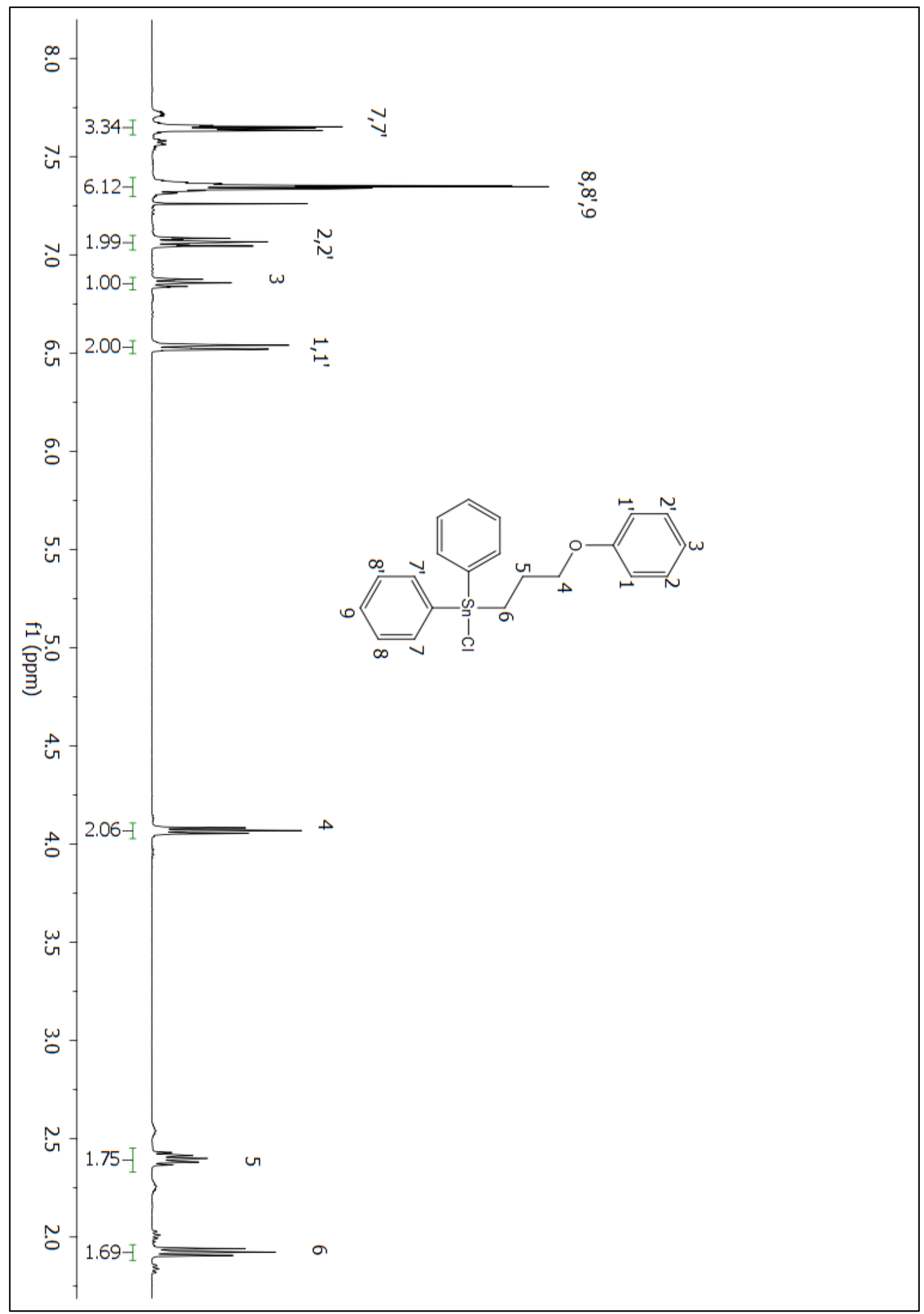

Figure A 26: ${ }^{1} \mathrm{H}$ NMR $\left(\mathrm{CDCl}_{3}\right)$ spectrum of compound 200. 


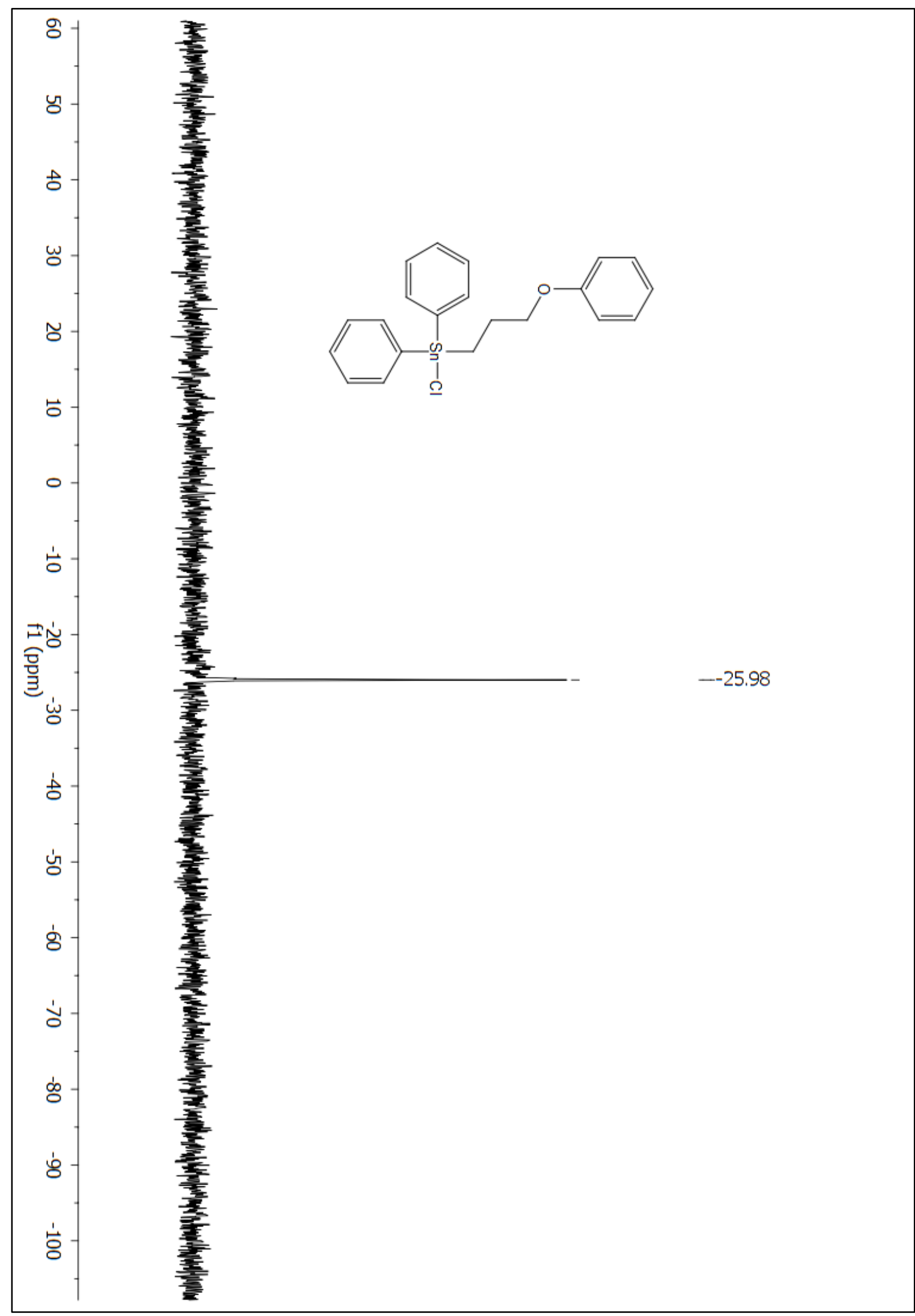

Figure A 27: ${ }^{119} \mathrm{Sn}$ NMR $\left(\mathrm{CDCl}_{3}\right)$ spectrum of compound 200. 


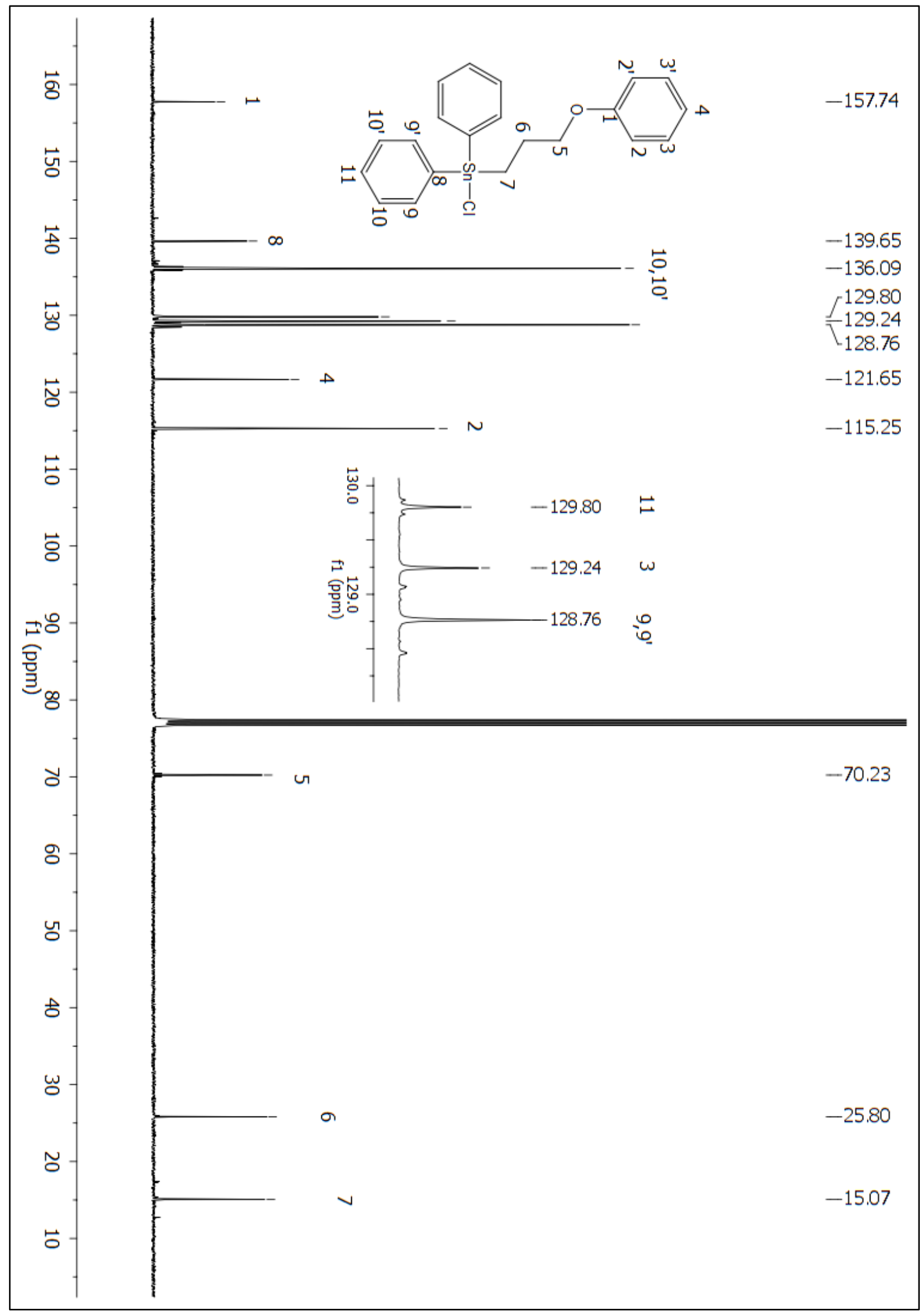

Figure A 28: ${ }^{13} \mathrm{C}$ NMR $\left(\mathrm{CDCl}_{3}\right)$ spectrum of compound 200. 


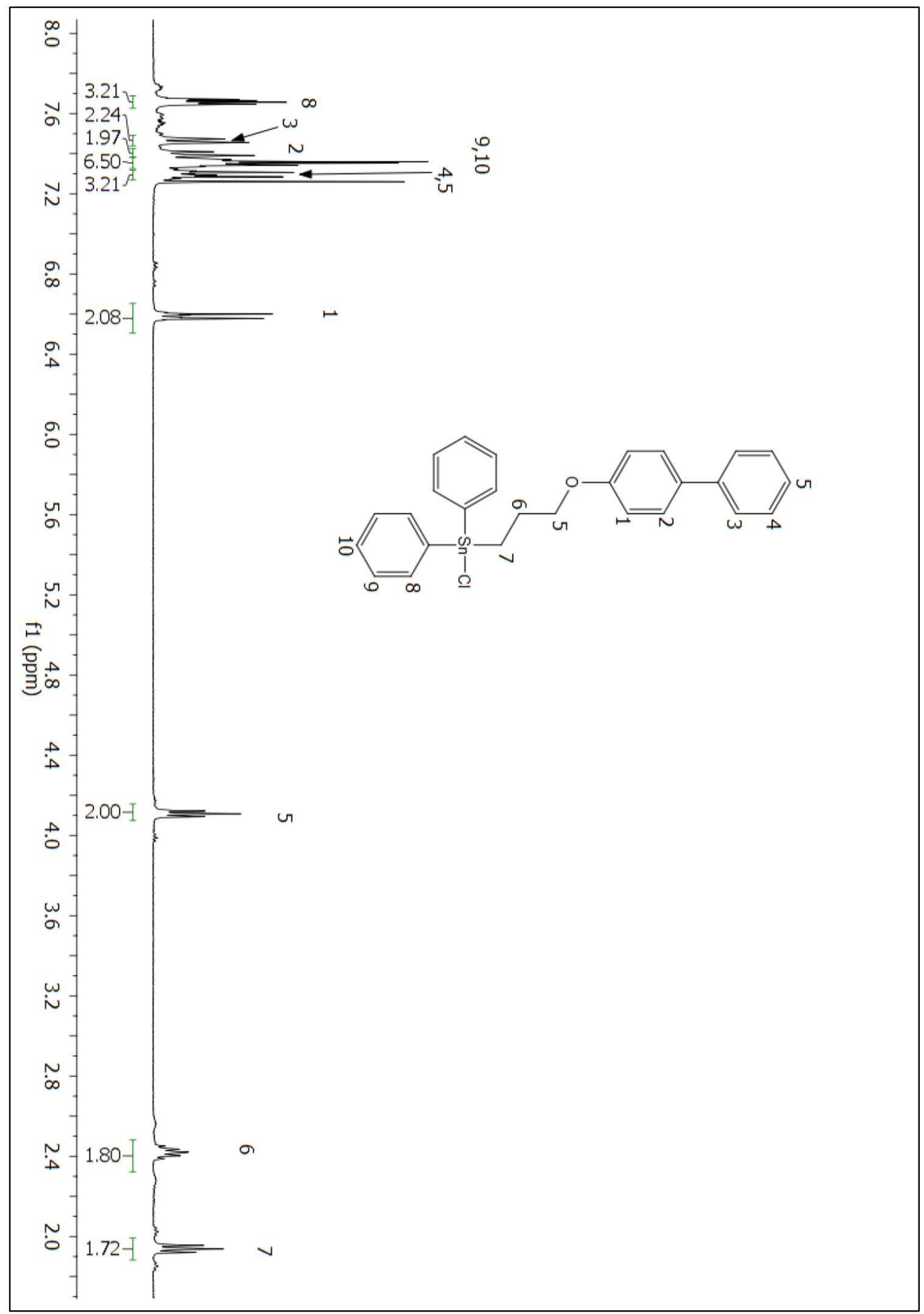

Figure A 29: ${ }^{1} \mathrm{H}$ NMR $\left(\mathrm{CDCl}_{3}\right)$ spectrum of compound 201. 


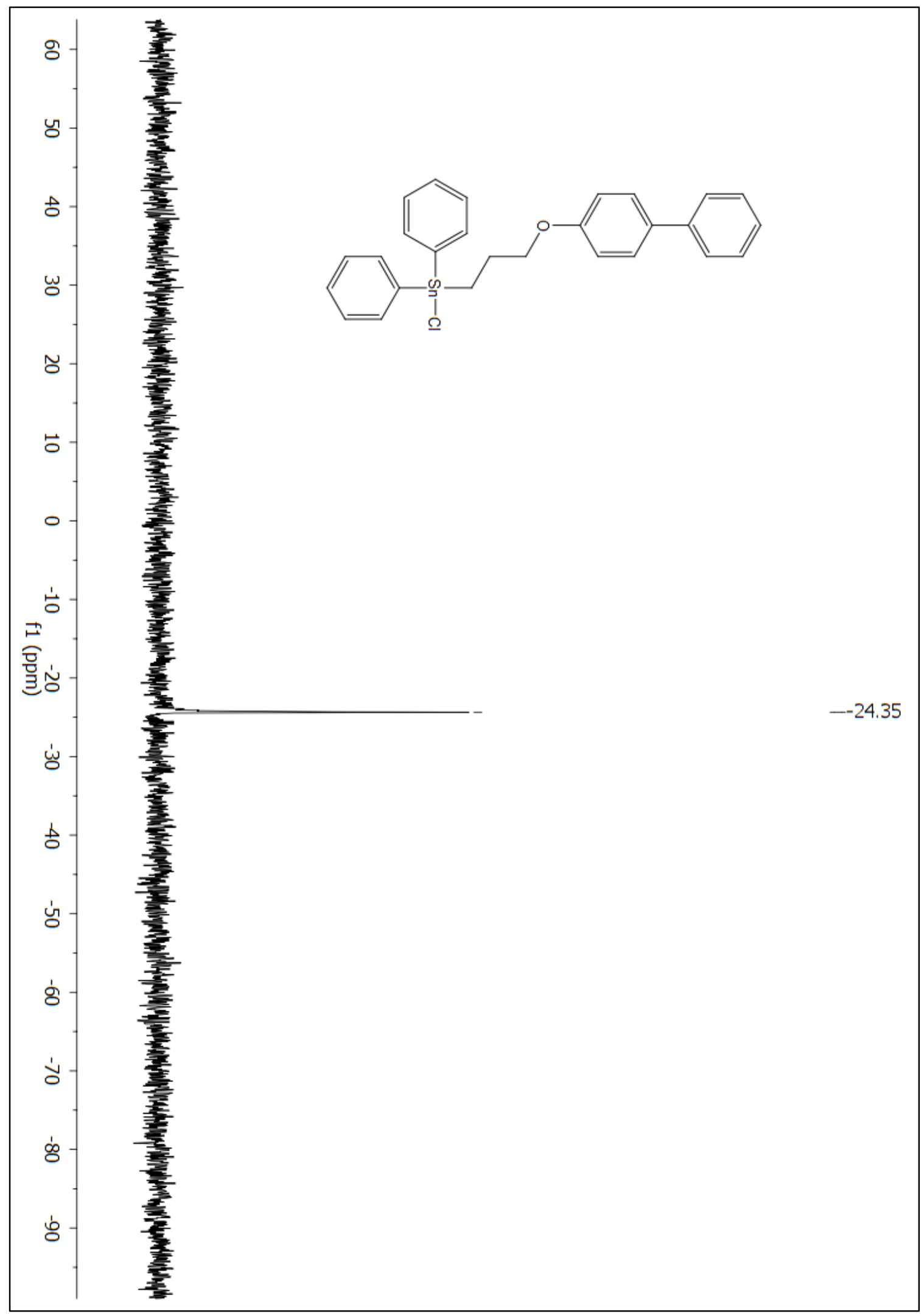

Figure A 30: ${ }^{119} \mathrm{Sn} \mathrm{NMR}\left(\mathrm{CDCl}_{3}\right)$ spectrum of compound 201. 


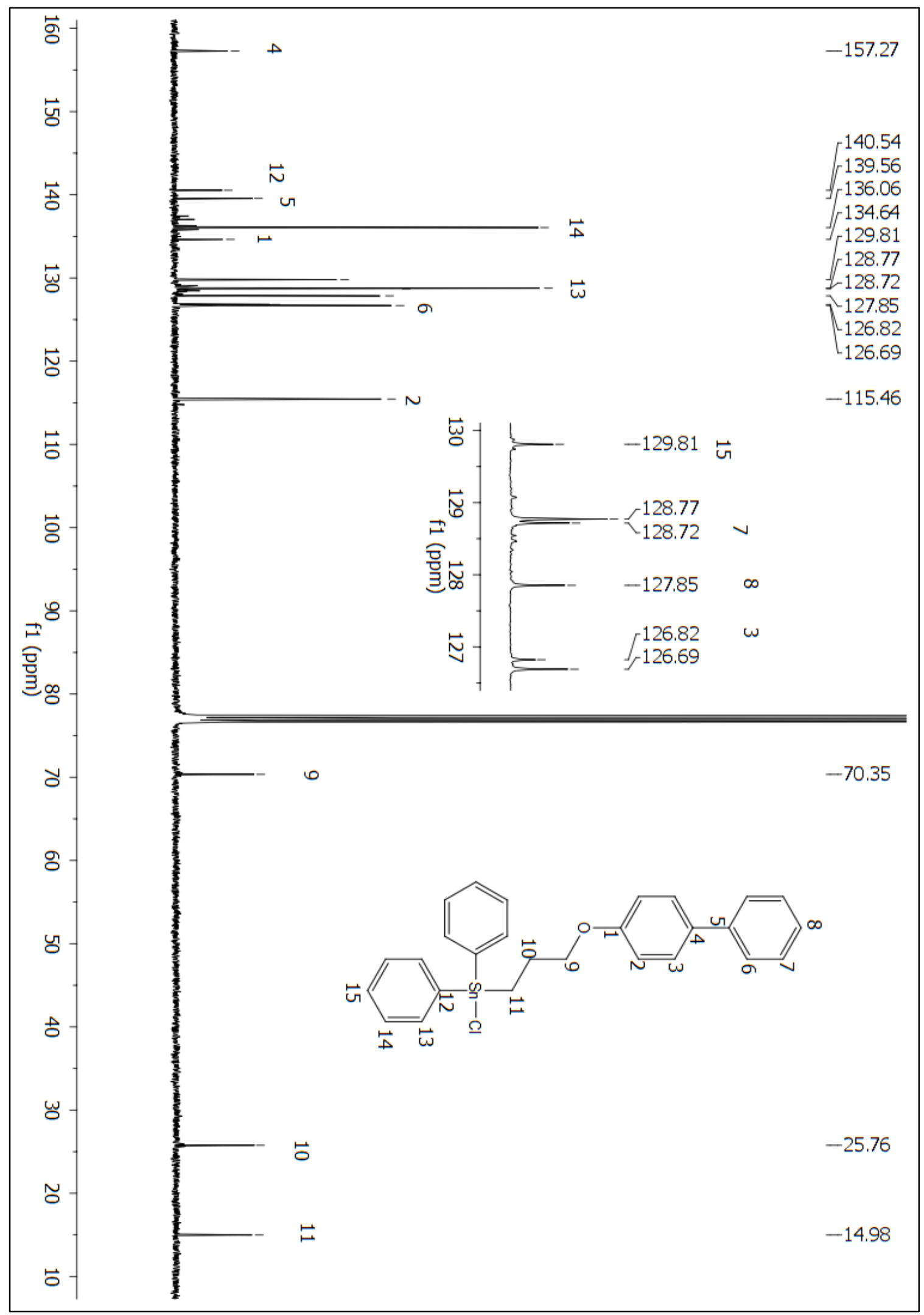

Figure A 31: ${ }^{13} \mathrm{C}$ NMR $\left(\mathrm{CDCl}_{3}\right)$ spectrum of compound 201. 


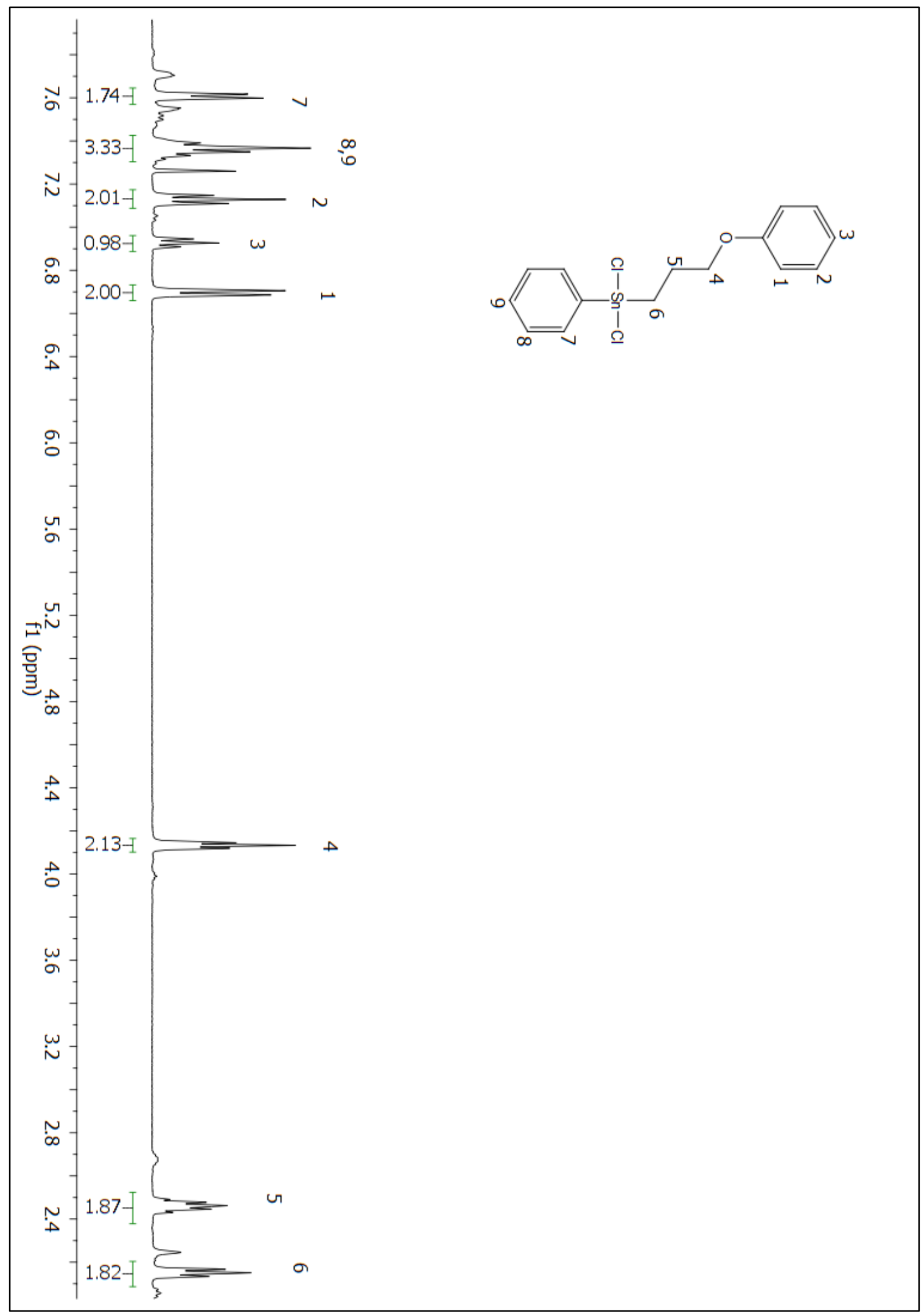

Figure A 32: ${ }^{1} \mathrm{H}$ NMR $\left(\mathrm{CDCl}_{3}\right)$ spectrum of compound 202. 


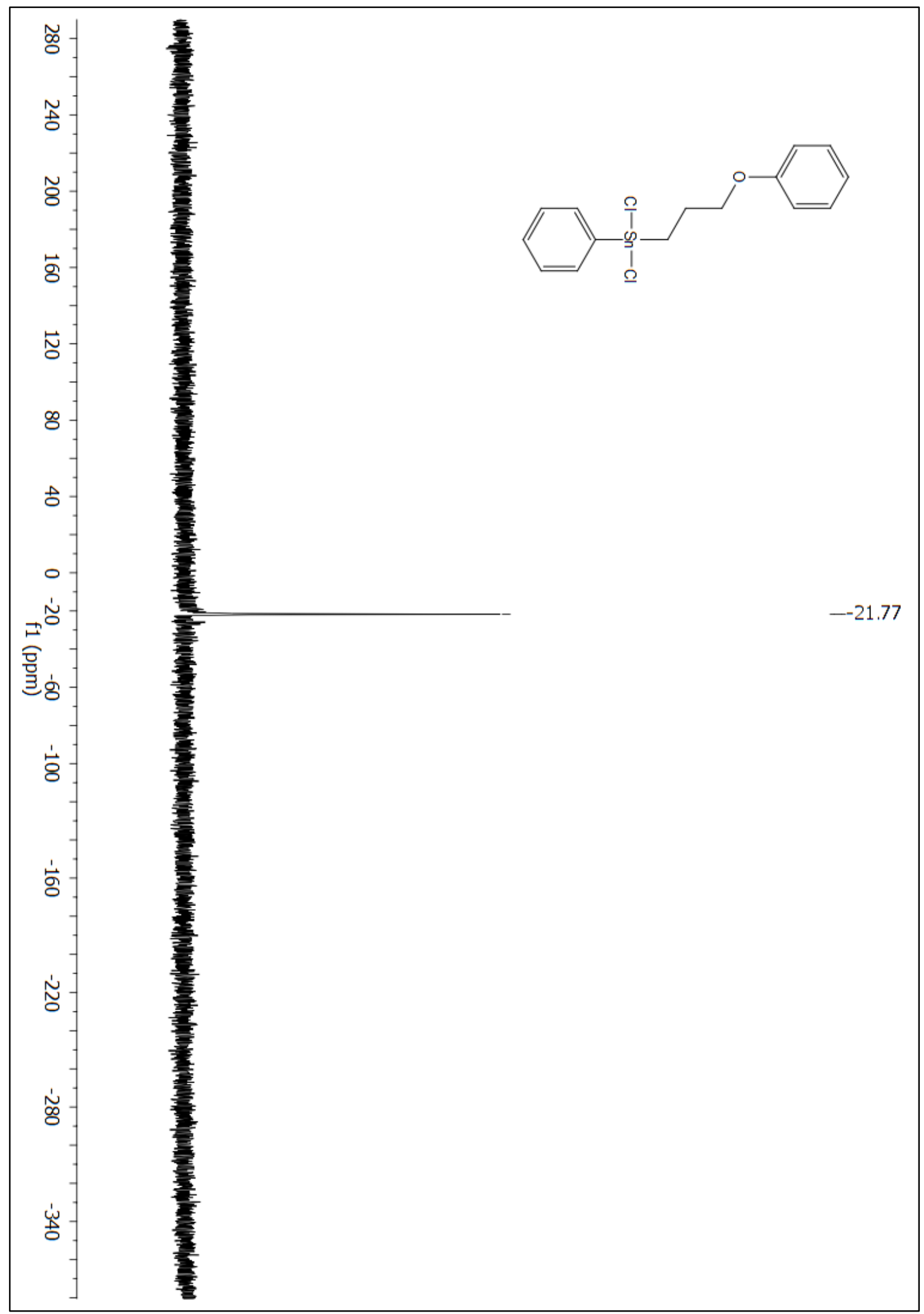

Figure A 33: ${ }^{119} \mathrm{Sn} \mathrm{NMR}\left(\mathrm{CDCl}_{3}\right)$ spectrum of compound 202. 


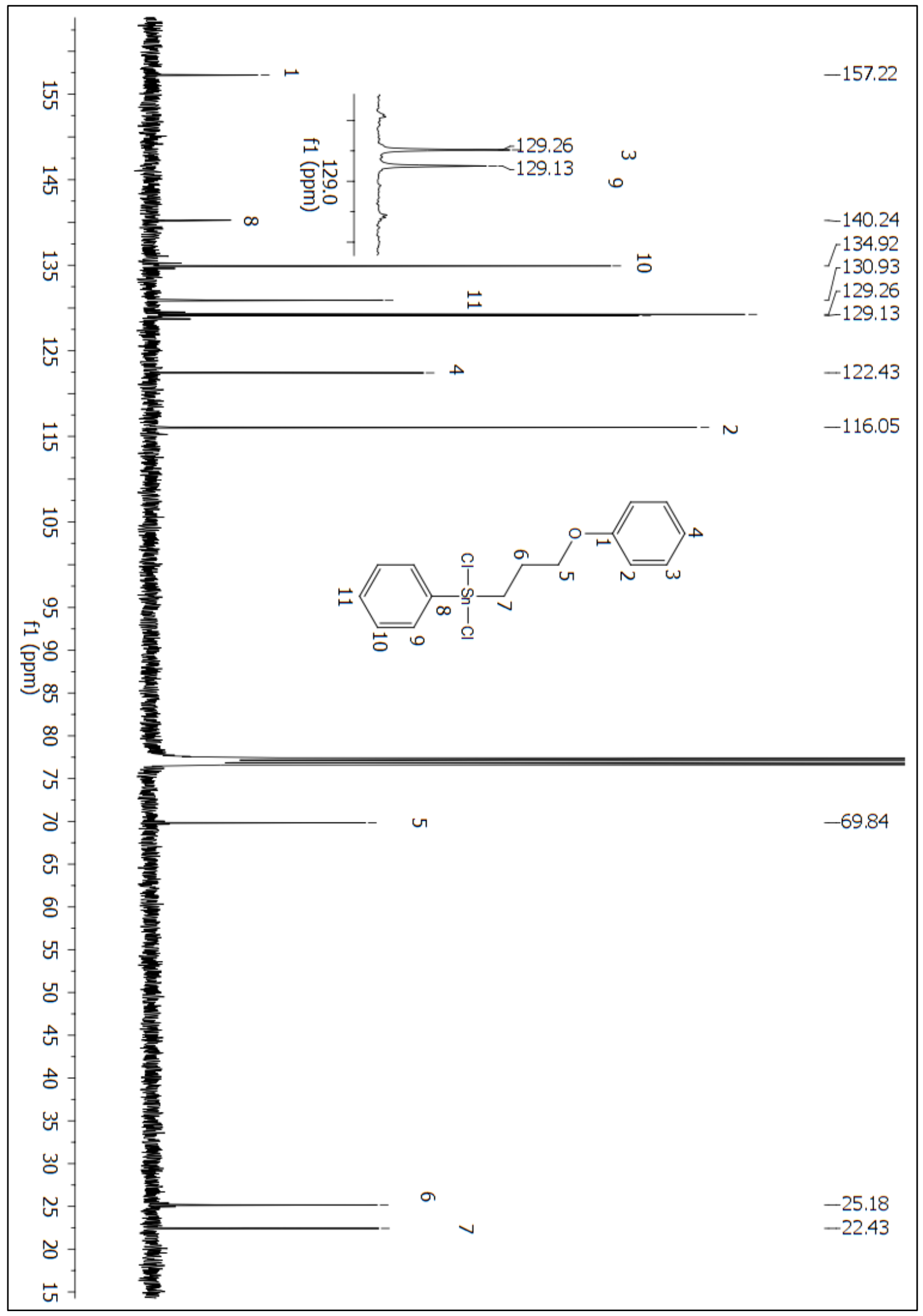

Figure A 34: ${ }^{13} \mathrm{C}$ NMR $\left(\mathrm{CDCl}_{3}\right)$ spectrum of compound 202. 


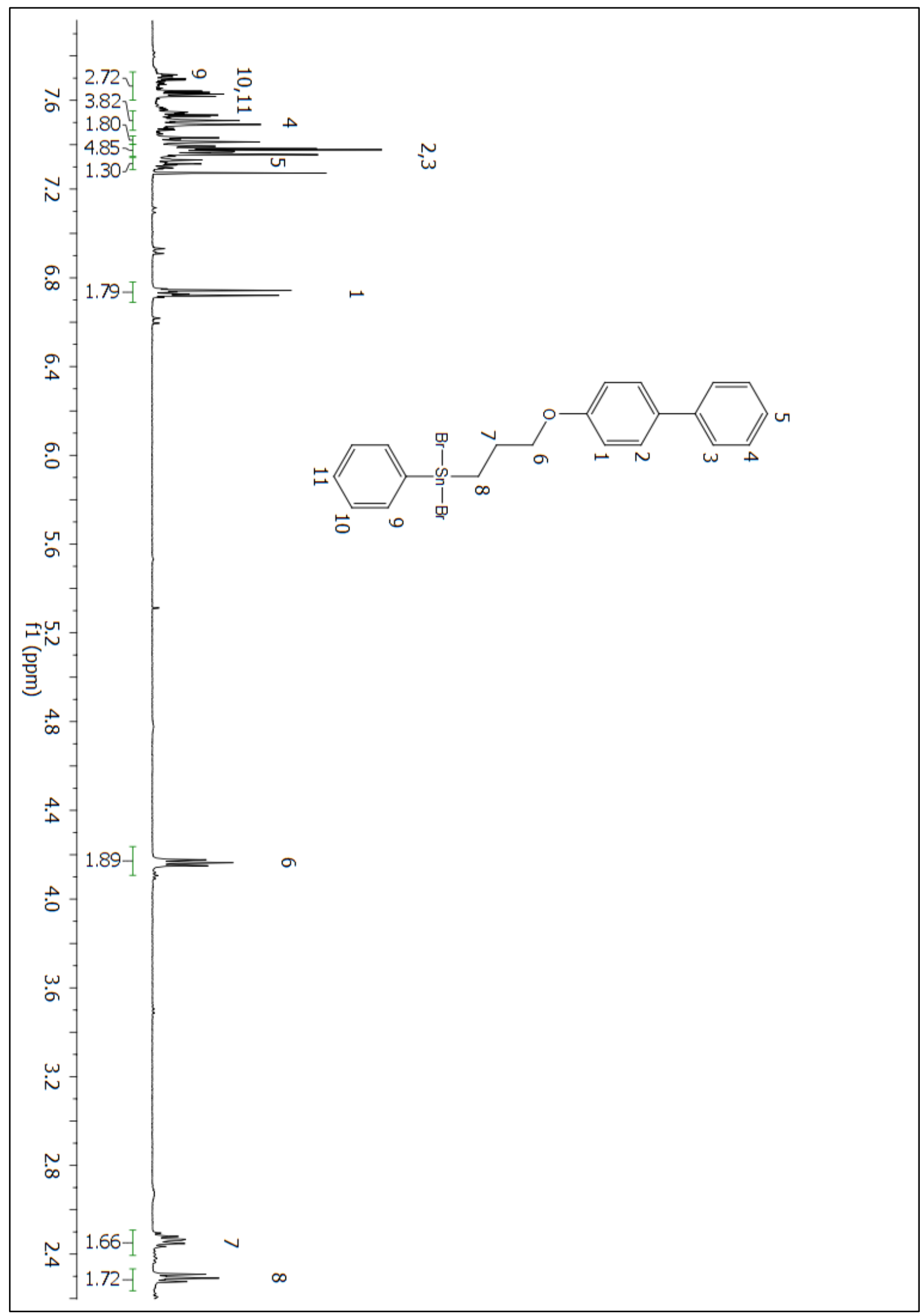

Figure A 35: ${ }^{1} \mathrm{H}$ NMR $\left(\mathrm{CDCl}_{3}\right)$ spectrum of compound 146. 


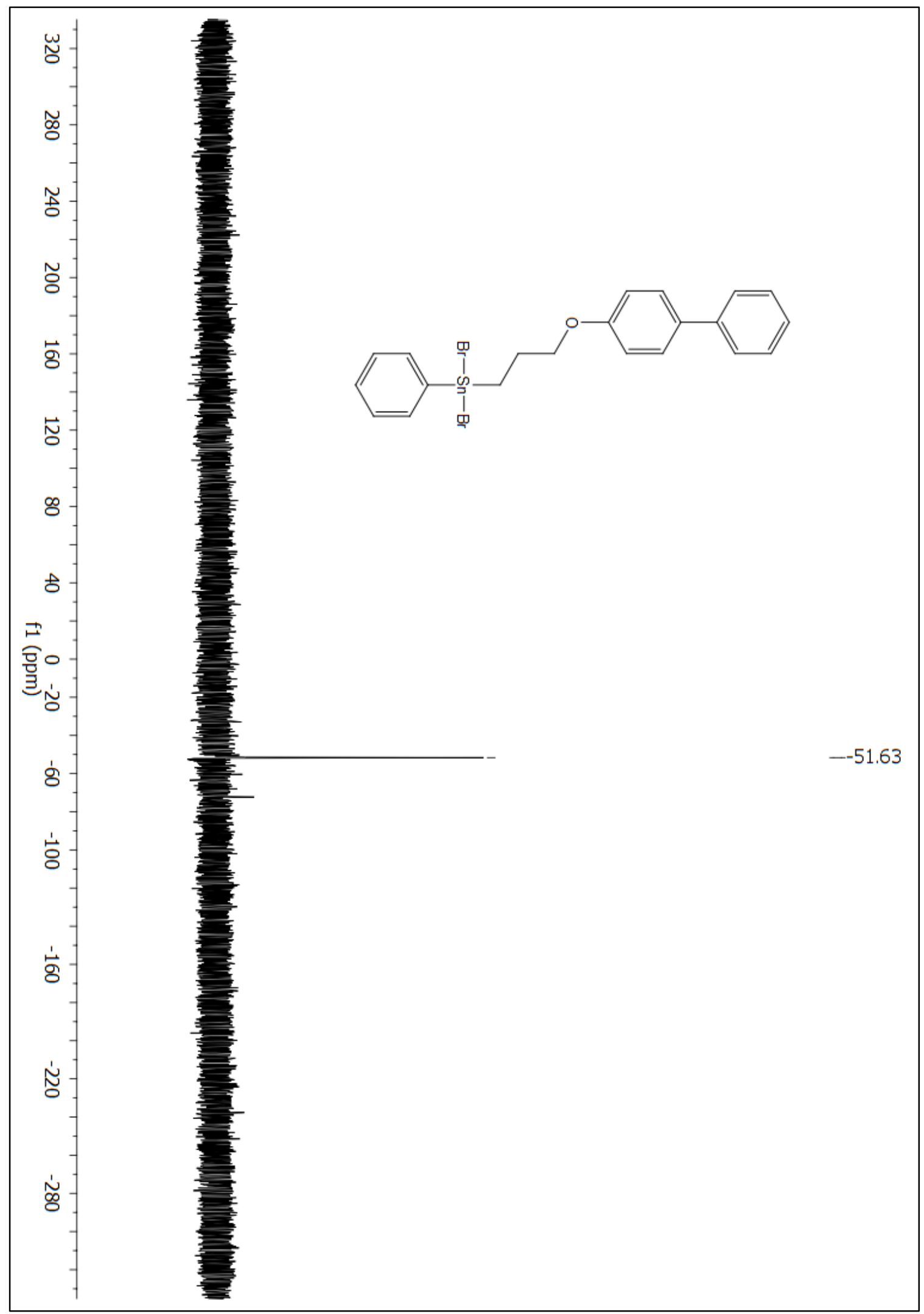

Figure A 36: ${ }^{119} \mathrm{Sn}$ NMR $\left(\mathrm{CDCl}_{3}\right)$ spectrum of compound 146. 


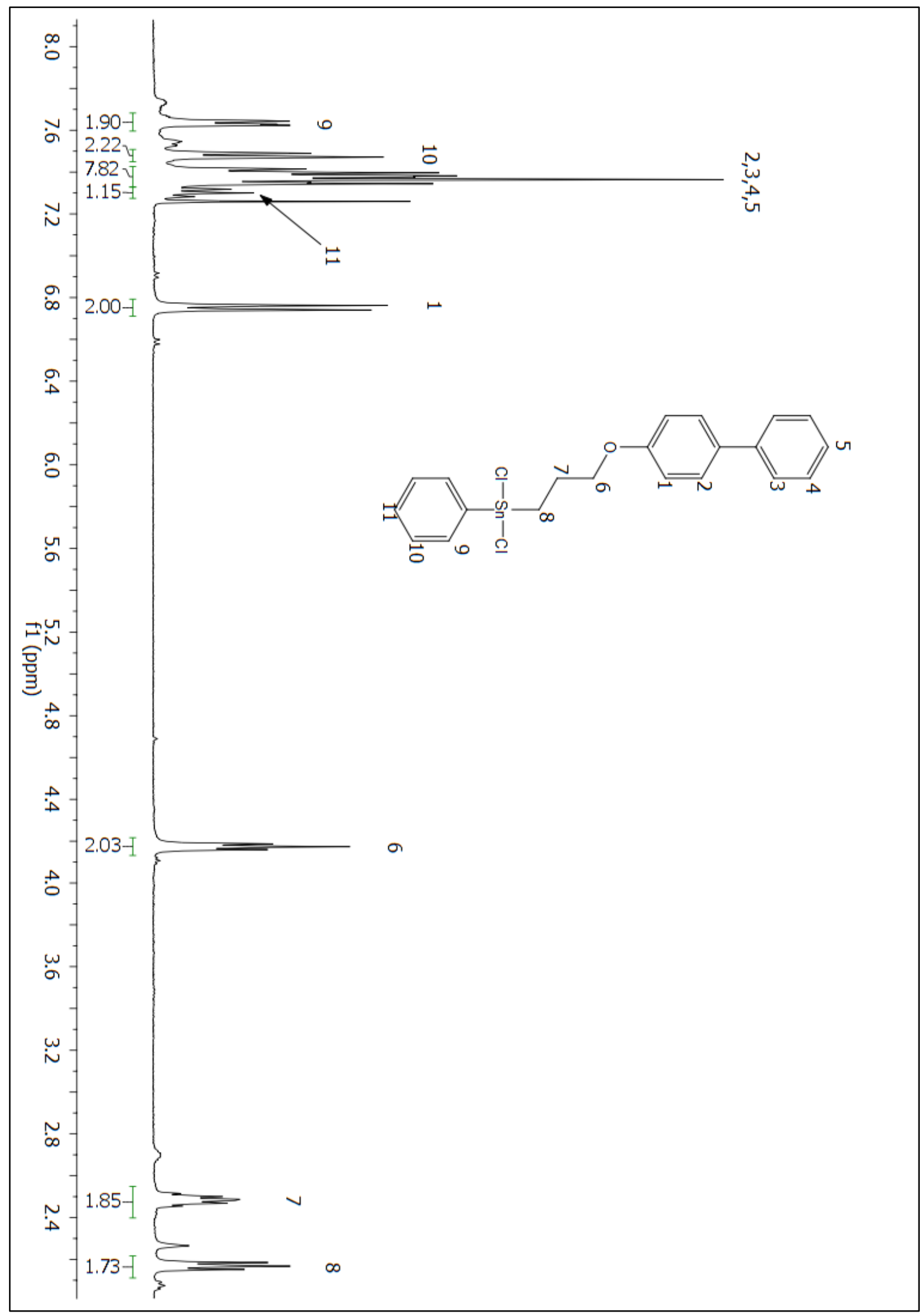

Figure A 37: ${ }^{1} \mathrm{H}$ NMR $\left(\mathrm{CDCl}_{3}\right)$ spectrum of compound 203. 


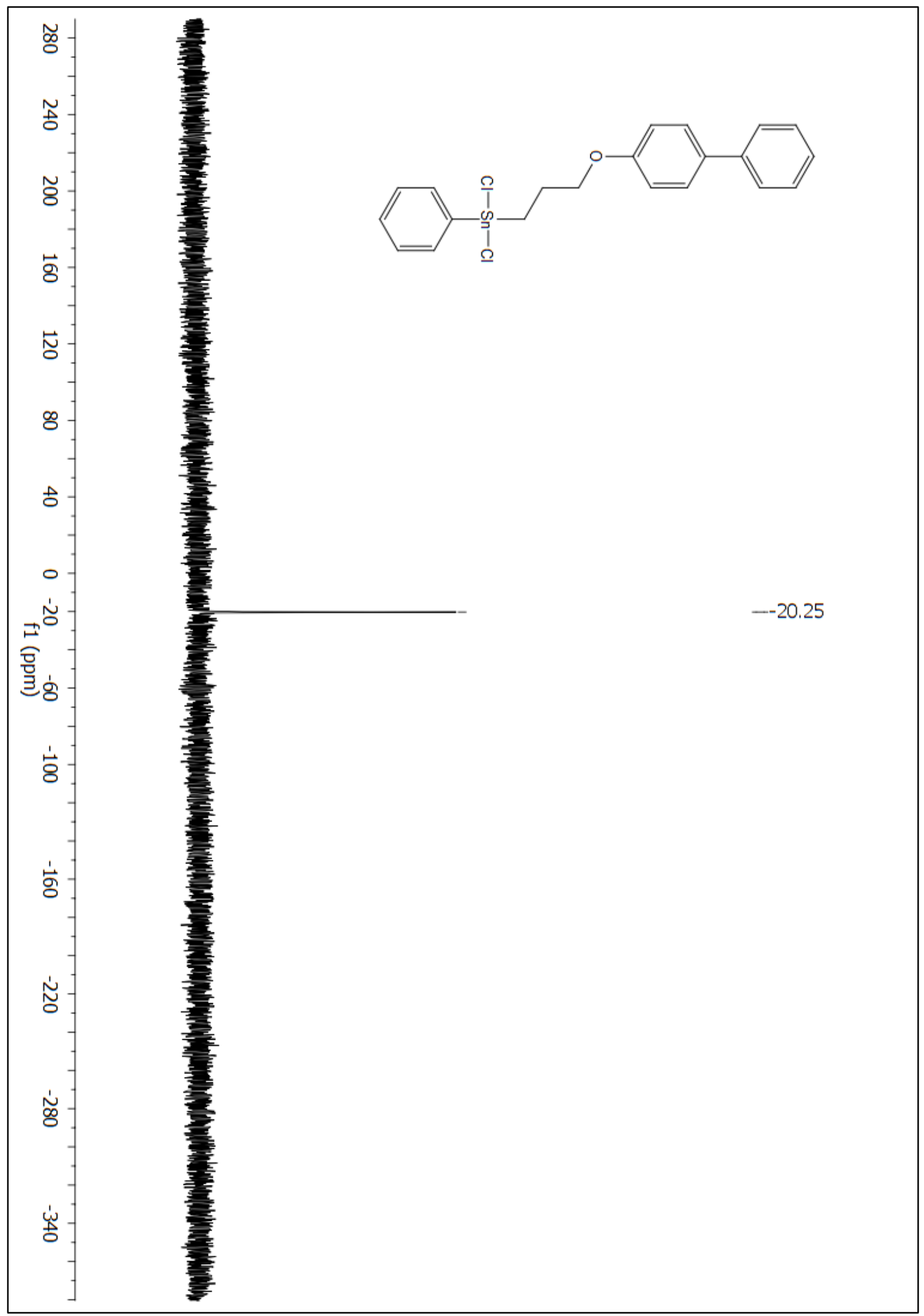

Figure A 38: ${ }^{119} \mathrm{Sn}$ NMR $\left(\mathrm{CDCl}_{3}\right)$ spectrum of compound 203. 


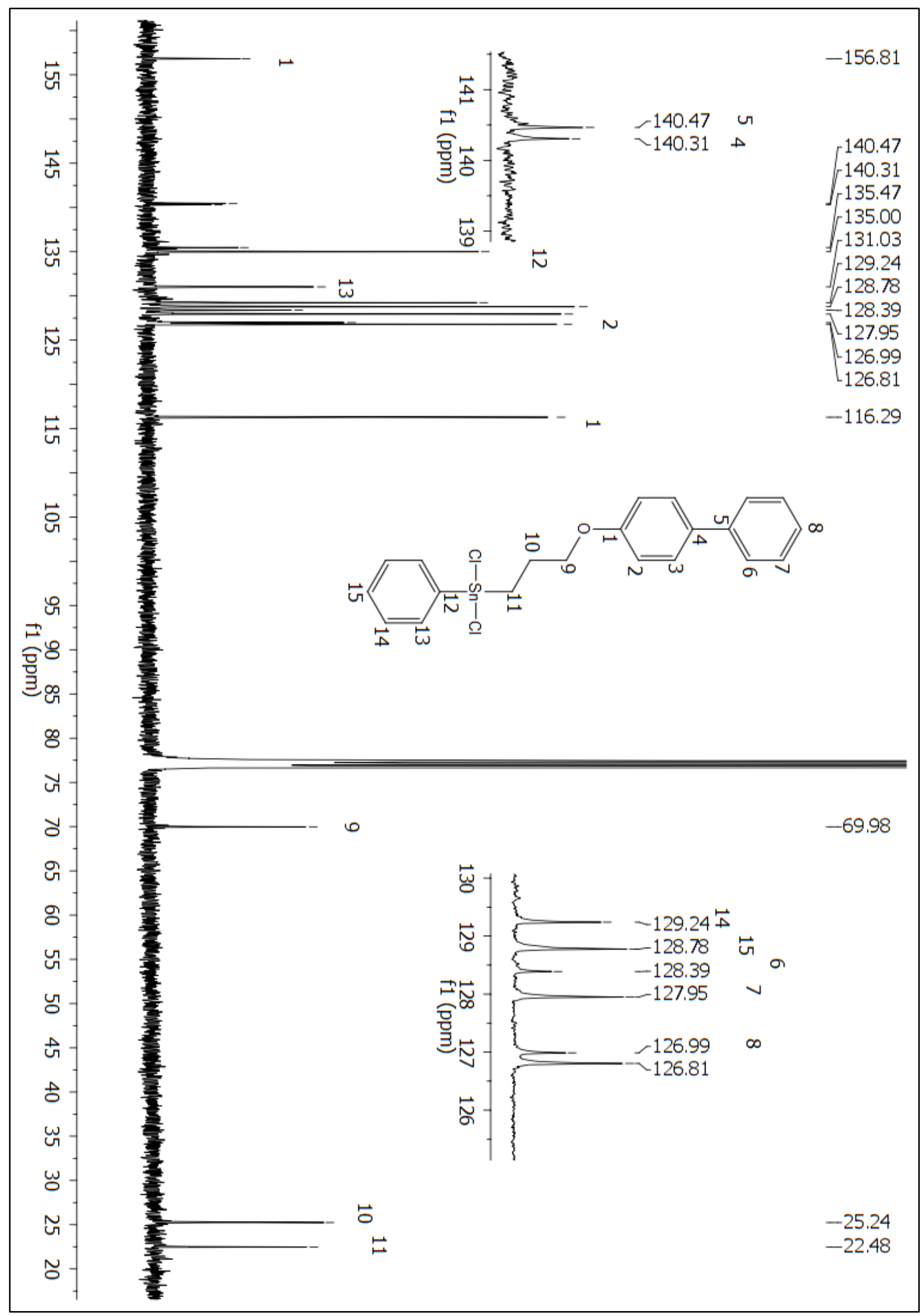

Figure A 39: ${ }^{13} \mathrm{C}$ NMR $\left(\mathrm{CDCl}_{3}\right)$ spectrum of compound 203. 


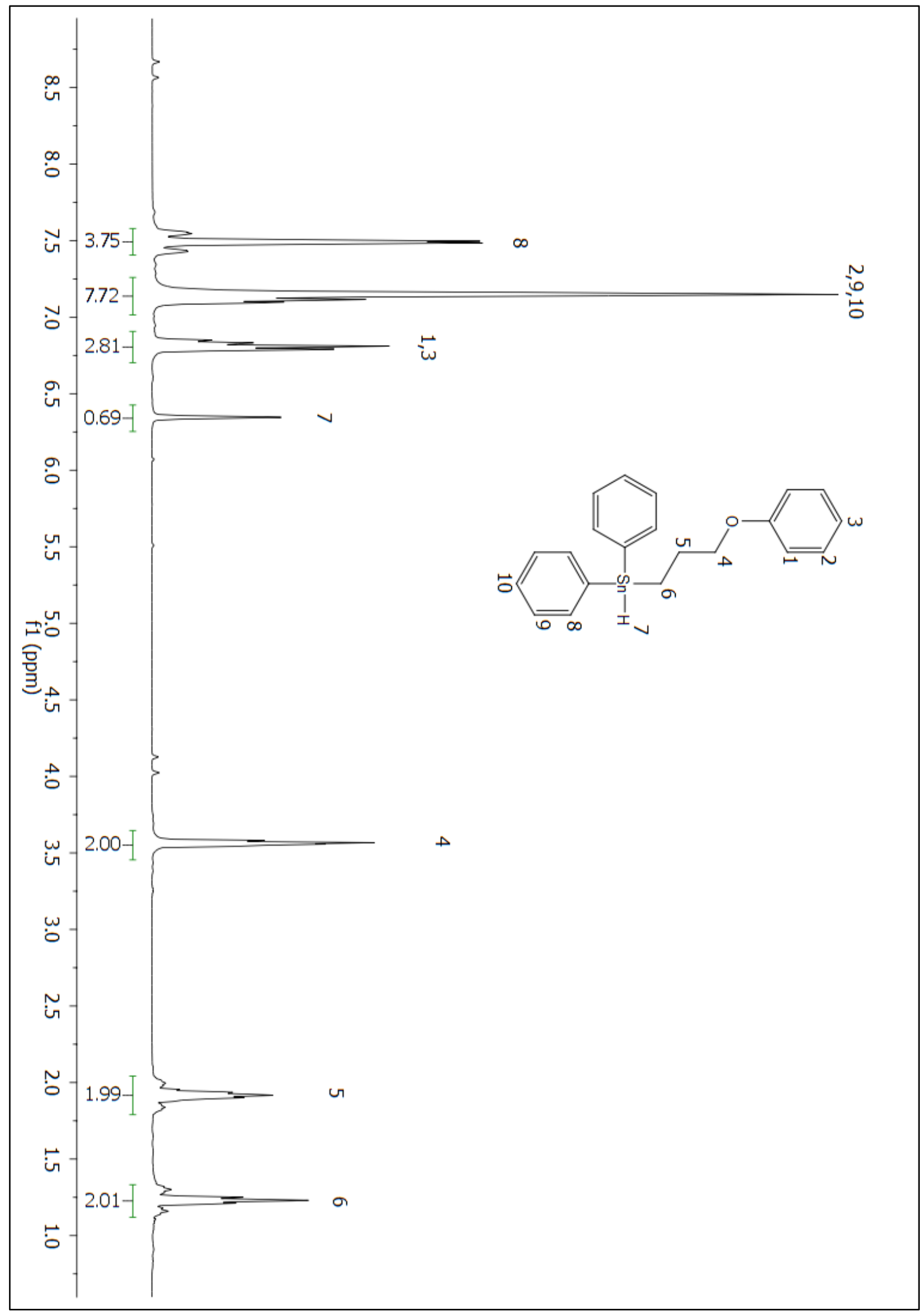

Figure A 40: ${ }^{1} \mathrm{H}$ NMR ( $\left.{ }_{6} D_{6}\right)$ spectrum of compound 204. 


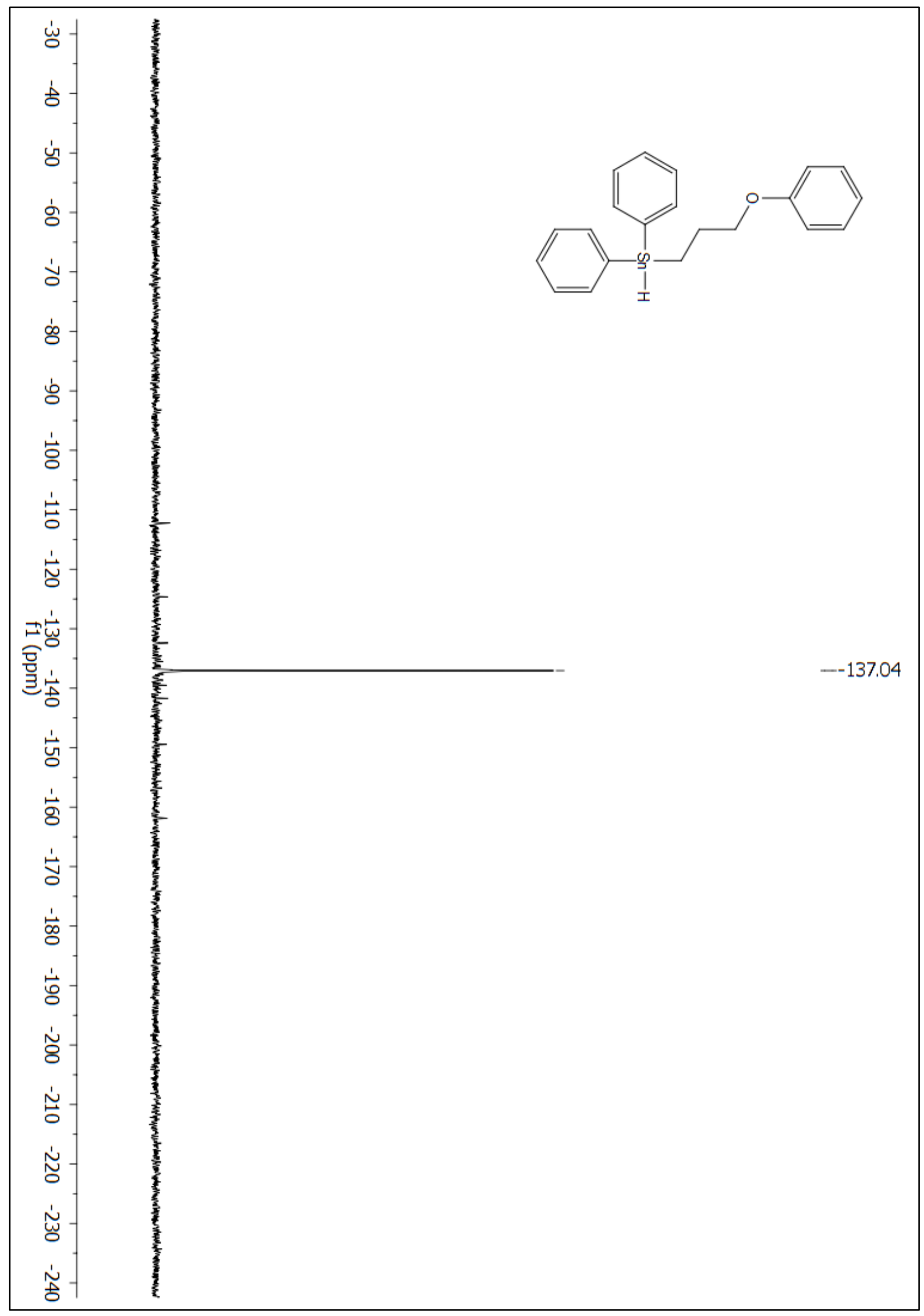

Figure A 41: ${ }^{119} \mathrm{Sn}$ NMR $\left(\mathrm{C}_{6} \mathrm{D}_{6}\right)$ spectrum of compound 204. 


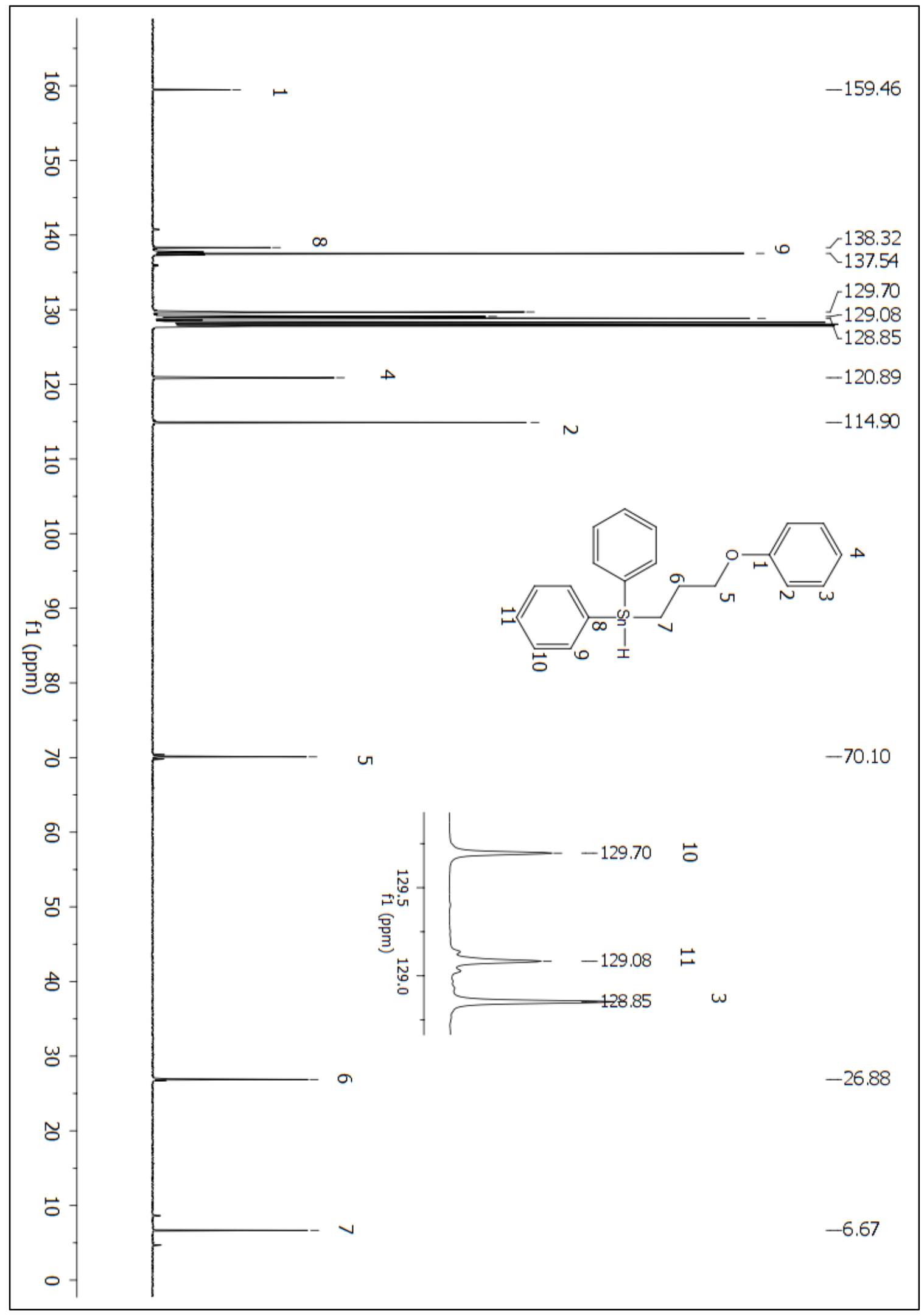

Figure A 42: ${ }^{13} \mathrm{C}$ NMR ( $\left.\mathrm{C}_{6} \mathrm{D}_{6}\right)$ spectrum of compound 204. 


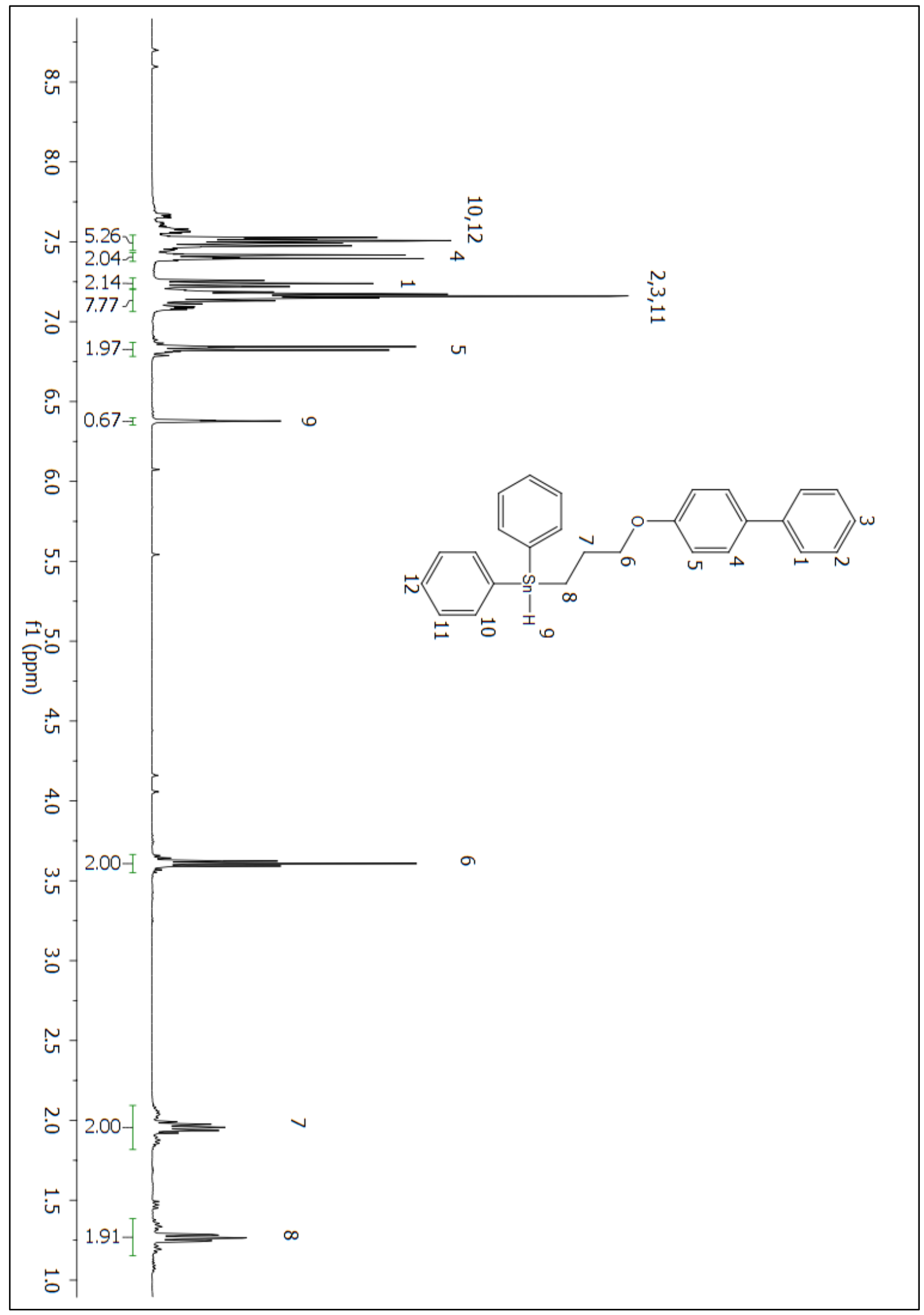

Figure A 43: ${ }^{1} \mathrm{H}$ NMR (C6D6) spectrum of compound 205. 


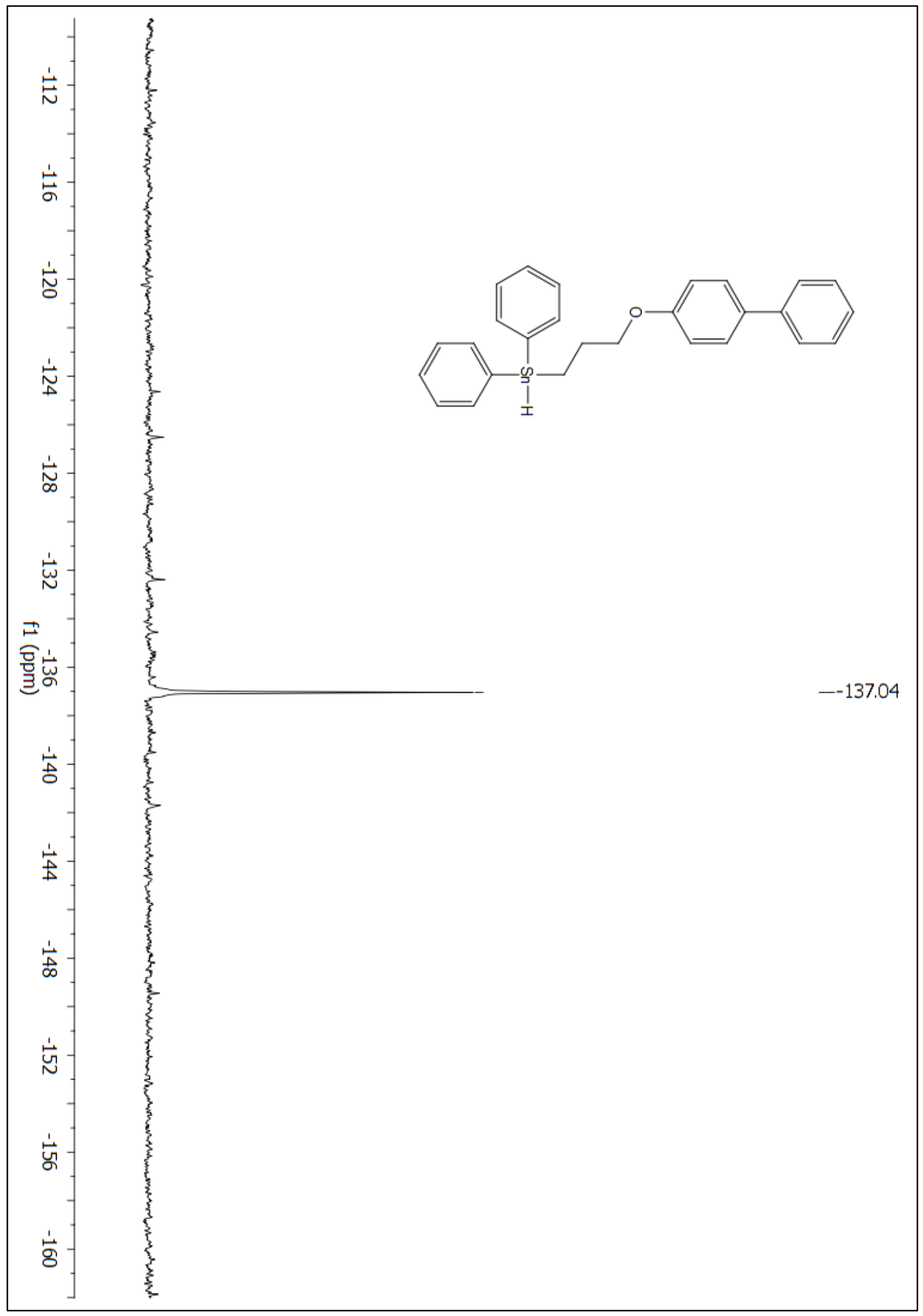

Figure A 44: ${ }^{119} \mathrm{Sn}$ NMR $\left(\mathrm{C}_{6} \mathrm{D}_{6}\right)$ spectrum of compound 205. 


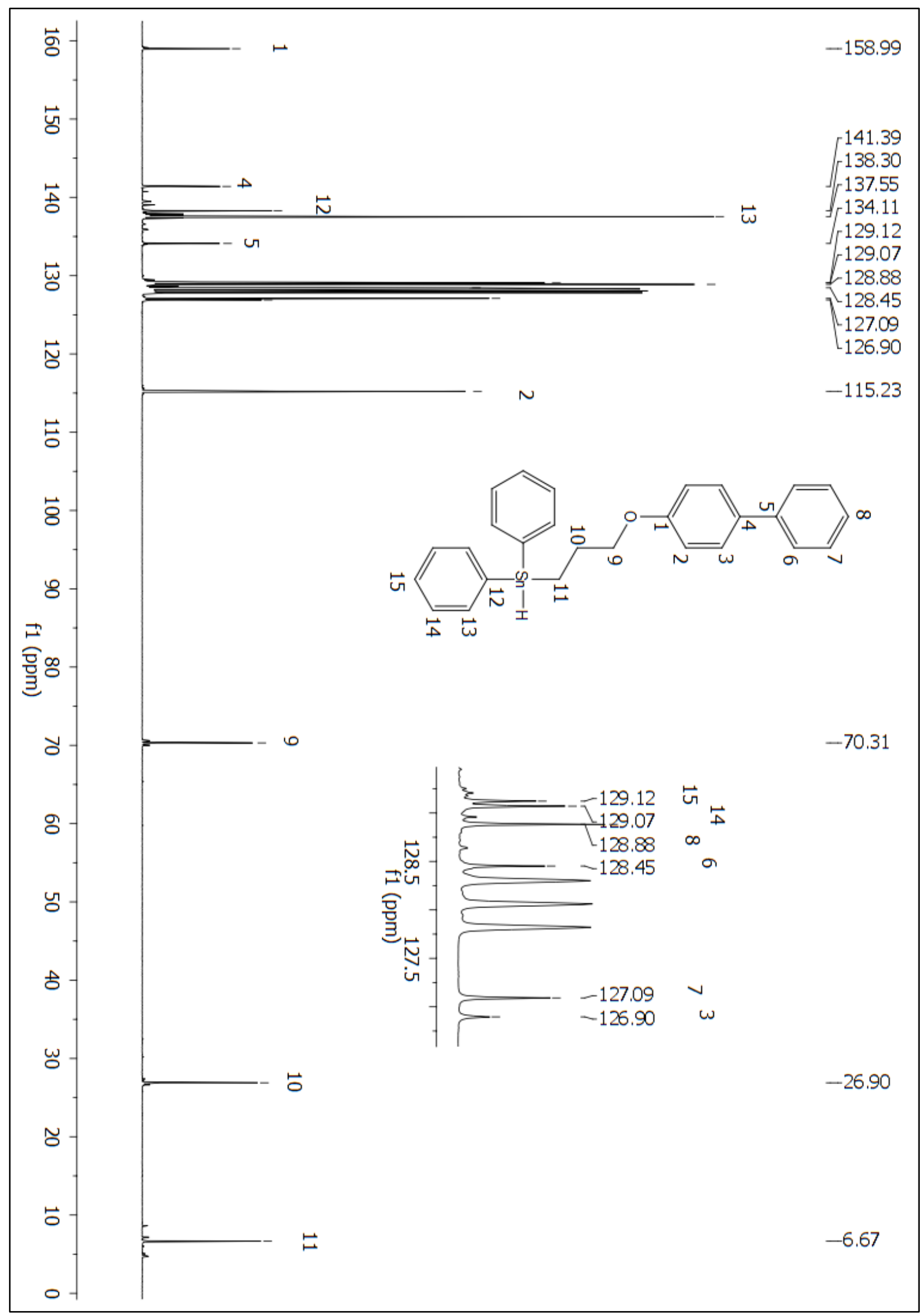

Figure A 45: ${ }^{13} \mathrm{C}$ NMR ( $\left.\mathrm{C}_{6} \mathrm{D}_{6}\right)$ spectrum of compound 205. 


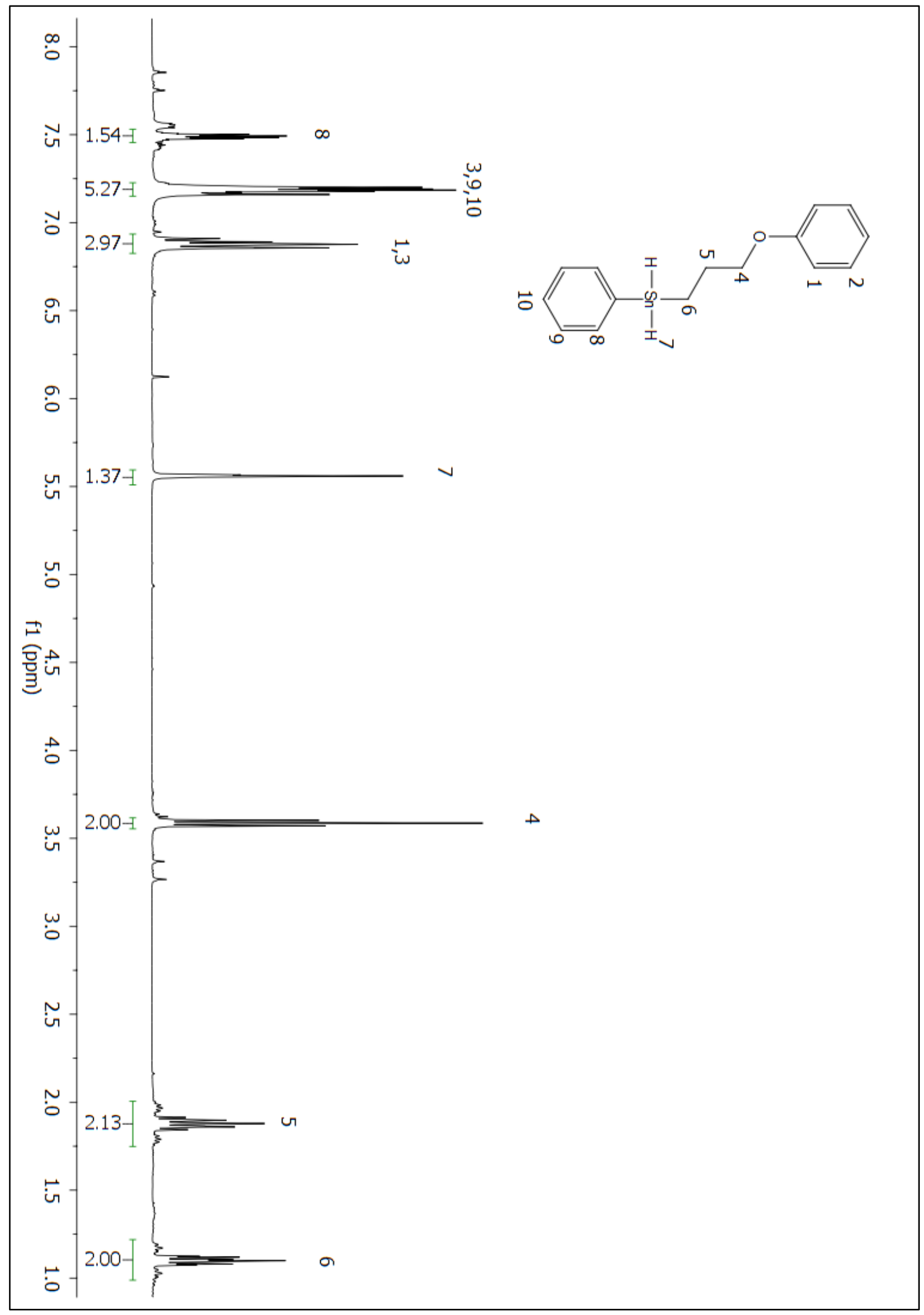

Figure A 46: ${ }^{1} \mathrm{H}$ NMR $\left(\mathrm{C}_{6} \mathrm{D}_{6}\right)$ spectrum of compound 206. 


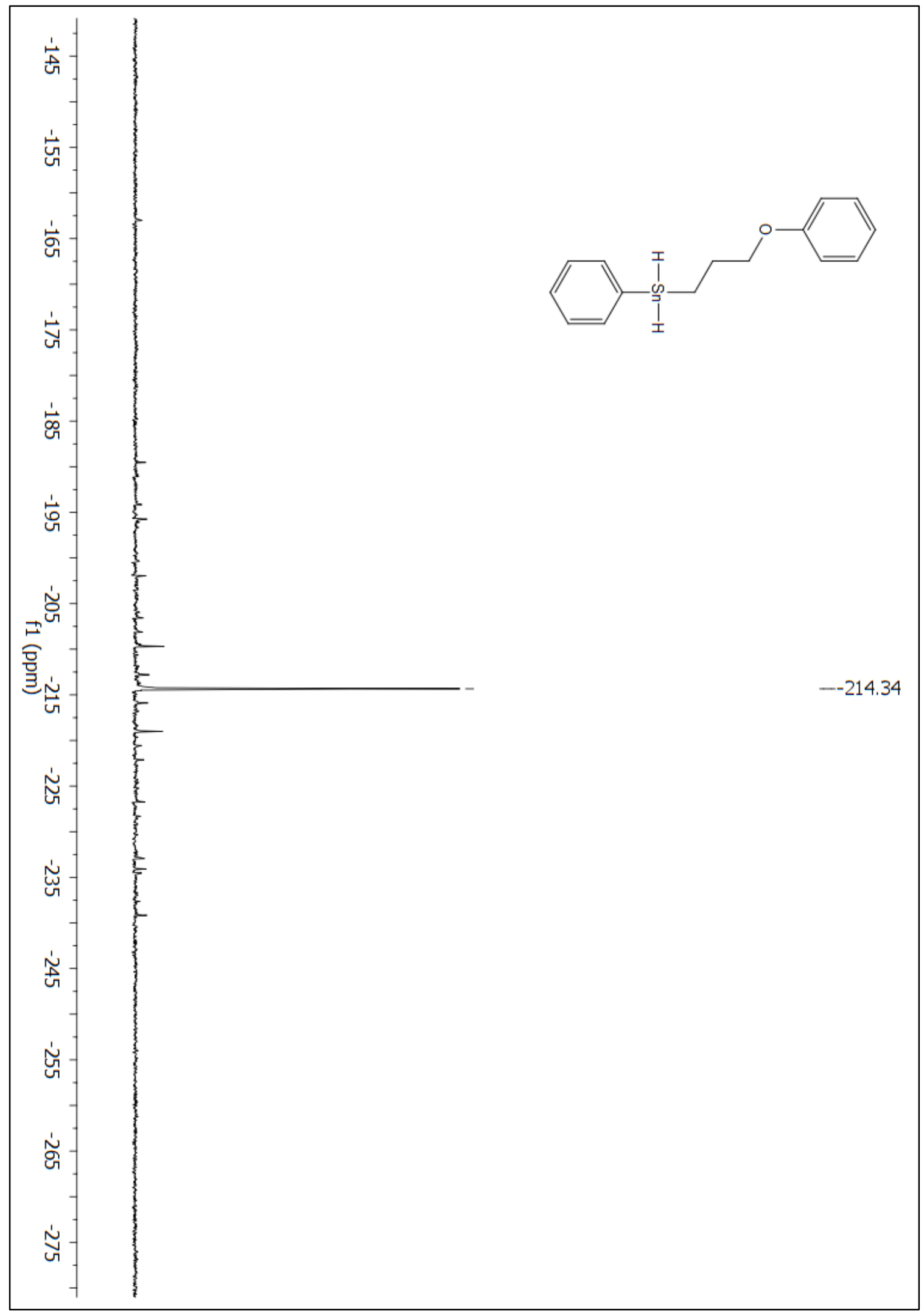

Figure A 47: ${ }^{119} \mathrm{Sn}$ NMR $\left(\mathrm{C}_{6} \mathrm{D}_{6}\right)$ spectrum of compound 206. 


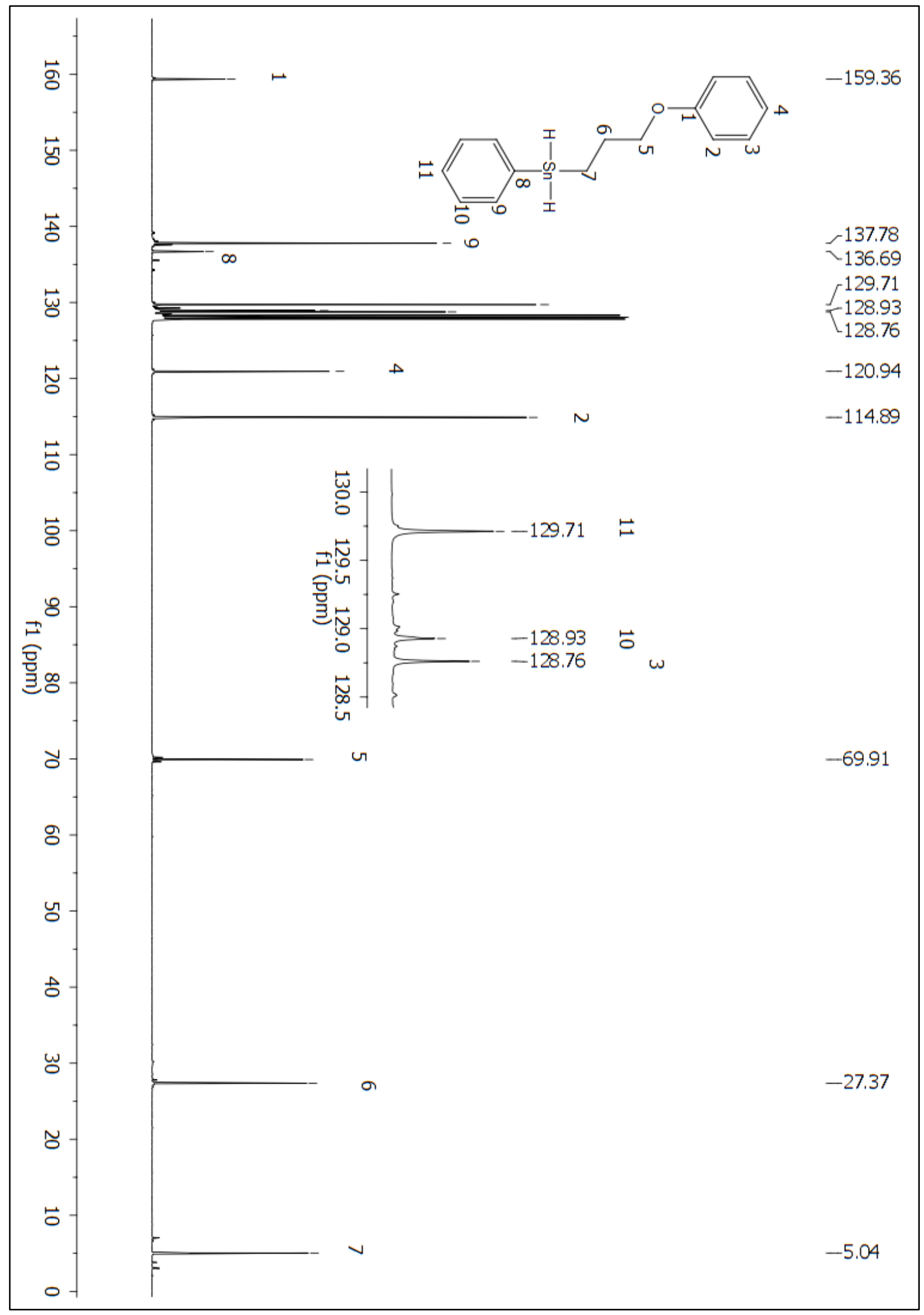

Figure A 48: ${ }^{13} \mathrm{C}$ NMR $\left(\mathrm{C}_{6} \mathrm{D}_{6}\right)$ spectrum of compound 206. 


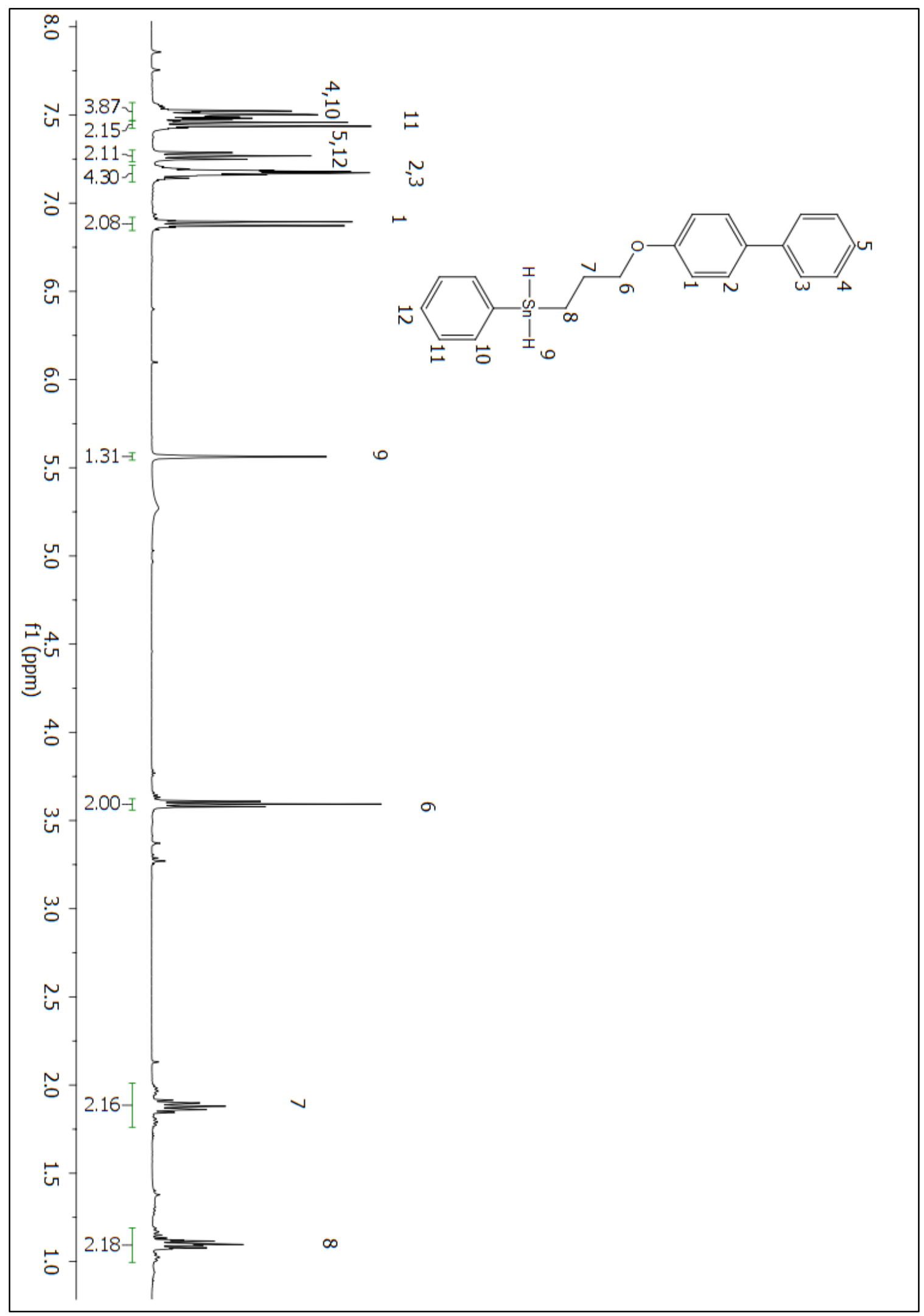

Figure A 49: ${ }^{1} \mathrm{H}$ NMR $\left(\mathrm{C}_{6} \mathrm{D}_{6}\right)$ spectrum of compound 207. 


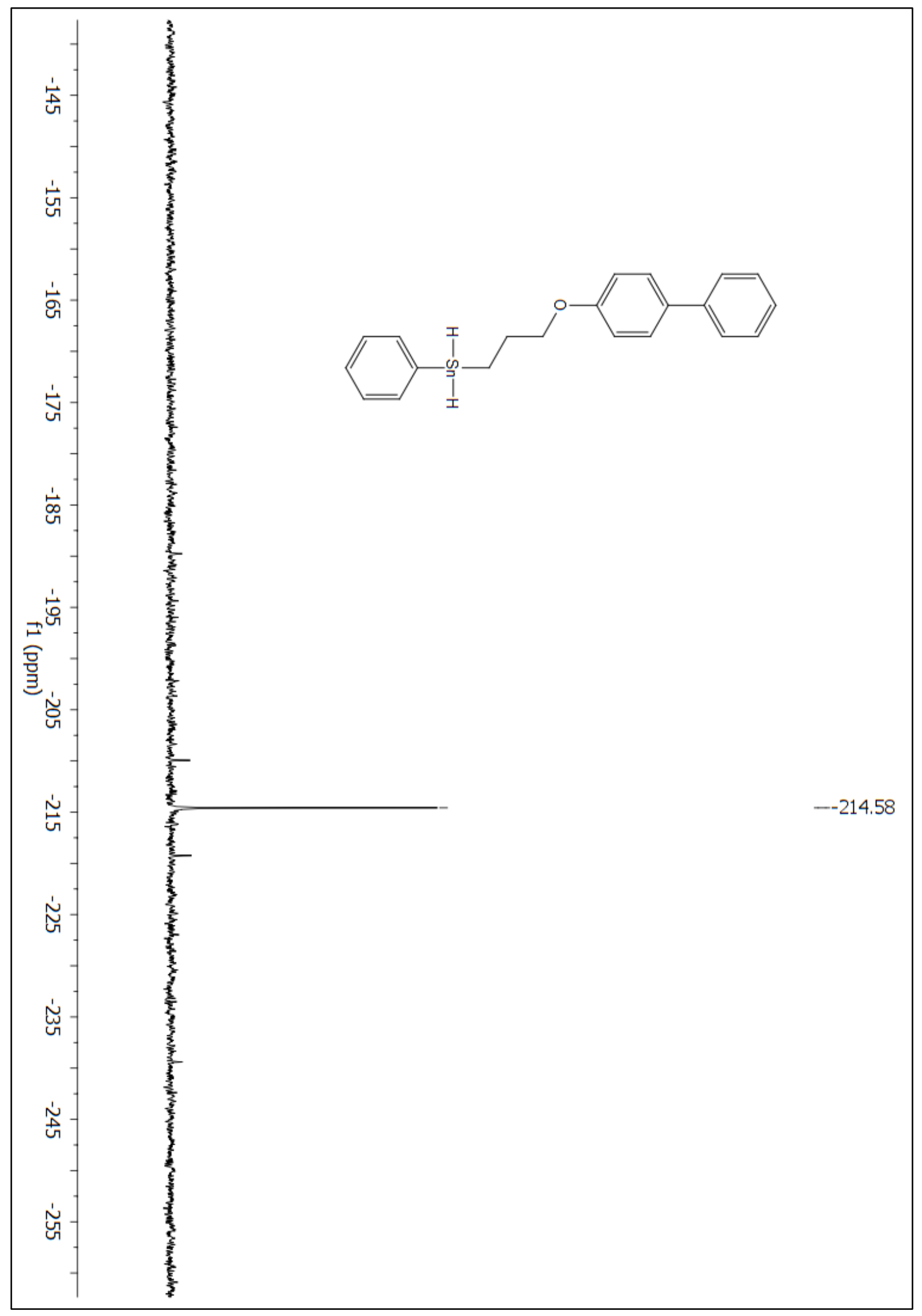

Figure A 50: ${ }^{119} \mathrm{Sn}$ NMR $\left(\mathrm{C}_{6} \mathrm{D}_{6}\right)$ spectrum of compound 207. 


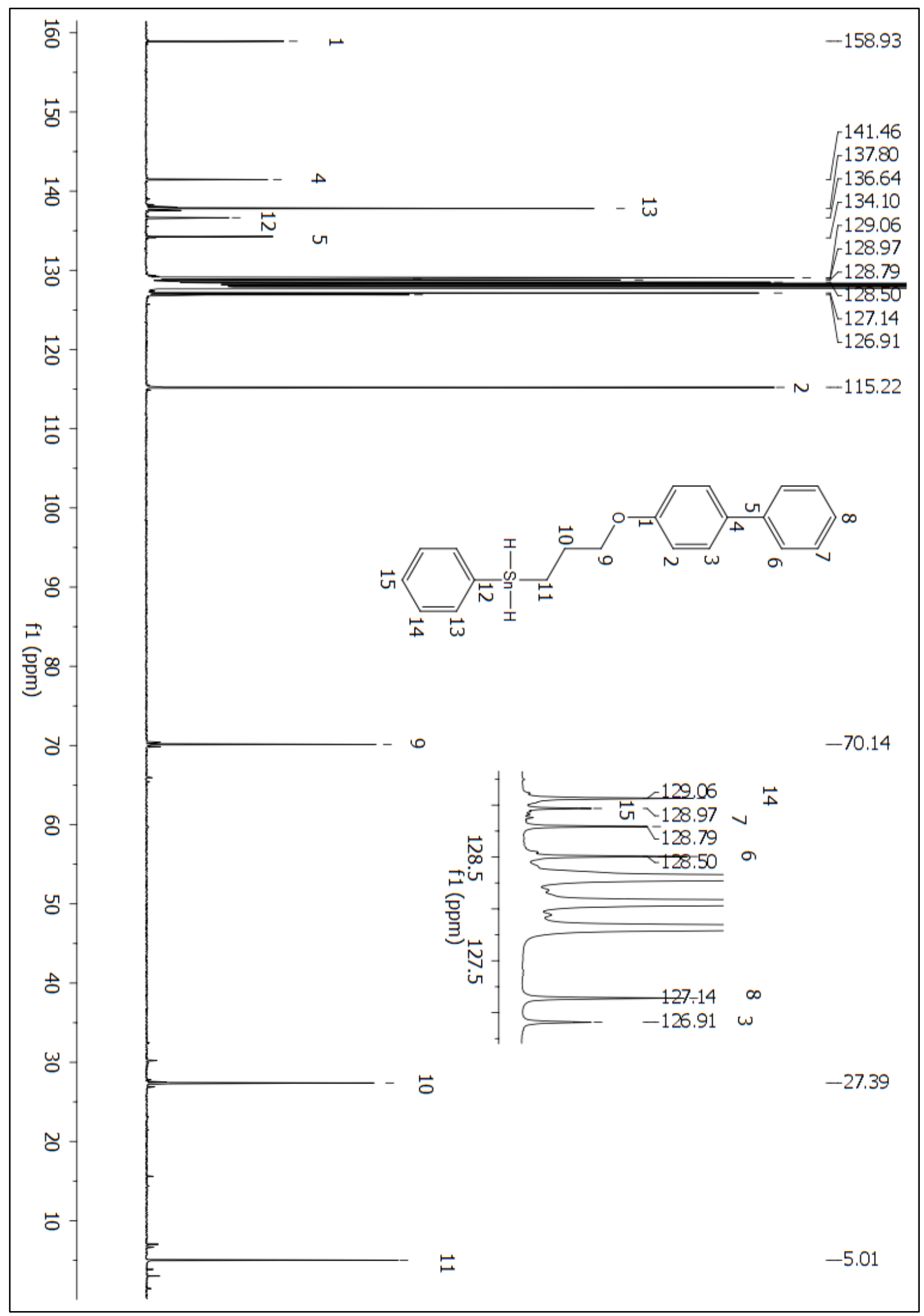

Figure A 51: ${ }^{13} \mathrm{C}$ NMR ( $\left.\mathrm{C}_{6} \mathrm{D}_{6}\right)$ spectrum of compound 207. 


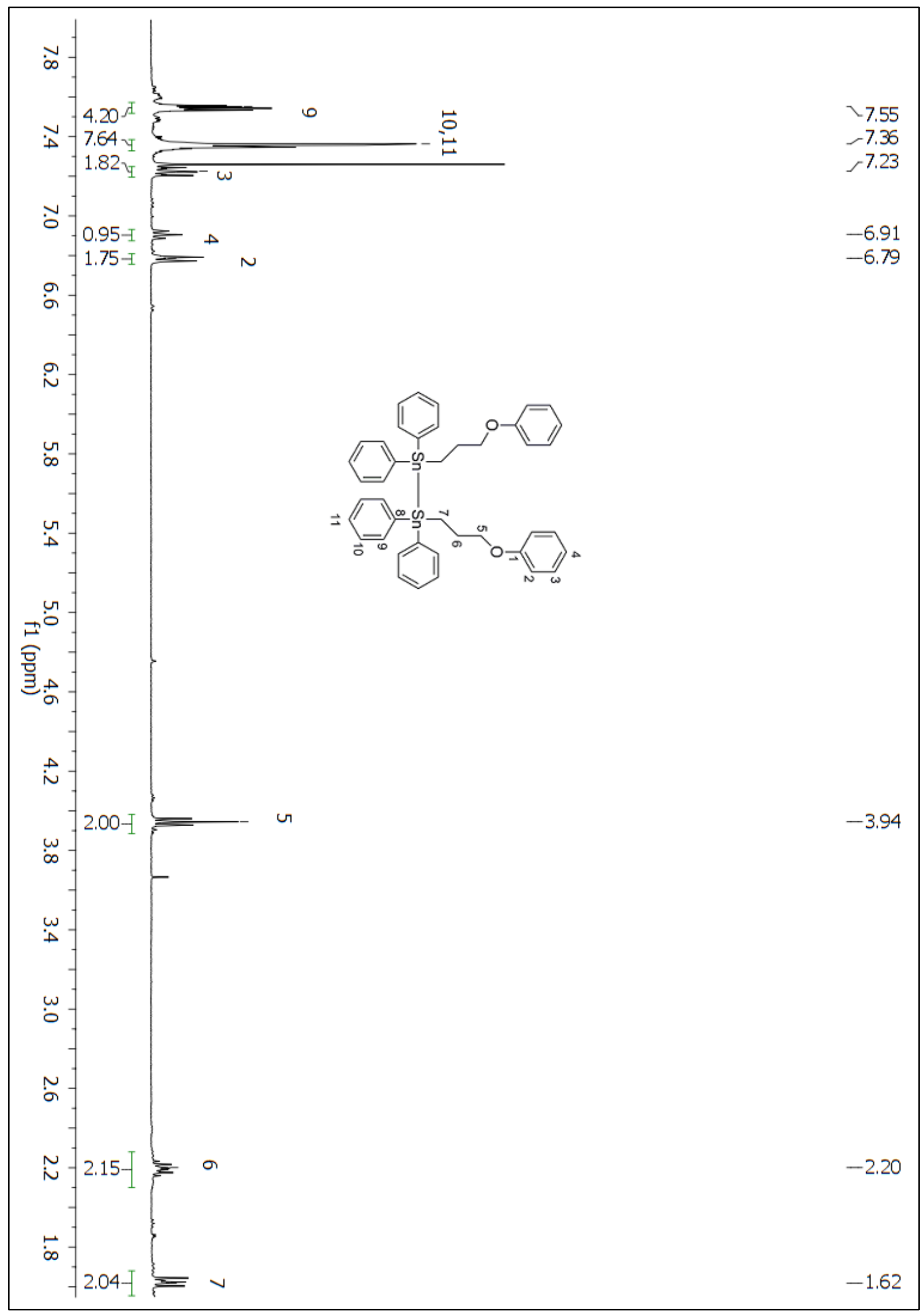

Figure A 52: ${ }^{1} \mathrm{H}$ NMR $\left(\mathrm{C}_{6} \mathrm{D}_{6}\right)$ spectrum of compound 208. 


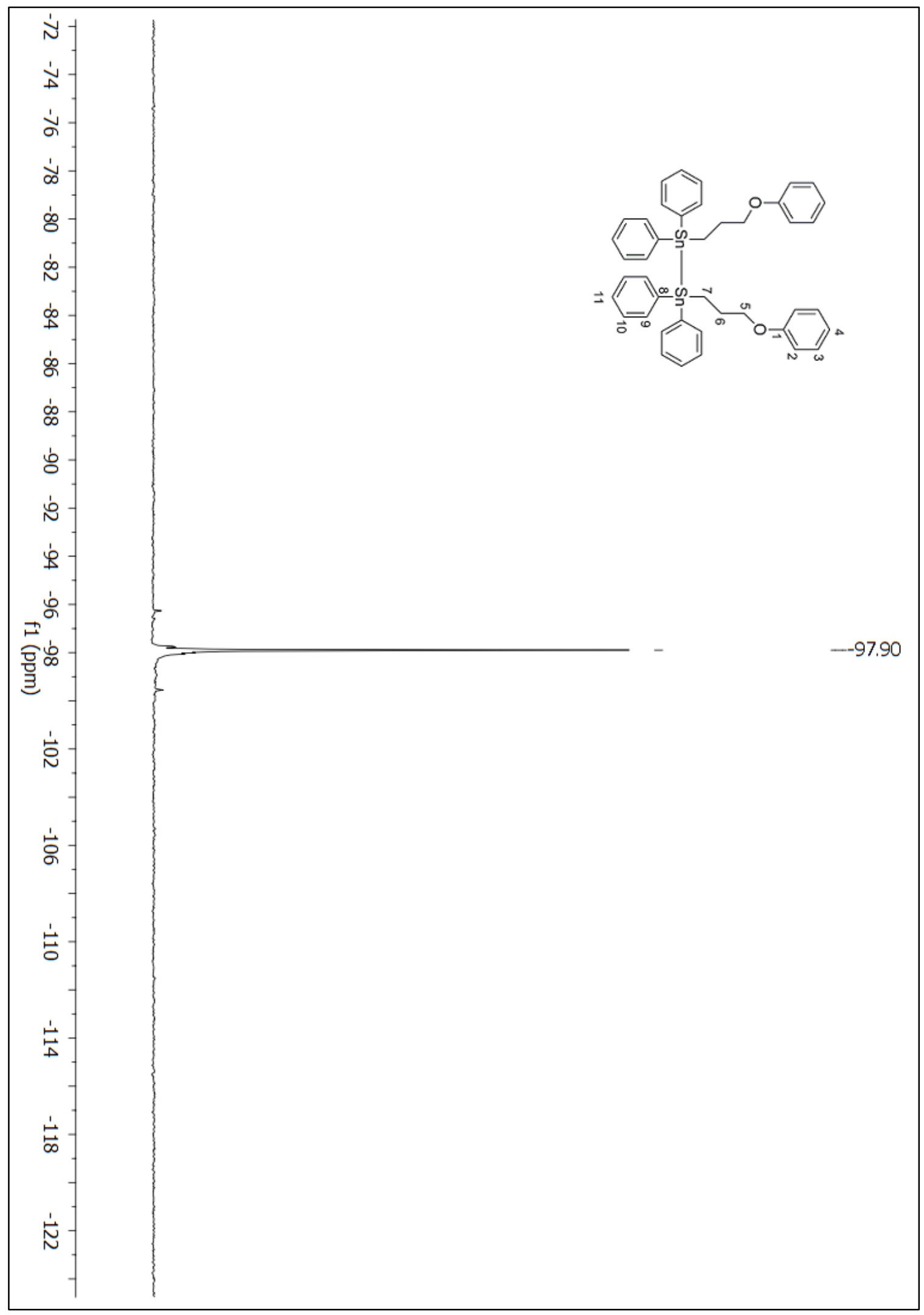

Figure A 53: ${ }^{119} \mathrm{Sn}$ NMR $\left(\mathrm{C}_{6} \mathrm{D}_{6}\right)$ spectrum of compound 208. 


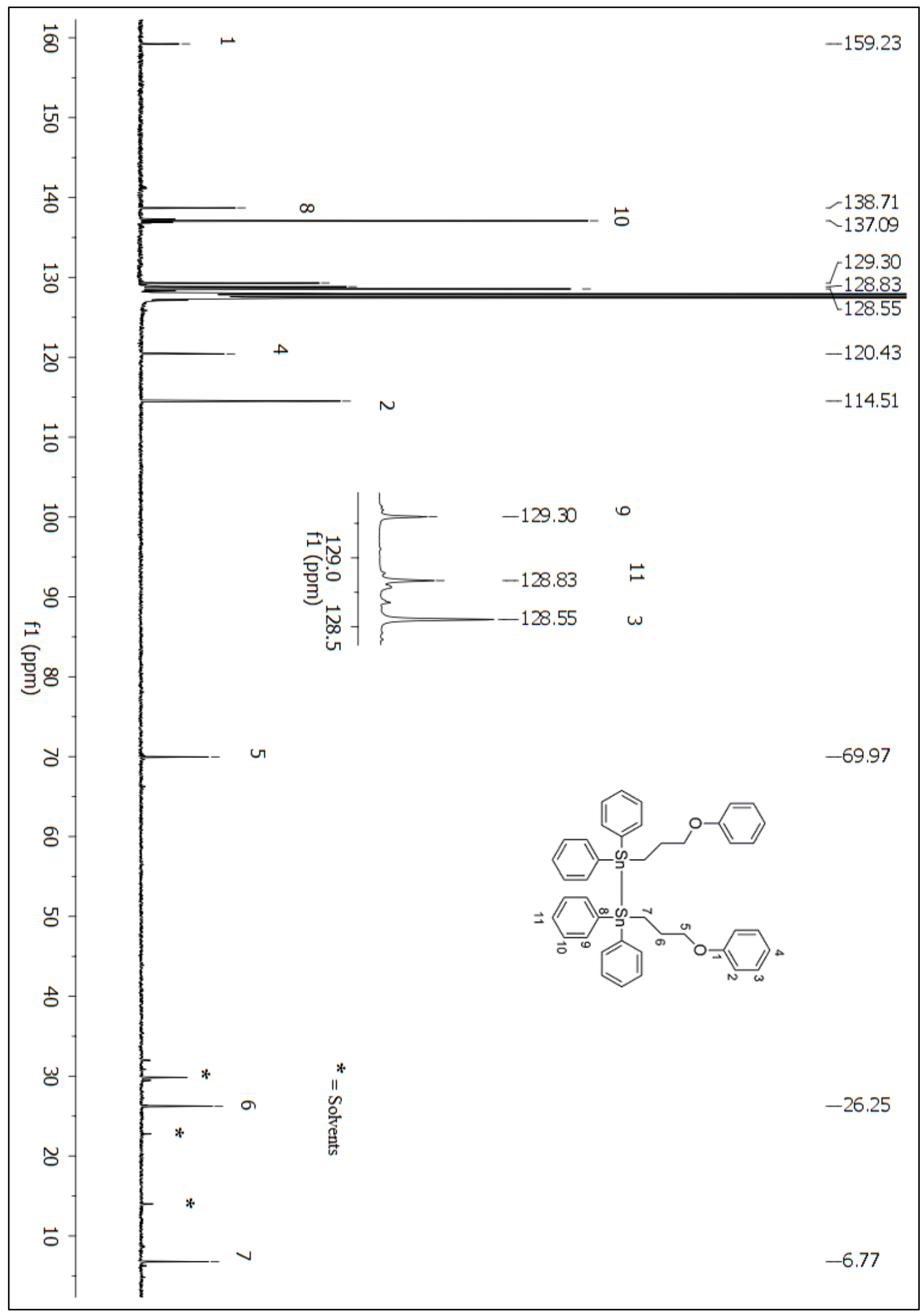

Figure A 54: ${ }^{13} \mathrm{C}$ NMR $\left(\mathrm{C}_{6} \mathrm{D}_{6}\right)$ spectrum of compound 208. 


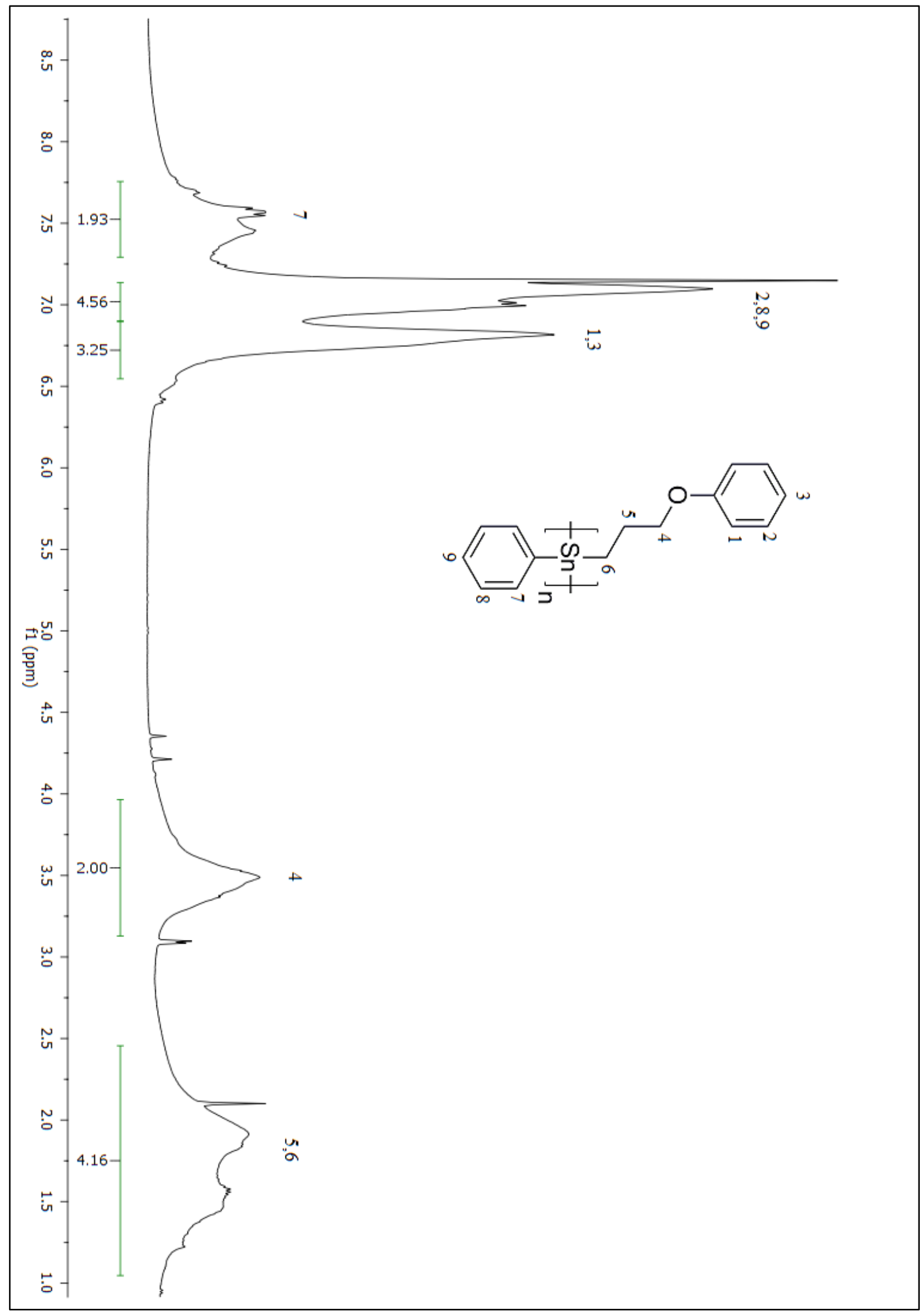

Figure A 55: ${ }^{1} \mathrm{H}$ NMR ( $\left.\mathrm{C}_{6} \mathrm{D}_{6}\right)$ spectrum of compound 249. 


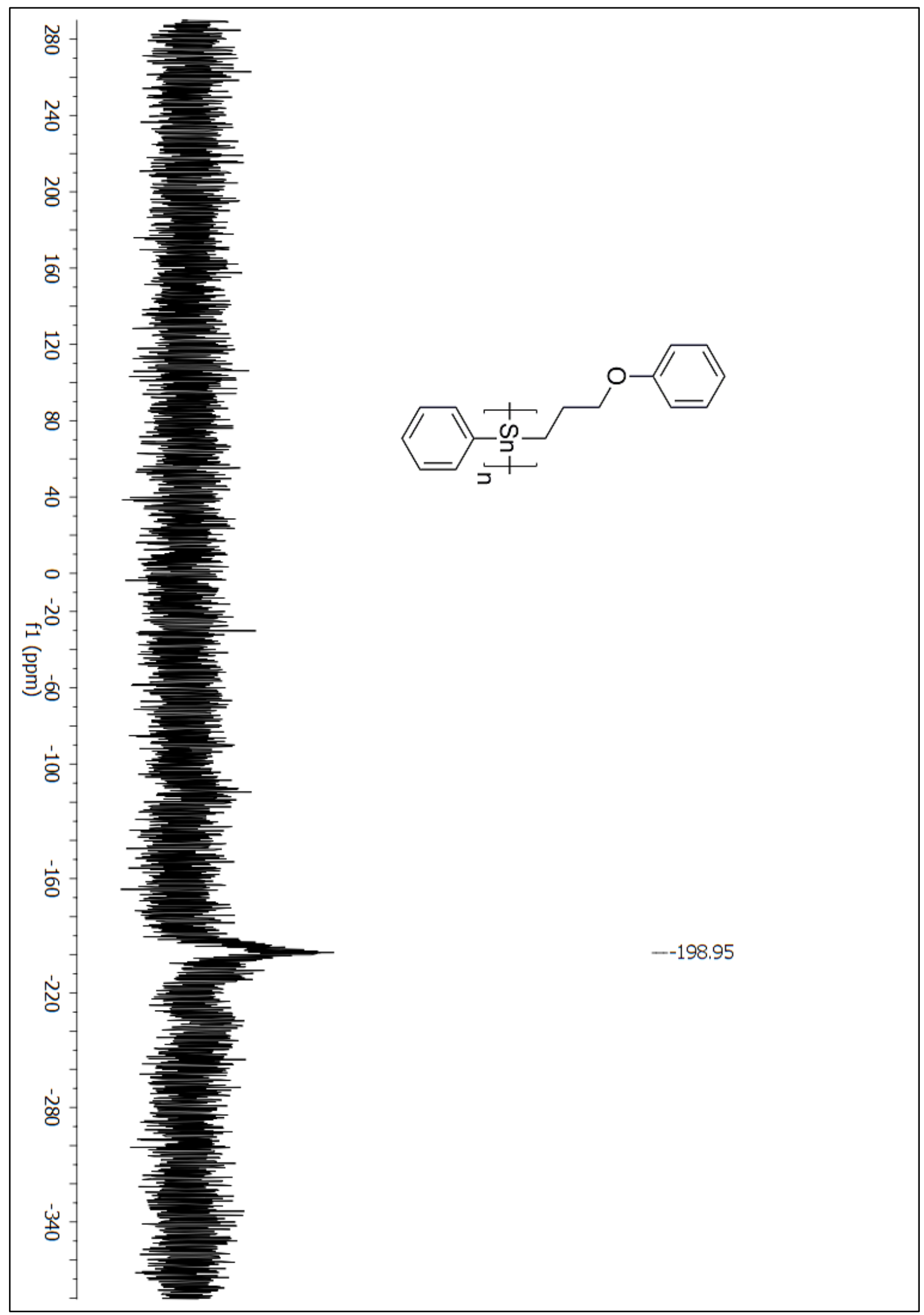

Figure A 56: ${ }^{119} \mathrm{Sn}$ NMR $\left(\mathrm{C}_{6} \mathrm{D}_{6}\right)$ spectrum of compound 249. 


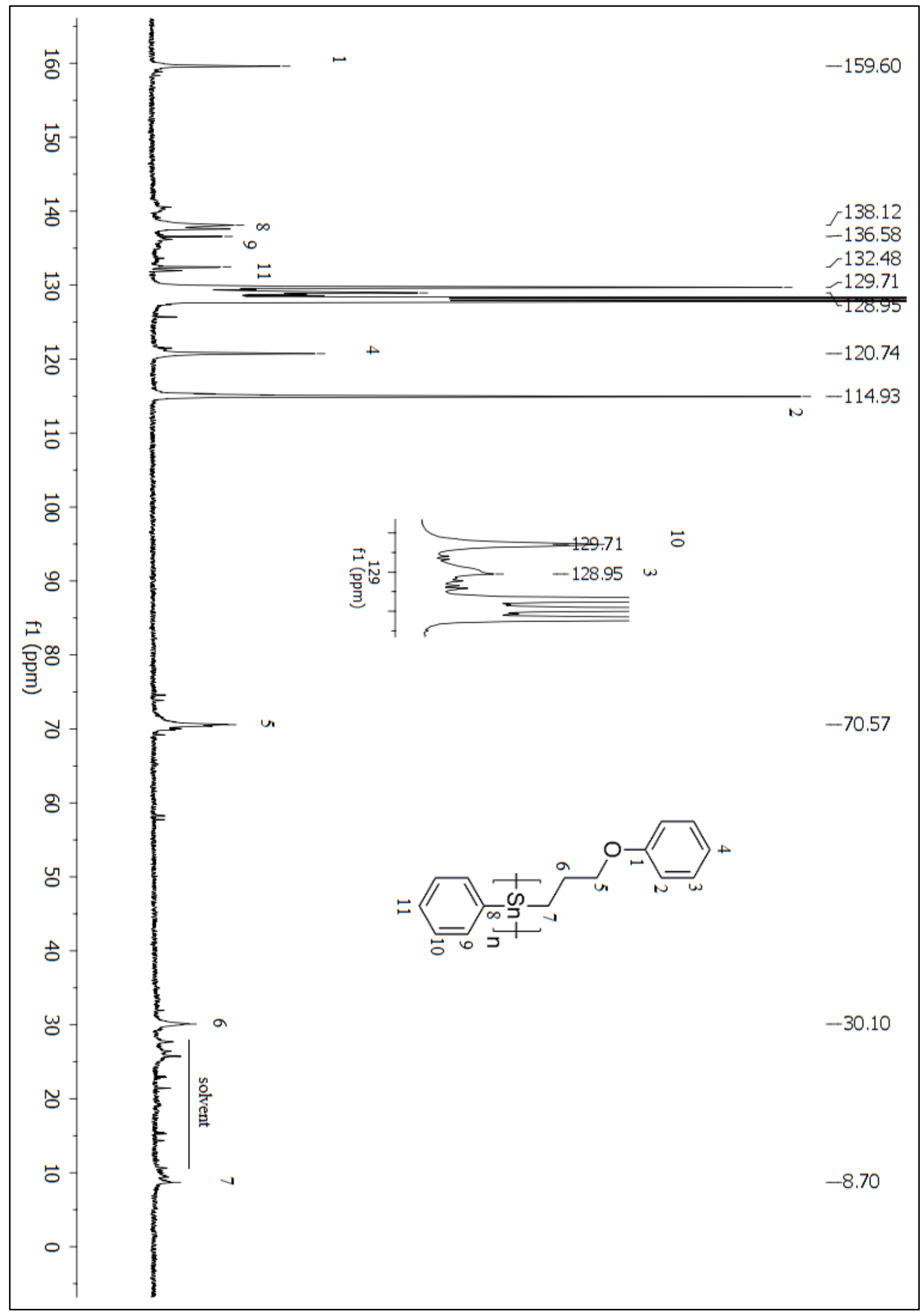

Figure A 57: ${ }^{13} \mathrm{C}$ NMR ( $\left.\mathrm{C}_{6} \mathrm{D}_{6}\right)$ spectrum of compound 249. 


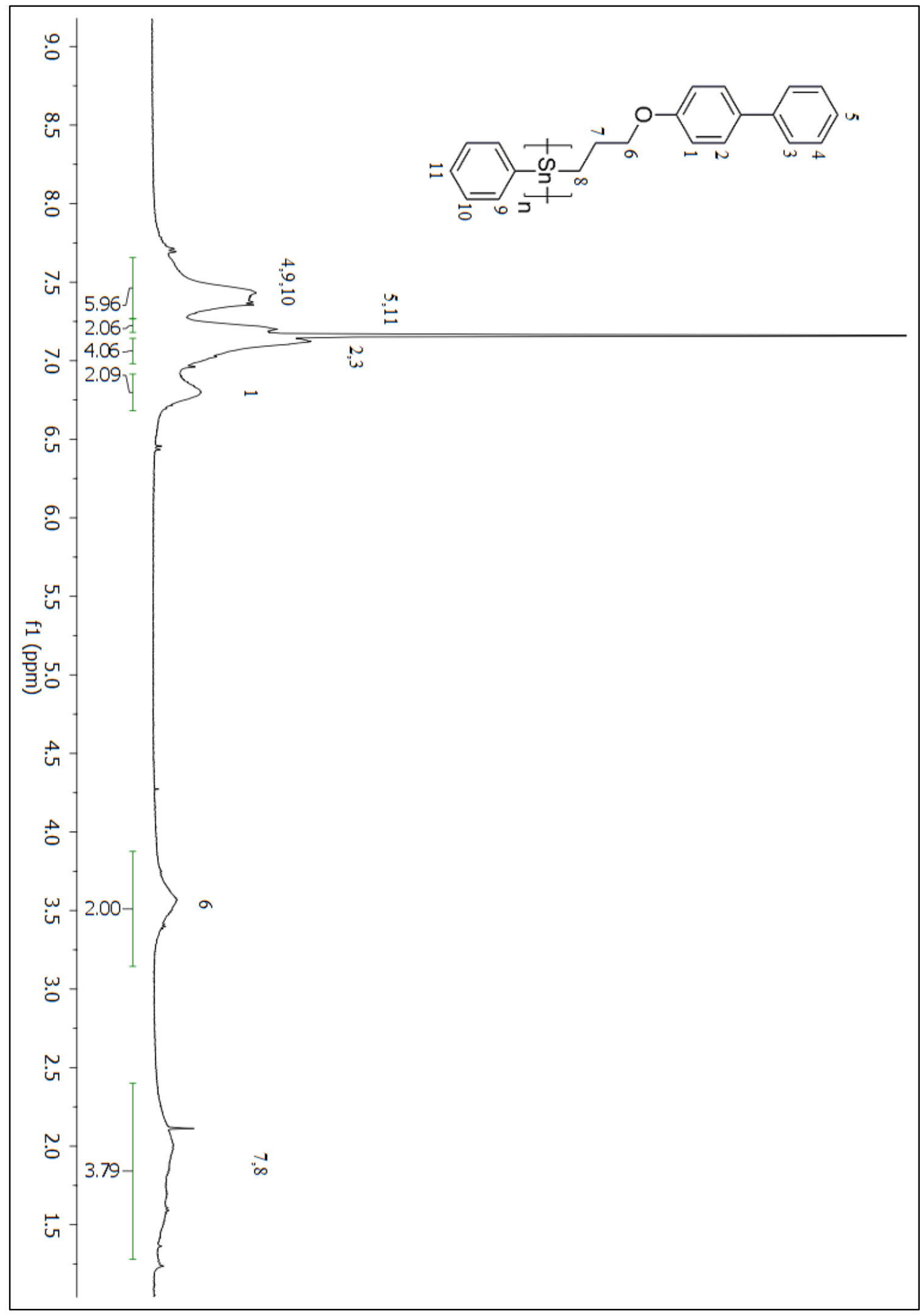

Figure A 58: ${ }^{1} \mathrm{H}$ NMR $\left(\mathrm{C}_{6} \mathrm{D}_{6}\right)$ spectrum of compound 250. 


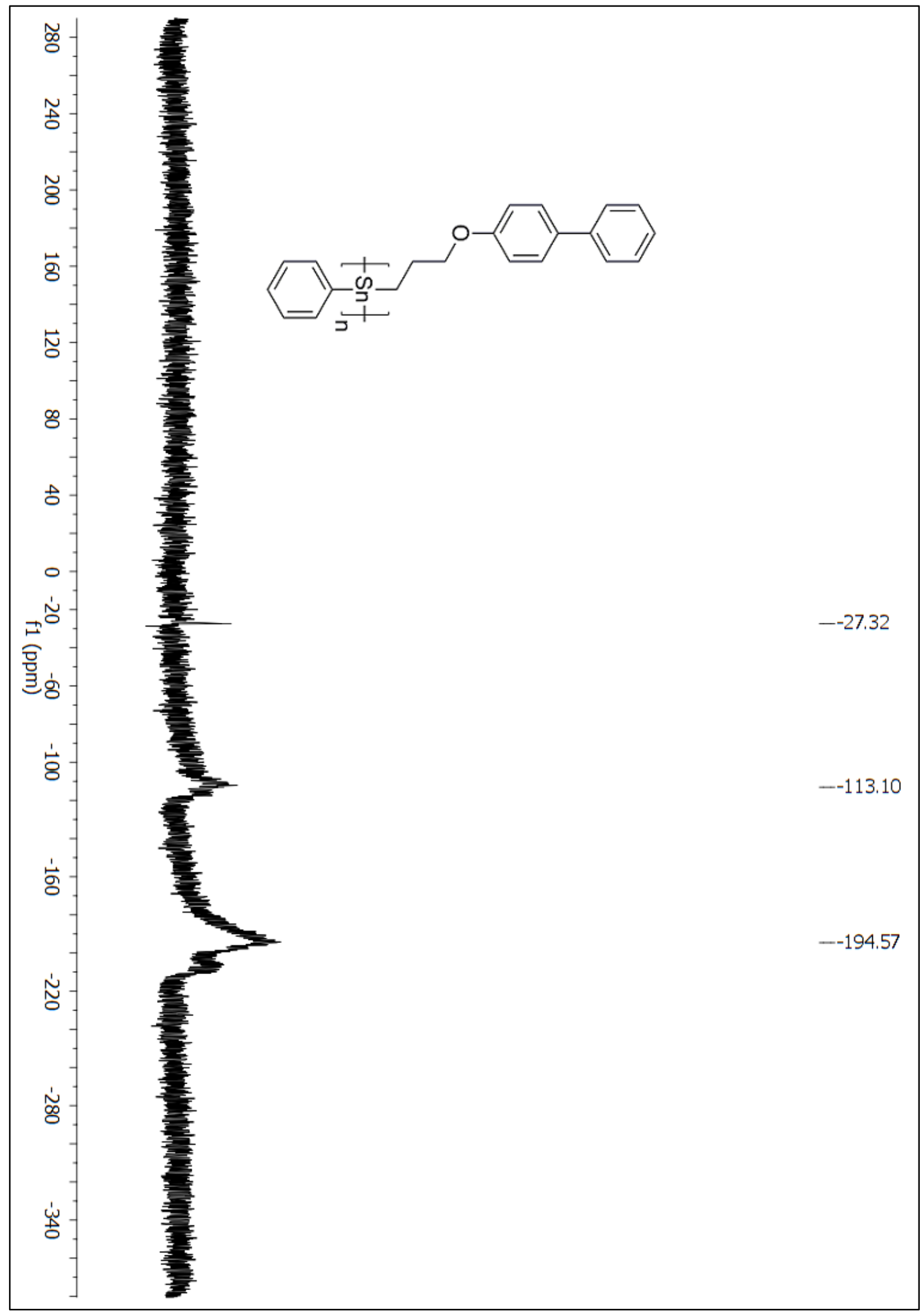

Figure A 59: ${ }^{119} \mathrm{Sn}$ NMR $\left(\mathrm{C}_{6} \mathrm{D}_{6}\right)$ spectrum of compound 250. 


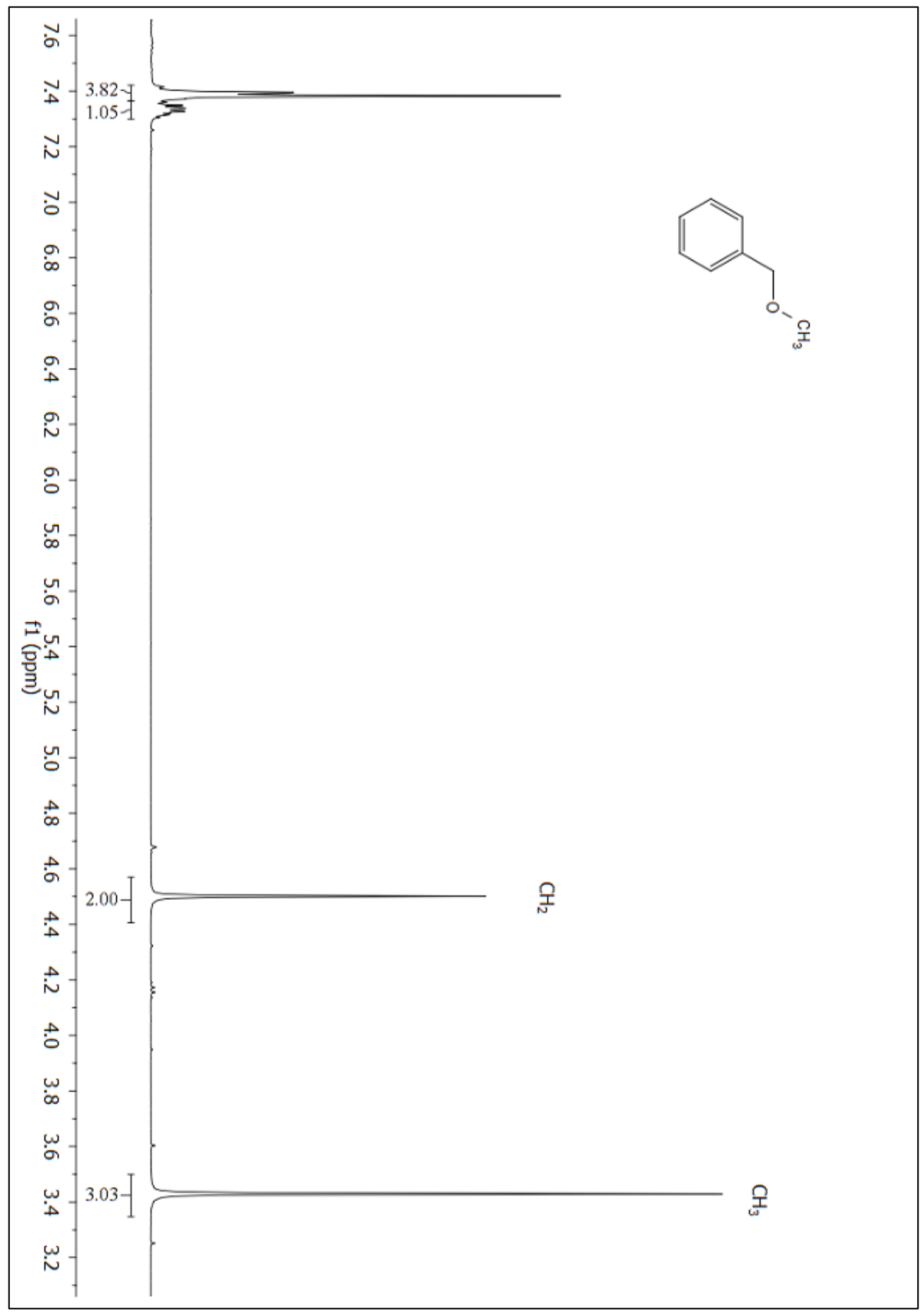

Figure A 60: ${ }^{1} \mathrm{H}$ NMR $\left(\mathrm{CDCl}_{3}\right)$ spectrum of compound 214. 


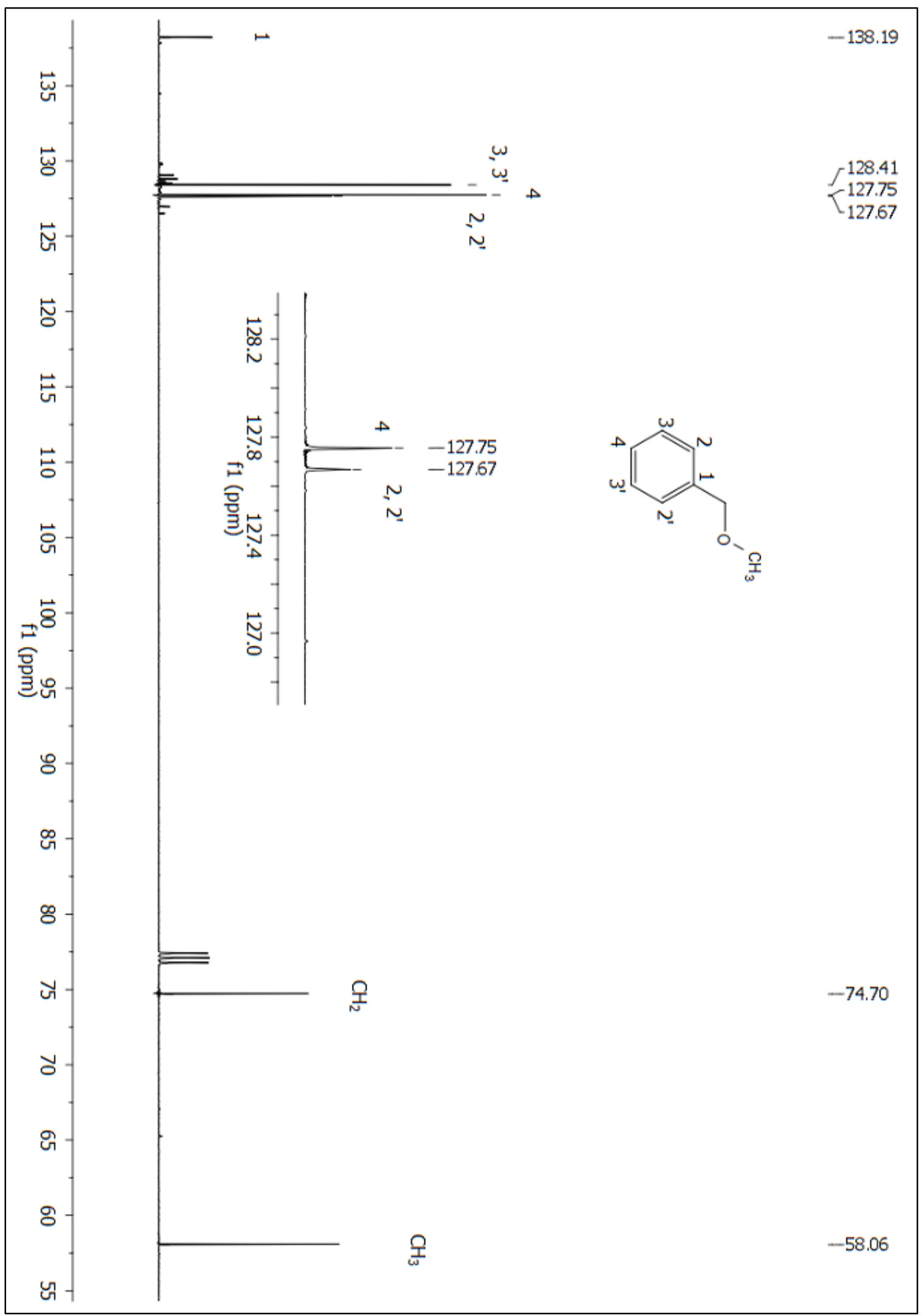

Figure A 61: ${ }^{13} \mathrm{C}$ NMR $\left(\mathrm{CDCl}_{3}\right)$ spectrum of compound 214. 


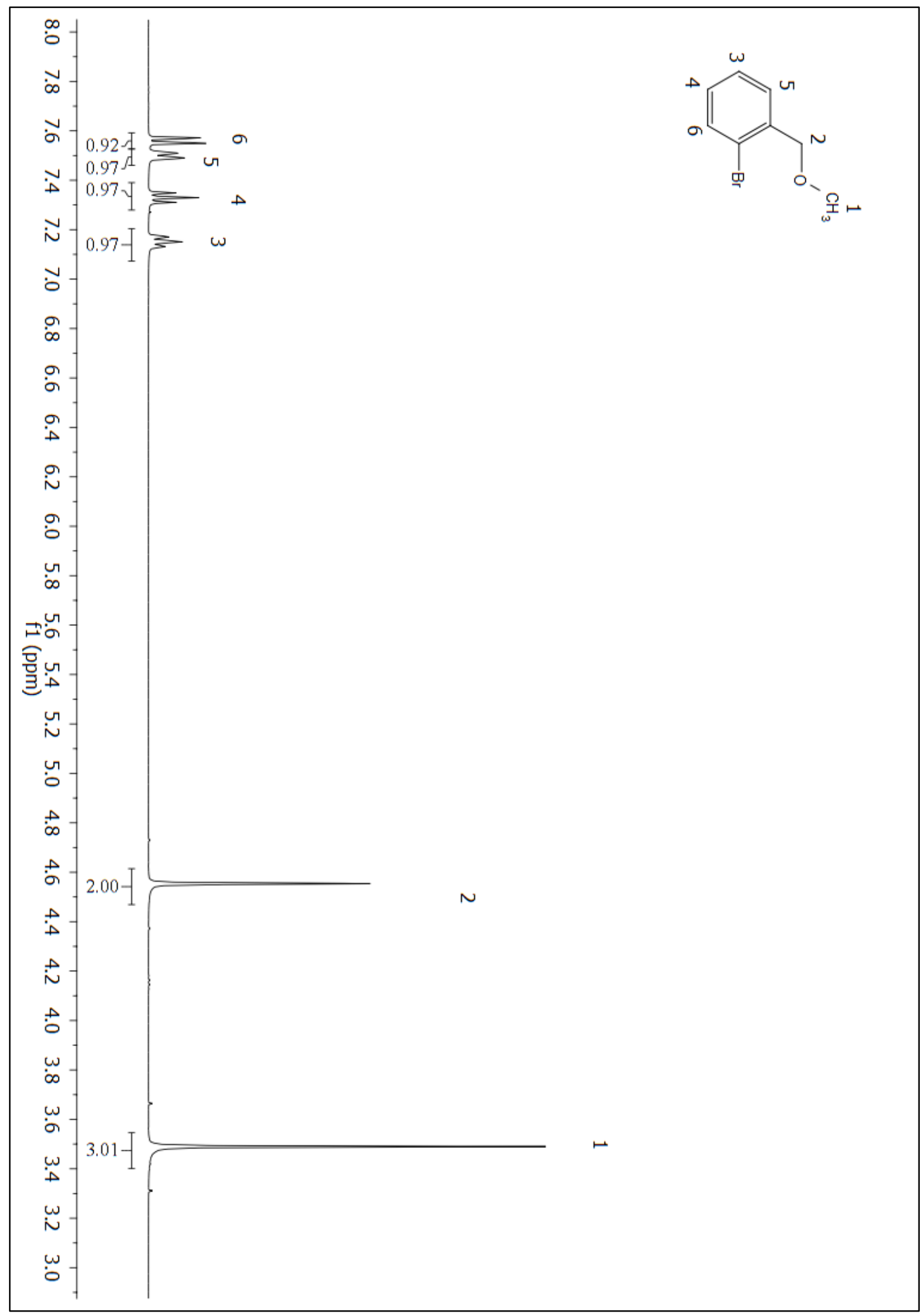

Figure A 62: ${ }^{1} \mathrm{H}$ NMR $\left(\mathrm{CDCl}_{3}\right)$ spectrum of compound 215. 


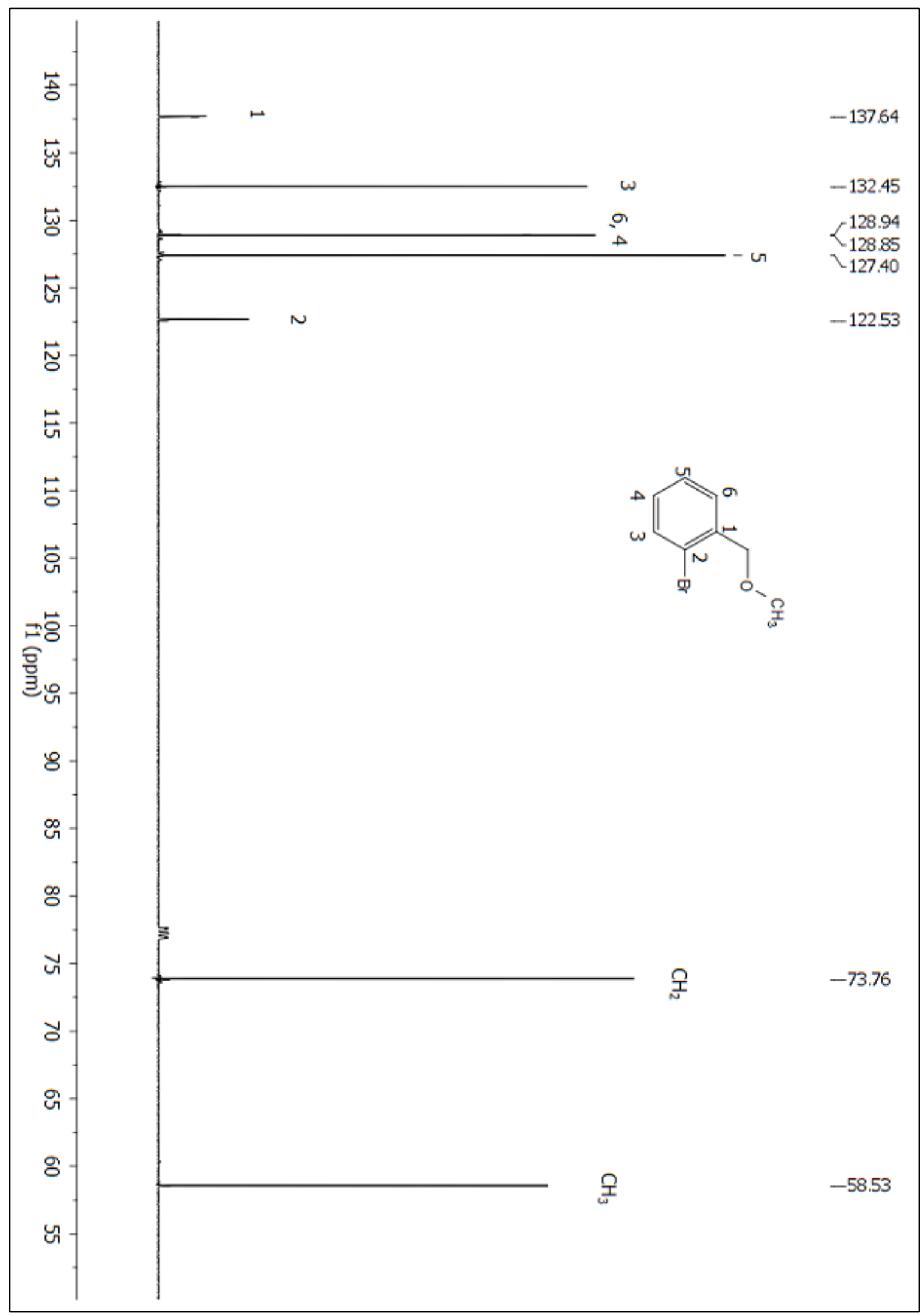

Figure A 63: ${ }^{13} \mathrm{C}$ NMR $\left(\mathrm{CDCl}_{3}\right)$ spectrum of compound 215. 


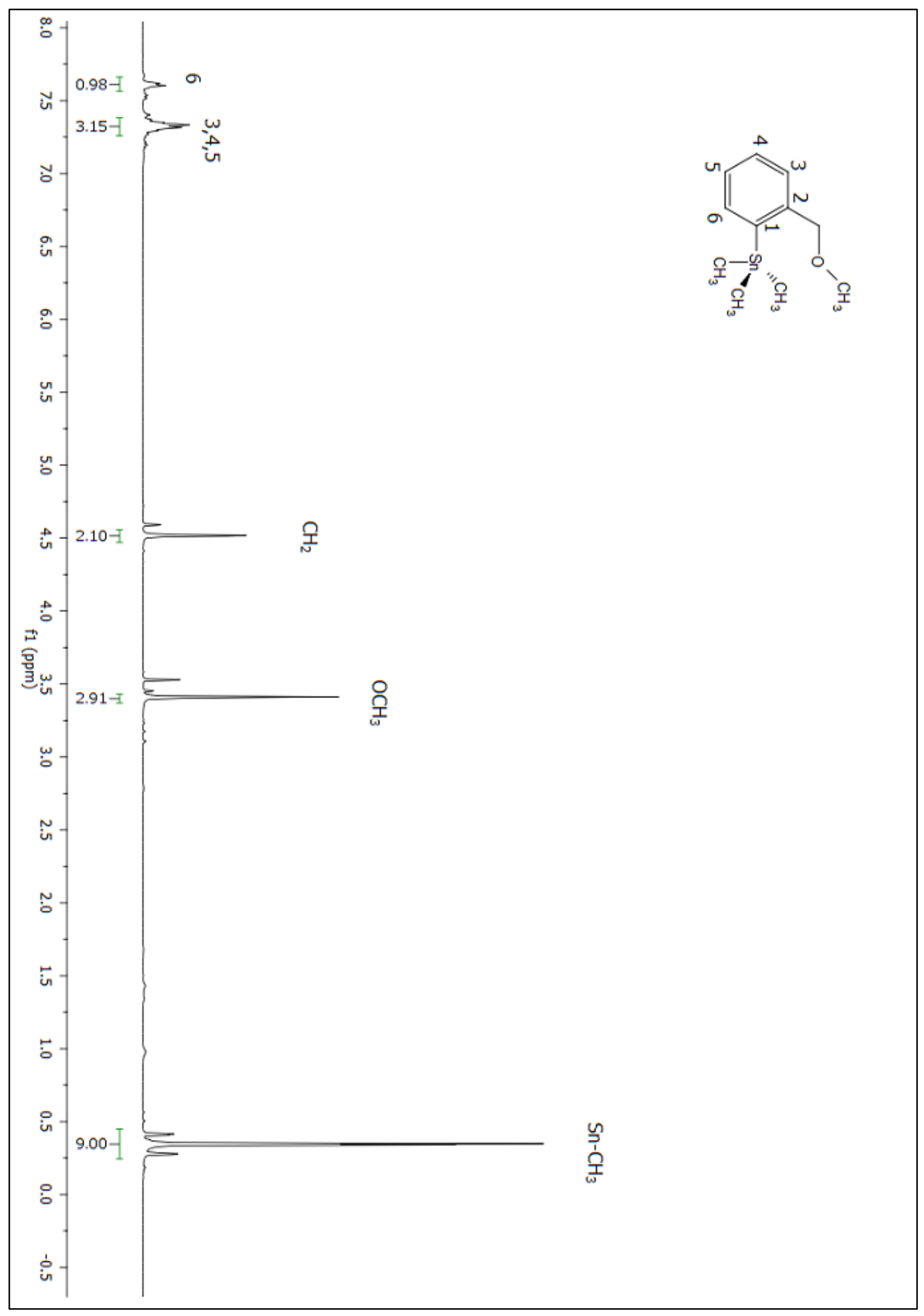

Figure A 64: ${ }^{1} \mathrm{H}$ NMR $\left(\mathrm{CDCl}_{3}\right)$ spectrum of compound 113. 


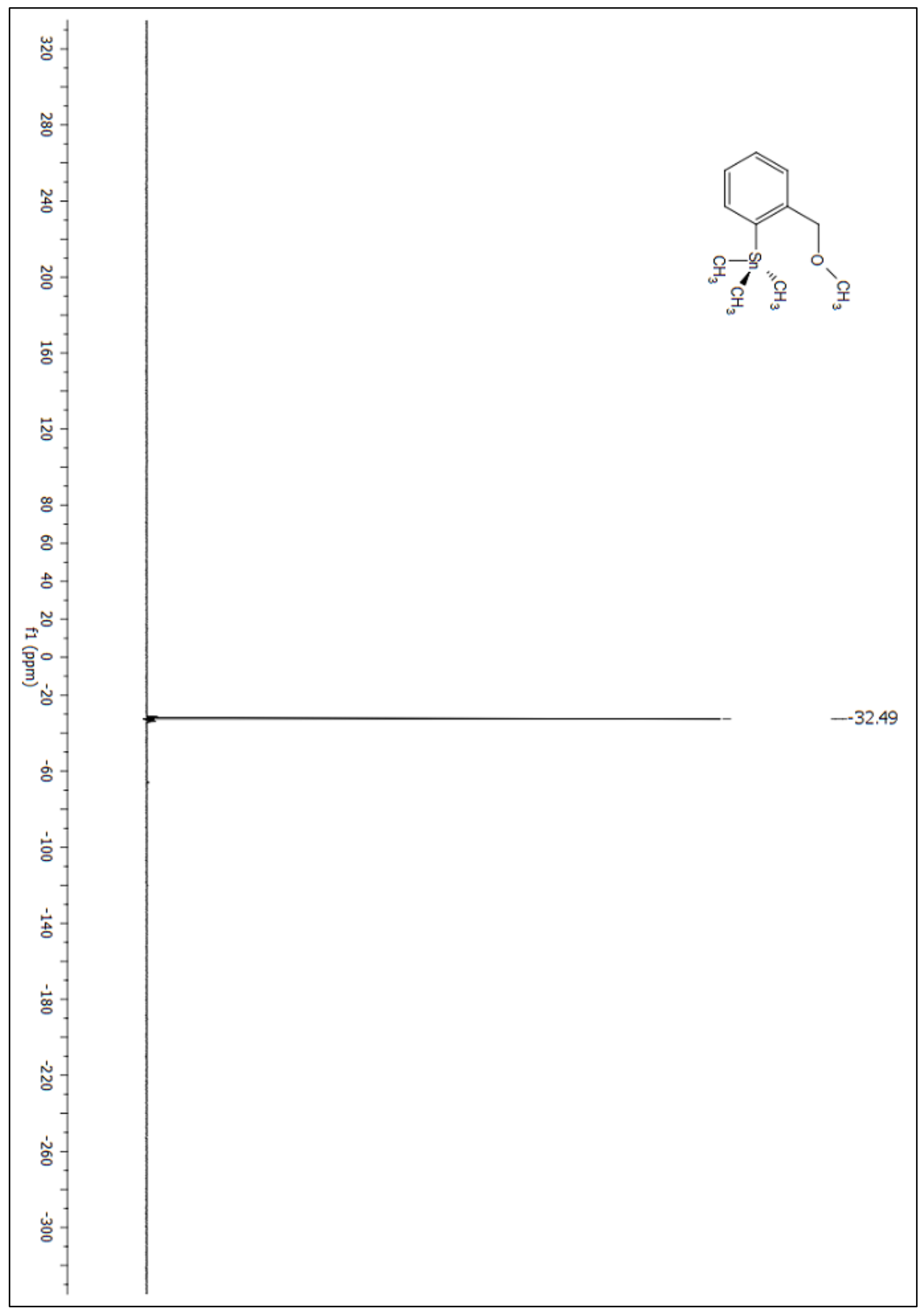

Figure A 65: ${ }^{119} \mathrm{Sn}$ NMR $\left(\mathrm{CDCl}_{3}\right)$ spectrum of compound 113. 


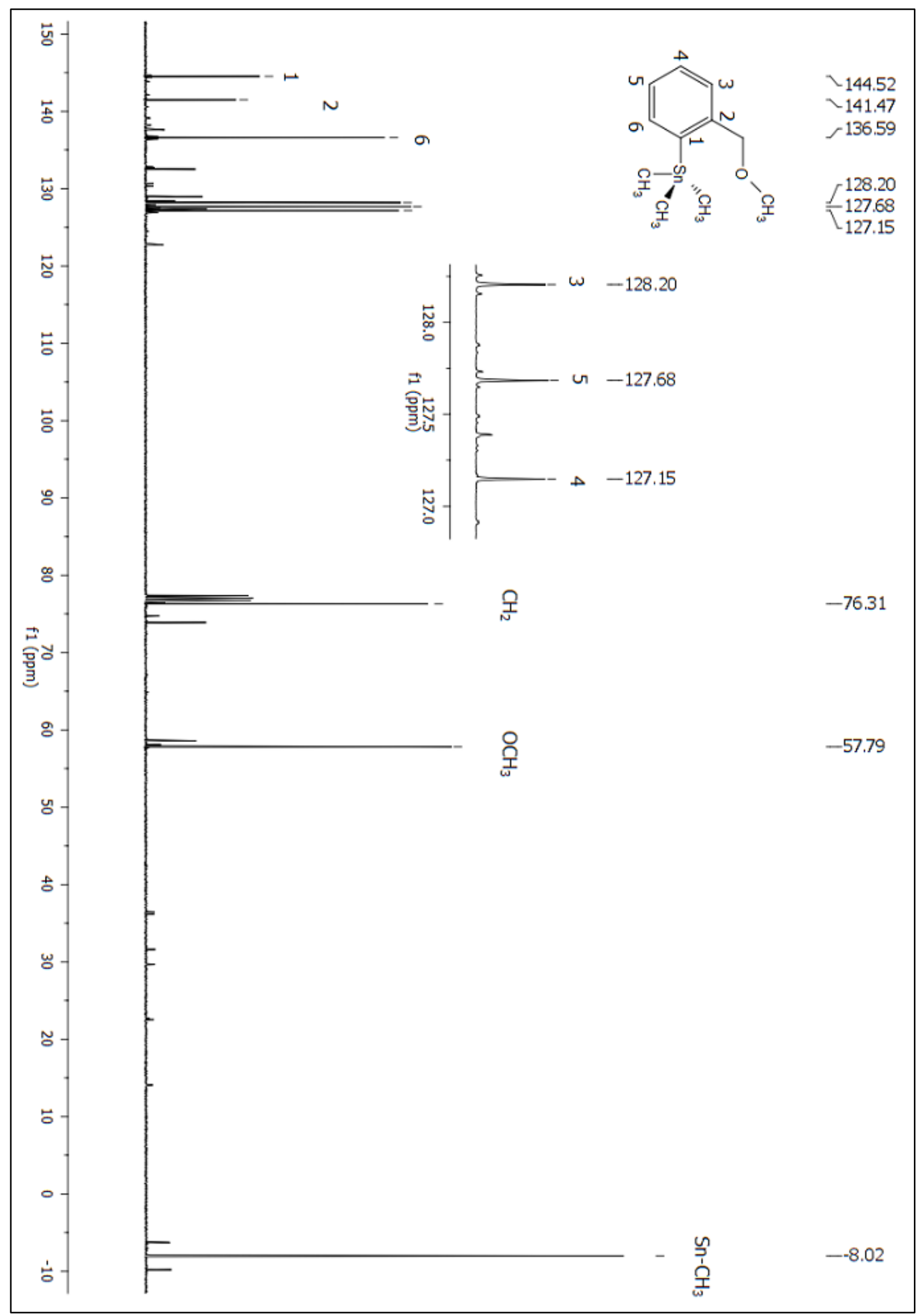

Figure A 66: ${ }^{13} \mathrm{C} \mathrm{NMR}\left(\mathrm{CDCl}_{3}\right)$ of spectrum compound 113. 


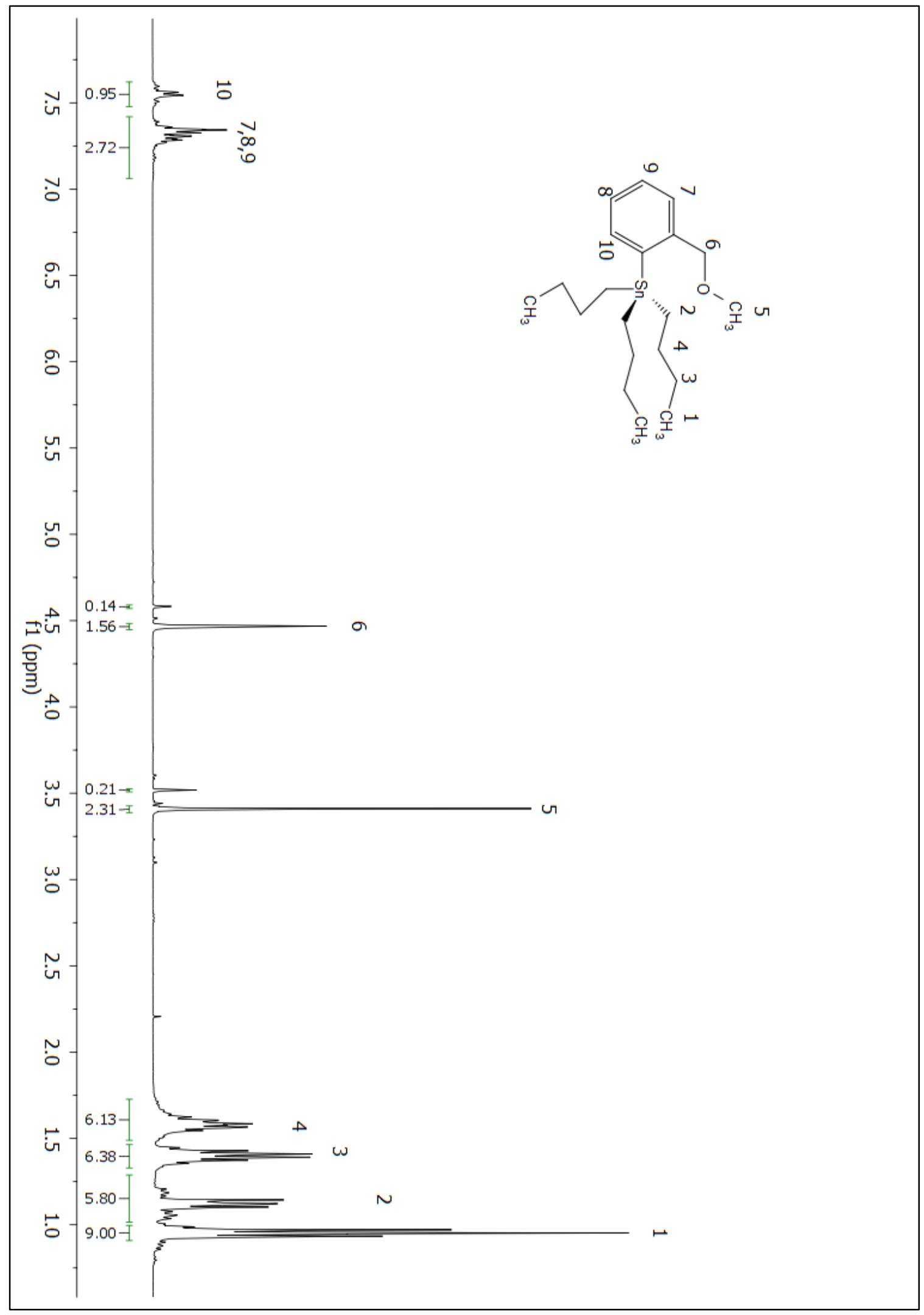

Figure A 67: ${ }^{1} \mathrm{H}$ NMR $\left(\mathrm{CDCl}_{3}\right)$ spectrum of compound 217. 


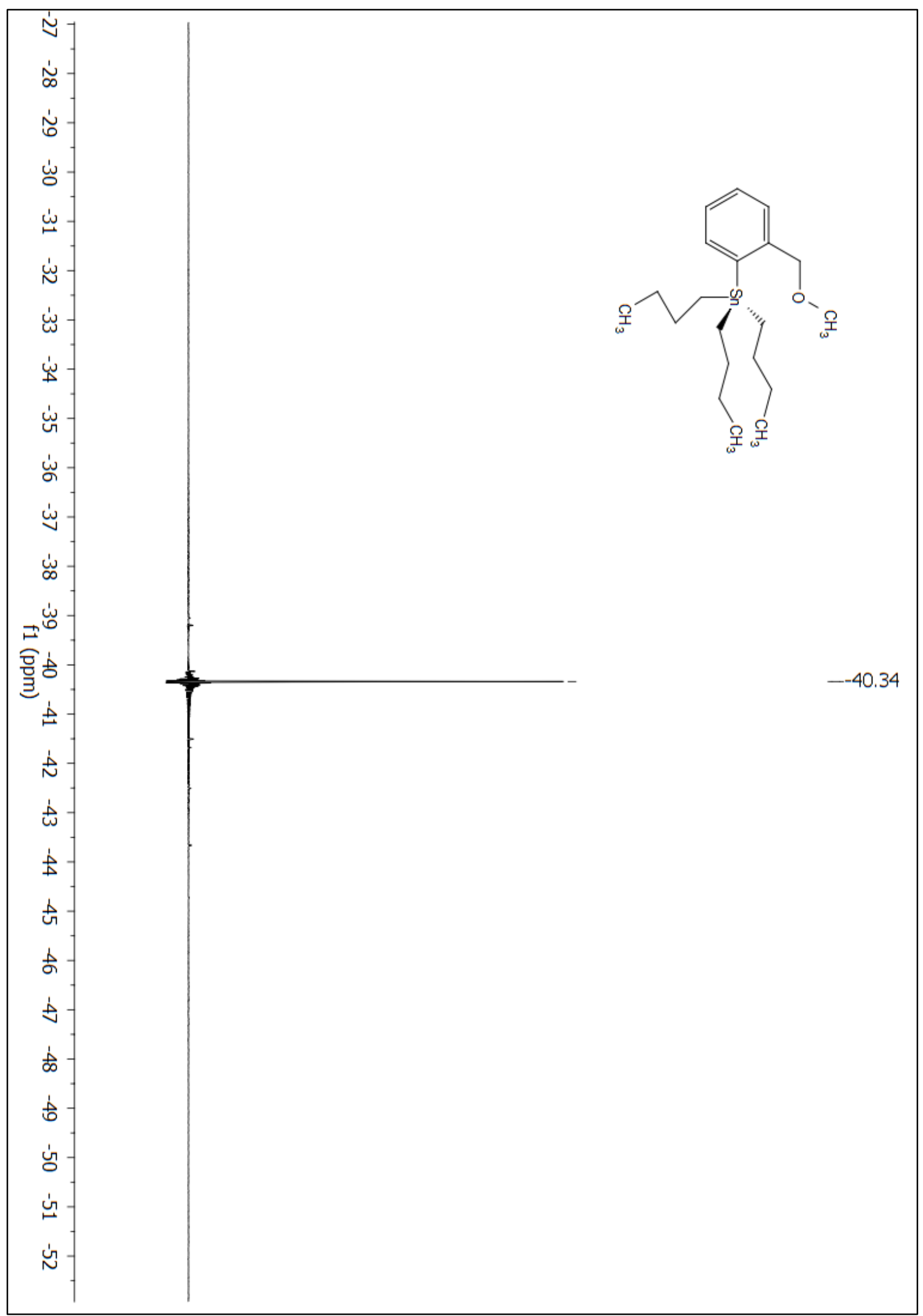

Figure A 68: ${ }^{119} \mathrm{Sn} \mathrm{NMR}\left(\mathrm{CDCl}_{3}\right)$ spectrum of compound 217. 


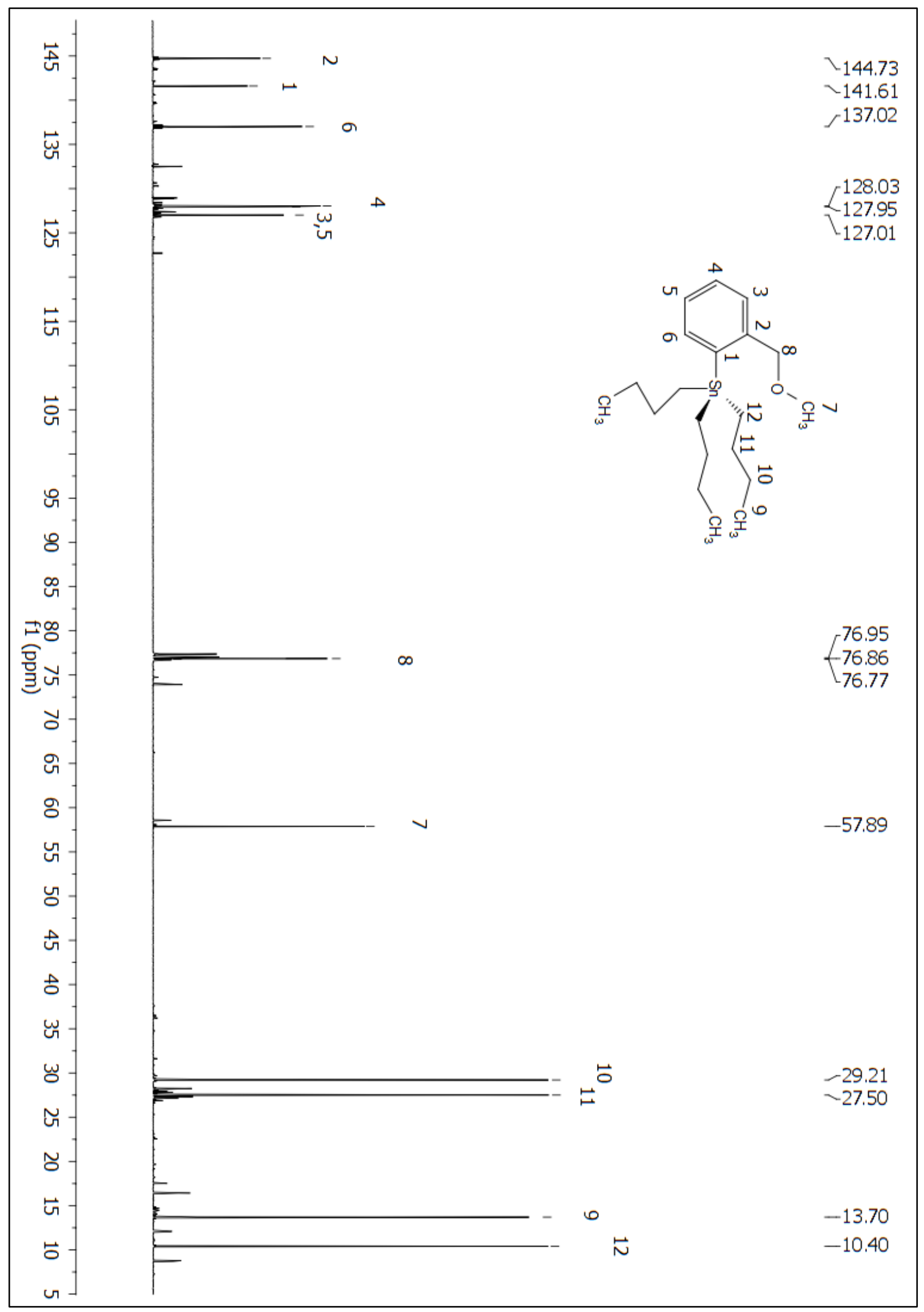

Figure A 69: ${ }^{13} \mathrm{C}$ NMR $\left(\mathrm{CDCl}_{3}\right)$ spectrum of compound 217. 


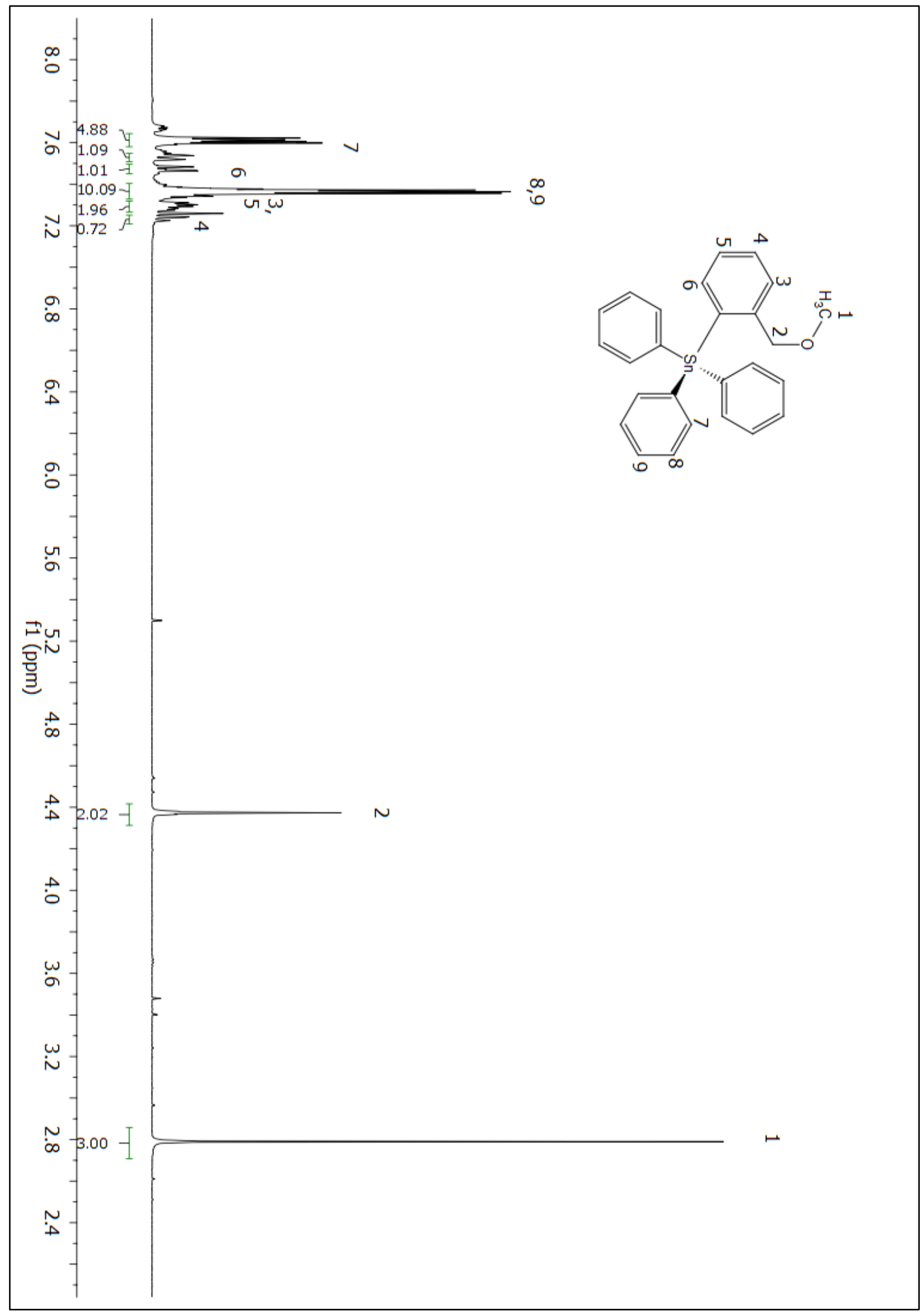

Figure A 70: ${ }^{1} \mathrm{H}$ NMR $\left(\mathrm{CDCl}_{3}\right)$ spectrum of compound 112. 


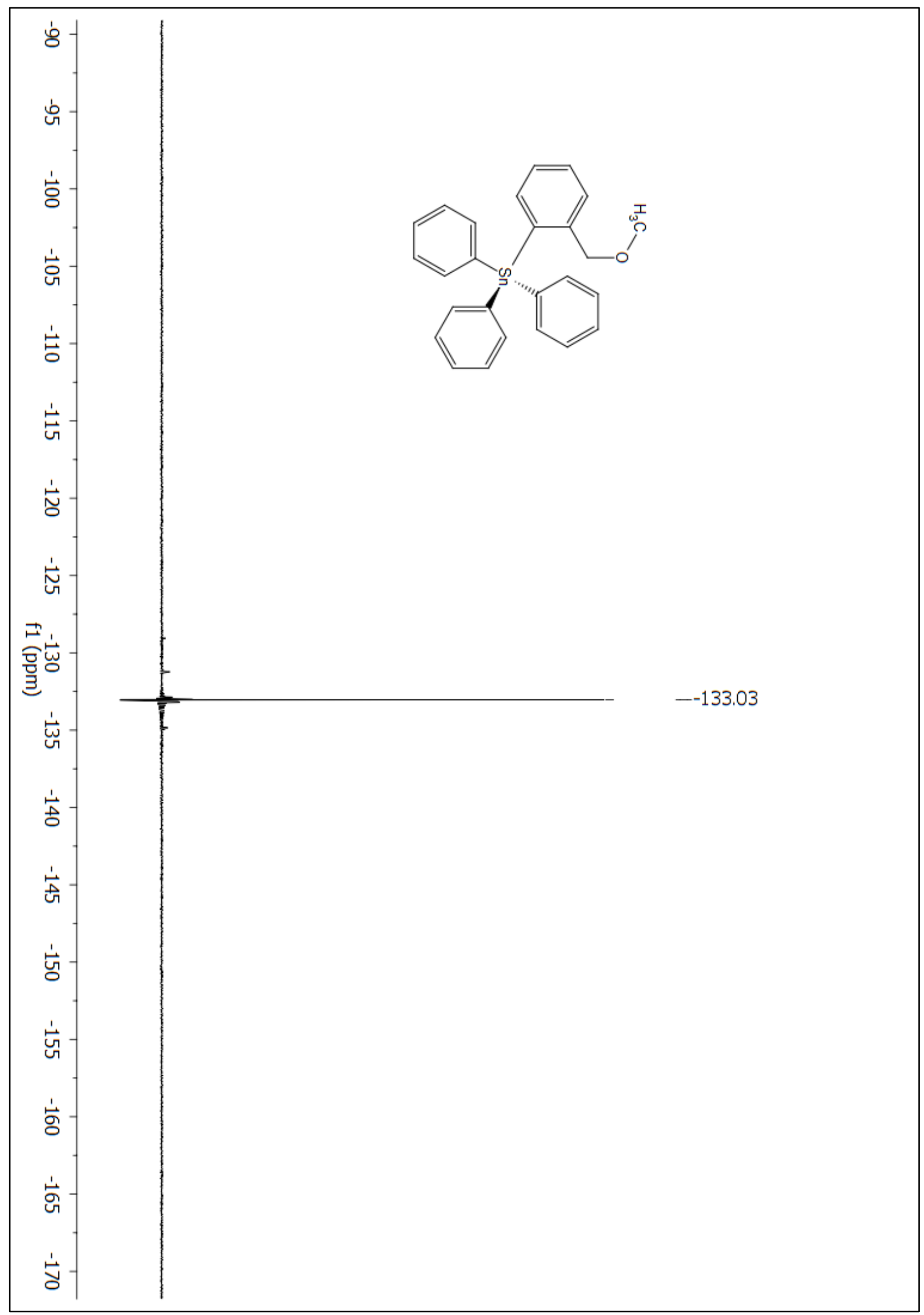

Figure A 71: ${ }^{119} \mathrm{Sn}$ NMR $\left(\mathrm{CDCl}_{3}\right)$ spectrum of compound 112. 


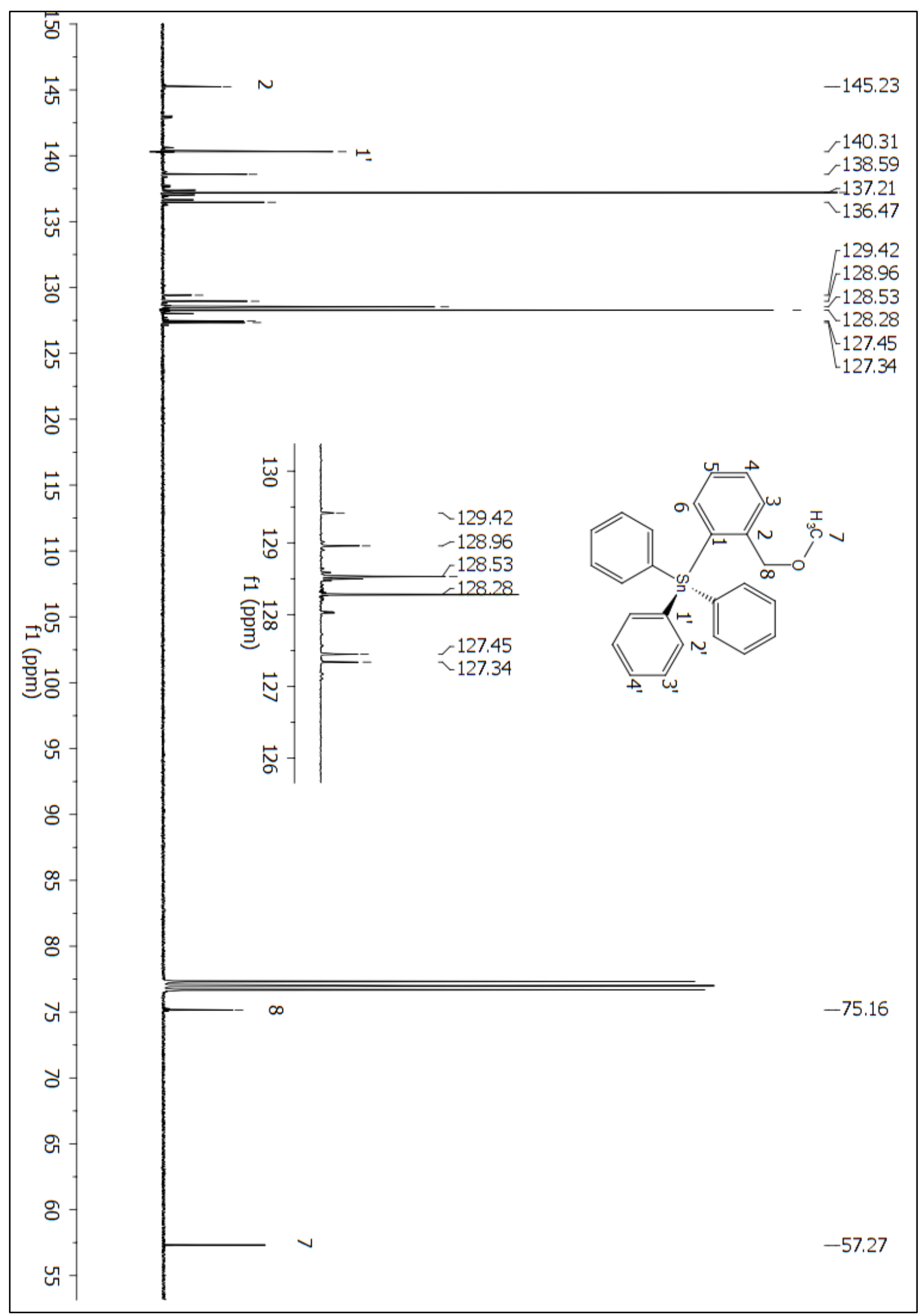

Figure A 72: ${ }^{13} \mathrm{C}$ NMR $\left(\mathrm{CDCl}_{3}\right)$ spectrum of compound 112. 


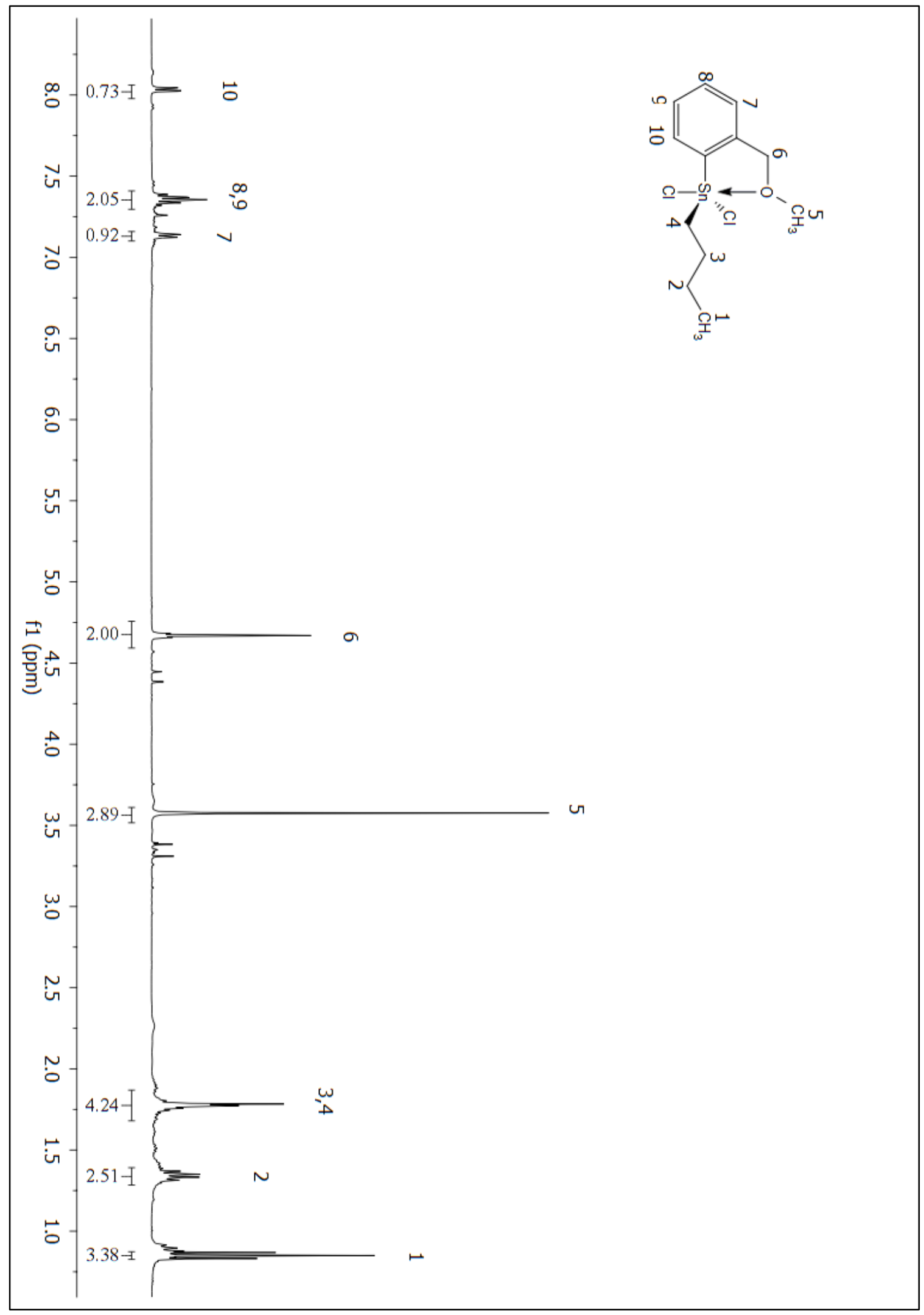

Figure A 73: ${ }^{1} \mathrm{H}$ NMR $\left(\mathrm{CDCl}_{3}\right)$ spectrum of compound 219. 


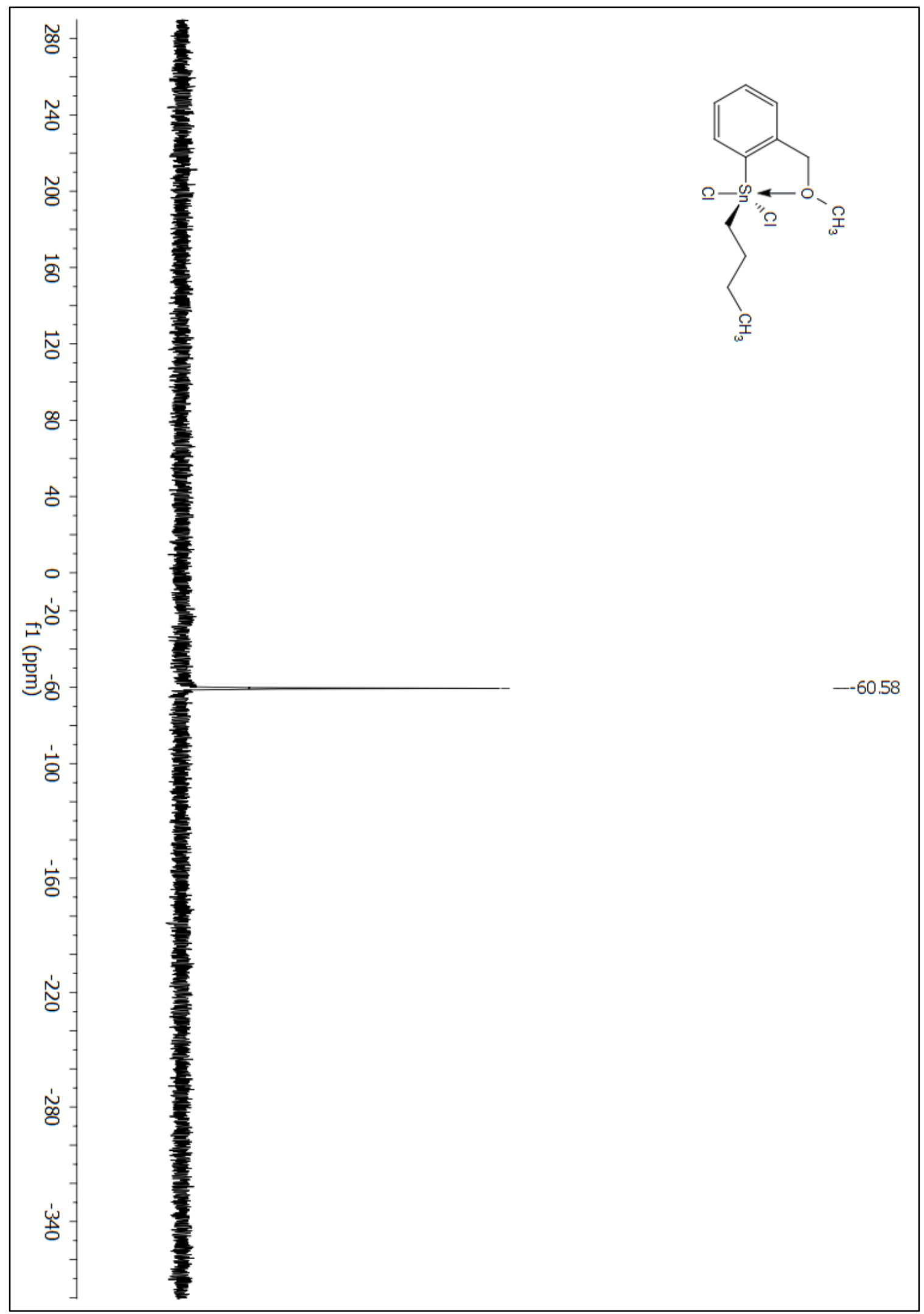

Figure A 74: ${ }^{119} \mathrm{Sn}$ NMR $\left(\mathrm{CDCl}_{3}\right)$ spectrum of compound 219. 


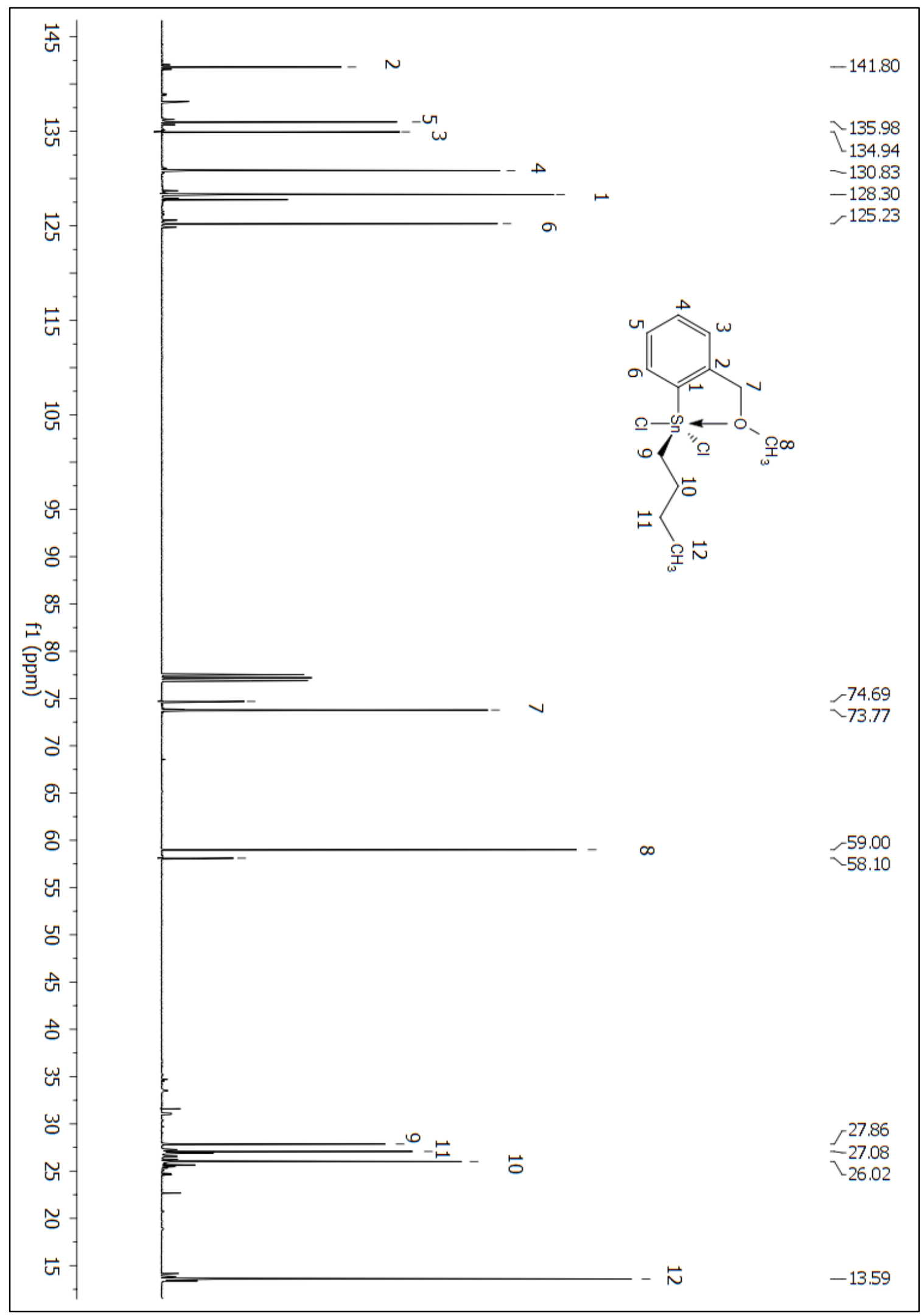

Figure A 75: ${ }^{13} \mathrm{C}$ NMR $\left(\mathrm{CDCl}_{3}\right)$ spectrum of compound 219. 


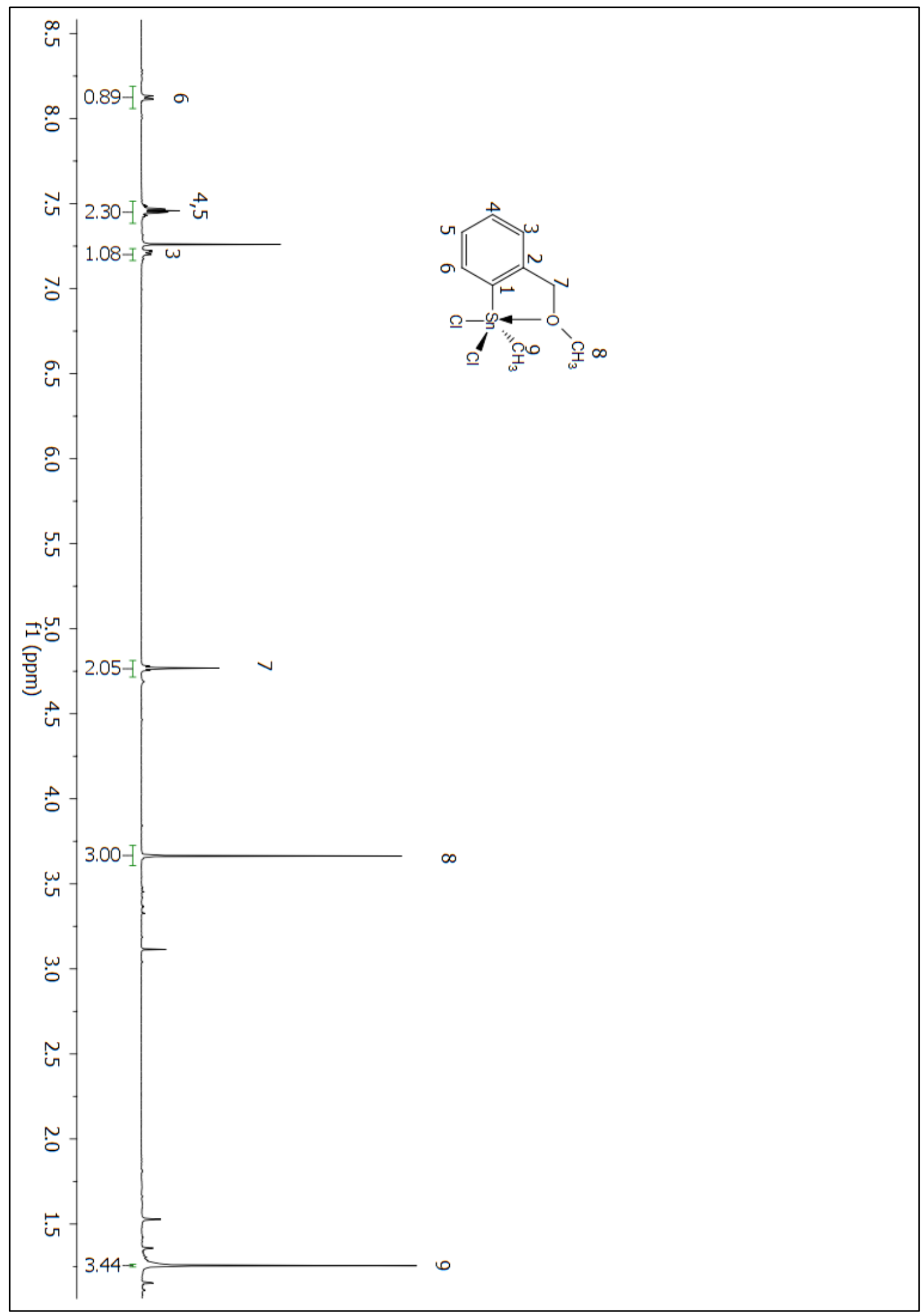

Figure A 76: ${ }^{1} \mathrm{H}$ NMR $\left(\mathrm{CDCl}_{3}\right)$ spectrum of compound 218. 


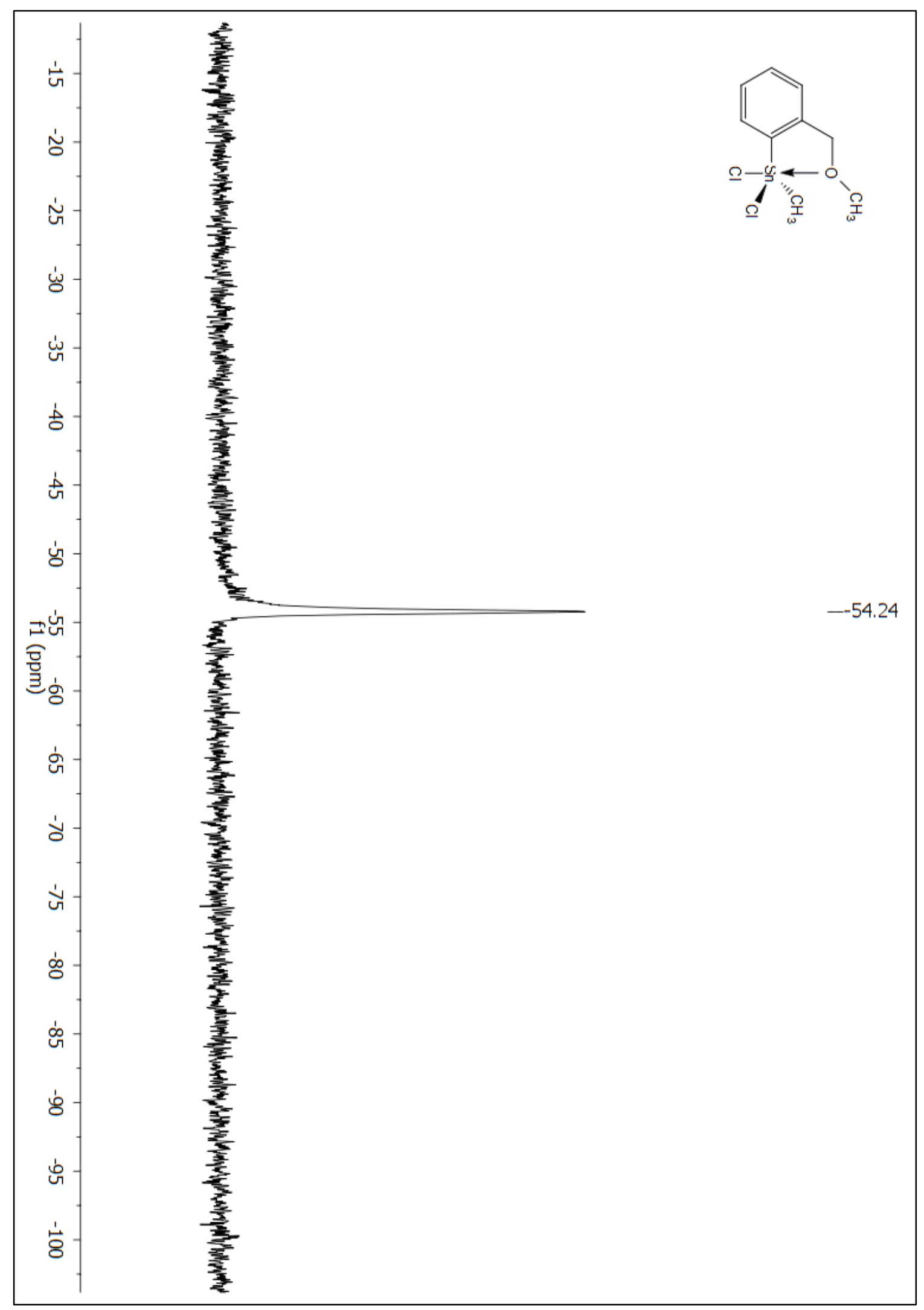

Figure A 77: ${ }^{119} \mathrm{Sn}$ NMR $\left(\mathrm{CDCl}_{3}\right)$ spectrum of compound 218.

304 


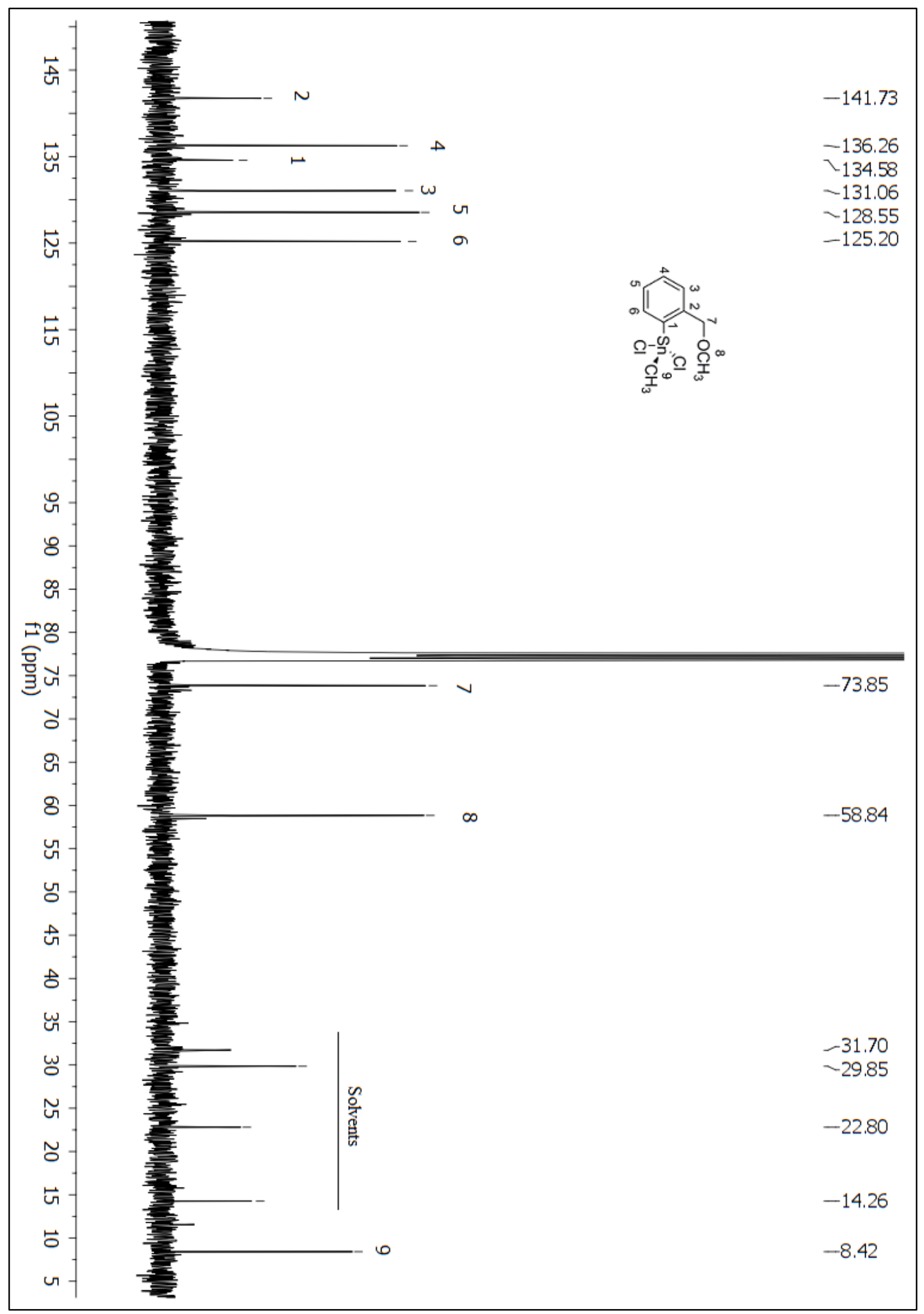

Figure A 78: ${ }^{13} \mathrm{C}$ NMR $\left(\mathrm{CDCl}_{3}\right)$ spectrum of compound 218. 


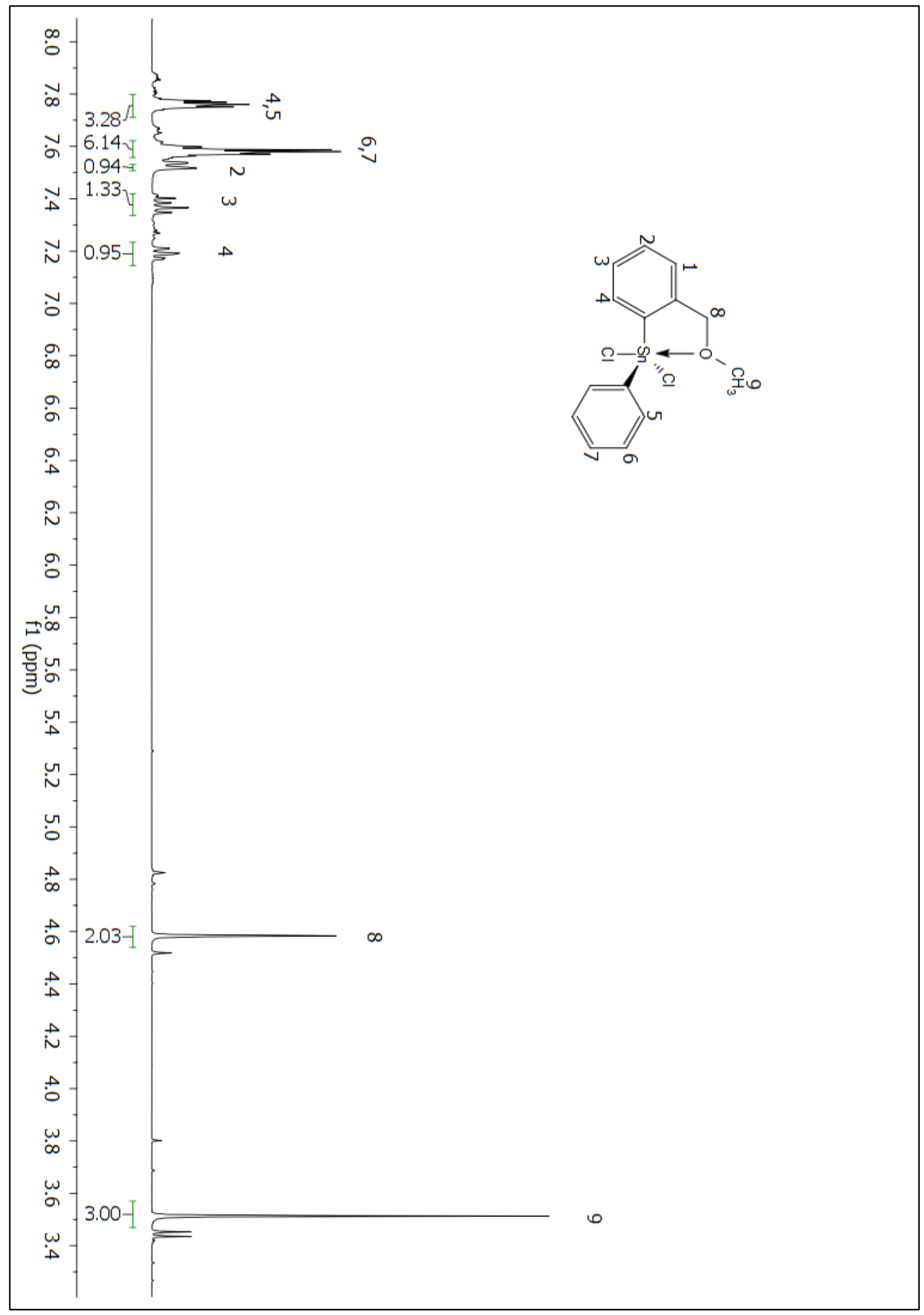

Figure A 79: ${ }^{1} \mathrm{H}$ NMR $\left(\mathrm{CDCl}_{3}\right)$ spectrum of compound 220. 


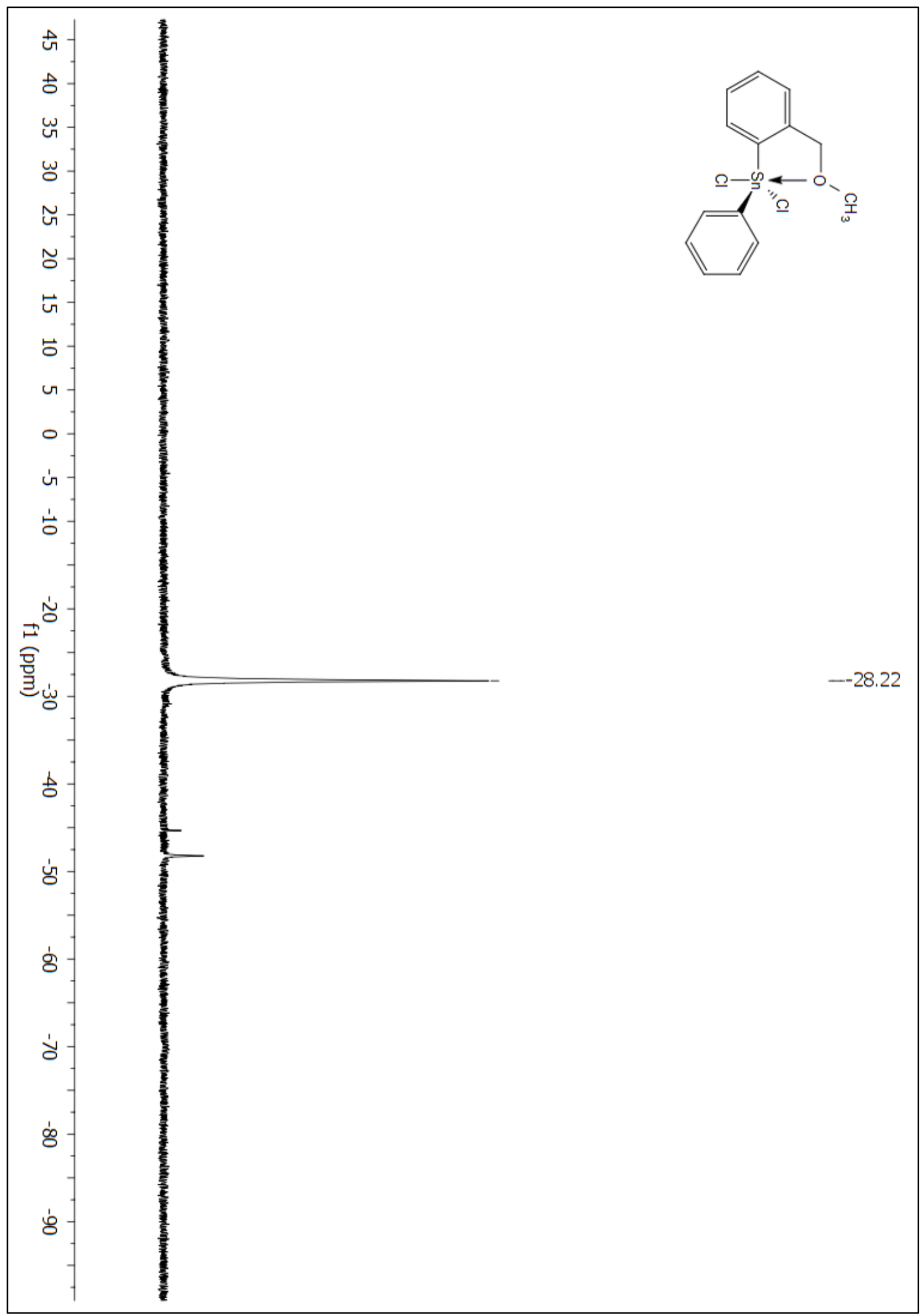

Figure A 80: ${ }^{119} \mathrm{Sn} \mathrm{NMR}\left(\mathrm{CDCl}_{3}\right)$ spectrum of compound 220. 


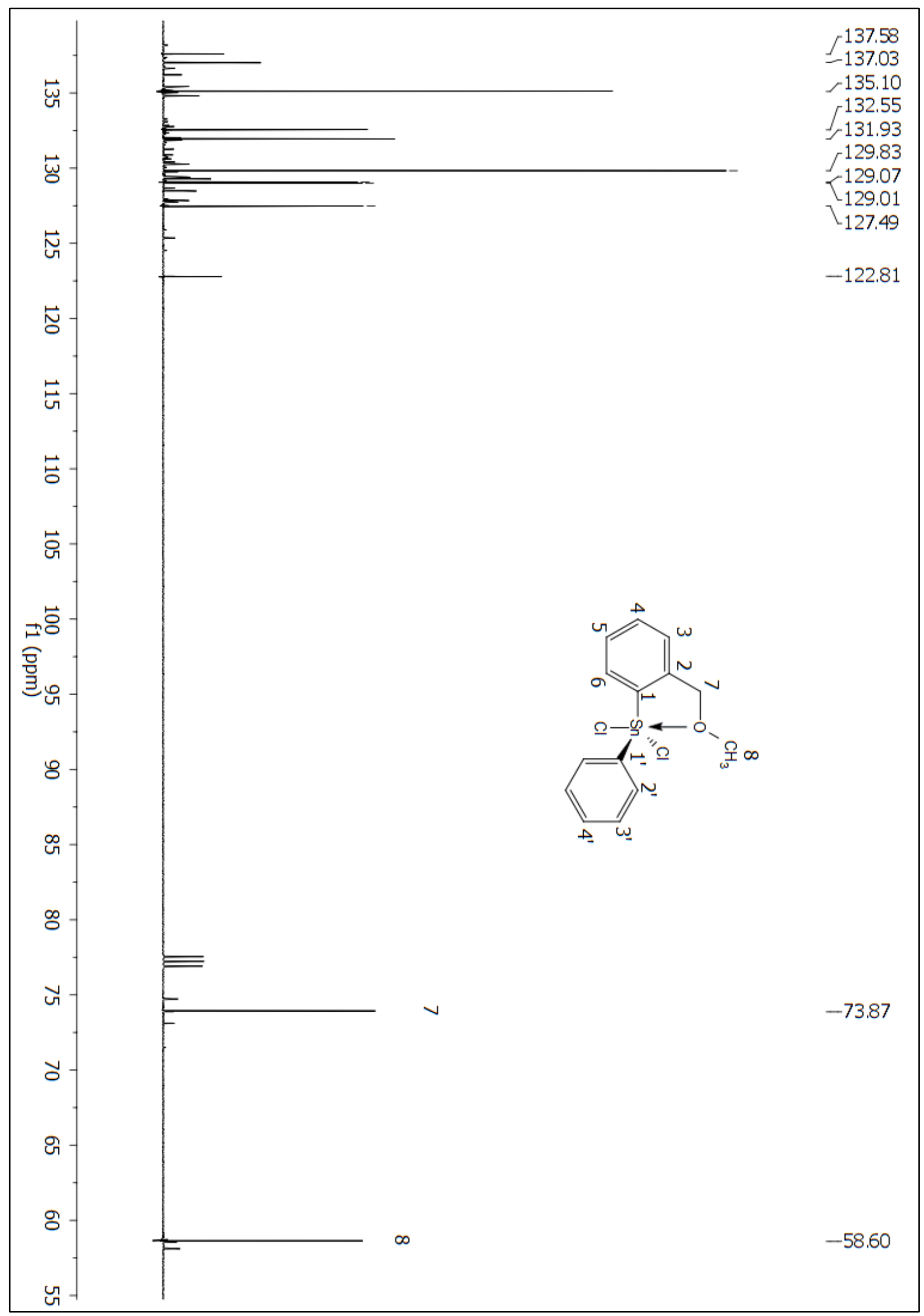

Figure A 81: ${ }^{13} \mathrm{C}$ NMR $\left(\mathrm{CDCl}_{3}\right)$ spectrum of compound 220. 


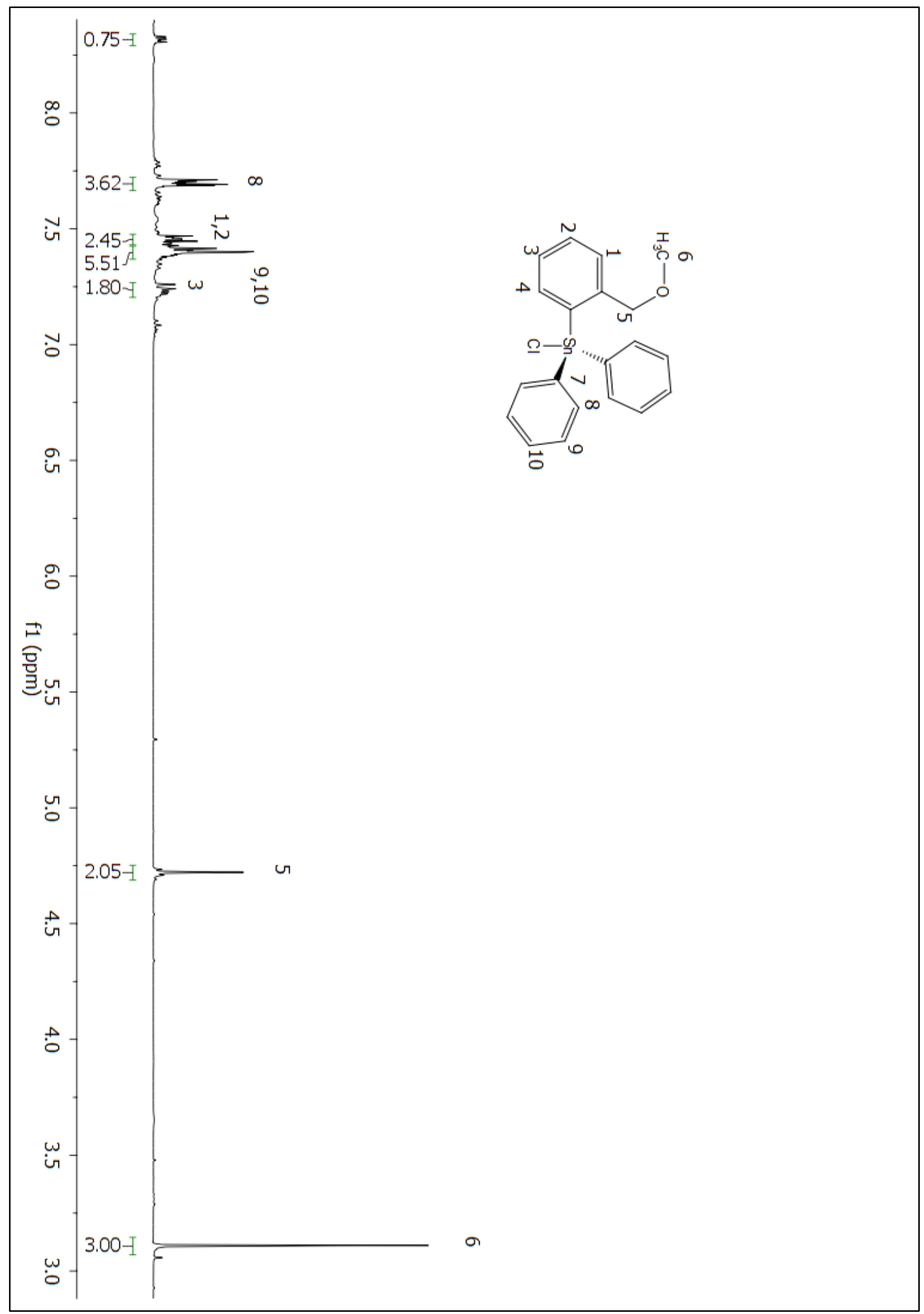

Figure A 82: ${ }^{1} \mathrm{H}$ NMR $\left(\mathrm{CDCl}_{3}\right)$ spectrum of compound 221. 


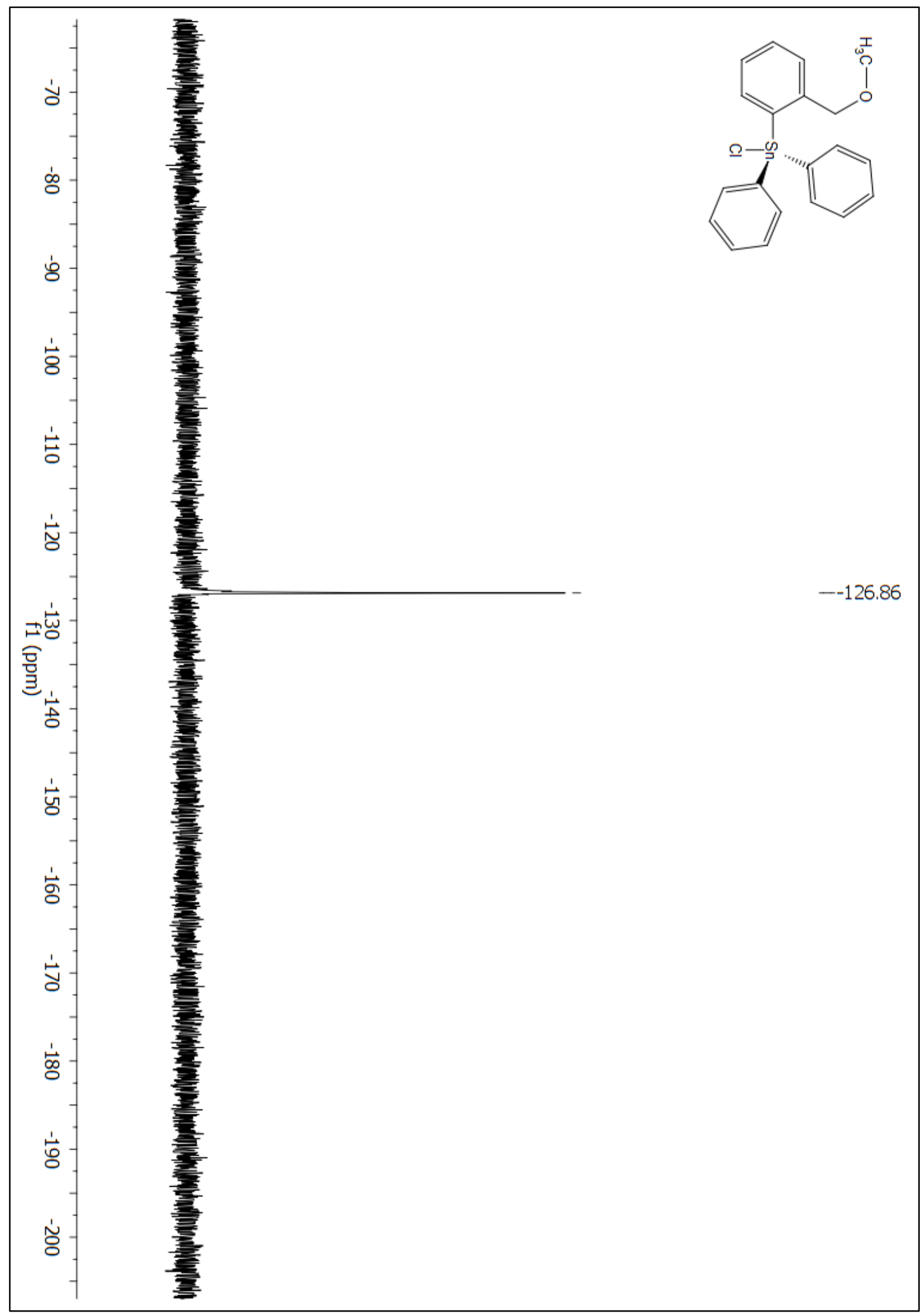

Figure A 83: ${ }^{119} \mathrm{Sn} \mathrm{NMR}\left(\mathrm{CDCl}_{3}\right)$ spectrum of compound 221. 


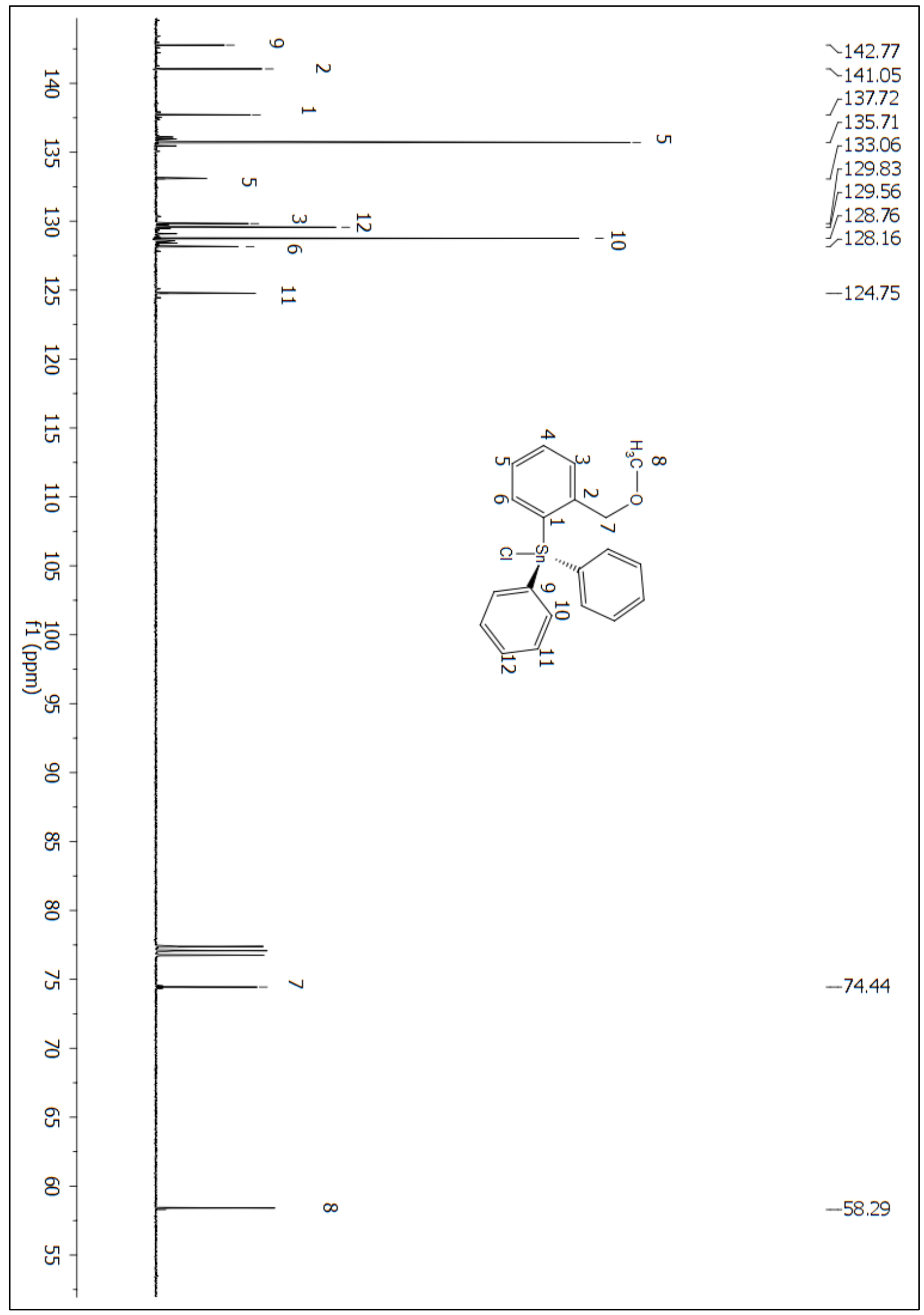

Figure A 84: ${ }^{13} \mathrm{C}$ NMR $\left(\mathrm{CDCl}_{3}\right)$ spectrum of compound 221. 


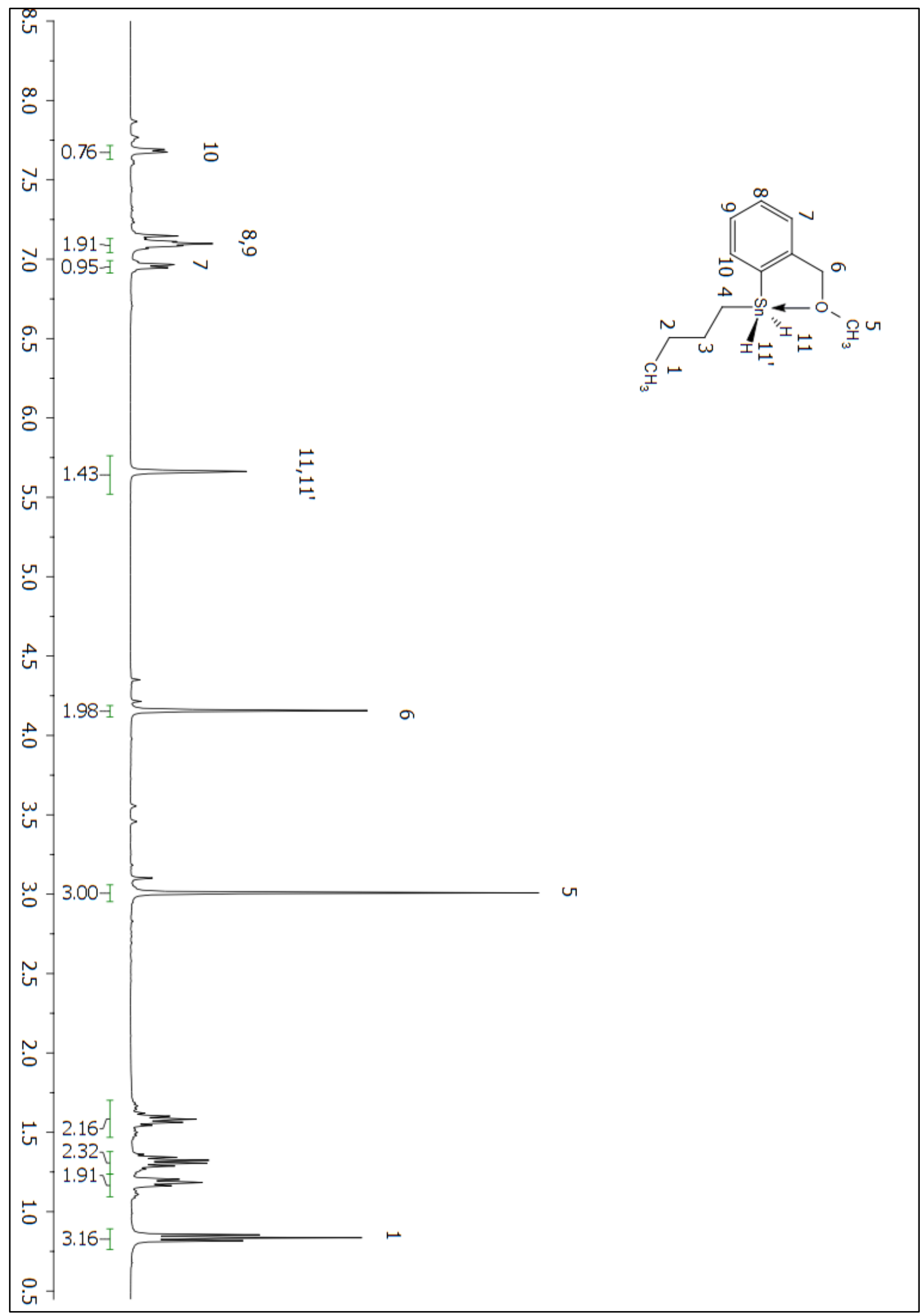

Figure A 85: ${ }^{1} \mathrm{H}$ NMR $\left(\mathrm{C}_{6} \mathrm{D}_{6}\right)$ spectrum of compound 227. 


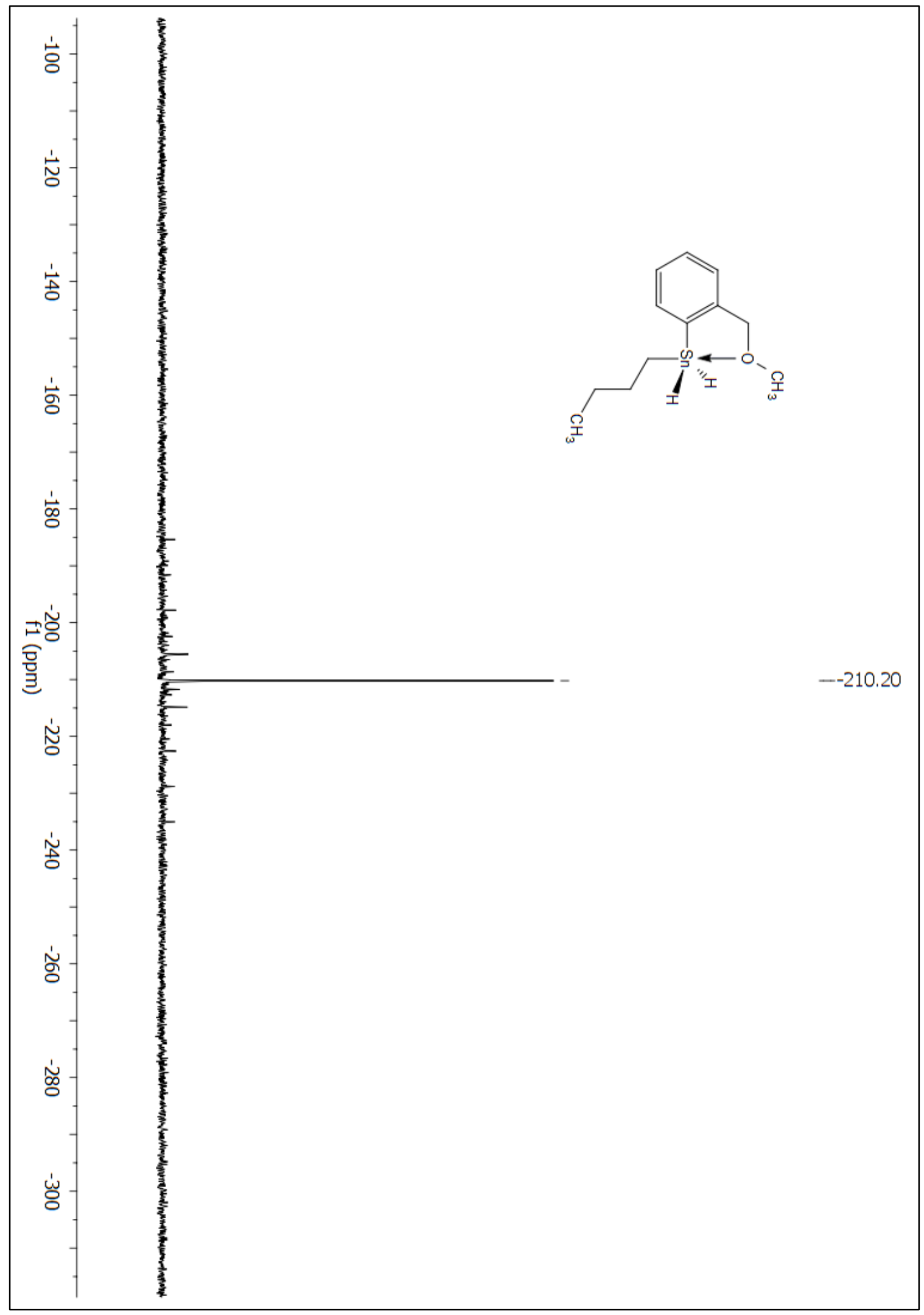

Figure A 86: ${ }^{119} \mathrm{Sn}$ NMR $\left(\mathrm{C}_{6} \mathrm{D}_{6}\right)$ spectrum of compound 227. 


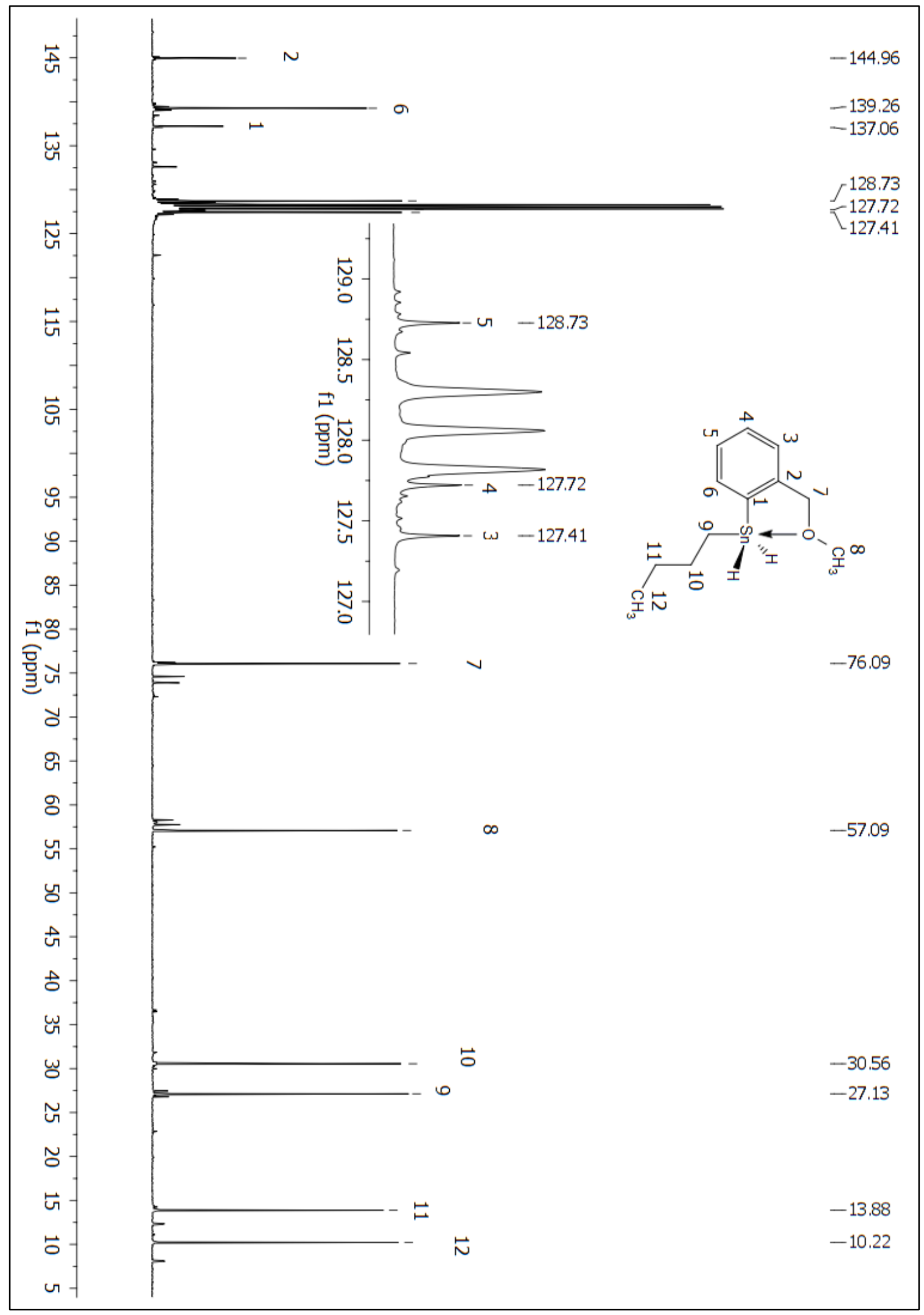

Figure A 87: ${ }^{13} \mathrm{C}$ NMR $\left(\mathrm{C}_{6} \mathrm{D}_{6}\right)$ spectrum of compound 227. 


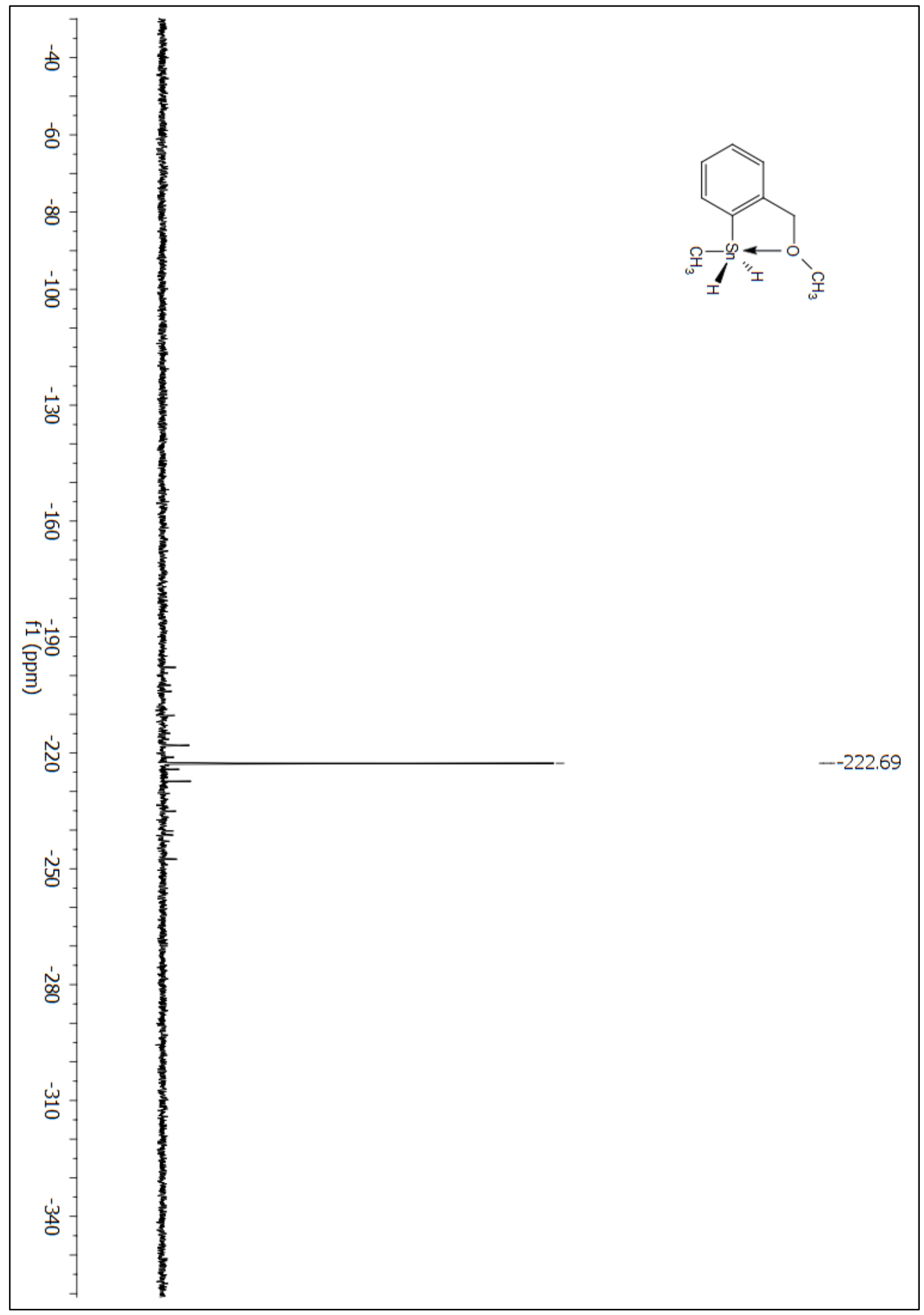

Figure A 88: ${ }^{119} \mathrm{Sn}$ NMR $\left(\mathrm{C}_{6} \mathrm{D}_{6}\right)$ spectrum of compound 226. 


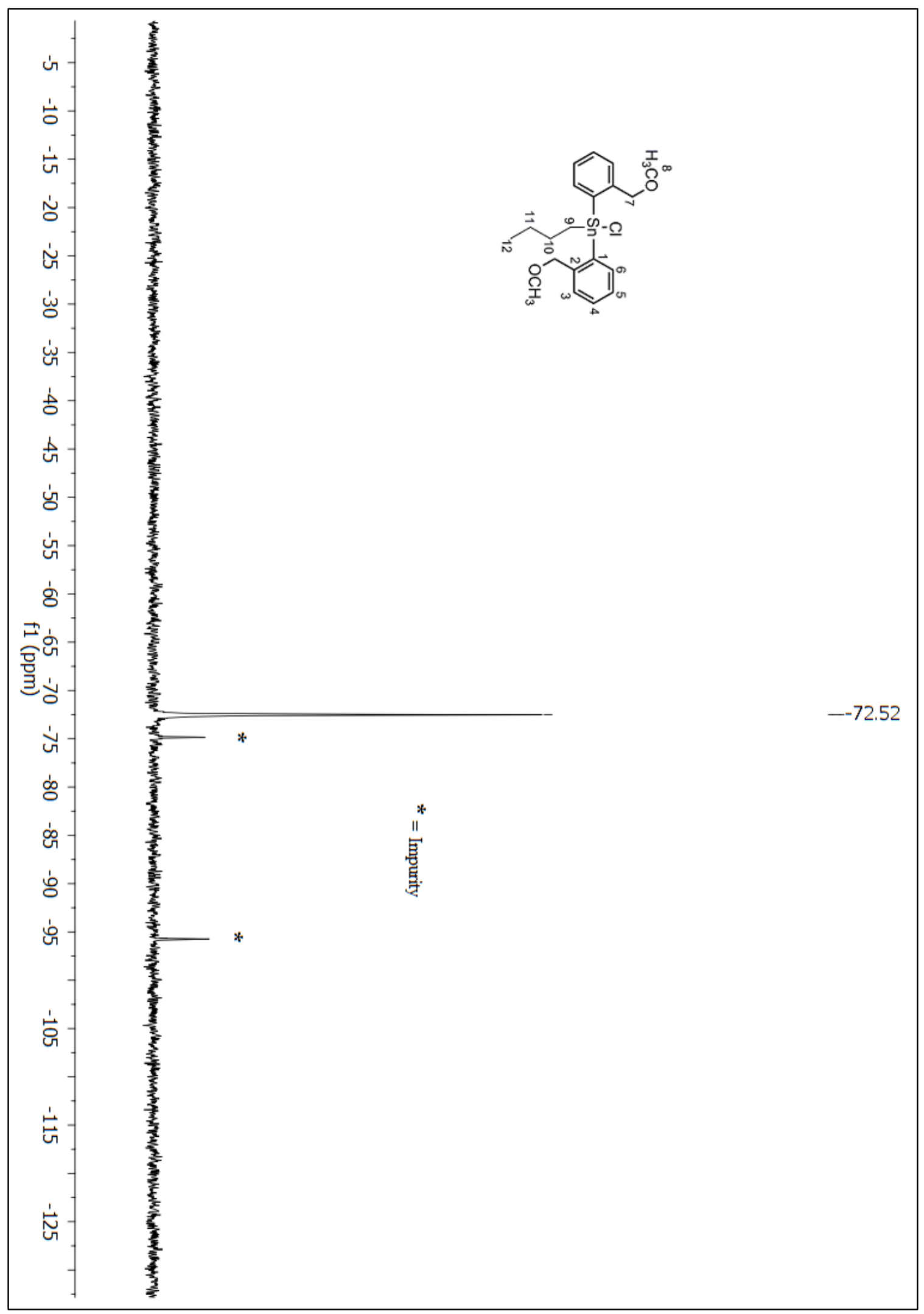

Figure A 89: ${ }^{119} \mathrm{Sn} \mathrm{NMR}\left(\mathrm{CDCl}_{3}\right)$ spectrum of compound 224 


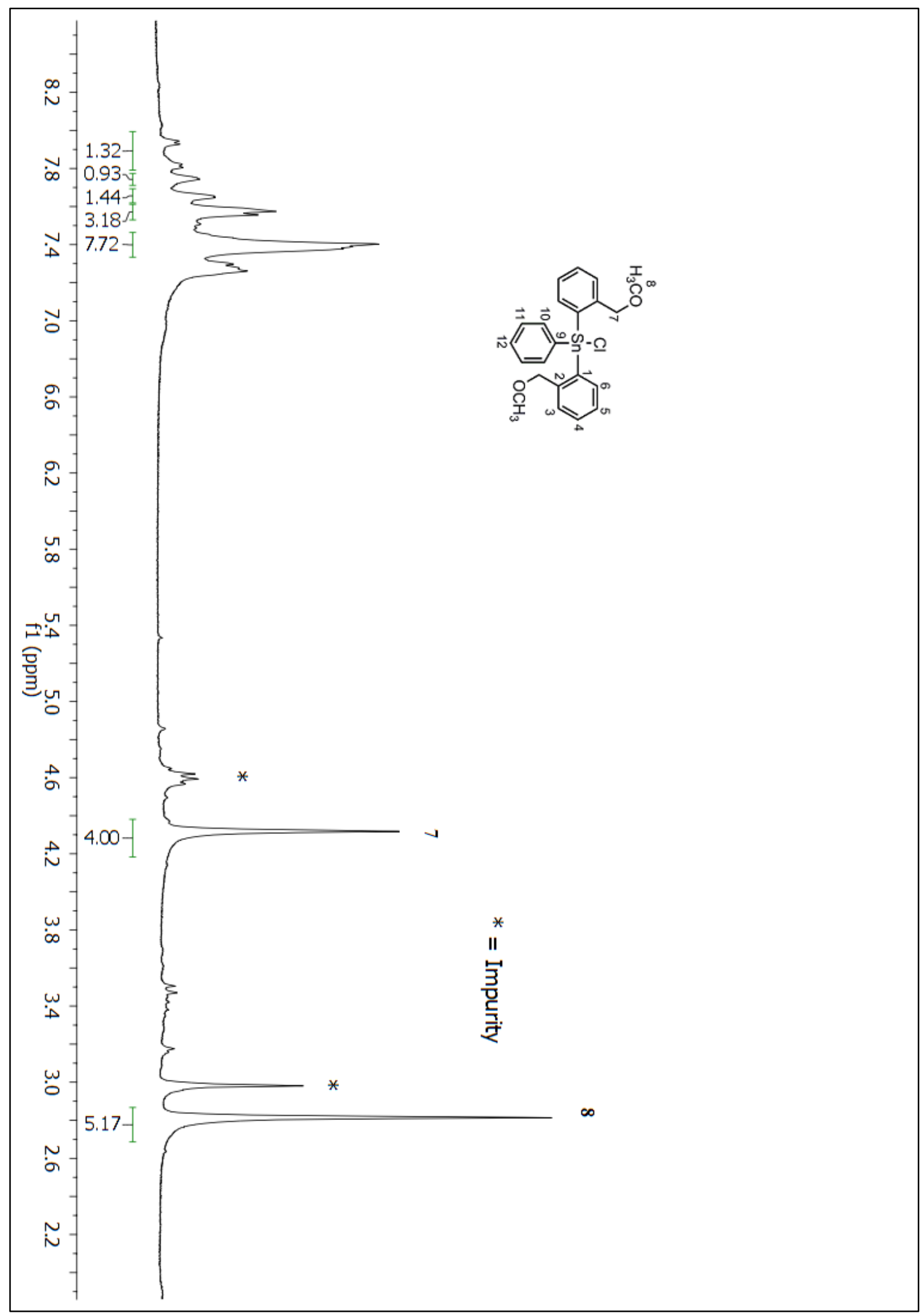

Figure A 90: ${ }^{1} \mathrm{H}$ NMR $\left(\mathrm{CDCl}_{3}\right)$ spectrum of compound 225 


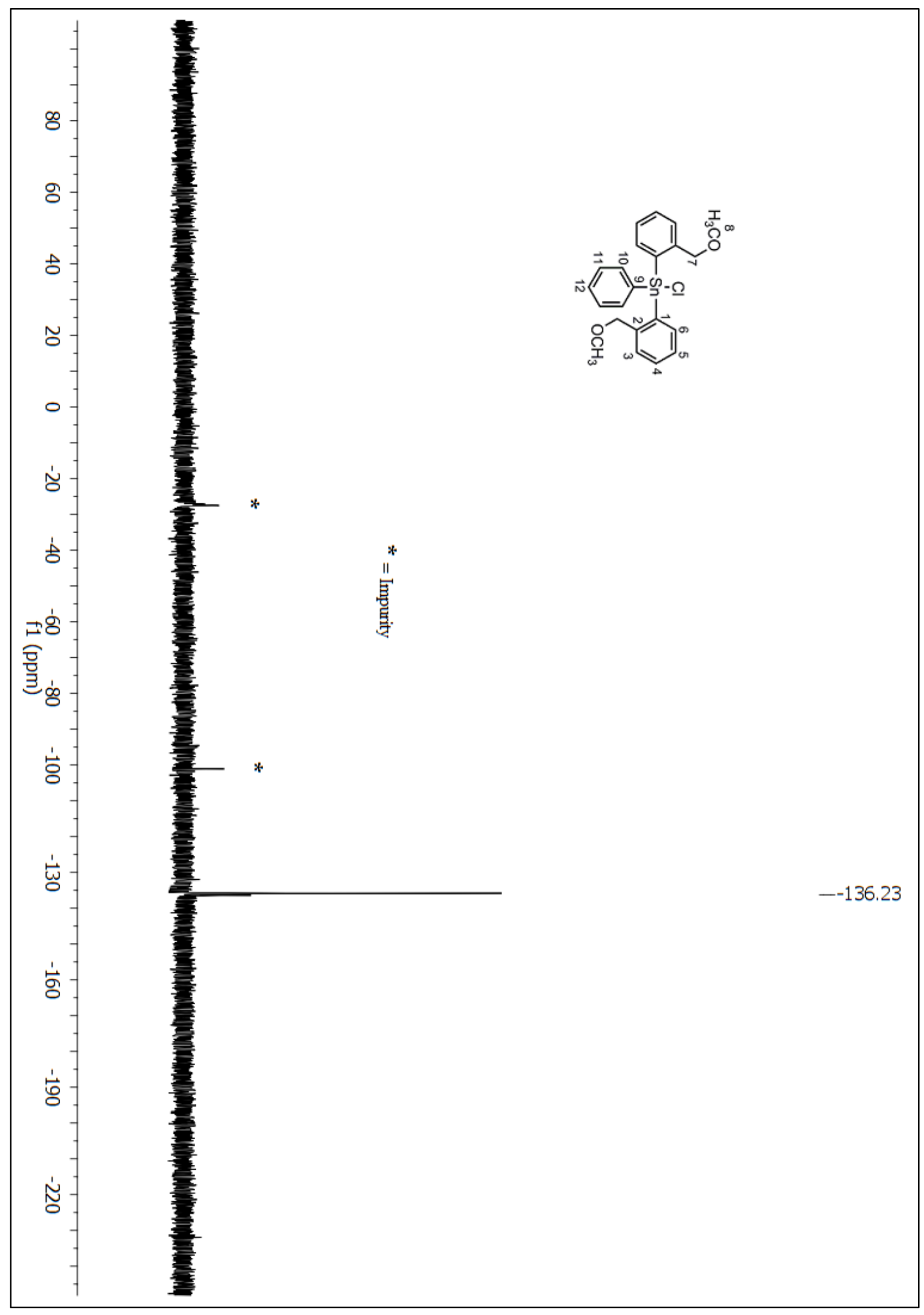

Figure A 91: ${ }^{119} \mathrm{Sn} \mathrm{NMR}\left(\mathrm{CDCl}_{3}\right)$ spectrum of compound 225 


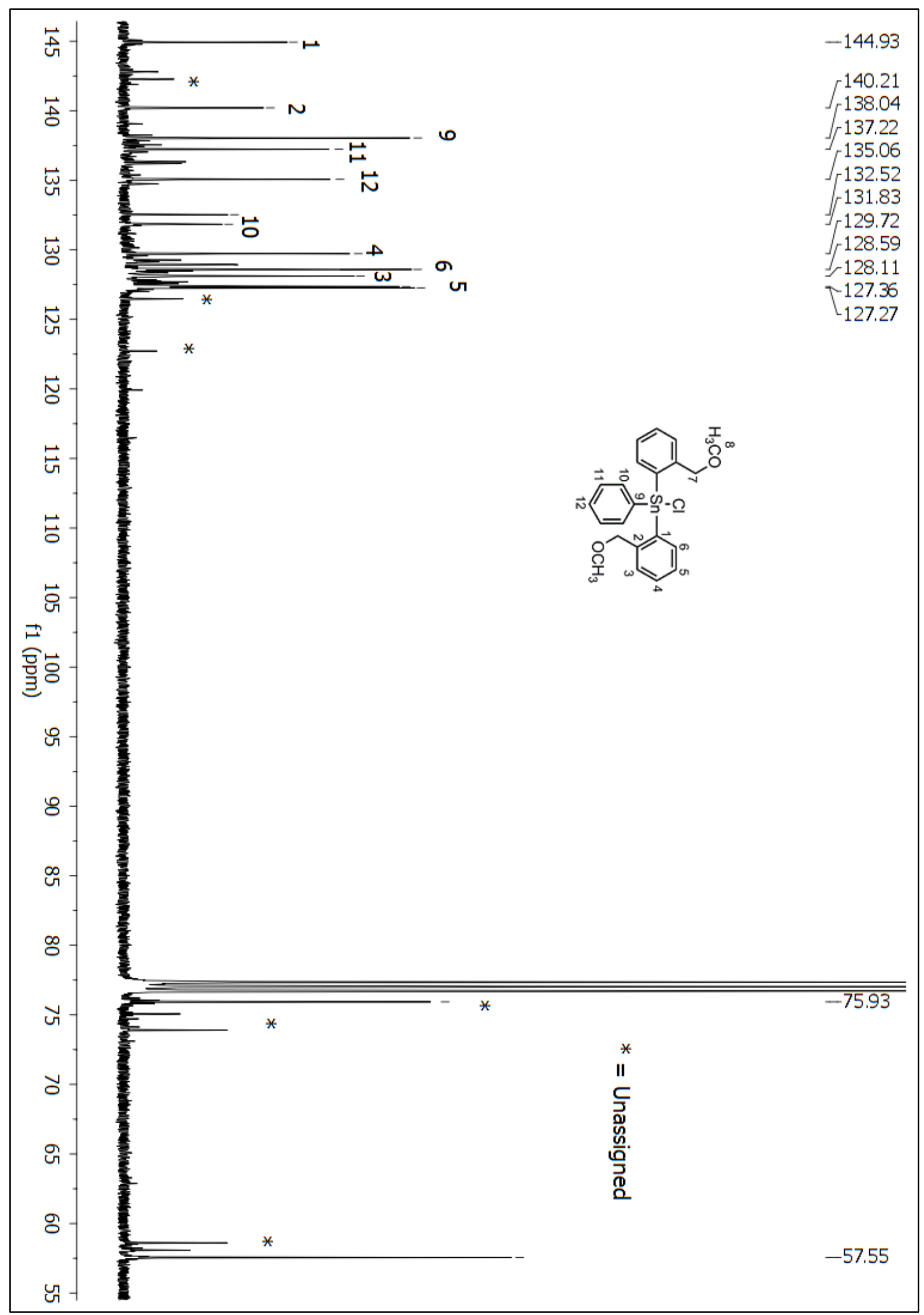

Figure A 92: ${ }^{13} \mathrm{C}$ NMR $\left(\mathrm{CDCl}_{3}\right)$ spectrum of compound 225 


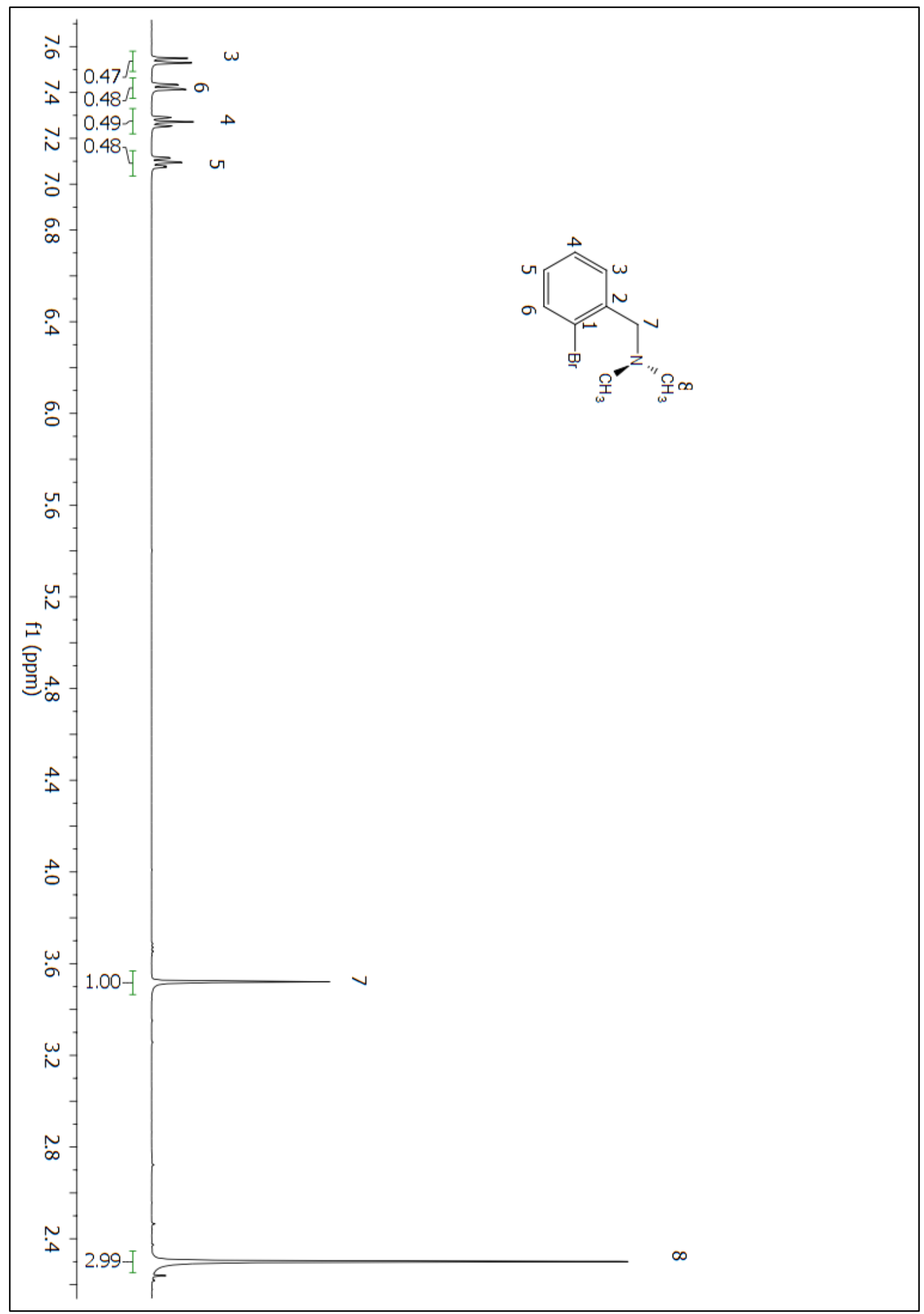

Figure A 93: ${ }^{1} \mathrm{H}$ NMR CDCl 3 spectrum of compound 228 


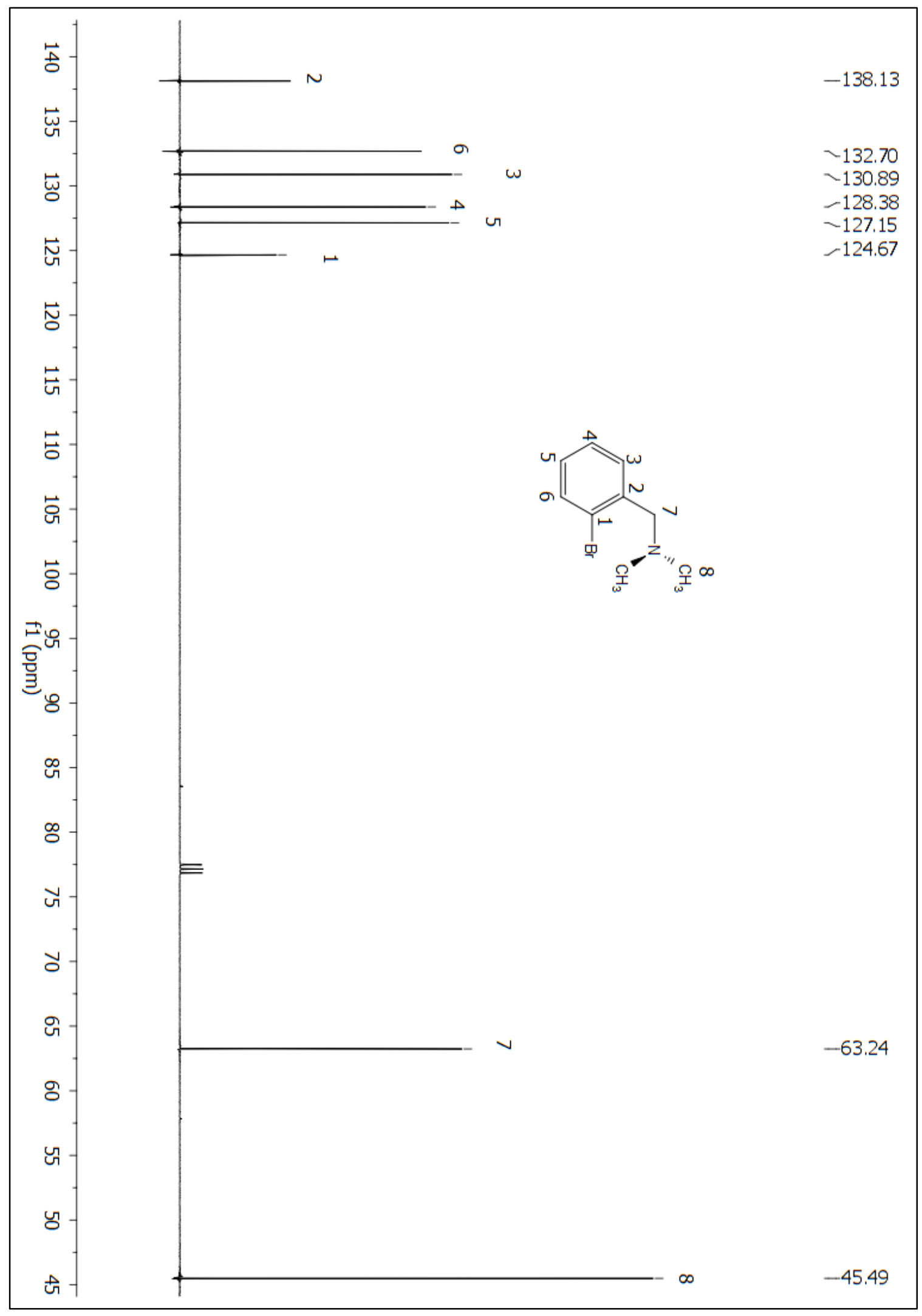

Figure A 94: ${ }^{13} \mathrm{C}\left(\mathrm{CDCl}_{3}\right) \mathrm{NMR}$ spectrum of compound 228 


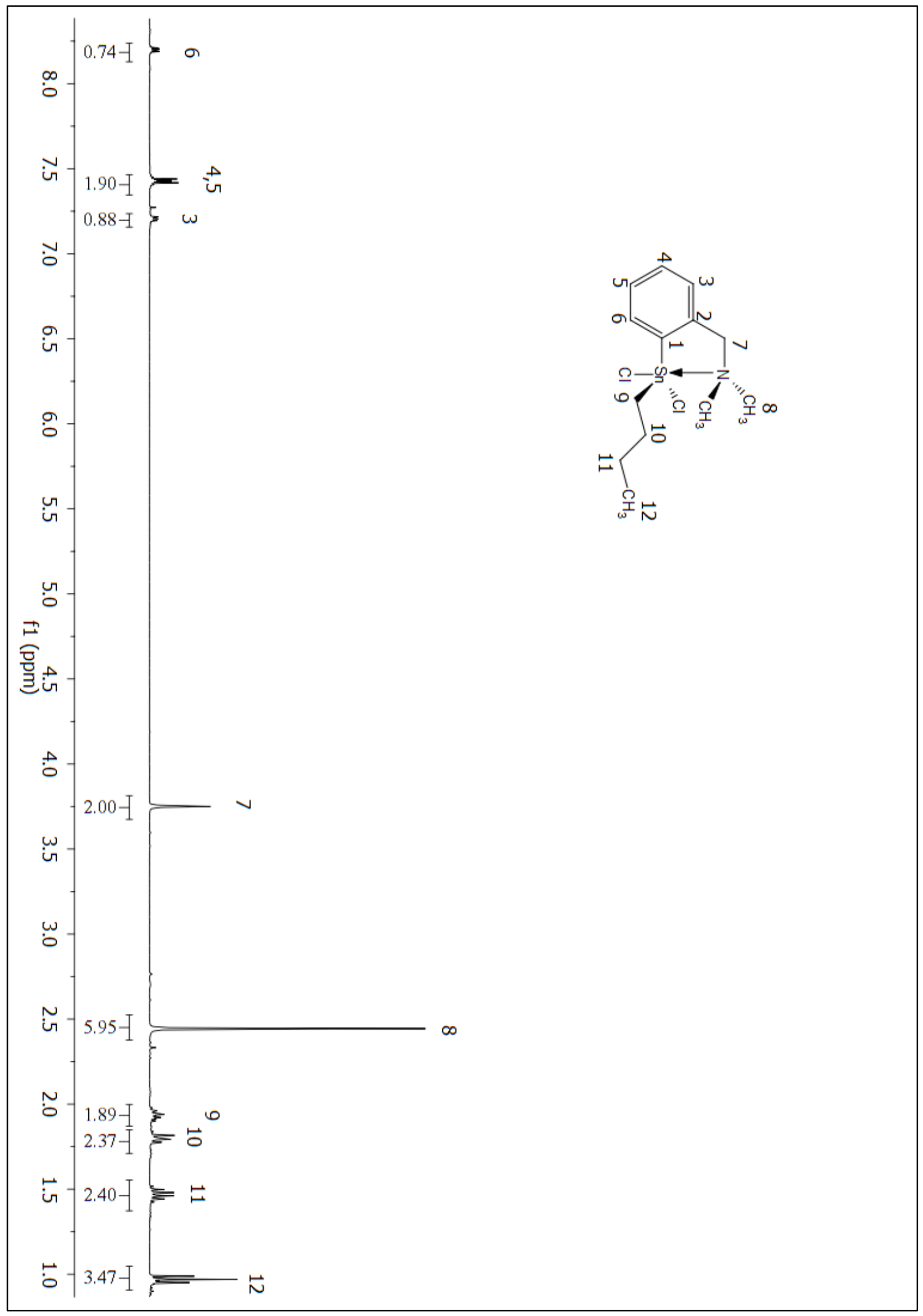

Figure A 95: ${ }^{1} \mathrm{H}$ NMR $\left(\mathrm{CDCl}_{3}\right)$ spectrum of compound 37. 


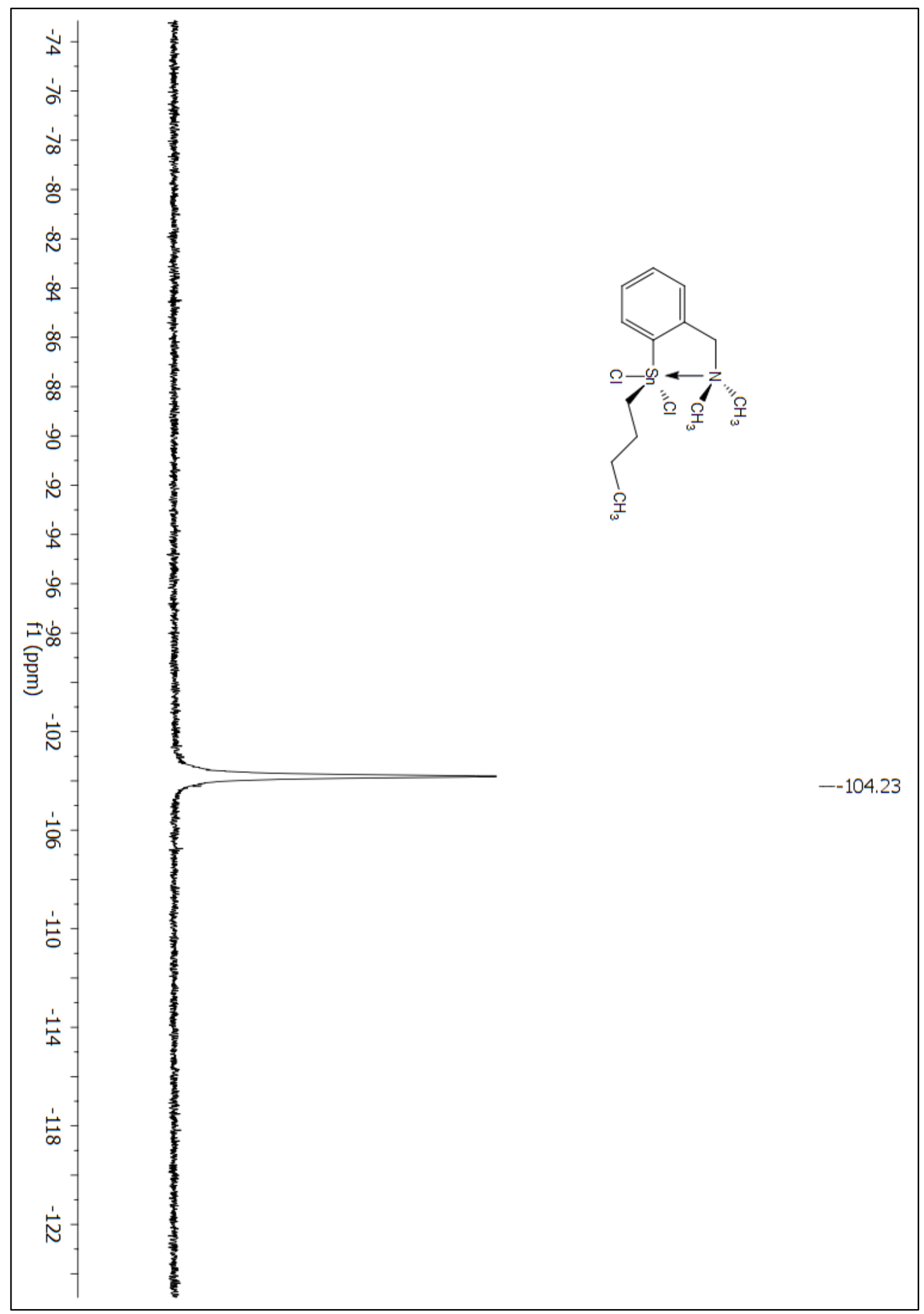

Figure A 96: ${ }^{119} \mathrm{Sn}$ NMR $\left(\mathrm{CDCl}_{3}\right)$ spectrum of compound 37. 


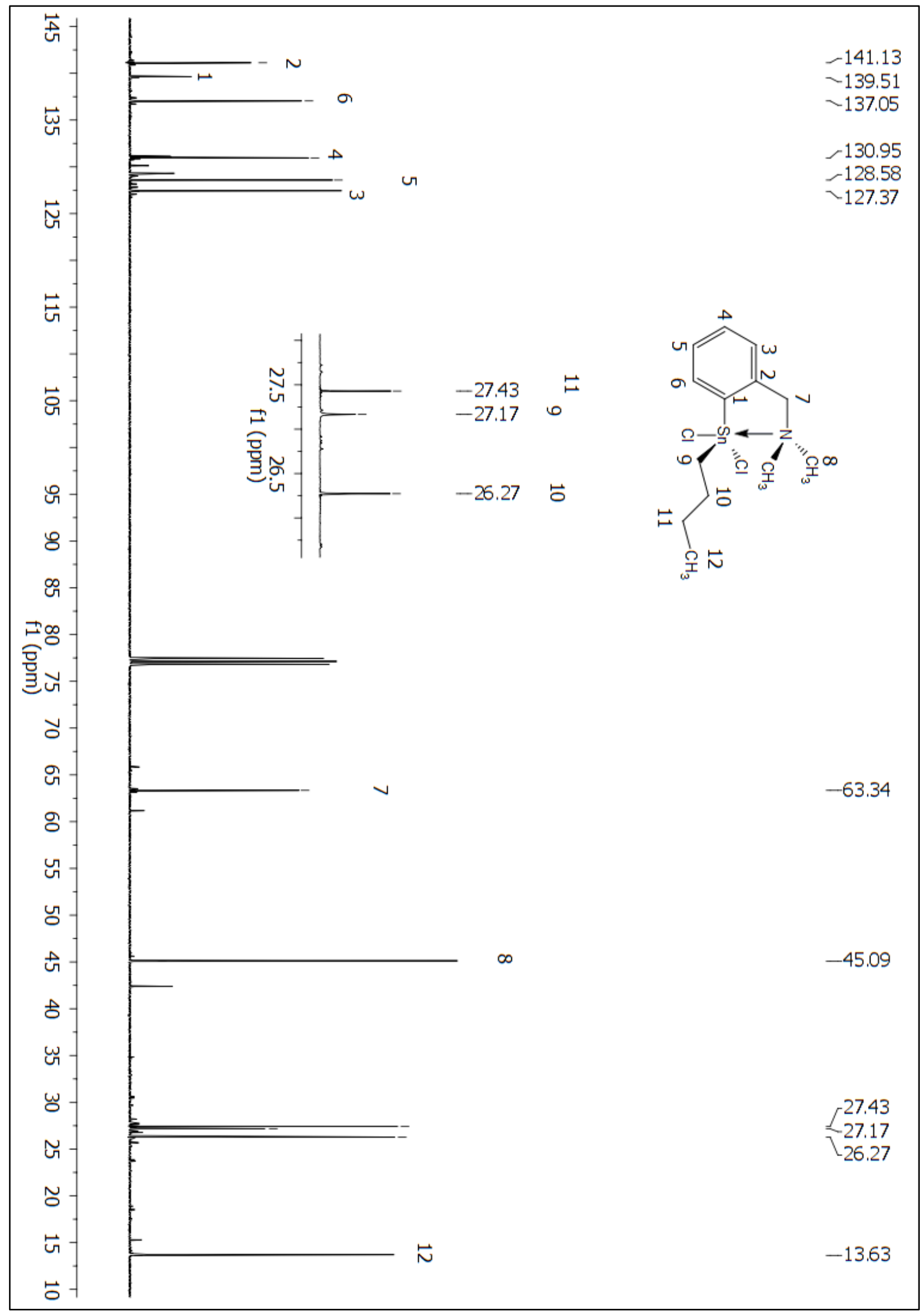

Figure A 97: ${ }^{13} \mathrm{C} \mathrm{NMR}\left(\mathrm{CDCl}_{3}\right)$ of compound 37. 


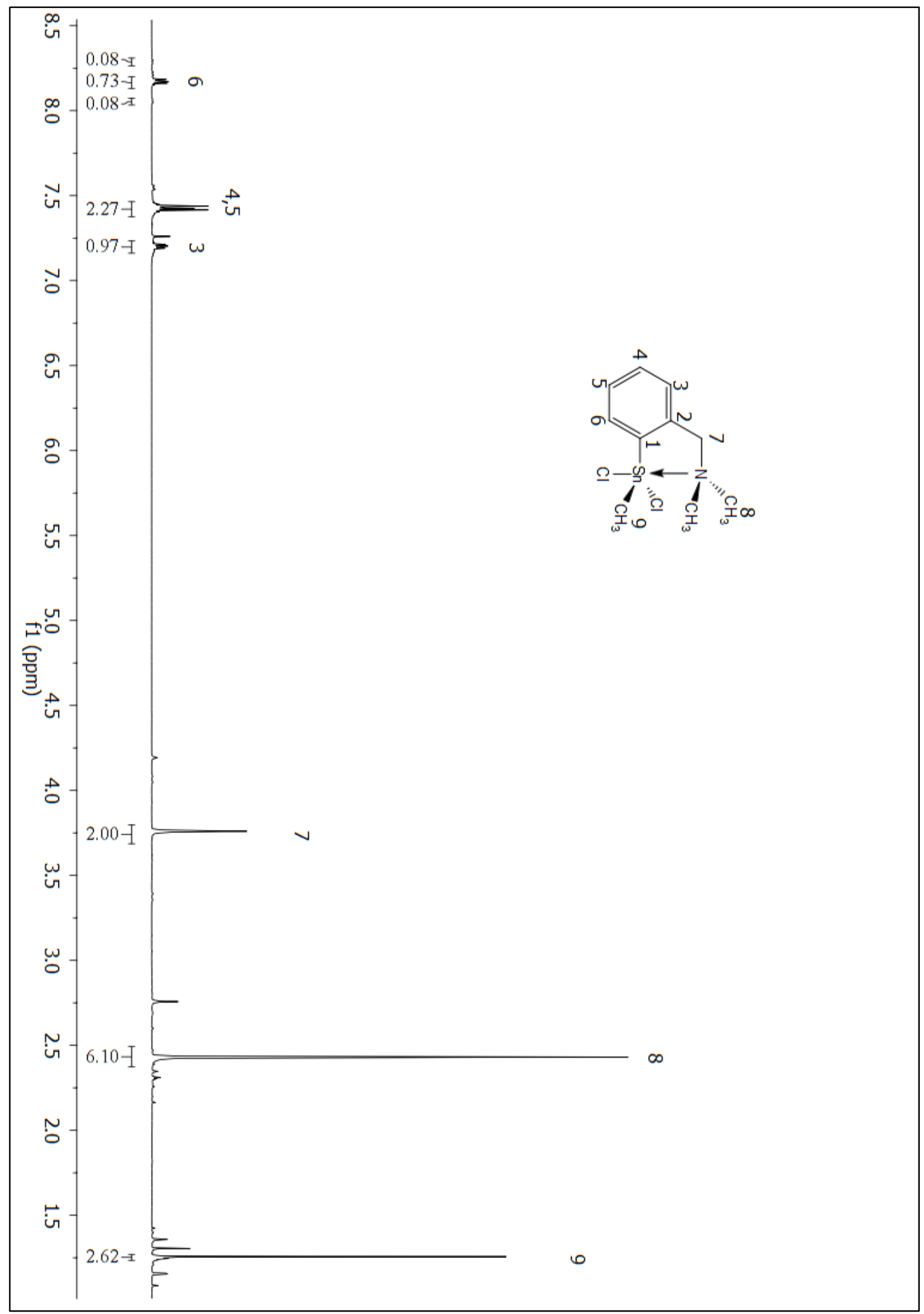

Figure A 98: ${ }^{1} \mathrm{H}$ NMR $\left(\mathrm{CDCl}_{3}\right)$ spectrum of compound 33. 


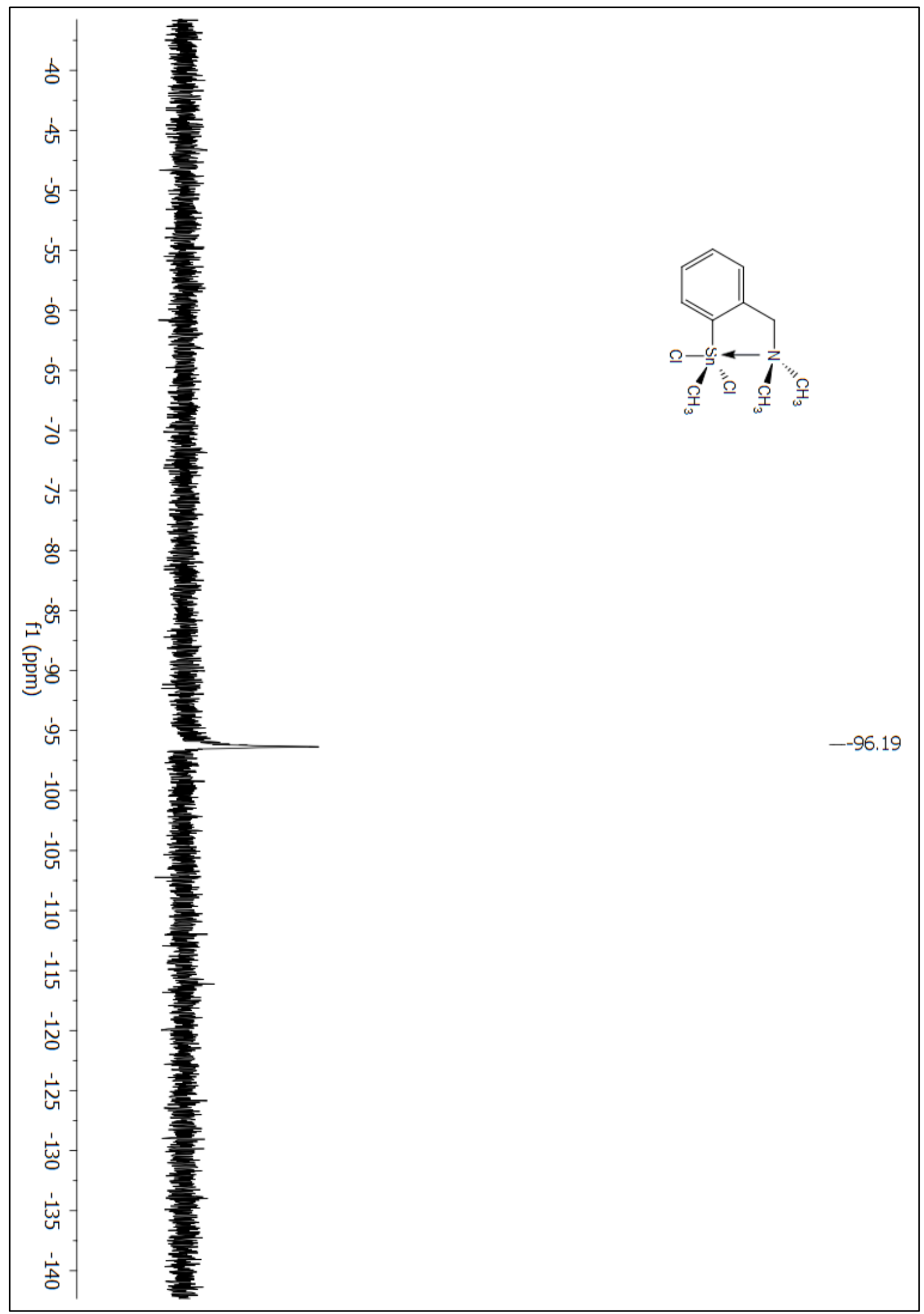

Figure A 99: ${ }^{119} \mathrm{Sn}$ NMR $\left(\mathrm{CDCl}_{3}\right)$ spectrum of compound 33. 


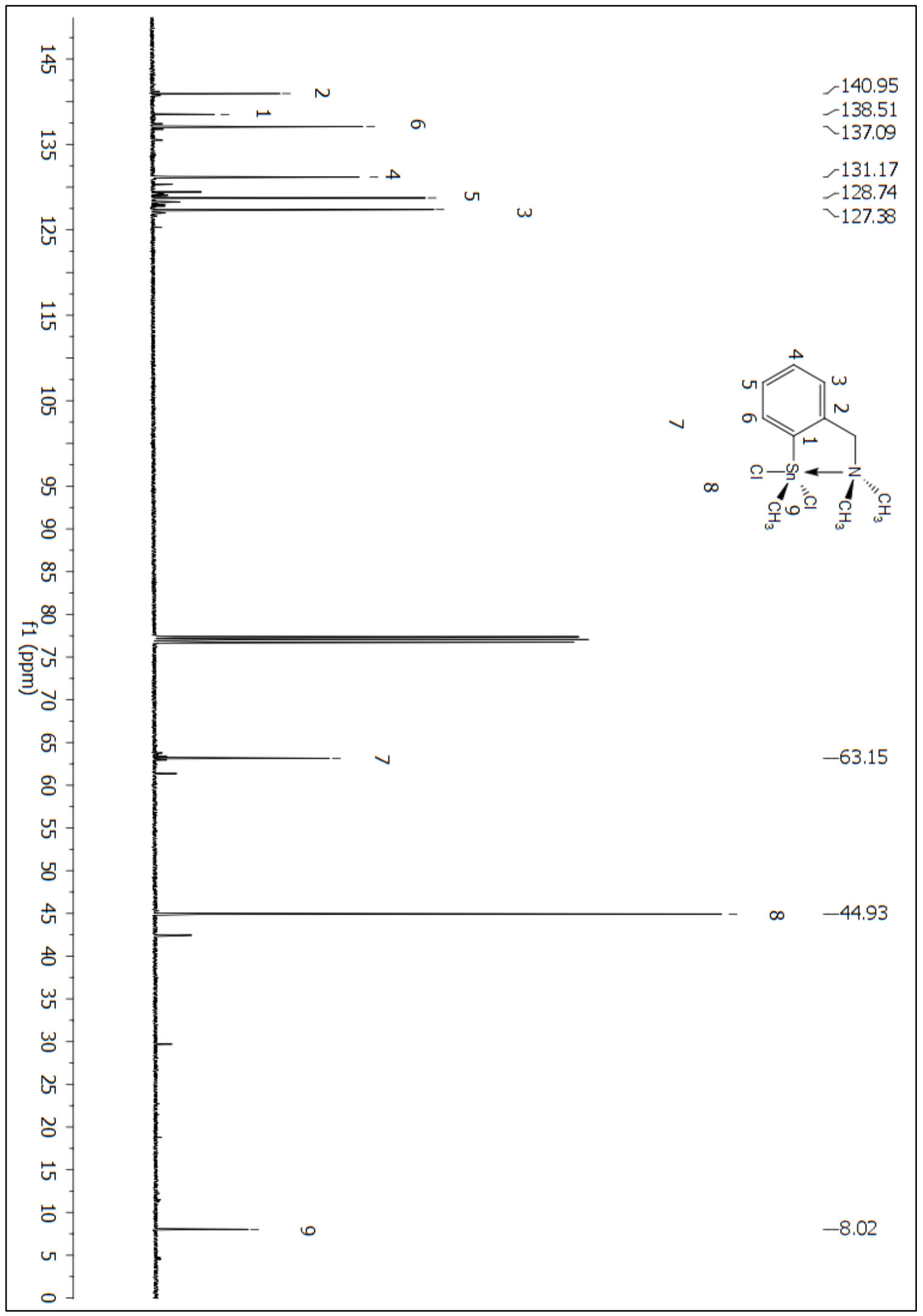

Figure A 100: ${ }^{13} \mathrm{C}$ NMR $\left(\mathrm{CDCl}_{3}\right)$ spectrum of compound 33. 


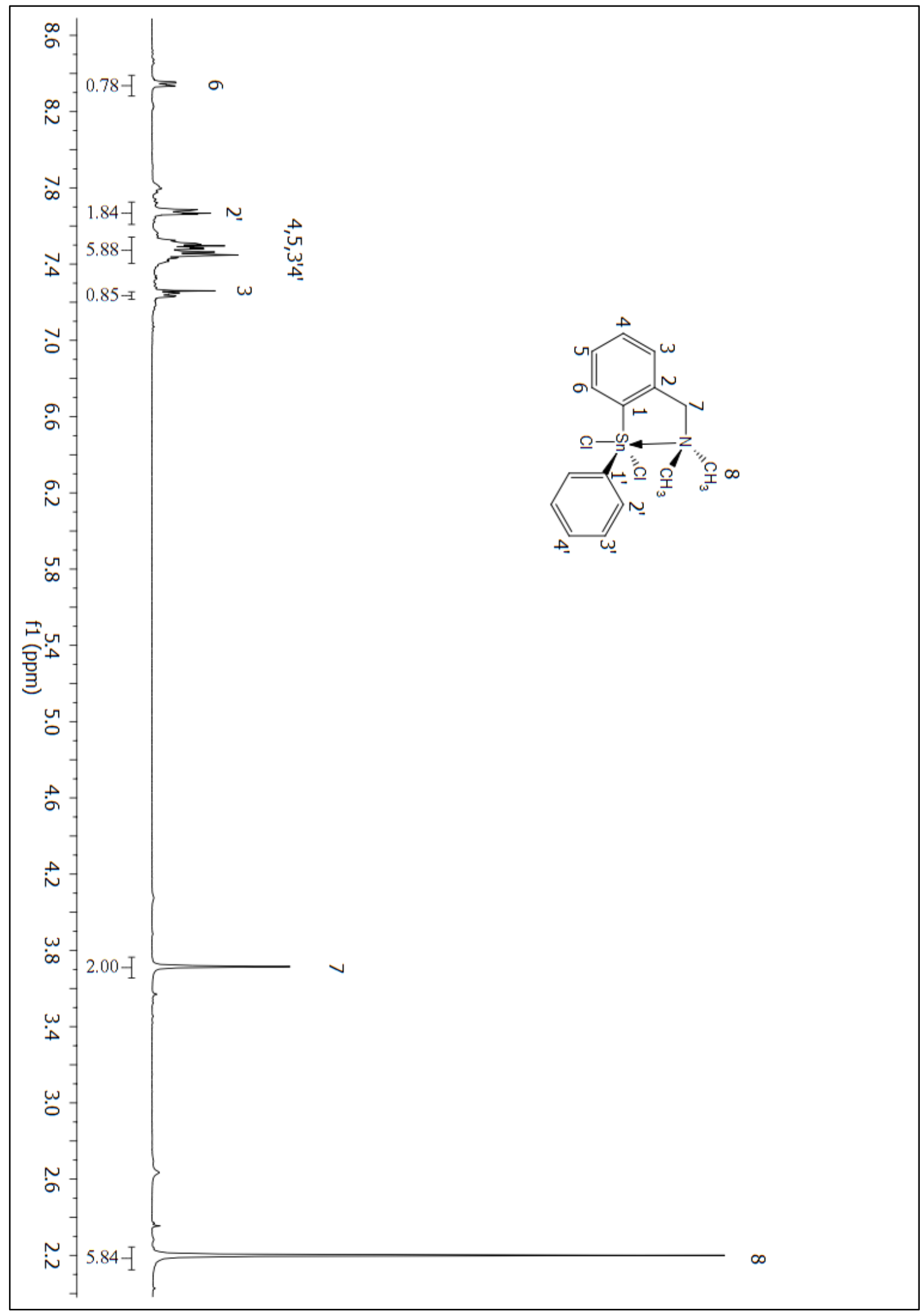

Figure A 101: ${ }^{1} \mathrm{H}$ NMR $\left(\mathrm{CDCl}_{3}\right)$ spectrum of compound 35. 


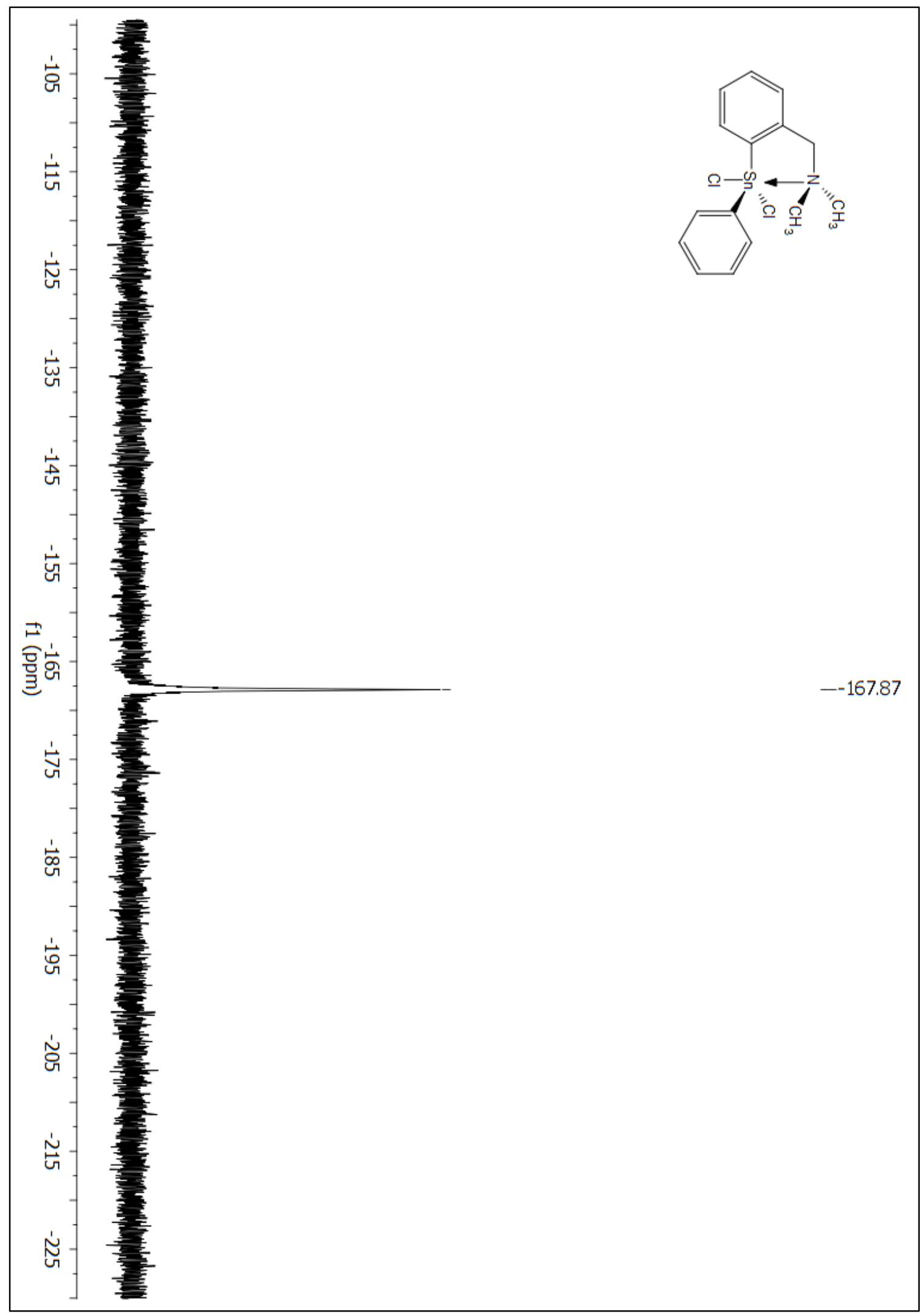

Figure A 102: ${ }^{119} \mathrm{Sn} \mathrm{NMR}\left(\mathrm{CDCl}_{3}\right)$ spectrum of compound 35. 


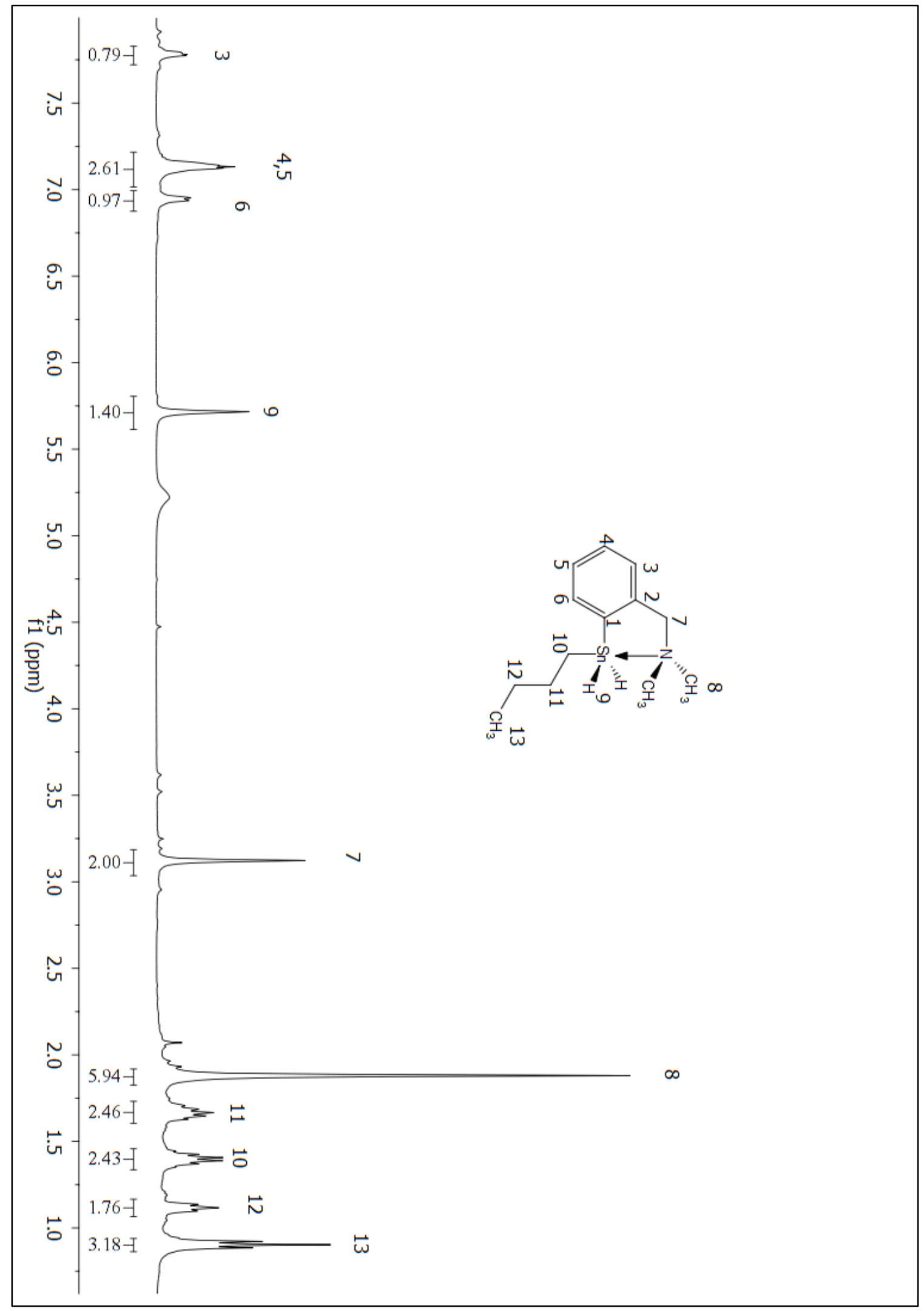

Figure A 103: ${ }^{1} \mathrm{H}$ NMR $\left(\mathrm{C}_{6} \mathrm{D}_{6}\right)$ spectrum of compound 231. 


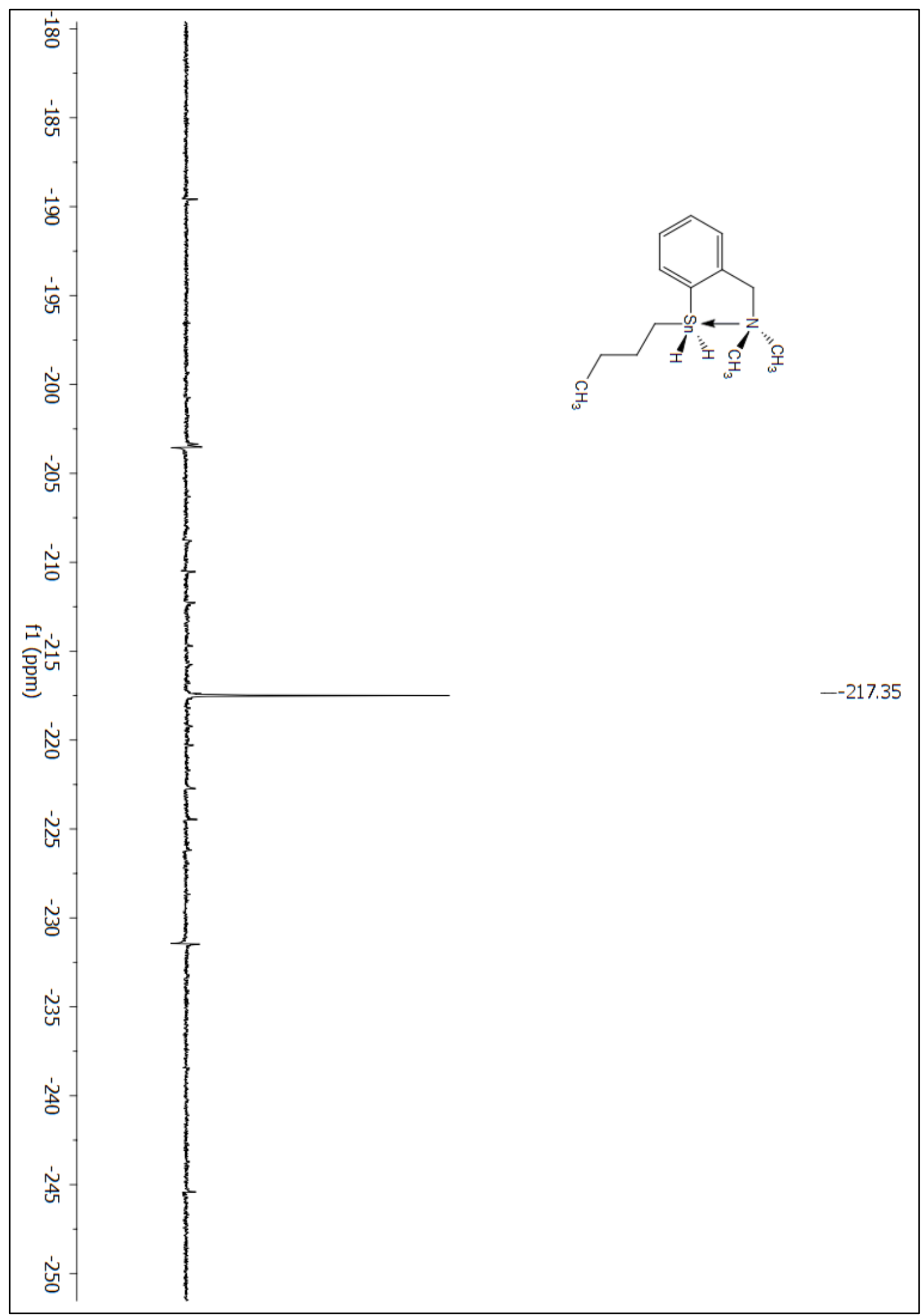

Figure A 104: ${ }^{119}$ Sn NMR $\left(\mathrm{C}_{6} \mathrm{D}_{6}\right)$ spectrum of compound 231. 


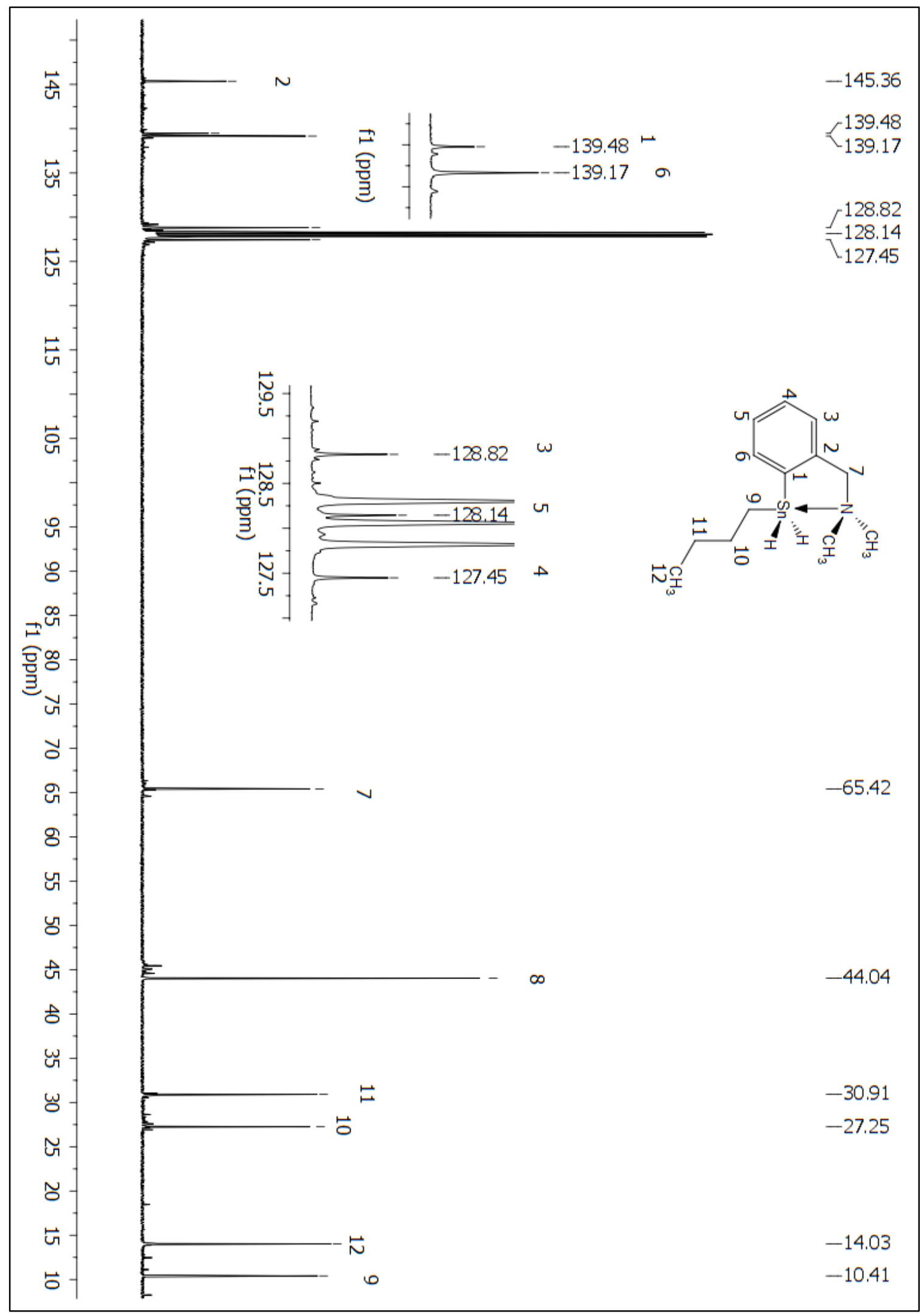

Figure A 105: ${ }^{13} \mathrm{C}$ NMR (C6D6) spectrum of compound 231. 


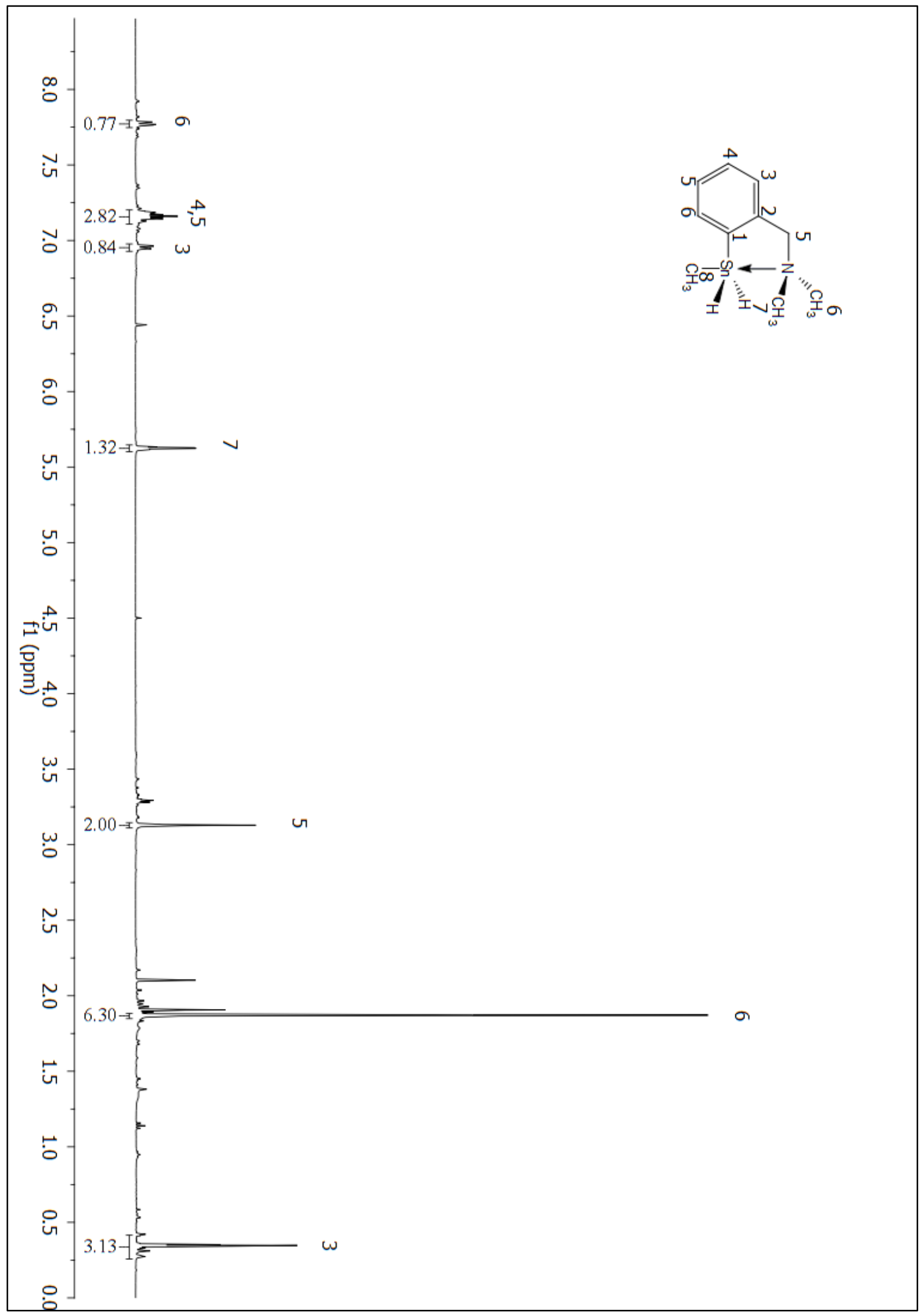

Figure A 106: ${ }^{1} \mathrm{H}$ NMR $\left(\mathrm{C}_{6} \mathrm{D}_{6}\right)$ spectrum of compound 230. 


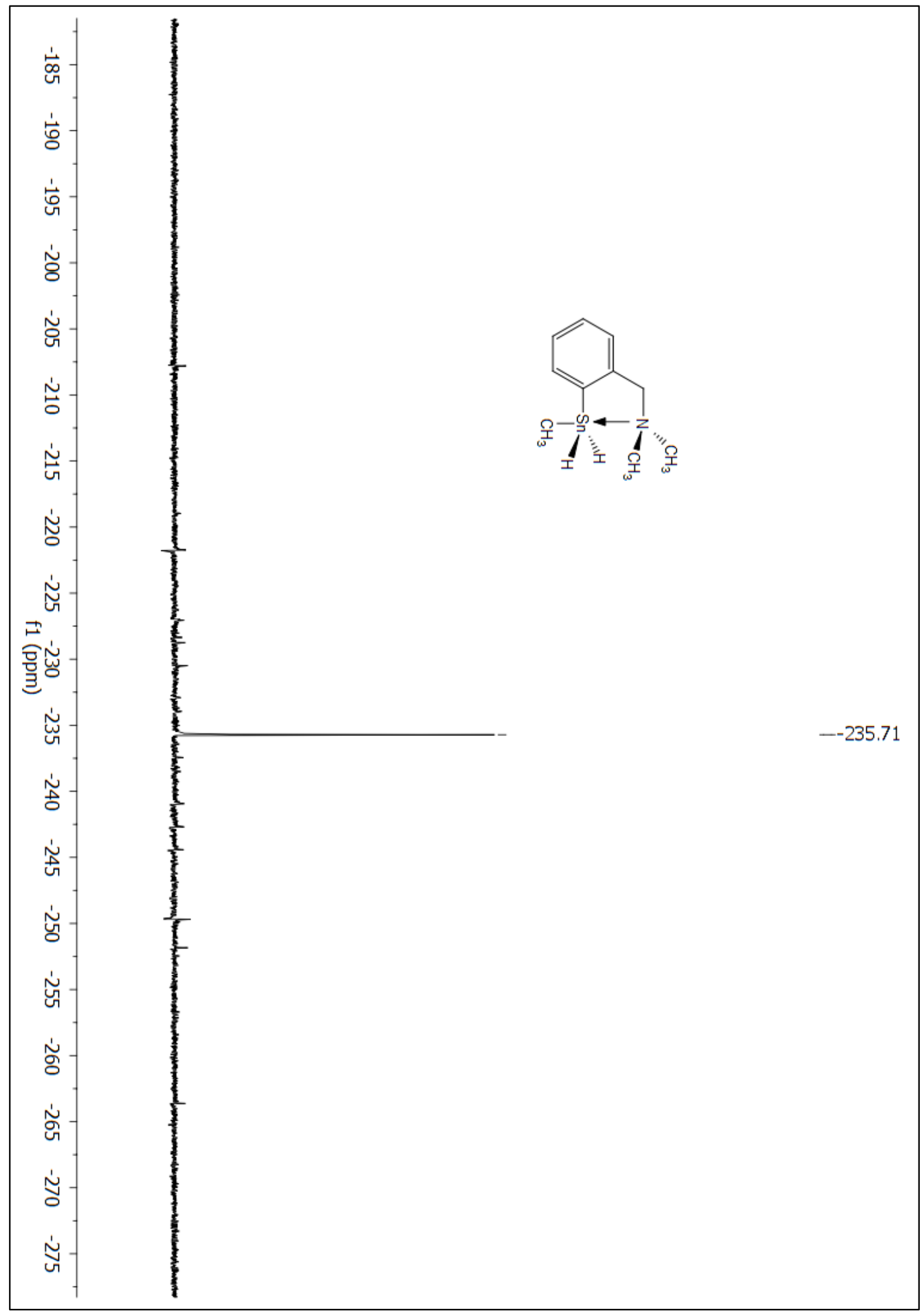

Figure A 107: ${ }^{119} \mathrm{Sn}$ NMR $\left(\mathrm{C}_{6} \mathrm{D}_{6}\right)$ spectrum of compound 230. 


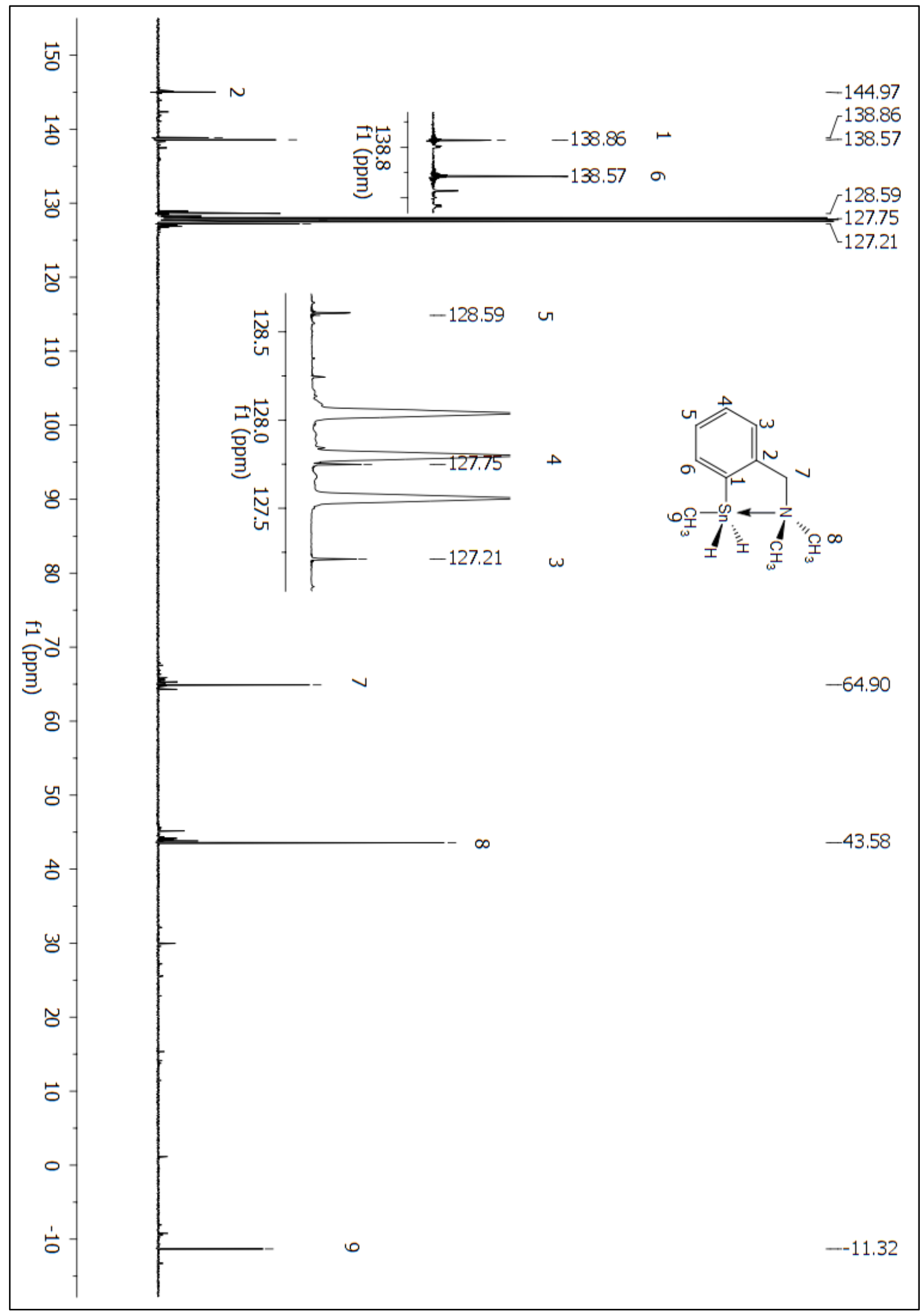

Figure A 108: ${ }^{13} \mathrm{C}$ NMR $\left(\mathrm{C}_{6} \mathrm{D}_{6}\right)$ spectrum of compound 230. 


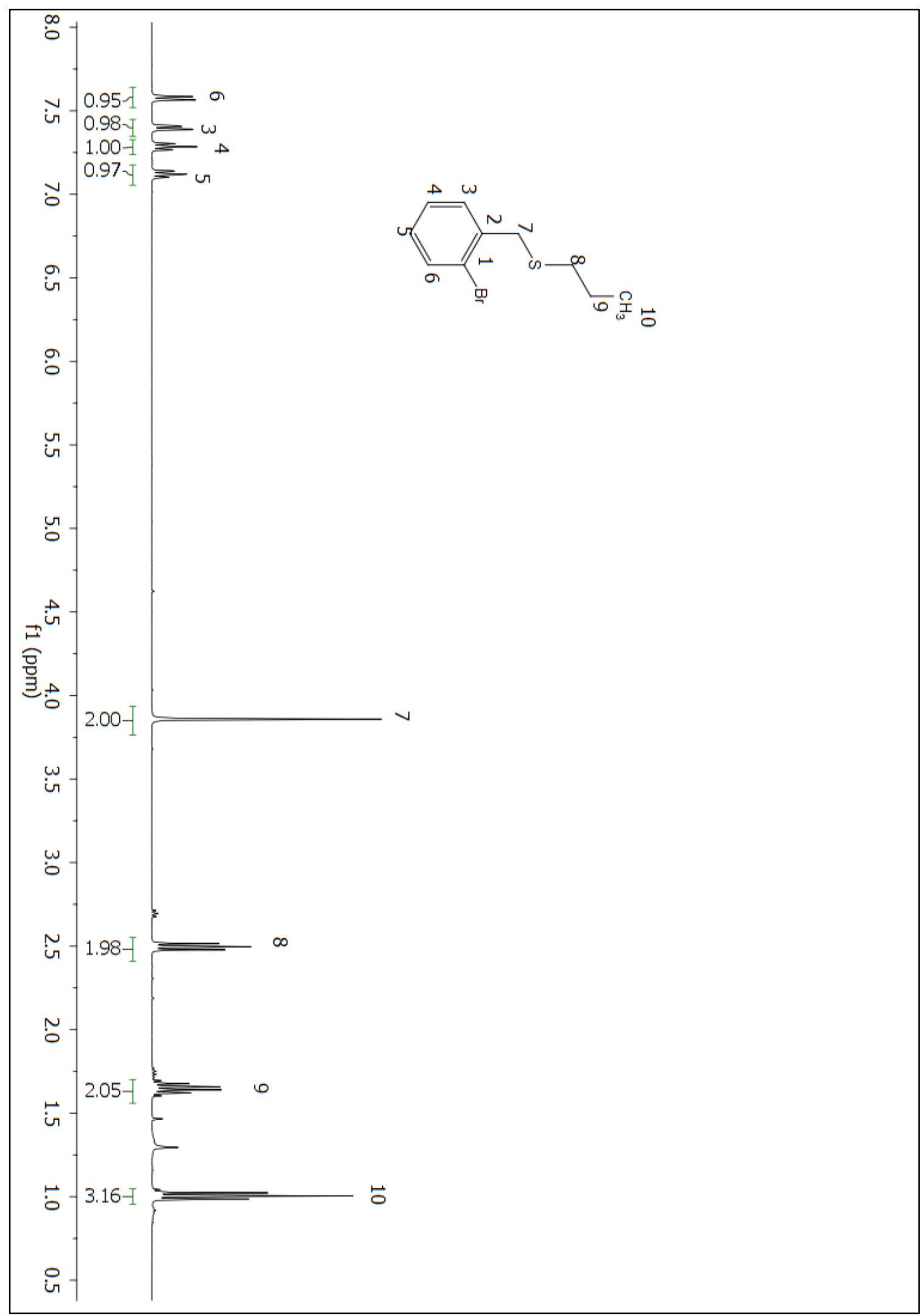

Figure A 109: ${ }^{1} \mathrm{H}$ NMR $\left(\mathrm{CDCl}_{3}\right)$ spectrum of compound 233. 


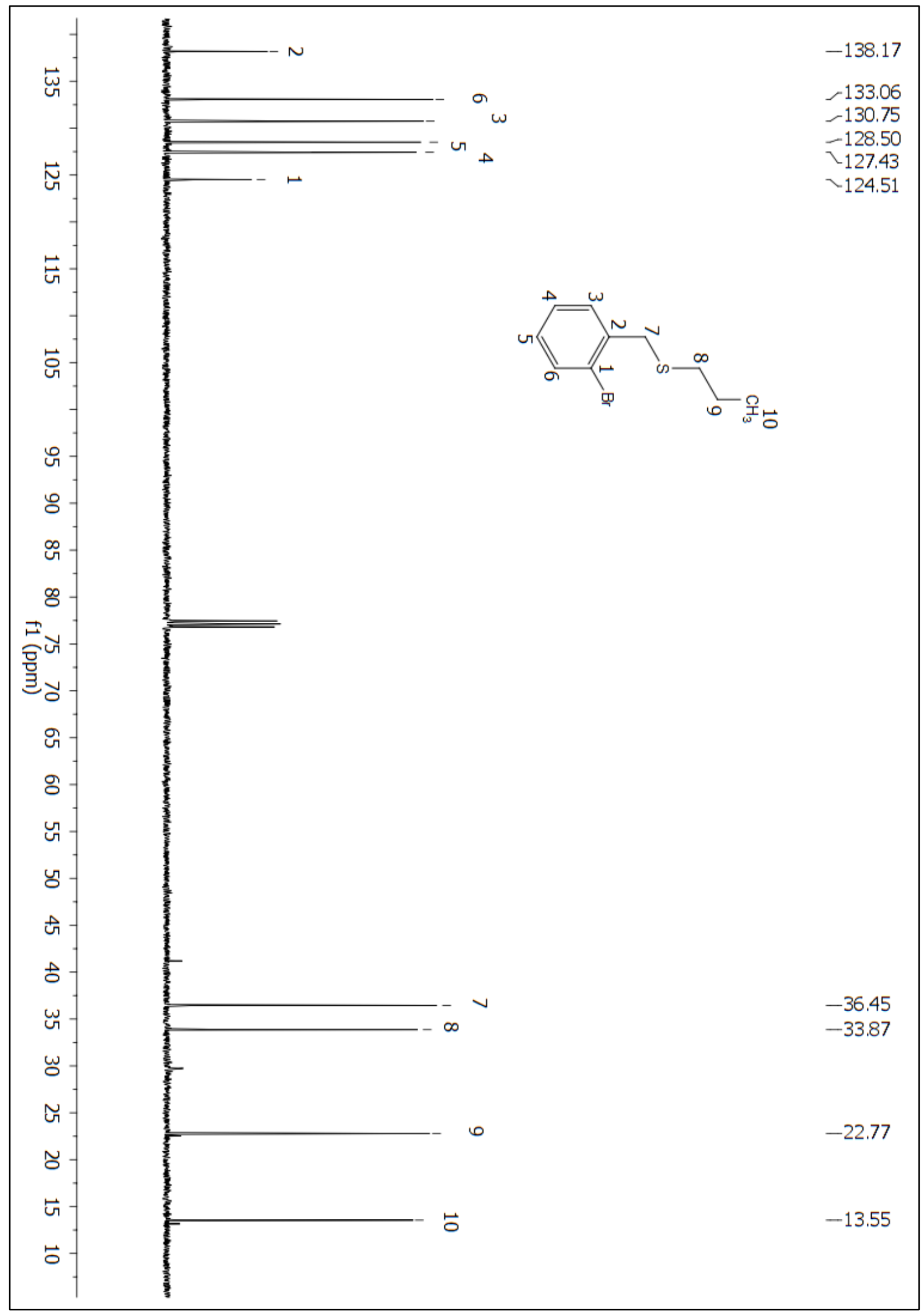

Figure A 110: ${ }^{13} \mathrm{C}$ NMR $\left(\mathrm{CDCl}_{3}\right)$ spectrum of compound 233. 


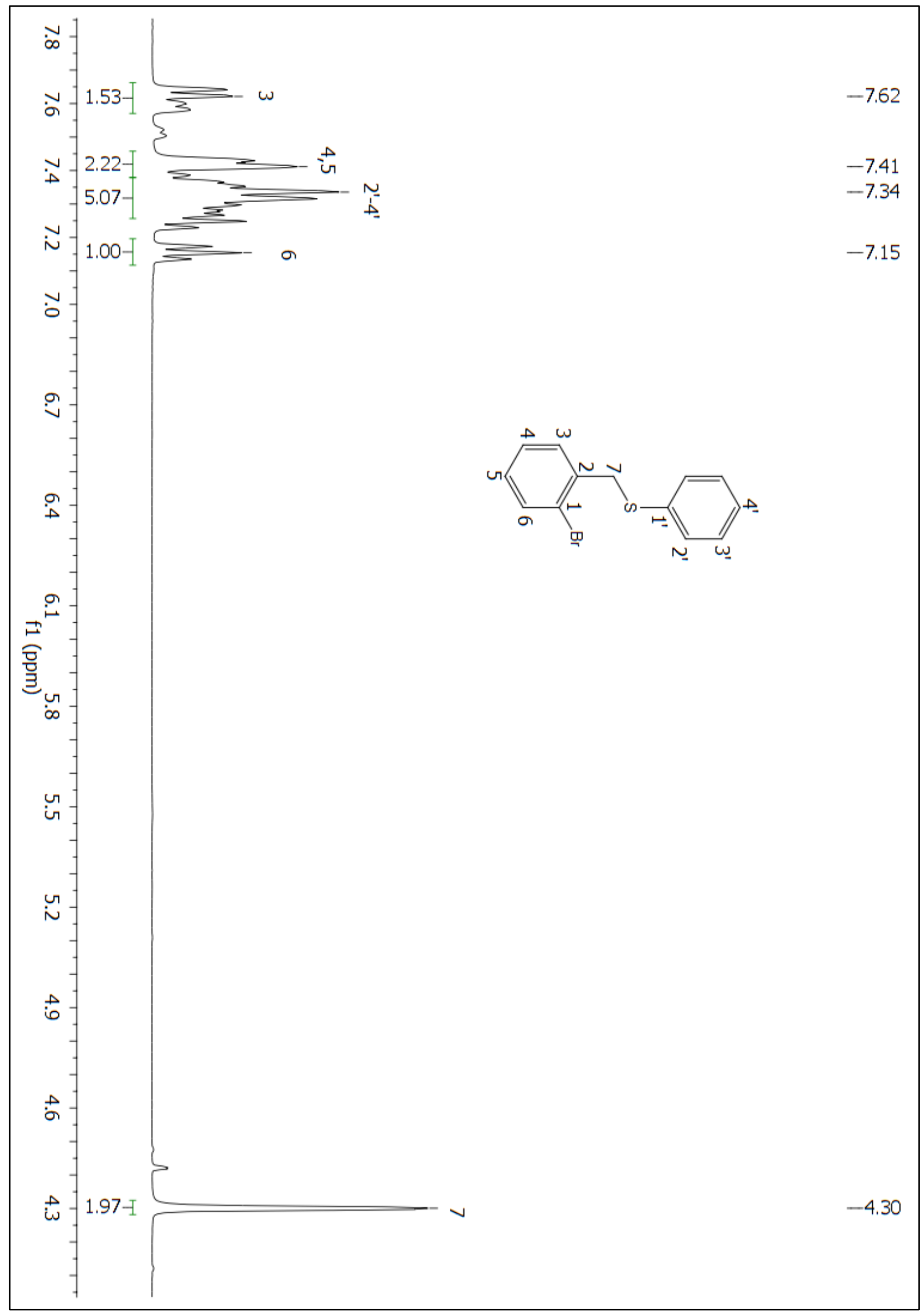

Figure A 111: ${ }^{1} \mathrm{H}$ NMR $\left(\mathrm{CDCl}_{3}\right)$ spectrum of compound 234. 


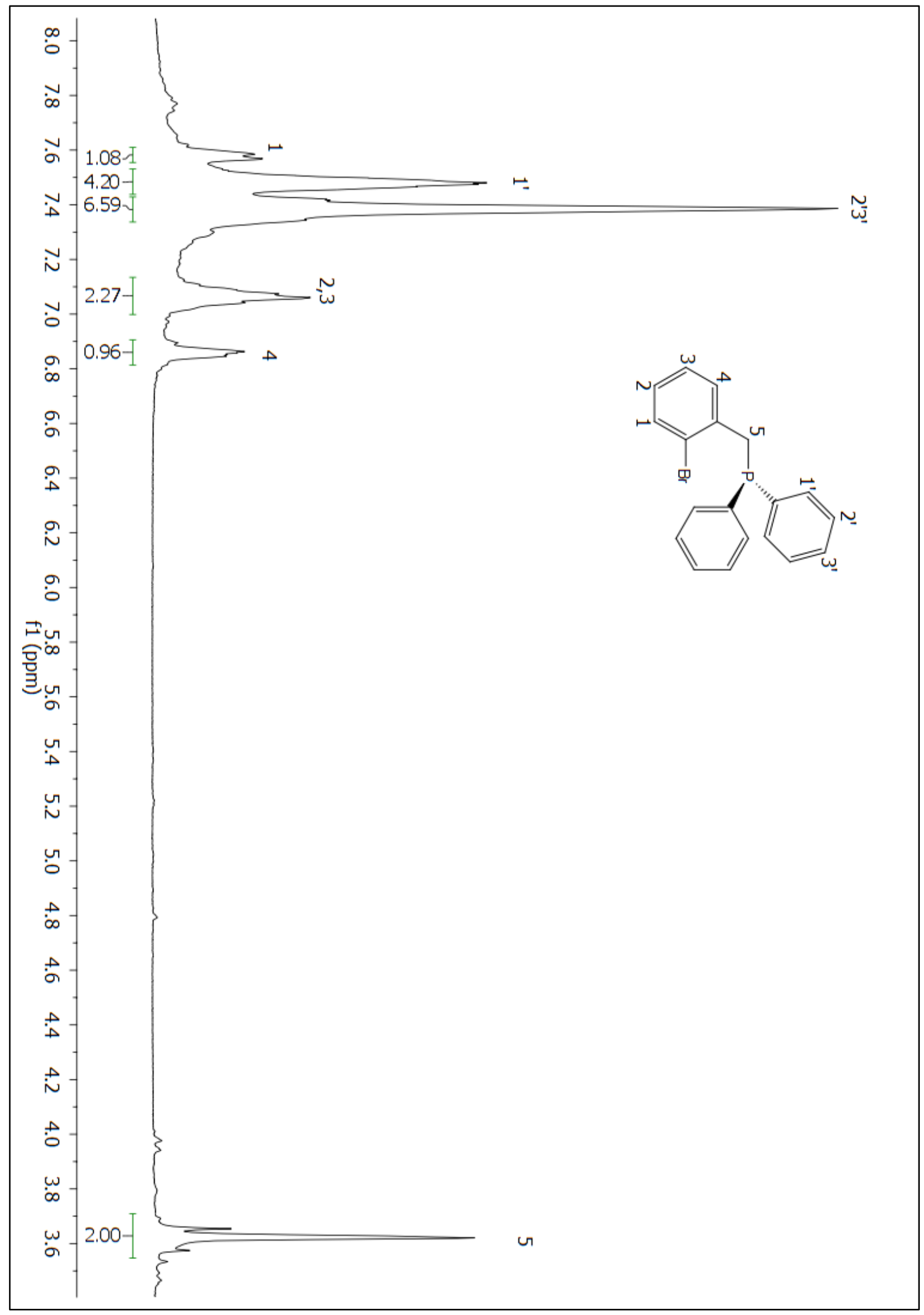

Figure A 112: ${ }^{1} \mathrm{H}$ NMR $\left(\mathrm{CDCl}_{3}\right)$ spectrum of compound 237. 


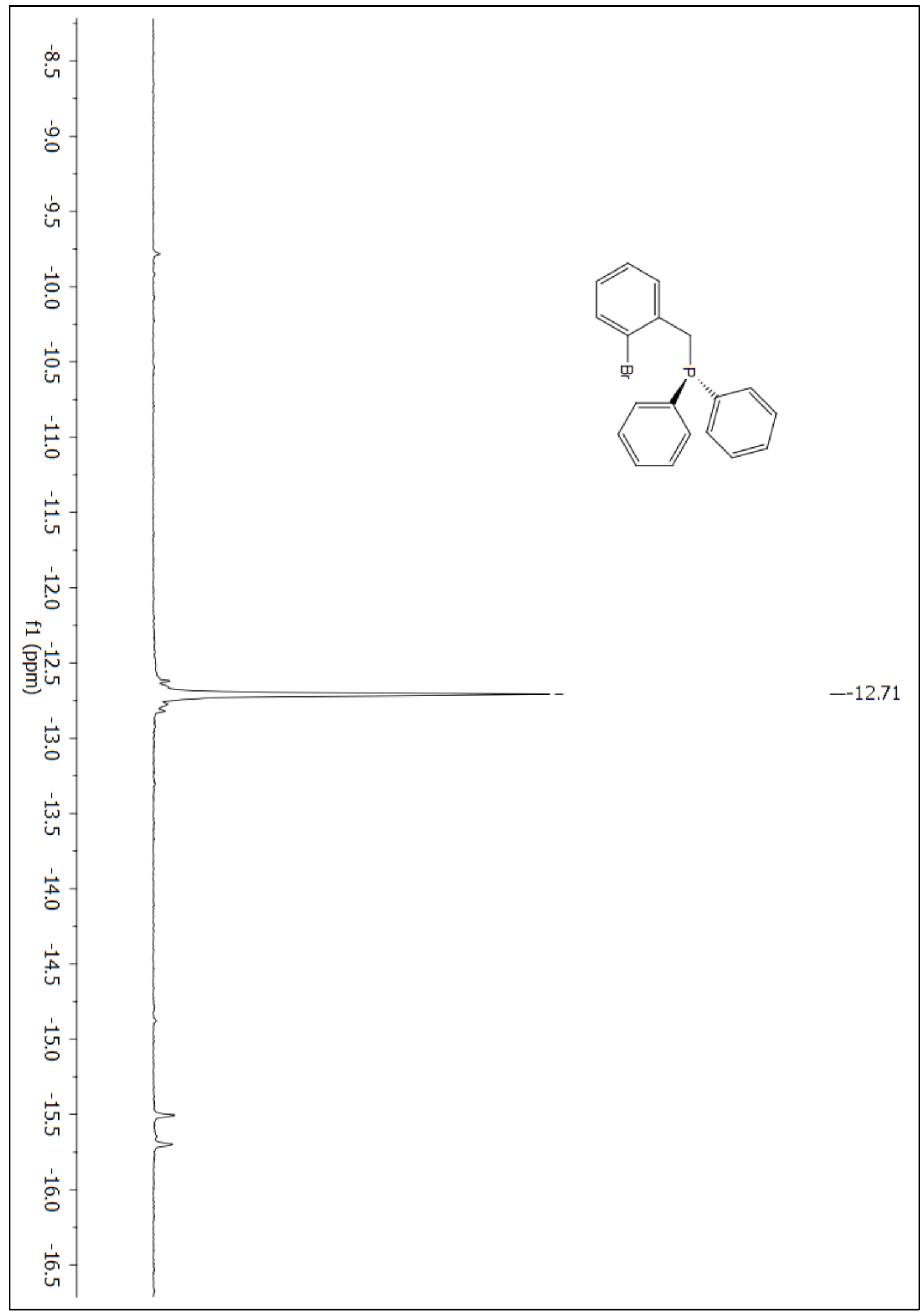

Figure A 113: ${ }^{31} \mathrm{P}$ NMR $\left(\mathrm{CDCl}_{3}\right)$ spectrum of compound 237. 


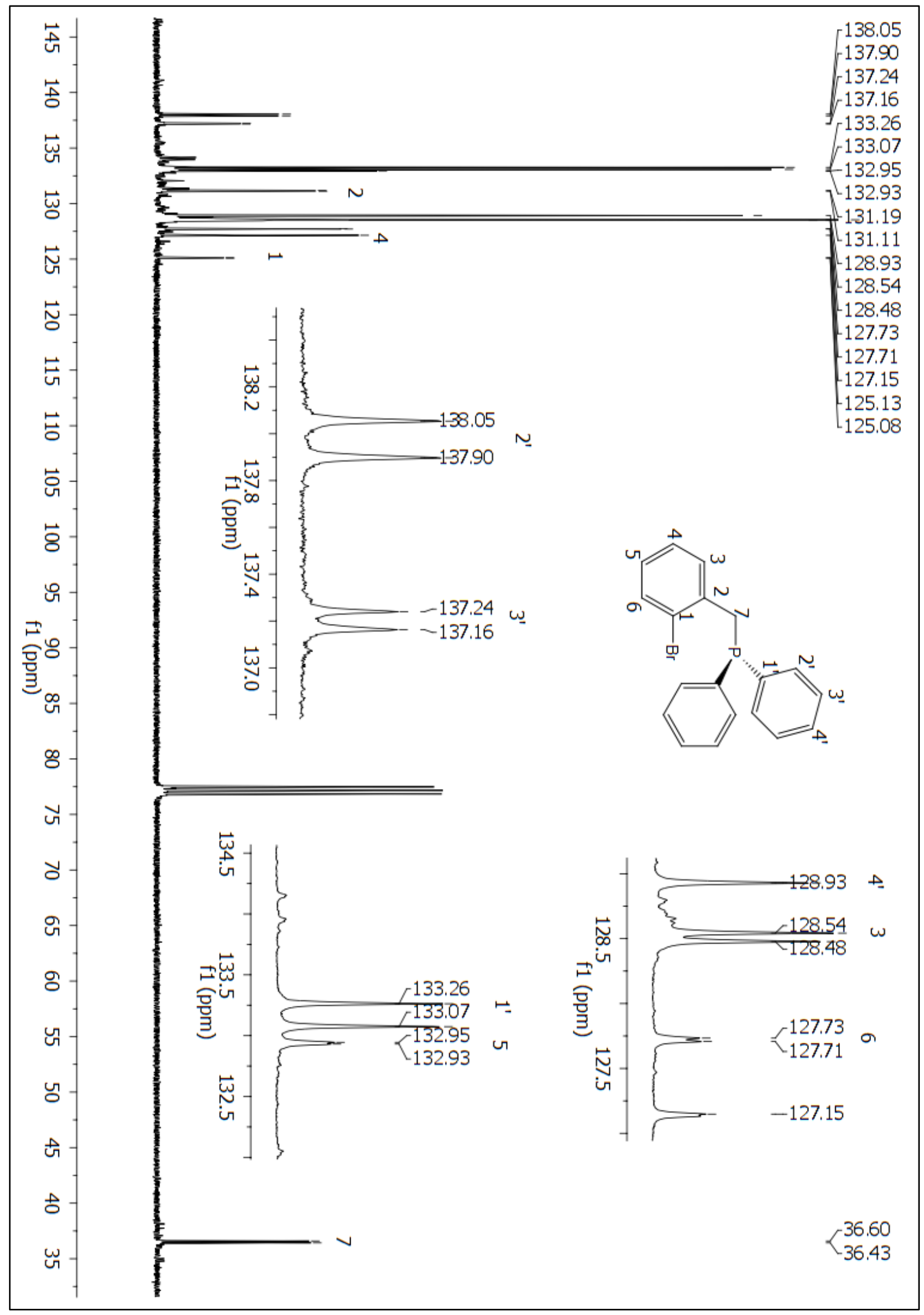

Figure A 114: ${ }^{13} \mathrm{C}$ NMR $\left(\mathrm{CDCl}_{3}\right)$ spectrum of compound 237. 


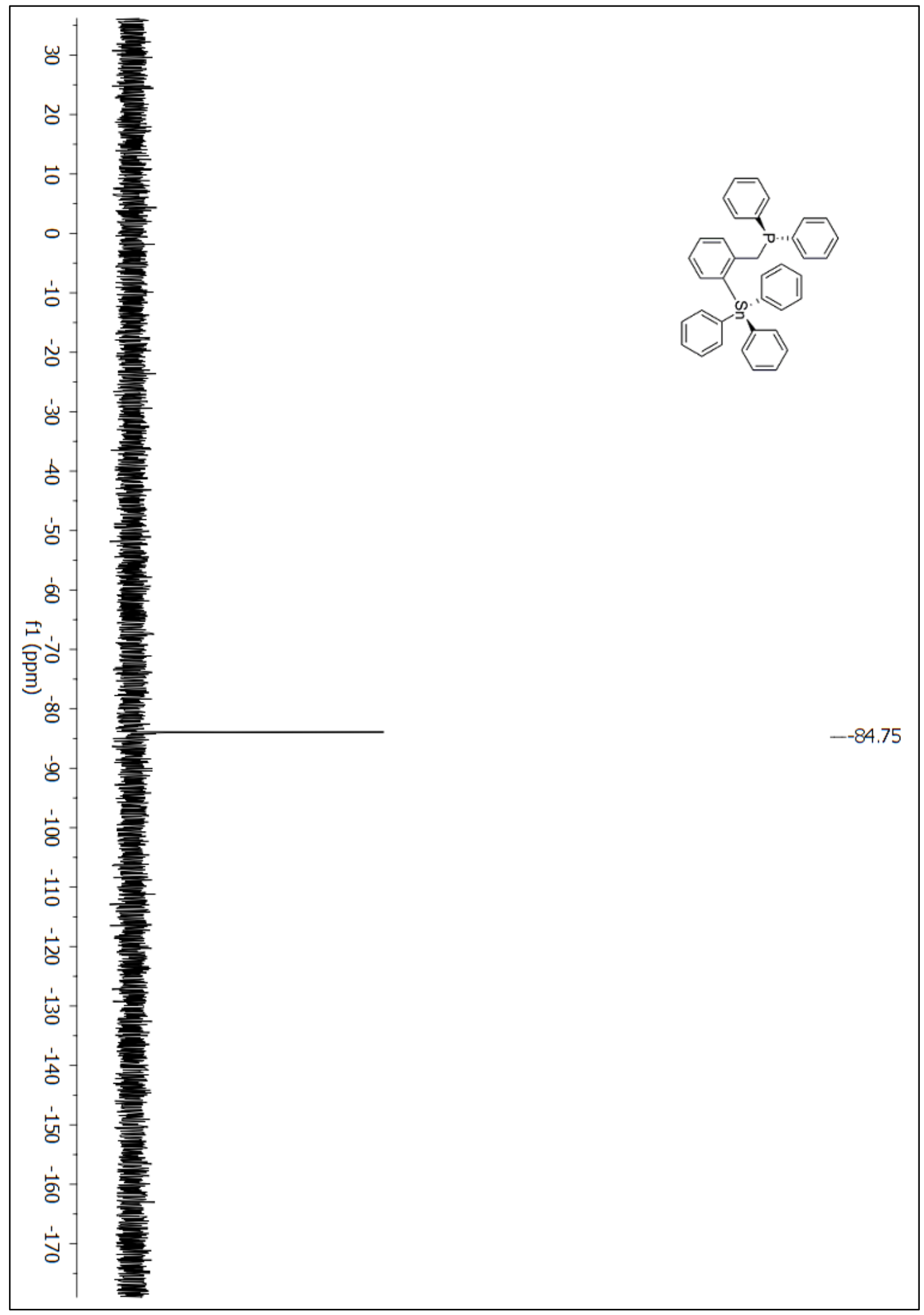

Figure A 115: ${ }^{119} \mathrm{Sn}$ NMR $\left(\mathrm{CDCl}_{3}\right)$ spectrum of compound 241. 


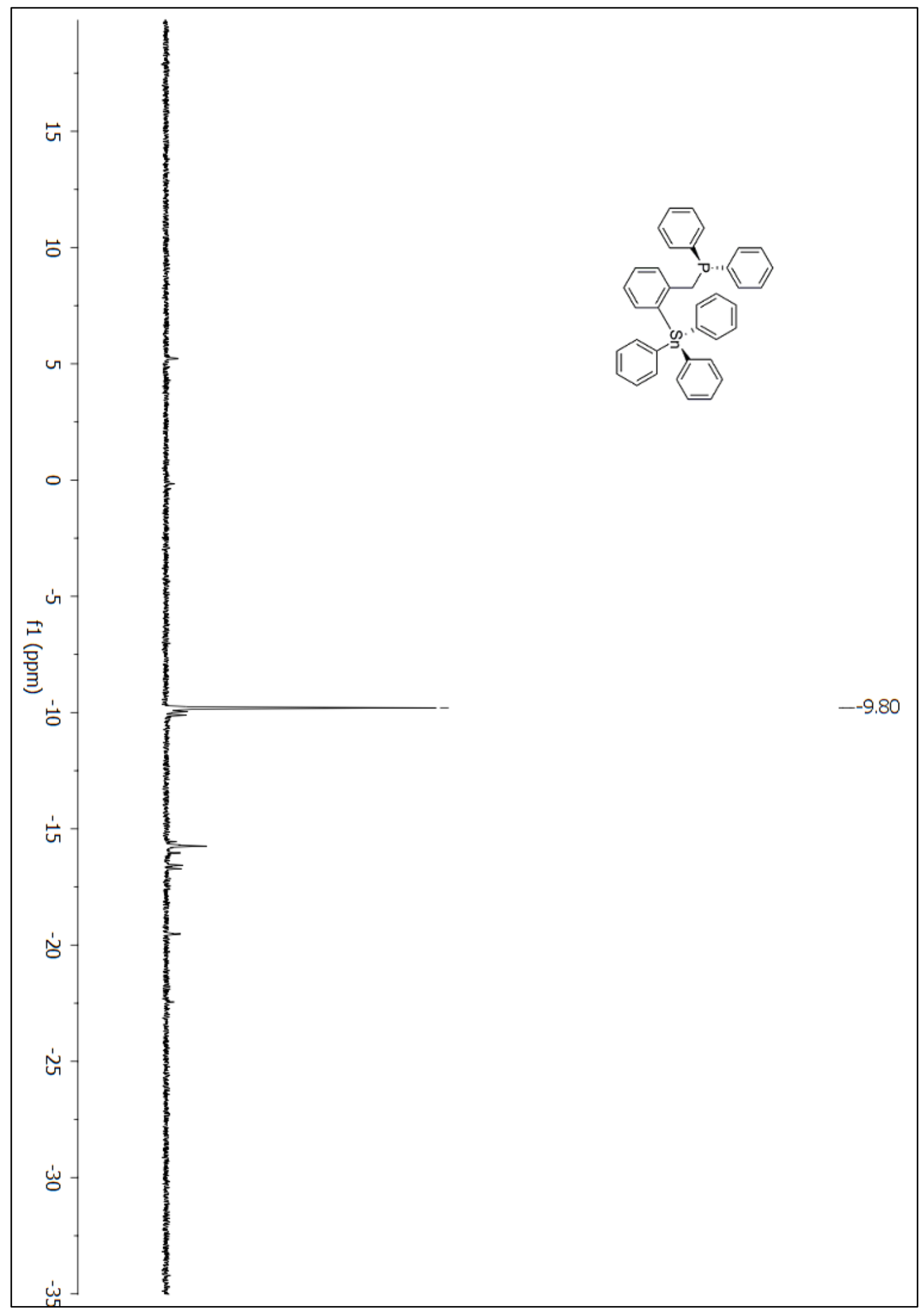

Figure A 116: ${ }^{31} \mathrm{P}$ NMR $\left(\mathrm{CDCl}_{3}\right)$ spectrum of compound 241. 


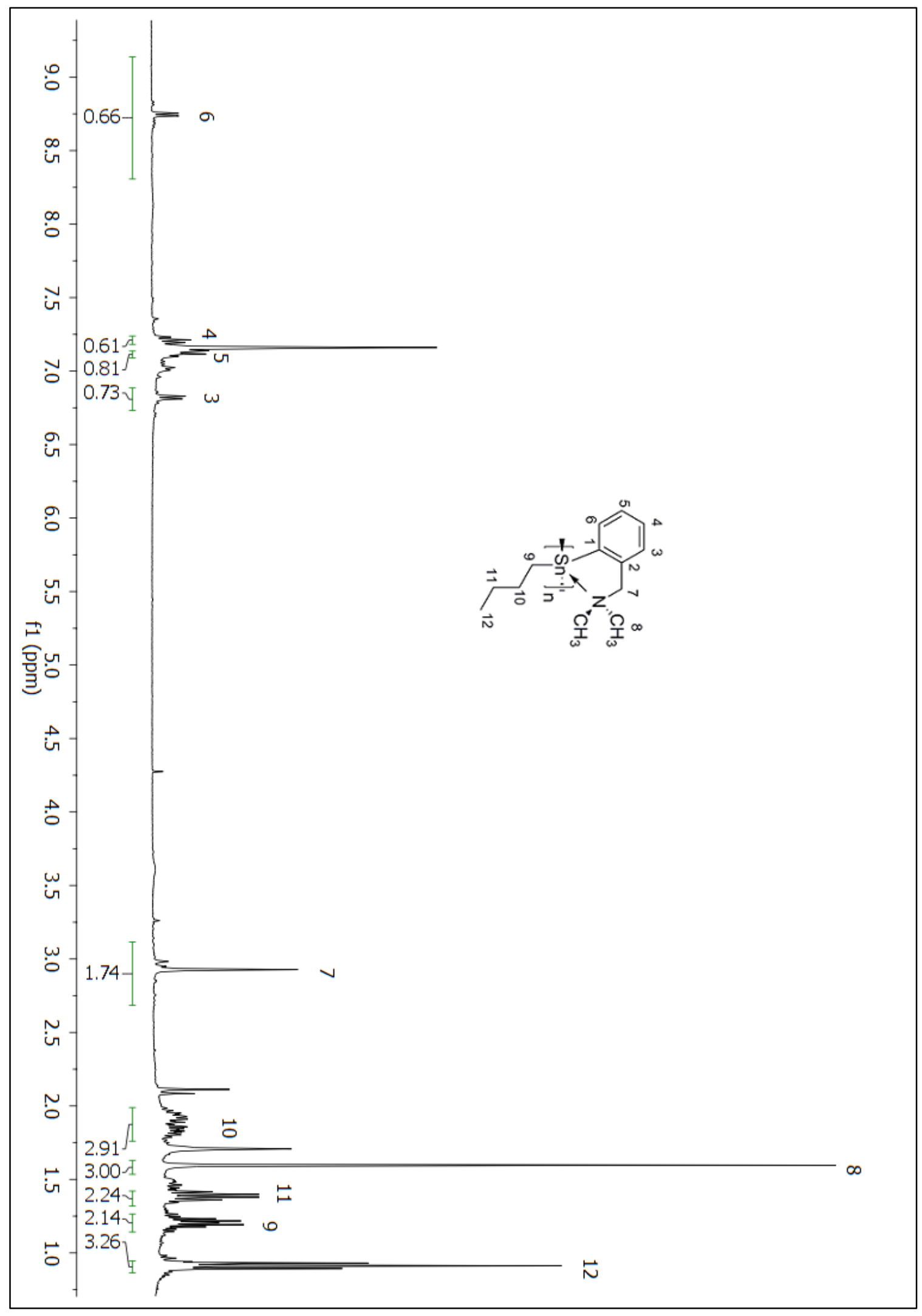

Figure A 117: ${ }^{1} \mathrm{H}$ NMR $\left(\mathrm{C}_{6} \mathrm{D}_{6}\right)$ spectrum of compound 248 


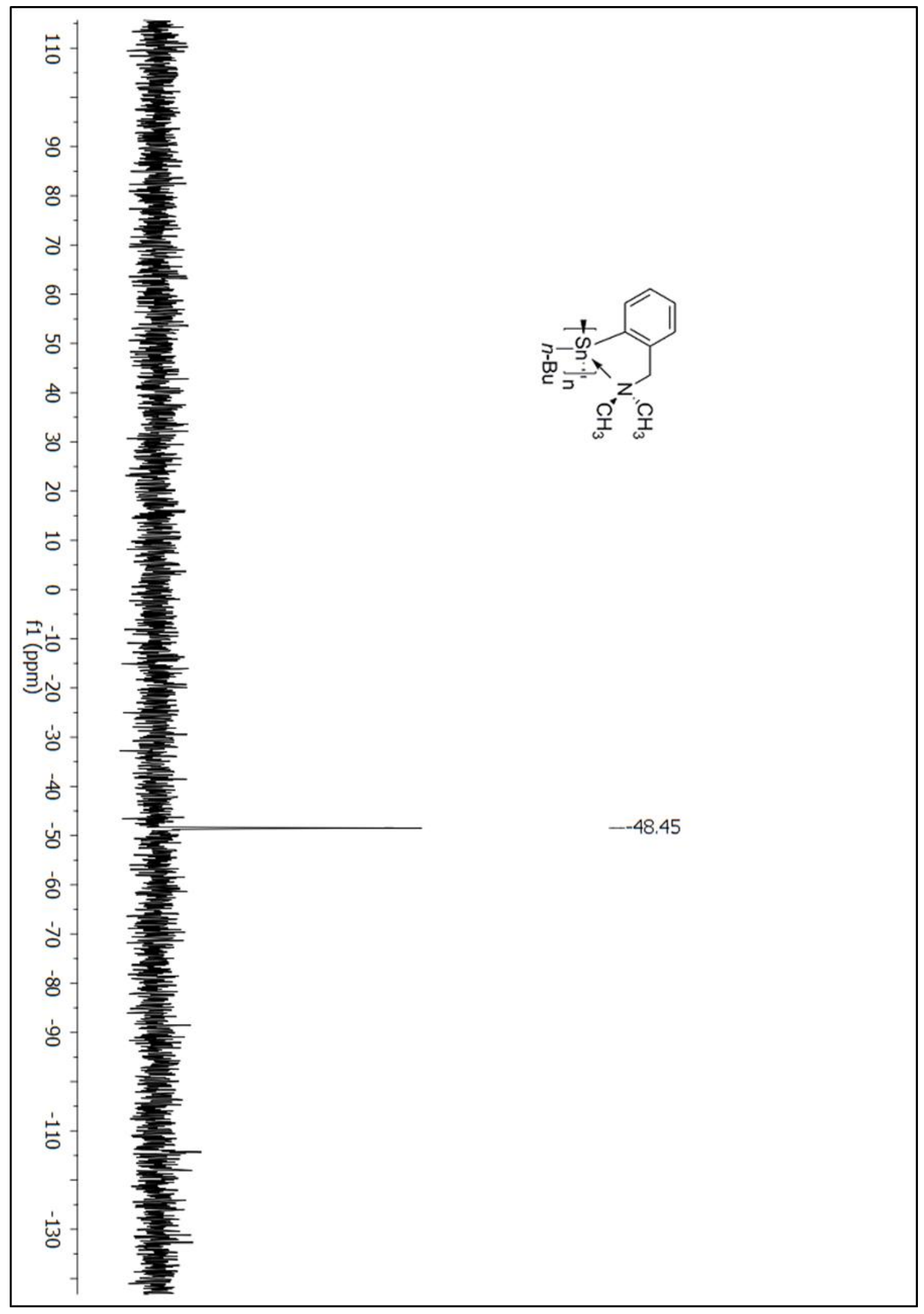

Figure A 118: ${ }^{119} \mathrm{Sn}$ NMR $\left(\mathrm{C}_{6} \mathrm{D}_{6}\right)$ spectrum of compound 248 


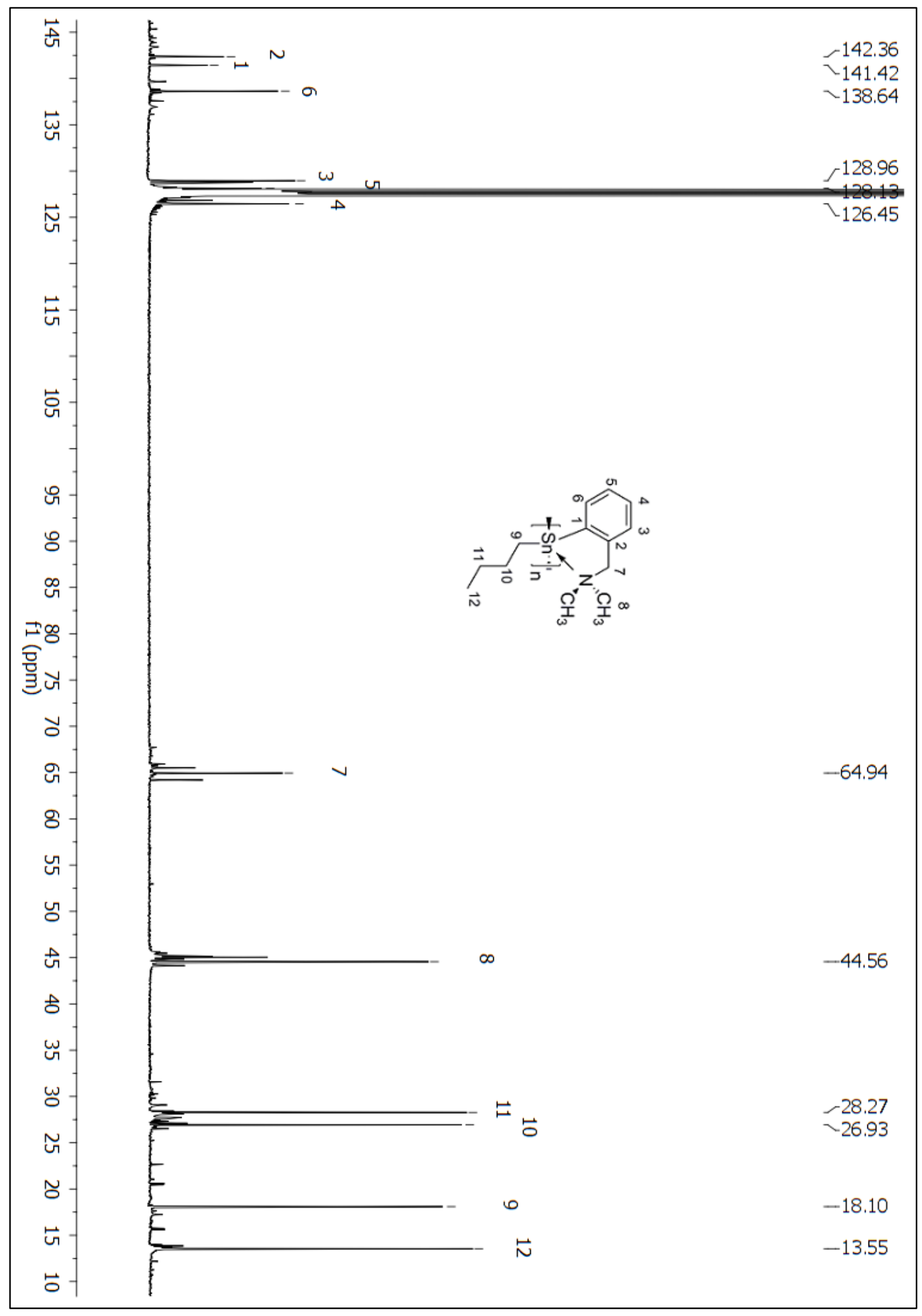

Figure A 119: ${ }^{13} \mathrm{C}$ NMR ( $\left.\mathrm{C}_{6} \mathrm{D}_{6}\right)$ spectrum of compound 248 


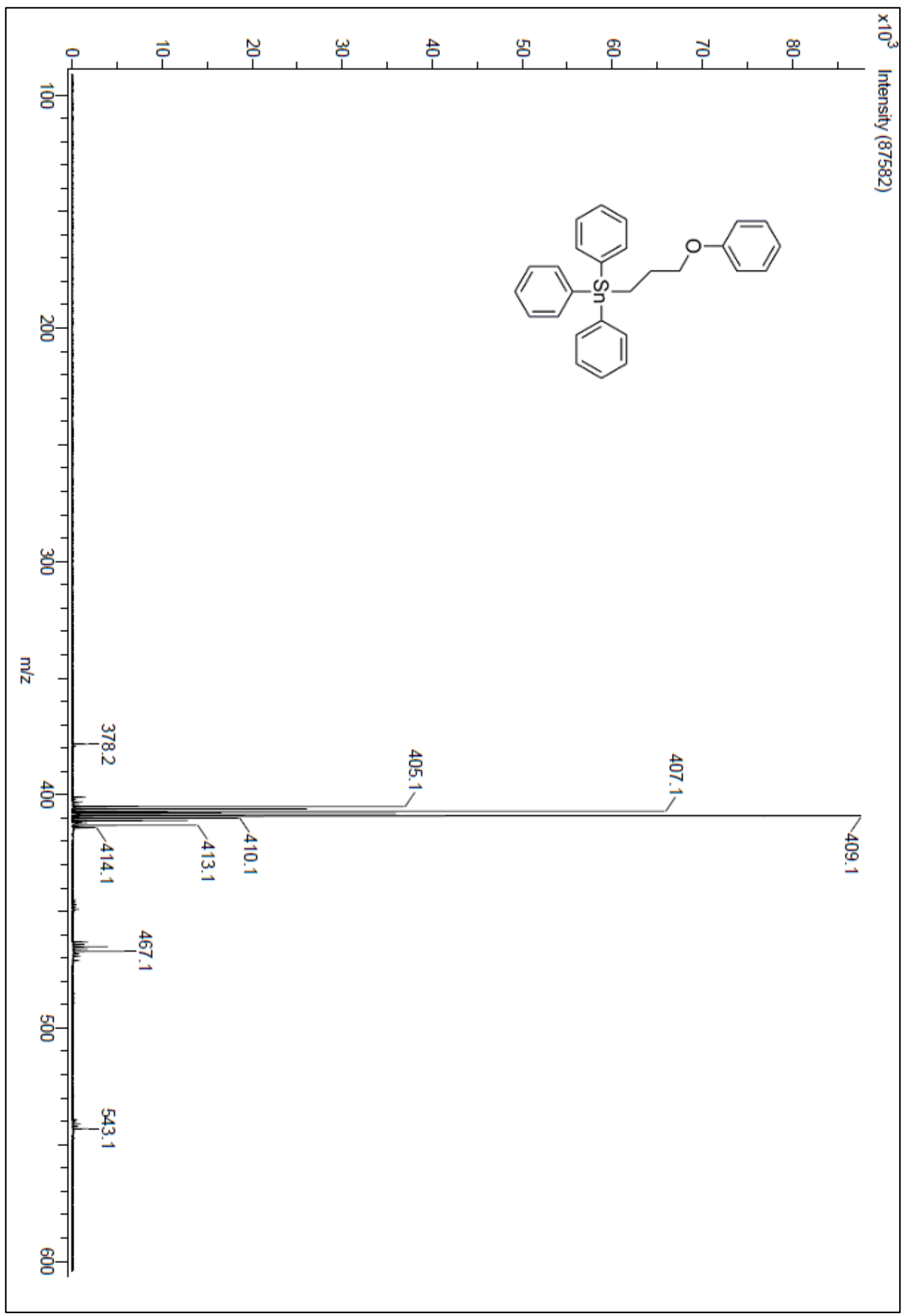

Figure A 120: DART spectrum of compound 197 


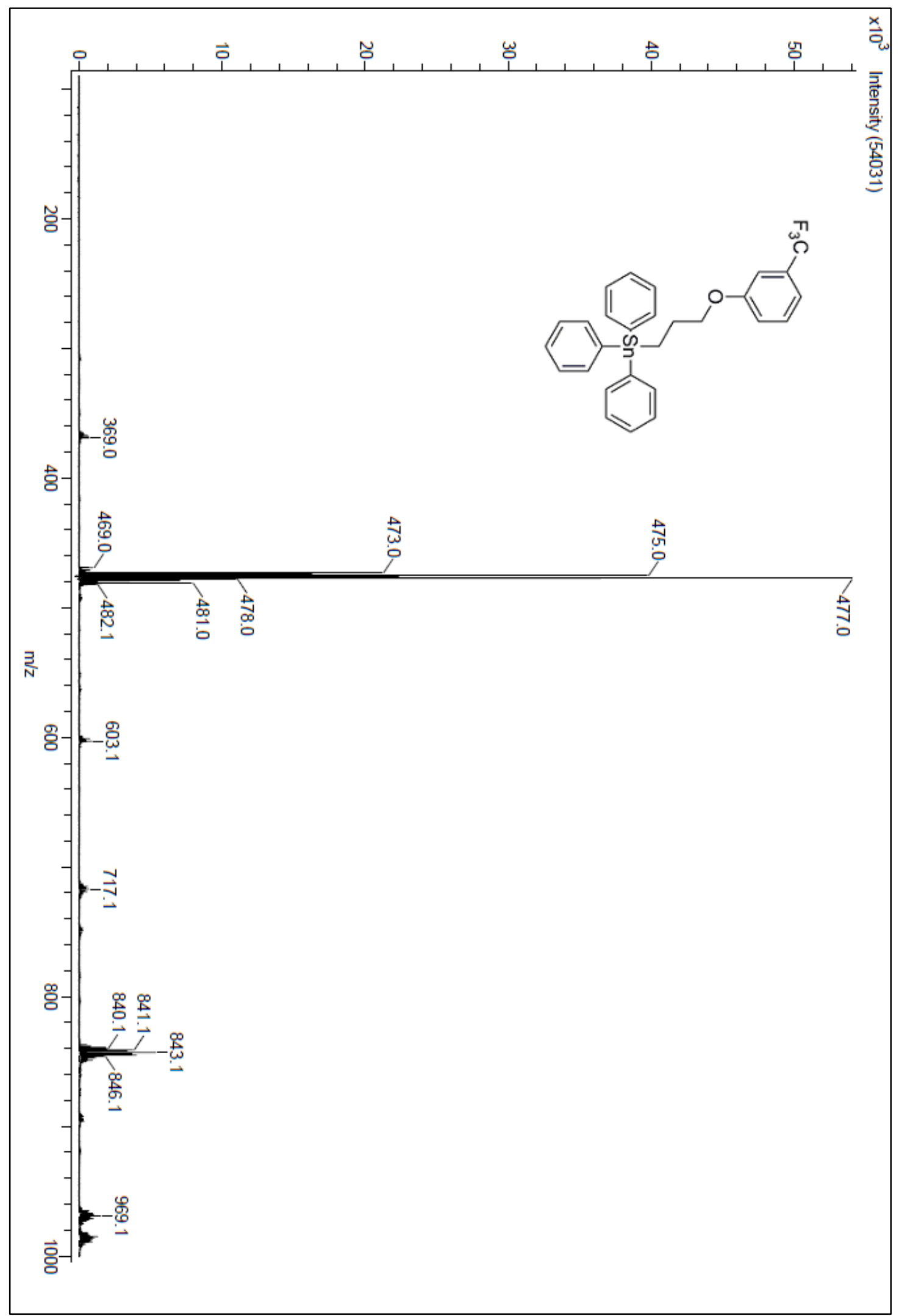

Figure A 121: DART spectrum of compound 198 


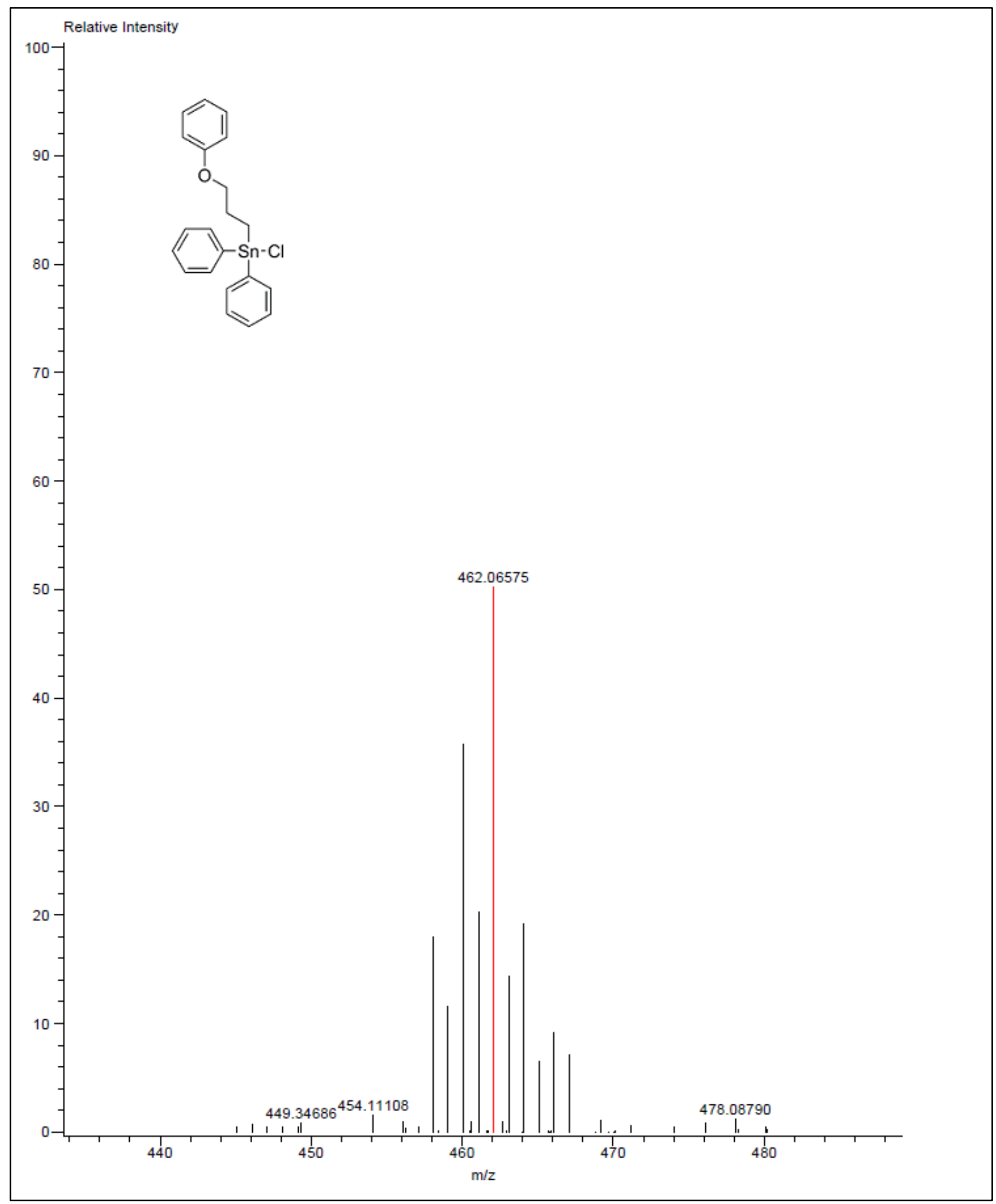

Figure A 122: HRMS-DART spectrum of compound 200 


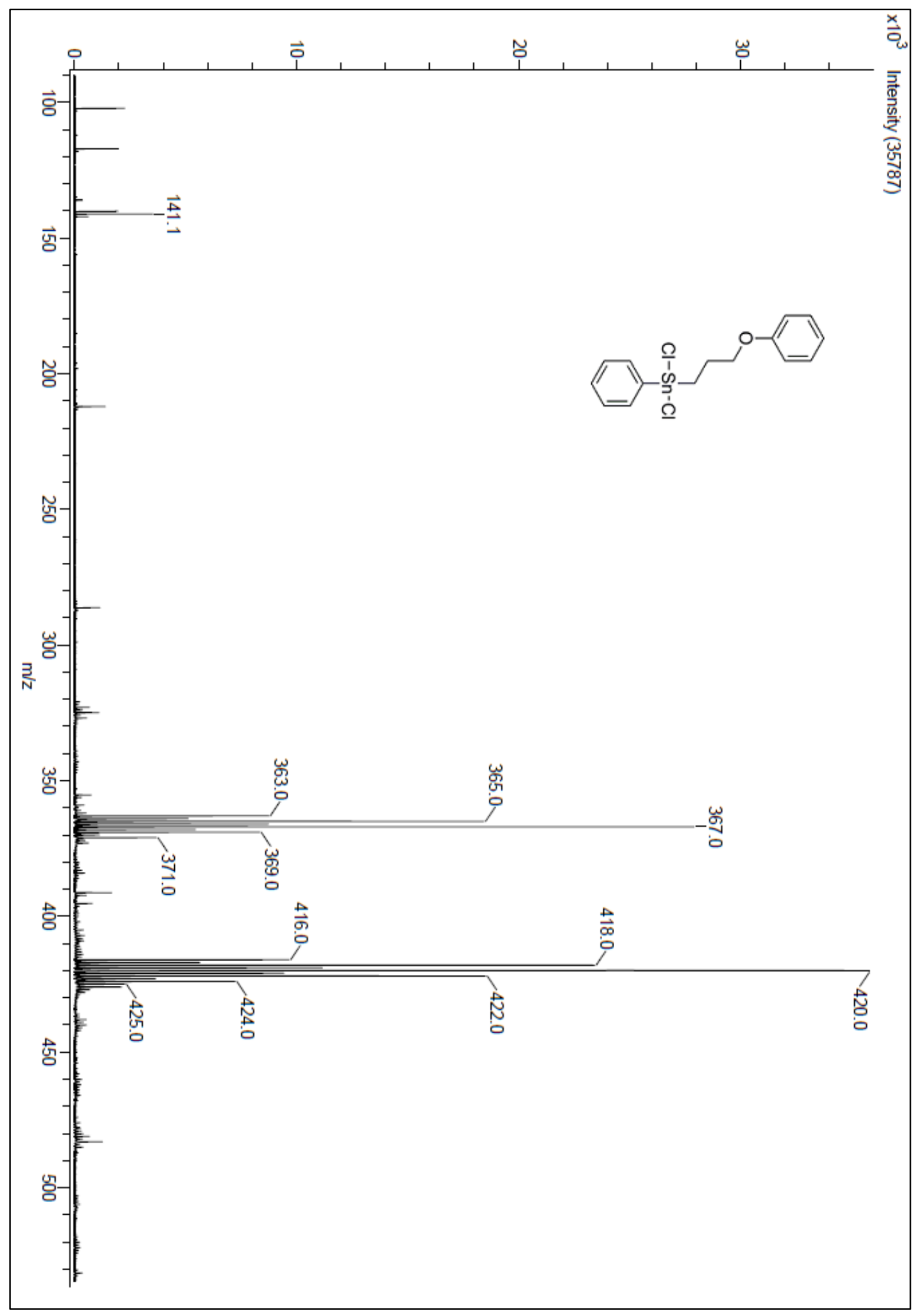

Figure A 123: DART spectrum of compound 202 


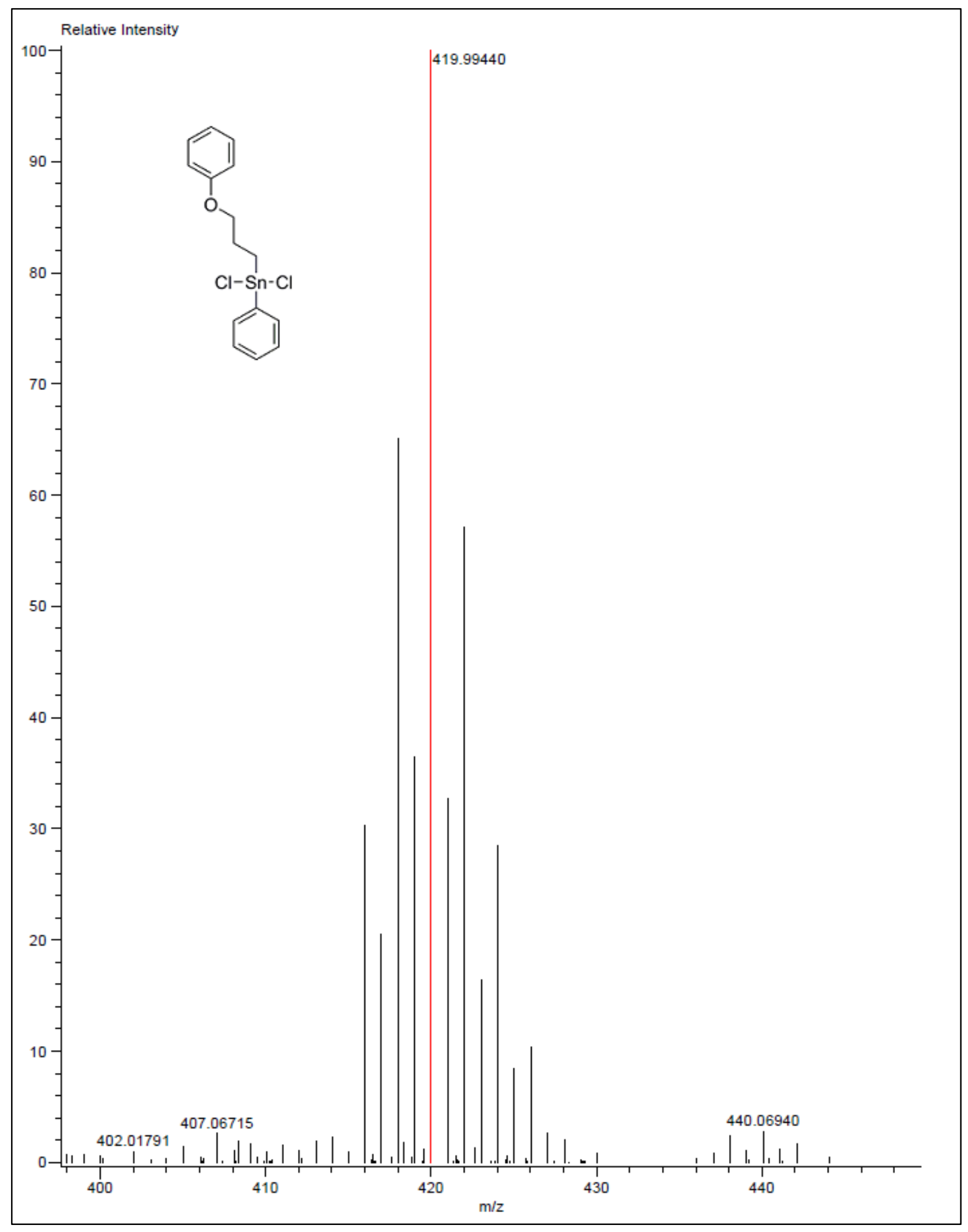

Figure A 124: HRMS-DART spectrum of compound 202 


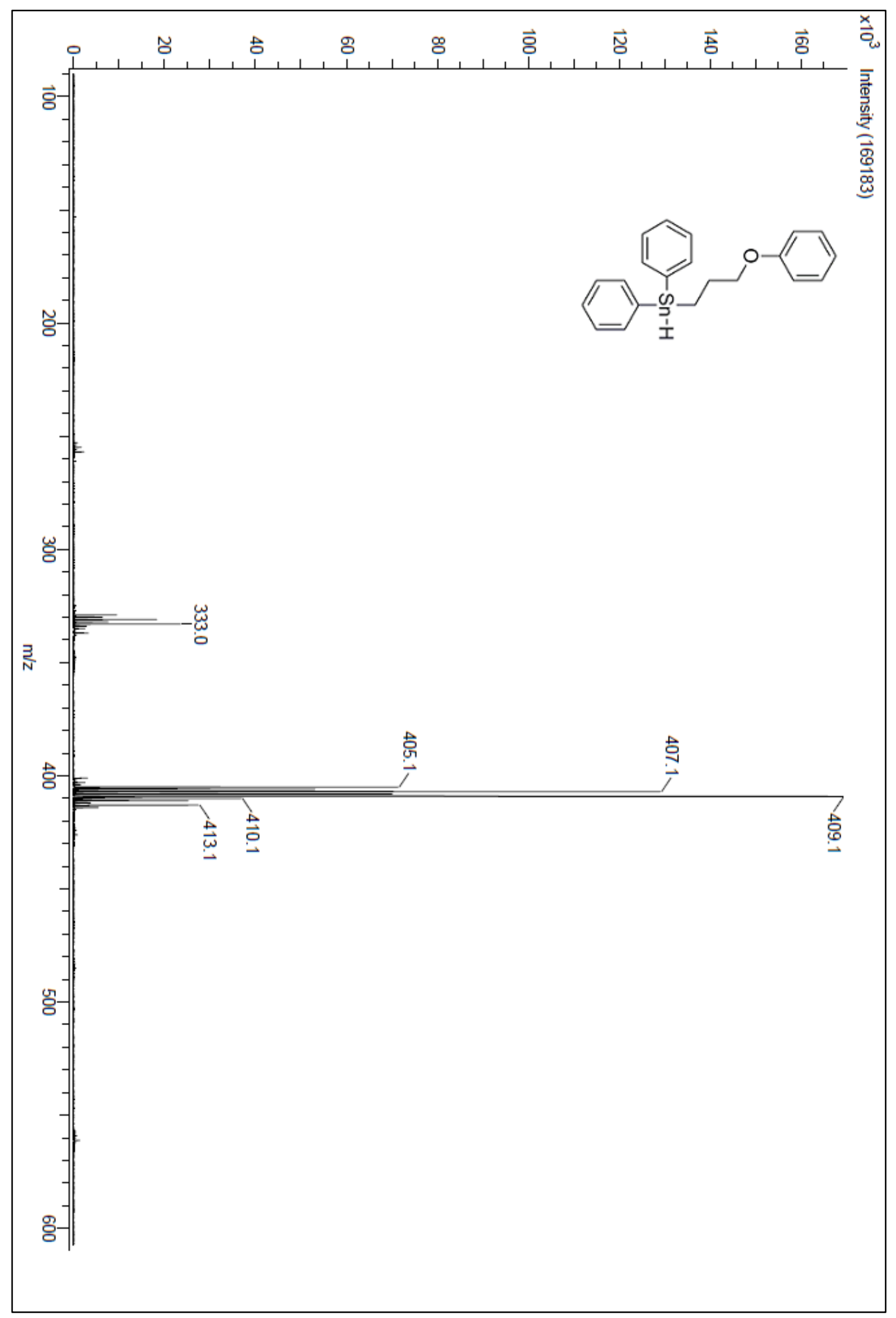

Figure A 125: DART spectrum of compound 204 


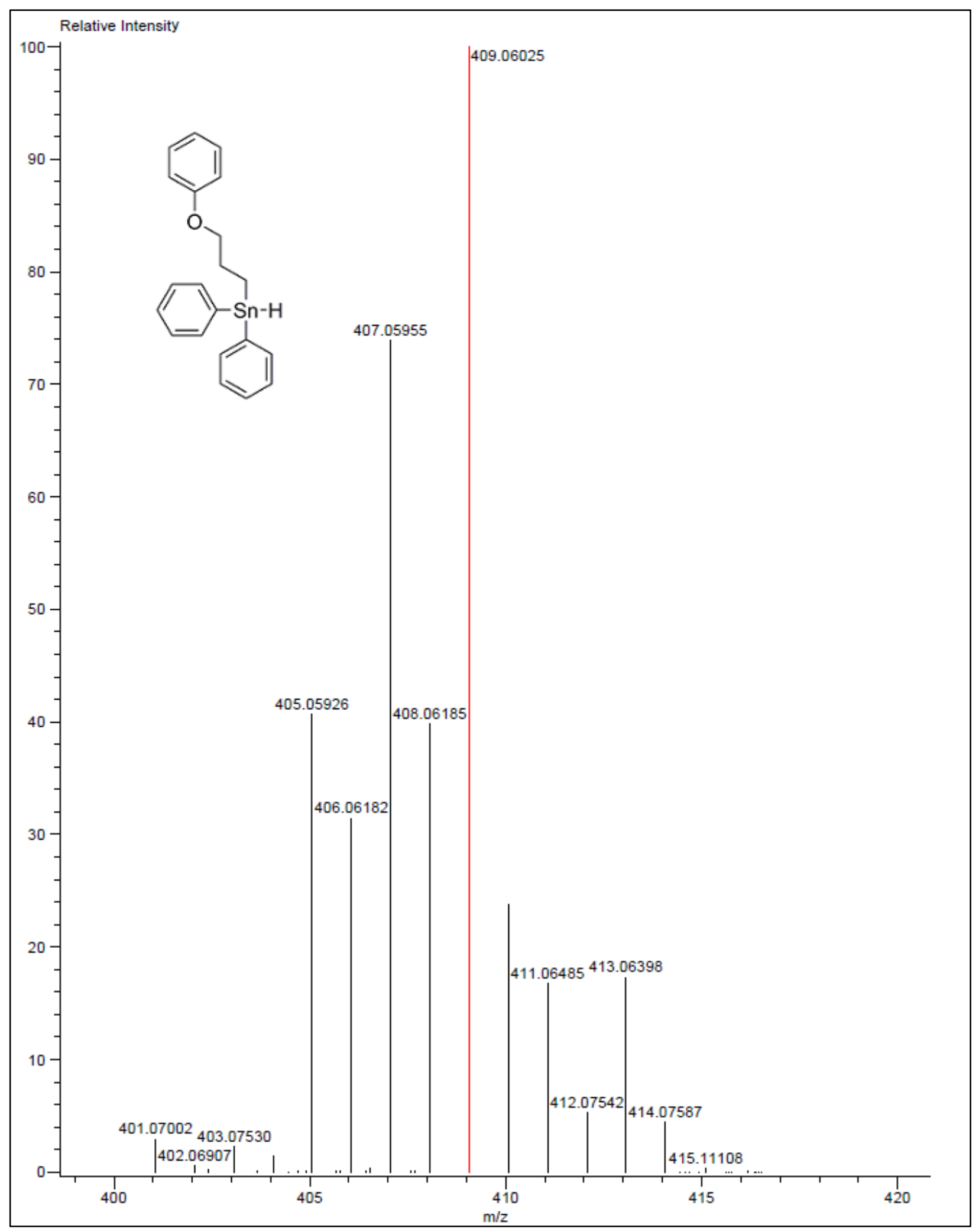

Figure A 126: HRMS-DART spectrum of compound 204 


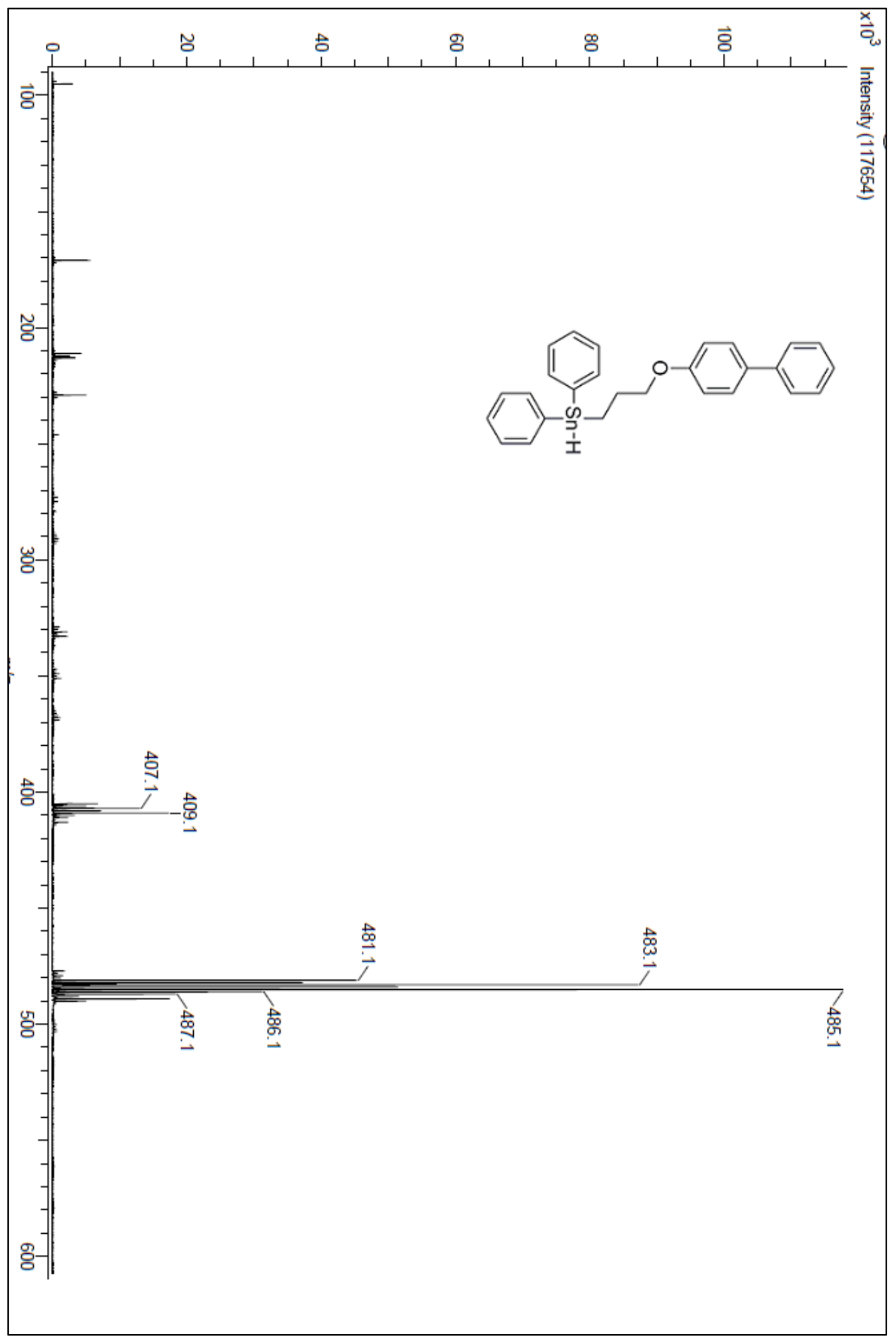

Figure A 127: DART spectrum of compound 205 


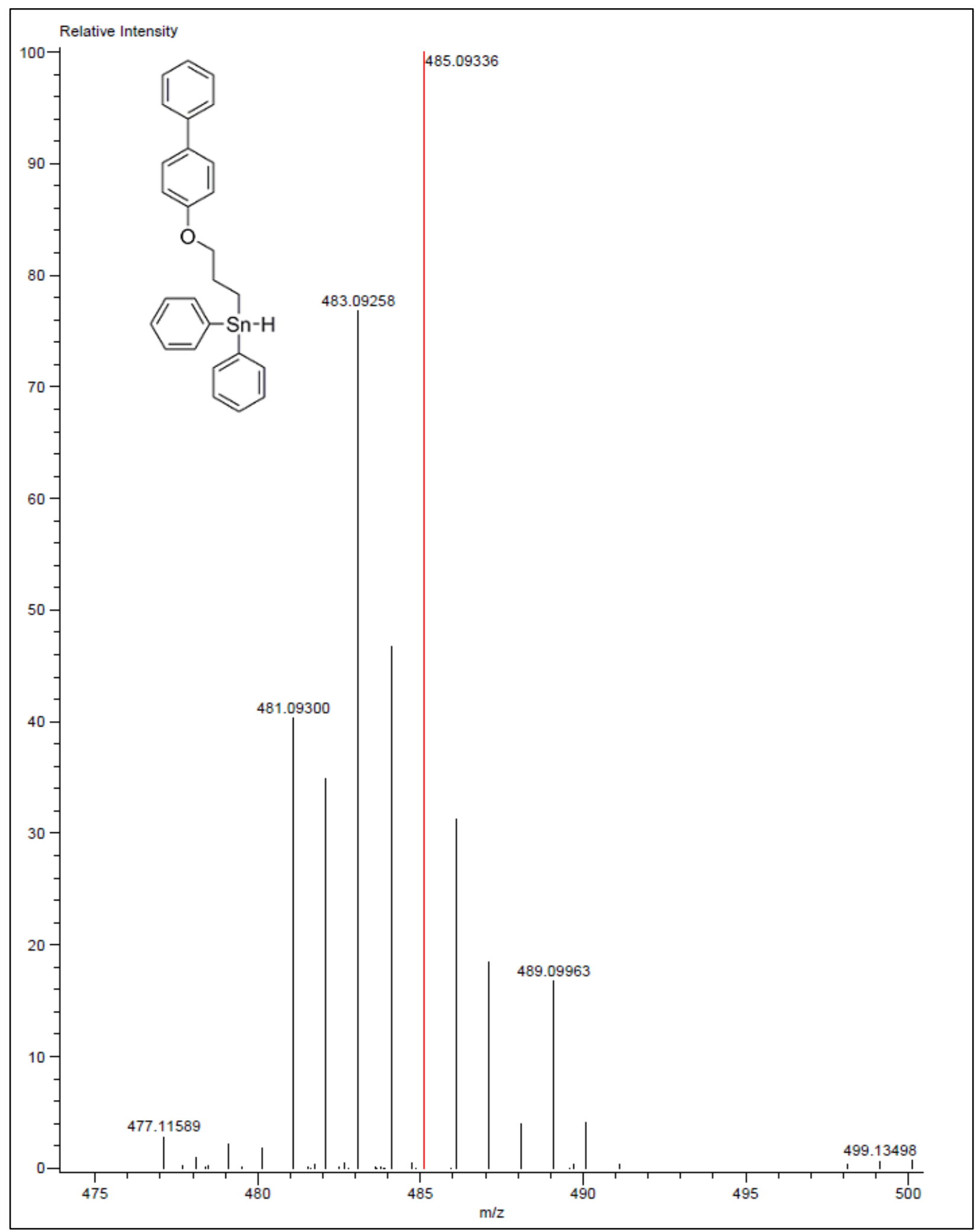

Figure A 128: HRMS-DART spectrum of compound 205 


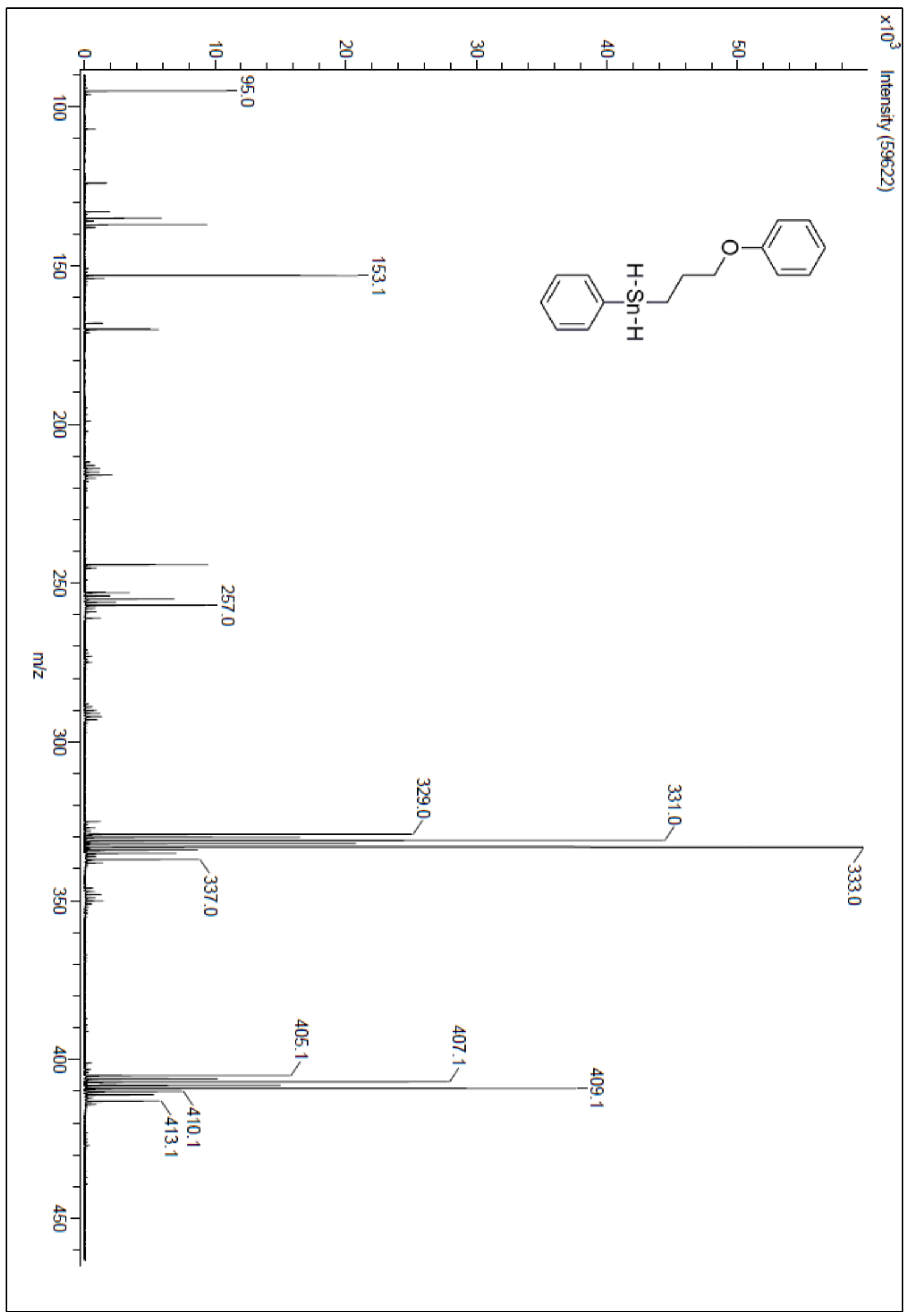

Figure A 129: DART spectrum of compound 206 


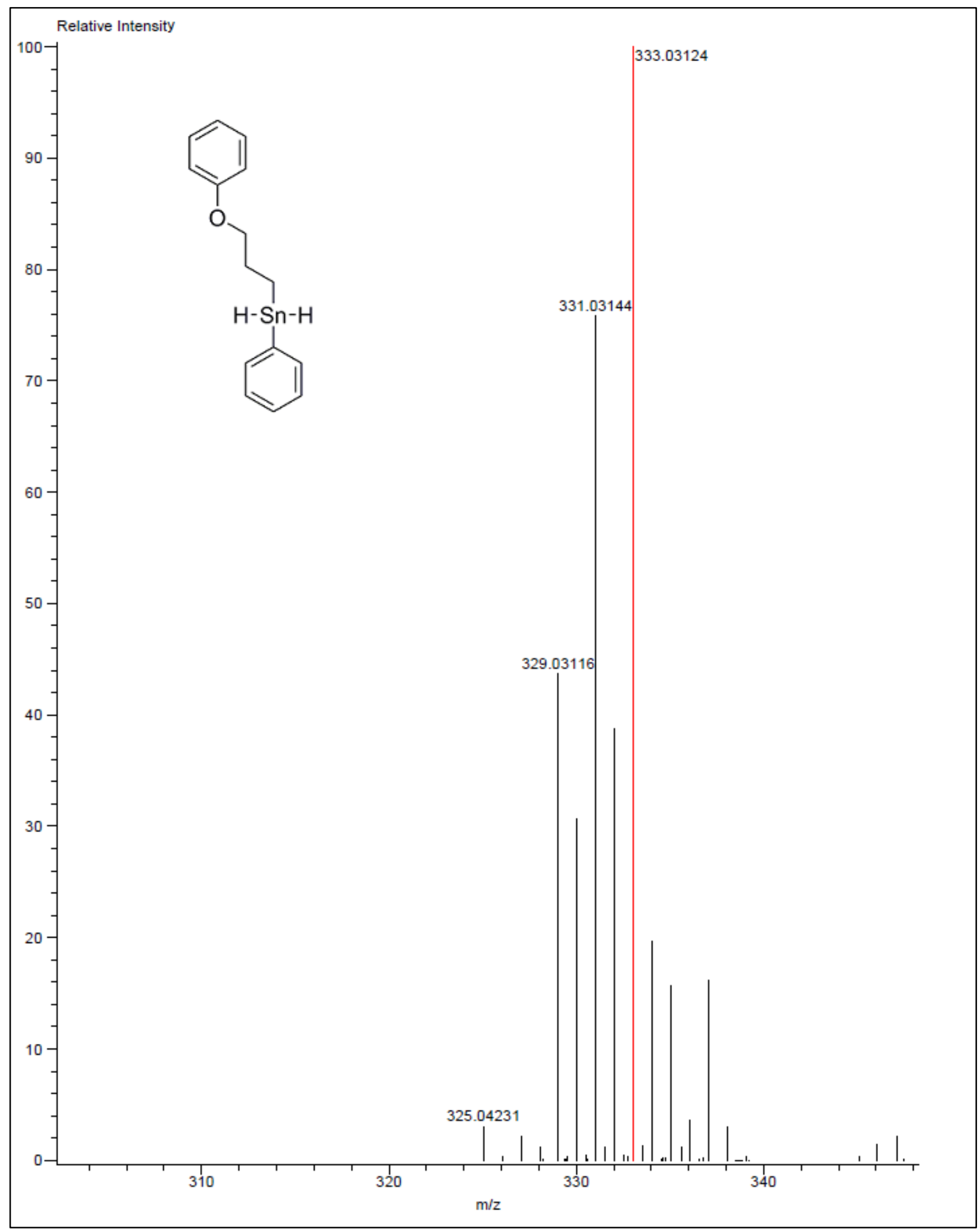

Figure A 130: HRMS-DART spectrum of compound 206 


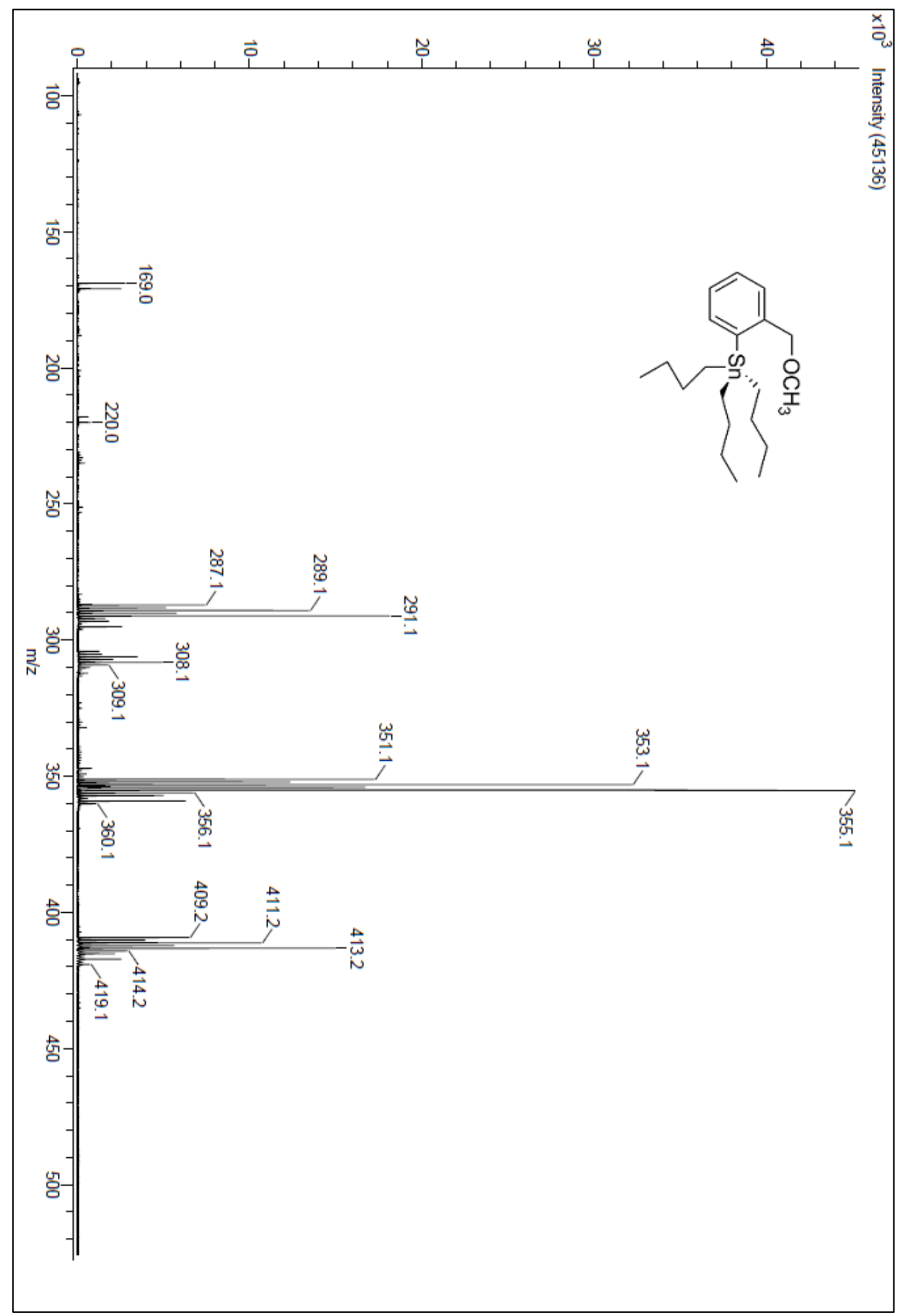

Figure A 131: DART spectrum of compound 217 


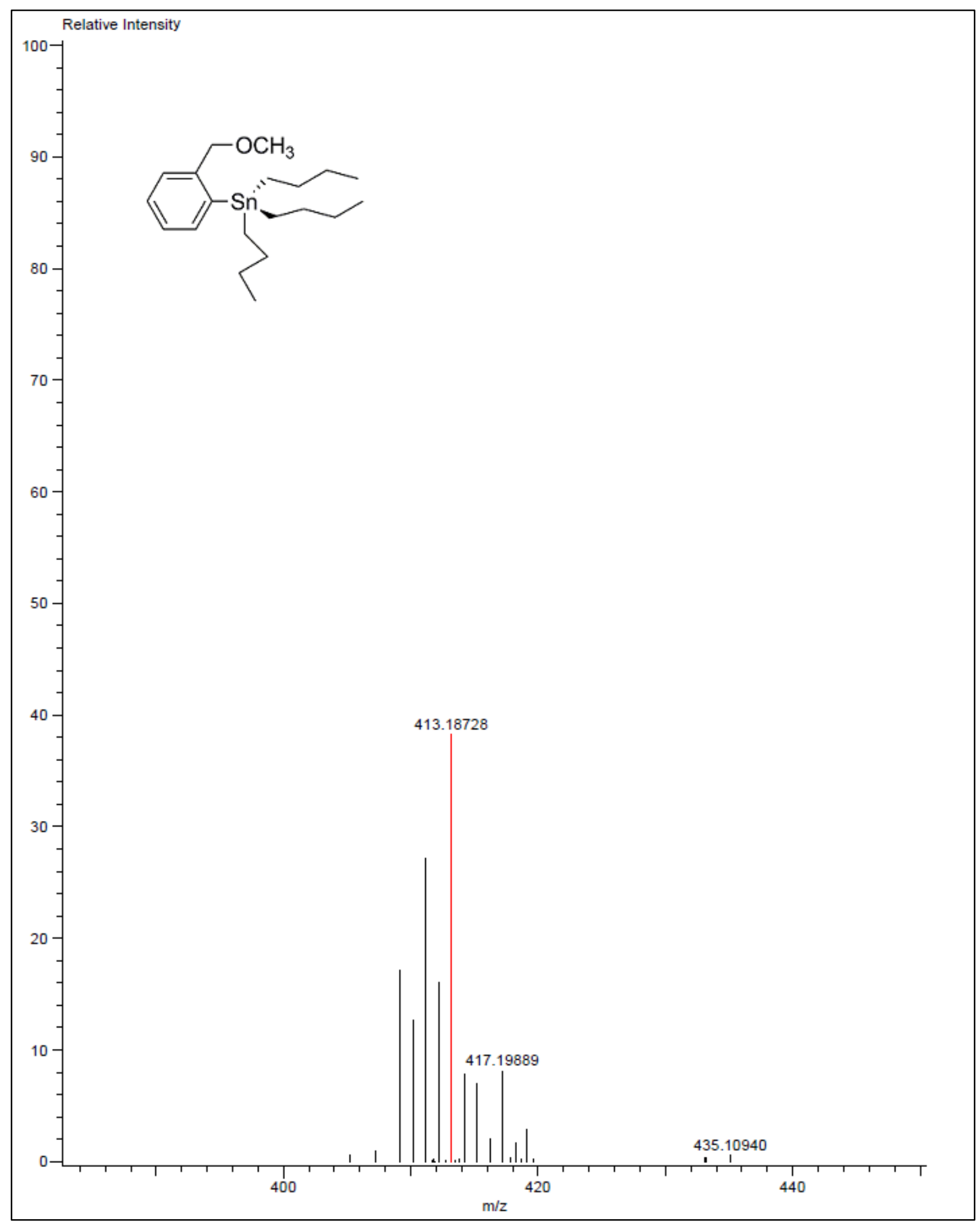

Figure A 132: HRMS-DART spectrum of compound 217 


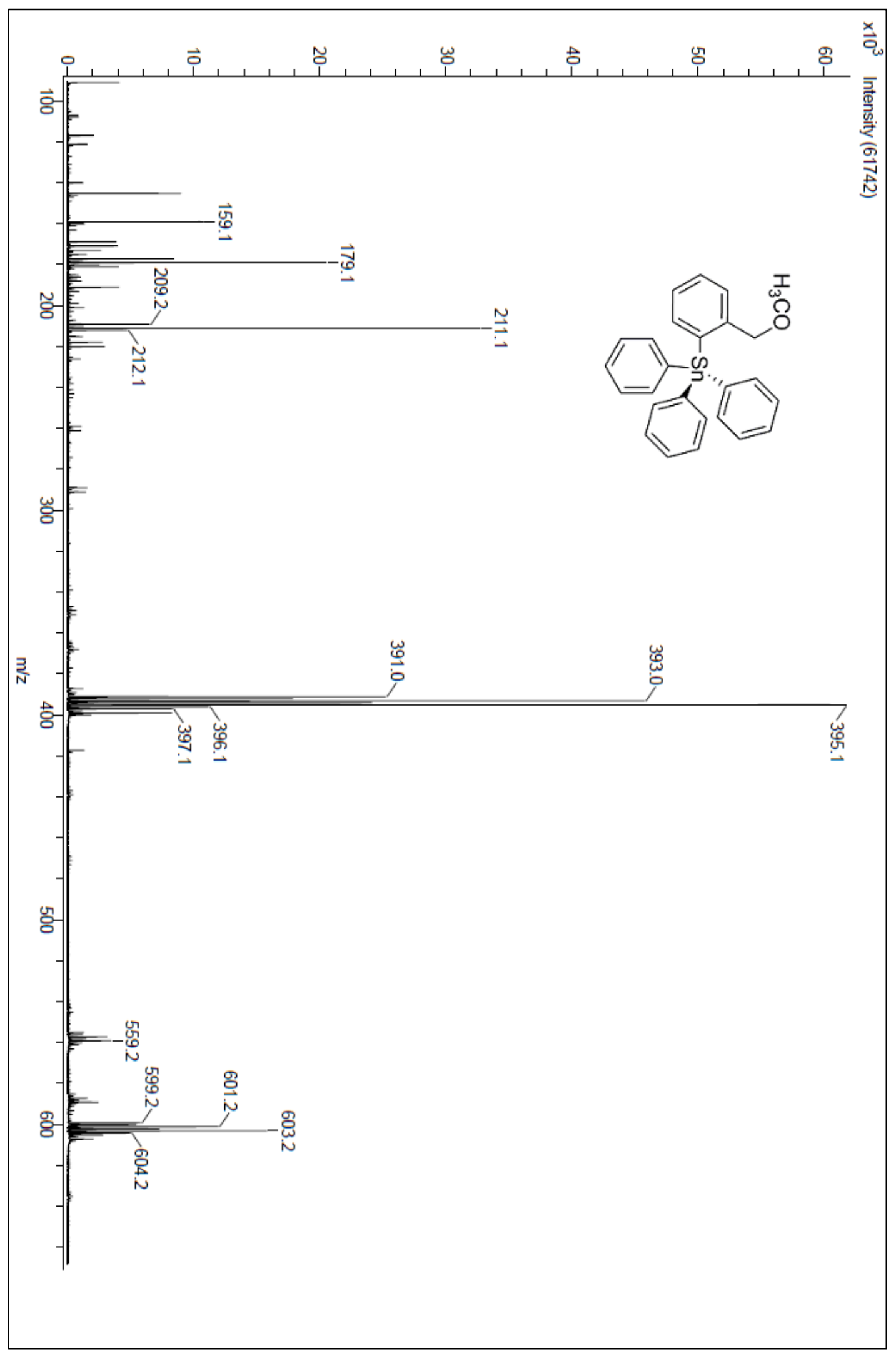

Figure A 133: DART spectrum of compound 112 


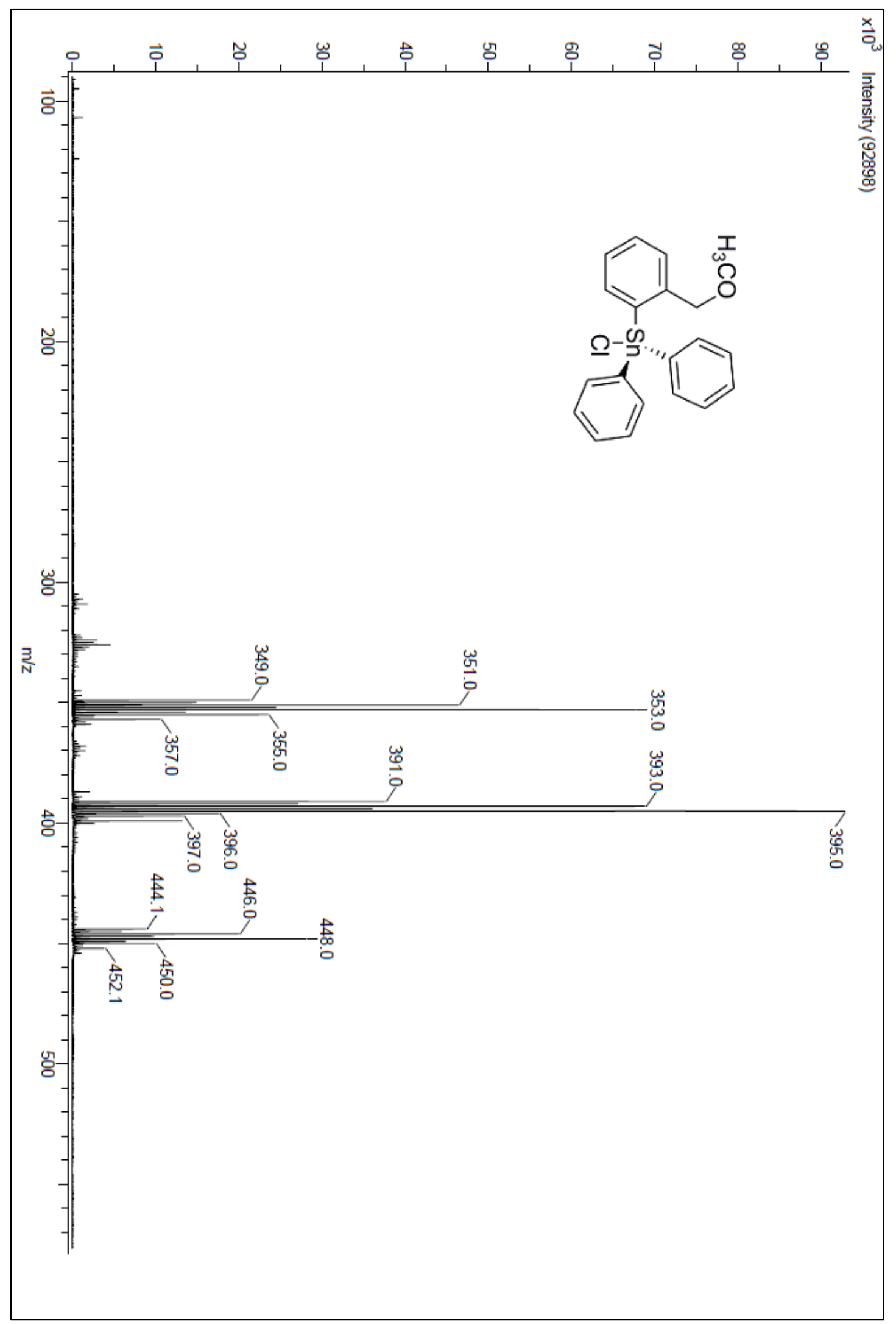

Figure A 134: DART spectrum of compound 112 


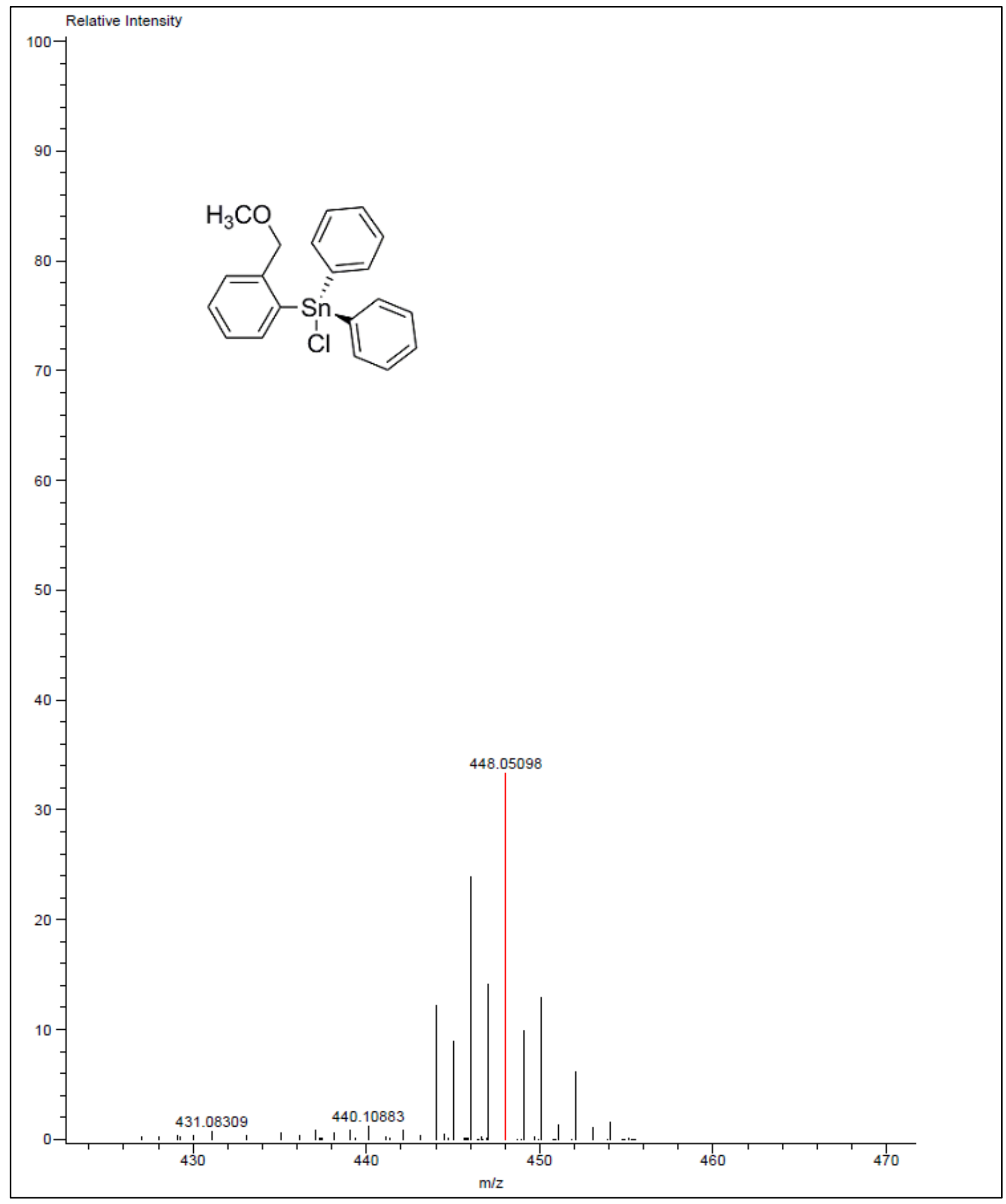

Figure A 135: HRMS-DART spectrum of compound 112 


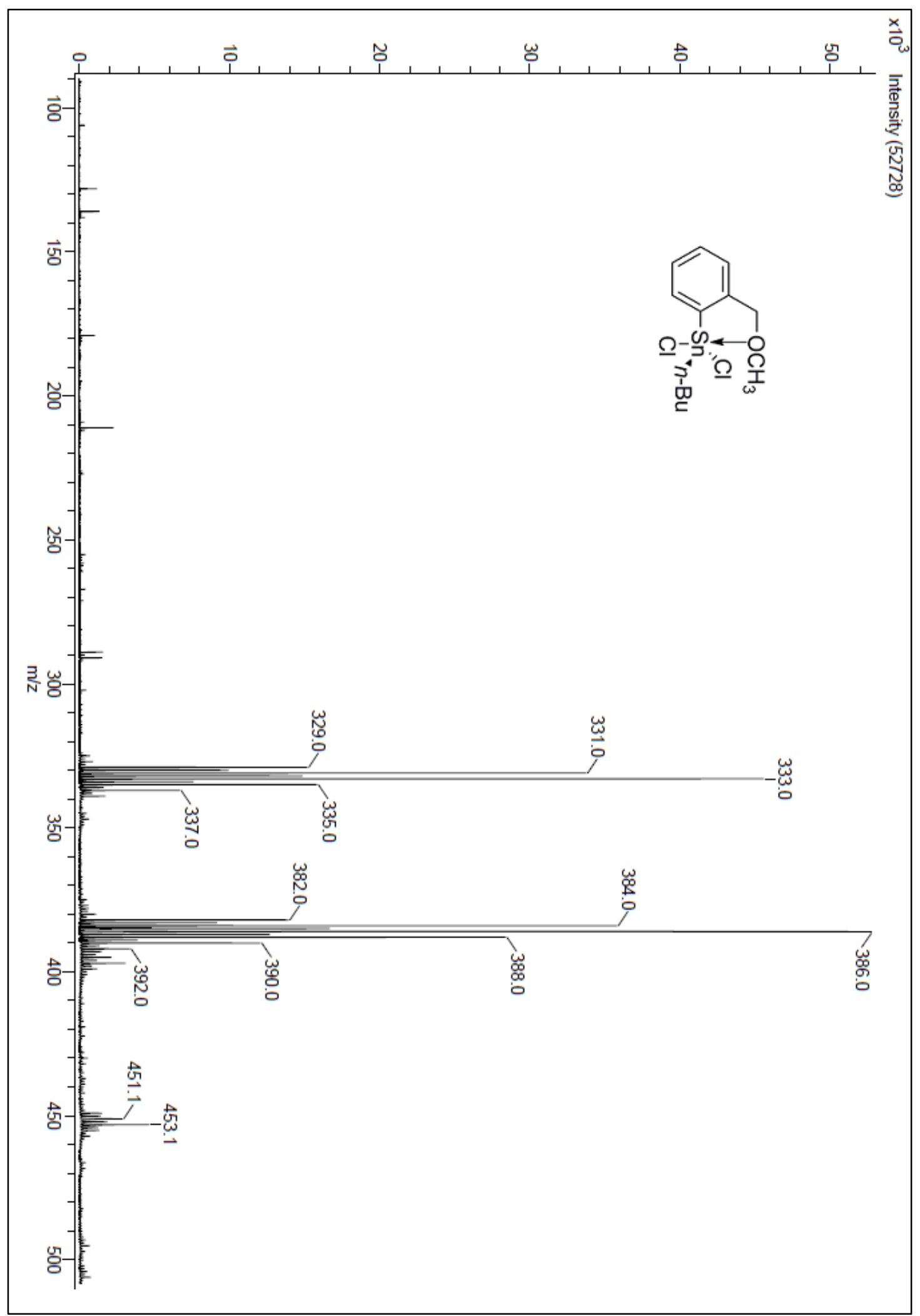

Figure A 136: DART spectrum of compound 219 


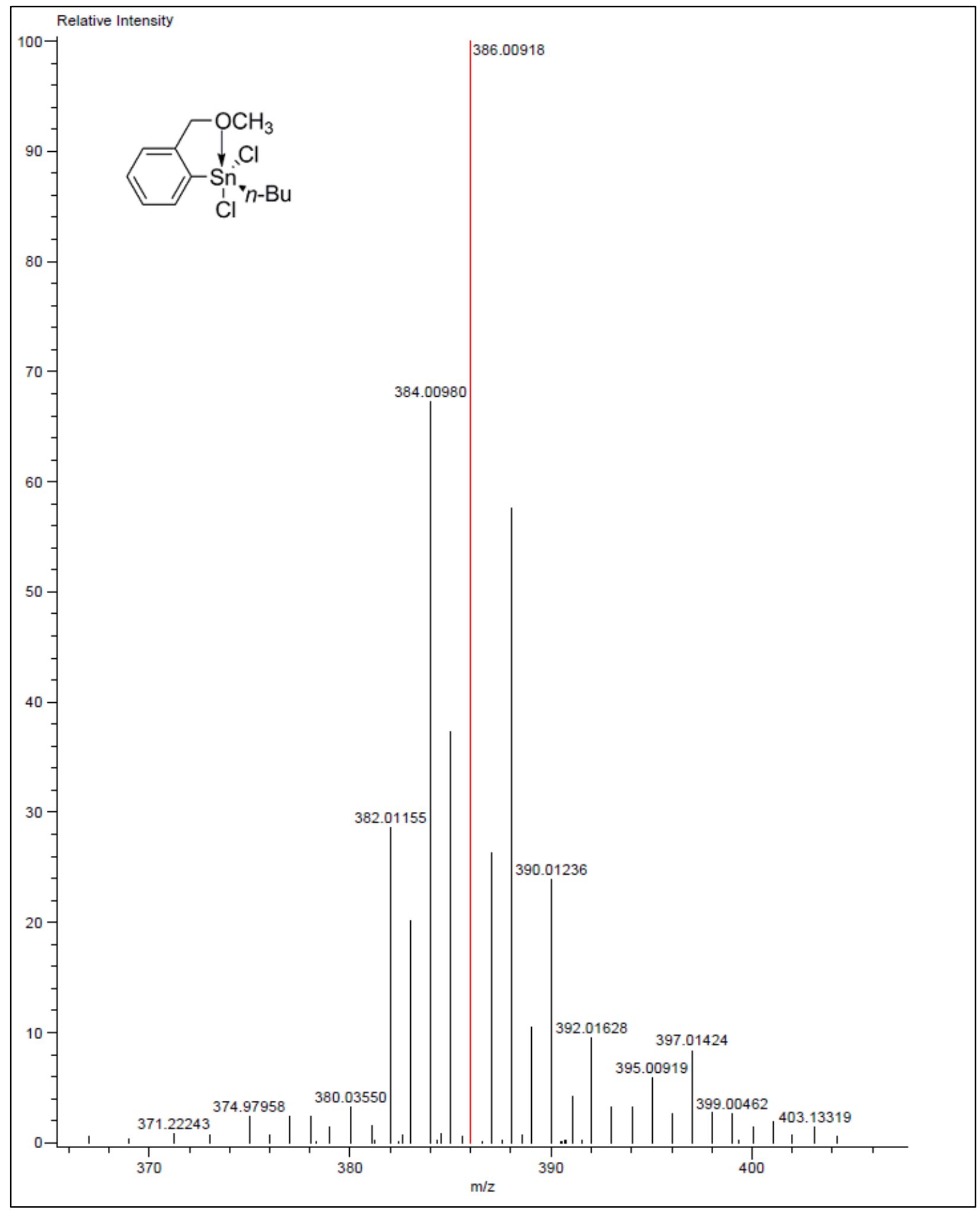

Figure A 137: HRMS-DART spectrum of compound 219 


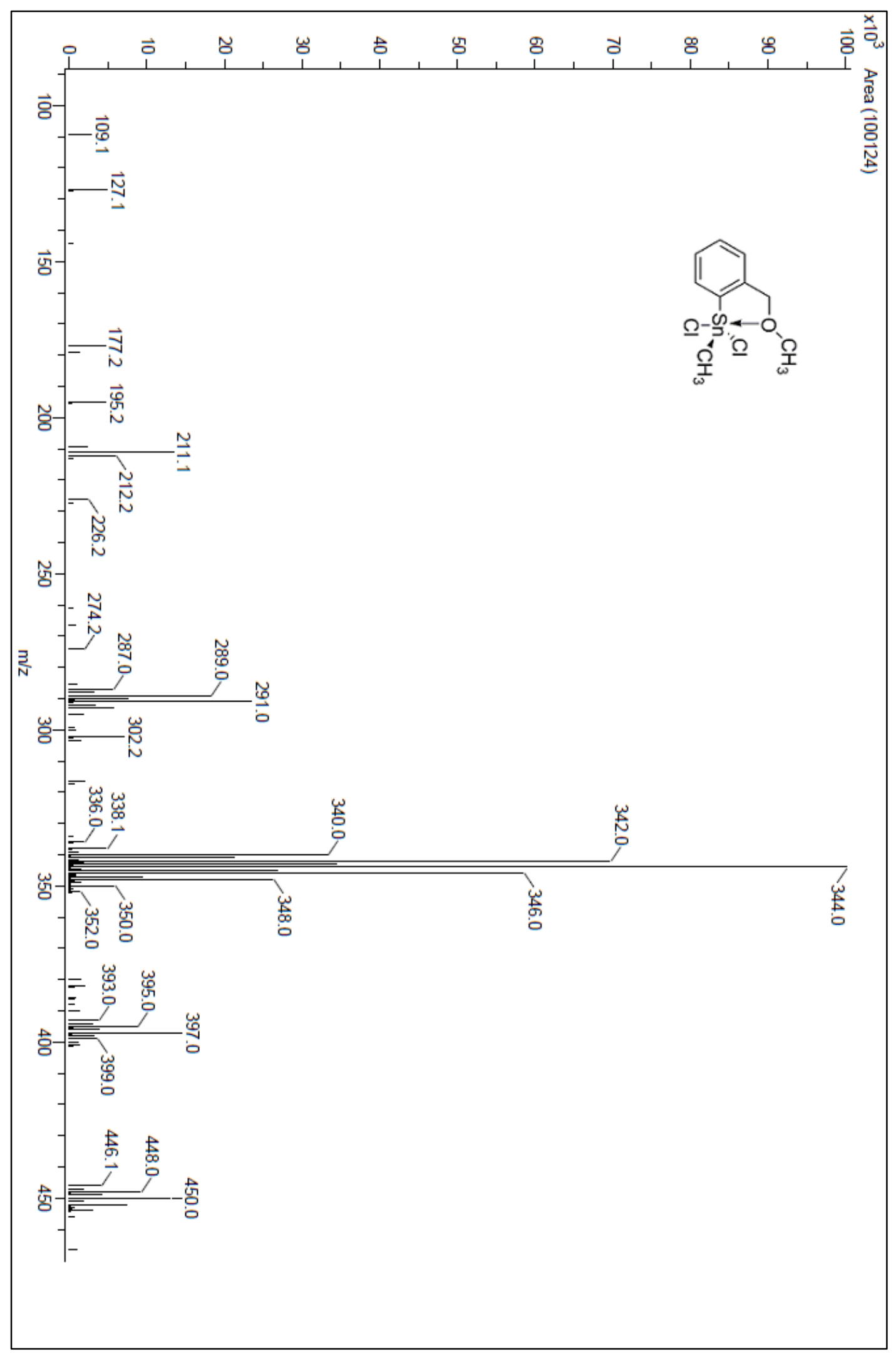

Figure A 138: DART spectrum of compound 218 


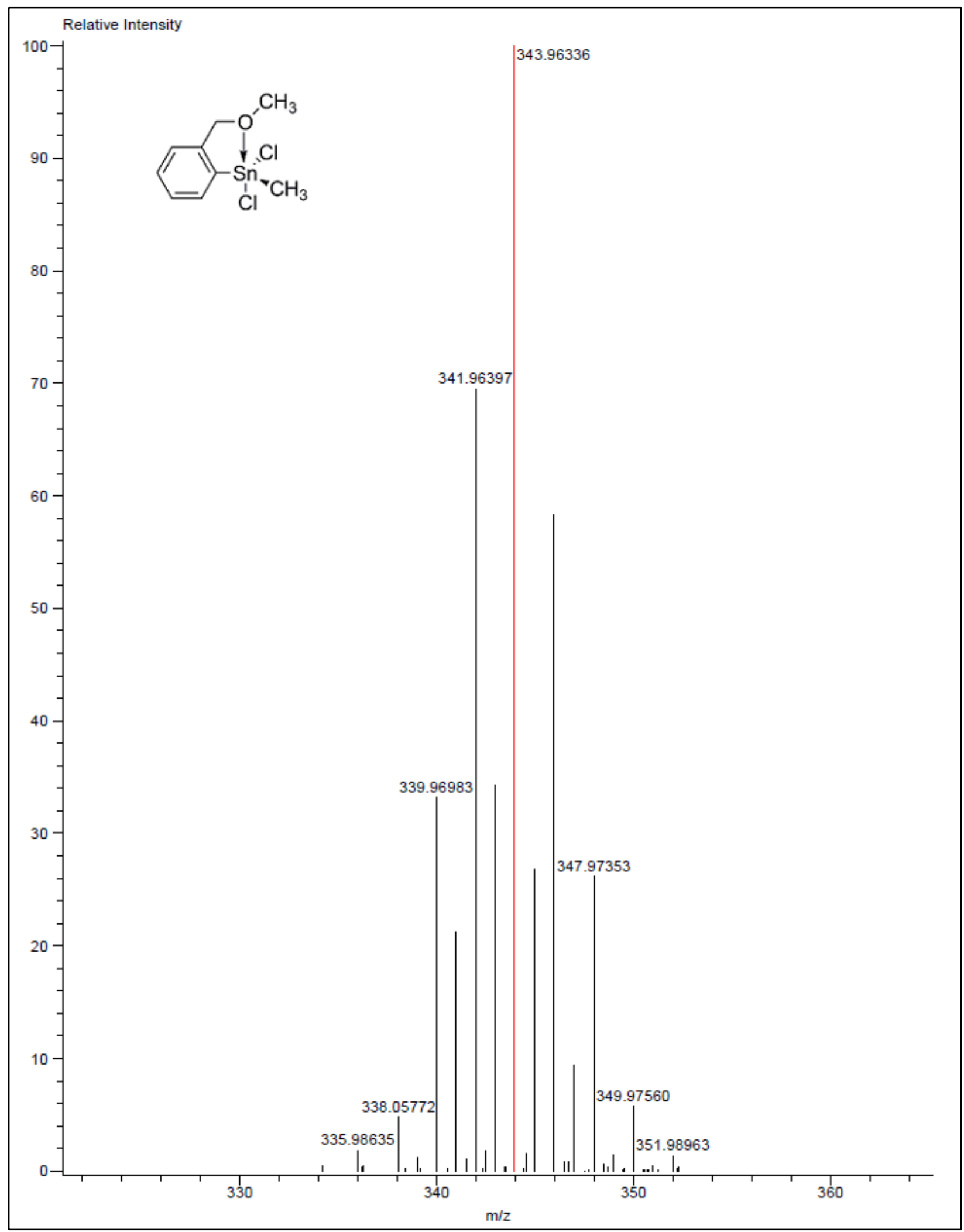

Figure A 139: HRMS-DART spectrum of compound 218 


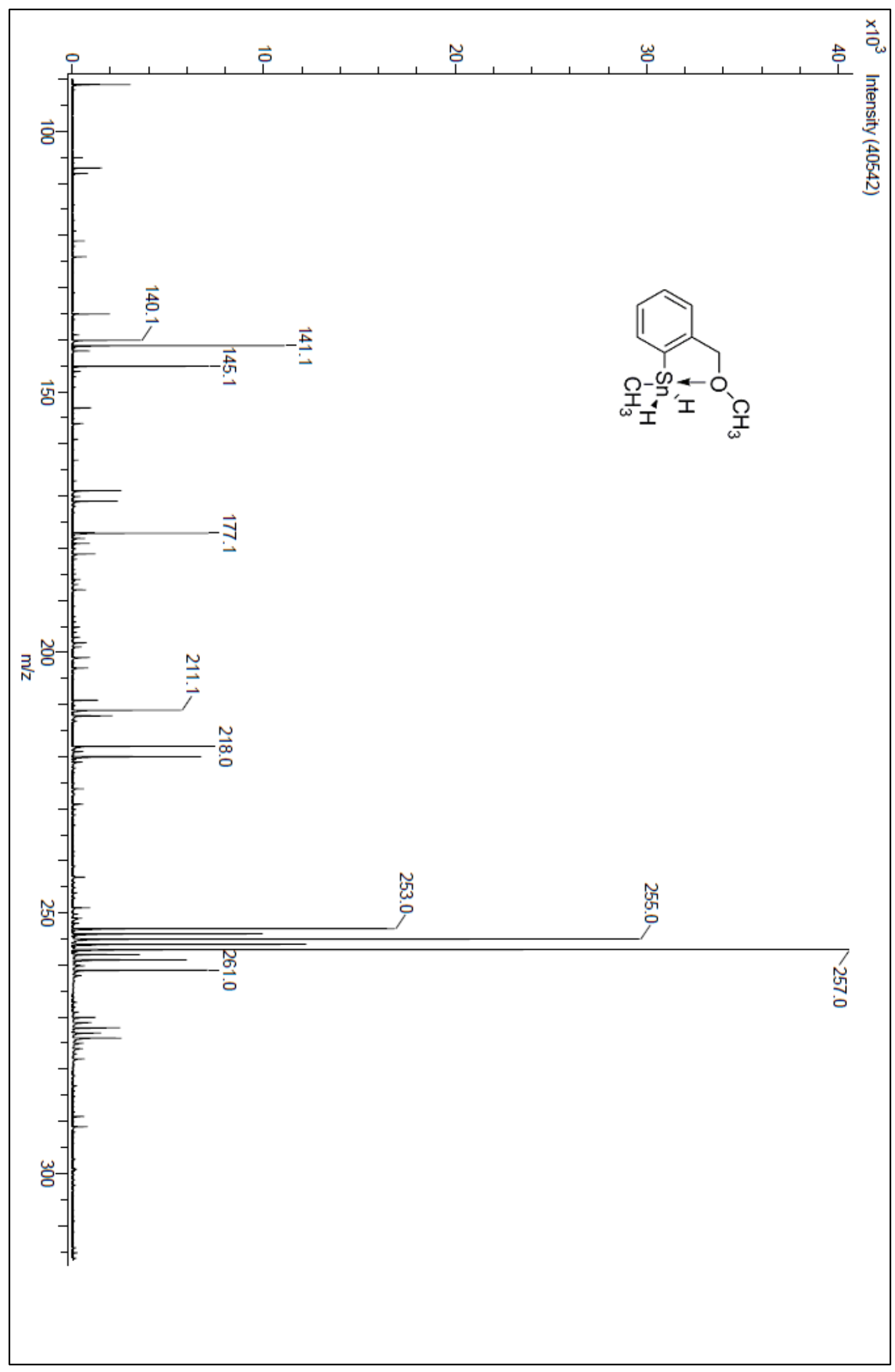

Figure A 140: DART spectrum of compound 226 


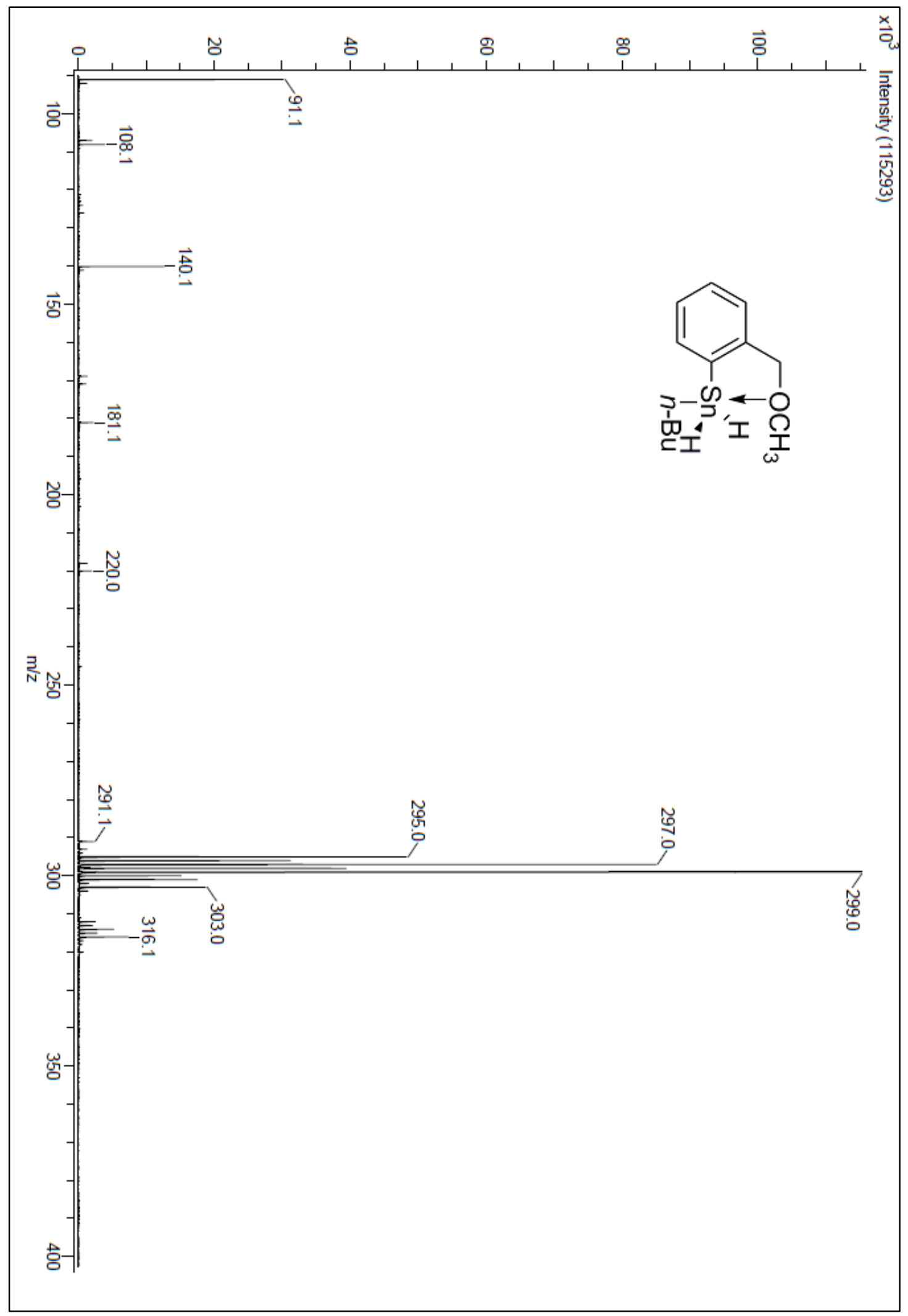

Figure A 141: DART spectrum of compound 227 


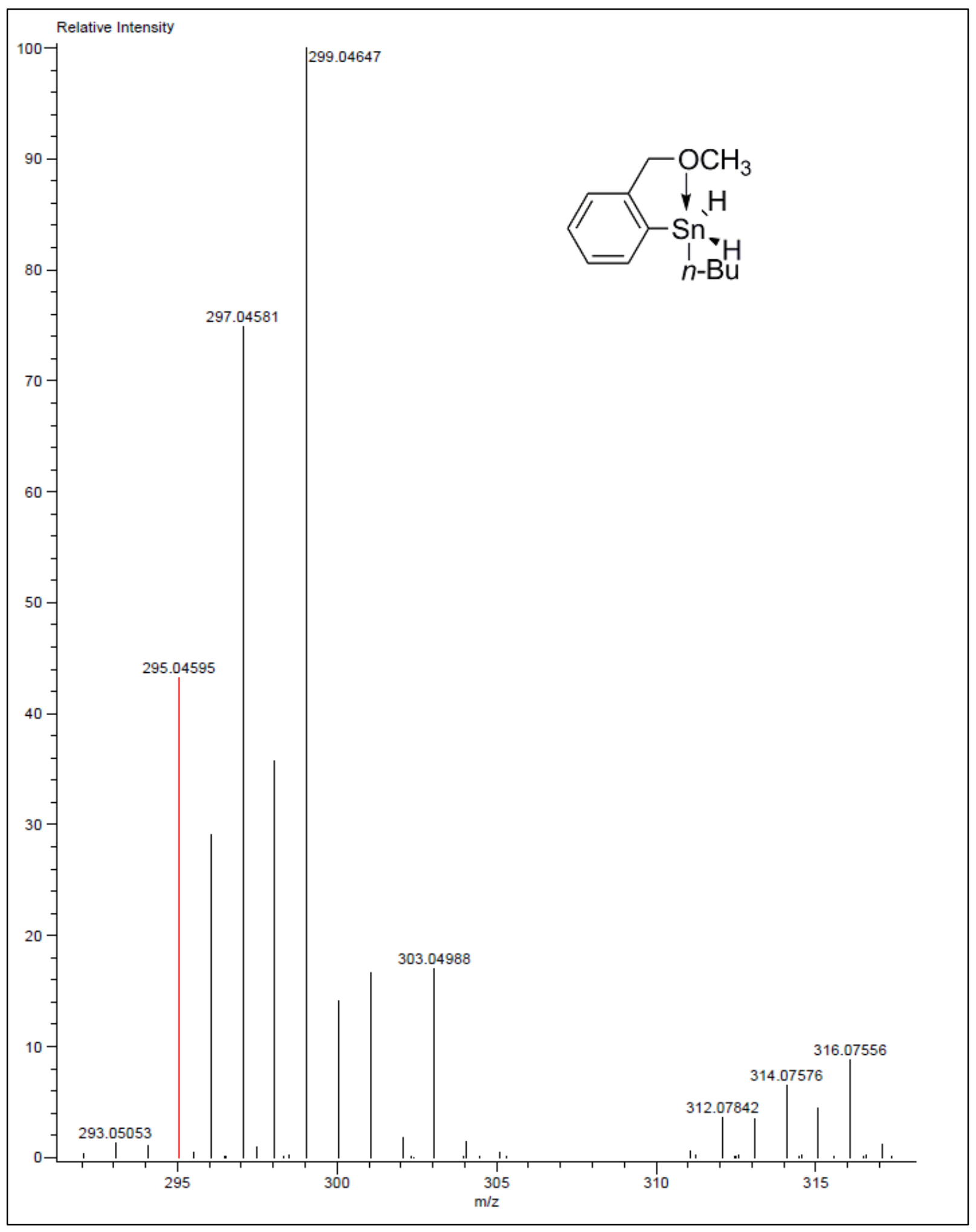

Figure A 142: HRMS-DART spectrum of compound 227 


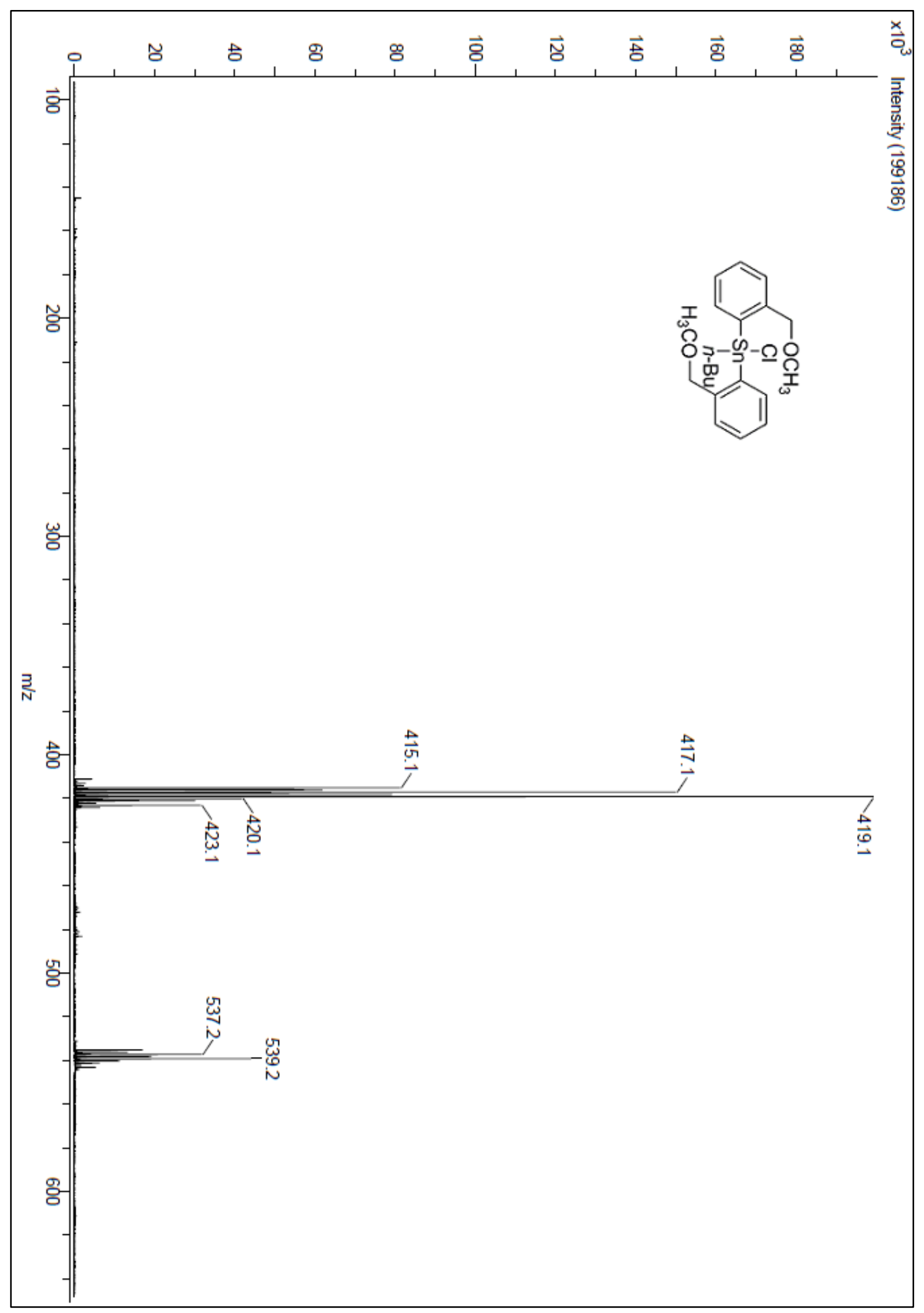

Figure A 143: DART spectrum of compound 224 


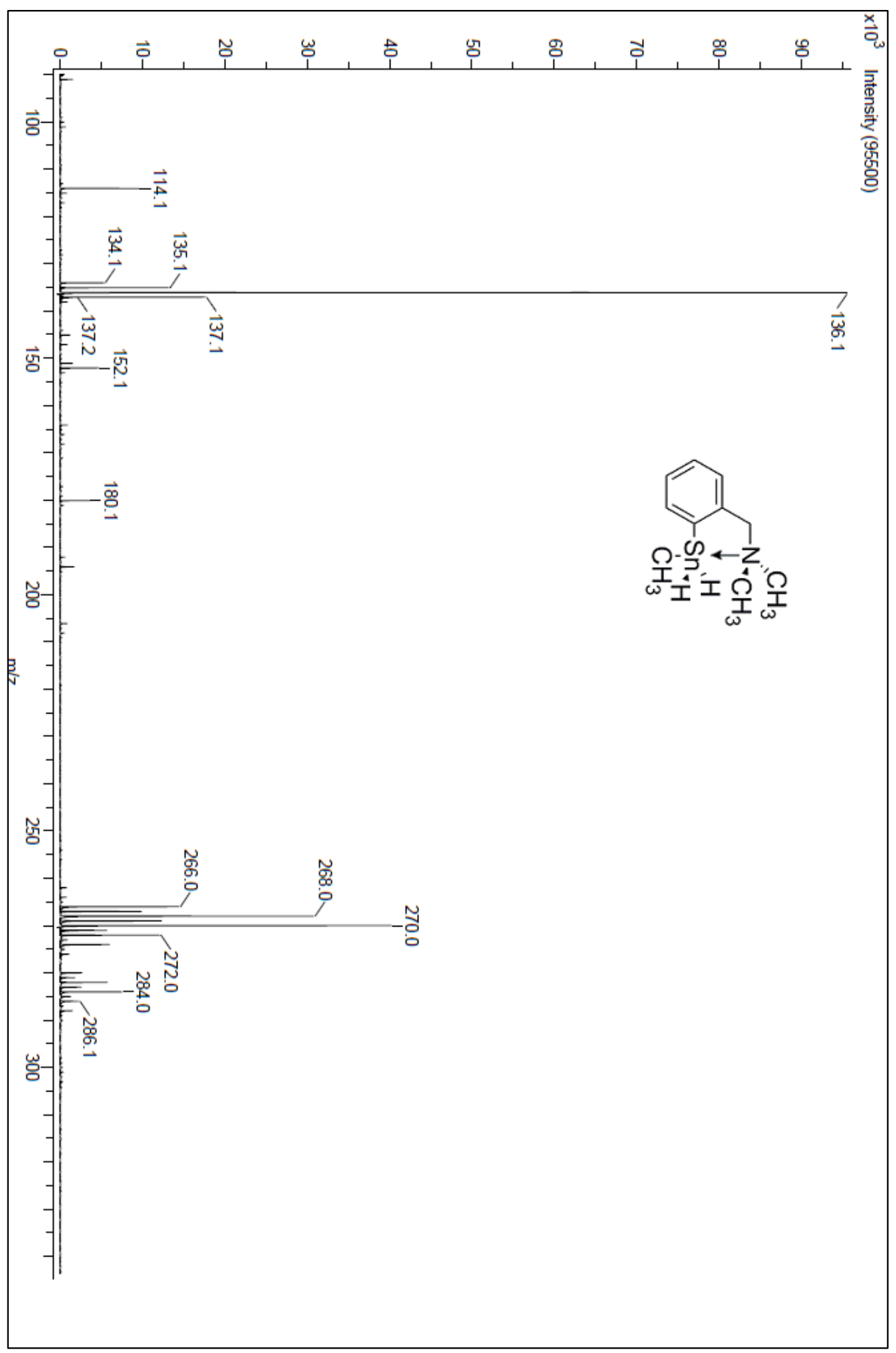

Figure A 144: DART spectrum of compound 230 


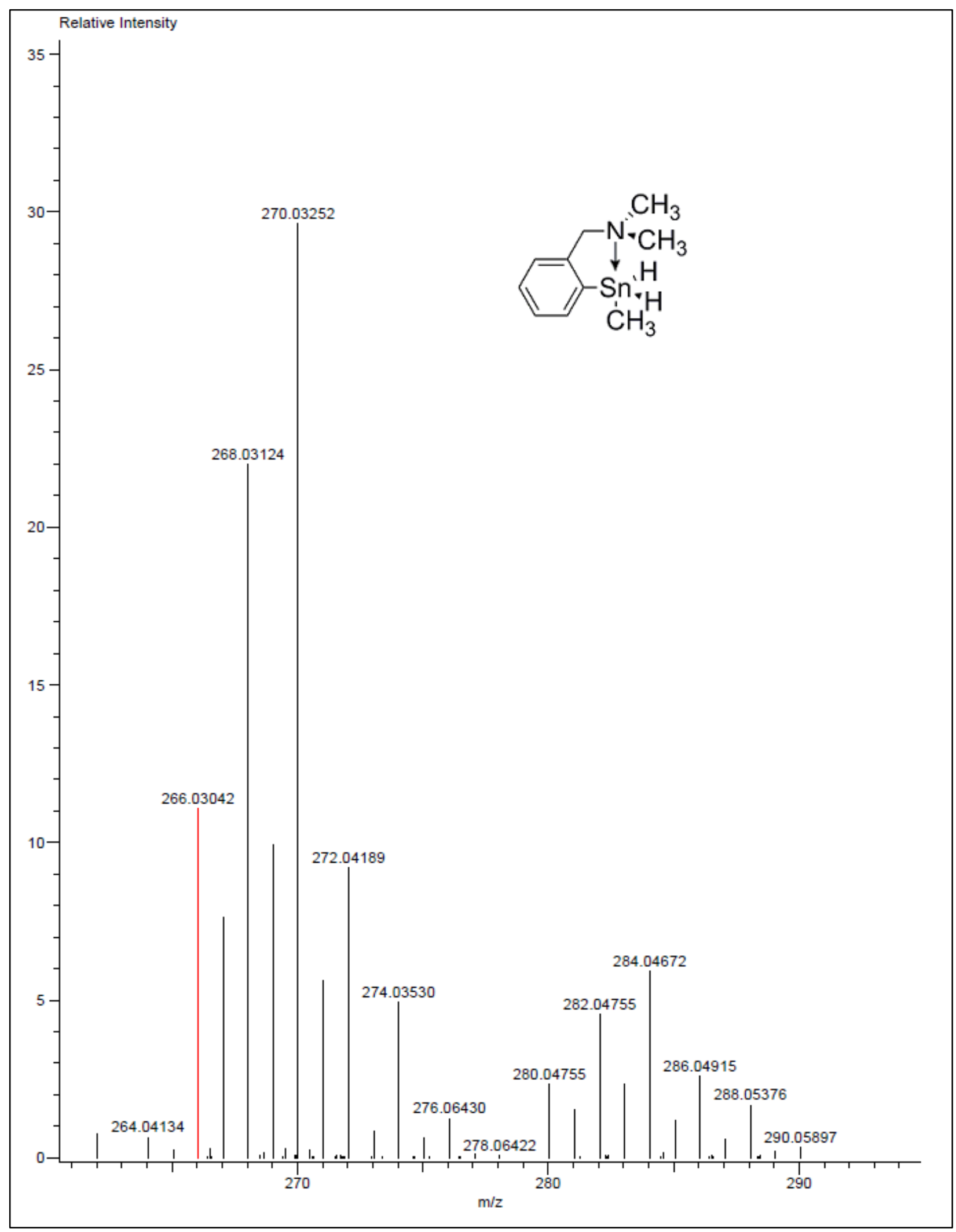

Figure A 145: HRMS-DART spectrum of compound 230 


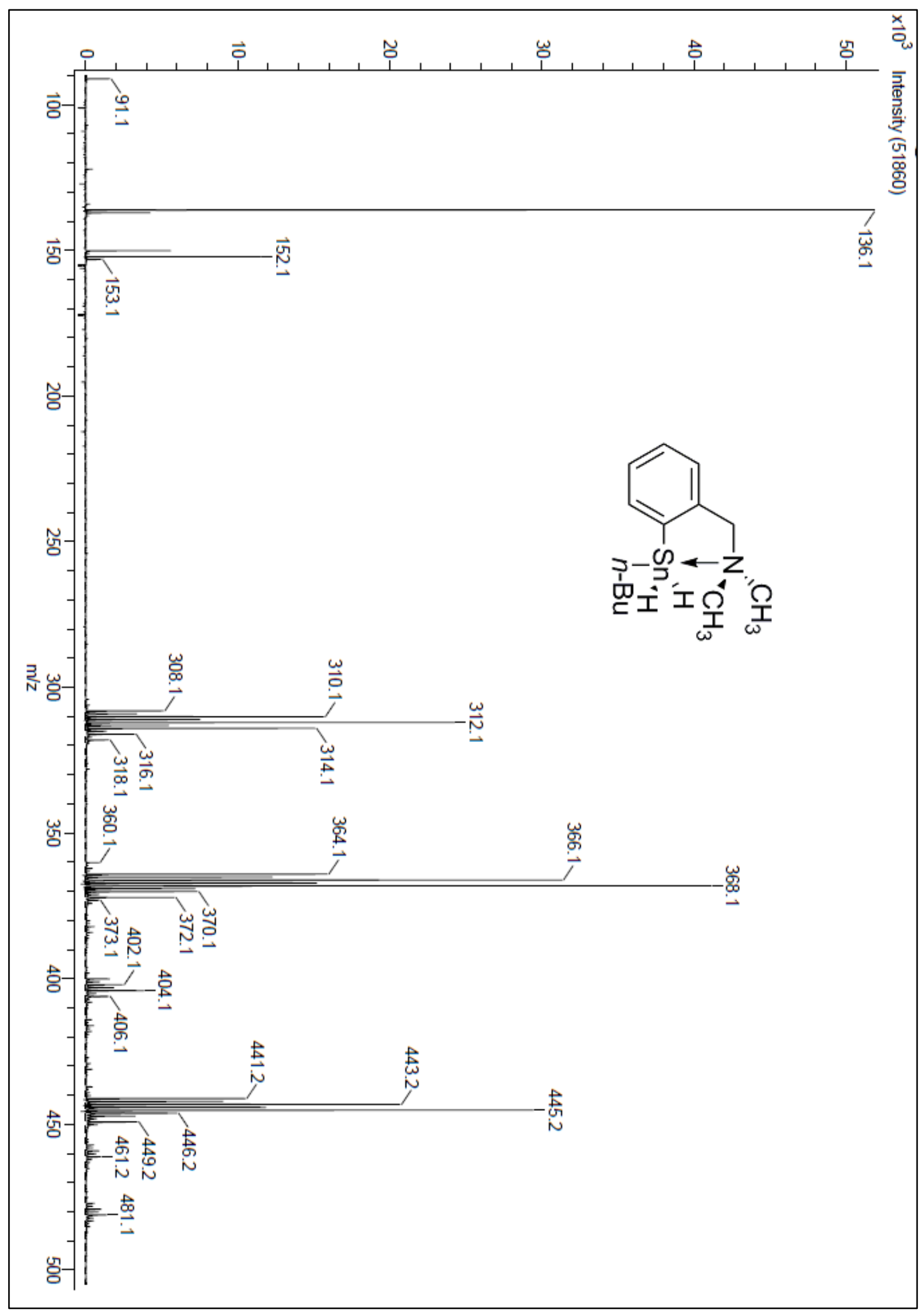

Figure A 146: DART spectrum of compound 231 


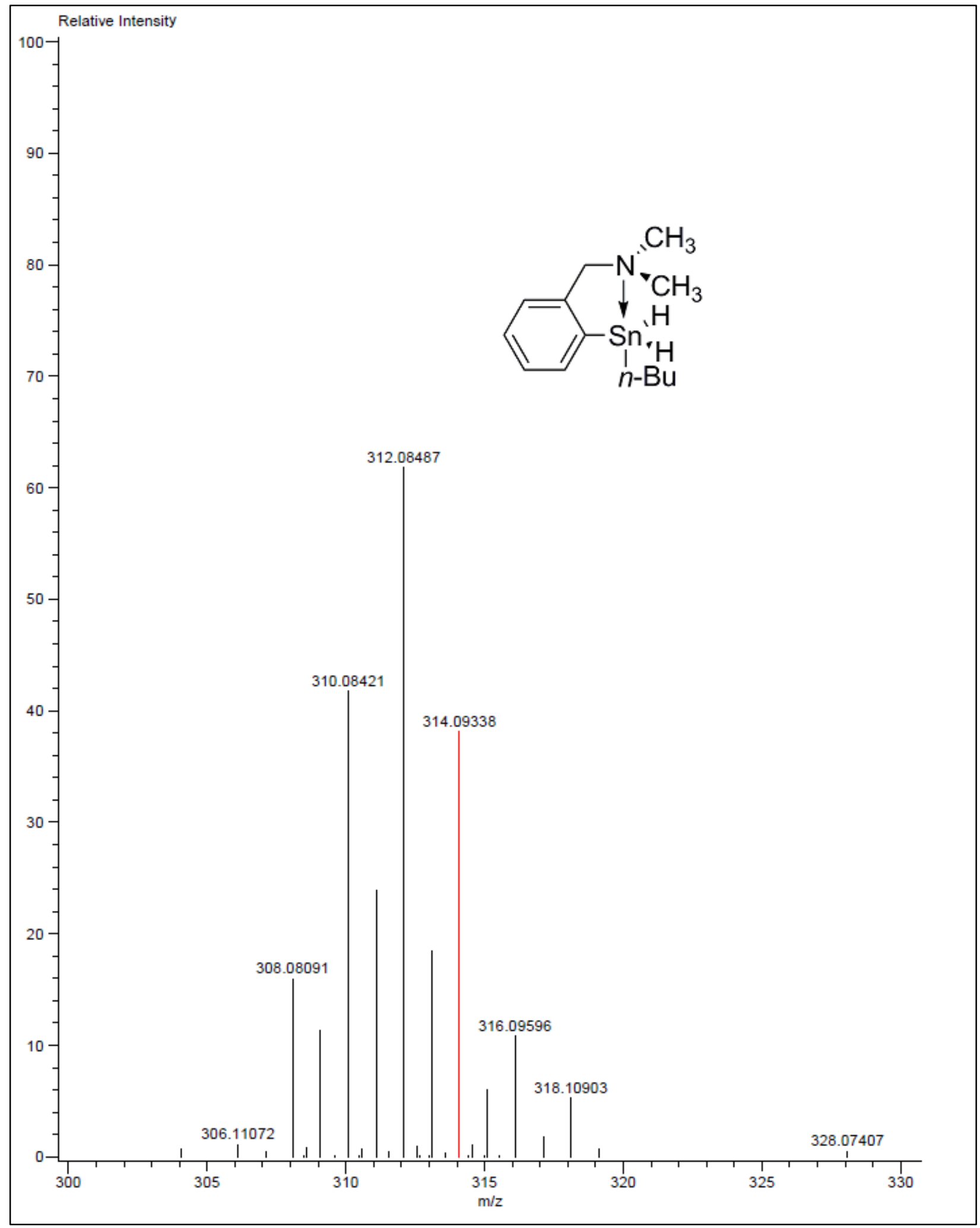

Figure A 147: HRMS-DART spectrum of compound 231 


\subsection{References:}

1. Chaudhary, A.; Singh, R. V. Main Group Met. Chem. 2008, 31, 107.

2. Tin Chemistry: Fundamental, Frontiers, and Applications, Edited by Gielen, M.; Davies, A.; Pannell, K.; Tiekink, E. John Willey \& Sons, Ltd., New York, 2000.

3. Frankland, E. Q. J. Chem. Soc. 1850, 2, 263.

4. Löwig, C. Liebigs, Ann. Chem. 1852, 84, 308.

5. Pope, W. J.; Peachy, S. J. Proc. Chem. Soc. 1903, 19, 290.

6. Kocheshkov, K. A. Ber., 1929, 62, 996.

7. Krause, E.; Von Grosse, A. Die Chemie der Metal-organization verbindungen, Verlag Borntraeger, Berlin, 1937.

8. Akiba, K.-Y. Chemistry of hypervalent compounds, John Willey \& Sons, Ltd. New York, 1999.

9. Tandura, S. N.; Voronkov, M. G.; Alekseev, N. A. Top. Curr. Chem. 1986, 131, 101

10. Chuit, C.; Corriu, R. J. P.; Reye, C.; Young. J. C. Chem. Rev. 1993, 93, 1371.

11. Holmes, R. R. Chem. Rev. 1996, 96, 927.

12. Voronkov, M. G.; Trofimova, O. M.; Bolgova, Y. I.; Chernov, N. F. Russ. Chem. Rev. 2007, 76, 825 .

13. Baukov, Y, I.; Tandura, S. N. The Chemistry of Organic Germanium, Tin and Lead Compounds, Rappoport, Z., Eds.; John Willey \& Sons, Ltd., New York, 2002; Vol. 2.

14. Jastrzebski, J. T. B. H.; van Koten, G. Adv. Organometal. Chem. 1993, 35, 241.

15. Janseen, M. J.; Lujiten, J. G. A.; van der Kerk, G. J. M.; J. Organometal. Chem. 1964, 1, 286.

16. Beattie, I. R.; Gilson, T.; J. Chem. Soc. 1961, 2582. 
17. Musher, J. I. Angew. Chem. Int. Ed. Engl. 1969, 8, 54.

18. Hulme, R. J. Chem. Soc. 1963, 1524.

19. Perkins, C. W.; Martin, J. C.; Arduengo, A. J.; Lau, W.; Alegria, A.; Koci, J. K. J. Am. Chem. Soc. 1985, 107, 6359.

20. (a) Pimentel, G. C. J. Chem. Phys. 1951, 19, 446. (b) Hackand, R. J.; Rundle, R. E. J. Am. Chem. Soc. 1951, 73, 4321.

21. Kutzelning, W. Angew. Chem. Int. Ed. Engl. 1984, 23, 272.

22. Reed, A. E.; Schleyer, P. V. R. J. Am. Chem. Soc. 1990, 112, 134.

23. Janseen, M. J.; Lujiten, J. A.; van der Kerk, G. J. M. J. Organomet. Chem. 1964, 1, 286.

24. Colton, R.; Dakternieks, D. Inorg. Chim. Acta. 1988, 31, 148.

25. Li, Q.; Yang, P.; Hau, E.; Tian, C. J. Coord. Chem. 1996, 40, 227.

26. Jastrzebski, J. T. B. H.; Grove, D. M.; Boersma, J.; van Koten, G. J. Magn. Reson. 1991, 29, S25.

27. Jastrzebski, J. T. B. H.; Knapp, C. T.; van Koten, G. J. Organomet. Chem. 1983, 255, 287.

28. Jastrzebski, J. T. B. H.; Boersma, J.; Esch, P. M.; van Koten, G. Organometallics 1991, $10,930$.

29. a) Novák, P.; Padělková, Z.; Kolářová, L.; Císařová, I; Růžička, A.; Holeček, J. Appl. Organometal. Chem. 2005, 19, 1101. b) Růžička, A.; Pejchal, V.; Holeček, J.; Lyčka, A.; Jacob, K. Collect. Czech. Chem. Commun. 1998, 63, 977.

30. Mitchell, T. N. J. Organomet. Chem. 1973, 59, 189.

31. Gielen, M. Pure Appl. Chem. 1980, 52, 657.

32. Jastrzebski, J. T. B. H.; van Koten, G.; Knaap, C. T.; Schreurs, A. M. M.; Kroon, J.; Spek, A. L. Organometallics 1986, 5, 1551. 
33. van Koten, G.; Jastnebki, J. T. B. H.; Noltes, J. G.; Pontenagel, W. M. G. F.; Kroon, J.; Spek, A. L. J. Am. Chem. Soc. 1978, 100, 5021.

34. Jastrzebski, J. T. B. H. Ph.D. Thesis, University of Utrecht, The Netherland, 1991.

35. Rippstein, R.; Kickelbick, G.; Schubert, U. Inorg. Chim. Acta 1999, 290, 100.

36. Kitching, W.; Drew, D.; Adcock, W.; Abeywickrema, A. N. J. Org. Chem. 1981, 46, 2252.

37. Gielen, M. Bull. Soc. Chim. Belg. 1983, 92, 409.

38. Kroth, H. J.; Schumann, H.; Kuivila, H. G.; Schaeffer, C. D.; Zuckerman, J. J. J. Am. Chem. Soc. 1975, 97,1754.

39. Hunter, B. K.; Reeves, L. W. Can. J. Chem. 1968, 46, 1399.

40. Choffat, F.; Kaser, S.; Wolfer, P.; Schmid, D.; Mezzenga, R.; Smith, P.; Caseri, W. Macromolecules 2007, 40, 7878.

41. Choffat, F.; Buchmuller, Y.; Mensing, C.; Smith, P.; Caseri, W. J. Inorg. Organomet. Polym. 2009, 19, 715.

42. Zeppek, C.; Pichler, J.; Torvisco, A.; Flock, M.; Uhlig, F. J. Organomet. Chem. 2013, 740, 41.

43. Deacon, P. R.; Devylder, N.; Hill, M.; Mahon, M. F.; Molloy, K. C.; Price, G. J. J. Organomet. Chem. 2003, 687, 46.

44. Jastrzebski J. T. B. H, van Koten, G. Adv. Organomet. Chem. 1993, 35, 241.

45. Růžička, A.; Jambor. R.; Brus, J.; Císařová, I.; Holeček, J. Inorg. Chim. Acta 2001, 323, 163.

46. Růžička, A.; Jambor, R.; Císařová, I.; Holeček, J. Chem. Eur. J. 2003, 9, 2411.

47. van Koten, G.; Schaap, C. A.; Noltes, J. G. J. Organomet. Chem. 1975, 99, 157.

48. Rippstein, R.; Kickelbick, G.; Schubert, U. Monatsh. Chem. 1999, 130, 385. 
49. Varga, R. A.; Rotar, A.; Schürmann, M.; Jurkschat, K.; Silvestru, C. Eur. J. Inorg. Chem. 2006, 1475.

50. van Koten, G.; Jastrzebski, J. T. B. H.; Noltes, J. G.; Godefridus J. V.; Anthony L. S.; and Jan K. J.Chem. Soc. Dalton Trans. 1980, 1352.

51. Varga, R. A.; Jurkschat, K.; Silvestru, C. Eur. J. Inorg. Chem. 2008, 708.

52. Novák, P.; Císařová, I; Jambor, R.; Růžička, A.; Holeček, J. Appl. Organomet. Chem. 2004, 18, 241.

53. Oki, M.; Ohira, M. Chem. Lett. 1982, 11, 1267.

54. $\quad$ van Koten, G.; Noltes, J. G.; Spek, A. L.; J. Organomet. Chem. 1976, 118, 183.

55. van Koten, G.; Noltes, J. G. J. Am. Chem. Soc. 1976, 98, 5393.

56. a)Jastrzebski, J. T. B. H.; Boersma, J.; Esch, P. M.; van Koten, G. J. Organomet. Chem. 1991, 413, 43. b) Wrackmeyer, B. Annu. Reports NMR Spectrosc. 1985, 16, 73.

57. van Koten, G.; Noltes, J. G. J. Am. Chem. Soc. 1976, 98, 5393.

58. Švec, P.; Padělková, Z.; Císařová, I; Růžička, A.; Holeček, J. Main group met. Chem. 2008, 31,305 .

59. van Koten, G.; Jastrzebski, J. T. B. H.; Noltes, J. G. J. Organometal. Chem. 1979, 177, 283.

60. Padělková, Z.; Brus, J.; Císařová, I; Růžička, A.; Holeček, J. J. Fluorine Chem. 2005, 126, 1531.

61. Novák, P.; Padělková, Z.; Císařová, I; Kolářová, L.; Růžička, A.; Holeček, J. Appl. Organomet. Chem. 2006, 20, 226.

62. Pejchal, V.; Holeček, J.; Lyčka, A. Sci. Pap. Univ. Pardubice Ser. A 1996, 2, 35. [Chem. Abstr. 1997, 126] 
63. Kraus, C. A.; Greer, N. W. J. Am. Chem. Soc. 1922, 44, 2629.

64. Finholt, A. E.; Bond, A. C.; Wilzbach Jr., K. E.; Schlesinger, H. I. J. Am. Chem. Soc. 1947, $69,2692$.

65. Komsta, Z.; Cmoch, P.; Staliński, K. Polish J. Chem. 2006, 80, 1259.

66. Turek, J.; Padělková, Z.; Černošek, Z.; Milan, E.; Lyčka, A.; Nechaev, M. S.; Císařová, I; Růžička, A.; J. Organomet. Chem. 2009, 694, 3000.

67. Vedejs, E.; Duncan, S. M.; Haight. A. R. J. Org. Chem. 1993, 58, 3046.

68. Dakternieks, D.; Dunn, K.; Carl H. Schiesser, C. H.; Tiekink, E. R. T. J. Organomet. Chem. 2000, 605, 209.

69. Cmoch, P.; Urbańczyk-Lipkowski, Z.; Petrosyan, A.; Stępień, A.; Staliński, K. J. Mol. Struct. 2005, 733, 29.

70. Rupnicki, L.; Urbańczyk-Lipkowski, Z.; Stępień, A.; Cmoch, P.; Pianowski, Z.; Staliński, K. J. Organomet. Chem. 2005, 690, 3690.

71. Staliński, K.; Urbańczyk-Lipkowski, Z.; Cmoch, P.; Rupnicki, L.; Grachev, A. J. Organomet. Chem. 2006, 691, 2394.

72. Matkowska, D.; Gola, M.; Śnieżek, M.; Cmoch, P.; Staliński, K. J. Organomet. Chem. 2007, 692, 2036.

73. Birchall, T.; Pereira, A. R. J. Chem. Soc., Dalton Trans. 1975, 1087.

74. Blunden, S. J.; Cusack, P. A.; Hill, R. The Industrial Uses of Tin Chemicals, Royal Society of Chemistry, London, 1985.

75. Gielen, M.; Willem, R.; Holeček, J.; Lyčka, A. Main Group Met. Chem. 1993, 16, 29.

76. Schumann, H.; Wassermann, B. C.; Ekkehardt H. F. Organometallics 1992, 11, 2803. 
77. a) Gay-Lussac, J. L.; Thenard, L. J. Mémoires de Physique et de Chimie de la Société d' Arceuil, 1809, 2, 317. b) Davy, J. Philos. Trans. 1812, 2, 352; Liebigs Ann. Chem. 1813, $86,178$.

78. Boyer, J.; Breliére, C.; Carré, F.; Corriu, R. J. P.; Kpoton, A.; Poirier, M.; Royo, G.; Young, J. C. J. Chem. Soc. Dalton Trans. 1989, 43.

79. Klebe, G.; Nix, M.; Hensen, K. J. Chem. Soc. Dalton Trans. 1985, 5.

80. Klebe, G. J. Organomet. Chem. 1987, 332, 35.

81. Breliere, C.; Carre, F.; Corriu, R. J. P.; De Saxce, A.; Poirier, M.; Royo, G. J. Organomet. Chem. 1981, 205, C1.

82. Klebe, G.; Nix, M.; Hensen, K. Chem. Ber. 1984, 117, 797.

83. Klanberg, F.; Muetterties, E. L. Inorg. Chem. 1968, 7, 155.

84. De Wit, P. P.; Van Der Kooi, H. O.; Wolters, J. J. Organomet. Chem. 1981, 216, C9.

85. Christea, A.; Silvestru, A.; Silvestru, C. Studia Universitatis Babeş-Bolyai, Chemia, 2009, $L I V, 4$.

86. Krause, E.; Reissaus, G. G. Ber. 1922, 55, 888.

87. GiIman, H.; Summers, L.; Leeper. R. W. J. Org. Chem. 1922, 17, 630.

88. Glockling, F.; Hooton, K.; Kingston, D. J. Chem. Soc. 1961, 4405.

89. Appeerson. L. D. Coll. J. Sci. 1941, 16, 7; Che. Abstr. 1942, 36, 4476.

90. Willemsens, L. C.; van der Kerk. G. J. M. J. Organomet. Chem. 1964, 2, 271.

91. Lenka Kolářová, L.; Holčapek, M.; Jambor, R.; Dostál, L.; Nádvorník, M.; Růžička, A. J. Mass Spectrom. 2004, 39, 621.

92. Jambor, R.; Dostál, L.; Růžička, A.; Císařová, I; Brus, J.; Holčapek, M.; Holeček, J. Organometallics 2002, 21, 3996. 
93. Mehring, M.; Schurmann, M.; Jurkschat, K. Organometallics 1998, 17, 1227.

94. GiIman, H.; Artzen, C. E. J. Am. Chem. Soc. 1950, 3823.

95. Reich, H. J.; Goldenberg, W. S.; Sanders, A. W.; Jantzi, K. L.; Tzschucke, C. C. J. Am. Chem. Soc. 2003, 125, 3509.

96. Munguia, T.; Lopez-Cardoso, M.; Cervantes-Lee, F.; Pannell, K. H. Inorg. Chem. 2007, $46,1305$.

97. Jousseaume, B.; Duboudin, J. G. J. Organomet. Chem. 1982, 238, 171.

98. Dostál, L.; Jambor, R.; Růžička, A.; Císařová, I; Holeček, J.; Biesemans, M.; Willem, R.; De Proft. F.; Geerlings, P. Organometallics 2007, 26, 6312.

99. Kemmer, M.; Biesemans, M.; Gielen, M.; Tiekink, E. R. T.; Willem, R. J. Organomet. Chem. 2001, 634, 55.

100. Susperregui, J.; Bayle, M.; Leger, J. M.; Deleris, G.; Biesemans, M.; Willem, R.; Kemmer, M.; Gielen, M. J. Organomet. Chem. 1997, 545, 559.

101. Jurkschat, K.; Schilling, J.; Mugge, C.; Tzschach, A.; Meunier- Piret, J.; van Meerscsche, M. Gielen, M.; Willem, R. Organometallics 1988, 7, 38.

102. Takeuchi, Y.; Yamamoto, H.; Tanaka, K.; Ogawa, K.; Harada, J.; Iwamoto, T.; Yuge, H. Tetrahedron 1998, 54, 5822.

103. Traynelis, V. J.; Borgnaes. D. M. J. Org. Chem. 1972, 37, 3824.

104. Yan, Y. Y.; Rajan Babu, T. V. J. Org. Chem. 2000, 65, 900.

105. Takeda, N.; Nakamura, T.; Imamura, A.; Unno, M. Heteroatom Chem. 2011, 22, 438.

106. Munguia, T.; Pavel. L. S.; Kapoor, A. N.; Cervantes-Lee, F.; Párkányi, L.; Pannell, K. H. Inorg. Chem. 2003, 81, 1388. 
107. Berlekamp, U. H.; Mix, A.; Neumann, B.; Stammler, H.-G.; Jutzi. P. J. Organomet. Chem. 2003, 667, 167.

108. Williams, E. A. In The Chemistry of Organic Silicon Compounds, Part 1; Patai, S.; Rappoport, Z. (Eds.); 1989, Wiley: Chichester, UK,.

109. Schoeller, W. W.; Rozhenko, A. Eur. J. Inorg. Chem. 2000, 375.

110. Lin, T. -P.; Gualco, P.; Ladeira, S.; Amgoune, A.; Bourissou, D.; Gabbaï, F. P. C. R. Chimie 2010, 13, 1168.

111. Weichmann, H.; Meunier-Piret, J.; Van Meerssche, M. J. Organomet. Chem. 1986, 309, 267.

112. von Abicht, H.-P.; Mügge, C.; Weichmann, H. Z. Anorg. Allg. Chem. 1980, 467, 203.

113. Gualco, P.; Lin, T. -P.; Sircoglou, M.; Mercy. M.; Ladeira, S.; Bouhadir, G.; Perez, L. M.; Amgoune, A. Maron, L.; Gabbaï, F. P.; Bourissou, D. Angew. Chem. Int. Ed. 2009, 48, 9892.

114. Hoppe, S.; Weichmann, H.; Jurkschat, K.; Schneider-Koglin. C.; Dräger, M. J. Organomet. Chem. 1995, 505, 63.

115. Gossage, R. A.; McLennan, G. D.; Stobart, S. R. Inorg. Chem. 1996, 35, 1729.

116. Lu, V.; Tilley, T. D., Marcromolecules 2000, 33, 2403.

117. Adams, S.; Dräger, M. Angew, Chem. Int. Ed. Engl. 1987, 26, 1255.

118. Imori, T.; Lu, V.; Cai, H.; Tilley, T. D. J. Am. Chem. Soc. 1995, 117, 9931.

119. Choffat, F.; Smith, P.; Caseri, W. J. Mater. Chem. 2005, 15, 1789.

120. Choffat, F.; Käser, S.; Wolfer, P.; Schmid, D.; Mezzenga, R.; Smith, P.; Caseri, W. Macromolecules 2007, 40, 7878. 
121. Babcock, J. R.; Sita, L. R. J. Am. Chem. Soc. 1996, 118, 12481.

122. Choffat, F.; Wolfer, P.; Smith, P.; Caseri, W. Macromol. Mater. Eng. 2010, 295, 210.

123. Pfeiffer, P.; Prade, R.; Rekate, H. Chem. Ber. 1911, 44, 1269.

124. Trummer, M.; Nauser, T.; Lechner, M-L.; Uhlig, F.; Caseri, W. Polym. Degrad. Stab. 2011, $96,1841$.

125. Kipping, F. S. J. Chem. Soc. 1924, 125, 2291.

126. Zou, W. K.; Yang, N.-L. Polym. Prepr. (Am. Chem. Soc. Div. Polym.Chem.) 1992, 33, 188.

127. Devylder, N.; Hill, M.; Molloy, K. C.; Price, G. J. Chem. Commun. 1996, 711.

128. Miles, D., Burrow. T.; Lough, A.; Foucher, D. J. Inorg. Organomet. Polym. 2010, $20,544$.

129. Trummer, M.; Zemp, J.; Sax, C.; Smith, P.; Caseri, W. J. Organomet. Chem. 2011, 696, 3041.

130. Lechner, M-L.; Trummer, M.; Bräunlich, I.; Smith, P.; Caseri, W. J. Appl. Organometal. Chem. 2011, 25, 769.

131. (a) Trefonas, P., III; Damewood, J. R., Jr; West, R.; Miller, R. D.; Organometallics 1985, 4, 1318. (b) Harrah, L. A.; Zeigler, J. M.; Macromolecules 1987, 20, 601. (c) Fujino, M.; Hishaki, T.; Fujiki, M.; Matsumoto, N. Macromolecules 1992, 25, 1079.

132. Okano, M.; Matsumoto, N.; Arakawa, M.; Tsuruta, T.; Hamano, H. Chem. Commun. 1998, 1799.

133. Elangovan, M.; Muthukumaran, A.; Kulandainathan, M. A. Mat. Lett. 2006, 60, 1099.

134. Okano, M.; Watanabe, K.; Totsuka, S. Chem. Commun. 2003, 4, 257.

135. Harrod, J. F.; MU, Y. Polyhedron 1991, 10, 123.

136. Imori, T.; Tilley, T. D. J. Chem. Soc., Chem. Commun. 1993, 1607. 
137. Woo, H-G.; Park, J-M.; Song, S-J.; Yang, S-Y.; Kim, I-S, Kim, W-G. Bull. Korean Chem. Soc. 1997, 18, 1291.

138. Neale, N. R.; Tilley, T. D. Tetrahedron, 2004, 60, 7247.

139. Thompson, S. M.; Schubert, U. Inorg. chim. Acta 2004, 357, 1959.

140. Choffat, F.; Buchmuller, Y.; Mensing, C.; Smith, P.; Caseri, W. J. Inorg. Organomet. Poylm. 2009, 19, 715 .

141. Choffat, F.; Smith, P.; Caseri, W. Adv. Mater. 2008, 20, 2225.

142. Beckmann, J.; Duthie, A.; Grassmann, M.; Semisch, A. Organometallics 2008, 27, 1495.

143. Mochida, K.; Azemi, T.; Hayakawa, M.; Yokoyama, Y. J. Chem. Soc., Chem. Commun. $1995,2275$.

144. Hayashi, H.; Wasaka, M.; Yokoyama, Y.; Tsuchikawa, T.; Hayakawa, M.; Mochida, K. Chem. Lett. 1998, 91.

145. Drenth, W.; Noltes, J. G.; Bulten, E. J.; Creemers, H. M. J. C. J. Organomet. Chem. 1969, $17,173$.

146. Sita, L.R. Organometallics 1992, 11, 1442.

147. Brunclich, I.; Trummer, M.; Lechner, M-L.; Smith, P.; Caseri, W.; Uhlig, F. Appl. Organomet. Chem. 2011, 25, 769.

148. Sanford, E. M.; Lis, C. C.; McPherson, N. R. J. Chem. Edu. 2009, 86 (12), 1422.

149. Brimble, M. A.; Flowers, C. L.; Trzoss, M.; Tsang, K. Y. Tetrahedron 2006, 62 (25), 5883.

150. Porosa, L. Detection and antimirobial avtivity of immobilized quaternary ammonium anitmicrobial monolayers on porous and non-porous surfaces. Ph.D. thesis, Ryerson University, 2014.

151. Mitchell, T. N.; Walter, G. J. Chem. Soc., Perkin Trans. 2, 1977, 1842. 
152. a) Tzeng, D.; Weber, W. P. J. Org. Chem. 1981, 46, 265. b) Tsai, C.-Y.; Robert Sung, R.; Zhuang, B.-R.; Sung, K. Tetrahedron 2010, 66, 6869.

153. Dutta, D. K.; Deb, B.; Hua, G.; Woollins, J. D. J. Mol. Catal. A 2012, 7, 353.

154. Lloyd, N. C.; Brian K. Nicholson, B. K.; Wilkins, A. L. J. Organomet. Chem. 2006, 691, 2757.

155. Wrackmeyer, B. Annu. Reports NMR Spectrosc. 1999, 38, 203.

156. Wrackmeyer, B.; Vosteen, M.: Storch, W. J. Mol. Struct. 2002, 177, 602.

157. Sonika.; Narula, A. K. Int. J. Chem. Sci. 2003, 1, 141.

158. Mahmud, T.; Iqbal, J.; Imran, M.; Mckee, V. J. Appl. Sci. 2007, 7, 1347.

159. Šindelář, K.; Holubek, J.; Svátek, E.; Matoušová, O.; Metyšová, J.; Protiva, M. J. Heterocyclic Chem. 1989, 26, 1325.

160. Biesemans, M.; Martin, J. C.; Willem, R.; Lyčka, A.; Růžička, A.; Holeček, J. Magn. Reson. 2002, 40, 65.

161. Zhou, D.; Reiche, C.; Nag, M.; Soderquist, J. A.; Gaspar, P. P. Organometallics, 2009, $28,2595$.

162. Müller, G.; Waldkirch, M.; Winkler, M. Z. Naturforsch. Teil. 1994, B 49, 106.

163. Morales-Morales, D.; Redón, R.; Zheng, Y.; Dilworth, J. R. Inorg. Chim. Acta 2002, 328, 39.

164. Tolleson, G. S.; Puckette, T. A. U.S. Patent 20090299099, 03 Dec 2009.

165. O'Keefe, M.; Brese, N. E. J. Am. Chem. Soc. 1991, 113, 3226.

166. (a) Dräger, M.; Guttmann, H. J. J. Organomet. Chem. 1981, 212, 171. (b) Kolb, U.; Beuter, M.; Dräger, M. Organometallics 1994, 13, 4413. 
167. Kolb, U.; Beuter, M.; Drager, M. Inorg. Chem. 1994, 33, 4522.

168. Kolb, U.; Dräger, M.; Jousseaume, B. Organometallics 1991, 10, 2737.

169. Cox, P. J.; Doidge-Harrison, S. M. S. V.; Nowell, I. W.; Howie, R. A.; Wardell, J. L.; Wigzell, J. M. Acta Crystallogr. 1990, C46, 1015.

170. Dräger, M. Z. Anorg. Allg. Chem. 1976, 423, 53.

171. Turek, J.; Padělková, Z.; Nechaev, M. S.; Růžička, A.; J. Organometal. Chem. 2010, 695, 1843.

172. (a) Farrar, W. V.; Skinner, H. A. J. Organomet. Chem. 1964, 1, 434. (b) Neumann, W. P.; Pedain, J.; Sommer, R. Ann. Chem. 1966, 694, 9.

173. Davies, A. G.; Osei-Kissi, D. K. J. Organomet. Chem. 1994, 474, C8.

174. Woo, B. Y. PCT Int. Appl., 2010002209, 07 Jan 2010.

175. El-Qisairi, A. K.; Qaseer, H. A.; Henry, P. M. J Organomet. Chem. 2002, 656, 168.

176. Manzer, L. E. J. Am. Chem. Soc. 1978, 100, 8068. 